Silvia Danne

\title{
Messebeteiligungen von Hochschulen
}




\section{Silvia Danne}

\section{Messebeteiligungen von Hochschulen}

Angesichts veränderter Rahmenbedingungen sehen sich Hochschulen vielfältigen Herausforderungen gegenübergestellt. In diesem Zusammenhang gewinnen Messebeteiligungen zunehmend an Bedeutung, da sie als multifunktionales Marketing-Instrument nicht nur die Marktorientierung von Hochschulen unterstützen, sondern darüber hinaus auch die Drittmitteleinwerbung über den Austausch von Transferleistungen forcieren. Aufgrund des expandierenden Messeangebots bei gleichzeitig hohen Messebeteiligungskosten und knappen Hochschulbudgets nimmt insbesondere die Kontrolle des Messebeteiligungserfolgs einen zunehmenden Stellenwert ein. Vor diesem Hintergrund verfolgt die Arbeit das Ziel, den Erfolg von Hochschul-Messebeteiligungen zu überprüfen. Dabei bildet die Analyse hochschulspezifischer Messezielsetzungen und -zielgruppen den Ausgangspunkt der Erfolgskontrolle. Auf Basis der theoretisch und empirisch gewonnenen Erkenntnisse werden abschließend Implikationen sowohl für Hochschul-Exponenten als auch für Finanzmittelgeber von HochschulMessebeteiligungen abgeleitet.

Silvia Danne wurde 1970 in Arnsberg geboren. Von 1991 bis 1996 studierte sie Betriebswirtschaftslehre an der Universität Münster mit den Schwerpunkten Marketing und Internationales Management. Nach ihrem Diplom arbeitete sie bis zum Mai 2000 am Institut für Marketing bei Prof. Dr. Dr. h.c. mult. Heribert Meffert als Wissenschaftliche Mitarbeiterin. 
Messebeteiligungen

von Hochschulen 


\section{SCHRIFTEN ZU \\ MARKETING UND MANAGEMENT}

Herausgegeben von Prof. Dr. Dr. h.c. mult. Heribert Meffert

Band 38

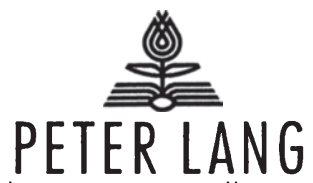

Frankfurt am Main - Berlin - Bern - Bruxelles - New York - Oxford - Wien 


\section{Silvia Danne}

\section{Messebeteiligungen von Hochschulen}

Ziele und Erfolgskontrolle

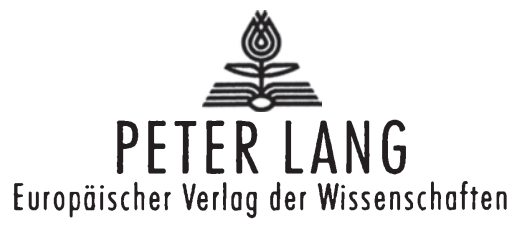


Die Deutsche Bibliothek - CIP-Einheitsaufnahme

Spermann, Alexander:

Kommunales Krisenmanagement : Reaktionen badenwürttembergischer Stadtkreise auf steigende Sozialhilfekosten und Einnahmenausfälle (1980 - 1992) / Alexander Spermann. Frankfurt am Main ; Berlin ; Bern ; New York ; Paris ; Wien : Lang, 1993

(Finanzwissenschaftliche Schriften ; Bd. 57)

Zugl.: Freiburg (Breisgau), Univ., Diss., 1992

ISBN 3-631-46170-4

NE: GT

Open Access: The online version of this publication is published on www.peterlang.com and www.econstor.eu under the international Creative Commons License CC-BY 4.0. Learn more on how you can use and share this work: http://creativecommons. org/licenses/by/4.0.

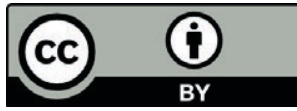

This book is available Open Access thanks to the kind support of ZBW - Leibniz-Informationszentrum Wirtschaft.

\author{
D 25 \\ ISSN $0170-8252$ \\ ISBN 3-631-46170-4 \\ ISBN 978-3-631-75076-6 (eBook) \\ (C) Peter Lang GmbH \\ Europäischer Verlag der Wissenschaften \\ Frankfurt am Main 1993 \\ Alle Rechte vorbehalten.
}

Das Werk einschließlich aller seiner Teile ist urheberrechtlich geschützt. Jede Verwertung außerhalb der engen Grenzen des Urheberrechtsgesetzes ist ohne Zustimmung des Verlages unzulässig und strafbar. Das gilt insbesondere für Vervielfältigungen, Übersetzungen, Mikroverfilmungen und die Einspeicherung und Verarbeitung in elektronischen Systemen. 
Meinen Eltern 



\section{Vorwort des Herausgebers}

Hochschulen sehen sich aufgrund dynamischer Umweltveränderungen einer Vielzahl von Herausforderungen gegenübergestellt, die insbesondere durch steigende Studierendenzahlen sowie erhöhte Anforderungen an Forschung und Lehre bei gleichzeitiger Verknappung öffentlicher Finanzmittel hervorgerufen werden. Angesichts dieser Entwicklungen steigt die Notwendigkeit einer Marktorientierung des Hochschulmanagement und der Erschließung zusätzlicher Finanzierungsquellen. In diesem Zusammenhang gewinnen Messebeteiligungen von Hochschulen einen zunehmenden Stellenwert. Messebeteiligungen unterstützen als multifunktionales Marketing-Instrument nicht nur die Marktorientierung von Hochschulen, sondern fördern darüber hinaus auch die Drittmitteleinwerbung über den Austausch von Transferleistungen zwischen Hochschulen und an Forschungsleistungen interessierten Anspruchsgruppen. Angesichts des expandierenden Messeangebots bei gleichzeitig hohen Messebeteiligungskosten und knappen Hochschulbudgets wird bei Hochschul-Messebeteiligungen neben einer verstärkten Messeselektion insbesondere auch die Überprüfung des Messebeteiligungserfolgs unabdingbar.

Vor diesem Hintergrund analysiert die Verfasserin den Erfolg von Hochschul-Messebeteiligungen, wobei die Betrachtung der hochschulspezifischen Messeziele und -zielgruppen als Grundlage der Erfolgskontrolle den Ausgangspunkt des Forschungsprojektes bildet. Die vorgenommene empirische Ermittlung der Bedeutung hochschulspezifischer Messezielsetzungen zeigt eine generell hohe Wichtigkeit der theoretisch abgeleiteten Messebeteiligungsziele aus Exponentensicht. Aus der hohen Anzahl hochschulspezifischer Messeziele werden im Rahmen einer empirischen Untersuchung fünf eigenständige, hochschulspezifische Messezieldimensionen identifiziert. Auf Basis dieser Messezieldimensionen leitet die Autorin drei hochschulspezifische Exponententypen empirisch ab, die sich in den Priorisierungen der Messezieldimensionen signifikant voneinander unterscheiden.

Aufbauend auf der Analyse hochschulspezifischer Messeziele überprüft die Verfasserin hochschulspezifische Anspruchsgruppen als Zielgruppen von HochschulMessebeteiligungen. Dabei wird ebenfalls das Verhalten der Hochschul-Messestand-Besucher analysiert, das durch die mit dem Besuch eines HochschulMessestandes verfolgten Zielsetzungen determiniert wird. Auf Grundlage der theoretisch abgeleiteten hochschulspezifischen Besucherzielsetzungen werden empirisch drei unterschiedliche Besuchertypen auf Hochschul-Messeständen

gebildet. Dabei ist festzustellen, daß das Verhalten dieser Besuchertypen kom- 
plementär zu den von den Hochschul-Exponenten hoch priorisierten, auf die jeweiligen Zielgruppen fokussierten Messeeinzelzielsetzungen stehen und daß dadurch grundsätzlich die Basis für eine erfolgreiche Hochschul-Messebeteiligung geschaffen wird.

Eine Hochschul-Messebeteiligung ist dann effizient, wenn der erzielte Nutzen einer Messebeteiligung die entstandenen Messekosten übersteigt und aufgrund einer alternativen Mittelverwendung kein höherer Nutzen hätte erzielt werden können. Folglich nimmt die Autorin eine zweistufige Erfolgskontrolle vor. Zunächst wird der Zielerreichungsgrad der verfolgten Messebeteiligungsziele analysiert und damit eine Nutzenbetrachtung im Sinne einer Effektivitätskontrolle durchgeführt. Sodann erfolgt eine Effizienzkontrolle, bei der Kosten-Nutzen-Relationen gebildet werden. Abschließend werden intra- und inter-instrumentelle Vergleiche mit ausgewählten Vergleichsobjekten vorgenommen.

Auf Basis der aufgezeigten Untersuchungsergebnisse leitet die Verfasserin sowohl strategische als auch operative Implikationen für Hochschul-Exponenten ab. In diesem Zusammenhang stellt die Autorin heraus, daß neben dem gezielten und abgestimmten Einsatz der aufgezeigten Strategien und Maßnahmen der Erfolg einer Messebeteiligung insbesondere vom integrierten Einsatz des Messeengagements im Rahmen des Hochschulmarketing abhängig ist. Der hierzu notwendige Koordinationsbedarf bezieht sich sowohl auf die Koordination des Marketing mit den anderen hochschulspezifischen Funktionsbereichen als auch auf die Abstimmung der Aktivitäten innerhalb des Hochschulmarketing im Sinne der Koordination der einzelnen Marketing-Mix-Instrumente. Messebeteiligungen sind dabei sowohl im Planungs- und Koordinationsprozeß als auch im Kontrollprozeß des Hochschulmarketing zu berücksichtigen und entsprechend zu integrieren.

Neben den Implikationen für Hochschul-Exponenten leitet die Autorin ebenfalls Implikationen für Finanzmittelgeber von Hochschul-Messebeteiligungen ab. Die von den jeweiligen finanzmittelgebenden öffentlichen oder kommerziellen Institutionen zu unterstützenden Messebeteiligungen sind danach auszuwählen, inwieweit das von den Hochschul-Exponenten geplante Messeengagement zum Erfolg führen wird. Die Auswahl sollte ihres Erachtens nicht zuletzt auch deshalb im aufgezeigten Sinne vorgenommen werden, weil der Messeerfolg der Exponenten letztlich den Erreichungsgrad der von den Finanzmittelgebern angestrebten Zielsetzungen determiniert. 
Die vorliegende Untersuchung liefert mit ihrer empirisch fundierten Analyse zu Messebeteiligungen im Hochschulbereich eine umfassende Bestandsaufnahme zu den Zielen und der Erfolgskontrolle von Hochschul-Messebeteiligungen. Der vorliegenden Arbeit kommt damit der Charakter einer Basisuntersuchung zu. Sie liefert eine wertvolle Grundlage für weiterführende Forschungsarbeiten im Bereich der Messebeteiligung von Hochschulen.

Die empirische Analyse der vorliegenden Arbeit erfolgte auf Basis des Datenmaterials einer vom Institut für Marketing der Westfälischen Wilhelms-Universität Münster in Zusammenarbeit mit dem Ministerium für Schule und Weiterbildung, Wissenschaft und Forschung des Landes Nordrhein-Westfalen durchgeführten Studie. Dieser Unterstützung und Kooperationsbereitschaft gilt mein besonderer Dank. 



\section{Vorwort der Verfasserin}

Angesichts veränderter Rahmenbedingungen sehen sich Hochschulen neben steigenden Studierendenzahlen sowie erhöhten Anforderungen an Forschung und Lehre insbesondere auch der Verknappung öffentlicher Finanzmittel gegenübergestellt. Den sich hieraus ergebenden Herausforderungen ist durch eine zunehmende Marktorientierung des Hochschulmanagement und der Erschließung zusätzlicher Finanzierungsquellen zu begegnen. In diesem Zusammenhang gewinnen Messebeteiligungen von Hochschulen zunehmend an Bedeutung, da sie als multifunktionales Marketing-Instrument nicht nur die Marktorientierung von Hochschulen unterstützen, sondern darüber hinaus auch die Drittmitteleinwerbung über den Austausch von Transferleistungen zwischen Hochschulen und an Forschungsleistungen interessierten Anspruchsgruppen forcieren. Aufgrund des expandierenden Messeangebots bei gleichzeitig hohen Messebeteiligungskosten und knappen Hochschulbudgets nimmt bei Hochschul-Messebeteiligungen insbesondere die Überprüfung des Messebeteiligungserfolgs einen zunehmenden Stellenwert ein.

Ausgangspunkt der Erfolgskontrolle von Hochschul-Messebeteiligungen bildet die Analyse hochschulspezifischer Messezielsetzungen und -zielgruppen. Vor diesem Hintergrund werden in der vorliegenden Arbeit zunächst hochschulspezifische Messezielsetzungen theoretisch hergeleitet und in einer empirischen Untersuchung zu fünf hochschulspezifischen Messezieldimensionen aggregiert. Diese Messezieldimensionen dienen zur Ableitung von drei hochschulspezifischen Exponententypen, die sich in den Priorisierungen der Messezieldimensionen signifikant voneinander unterscheiden. Im Anschluß daran werden hochschulspezifische Anspruchsgruppen dargestellt und ihre Relevanz für Messebeschickungen im Hochschulbereich überprüft. Im Rahmen der Diskussion finden ebenfalls verhaltensorientierte Kriterien Berücksichtigung. Die in diesem Zusammenhang theoretisch hergeleiteten fünf hochschulspezifischen Besucherzielsetzungen bilden die Basis zur empirischen Ableitung von drei Besuchertypen auf HochschulMesseständen.

Auf Grundlage der Ergebnisse zu den hochschulspezifischen Messebeteiligungszielen und -zielgruppen wird der Erfolg von Hochschul-Messebeteiligungen untersucht. Dazu erfolgt zunächst eine Effektivitätskontrolle, bei der aus den hochschulspezifischen Messezielsetzungen Erfolgskriterien systematisch abgeleitet und ausgewählte Methoden zur Messeerfolgskontrolle eingesetzt werden. Die 
anschließend vorgenommene Effizienzbetrachtung basiert auf Wirtschaftlichkeitsanalysen. Zum Abschluß der Messeerfolgskontrolle werden intra- und interinstrumentelle Vergleiche mit ausgewählten Vergleichsobjekten durchgeführt. Auf Basis der theoretisch und empirisch gewonnenen Erkenntnisse werden abschlieBend Implikationen sowohl für Hochschul-Exponenten als auch für Finanzmittelgeber von Hochschul-Messebeteiligungen abgeleitet.

Das Entstehen dieser Arbeit war nur mit der Unterstützung zahlreicher Personen und Institutionen möglich. Mein besonderer Dank gilt zunächst meinem akademischen Lehrer, Herrn Prof. Dr. Dr. h.c. mult. Heribert Meffert, der meine fachliche und persönliche Entwicklung als wissenschaftliche Mitarbeiterin am Institut für Marketing umfassend förderte und die Erstellung der Arbeit in allen Phasen ihrer Entstehung unterstützte. Herrn Prof. Dr. Heinz Lothar Grob danke ich vielmals für die Übernahme des Zweitgutachtens.

Die empirische Analyse der vorliegenden Arbeit basiert auf einer vom Institut für Marketing der Westfälischen Wilhelms-Universität Münster in Zusammenarbeit mit dem Ministerium für Schule und Weiterbildung, Wissenschaft und Forschung des Landes Nordrhein-Westfalen durchgeführten Studie. Für die freundliche Unterstützung durch das Ministerium und dessen Mitarbeiter, ohne die der empirische Teil dieser Arbeit nicht möglich gewesen wäre, möchte ich mich nachdrücklich bedanken. Mein Dank gilt schließlich auch allen an diesem umfangreichen Forschungsprojekt beteiligten Interviewerinnen und Interviewern, die durch ihren unermüdlichen Einsatz zu der bemerkenswerten Messebesucher-Stichprobe beigetragen haben.

Bedanken möchte ich mich nicht zuletzt bei allen aktuellen und ehemaligen Kolleginnen und Kollegen sowie Freundinnen und Freunden am Institut für Marketing der Universität Münster, die mich während der Erstellung der Arbeit in vielfältiger Weise unterstützt haben. Besonders hervorheben möchte ich Herrn Dr. Dr. Helmut Schneider, dessen kritische Diskussionsbereitschaft und fachliche Kompetenz wesentlich zur Qualität der Arbeit beigetragen hat. Ferner gilt mein Dank Herrn Dipl.-Kfm. Michael Bongartz und Herrn Dipl.-Kfm. Michael Schleusener für wertvolle Anregungen bei der Durchsicht des Manuskripts. Dank schulde ich auch meinen Freundinnen und Freunden außerhalb des Instituts, die mir während der gesamten Promotionszeit zur Seite gestanden und mir in dieser Zeit ein Höchst$\mathrm{maß}$ an Verständnis entgegengebracht haben. 
Ganz besonderer Dank gilt meiner Familie, die mir während meiner Ausbildungszeit stets den notwendigen Rückhalt gegeben hat. Danken möchte ich meinen Geschwistern Frank, Reinhild und William sowie ihren Lebenspartnern, die mich immer hilfsbereit unterstützten. Meinem Patenkind Philipp verdanke ich viele Stunden der Heiterkeit und Entspannung sowie das notwendige Maß an Distanz zur eigenen Arbeit. In Gedanken werde ich auch immer meiner Schwester Marion ( $\dagger$ ) dankbar sein, die mich in meiner frühen Ausbildungszeit für eine akademische Laufbahn motivierte, den Tag meiner Promotion jedoch nicht miterleben sollte.

Schließlich ist es mir ein besonderes Anliegen, meinen Eltern zu danken, die mich in allen Phasen meiner Ausbildung in vielerlei Hinsicht stets liebevoll unterstützt haben und mir dabei immer die Freiheit eigener Entscheidungen ließen. Sie haben nicht nur die Grundlage für die Erstellung dieser Arbeit geschaffen, sondern darüber hinaus durch ihre Unterstützung und ihr Verständnis den erfolgreichen Abschluß meiner Arbeit erst ermöglicht. Innen widme ich diese Arbeit. 



\section{Inhaltsverzeichnis}

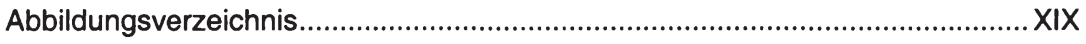

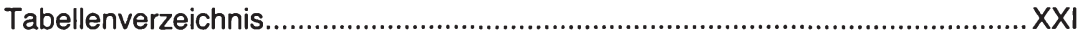

Abkürzungsverzeichnis ................................................................ XXII

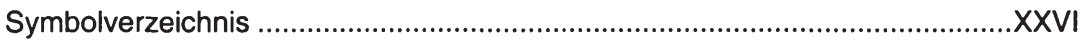

A. Messebeteiligungen als Herausforderung an das Marketing im Hochschulbereich............................................................................ 1

1. Bedeutung von Messebeteiligungen für Hochschulen ................................

2. Besonderheiten der Messebeteiligung von Hochschulen ............................8

2.1 Messebeteiligungsrelevante Spezifika von Hochschulen ......................8

2.2 Messebeteiligungen im Rahmen des Hochschulmarketing ................... 18

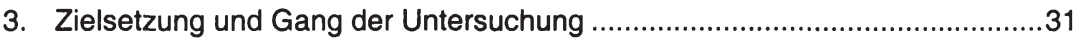

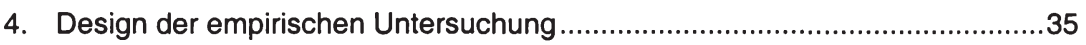

B. Analyse hochschulspezifischer Messeziele und Messezielgruppen .......37

1. Zielsystem von Hochschulen bei Messebeteiligungen.................................37

1.1 Zielsetzungen von Messebeteiligungen im Hochschulbereich ...............38

1.11 Hochschulspezifische Messeziele auf verschiedenen Ebenen des Hochschulbereichs .............................................38

1.12 Empirische Analyse der Bedeutung hochschulspezifischer Messebeteiligungsziele. 
1.2 Zieldimensionen von Hochschul-Messebeteiligungen

1.21 Systematisierung hochschulspezifischer Messebeteiligungsziele

1.22 Empirische Ermittlung hochschulspezifischer

Messezieldimensionen auf Basis einer Faktorenanalyse

1.3 Ableitung von Hochschul-Exponententypen auf Basis hochschulspezifischer Messezieldimensionen

1.31 Darstellung exponentenspezifischer Grundhaltungstypen

1.32 Clusteranalytische Bestimmung hochschulspezifischer Exponententypen und diskriminanzanalytische Überprüfung .55

2. Zielgruppen der Messebeteiligung von Hochschulen

2.1 Anspruchsgruppen als Zielgruppen von HochschulMessebeteiligungen

2.11 Systematisierung hochschulspezifischer Anspruchsgruppen

2.12 Empirische Analyse der Bedeutung hochschulspezifischer Messezielgruppen

2.2 Ziele von Messebesuchern als komplementäres Zielgruppenkriterium für hochschulspezifische Messezielgruppen

2.21 Messebesucherziele als Determinante des Messebesucherverhaltens

2.22 Clusteranalytische Bestimmung von Hochschul-MessestandBesuchertypen und diskriminanzanalytische Überprüfung

2.3 Integrierte Betrachtung von hochschulspezifischen Messezielgruppen und Hochschul-Messestand-Besuchertypen

C. Erfolgskontrolle der Messebeteiligung von Hochschulen

1. Unsicherheit über die Effizienz von Hochschul-Messebeteiligungen

2. Indirekte Erfolgskontrolle von Hochschul-Messebeteiligungen

2.1 Messezielsetzungen als Grundlage zur indirekten Erfolgskontrolle von Hochschul-Messebeteiligungen 
2.11 Erfolgskriterien zur Kontrolle von Informationszielen ...................98

2.12 Erfolgskriterien zur Kontrolle von Beeinflussungszielen ..............99

2.13 Erfolgskriterien zur Kontrolle von Projektzielen.........................100

2.2 Analyse des indirekten Erfolgs von Hochschul-Messebeteiligungen durch Einsatz von Methoden zur Messeerfolgskontrolle

2.21 Einsatz von Methoden zur indirekten Messeerfolgskontrolle bzw. -prognose in der Vor-Messe-Phase.

2.22 Einsatz von Methoden zur indirekten Messeerfolgskontrolle in der Messe-Phase

2.23 Einsatz von Methoden zur indirekten Messeerfolgskontrolle in der Nach-Messe-Phase

3. Direkte Erfolgskontrolle von Hochschul-Messebeteiligungen

3.1 Kostenanalyse als Voraussetzung zur Ermittlung des direkten Erfolgs von Hochschul-Messebeteiligungen.

3.2 Analyse des direkten Erfolgs von Hochschul-Messebeteiligungen auf Grundlage von Kosten-Nutzen-Relationen.

3.21 Einsatz von Kennziffernanalysen .............................................156

3.22 Einsatz von Messekontaktwertrechnungen...............................159

3.3 Zentrale Ergebnisse der Erfolgskontrolle von HochschulMessebeteiligungen.

4. Effizienzvergleiche von Hochschul-Messebeteiligungen mit ausgewählten Vergleichsobjekten.

4.1 Intra-instrumentelle Vergleiche zwischen verschiedenen

Messebeteiligungen.

4.2 Inter-instrumentelle Vergleiche zwischen Messebeteiligungen und alternativen Kommunikationsinstrumenten 
1. Implikationen für Hochschul-Exponenten …............................................172

1.1 Entscheidungen über die Beteiligung an Messen ...............................172

1.11 Auswahl des Messetyps .........................................................172

1.12 Auswahl hochschulspezifischer Messebeteiligungsformen .......176

1.2 Entscheidungen über die Gestaltung von Hochschul-

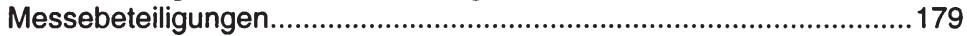

1.21 Maßnahmen in der Vor-Messe-Phase ......................................180

1.22 Maßnahmen in der Messe-Phase ...........................................186

1.23 Maßnahmen in der Nach-Messe-Phase ..................................191

1.3 Implikationen für die Integration von Messebeteiligungen in das Hochschulmarketing

2. Implikationen für Finanzmittelgeber von Hochschul-Messebeteiligungen.....199

E. Zusammenfassung und Ausblick

1. Zusammenfassende Darstellung der Untersuchungsergebnisse

2. Ansatzpunkte für weiterführende Forschungsarbeiten 


\section{Abbildungsverzeichnis}

Abb. 1: Systemorientierte Betrachtung der Hochschule ......................................12

Abb. 2: Zielsystem von Hochschulen .................................................................15

Abb. 3: Einordnung von Messebeteiligungen in den Managementproze $B$ des Hochschulmarketing .........................................................................22

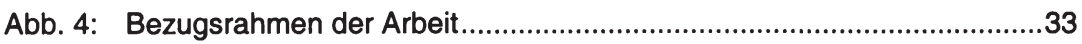

Abb. 5: Hochschulspezifische Messezielsetzungen auf verschiedenen Ebenen des Hochschulbereichs ..........................................................44

Abb. 6: Bedeutung hochschulspezifischer Messebeteiligungsziele .....................45

Abb. 7: Faktorenanalytische Ermittlung der Messezieldimensionen .....................49

Abb. 8: Bedeutung hochschulspezifischer Messeziele und Messezieldimensionen

Abb. 9: Fusionierungsprozeß des Ward-Verfahrens und Bestimmung der Exponenten-Clusterlösung

Abb. 10: Varianzanalytische Prüfung auf Bewertungsunterschiede bei Zieldimensionen zwischen hochschulspezifischen Exponententypen.

Abb. 11: Zieldimensionen und Exponententypen der Messebeteiligung von Hochschulen

Abb. 12: Systematisierung von Anspruchsgruppen zur Ableitung hochschulspezifischer Messebeteiligungszielgruppen ...

Abb. 13: Hochschulspezifische Zielgruppen und die auf sie gerichteten hochschulspezifischen Messezielsetzungen

Abb. 14: Bedeutung hochschulspezifischer Messezielgruppen ............................72

Abb. 15: Exponententypspezifische Bedeutung hochschulspezifischer Messezielgruppen

Abb. 16: Fusionierungsprozeß des Ward-Verfahrens und Varianzkriterium zur Bestimmung der Messestand-Besucher-Clusterlösung.

Abb. 17: Varianzanalytische Prüfung auf Bewertungsunterschiede bei Besucherzielsetzungen zwischen Hochschul-Messestand-Besuchertypen....82

Abb. 18: Systematisierung hochschulspezifischer Messezielgruppen unter Berücksichtigung verhaltensorientierter Kriterien

Abb. 19: Hochschulspezifische Messezielsetzungen und ihr Fokus auf unterschiedliche Messezielgruppen unter Berücksichtigung verhaltensorientierter Kriterien

Abb. 20: Integration von Hochschul-Exponenten- und HochschulMessestand-Besucher-Perspektive

Abb. 21: Erfolgskriterien zur Kontrolle von Informationszielen.............................98

Abb. 22: Erfolgskriterien zur Kontrolle von Beeinflussungszielen .........................99 


\section{$X X$}

Abb. 23: Erfolgskriterien zur Kontrolle von Projektzielen

Abb. 24: Erfolgskriterien und Methoden zur Erfolgskontrolle von Hochschul-Messebeteiligungen

Abb. 25: Wichtigkeiten und Besucheranteile hochschulspezifischer Messezielgruppen

Abb. 26: Messetypspezifische Betrachtung von Wichtigkeiten und Besucheranteilen hochschulspezifischer Messezielgruppen.

Abb. 27: Beurteilung der von den Hochschul-Exponenten präsentierten Exponate aus Sicht der Hochschul-Messestand-Besucher.

Abb. 28: Messetypspezifische Beurteilung der von den HochschulExponenten präsentierten Exponate aus Sicht der HochschulMessestand-Besucher.

Abb. 29: Vergleich der Hochschul-Messestand-Besucherstruktur mit der Gesamtbesucherstruktur der betrachteten Messen

Abb. 30: Zufriedenheit der Hochschul-Exponenten mit der Messebeteiligung ...140

Abb. 31: Messetypspezifische Zufriedenheit der Hochschul-Exponenten mit der Messebeteiligung.

Abb. 32: Exponententypspezifische Zufriedenheit mit der Messebeteiligung .....142

Abb. 33: Messetypspezifische Messebeteiligungs-Nutzen-Indizes

Abb. 34: Exponententypspezifische Messebeteiligungs-Nutzen-Indizes 145

Abb. 35: Angebahnte Projekte aufgrund des von Hochschul-Exponenten realisierten Messeengagements

Abb. 36: Messetypspezifisch angebahnte Projekte aufgrund des von Hochschul-Exponenten realisierten Messeengagements.

Abb. 37: Exponententypspezifisch angebahnte Projekte aufgrund des von Hochschul-Exponenten realisierten Messeengagements

Abb. 38: Intra-instrumentelle Vergleiche zwischen verschiedenen Messebeteiligungen.

Abb. 39: Inter-instrumentelle Vergleiche zwischen Messebeteiligungen und alternativen Kommunikationsinstrumenten.

Abb. 40: Identität einer Hochschule als Marke 


\section{Tabellenverzeichnis}

Tab. 1: Hochschulspezifische Messebeteiligungsformen im Vergleich ..............29

Tab. 2: Klassifizierungsmatrix der diskriminanzanalytischen Überprüfung der Exponenten-Clusterlösung

Tab. 3: Diskriminanzanalytische Bedeutung der hochschulspezifischen Messezieldimensionen für Exponententypen

Tab. 4: Beziehungen zwischen Messebeteiligungszielen von Hochschul-Exponenten und Zielsetzungen von Hochschul-Messestand-Besuchern ...80

Tab. 5: Art und durchschnittliche Anzahl der geführten Gespräche von Hochschul-Messestand-Besuchertypen mit Hochschul-Exponenten

Tab. 6: Klassifizierungsmatrix der diskriminanzanalytischen Überprüfung der Besucher-Clusterlösung.....

Tab. 7: Diskriminanzanalytische Bedeutung der hochschulspezifischen Besucherzielsetzungen für Besuchertypen

Tab. 8: Zusammensetzung hochschulspezifischer Messezielgruppen nach Hochschul-Messestand-Besuchertypen

Tab. 9: Systematisierung der Methoden zur indirekten Erfolgskontrolle von Hochschul-Messebeteiligungen

Tab. 10: Messetypspezifische Betrachtung ausgegebener Informationsmaterialien im Rahmen von Hochschul-Messebeteiligungen .....

Tab. 11: Exponententypspezifische Betrachtung ausgegebener Informationsmaterialien im Rahmen von Hochschul-Messebeteiligungen

Tab. 12: Kontaktqualität und -quantität der zwischen Hochschul-Exponenten und Hochschul-Messestand-Besuchern geführten Gespräche

Tab. 13: Messetypspezifischer Vergleich der Kontaktqualität und -quantität der geführten Hochschul-Messestand-Gespräche

Tab. 14: Exponententypspezifischer Vergleich der Kontaktqualität und -quantität der geführten Hochschul-Messestand-Gespräche

Tab. 15: Analyse der Kontakte zwischen Hochschul-Exponenten und hochschulspezifischen Messezielgruppen

Tab. 16: Messetypspezifische Analyse der Kontakte zwischen HochschulExponenten und hochschulspezifischen Messezielgruppen

Tab. 17: Analyse der Kontakte zwischen Hochschul-Exponententypen und hochschulspezifischen Messezielgruppen.

Tab. 18: Wirtschaftsvertreter auf den Hochschul-Messeständen und ihre Entscheidungskompetenz innerhalb der Unternehmen.

Tab. 19: Kostenbelastungen für Hochschul-Exponenten in Abhängigkeit der hochschulspezifischen Messebeteiligungsform. 
Tab. 20: Kostenstruktur der Messebeteiligung von Hochschulen 155

Tab. 21: Kennziffern der Messebeteiligung von Hochschulen. 158

Tab. 22: Medienbeiträge zu Hochschul-Messebeteiligungen 159

Tab. 23: Methoden zur Erfolgskontrolle von Hochschul-Messebeteiligungen und deren messetypspezifische Ergebnisse

Tab. 24: Messetypspezifische und exponententypspezifische Analyse des Erfolgs von Hochschul-Messebeteiligungen.

Tab. 25: Eignung ausgewählter inter- und intra-instrumenteller Vergleichsobjekte zur Erreichung hochschulspezifischer Messeziele und -zielgruppen.

Tab. 26: Eignung verschiedener, nach Angebotsbreite klassifizierter Messetypen zur Erreichung exponententypspezifischer Messezielsetzungen ..173

Tab. 27: Eignung verschiedener, nach Angebotsbreite klassifizierter Messetypen zur Erreichung hochschulspezifischer Messezielgruppen

Tab. 28: Eignung verschiedener, nach Angebotsbreite klassifizierter Messetypen zur Erreichung von Hochschul-Messestand-Besuchertypen ......176

Tab. 29: Hochschulspezifische Messebeteiligungsformen und ihre Eignung für Hochschul-Exponententypen

Tab. 30: Hochschulspezifische Messebeteiligungsformen und ihre Eignung zur Erreichung hochschulspezifischer Messezielgruppen

Tab. 31: Maßnahmen in der Vor-Messe-Phase und deren Einflußmöglichkeiten auf die Erreichung hochschulspezifischer Messeziele und -zielgruppen

Tab. 32: Maßnahmen in der Messe-Phase und deren Einflußmöglichkeiten auf die Erreichung hochschulspezifischer Messeziele und -zielgruppen

Tab. 33: Maßnahmen in der Nach-Messe-Phase und deren Einflußmöglichkeiten auf die Erreichung hochschulspezifischer Messeziele und -zielgruppen 


\section{Abkürzungsverzeichnis}

$\begin{array}{ll}\text { a.a.O. } & \text { am angegebenen Ort } \\ \text { Abb. } & \text { Abbildung } \\ \text { Abs. } & \text { Absatz } \\ \text { AG } & \text { Aktiengesellschaft } \\ \text { AMR } & \text { Academy of Management Review } \\ \text { asw } & \text { Absatzwirtschaft } \\ \text { Art. } & \text { Artikel } \\ \text { Aufl. } & \text { Auflage } \\ \text { AUMA } & \text { Ausstellungs- und Messe-Ausschuss der } \\ & \text { Deutschen Wirtschaft e.V. }\end{array}$

Bd.

BddW

Band

$\mathrm{BH}$ bspw. bzw.

Blick durch die Wirtschaft Business Horizons beispielsweise beziehungsweise

ca. circa

CAL

CAT Computer Assisted Learning

$\mathrm{CHL}$ Computer Assisted Teaching computergestützte Hochschullehre

DAAD

d.h.

Deutscher Akademischer Austauschdienst e.V.

DBW

DFG

Diss.

DM

DUZ das heißt

Die Betriebswirtschaft

Deutsche Forschungsgemeinschaft

Dissertation

Deutsche Mark

DWG

Deutsche Universitätszeitung

Deutsche Werbewissenschaftliche Gesellschaft e.V.

Ed.

et al.

etc.

Edition, Editor

e.V.

et alii, et alia, et alteri

et cetera

f., ff.

eingetragener Verein

FAMAB

folgende, fortfolgende

FAZ

FB

Fachverband Messe- und Ausstellungsbau e.V.

Frankfurter Allgemeine Zeitung

Fachbereich

FKM

Gesellschaft zur freiwilligen Kontrolle von Messe- und Ausstellungszahlen 
GfK

GG

ggf.

$\mathrm{GmbH}$

$\mathrm{HB}$

HBR

HRG

Hrsg.

HWA

i.d.R.

i.e.S.

III.

IMM

Inst.

i.S.

i.S.e.

i.S.d.

i.S.v.

i.w.S

J.

Jg.

JoM

JoMR

JoMRS

JoR

Lst.

k.A.

Kap.

MA

Mio.

Mrd.

$\mathrm{m}+\mathrm{a}$

n.s.

No.

Nr.

NY
Gesellschaft für Konsum-, Markt- und Absatzforschung

Grundgesetz

gegebenenfalls

Gesellschaft mit beschränkter Haftung

Handelsblatt

Harvard Business Review

Hochschulrahmengesetz

Herausgeber

Handwörterbuch der Absatzwirtschaft

in der Regel

im engeren Sinne

Illinois

Industrial Marketing Management

Institut

im Sinne

im Sinne einer (s)

im Sinne der (s)

im Sinne von

im weiteren Sinne

Jahr (e)

Jahrgang

Journal of Marketing

Journal of Marketing Research

Journal of the Market Research and Society

Journal of Retailing

Lehrstuhl

keine Angabe

Kapitel

Massachusetts

Millionen

Milliarden

Messen und Ausstellungen

nicht signifikant

Number

Nummer

New York 
0.9 .

o.J.

o.Nr.

o.O.

o.V.

OWC

PA

PuA

qm

S.

sog.

Sp.

SPSS

Tab.

TU

u.a.

Univ.

USA

vgl.

VM

Vol.

vs.

WHU

WWW

z.B.

ZfB

$\mathrm{ZfbF}$

ZFP

$\mathrm{ZfO}$

z.T. oben genannte $(n, r, s)$

ohne Jahr

ohne Nummer

ohne Ort

ohne Verfasser

Ost-West-Contact

Pennsylvania

Planung und Analyse

Quadratmeter

Seite

sogenannte $(n, r, s)$

Spalte

Superior Performing Software Systems

Tabelle

Technische Universität

und andere, unter anderem

Universität

United States of America

vergleiche

Verkaufsmanagement

Volume

versus

Wissenschaftliche Hochschule für Unternehmensführung

World Wide Web

zum Beispiel

Zeitschrift für Betriebswirtschaft

Zeitschrift für betriebswirtschaftliche Forschung

Zeitschrift für Forschung und Praxis

Zeitschrift für Organisation

zum Teil 
$X X V I$

\section{Symbolverzeichnis}

$\begin{array}{ll}\mathrm{g} & \text { Person } \\ \mathrm{h} & \text { Faktor } \\ \mathrm{i} & \text { Variable } \\ \mathrm{m}_{\mathrm{h}} & \text { Zahl der Variablen i, die den Faktor } \mathrm{h} \text { bilden } \\ \mathrm{x}_{\mathrm{gi}} & \text { Ausprägung der Person } \mathrm{g} \text { in der Variablen } \mathrm{i} \\ \mathrm{y}_{\mathrm{gh}} & \text { mittlerer Gesamtpunktwert einer Person } \mathrm{g} \text { im Faktor } \mathrm{h}\end{array}$




\section{A. Messebeteiligungen als Herausforderung an das Marketing im Hochschulbereich}

\section{Bedeutung von Messebeteiligungen für Hochschulen}

Hochschulen sehen sich angesichts dynamischer Umweltveränderungen einer Vielzahl von Herausforderungen gegenübergestellt, die insbesondere durch steigende Studierendenzahlen sowie erhöhte Anforderungen an Forschung und Lehre bei gleichzeitiger Verknappung öffentlicher Finanzmittel hervorgerufen werden.

Seit Mitte der 70er Jahre können deutsche Hochschulen steigende Studierendenzahlen verzeichnen. So erhöhte sich die Zahl der Studierenden in den alten Bundesländern vom Wintersemester 1986/87 bis zum Wintersemester 1991/92 von 1,366 Mio. um über $20 \%$ auf 1,647 Mio. bzw. bezogen auf das gesamte Bundesgebiet auf 1,783 Mio. Studierende. ${ }^{1}$ Vom Wintersemester 1991/92 bis zum Wintersemester 1998/99 konnte - trotz der von 1990 bis 1995 rückläufigen Studienanfängerzahlen ${ }^{2}$ - abermals ein Anstieg auf 1,801 Mio. Studierende festgestellt werden. ${ }^{3}$ Die Gründe für diese Entwicklung liegen vor allem in einer gestiegenen Bildungsbeteiligung ${ }^{4}$ und der im internationalen Vergleich langen Studienzeit an deutschen Hochschulen. ${ }^{5}$

Vgl. Möncke, A. F., Studenten im Wintersemester 1991/92, in: Wirtschaft und Statistik, 44. Jg., Heft 4, 1992, S. 247.

2

Vgl. Mösch, B., Studierende im Wintersemester 1995/96, in: Wirtschaft und Statistik, 48. Jg., Heft 6, 1996, S. 380.

3 Vgl. Statistisches Bundesamt (Hrsg.), Hochschulen, in: Internet, http://www.statistikbund.de/ basis/d/biwiku/hochtxt.htm, Stand: 03.12.1999.

4 Sowohl der Anteil der Studienberechtigten an der Bevölkerung als auch der Anteil der Studierenden und Studienanfänger an den Studienberechtigten sind gestiegen. Vgl. Lammert, N., Zwischen Lebenslügen und Überlebensstrategien - Vorschläge zur Reform des deutschen Hochschulwesens, in: Wirtschaftsdienst, 74. Jg., Heft 5, 1994, S. 223.

5 Die durchschnittliche Studiendauer der Studienabsolventen lag 1996 in Deutschland bei 5,7 Jahren (Studiendauer nach Hochschulsemestern) bzw. bei 5,2 Jahren (Studiendauer nach Fachsemestern). Vgl. Bundesministerium für Bildung und Forschung (Hrsg.), "Zahlenbarometer im Internet“, in: Internet, http://192.76.176.139/deutsch/kap/s39.htm, Stand: 03.12.1999. Im internationalen Vergleich werden damit mit die längsten Studienzeiten realisiert. So liegt die Studiendauer der deutschen Hochschulabsolventen bspw. höher als die universitäre Ausbil- 
Zudem sind erhöhte Anforderungen an Forschung und Lehre der Hochschulen zu verzeichnen, die nicht zuletzt auf die Verschärfung des internationalen Standortwettbewerbs und der damit u.a. einhergehenden Notwendigkeit zur Ausbildung qualifizierter Fachkräfte zurückzuführen sind. Hochschulen sind in diesem Zusammenhang dazu aufgefordert, durch eine entsprechende Spezialisierung und Intensivierung der Forschung und Lehre diesen erhöhten Ansprüchen gerecht zu werden. ${ }^{6}$

Trotz dieser Entwicklungen erfolgte keine entsprechende Anpassung der Hochschulressourcen. ${ }^{7}$ Demzufolge konnte eine Vielzahl von Hochschulen eine Bereitstellung ihrer Lehr- und Forschungsleistungen in der notwendigen Qualität sowie Quantität nicht im vollem Umfang gewährleisten ${ }^{8}$ und folglich den gewachsenen gesellschaftlichen Ansprüchen nur eingeschränkt gerecht werden. ${ }^{9}$ Den vor diesem Hintergrund gestellten Forderungen nach Ausweitung der öffentlichen Bildungsetats stehen insgesamt knapper werdende öffentliche Finanzmittel

dungszeit von Japanern, Briten oder Amerikanern. Vgl. Krumrey, H., Canibol, H. P., Studium für Maurer, in: Wirtschaftswoche, 46. Jg., Heft 18, 1992, S. 42.

6 Vgl. Meffert, H., Einführung in die Themenstellung, in: Meffert, H., Wagner, H., Backhaus, K. (Hrsg.), Entwicklungsperspektiven der wirtschaftswissenschaftlichen Hochschulausbildung, Dokumentationspapier Nr. 102 der Wissenschaftlichen Gesellschaft für Marketing und Unternehmensführung e.V., Münster 1996, S. 1 ff.; Meffert, H., Herausforderungen an die Betriebswirtschaftslehre - Die Perspektive der Wissenschaft, in: DBW, 58. Jg., Heft 6, 1998, S. 709 ff. In diesem Zusammenhang gewinnt die computergestützte Lehre eine zunehmende Bedeutung. Vgl. Grob, H. L., Grießhaber, W., Computergestützte Lehre an der Universität, Arbeitsbericht Nr. 1 der Reihe CAL + CAT des Lehrstuhls für Wirtschaftsinformatik und Controlling, Münster 1995, S. 1. Zu Ausgestaltungsformen der computergestützten Hochschullehre vgl. Grob, H. L., Das Internet im Mittelpunkt einer computergestützten Hochschullehre (cHL), in: Hauff, M. (Hrsg.), media@ uni-multi.media? - Entwicklung - Gestaltung - Evaluation neuer Medien, Münster u.a. 1998, S. 101-112; Grob, H. L., CAL + CAT, Arbeitsbericht Nr. 2 der Reihe CAL + CAT des Lehrstuhls für Wirtschaftsinformatik und Controlling, Münster 1995; Grob, H. L., Multimediale Lehre an der Universität, in: Forschungsjournal der Westfälischen Wilhelms-Universität Münster, 4. Jg., Heft 1, 1995, S. 38-42.

Während - bezogen auf die alten Bundesländer - die Zahl der Studierenden im Zeitraum von 1980 bis 1990 um $61 \%$ anstieg, wurden die Grundmittel für Forschung und Lehre real nur um $4,2 \%$ erhöht. Vgl. o.V., Hochschulfinanzen: Krisenmanagement, in: Informationsdienst der deutschen Wirtschaft, 20. Jg., Heft 18, 1994, S. 5.

Vgl. Ziegele, F., Hochschule und Finanzautonomie: Grundlagen und Anwendung einer politisch-ökonomischen Theorie der Hochschule, 2. Aufl., Frankfurt am Main u.a. 1998, S. 20.

Vgl. Amrhein, D., Die Universität als Dienstleistungsunternehmen: Innovative Organisationsstrukturen und Motivationskonzepte, Wiesbaden 1998, S. 10 ff.; Nowotny, H., Mass Higher Education and Social Mobility: A Tenuous Link, in: Dill, D., Sporn, B. (Ed.), Emerging Patterns of Social Demand and University Reform: Through a Glass Darkly, Oxford, New York, Tokyo 1995, S. 72 f. 
gegenüber. ${ }^{10}$ Durch zunehmende Finanzierungsengpässe steigt der Druck auf die Bildungsetats des Bundes und der für die Hochschulpolitik zuständigen Bundesländer. ${ }^{11}$ Bei gleichzeitig steigenden Kosten im Bildungs- und Forschungsbereich sind Hochschulen zunehmend darauf angewiesen, zusätzliche Finanzmittel einzuwerben. ${ }^{12}$ In diesem Zusammenhang ist ein erhöhter Wettbewerb ${ }^{13}$ zwischen Hochschulen insbesondere in bezug auf die über den regulären Haushalt hinausgehende Einwerbung öffentlicher als auch nicht-öffentlicher Finanzmittel zu verzeichnen.

Angesichts der aufgezeigten Umweltveränderungen sehen sich Hochschulen zunehmend dazu veranlaßt, neben gemeinwohlorientierten Zielsetzungen - unter Berücksichtigung des gesetzlichen Auftrags - auch quasi-ökonomische Ziele im Rahmen des Hochschulmarketing zu verfolgen. Neben psychographischen Zielen nimmt insbesondere das Ziel der Drittmitteleinwerbung über eine Nachfragesteigerung im Bereich der Forschungsdienstleistungen als ökonomisches Ziel einen zunehmenden Stellenwert ein. Dies impliziert eine notwendige Orientierung der

Vgl. De Gijsel, P., Ziesemer, T., Hochschulreform in den Niederlanden in den achtziger und neunziger Jahren und ihre Implementierung, in: Grötzinger, G., Hödl, E. (Hrsg.), Hochschulen im Niedergang? Zur politischen Ökonomie von Lehre und Forschung, Marburg 1994, S. 101.

Vgl. Peisert, H., Framhein, G., Das Hochschulsystem in Deutschland, hrsg. vom Bundesministerium für Bildung, Wissenschaft, Forschung und Technologie, 2. Aufl., Bonn 1997, S. 32. Die Bundesländer regeln den Hochschulbereich durch Gesetze, Verordnungen und Verwaltungsakte, wobei das in Bundeskompetenz fallende Hochschulrahmengesetz den Hochschulgesetzen der Länder als Grundlage dient und trotz länderspezifischer Regelungen für die erforderliche Einheitlichkeit des Hochschulbereichs sorgt. Vgl. Gaugler, E., Hochschulen, in: Chmielewicz, K., Eichörn, P. (Hrsg.), Handwörterbuch der öffentlichen Betriebswirtschaft, Stuttgart 1989, Sp. 583. Aufgrund dieser engen rechtlichen Beziehungen besteht eine hohe Abhängigkeit der Hochschulen vom Staat, die sich insbesondere im Rahmen der Finanzierung und der Haushaltsverwaltung niederschlägt. Vgl. Schober, K., Grundlagen des Fakultätsmarketing, Arbeitspapier Nr. 56 des Lehrstuhls für Marketing, WiSo-Fakultät Nürnberg, Erlangen-Nürnberg 1998, S. $3 \mathrm{f}$.

Vgl. Trogele, U., Strategisches Marketing für deutsche Universitäten: Die Anwendung von Marketing-Konzepten amerikanischer Hochschulen in deutschen Universitäten, Frankfurt am Main u.a. 1995, S. 1; o.V., Zwischen Erkenntnis und Vermarktung, in: Wirtschaftsspiegel, 53. Jg., Heft 8, 1998, S. $10 \mathrm{ff}$.

Der Wettbewerb zwischen den Hochschulen wird im allgemeinen als effizienzsteigernd für das Hochschulmanagement angesehen. Bereits Mitte der 80er Jahre wurden diesbezüglich vom Wissenschaftsrat Empfehlungen gegeben. Vgl. Wissenschaftsrat (Hrsg.), Empfehlungen zum Wettbewerb im deutschen Hochschulsystem, Köln 1985, S. 23 ff.; Turner, G., Mangelnder Ansporn, in: Wirtschaftswoche, 46. Jg., Heft 42, 1992, S. 74. Vgl. hierzu auch Wissenschaftliche Gesellschaft für Marketing und Unternehmensführung e.V. (Hrsg.), Memorandum zur wirtschaftswissenschaftlichen Ausbildung in Deutschland, in: Meffert, H., Wagner, H., Backhaus, K. (Hrsg.), Entwicklungsperspektiven der wirtschaftswissenschaftlichen Hochschulausbildung, a.a.O., S. $57 \mathrm{f}$. 
Forschungsaktivitäten an den Interessen der Wirtschaft sowie anderen hochschulspezifischen Anspruchsgruppen.

\section{Vor diesem Hintergrund steigt die Notwendigkeit einer Marktorientierung des} Hochschulmanagement, wenngleich öffentliche Hochschulen grundsätzlich nicht-marktlichen Koordinationsmechanismen unterliegen. ${ }^{14}$ Zur Marktorientierung von Hochschulen werden in der Literatur zahlreiche Ansätze diskutiert. ${ }^{15}$ Während in der angelsächsischen Literatur - nicht zuletzt aufgrund der Unterschiede zwischen deutschen und amerikanischen Hochschulsystemen und deren spezifischen Rahmenbedingungen - der Schwerpunkt auf der Betrachtung des langfristigen strategischen Marketingansatzes für Hochschulen liegt, ${ }^{16}$ wird in der deutschsprachigen Literatur der Marketingansatz stark auf ein einheitliches Erscheinungsbild fokussiert und damit auf ein Selbstdarstellungskonzept reduziert. ${ }^{17}$ Im

Das Hochschulmarketing läßt sich aus institutioneller Sicht in den Bereich des Marketing für Nonprofit-Organisationen, dem Marketing für öffentliche Betriebe - strenggenommen dem Marketing für öffentliche Verwaltungen - zuordnen. Vgl. Raffée, H., Fritz, W., Wiedmann, K.-P., Marketing für öffentliche Betriebe, Stuttgart, Berlin, Köln 1994, S. 19 ff. Zum Marketing für öffentliche Verwaltungen vgl. Bargehr, B., Marketing in der öffentlichen Verwaltung: Ansatzpunkte und Entwicklungsperspektiven, Stuttgart 1991. Zum Nonprofit-Marketing vgl. Kotler, P., Andreasen, A. R., Marketing for Nonprofit Organisations, 5th Ed., New Jersey 1996. Der Transfer des Marketinggedankens auf nicht-kommerzielle Bereiche ist seit seiner unter den Schlagwörtern Deepening und Broadening des Marketing diskutierten Einführung Ende der 60 er Jahre inzwischen in der Marketingwissenschaft allgemein anerkannt. Vgl. Meffert, $\mathrm{H}$., Marketing - Grundlagen marktorientierter Unternehmensführung, 8. Aufl., Wiesbaden 1998, S. $1183 \mathrm{ff}$. Zur Vertiefung des kommerziellen Marketing (Deepening) vgl. Dawson, L. M., The human concept: New philosophy for business, in: BH, Vol. 12, No. 12, 1969, S. 29 ff. Zur Ausweitung des klassischen Marketinggedankens auf nicht-kommerzielle Bereiche (Broadening) vgl. Kotler, P., Levy, S. J., Broadening the concept of Marketing, in: Marketing Journal, Vol. 33, No. 1,1969 , S. $10 \mathrm{ff}$.

Vgl. z.B. Alewell, K., Marketing-Management für Universitäten: Umweltbezogene Führung von Universitäten, in: ZłO, 46. Jg., Heft 5, 1977, S. 263-274; Wangen-Goss, M., Marketing für Universitäten: Möglichkeiten und Grenzen der Übertragbarkeit des Marketing-Gedankens auf den universitären Bereich, Spardorf 1983.

Vgl. Doyle, P., Lynch, J. E., A Strategic Model for University Planning, in: Journal of the Operational Research Society, Vol. 30, No. 7, 1979, S. 603-609; Keller, G., Academic Strategy: The Management Revolution of American Higher Education, Baltimore, London 1983; Kotler, P., Fox, K. F. A., Strategic Marketing for Educational Institutions, 2nd Ed., New Jersey 1995; Doyle, P., Newbould, G. D., A Strategic Model for University Planning, in: Ryans, C., Shanklin, W. (Ed.), Strategic Planning, Marketing, Public Relations and Fund Raising in Higher Education, London 1986, S. 15-23.

Vgl. Simon, H., Kucher, E., Akademischer Wettbewerb, in: asw, 31. Jg., Heft 31, 1988, S. 144147; Block, H. J., Maßnahmen für eine Förderung des leistungssteigernden Wettbewerbs im deutschen Hochschulsystem, in: Helberger, C. (Hrsg.), Ökonomie der Hochschule I, Berlin 1989, S. 153-179; Frackmann, E., Informationsmanagement der Hochschule im Wettbewerb: Amerikanische Erfahrungen zu einer Betriebswirtschaftslehre der Hochschule, in: DBW, 47. Jg., Heft 6, 1987, S. 717-736; Frackmann, E., Die Bedeutung von „Rankings“ für den 
operativen Bereich wird dabei vor allem die Öffentlichkeitsarbeit als ein wichtiges Instrument des Marketing-Mix von Hochschulen herausgestellt. ${ }^{18}$

Zur Realisierung der Einwerbung von Drittmitteln bedarf es des Austausches von Transferleistungen zwischen Hochschulen und an Forschungsleistungen interessierten Anspruchsgruppen. Dabei kommt dem Kontakt zwischen den Transaktionspartnern eine zentrale Bedeutung zu. Nicht zuletzt aufgrund der fehlenden Institutionalisierung derartiger Kontakte im Hochschulbereich gewinnen Messen als Ort des Zusammentreffens zwischen Ausstellern als Anbietern und Messebesuchern als Nachfragern von Forschungsergebnissen eine besondere Bedeutung. ${ }^{19}$ Messen zeichnen sich vor allem durch ein hohes Maß an persönlichem Kontakt und Interaktivität zwischen Ausstellern und Messebesuchern aus und bieten vielfältige Möglichkeiten zur Besichtigung sowie Erklärung von Ausstellungsobjekten. Sowohl für Aussteller als auch für Messebesucher haben sie einen hohen Erlebniswert und verfügen für beide Gruppen über einen umfangreichen Informationsnutzen aufgrund der unmittelbaren und i.d.R. umfassenden Möglichkeit zu Konkurrenzvergleichen. ${ }^{20}$ Deshalb werden Messen oftmals auch als "Markt für Informationen" bezeichnet. ${ }^{21}$

Wettbewerb im Hochschulbereich, in: Betriebswirtschaftliche Forschung und Praxis, 40. Jg., Heft 2, 1988, S. 139-162; Harpenau, J., Selbstdarstellung als Instrument im Wettbewerb zwischen Hochschulen: Ein Beitrag aus regionalwirtschaftlicher Sicht am Beispiel der Universität Hannover, Bern 1991.

Vgl. Topf, C., Öffentlichkeitsarbeit im Rahmen des Hochschulmarketing - Interne und externe Informations- und Kommunikationsbeziehungen der Hochschulen, Frankfurt am Main 1986.

Dies gilt insbesondere für einen technisch orientierten Austausch zwischen Wissenschaft und Praxis. Vgl. Meier, H.-G., Technologietransfer via Messen und Ausstellungen am Beispiel des Forschungsmarktes Berlin, in: TU International, 28./29. Mai 1995, S. 4 ff.; Goehrmann, K. E., Natur- und ingenieurwissenschaftliche Hochschulinstitute sowie andere Forschungsstellen leisten wichtige Beiträge - Biotechnika: Horizonte rücken näher, in: HB, Nr. 182, 23.09.1987, S. 16; Goehrmann, K. E., Die ungenutzten Instrumente der Wirtschafts-, Technologie- und Strukturpolitik aktivieren - Messen können Technologie-Akzente gezielt setzen, indem sie Ergebnisse aus Forschungsprogrammen umsetzen helfen, in: HB, Nr. 249, 31.12.1985, S. 30.

Vgl. Backhaus, K., Messen als Institutionen der Informationspolitik, in: Strothmann, K.-H., Busche, M. (Hrsg.), Handbuch Messemarketing, Wiesbaden 1992, S. 91 ff.; Rost, D., Messen als Kommunikations-Problem, in: Deutsche Werbewissenschaftliche Gesellschaft e.V. (DWG) (Hrsg.), Messen als Marketinginstrument, Bonn 1983, S. $35 \mathrm{ff}$.

Vgl. Meffert, H., Neuere Entwicklungen in Kommunikation und Vertrieb, in: Meffert, H., Necker, T., Sihler, H. (Hrsg.), Märkte im Dialog: Die Messen der dritten Generation, Leipzig 1997, S. 50; Necker, T., Globale Herausforderung, Dienstleistungs- und Informationszeitalter, in: Meffert, H., Necker, T., Sihler, H. (Hrsg.), Märkte im Dialog: Die Messen der dritten Generation, a.a.O., S. 30; Backhaus, K., Messen als Institutionen der Informationspolitik, a.a.O., S. $89 \mathrm{f}$. 
Im Hochschulbereich wurden diese vielfältigen Messepotentiale in den letzten Jahren zunehmend erkannt. Während bspw. auf der Hannover Messe, die eine internationale, thematisch breit angelegte Messe des Industriebereichs darstellt, im Jahre 1991 nur zwei Hochschulen ihre Exponate ausstellten, präsentierten sich im Jahre 1995 bereits 139 Hochschulen mit ihren Forschungsergebnissen. Im Zeitraum von 1995 bis 1999 stieg die Anzahl der Hochschul-Exponenten auf der Hannover Messe nochmals um $12 \%$ auf 155 ausstellende Hochschulen. Ähnliche Entwicklungen sind auf spezialisierteren Messen zu verzeichnen. Auf der CeBIT bspw., die als eine auf den IT-Bereich spezialisierte Messe gilt, stieg die Zahl der Hochschul-Exponenten von 107 im Jahr 1995 um $15 \%$ auf 123 im Jahre 1999. ${ }^{22}$

Im Rahmen ihrer Messebeteiligungen werden Hochschulen mit zahlreichen Entwicklungen des Messewesens konfrontiert, die sich insbesondere in einer Angebotsausweitung $^{23}$ und Internationalisierung ${ }^{24}$ der Messen äußern. Darüber hinaus werden bestehende Messen zunehmend einer Differenzierung durch Abspaltung bestimmter Aussteller- und Besuchersegmente in eigens konzipierte Messeveranstaltungen unterzogen. Infolge dieser Entwicklungen werden derzeit für nahezu alle Wirtschaftsbereiche Messen mit jeweils spezifischen Themenschwerpunkten ausgerichtet. ${ }^{25}$

Angesichts des expandierenden Messeangebots bei gleichzeitig hohen Messebeteiligungskosten und knappen Hochschulbudgets wird neben einer verstärkten Messeselektion insbesondere auch die Überprüfung des Erfolgs von

Die Daten wurden nicht veröffentlichten, internen Studien der Deutschen Messe AG, Hannover, entnommen.

In Deutschland wurden allein für das Jahr 1997 insgesamt 101 neue Messeveranstaltungen gemeldet, von denen 87 Veranstaltungen durchgeführt wurden. Vgl. Ausstellungs- und MesseAusschuss der Deutschen Wirtschaft e.V. (AUMA) (Hrsg.), Die Messewirtschaft 1997/1998, Bergisch Gladbach 1998, S. 18.

Vgl. Krebs, H., Messe als internationaler Marktplatz, in: Messen und Ausstellungen, Beilage der FAZ, Nr. 15, 19.01.1999, S. B3. Zum Angebot an internationalen Messen vgl. Ausstellungs- und Messe-Ausschuss der Deutschen Wirtschaft e.V. (AUMA) (Hrsg.), AUMA-Handbuch International 1999, Bergisch Gladbach 1998.

Zum Angebot an überregionalen Messen vgl. Ausstellungs- und Messe-Ausschuss der Deutschen Wirtschaft e.V. (AUMA) (Hrsg.), AUMA-Handbuch Messeplatz Deutschland 1999, Bergisch Gladbach 1998. Zum Angebot an regionalen Messen vgl. ders., AUMA-Handbuch Regional 1999, Bergisch Gladbach 1998. Zum Angebot an Fachmessen vgl. ders., Messeplatz Deutschland 1999/2000 - Fachmessen, Termine, Fakten, Bergisch Gladbach 1998. 
Hochschul-Messebeteiligungen unabdingbar. ${ }^{26}$ Bis heute fehlt eine wissenschaftliche Auseinandersetzung mit Messebeteiligungen von Hochschulen, obwohl die ältesten wissenschaftlichen Veröffentlichungen zum Messewesen bis in das 19. Jahrhundert zurückreichen. ${ }^{27}$ Bis zum Ende der 70er Jahre wurden sowohl in der wissenschaftlichen als auch populärwissenschaftlichen Literatur Messebeteiligungen eher vernachlässigt. ${ }^{28}$ Seitdem wurden Messebeteiligungen kommerzieller Institutionen in der Literatur ausführlich diskutiert, ${ }^{29}$ wohingegen Messebeteiligungen nicht-kommerzieller Institutionen weitgehend unberücksichtigt blieben. Gegenüber kommerziellen Messebeteiligungen ergeben sich aufgrund hochschulspezifischer Rahmenbedingungen und Strukturen zahlreiche Besonderheiten, die eine spezifische Betrachtung von Hochschul-Messebeteiligungen notwendig erscheinen lassen.

Belegt wird diese Aussage nicht zuletzt auch dadurch, daß Evaluierungen von HochschulMessebeteiligungen zunehmend in der Diskussion von Experten stehen. Vgl. Expertenworkshop zu Messebeteiligungen von Hochschulen, Kiel 1999, Anhang I.

Vgl. bspw. Exner, W. F., Der Aussteller und die Ausstellungen - Erörterungen vor allem über die Messe von Wien 1873, Weimar 1873.

Vgl. Meffert, H., Einführung in die Problemstellung, in: Meffert, H., Wagner, H. (Hrsg.), Messemarketing - Bestandsaufnahme und Perspektiven, Dokumentationspapier Nr. 52 der Wissenschaftlichen Gesellschaft für Marketing und Unternehmensführung e.V., Münster 1989, S. 1; Meffert, H., Gass, C., Messen und Ausstellungen im System des Kommunikationsmix ein entscheidungsorientierter Ansatz, Arbeitspapier Nr. 33 des Instituts für Marketing, Münster 1985, S. 1.

In der deutschsprachigen Literatur vgl. z.B. Huckemann, M., ter Weiler, D. S., Messen Meßbar Machen - Die 5 trojanischen Pferde des Messe-Marketing, 2. Aufl., Neuwied u.a. 1998; Naumann, C., Erfolgreich auf der Messe: Handbuch für die Beteiligung an Messen und Ausstellungen, Heidelberg 1993; Zotter, R., Erfolgreiches Messemarketing: Leitfaden für Analyse, Planung, Durchführung, Nachbearbeitung, Erfolgskontrolle bei Messebeteiligungen im In- und Ausland, Zürich 1993; Zerres, R., Ausstellen und Gewinnen: Der Kompass für die erfolgreiche Messepraxis, Woldert 1992; Földy, R., Die Messe als Markt - Beteiligungsstrategien für Aussteller, Wien 1990; Goschmann, K., Die erfolgreiche Beteiligung an Messen und Ausstellungen von A-Z, Landsberg am Lech 1988; Scheitlin, V., Messebeteiligung erfolgreich gestalten, St. Gallen, Stuttgart, Steyr 1984; Kustenaar, J., Messehandbuch - ein Leitfaden für Messebeteiligungen, Bern, Stuttgart 1983; Roth, G. D., Messen und Ausstellungen verkaufswirksam planen und durchführen, Landsberg am Lech 1981; Hoche, K., Handbuch für Aussteller, 111 Messetips, München 1974; Haeberle, K. E., Erfolg auf Messen und Ausstellungen: Handbuch für Teilnahme, Organisation, Gestaltung, Technik, Stuttgart 1967. In der angloamerikanischen Literatur vgl. z.B. Siskind, B., The Successful Exhibitor's Handbook: Trade Show Techniques for Beginners and Pros, 2. Ed., Vancouver, Bellingham 1994; Christman, C., The Complete Handbook of Profitable Trade Show Exhibiting, Englewood Cliffs 1991; Dudley, J. W., Successful Exhibiting, Holbrook 1991; Weintraub, D. K., Trade Show Exhibiting: The Insider's Guide for Entrepreneurs, o.O. 1991; Chapman, E. A., Exhibit Marketing: A Survival Guide for Managers, New York u.a. 1987. 


\section{Besonderheiten der Messebeteiligung von Hochschulen}

Grundlage einer wissenschaftlichen Auseinandersetzung mit der Messebeteiligung von Hochschulen ist die Definition und Abgrenzung der für die Untersuchung relevanten Begriffe. In diesem Zusammenhang ist es erforderlich, die hochschulspezifischen Rahmenbedingungen und Strukturen darzustellen, um darauf aufbauend Besonderheiten der Messebeteiligung von Hochschulen zu diskutieren.

\subsection{Messebeteiligungsrelevante Spezifika von Hochschulen}

Hochschulen haben sich zu Institutionen entwickelt, die in vielfältigen Beziehungen zu anderen Institutionen und Anspruchsgruppen stehen und durch zunehmende Komplexität innerer Strukturen geprägt sind. ${ }^{30}$ Zur Analyse der hochschulspezifischen Besonderheiten, die z.T. erheblichen Einfluß auf Messebeteiligungen im Hochschulbereich nehmen können, ist es somit notwendig, sowohl die einzelnen Strukturelemente der Hochschule abzubilden als auch deren Beziehungen untereinander und zu ihrer Umwelt zu verdeutlichen. Für dieses Vorhaben bietet sich eine systemorientierte Betrachtung von Hochschulen an. Der Mehrzahl systemtheoretischer Forschungsansätze folgend, soll hier zwischen globaler Umwelt und Aufgabenumwelt der Hochschule unterschieden werden. ${ }^{31}$

Die globale Umwelt der Hochschule beinhaltet nichtkontrollierbare Faktoren, die sowohl das Verhalten von Hochschulen als auch von Transaktionspartnern der Aufgabenumwelt mittelbar beeinflussen können. Diese Einflüsse, die auch das Messeengagement von Hochschulen tangieren, liegen in ökonomischen, technologischen, ökologischen, soziokulturellen und politisch-rechtlichen Umweltentwicklungen begründet. ${ }^{32}$ Letztere nehmen aufgrund des starken Steuerungseinflusses, den der Staat auf die Gestaltung der Strukturen und Inhalte der Aktivitäten von

Vgl. Bolsenkötter, H., Ökonomie der Hochschule: Eine betriebswirtschaftliche Untersuchung (Bd. I), Baden-Baden 1976, S. 1 ff.

Vgl. Meffert, H., Marketing - Grundlagen marktorientierter Unternehmensführung, a.a.O., S. 28.

32 Vgl. Heinzel, M., Anforderungen deutscher Unternehmen an betriebswirtschaftliche Hochschulabsolventen - Zur Marktorientierung von Hochschulen, Wiesbaden 1997, S. 38 f.; Trogele, U., Strategisches Marketing für deutsche Universitäten: Die Anwendung von MarketingKonzepten amerikanischer Hochschulen in deutschen Universitäten, a.a.O., S. 34 ff. 
Hochschulen ausübt, eine besondere Stellung in der globalen Umwelt von Hochschulen ein.

Die Aufgabenumwelt bildet die Beziehungen von Hochschulen zu externen Transaktionspartnern ab. ${ }^{33}$ Diese Betrachtung basiert auf transaktionstheoretischen und aus der Anreiz-/Beitragstheorie abgeleiteten Erkenntnissen. Die Umweltbeziehungen einer Hochschule lassen sich als Austauschbeziehungen kennzeichnen, ${ }^{34}$ durch welche Hochschulen die an sie herangetragenen Ansprüche verschiedener Transaktionspartner zu befriedigen versuchen. Damit kann jede Bezugsgruppe auch als Anspruchsgruppe bezeichnet werden. ${ }^{35}$ Bei den zur Erfüllung der Ansprüche initiierten Transaktionen einer Hochschule handelt es sich um Austauschprozesse, die auf dem Prinzip der Gegenseitigkeit beruhen und auch im Rahmen von Messebeteiligungen zu berücksichtigen sind. ${ }^{36}$ Die Transaktionen können dabei auf verschiedenen Teilmärkten erfolgen, wobei diese zum einen dem Beschaffungs- und zum anderen dem Leistungsmarkt der Hochschulen zuzuordnen sind. Grundsätzlich können Hochschulen durch Messebeteiligungen Transaktionsprozesse auf beiden Märkten initiieren.

\section{Der Beschaffungsmarkt von Hochschulen dient einerseits der Akquirierung von} „Human Ressources“ wie Wissenschaftlern, Studierenden und Hochschulverwaltungsangestellten sowie andererseits der Beschaffung von Finanz- und Sachmitteln. ${ }^{37}$ In bezug auf die Finanzmittelbeschaffung lassen sich öffentliche Haushaltsmittel und Drittmittel unterscheiden. Insbesondere die Beschaffung von Drittmitteln, die sowohl aus der öffentlichen Hand (z.B. öffentliche Förderung) als

Vgl. Meffert, H., Marketing - Grundlagen marktorientierter Unternehmensführung, a.a.O., S. 28.

Vgl. Levy, S. J., Zaltman, G., Marketing, Society and Conflict, Englewood Cliffs 1975, S. 40 ff.; Bagozzi, R., Marketing as an Organized Behavioral System of Exchange, in: JoM, Vol. 38, No. 4, 1974, S. 77 ff.; Homans, G. C., Soziales Verhalten als Austausch, in: Hartmann, H. (Hrsg.), Moderne Amerikanische Soziologie: Neuere Beiträge zur Soziologischen Theorie, 2. Aufl., Stuttgart 1973, S. $245 \mathrm{ff}$.

Vgl. Meffert, H., Kirchgeorg, M., Marktorientiertes Umweltmanagement, 3. Aufl., Stuttgart 1998, S. 94 ff.; Dyllick, T., Management der Umweltbeziehungen - Öffentliche Auseinandersetzung als Herausforderung, Wiesbaden 1989, S. $42 \mathrm{ff}$.

Zu Anreiz-Beitrags-Relationen von Messen vgl. Robertz, G., Strategisches Messemanagement im Wettbewerb - ein Beitrag aus markt-, ressourcen- und koalitionsorientierter Sicht, Wiesbaden 1999, S. $50 \mathrm{ff}$. 
auch dem wirtschaftlichen Bereich (private und gewerbliche Mittel) stammen, ${ }^{38}$ kann durch Messebeteiligungen entsprechend forciert werden. Die Sachmittelbeschaffung, die bspw. zur literarischen und technischen Ausstattung der Hochschule dient, ${ }^{39}$ ist hingegen durch Messebeteiligungen i.d.R. nur indirekt über die Einwerbung der Finanzmittel realisierbar.

Auf dem Leistungsmarkt lassen sich die Hochschulleistungen funktionell - nicht jedoch organisatorisch und finanziell - in die drei Sektoren Forschung, Lehre und sonstige Dienstleistungen untergliedern. ${ }^{40}$ Eine der zentralen Leistungen von Hochschulen ist die Forschung, d.h. die Gewinnung neuer Erkenntnisse in methodischer und nachprüfbarer Weise einschließlich der Entwicklung von Verfahren zur Anwendung dieser Erkenntnisse. ${ }^{41}$ Dabei wird zwischen Grundlagenforschung und angewandter Forschung unterschieden. ${ }^{42}$ Während bei der Grundlagenforschung die Leistungsverwertung vorerst unbestimmt ist, sollen die Erkenntnisse der angewandten Forschung unmittelbar in die Praxis transferiert werden. ${ }^{43}$ Folglich kommt gerade inr eine besondere Bedeutung für Messebeschickungen zu. Die Lehre ${ }^{44}$, die in erster Linie der Vorbereitung von Studierenden auf ein berufliches Tätigkeitsfeld und der Vermittlung der dafür erforderlichen fachlichen Kenntnisse, Fähigkeiten sowie Methoden auf wissenschaftlicher Grundlage $^{45}$ dient, eignet sich hingegen im Vergleich zu Forschungsergebnissen

Zu den verschiedenen Formen von Finanzmitteln und deren Beschaffung vgl. Bolsenkötter, $H$., Ökonomie der Hochschule: Eine betriebswirtschaftliche Untersuchung (Bd. II), Baden-Baden 1976, S. $478 \mathrm{ff}$.

Zur Sachmittelbeschaffung vgl. Bolsenkötter, H., Ökonomie der Hochschule: Eine betriebswirtschaftliche Untersuchung (Bd. II), a.a.O., S. 603 ff.

Vgl. Alewell, K., Marketing-Management für Universitäten: Umweltbezogene Führung von Universitäten, a.a.O., S. 264.

Vgl. Wissenschaftsrat (Hrsg.), Empfehlungen zur Organisation, Planung und Förderung der Forschung, Bonn 1975, S. 25. Zur Diskussion der Hochschule als Institution der Forschung vgl. Bolsenkötter, H., Ökonomie der Hochschule: Eine betriebswirtschaftliche Untersuchung (Bd. I), a.a.O., S. $295 \mathrm{ff}$.

Aufgrund der oftmals nicht möglichen klaren Abgrenzung zwischen den Forschungsarten ist die Unterteilung der Forschung in Grundlagen- und angewandte Forschung nicht unbestritten. Vgl. Bolsenkötter, H., Ökonomie der Hochschule: Eine betriebswirtschaftliche Untersuchung (Bd. I), a.a.O., S. $295 \mathrm{ff}$.

Vgl. Schober, K., Grundlagen des Fakultätsmarketing, a.a.O., S. 5.

Unter dem von der Gesetzgebung übernommenen Terminus "Lehre“ werden alle Formen der Aus- und Weiterbildung subsumiert.

45 Vgl. $\$ 7$ HRG. Die Gesetzgebung unterscheidet zwischen „Lehre und Studium“, wobei beide Termini letztendlich dasselbe darstellen und sich nur durch die Sichtweise unterscheiden.

(Fortsetzung der Fußnote auf der nächsten Seite) 
vor allem aufgrund ihrer Immaterialität ${ }^{46}$ weniger zur Präsentation auf Messen. Zudem ist die Darstellung der Lehre angesichts der eher nur bedingten Transfereignung in bezug auf die direkte Drittmitteleinwerbung weniger zielführend. Neben der Forschung und Lehre, die die Hauptleistungen des Hochschulbereichs darstellen, ${ }^{47}$ bieten die Hochschulen sonstige Dienstleistungen an. $\mathrm{Zu}$ diesen zählen bspw. klinische Leistungen sowie Beratungs- und Gutachtertätigkeiten, ${ }^{48}$ die zur Messepräsentation insbesondere aufgrund ihrer Immaterialität i.d.R. weniger geeignet sind als Ergebnisse der angewandten Forschung.

In Abb. 1 werden die zentralen Elemente der systemorientierten Betrachtung von Hochschulen zusammenfassend dargestellt sowie die Struktur der Institution Hochschule skizziert, auf die im folgenden näher eingegangen wird.

Während bei der Lehre die leistende Hochschule in den Vordergrund gestellt wird, steht beim Studium der Studierende im Mittelpunkt der Betrachtung. Vgl. Weber, J., Hochschulcontrolling: Das Modell der WHU, Stuttgart 1996, S. 25. Zur Diskussion der Hochschule als Institution der Lehre vgl. Bolsenkötter, H., Ǒkonomie der Hochschule: Eine betriebswirtschaftliche Untersuchung (Bd. I), a.a.O., S. $173 \mathrm{ff}$.

Zur Immaterialität von Dienstleistungen als eine dienstleistungsspezifische Besonderheit sowie zu weiteren Besonderheiten von Dienstleistungen vgl. Meffert, H., Bruhn, M., Dienstleistungsmarketing: Grundlagen - Konzepte - Methoden, 2. Aufl., Wiesbaden 1997, S. 59 ff.

Vgl. § 2 Abs. 1 HRG. Empirische Untersuchungen bestätigen enge Verbindungen zwischen Forschung und Lehre. Vgl. Backes-Gellner, U., Zanders, E., Lehre und Forschung als Verbundproduktion, in: ZfB, 59. Jg., Heft 3, 1989, S. 271 ff. Im Laufe der Zeit haben sich Forschung und Lehre in Deutschland jedoch aufgrund der stark gestiegenen Spezialisierung und Fragmentierung des akademischen Wissens und der Überfüllung der Studiengänge, die forschungsnahe Lehrgespräche mit Studierenden verhindern, auseinanderentwickelt. Vgl. Weber, J., Hochschulcontrolling: Das Modell der WHU, a.a.O., S. 26.

Vgl. Alewell, K., Wissenschaftsmarketing, in: Tietz, B., Köhler, K., Zentes, J. (Hrsg.), Handwörterbuch des Marketing, Stuttgart 1995, Sp. 2779. Zur Diskussion sonstiger Dienstleistungen von Hochschulen vgl. Bolsenkötter, H., Ökonomie der Hochschule: Eine betriebswirtschaftliche Untersuchung (Bd. II), a.a.O., S. $398 \mathrm{ff}$. 


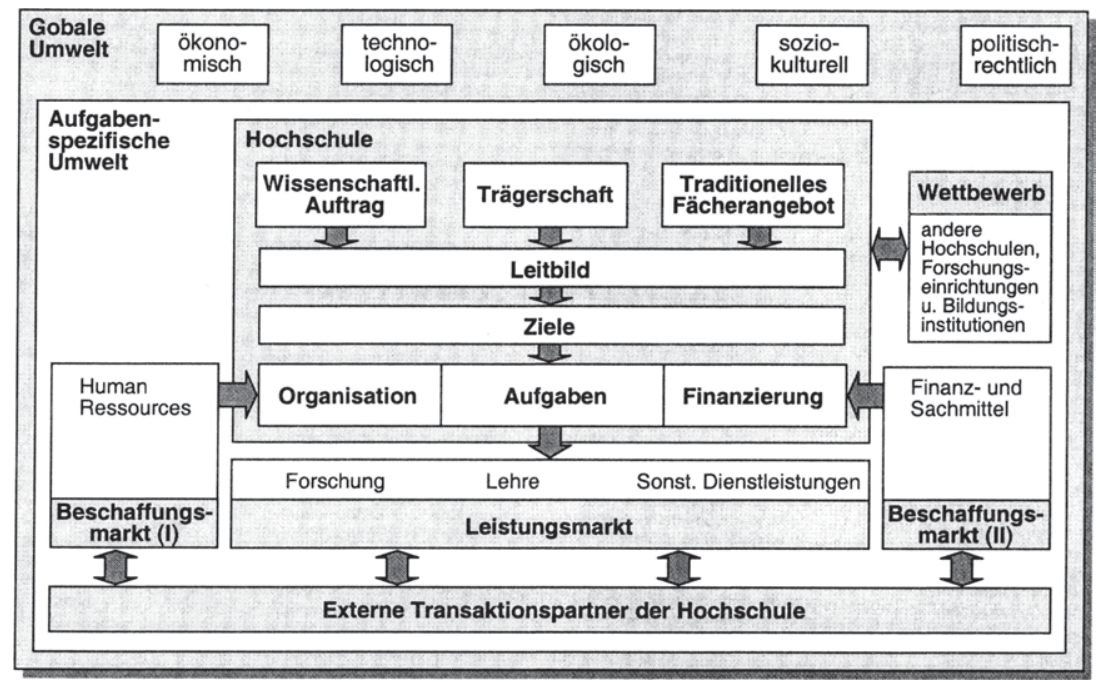

Abb. 1: Systemorientierte Betrachtung der Hochschule

In Deutschland können nach dem wissenschaftlichen Auftrag verschiedene Hochschularten unterschieden werden. $^{49}$ Universitäten, Gesamthochschulen, Theologische sowie Pädagogische Hochschulen und i.w.S. auch Kunsthochschulen sowie Technische Hochschulen gelten als wissenschaftliche Hochschulen. ${ }^{50}$ Universitäten sehen als rein wissenschaftliche Hochschulen die Forschung und Lehre als gleichrangig an. Fachhochschulen ${ }^{51}$ und auch Verwaltungsfachhochschulen stellen den Praxisbezug in den Vordergrund ihrer Ausbildung. Charakteristisch für Gesamthochschulen ist die Integration von Universitäts- und Fachhochschulleistungen. Grundsätzlich besitzen damit alle Hochschularten das Potential, Forschungserkenntnisse auf Messen zu präsentieren.

Vgl. Gaugler, E., Hochschulen, a.a.O., Sp. 581 f.; Hermeier, B., Konzept eines marketingorientierten Hochschulmanagement - theoretische Ansätze und empirische Befunde, Essen 1992, S. 25.

51 Vgl. Bundesministerium für Bildung und Wissenschaft (Hrsg.), Die Fachhochschulen in Deutschland, Bonn 1994, S. 6. 
In bezug auf die Trägerschaft werden staatliche Hochschulen und nicht-staatliche bzw. private Hochschulen unterschieden. ${ }^{52}$ Im Vergleich zu anderen Staaten (z.B. USA, Japan, Korea) sind die Hochschulen in deutschsprachigen Ländern (Deutschland, Schweiz, Österreich) zum größten Teil staatliche Einrichtungen. ${ }^{53}$ Aus diesem Grund stehen staatliche Hochschulen im Mittelpunkt der vorliegenden Untersuchung, da gerade für sie Messebeteiligungen zur Begegnung der aufgezeigten Entwicklungen - insbesondere der Verknappung öffentlicher Finanzmittel von besonderer Relevanz sind.

Hochschulen können darüber hinaus nach dem Fächerangebot, das einerseits in der Breite und andererseits in der Gestaltung inhaltlicher Schwerpunkte zum Ausdruck kommt, unterschieden werden. Während die Angebotsbreite, nach der Hochschulen mit breitem Fächerangebot von Spezialhochschulen mit eher engem Fächerangebot zu differenzieren sind, ${ }^{54}$ für eine Messebeteiligung eher von untergeordneter Bedeutung ist, kann die Gestaltung inhaltlicher Schwerpunkte auf das Messeengagement erheblichen Einfluß nehmen. Vor dem Hintergrund der Zielsetzung Drittmitteleinwerbung haben bspw. natur- und ingenieurwissenschaftliche Fachrichtungen oftmals geeignetere Forschungserkenntnisse zur Messepräsentation zu bieten als geisteswissenschaftliche Fachrichtungen, weil deren Transfereignung i.d.R. höher ausfällt als bei Erkenntnissen geisteswissenschaftlicher Fachrichtungen.

Vgl. Heinzel, M., Anforderungen deutscher Unternehmen an betriebswirtschaftliche Hochschulabsolventen - Zur Marktorientierung von Hochschulen, a.a.O., S. 36.

Unter den im Wintersemester 1994/95 existierenden 325 Hochschulen waren nur 57 private Hochschulen vertreten. Vgl. Bundesministerium für Bildung, Wissenschaft, Forschung und Technologie (Hrsg.), Grund- und Strukturdaten 1995/96, Referat Öffentlichkeitsarbeit, Bonn 1995, S. 135. Infolge der grundsätzlich verankerten Kulturhoheit der Bundesländer (Art. 30 GG) fungiert der Bund nur in Ausnahmefällen als Träger von Hochschulen (z.B. Universität der Bundeswehr). Träger privater Hochschulen sind neben kirchlichen Institutionen privatrechtlich organisierte Zusammenschlüsse aus der Wirtschaft bzw. gesellschaftlichen Gruppen. Vgl. Gaugler, E., Hochschulen, a.a.O., Sp. 582.

Als Indikator der Angebotsbreite wird i.d.R. die Anzahl der Studiengänge herangezogen. Vgl. Peisert, H., Framhein, G., Das Hochschulsystem in Deutschland, a.a.O., S. 42. Während früher von der Hochschulart auf die Breite des Fächerangebotes geschlossen werden konnte, ist dies heute aufgrund der Einführung von Gesamthochschulen, von Neugründungen oder durch Erweiterung bestehender Hochschulen nur noch erschwert möglich. Vgl. Erb, W. D., Differenzierung der wissenschaftlichen Hochschulen nach Größe, Alter und Angebotsstruktur, in: Giese, E. (Hrsg.), Aktuelle Beiträge zur Hochschulforschung, Giessener Geographische Schriften, Heft 62, Giessen 1987, S. 117. 
Sowohl das Fächerangebot und die Trägerschaft als auch der wissenschaftliche Auftrag bilden den Ausgangspunkt von Leitbild- und Zielfestlegung der Hochschulen. Das grundsätzliche Leitbild aller Hochschulen, das in Deutschland stark historisch geprägt ist, spiegelt sich im Hochschulrahmengesetz wider. ${ }^{55}$ Innerhalb dieses vorgegebenen gesetzlichen Rahmens kann sowohl auf der Ebene der einzelnen Hochschulen als auch auf den jeweiligen hochschulinternen Ebenen (bspw. der Fakultätsebene) eine Konkretisierung des Leitbildes vorgenommen werden, ${ }^{56}$ welches bei der Planung des Messeengagements entsprechend zu berücksichtigen ist.

Vom Leitbild determiniert werden die Ziele, die primär in der - für die Messebeteiligung besonders relevanten - Gewinnung wissenschaftlicher Erkenntnisse durch Forschung und in der - für eine Messebeteiligung eher weniger bedeutenden - Bildung des akademischen Nachwuchses durch Lehre liegen. ${ }^{57}$ Im Zielsystem der Hochschulen besitzen Wirtschaftlichkeitsziele als Formalziele keinen originären Charakter. ${ }^{58}$ Ihre Berücksichtigung bildet jedoch eine notwendige Voraussetzung zur Erreichung der im Mittelpunkt stehenden Sachziele, die sich aus den Hauptzielen Forschung und Lehre sowie aus den Nebenzielen, z.B. Erstellung sonstiger Dienstleistungen, zusammensetzen. ${ }^{59}$ Das Zielsystem von Hochschulen

Vgl. Heinzel, M., Anforderungen deutscher Unternehmen an betriebswirtschaftliche Hochschulabsolventen - Zur Marktorientierung von Hochschulen, a.a.O., S. 39. Nach dem Hochschulrahmengesetz fungiert die Wissenschaftsorientierung im Sinne der "Bildung durch Wissenschaft" als ein wesentliches Element des Leitbildes deutscher Hochschulen. Vgl. § 2 Abs. 1 HRG. Darüber hinaus sind die "Einheit von Forschung und Lehre" als Ergebnis der Humboldtschen Universitätsreform und die im Grundgesetz sowie im Hochschulrahmengesetz explizit formulierte, auf Forschung und Lehre bezogene "Freiheit der Wissenschaft" als weitere Bestandteile des Leitbildes hervorzuheben. Vgl. Art. 5 Abs. 3 GG.

Vgl. Heinzel, M., Anforderungen deutscher Unternehmen an betriebswirtschaftliche Hochschulabsolventen - Zur Marktorientierung von Hochschulen, a.a.O., S. $42 \mathrm{f}$. Das für die jeweilige Hochschule festgelegte Leitbild bestimmt die globale Richtung der Institution Hochschule und dient als Rahmen und Leitlinie für Hochschulleitung, -verwaltung, -dekane und -professoren. Vgl. Trogele, U., Strategisches Marketing für deutsche Universitäten: Die Anwendung von Marketing-Konzepten amerikanischer Hochschulen in deutschen Universitäten, a.a.O., S. 18.

Vgl. § 2 Abs. 1 HRG.

Vgl. Gaugler, E., Hochschulen, a.a.O., Sp. 585 f. In der betriebswirtschaftlichen Literatur wird zwischen Formal- und Sachzielen unterschieden, wobei sich die Sachziele auf die Art, die Menge und den Zeitpunkt der am Markt abzusetzenden Leistungen beziehen und die Formalziele auf die Wirtschaftlichkeit - vor allem die Rentabilität - gerichtet sind. Vgl. Kosiol, E., Die Unternehmung als wirtschaftliches Aktionszentrum: Einführung in die Betriebswirtschaftslehre, Reinbek/Hamburg 1972, S. 223.

59 Auf der Ebene der Hochschulen kann eine weitere Ausdifferenzierung der jeweiligen Ziele vorgenommen werden. Nicht zuletzt aufgrund des starken Steuerungseinflusses des Staates 
wird darüber hinaus von Auflagen und Nebenbedingungen geprägt, ${ }^{60}$ die insbesondere in der öffentlich-rechtlichen Stellung der Institution Hochschule begründet liegen. Die folgende Abbildung stellt die Struktur des vom Leitbild determinierten Hochschul-Zielsystems, das im Rahmen des Zielbildungsprozesses bei Messebeteiligungen Berücksichtigung finden sollte, schematisch dar und deutet auf die daraus abzuleitenden Hochschulaufgaben hin.

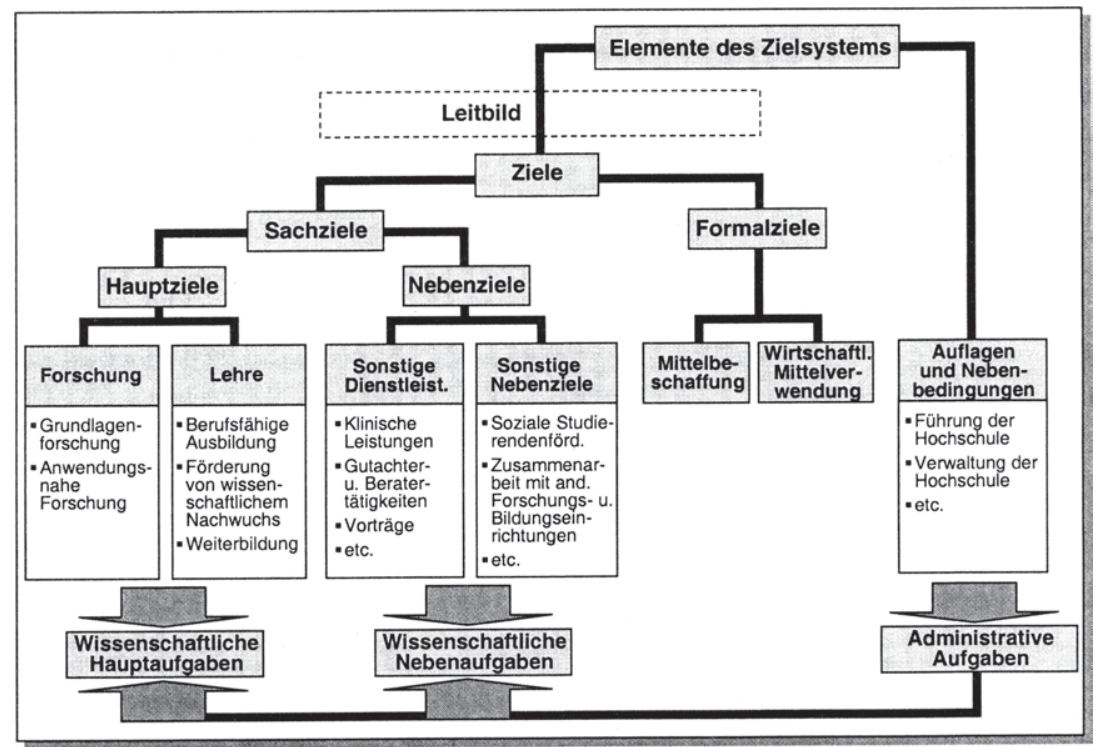

\section{Abb. 2: Zielsystem von Hochschulen}

(Quelle: In Anlehnung an Bolsenkötter, H., Die Hochschule als Betrieb, a.a.O., S. 651; Topf, C., Öffentlichkeitsarbeit im Rahmen des Hochschulmarketing - Interne und externe Informations- und Kommunikationsbeziehungen der Hochschulen, a.a.O., S. 99.)

ist ein solcher hochschulinterner Zielbildungsprozeß in Deutschland - im Gegensatz zu den USA - zumindest in staatlichen Hochschulen vergleichsweise gering ausgeprägt. Vgl. Heinzel, M., Anforderungen deutscher Unternehmen an betriebswirtschaftliche Hochschulabsolventen Zur Marktorientierung von Hochschulen, a.a.O., S. 42 f. Als Beispiel für die Realisierung eines hochschulinternen Zielbildungsprozesses ist die privatwirtschaftlich geführte WHU Koblenz zu nennen. Vgl. Weber, J., Hochschulcontrolling: Das Modell der WHU, a.a.O., S. $41 \mathrm{ff}$.

Vgl. Bolsenkötter, H., Die Hochschule als Betrieb, in: DUZ - Das Hochschulmagazin, 32. Jg., Heft 12, 1976, S. 651. 
Die Aufgaben der Hochschulen sind zum einen wissenschaftlich und zum anderen administrativ ausgerichtet. ${ }^{61}$ Während sich die wissenschaftlichen Aufgaben vor allem auf die Forschung und Lehre beziehen, ergeben sich die administrativen Aufgaben „aus der Leitung (Führung) und Verwaltung der Hochschule als körperschafts-rechtliche Institution des öffentlichen Dienstes “62. Die letzteren werden in bezug auf Messebeteiligungen weniger, die forschungsbezogenen Aufgaben hingegen stärker tangiert.

Die Aufgabenerfüllung wird stark von der Organisationsstruktur der Hochschule beeinflußt, ${ }^{63}$ bei der mit dem wissenschaftlichen Bereich, dem wissenschaftlichen Nebenbetrieb, dem Hilfsbetrieb und der Hochschulverwaltung vier Funktionsbereiche unterschieden werden. ${ }^{64}$ Der wissenschaftliche Bereich umfaßt dabei drei Ebenen: die Hochschulleitung, die Fachbereichs- bzw. Fakultätsebene sowie das Institut bzw. die Lehrstuhlebene. Die organisatorische Grundeinheit der Hochschule bildet der Fachbereich, der sich aus mehreren Instituten bzw. Lehrstühlen zusammensetzt. ${ }^{65} \mathrm{Zu}$ den Hilfs- und Nebenbetrieben zählen bspw. Hochschulbibliotheken, Rechenzentren und Studentenwerke, während die Hochschulverwaltung die Organisationseinheiten zur Erfüllung der allgemeinen administrativen

Die Aufgaben der Hochschulen sind im 1. Kapitel des Hochschulrahmengesetzes festgehalten. Vgl. $\S \S 2$ bis 26 HRG.

62 Stieger, H., Zur Ökonomie der Hochschule, Gießen 1980, S. 41. Die wissenschaftlichen Hochschulaufgaben umfassen gemäß des Zielsystems Haupt- und Nebenaufgaben, wobei sich erstere auf Forschung sowie Lehre und letztere auf sonstige Dienstleistungen beziehen. Dagegen sind die administrativen Aufgaben, die wiederum die wissenschaftlichen Aufgaben beeinflussen, insbesondere auf die Erfüllung des gesetzlichen Auftrags im Hinblick auf die Selbstverwaltung der Hochschule, auf das Zusammenwirken von Hochschule und Staat sowie auf die Hochschulorganisation gerichtet. Zum Selbstverwaltungsrecht der Hochschule vgl. § 58 HRG. Zur Rechtsaufsicht des Landes vgl. §59 HRG.

Die Organisation der Hochschule wird erheblich von den Mitgliedern der Hochschule geprägt. Zum Begriff der Organisation von Hochschulen vgl. Bolsenkötter, H., Ökonomie der Hochschule: Eine betriebswirtschaftliche Untersuchung (Bd. I), a.a.O., S. 71. Zu den Mitgliedern einer Hochschule zählen das wissenschaftliche und nicht-wissenschaftliche Personal sowie die Studierenden. Das wissenschaftliche Personal umfaßt Professoren, Hochschulassistenten, Wissenschaftliche Mitarbeiter, Lehrbeauftragte und Lehrkräfte für besondere Aufgaben sowie Tutoren. Vgl. §§ 42 bis 57 HRG.

Vgl. Gaugler, E., Hochschulen, a.a.O., Sp. 589.

65 Vgl. Heinzel, M., Anforderungen deutscher Unternehmen an betriebswirtschaftliche Hochschulabsolventen - Zur Marktorientierung von Hochschulen, a.a.O., S. 49 f. Größere wissenschaftliche Hochschulen setzen sich i.d.R. aus einer Vielzahl von Fachbereichen zusammen. Vgl. Gaugler, E., Hochschulen, a.a.O., Sp. 589. 
Aufgaben umfaßt. ${ }^{66}$ Unter der Zielsetzung der Drittmitteleinwerbung sind primär Einrichtungen des wissenschaftlichen Bereichs geeignet, Formen der zielgruppengerichteten Kommunikationspolitik zu nutzen und in diesem Zusammenhang Forschungsergebnisse auf Messen zu präsentieren.

Die Finanzierung der deutschen Hochschulen erfolgt vorwiegend - angesichts der überwiegend als staatliche Einrichtungen in Trägerschaft der Bundesländer fungierenden deutschen Hochschulen - über die jeweiligen Landeshaushalte. Die Finanzierungsmittel setzen sich dabei insbesondere aus Grund- und Drittmitteln sowie Verwaltungseinnahmen zusammen. ${ }^{67}$ Über die von den Länderhaushalten getragenen Grundmittel werden die laufenden Ausgaben für Personal und Sachmittel finanziert. ${ }^{68}$ Hochschulen haben zum Zwecke der Forschungsförderung die Möglichkeit, weitere Finanzmittel für die Durchführung von spezifischen Forschungsprojekten bei Dritten, wie bspw. der Deutschen Forschungsgemeinschaft (DFG), Stiftungen (Stifterverband etc.), einzuwerben. ${ }^{69}$ Weitere Drittmittelgeber können sowohl öffentliche als auch privatrechtliche Institutionen darstellen, zu deren Kontaktaufnahme sich Messebeteiligungen grundsätzlich anbieten können. Bei den Verwaltungseinnahmen der Hochschulen handelt es sich um die Erwirtschaftung eigener Hochschuleinnahmen, bspw. im Rahmen von klinischen Leistungen, ${ }^{70}$ deren Höhe nur im geringen Ausmaß durch Messebeteiligungen beeinflußbar ist.

Auf Grundlage der dargestellten Charakteristika von Hochschulen sowie dem Hochschulrahmengesetz ${ }^{71}$ folgend werden Hochschulen im folgenden verstanden als öffentlich-rechtliche Institutionen, die als nicht-kommerzielle Einrichtungen des tertiären Bildungssektors auf Grundlage von personellen, sachlichen und

Vgl. Bender, I., Henning, W. (Hrsg.), Universitätsmanagement, Trier 1980, S. 20 ff.; Gaugler, E., Hochschulen, a.a.O., Sp. 589.

Vgl. Heinzel, M., Anforderungen deutscher Unternehmen an betriebswirtschaftliche Hochschulabsolventen - Zur Marktorientierung von Hochschulen, a.a.O., S. $51 \mathrm{f}$.

Darüber hinaus werden Investitionen von Bauten und Großgeräten zu den Grundmitteln gezählt, die jedoch zur Hälfte vom Bund getragen werden. Vgl. Peisert, H., Framhein, G., Das Hochschulsystem in Deutschland, a.a.O., S. $54 \mathrm{ff}$.

Vgl. Bolsenkötter, H., Ökonomie der Hochschule: Eine betriebswirtschaftliche Untersuchung (Bd. II), a.a.O., S. $499 \mathrm{ff}$.

Vgl. Peisert, H., Framhein, G., Das Hochschulsystem in Deutschland, a.a.O., S. 54 ff. 
finanziellen Ressourcen Leistungen für die Gesellschaft im Bereich Forschung und Lehre, Wissenstransfer, Wirtschaftsförderung oder Kultur erbringen. Aufbauend auf diesem grundlegenden Verständnis von Hochschulen sollen im folgenden die hochschulspezifischen Besonderheiten einer Hochschul-Messebeteiligung und deren Einordnung in das Hochschulmarketing betrachtet werden.

\subsection{Messebeteiligungen im Rahmen des Hochschulmarketing}

Voraussetzung zur Einordnung von Messebeteiligungen in das Hochschulmarketing ist die Analyse des Marketingverständnisses im Hochschulbereich. Die Anwendung der Bezeichnung Marketing auf den Hochschulsektor verleitet nicht selten sowohl Wissenschaftler als auch Nicht-Wissenschaftler zu dem Mißverständnis, daß hier „Manipulations"-Instrumente aus dem „kommerziellen“ Bereich zu Hilfe genommen werden, was zum einen moralisch verwerflich, zum anderen nicht dem Hochschulzweck angemessen sei. ${ }^{72}$ Nicht zuletzt deshalb ist die Übertragung eines kommerziell-geprägten Marketing-Ansatzes auf die nicht-kommerzielle Institution Hochschule umstritten.

Wird jedoch Hochschulmarketing als nachfrage- und konkurrenzorientierte Managementkonzeption verstanden, welche die Analyse, Planung und Kontrolle aller Aktivitäten von Hochschulen umfaßt, die darauf abzielen, Leistungen an ausgewählte Zielgruppen zu vermitteln oder ihre Beziehungen zu relevanten Umweltbereichen zu pflegen, ${ }^{73}$ so werden die Chancen, die sich beim Einsatz des Marketing im Hochschulbereich ergeben können, offensichtlich. Im Rahmen des Hochschulmarketing werden nicht nur Probleme und Defizite im Hinblick auf die Aufgabenerfüllung identifiziert, sondern darüber hinaus Instrumente zur besseren Erfüllung des gesetzlichen Auftrags bereitgestellt. ${ }^{74}$ Des weiteren kann auf Basis der Analyse von Bedürfnissen und Wünschen der Transaktionspartner eine

72 Vgl. Meffert, H., Birkelbach, R., Frömbling, S., Die Westtälische Wilhelms-Universität Münster im Jahre 2010 - Bestandsaufnahme und Entwicklungsperspektiven, nicht veröffentlichtes Gutachten, Münster 1993, S. 1.

Vgl. Meffert, H., Birkelbach, R., Frömbling, S., Die Westfälische Wilhelms-Universität Münster im Jahre 2010 - Bestandsaufnahme und Entwicklungsperspektiven, nicht veröffentlichtes Gutachten, a.a.O., S. 1. 
höhere Zufriedenheit erzielt werden, welches wiederum die Ressourcenbeschaffung begünstigen kann.

Die Realisierung dieser Vorteile setzt im Hochschulbereich einen Management-

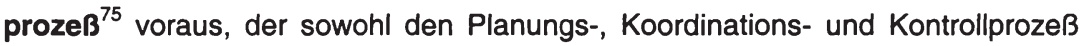
als auch den Transaktionsproze $\beta$ umfaßt und aus mehreren Phasen mit Rückkopplungsschleifen besteht. ${ }^{76}$ Ausgangspunkt bilden die Analyse der aktuellen und die Antizipation zukünftiger Umweltbedingungen der Hochschule, um Chancen und Risiken zu eruieren, sowie die Analyse interner Ressourcen (z.B. Personal, Finanz- und Sachmittel, organisatorische Strukturen) mit dem Ziel, Stärken und Schwächen der Hochschule zu ermitteln. Aufbauend auf diesen Erkenntnissen werden das spezifische Leitbild und die strategischen sowie operativen Ziele der Institution definiert. Auf dieser Basis können auf zu identifizierende Anspruchsgruppen zugeschnittene Strategien und operative Maßnahmen abgeleitet werden, die es anschließend zu realisieren gilt. Die Instrumente des Hochschulmarketing lassen sich in Anlehnung an das "klassische Marketing-Mix" ${ }^{77}$ den

Das Grundverständnis des betrieblichen Steuerungsprozesses der klassischen Managementlehre basiert auf dem Prozeß-Paradigma, wonach das Management als eine systematische Abfolge von Funktionen begriffen wird. Am Anfang steht die Planung als geistiger Entwurf der zukünftig zu erreichenden Ziele und der dazu zu ergreifenden Maßnahmen (Primat der Planung). Ihr folgen die Organisation als Strukturentwurf für den Aufgabenvollzug sowie die Führung als Veranlassung und Überwachung der Aufgabenrealisierung. Der Prozeß schließt mit der Kontrolle, die schließlich Informationen über den Zielerreichungsgrad an die Planung weitergibt, um bei zukünftigen Planungen Berücksichtigung zu finden. Zum Primat der Planung vgl. Adam, D., Planung und Entscheidung: Modelle - Ziele - Methoden; mit Fallstudien und Lösungen, 4. Aufl., Wiesbaden 1996, S. 12 Hf.; Griffith, R. W., Management, 2. Aufl., Boston 1987, S. $10 \mathrm{ff}$. Zur Einordnung der Prozeßfunktionen vgl. Gutenberg, E., Grundlagen der Betriebswirtschaftslehre, Bd. 1: Die Produktion, 24. Aufl., Berlin u.a. 1983, S. 235 f.; Gutenberg, E., Die Unternehmung als Gegenstand betriebswirtschaftlicher Theoriebildung, Frankfurt am Main 1967, S. 25 f.; Steinmann, H., Schreyögg, G., Management: Grundlagen der Unternehmensführung - Konzepte, Funktionen, Praxisfälle, Wiesbaden 1990, S. 103 ff.

Vgl. Meffert, H., Marketing - Grundlagen marktorientierter Unternehmensführung, a.a.O., S. 12 ff.; Kotler, P., Fox, K. F. A., Strategic Marketing for Educational Institutions, a.a.O., S. $93 \mathrm{ff}$.

Unter dem "klassischen Marketing-Mix" soll hier nach MefFent die Zuordnung der Marketinginstrumente zu den Mix-Bereichen Produkt-, Kontrahierungs- bzw. Gegenleistungs-, Distributions- und Kommunikationspolitik verstanden werden, die sich im Marketing allgemein durchgesetzt hat und auch für das Marketing öffentlicher Verwaltungen - und damit auch für Hochschulen - Gültigkeit besitzt. Vgl. Meffert, H., Marketing - Grundlagen marktorientierter Unternehmensführung, a.a.O., S. 317 ff. sowie S. 1172 ff. 
vier Bereichen Leistungs-, Gegenleistungs-, Distributions- und Kommunikationspolitik zuordnen. $^{78}$

Im Marketing-Mix von Hochschulen umfaßt die Leistungspolitik die tauschpartnergerechte Konzeption, Gestaltung und Differenzierung der Hochschulleistungen (Forschung, Lehre und sonstige Dienstleistungen) sowie die nachfragegerechte Bereitstellung dieser Leistungen. ${ }^{79}$ Im Bereich der Gegenleistungspolitik können für die meisten Transaktionen der Hochschulen keine eindeutigen Beziehungen zwischen Leistung und finanzieller Gegenleistung der Transaktionspartner festgelegt werden. So werden die Hauptaufgaben Forschung und Lehre bspw. überwiegend durch pauschale staatliche Zuwendungen abgegolten. ${ }^{80}$ Nur in wenigen Bereichen, wie der Drittmittelforschung, kann ein Preis als Gegenleistung für die Hochschulleistung verlangt werden. ${ }^{81}$ Die Distributionspolitik soll die Verfügbarkeit von Hochschulleistungen gewährleisten. ${ }^{82}$ Dabei haben die Hochschulen örtliche und räumliche Entscheidungen über Transaktionskanäle zu treffen, über die das Leistungsangebot an die Empfänger gelangen soll. ${ }^{83}$ Solche Transaktionskanäle sind bspw. Vorlesungen und Veröffentlichungen (z.B. allgemeine Publikationen, Dissertationen, Aufsätze in Fachzeitschriften). Im Rahmen der Kommunikationspolitik, der eine besondere Bedeutung als Instrument des Hochschulmarketing zugesprochen wird, ${ }^{84}$ steht die Verbreitung von Informationen im Vordergrund. Dabei geht es insbesondere um die sachgerechte Darstellung des spezifischen Leistungsprofils, der Qualität der Leistungserstellung und -ergebnisse sowie die Leistungsbereitschaft von Hochschulen gegenüber den verschiedenen Anspruchsgruppen. Hierdurch versuchen Hochschulen einerseits,

Vgl. Wangen-Goss, M., Marketing für Universitäten: Möglichkeiten und Grenzen der Übertragbarkeit des Marketing-Gedankens auf den universitären Bereich, a.a.O., S. $104 \mathrm{ff}$.

Vgl. Alewell, K., Wissenschaftsmarketing, a.a.O., Sp. 2781.

Vgl. Alewell, K., Marketing-Management für Universitäten: Umweltbezogene Führung von Universitäten, a.a.O., S. 268.

Vgl. Topf, C., Öffentlichkeitsarbeit im Rahmen des Hochschulmarketing - Interne und externe Informations- und Kommunikationsbeziehungen der Hochschulen, a.a.O., S. 50.

Vgl. Wangen-Goss, M., Marketing für Universitäten: Möglichkeiten und Grenzen der Übertragbarkeit des Marketing-Gedankens auf den universitären Bereich, a.a.O., S. 190.

Vgl. Gysin, R., Ansätze zur Universitätsplanung - dargestellt am Beispiel der Universität Basel, Basel 1973, S. 182.

Vgl. Nietiedt, T., Kommunikationspolitik für Hochschulen: Analyse und Instrumentarium, Frankfurt am Main 1996, S. 7 ff. 
bei aktuellen sowie potentiellen Nachfragern für ihr gesamtes Leistungsangebot Interesse zu wecken, und andererseits, die Erfüllung der ihr von der Gesellschaft übertragenen Aufgaben zu dokumentieren. ${ }^{85}$

Aus dieser instrumentellen Perspektive soll die Einordnung von Messebeteiligungen im dargestellten Marketing-Mix von Hochschulen diskutiert werden. In der allgemeinen Marketing-Literatur hat sich diesbezüglich nicht zuletzt aufgrund der sog. "Multifunktionalität ${ }^{466}$ von Messen keine einstimmig vertretene Sichtweise herauskristallisiert. Zahlreiche Autoren vertreten die Ansicht, daß Ausstellern mit Messebeteiligungen ein weitgehend eigenständiges Marketing-Instrument zur Verfügung steht, welches das „klassische Marketing-Mix" um einen zusätzlichen Instrumentalbereich erweitert. ${ }^{87}$ MEFFERT macht jedoch deutlich, daß eine Messebeteiligung im wesentlichen durch kommunikationspolitische Aktivitäten bestimmt wird. Demnach sind Messebeteiligungen dem kommunikationspolitischen Instrumentarium zuzuordnen ${ }^{88}$ und als eigenständiges Kommunikationsinstrument zu verstehen. Dieser Argumentation wird im Rahmen der vorliegenden Arbeit gefolgt.

Bei der Zuordnung von Messebeteiligungen in den Bereich der Kommunikationspolitik des Hochschulmarketing ist der spezifische Instrumentalcharakter von Messebeteiligungen zu berücksichtigen. Einerseits sind Messebeteiligungen zwar mehr als andere Kommunikationsinstrumente durch weitere Marketingmaßnahmen zu ergänzen, andererseits vermögen sie deren Wirkung aber auch viel-

Vgl. Tavenier, K., Strategisches Management in Universitäten, in: Bertelsmann Stiftung (Hrsg.), Evolution im Hochschulbereich, Gütersloh 1990, S. 55 ff.; Tavenier, K., MarketingManagement in Universitäten, in: Engelhardt, W. H., König, J., Nietiedt, T. (Hrsg.), Wissenschaftsmarketing, Hochschule und Region im Umbruch, Bochum 1993, S. $111 \mathrm{ff}$.

Unter Multifunktionalität wird hier die Möglichkeit zur persönlichen und unpersönlichen Kommunikation zwischen Aussteller und Messebesucher verstanden. Zur Multifunktionalität von Messen vgl. Groth, C., Lentz, I., Die Messe als Dreh- und Angelpunkt: Multifunktionales Instrument für erfolgreiches Marketing, Landsberg am Lech 1993, S. 1 ff.

Vgl. stellvertr. Selinski, H., Sperling U. A., Marketinginstrument Messe: Arbeitsbuch für Studium und Praxis, Köln 1995, S. 96 ff.; Groth, C., Lentz, I., Die Messe als Dreh- und Angelpunkt: Multifunktionales Instrument für erfolgreiches Marketing, a.a.O., S. 17 ff.; Jaspert, F., Das synergetische Potential ist groß - die Messe als Teil der betrieblichen Absatzpolitik, in: Küffner, G., Mortsiefer, J. (Hrsg.), Messeplatz Europa: Messen als Bestandteil des betrieblichen Marketings, Frankfurt am Main 1990, S. $36 \mathrm{ff}$.

Vgl. Meffert, H., Messen und Ausstellungen als Marketinginstrument, in: Verlag Wirtschaft und Finanzen (Hrsg.), Messen als Marketinginstrument, Düsseldorf 1988, S. 10. 
seitiger und z.T. gezielter zu unterstützen. ${ }^{89}$ So ist eine ausstellende Hochschule grundsätzlich in der Lage, auf dem Messestand gleichzeitig Beeinflussungswirkungen zu erzielen und i.S.d. Informationsgewinnung von Messebesuchern geäuBerte Erkenntnisse, Bedürfnisse sowie Probleme zur Verbesserung der Leistungspolitik aufzunehmen. Die daraus folgende Erfordernis einer Integration der Messeaktivitäten in weitere Kommunikationsmaßnahmen ${ }^{90}$ sowie die Abstimmung des gesamten Marketing-Mix weisen auf die Komplexität von Hochschul-Messebeteiligungen hin (vgl. Abb. 3). ${ }^{91}$

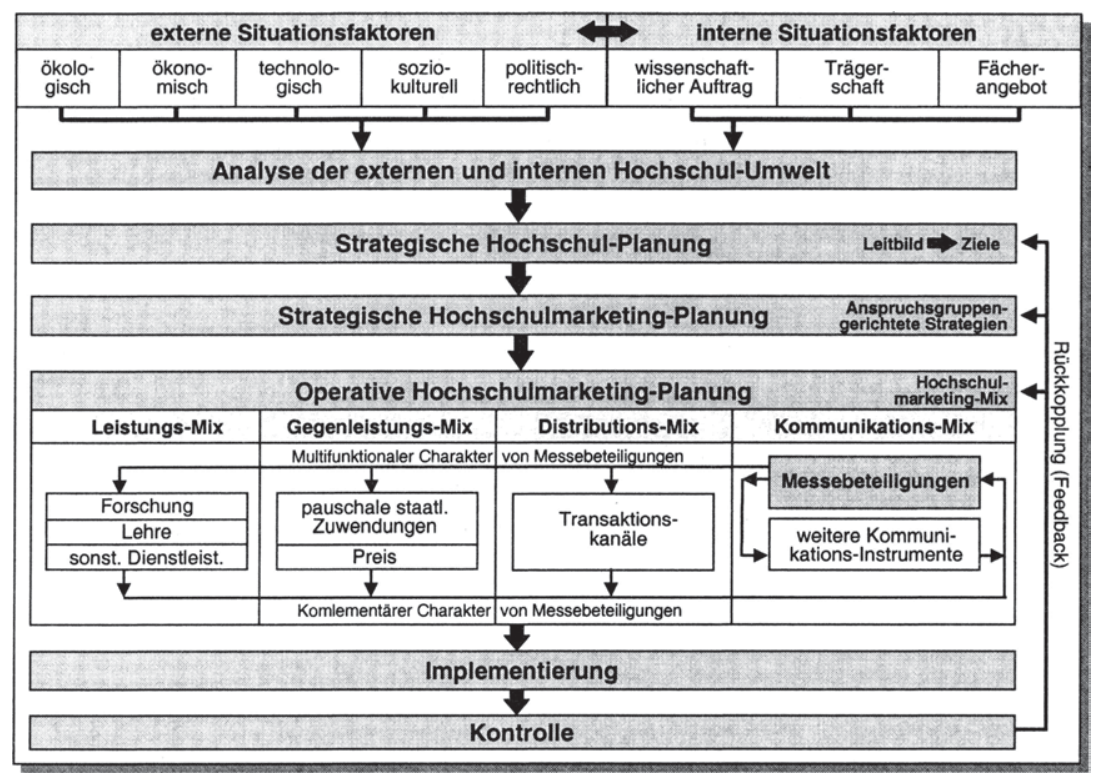

Abb. 3: Einordnung von Messebeteiligungen in den Managementprozeß des Hochschulmarketing

Vgl. Ueding, R., Management von Messebeteiligungen: Identifikation und Erklärung messespezifischer Grundhaltungen auf der Basis einer empirischen Untersuchung, Frankfurt am Main u.a. 1998, S. $10 \mathrm{ff}$.

Vgl. Raffée, H., Integrierte Kommunikation, in: Werbeforschung und Praxis, 36. Jg., Heft 3, 1991, S. $87 \mathrm{ff}$.

91 Vgl. Jaspert, F., Marketinginstrument Messe: Das synergetische Potential ist groß, in: BddW, Nr. 52, 1989 , S. 7. 
Im Hochschulbereich können alle drei Ebenen des wissenschaftlichen Bereichs (Hochschulleitung, Fachbereichs- bzw. Fakultätsebene oder Instituts- bzw. Lehrstuhlebene) Exponate ${ }^{92}$ auf Messen ${ }^{93}$ ausstellen. Unter einer Messebeteiligung von Hochschulen wird damit im folgenden die thematisch fokussierte Exponatpräsentation von Hochschulleitungen, Fachbereichen bzw. Fakultäten sowie Instituten bzw. Lehrstühlen auf Messen verstanden.

Die einzelnen Entscheidungstatbestände einer Messebeteiligung sind vor dem Hintergrund des jeweiligen situativen Kontextes einer ausstellenden Hochschule in einen systematischen Prozeß des Messebeteiligungs-Management zu überführen. ${ }^{94}$ Während die einer Situationsanalyse folgende konzeptionelle Planung der Festlegung von Messezielen und -strategien dient, sind der Maßnahmenplanung die stärker instrumentellen Bestandteile zuzuordnen. Die Realisation bzw. Durchsetzung umfaßt die Organisations-, Führungs- und Kontrollprozesse der Messebeteiligung. Die in dieser Phase gewonnenen Erkenntnisse gehen im Rahmen eines Feedbackprozesses in weiter vorgelagerte Planungsentscheidungen ein.

Als Exponate werden die auf den Messen und Ausstellungen präsentierten Produkte bzw. Entwicklungs- und Forschungsergebnisse bezeichnet.

In der betriebswirtschaftlichen Literatur werden Messen als Marktveranstaltungen gekennzeichnet, die darauf ausgerichtet sind, Marktaktivitäten zu generieren und zu stimulieren, indem Angebot und Nachfrage eines Marktes innerhalb eindeutig abgegrenzter Zeiträume in Kontakt gebracht werden. Dabei fungieren Märkte als Zwischensysteme der Aufgabenumwelt. Vgl. Meffert, H., Marketing - Grundlagen marktorientierter Unternehmensführung, a.a.O., S. 34. In der betriebswirtschaftlichen Literatur werden Messen als "Abbild des Marktes" bezeichnet. Vgl. Ziegler, R., Messen - ein makroökonomisches System, in: Strothmann, K.-H., Busche, M. (Hrsg.), Handbuch Messemarketing, a.a.O., S. $115 \mathrm{ff}$.

Im kommerziellen Bereich liegt der Schwerpunkt wissenschaftlicher Arbeiten zum Messebeteiligungs-Management auf Teilbereichen des Messebeteiligungsprozesses. Vgl. Gopalakrishna, S., Lilien, J. D., Sequeira, I. K., Do Trade Shows Pay Off?, in: JoM, Vol. 59, No. 7, 1995, S. 7583; Tanner, J. F., Chonko, L. B., Trade Show Objectives, Management, and Staffing Practices, in: IMM, Vol. 24, No. 4, 1995, S. 257-264; Spryß, W. M., Nerb, G., Reuter, J., Messeziele Messeeffizienz, Münster-Sarmsheim 1994; Gopalakrishna S., Williams J., Identifying Important Factors in Lead Efficiency at Trade Shows, in: Trade Show Bureau (Ed.), Report No. SM 23, Denver 1993; Head, J., Positioning, Objectives, and Selection Factors for Trade Shows, in: Trade Show Bureau (Ed.), Report No. MC24, Denver 1992; Schmalen, H., Wagner, A., Empirische Untersuchung zur Messebeteiligung von Unternehmen, Arbeitspapier, o.Nr., Passau 1990; Rosson, P., Seringhaus, F. H. R., Reaching Export Objectives through International Trade Fairs, in: Trade Show Bureau (Ed.), Report No. IN 6, Denver 1991, S. 2; Mortsiefer, J., Messen und Ausstellungen als Mittel der Absatzpolitik mittelständischer Herstellerbetriebe, Göttingen 1986; Täger, U. C., Ziegler, R., Die Bedeutung von Messen und Ausstellungen in der Bundesrepublik Deutschland für den Inlands- und Auslandsabsatz in ausgewählten Branchen, in: ifo-Institut für Wirtschaftsforschung e.V. (Hrsg.), ifo-Studien zu Handels- und Dienstleistungsfragen, Nr. 25, München 1984, S. 51; Gräbener, W., Die Messepolitik als Marketinginstrument - dargestellt am Beispiel von Investitionsgüterproduzenten, Göttingen 1981. 
Die verschiedenen Teilbereiche des damit grob gekennzeichneten Managementprozesses der Messebeteiligung von Hochschulen sind mit spezifischen Fragestellungen behaftet. Die zentralen Fragestellungen einer Hochschul-Messebeteiligung lassen sich anhand eines Paradigmas ${ }^{95}$ der Messebeteiligungsentscheidung ${ }^{96}$ verdeutlichen:

- Was wird mit einer Beteiligung

- an welchen Messen (Messetypen)

- wie

- zu welchen Kosten und mit welchen Wirkungen $\rightarrow$ Messebeteiligungsziele

$\rightarrow$ Messeauswahl

$\rightarrow$ Messebeteiligungsform

Messebeteiligungserfolg erreicht?

Die Formulierung von Messebeteiligungszielsetzungen als Ausgangspunkt des Managementprozesses von Hochschul-Messebeteiligungen gehört zu den prädisponierenden und damit entscheidenden Planungsschritten des Messeengagements im Hochschulbereich. An den festgelegten Messezielsetzungen, die sowohl in bezug auf die Zielinhalte als auch im Hinblick auf den Zielgruppenbezug dem öffentlich-rechtlichen Charakter der nicht-kommerziellen Institution Hochschule in geeigneter Weise Rechnung zu tragen haben, richten sich alle weiteren Entscheidungen im Managementprozeß der Messebeteiligung aus.

Grundsätzlich können Hochschulen im Rahmen der Messeauswahl zwischen einer Reihe verschiedener Messetypen ${ }^{97}$ wählen, ${ }^{98}$ wobei sich diese durch grundsätzliche Messemerkmale wie

Vgl. Meffert, H., Marketing, in: Management-Enzyklopädie, Bd. 4, München 1971, S. 392; Meffert, H., Marketingforschung und Käuferverhalten, 2. Aufl., Wiesbaden 1992, S. 22.

Im Rahmen dieses Paradigmas wird der grundsätzliche Entschluß zum Einsatz von Messebeteiligungen als Kommunikationsinstrument als positiv entschieden vorausgesetzt.

Zur Bedeutung der Bildung von Messe-Idealtypen als Grundlage wissenschaftlicher Arbeiten vgl. Doty, D. H., Glick, W., Typologies as a unique form of theory building: Towards improved understanding and modelling, in: AMR, No. 2, 1994, S. 230-251. Ein erster umfassender Ansatz zur Typologisierung von Messen und Ausstellungen wurde von TIETZ vorgenommen. Vgl. Tietz, B., Bildung und Verwendung von Typen in der Betriebswirtschaftslehre, dargestellt am Beispiel der Typologie der Messen und Ausstellungen, Köln, Opladen 1960.

In der Literatur sind zur Darstellung und Systematisierung von unterschiedlichen Messetypen verschiedene Ansätze zu finden, die teilweise erheblich divergieren. Einfache Varianten einer Messekategorisierung sind bei BONOMA und RICE zu finden. Vgl. Bonoma, T. V., Get more out of your trade shows, in: HBR, Vol. 61, No. 1, 1983, S. 80; Rice, G., Using the Interaction 
- Messefunktion

(Informations- und Ordermesse) $^{99}$,

- Angebotsschwerpunkt

(Dienstleistungs-, Investitionsgüter-, Konsumgütermessen) ${ }^{100}$,

- Aussteller- und Besucherreichweite

(regionale, überregionale, nationale und internationale Messen) ${ }^{101}$ sowie

- Angebotsbreite

(Universal-, Branchen-/Mehrbranchen-, Verbund- und Fachmessen)

unterscheiden. $^{102}$

Sowohl die nach Messefunktion als auch nach Angebotsschwerpunkt differenzierten Messetypen sind grundsätzlich alle für Hochschul-Messebeteiligungen geeignet. Zwar erscheinen für Hochschulen, die mit Forschung, Lehre und sonstigen Dienstleistungen klassische Anbieter von Dienstleistungen darstellen, Dienstleistungsmessen den Investitions- und Konsumgütermessen überlegen, aufgrund der hohen Relevanz von Forschungsleistungen für den Investitions- und Konsumgüterbereich sind jedoch generell alle drei Messetypen für Hochschul-Messebeschickungen von Bedeutung. Der Stellenwert der einzelnen Ausprägungen bzgl. Aussteller- und Besucherreichweiten hängt u.a. davon ab, welche Zielgruppe mit einer Hochschul-Messebeteiligung erreicht werden soll. Die Angebotsbreite kann - wie die folgenden Charakterisierungen andeuten - für die Messeauswahl

Approach to Understand International Trade Shows, in: IMR, Vol. 9, No. 4, 1992, S. 34. Detailliertere Ansätze der Kategorisierung von Messen wurden von FUNKE, STROTHMANN und ROLOFF sowie FLIEß vorgenommen. Vgl. Funke, K., Messeentscheidungen - Handlungsalternativen und Informationsbedarf, Frankfurt am Main u.a. 1987, S. 3 ff.; Strothmann, K.-H., Roloff, E., Charakterisierung und Arten von Messen, in: Berndt, R., Hermanns, A. (Hrsg.), Handbuch Marketing-Kommunikation: Strategien - Instrumente - Perspektiven, Wiesbaden 1993, S. 707 ff.; Fließ, S., Messeselektion - Entscheidungskriterien für Investitionsgüteranbieter, Wiesbaden 1994, S. $72 \mathrm{ff}$.

Häufig wird auf eine Verdrängung von Ordermessen zugunsten von Informationsmessen hingewiesen. Vgl. Tanner, J. F., Chonko, L. B., Trade Show Objectives, Management, and Staffing Practices, a.a.O., S. 259.

Vgl. Meffert, H., Messen und Ausstellungen als Marketinginstrument, a.a.O., S. 10; Stroth101 mann, K.-H., Roloff, E., Charakterisierung und Arten von Messen, a.a.O., S. 717.

Vgl. Ausstellungs- und Messe-Ausschuss der Deutschen Wirtschaft e.V. (AUMA) (Hrsg.), AUMA-Leitsätze zur Typologie von Messen und Ausstellungen, Köln 1996.

102 Vgl. Meffert, H., Messen und Ausstellungen als Marketinginstrument, a.a.O., S. 10. Vgl. dazu auch Funke, K., Messeentscheidungen - Handlungsalternativen und Informationsbedarf, a.a.O., S. 4 . 
von Hochschul-Exponenten nicht zuletzt vor dem Hintergrund ihres Fächerangebots und der damit thematisch mehr oder weniger fokussierten Forschungsergebnisse von besonderem Stellenwert sein. Charakteristisch für Universalmessen ist eine prinzipiell offene und damit nicht festgelegte thematische Schwerpunktsetzung. ${ }^{103}$ Branchenmessen bzw. Mehrbranchenmessen präsentieren dagegen eine fokussiertere Exponatauswahl, wobei sie das Angebot einer bzw. mehrerer Wirtschaftszweige zum Ausgangspunkt ihrer konzeptionellen Abgrenzung nehmen. Charakteristisch für Fachmessen ist eine große Angebotstiefe bei gleichzeitig geringer Angebotsbreite. ${ }^{104}$ Werden thematisch verwandte Fachmessen zu einer Veranstaltung zusammengefaßt, so wird diese Form als Verbundmesse bezeichnet. $^{105}$

Die aufgeführten Messetypen sind i.d.R. nicht in ihrer Reinform vorzufinden. Vielmehr haben sich Messen entwickelt, die verschiedene Messetypen in sich vereinen. Im Hochschulbereich bspw. haben sich in diesem Zusammenhang Wissenschafts- und Bildungsmessen herauskristallisiert. Wissenschaftsmessen lassen sich den Fach- und Dienstleistungsmessen zuordnen. Diese Messen ermöglichen Hochschulen, ihre Forschungsergebnisse einer eng fokussierten Zielgruppe zu

103

Universalmessen sind darauf angelegt, einen umfassenden Überblick über das Leistungspotential einer Volkswirtschaft zu geben. Sie sind in ihrer klassischen Prägung heute nicht mehr anzutreffen. Die großen Messeplätze des Ostblocks hatten bis zum Beginn der politischen und ökonomischen Transformationen i.d.R. pro Jahr zwei thematisch nur schwach konturierte Messen, die weitgehend dem Typus der Universalmesse entsprachen. Vgl. Meffert, $H$., Robertz, G., Hauptstädte werden als Messeorte interessant, in: OWC, Heft 2, 1998, S. 22-26.

Der Begriff Fachmesse wird in der Literatur einerseits im Sinne einer spezialisierten fachlichen Ausrichtung der Messe und andererseits als Oberbegriff für alle Messen, die sich an ein Fachpublikum richten, genutzt. Zur Verwendung als spezialisierte Messe vgl. stellvertr. Roloff, E., Messen und Medien: Ein sozialpsychologischer Ansatz der Öffentlichkeitsarbeit, Wiesbaden 1992, S. 9 ff. Zur Verwendung als Oberbegriff vgl. stellvertr. Taeger, M., Messemarketing Marketingmix von Messegesellschaften unter Berücksichtigung wettbewerbspolitischer Rahmenbedingungen, Göttingen 1993, S. 30. Die Konzeption von Fachmessen ist durch konsequente Themenorientierung geprägt, wobei spezifische Anwendungsprobleme und ein damit korrespondierender Überblick über mögliche Problemlösungskonzepte den Kern des Messeprogramms bilden. Vgl. Meffert, H., Neuere Entwicklungen in Kommunikation und Vertrieb, a.a.O., S. 52.

Vgl. Groth, C., Das Leistungspotential von Fachmessen, in: Deutsche Werbewissenschaftliche Gesellschaft e.V. (DWG) (Hrsg.), Messen als Marketinginstrument, a.a.O., S. 58. Durch Nutzung von Synergieeffekten wird mit einem derartigen Messetyp der Problematik geringer Aussteller- und Besucherzahlen auf spezialisierten Fachmessen entgegengewirkt. Gleichzeitig wird mit der Durchführung von Verbundmessen eine erhöhte Attraktivität der nun im Verbund stattfindenden und damit thematisch breiter angelegten Veranstaltungen angestrebt. Vgl. Selinski, H., Fair Play in Europa, in: Kliche, M. (Hrsg.), Investitionsgütermarketing, Wiesbaden 1990, S. 181. 
präsentieren. Bildungsmessen vereinen - wie Wissenschaftsmessen - Fach- und Dienstleistungsmessen, wobei sie weniger an transferorientierten als vielmehr an bildungspolitischen Aspekten ausgerichtet werden.

Eng verbunden mit der Auswahl des zu beschickenden Messetyps ist die Entscheidung über die Wahl der Messebeteiligungsform. Grundsätzlich können sich Hochschul-Exponenten allein auf eigenen Messeständen oder zusammen mit anderen Ausstellern auf Gemeinschaftsständen präsentieren. Zu hochschulspezifischen Integrationsformen zählen Gemeinschaftsstände, auf denen HochschulExponenten zusammen mit wissenschaftlichen und wirtschaftlichen sowie auch regionalen Institutionen ${ }^{106}$ ausstellen. Folglich bieten sich Hochschul-Exponenten zur Präsentation ihrer Exponate

- Hochschul-Einzel-Messestände,

- Wissenschafts-Gemeinschaftsstände,

Wirtschafts-Gemeinschaftsstände,

Regionen-Gemeinschaftsstände und/oder

- Landes-Gemeinschaftsstände.

Auf hochschulspezifischen Einzel-Messeständen stellen einzelne HochschulExponenten ihre Exponate allein aus. Diese Art der Messebeteiligung bietet den Exponenten die Möglichkeit zur selbstbestimmten Präsentation. Einzel-Messestände sind jedoch nur von größeren Institutionen des Hochschulbereichs realisierbar, da eine derartige Messebeteiligungsform entsprechende finanzielle und personelle Ressourcen sowie das notwendige organisatorische Know-how voraussetzt.

Präsentieren sich verschiedene Hochschul-Exponenten, d.h. also zwei oder mehrere Institute bzw. Lehrstühle, Fachbereiche oder Hochschulen zusammen auf einem Messestand, so bilden sie einen Hochschul-Gemeinschaftsstand. Bei einem derartigen Gemeinschaftsstand steht ausschließlich die Ebene der Hoch-

106 Unabhängig von dem jeweiligen Kooperationspartner der Messebeteiligung werden alle derartigen Messestände als Gemeinschaftsstände bezeichnet. Im folgenden werden zur besseren Unterscheidung dieser Gemeinschaftsstände in Abhängigkeit des jeweiligen Kooperationspartners die Bezeichnungen Wissenschafts-, Wirtschafts-, Regionen- und Landes-Gemeinschaftsstand verwendet. 
schulen bzw. der Fachbereiche, Institute oder Lehrstühle im Vordergrund des Messeengagements. Nach Vorstellungen der auf dem Gemeinschaftsstand ausstellenden Exponenten können sowohl einzelne Forschungsthemen als auch thematisch breit gestreute Forschungspotentiale der Hochschul-Exponenten im Mittelpunkt der Messebeteiligung stehen. ${ }^{107}$ Ein entscheidender Vorteil dieser Integrationsform gegenüber der Präsentation auf eigenen Messeständen liegt in der Aufteilung des organisatorischen und finanziellen Aufwands der Messebeteiligung unter den ausstellenden Hochschul-Exponenten.

Eine weitere Integrationsform von Hochschul-Messebeteiligungen ist zwischen Hochschul-Exponenten und Wirtschaftspartnern i.S.e. Wirtschafts-Gemeinschaftsstands vorzufinden. Dabei fungiert entweder ein Unternehmen als Gastaussteller auf einem Hochschul-Messestand oder ein Hochschul-Exponent ist ausstellender Gast auf dem Messestand eines Unternehmens. Der entscheidende Vorteil dieser Kooperationsform liegt in der anhand des Exponates als Forschungsergebnis unmittelbar dokumentierbaren, erfolgreichen Kooperation zwischen Wissenschaft und Praxis. Bei einer derartigen Integrationsform trägt der "Gastgeber" den größeren Teil des organisatorischen und finanziellen Aufwands. Folglich treten bei dieser Messebeteiligungsform eher Wirtschaftspartner denn Hochschulen als "Gastgeber" auf.

Der Regionen-Gemeinschaftsstand, auf dem ein oder mehrere HochschulExponenten mit einer regionalen Einrichtung ihre Exponate ausstellen, bildet eine weitere Integrationsform der Messebeteiligung im Hochschulbereich. Bei dieser Integrationsform stehen imagefördernde Aspekte einer Wirtschaftsregion im Vordergrund des Messeengagements. Die Darstellung des Ausbildungs- sowie Forschungspotentials der in der Region ansässigen Hochschulen nimmt damit eine besondere Bedeutung für die Messebeschickung ein. Der Aufwand regionaler Gemeinschaftsstände wird größtenteils von dem regionalen Partner getragen.

Vgl. hierzu sowie im folgenden Kayser, P., Typen von Messen und Formen der Messebeteiligung, in: Budach, W. et al. (Hrsg.), Hochschulen und Messen: Praktische Hinweise für Messebeteiligungen durch Hochschulen, Bad Honnef 1992, S. 58 f. 
Auf Landes-Gemeinschaftsständen stellen die Hochschulen eines Landes ${ }^{108}$ gemeinsam ihre Exponate auf einem Messestand aus. Die präsentierten Forschungsprojekte der Hochschul-Exponenten dienen dabei vorwiegend zur Darstellung des Forschungspotentials eines Landes ${ }^{109}$ und weniger zur eigenen Profilierung. Charakteristisch für Landes-Gemeinschaftsstände sind große Ausstellungsflächen, die letztlich zu einer höheren Aufmerksamkeitswirkung des gesamten Messestands führen. Die Organisation und Finanzierung derartiger Messestände wird größtenteils vom jeweiligen Land übernommen. ${ }^{110}$ Oftmals ermöglicht erst diese Integrationsform Hochschul-Exponenten eine Messebeschickung.

Die verschiedenen, sich den Hochschul-Exponenten bietenden Formen der Messebeteiligung und inre wesentlichen Charakteristika sind in der folgenden Tabelle zusammenfassend dargestellt.

\begin{tabular}{|c|c|c|c|c|}
\hline $\begin{array}{l}\text { Ausgewähl- } \\
\text { te Merk- } \\
\text { Mes- } \\
\text { sebeteili- male } \\
\text { gungsformen }\end{array}$ & $\begin{array}{l}\text { Primär verfolgte } \\
\text { Messestand- } \\
\text { Zielsetzungen }\end{array}$ & $\begin{array}{l}\text { Primär be- } \\
\text { rücksichtigte } \\
\text { Zielebene }\end{array}$ & $\begin{array}{l}\text { Trans- } \\
\text { ferorien- } \\
\text { tierung }\end{array}$ & $\begin{array}{l}\text { Hauptträger des orga- } \\
\text { nisatorischen und } \\
\text { finanziellen Aufwands }\end{array}$ \\
\hline $\begin{array}{l}\text { Hochschul- } \\
\text { Einzel- } \\
\text { Messestand }\end{array}$ & $\begin{array}{l}\text { Profil und Leistungspoten- } \\
\text { tial des Hochschul- } \\
\text { Exponenten darstellen }\end{array}$ & $\begin{array}{l}\text { Hochschul- } \\
\text { Exponent }\end{array}$ & mittel & $\begin{array}{l}\text { Hochschul- } \\
\text { Exponent }\end{array}$ \\
\hline $\begin{array}{l}\text { Wissenschafts- } \\
\text { Gemein- } \\
\text { schaftsstand }\end{array}$ & $\begin{array}{l}\text { Profile und Leistungspoten- } \\
\text { tiale der Hochschul- } \\
\text { Exponenten darstellen }\end{array}$ & $\begin{array}{l}\text { Hochschul- } \\
\text { Exponenten }\end{array}$ & mittel & $\begin{array}{l}\text { Aufteilung zwischen } \\
\text { Hochschul- } \\
\text { Exponenten }\end{array}$ \\
\hline $\begin{array}{l}\text { Wirtschafts- } \\
\text { Gemein- } \\
\text { schaftsstand }\end{array}$ & $\begin{array}{l}\text { Erfolgreiche Kooperationen } \\
\text { zwischen Wissenschaft und } \\
\text { Praxis präsentieren }\end{array}$ & $\begin{array}{l}\text { i.d.R. } \\
\text { Wirtschafts- } \\
\text { partner }\end{array}$ & hoch & $\begin{array}{l}\text { i.d.R. } \\
\text { Wirtschafts- } \\
\text { partner }\end{array}$ \\
\hline $\begin{array}{l}\text { Regionen- } \\
\text { Gemein- } \\
\text { schaftsstand }\end{array}$ & $\begin{array}{l}\text { Forschungs- und Entwick- } \\
\text { lungspotentiale der Wirt- } \\
\text { schaftsregion darstellen }\end{array}$ & Kommune & $\begin{array}{l}\text { i.d.R. } \\
\text { hoch }\end{array}$ & $\begin{array}{l}\text { Regionale } \\
\text { Einrichtung }\end{array}$ \\
\hline $\begin{array}{l}\text { Landes- } \\
\text { Gemein- } \\
\text { schaftsstand }\end{array}$ & $\begin{array}{l}\text { Forschungs- und Entwick- } \\
\text { lungspotentiale des Landes } \\
\text { darstellen }\end{array}$ & Land & $\begin{array}{l}\text { i.d.R. } \\
\text { gering }\end{array}$ & $\begin{array}{l}\text { Ministerium des } \\
\text { Landes }\end{array}$ \\
\hline
\end{tabular}

Tab. 1: Hochschulspezifische Messebeteiligungsformen im Vergleich

108 Auf Landes-Gemeinschaftsständen in Deutschland kann der einzelne Hochschul-Exponent einerseits gemeinsam mit Hochschulen aus der gesamten Bundesrepublik, andererseits zusammen mit Hochschulen eines Bundeslandes seine Exponate ausstellen.

109 Vgl. Kayser, P., Typen von Messen und Formen der Messebeteiligung, a.a.O., S. 57 f.

110 Vgl. Forschungsland Nordrhein-Westfalen (Hrsg.), Messehandbuch, Aachen 1997, Absatz 2. 
Präsentieren sich Hochschul-Exponenten auf Wirtschafts-, Regionen- oder Landes-Gemeinschaftsständen, so sind oftmals die Ausrichter der Gemeinschaftsstände und damit hochschulexterne Institutionen primäre Ansprechpartner, die Hochschul-Exponenten bzgl. ihrer Messebeteiligungen im aufgezeigten Sinne unterstützen. Darüber hinaus bieten sich diesen Exponenten zusätzlich hochschulinterne Institutionen, die in bezug auf den vom Hochschul-Exponenten noch selbst zu tragenden Messebeteiligungsaufwand unterstützende Leistungen bieten. Folglich nehmen diese Institutionen insbesondere auch für Exponenten von Hochschul-Einzel-Messeständen sowie Wissenschafts-Gemeinschaftsständen einen besonderen Stellenwert ein. Hochschulinterne Institutionen, zu denen Transferstellen, Pressestellen, Messebüros und Messebeauftragte im Nebenamt zählen, ${ }^{111}$ bieten Hochschul-Exponenten verschiedene Arten von unterstützenden Leistungen zur Messebeteiligung, wobei der Umfang im wesentlichen von der gewählten Messebeteiligungsform abhängt. ${ }^{112}$

Nach der auf Grundlage der vorgenommenen Auswahl des Messetyps und der Messebeteiligungsform durchgeführten Messebeteiligung stellt sich die Frage nach dem Messebeteiligungserfolg. In diesem Zusammenhang ist die Überprüfung der Effektivität i.S.d. Ermittlung des Messebeteiligungsnutzens und der Effizienz i.S.e. Kosten-Nutzen-Analyse erforderlich. Zur Erfassung des Messebeteiligungsnutzens, der durch die Erreichung verfolgter Messezielsetzungen determiniert wird, ist die Auseinandersetzung mit hochschulspezifischen Messezielsetzungen notwendig. Angesichts der aufgezeigten Entwicklungen, die eine Ermittlung des Erfolgs von Hochschul-Messebeteiligungen zur Rechtfertigung ihrer Existenz im Hochschulbereich erforderlich werden lassen, liegt der Fokus der vorliegenden Arbeit auf der Erfolgskontrolle von Hochschul-Messebeteiligungen sowie der dazu notwendigen Analyse hochschulspezifischer Messebeteiligungsziele.

Zu den einzelnen Institutionen vgl. Stracke, F., Personelle Organisation der Messezuständigkeit, in: Budach, W. et al. (Hrsg.), Hochschulen und Messen: Praktische Hinweise für Messebeteiligungen durch Hochschulen, a.a.O., S. $111 \mathrm{f}$.

112 Vgl. Stracke, F., Personelle Organisation der Messezuständigkeit, a.a.O., S. $111 \mathrm{ff}$; Meier, H.-G., Kosten - Finanzierung - Erfolg: Finanzierung, in: Budach, W. et al. (Hrsg.), Hochschulen und Messen: Praktische Hinweise für Messebeteiligungen durch Hochschulen, a.a.O., S. $144 \mathrm{f}$. 


\section{Zielsetzung und Gang der Untersuchung}

Hohe Messebeteiligungskosten und knappe Hochschulbudgets führen zur Notwendigkeit, die Effizienz von Hochschul-Messebeteiligungen i.S.e. Kosten-NutzenAnalyse zu überprüfen. Dies gilt um so mehr, da sowohl über die Kosten als auch über den Nutzen von Hochschul-Messebeteiligungen keine empirischen Erkenntnisse vorliegen. Hochschul-Exponenten streben mit ihrem Messeengagement i.d.R. nach einer Nutzenmaximierung, wobei sie aufgrund ihres begrenzten Messebudgets eine definierte Kostengrenze zu berücksichtigen haben. Im Rahmen der Erfolgskontrolle von Hochschul-Messebeteiligungen erscheint es demnach sinnvoll, zunächst eine Effektivitätsbetrachtung, d.h. eine Analyse des Nutzens i.S.d. Erreichung verfolgter Messebeteiligungsziele, vorzunehmen und darauf aufbauend Kosten-Nutzen-Relationen zu untersuchen. Vor dem aufgezeigten Hintergrund besteht das generelle Forschungsziel dieser Arbeit in der Überprüfung des Erfolgs von Hochschul-Messebeteiligungen, wobei die Analyse der hochschulspezifischen Messeziele und -zielgruppen als Grundlage der Erfolgskontrolle den Ausgangspunkt des Forschungsprojektes bildet.

Aus diesem generellen Forschungsziel lassen sich fünf Teilziele und damit folgende Forschungsschwerpunkte ableiten:

Analyse und Systematisierung hochschulspezifischer Messebeteiligungsziele von Hochschul-Exponenten und Ableitung hochschulspezifischer Exponententypen.

- Systematisierung hochschulspezifischer Messezielgruppen und Analyse ihrer Relevanz für Hochschul-Exponenten sowie ihrer Verhaltensweisen auf einem Hochschul-Messestand.

- Analyse von Ansätzen zur Erfolgskontrolle von Hochschul-Messebeteiligungen und konzeptionelle Überprüfung ihrer Relevanz für Hochschul-Messebeteiligungen.

- Durchführung einer Erfolgskontrolle von Hochschul-Messebeteiligungen zur Überprüfung der Effizienz sowie der Bedeutung von Messebeteiligungen im Hochschulbereich.

- Ableitung von Implikationen sowohl für das Messeengagement von HochschulExponenten als auch für die Finanzmittelgeber von Hochschul-Messebeteiligungen. 
Im Hinblick auf das theoretisch-methodische Vorgehen bildet der entscheidungsorientierte Ansatz der Betriebswirtschaftslehre ${ }^{113}$ den Bezugsrahmen der Arbeit (Abb. 4). Vor dem Hintergrund der aufgezeigten Zielsetzung der Arbeit wird die vorliegende Untersuchung auf die Entscheidungstatbestände des Managementprozesses fokussiert, die zur Ermittlung des Erfolgs von Hochschul-Messebeteiligungen i.S.v. Kosten-Nutzen-Analysen relevant sind. Grundlage bildet die Analyse hochschulspezifischer Messeziele und -zielgruppen, denen als Ausgangspunkt von Hochschul-Messebeteiligungen eine besondere Bedeutung zukommt. Zur Überprüfung der Zielerreichung, die als Nutzen den Erfolg einer Messebeteiligung wesentlich determiniert, bedarf es der Erfolgskontrolle. In diesem Zusammenhang sind neben der Ableitung von Erfolgskriterien und dem Einsatz von Methoden zur Messeerfolgskontrolle Messebeteiligungskosten zu analysieren sowie intra- und inter-instrumentelle Vergleiche mit geeigneten Vergleichsobjekten vorzunehmen. Auf Grundlage der gewonnenen Erkenntnisse können abschließend Implikationen für zukünftige Hochschul-Messebeteiligungen abgeleitet werden.

113 Zum entscheidungsorientierten Ansatz vgl. Heinen, E., Einführung in die Betriebswirtschaftslehre, 9. Aufl., Wiesbaden 1985, S. 259 ff.; Meffert, H., Die Leistungsfähigkeit der entscheidungs- und systemorientierten Marketinglehre, in: Kortzfleisch, G. von (Hrsg.), Wissenschaftsprogramm und Ausbildungsziele der Betriebswirtschaftslehre, Berlin 1971, S. 167 ff.; Witt, F. H., Theorietradition der Betriebswirtschaftlichen Forschung, Wiesbaden 1995, S. 29. 


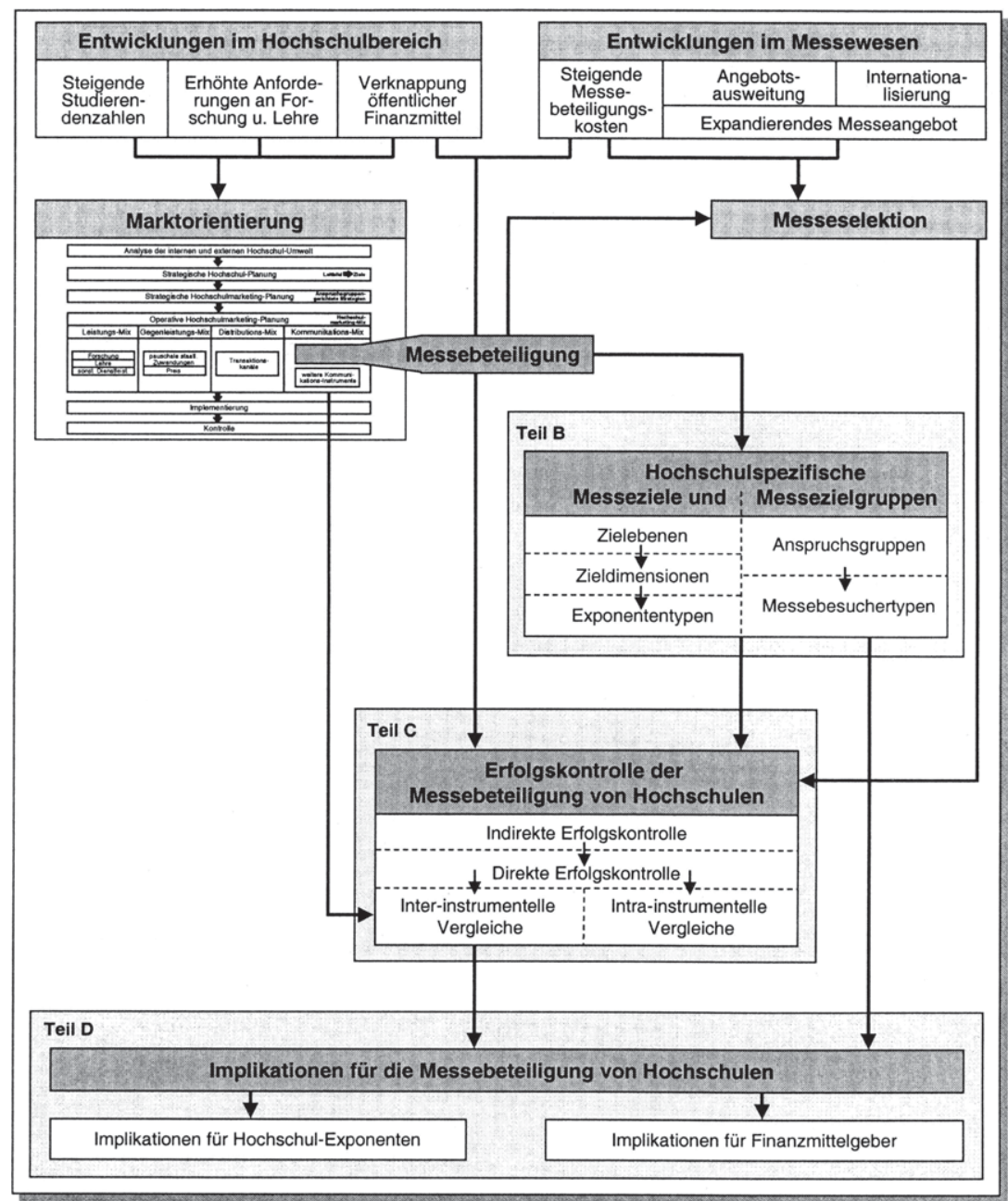

\section{Abb. 4: Bezugsrahmen der Arbeit}

Die Vorgehensweise dieser Arbeit ist konzeptionell-literaturgestützt ausgerichtet, wobei die konzeptionellen Überlegungen mit einer empirischen Untersuchung unterlegt werden. Mit den beschriebenen Zielsetzungen und dem aufgezeigten Bezugsrahmen ist der Gang der Untersuchung bereits vorgezeichnet: 
In Teil B der Arbeit erfolgt zunächst eine Betrachtung der hochschulspezifischen Messebeteiligungszielsetzungen. In diesem Rahmen werden spezifische Messebeteiligungsziele der verschiedenen Ebenen des Hochschulbereichs dargestellt und darauf aufbauend hochschulspezifische Zieldimensionen abgeleitet, die als Grundlage zur Bildung hochschulspezifischer Exponententypen dienen. Daran anschließend werden auf Grundlage hochschulspezifischer Anspruchsgruppen Zielgruppen einer Hochschul-Messebeteiligung gebildet, wobei diese Zielgruppensystematik um verhaltensorientierte Kriterien ergänzt wird. Die Ausführungen werden dabei sowohl hinsichtlich der hochschulspezifischen Messeziele als auch der hochschulspezifischen Zielgruppen empirisch unterlegt.

In Teil C wird auf Grundlage der Ergebnisse zur Analyse der Messebeteiligungsziele und -zielgruppen eine Erfolgskontrolle von Hochschul-Messebeteiligungen vorgenommen. Dazu wird zunächst die Effektivität von Hochschul-Messebeteiligungen im Rahmen einer indirekten Erfolgskontrolle überprüft, die den Erfolg als Grad der Erreichung von angestrebten Messezielsetzungen definiert. In diesem Zusammenhang werden die aus den hochschulspezifischen Messezielsetzungen abgeleiteten Erfolgskriterien systematisch dargestellt und ausgewählte Methoden zur indirekten Erfolgskontrolle auf ihre Eignung und Relevanz für Hochschul-Messebeteiligungen diskutiert und entsprechend eingesetzt. Die so ermittelten Ergebnisse bilden die Grundlage der Effizienzbetrachtung von Hochschul-Messebeteiligungen. Dazu wird eine direkte Erfolgskontrolle durchgeführt, die eine Wirtschaftlichkeitsanalyse von Hochschul-Messebeteiligungen vornimmt und damit neben dem Zielerreichungsgrad auch die im Rahmen einer Messebeteiligung anfallenden Kosten berücksichtigt. Die zur abschließenden Effizienzbewertung von Hochschul-Messebeteiligungen erforderlichen intra- und inter-instrumentellen Vergleiche bilden den Abschluß der Messeerfolgskontrolle.

Teil $\mathbf{D}$ dient der Ableitung von Implikationen für zukünftige Messebeteiligungen von Hochschulen. Dabei werden für Hochschul-Exponenten sowohl Empfehlungen für strategische als auch für operative Entscheidungsfelder gegeben. Darüber hinaus werden ebenfalls Implikationen für Finanzmittelgeber von Hochschul-Messebeteiligungen abgeleitet. Abschluß der Arbeit bildet Teil E, in dem eine Zusammenfassung der Untersuchungsergebnisse erfolgt sowie Ansatzpunkte für weiterführende Forschungsarbeiten aufgezeigt werden. 


\section{Design der empirischen Untersuchung}

Die empirische Analyse der Messebeteiligung von Hochschulen erfolgt auf Basis des Datenmaterials einer vom Institut für Marketing der Westfälischen WilhelmsUniversität Münster in Zusammenarbeit mit dem Ministerium für Schule und Weiterbildung, Wissenschaft und Forschung des Landes Nordrhein-Westfalen durchgeführten Studie zur "Evaluierung von Hochschul-Messebeteiligungen“. Im Rahmen der Untersuchung wurden auf einer ausgewählten Mehrbranchen- und Fachmesse einerseits schriftliche und mündliche Befragungen mit 47 HochschulExponenten ( $n=32$ Mehrbranchenmesse-Exponenten und $n=15$ FachmesseExponenten) und andererseits persönliche Interviews mit 1.218 Hochschul-Messestand-Besuchern ( $n=685$ Mehrbranchenmesse-Besucher und $n=533$ Fachmesse-Besucher) durchgeführt. Dabei fand die Feldphase von November 1997 bis Oktober 1998 statt.

Angesichts der verfolgten Zielsetzung, die Ziele und den Erfolg von HochschulMessebeteiligungen zu analysieren, bot sich zur Strukturierung des Untersuchungsobjektes die zeitbezogene Unterteilung in eine Vor-Messe-Phase, MessePhase sowie Nach-Messe-Phase an. Die mit einer Messebeteiligung verfolgten Zielsetzungen werden i.d.R. von den Hochschul-Exponenten vor der MessePhase definiert und wurden demzufolge in der Vor-Messe-Phase in teilstandardisierten schriftlichen und mündlichen Befragungen von Hochschul-Exponenten erhoben. ${ }^{114}$ Der Zielerreichungsgrad sowie die mit einer Messebeteiligung verbundenen Kosten sind hingegen erst nach Ablauf des Messegeschehens ermittelbar. Folglich wurden diese Aspekte in der Nach-Messe-Phase in persönlichen und telefonischen Exponenten-Interviews erfaßt. Die Messe-Phase galt der Befragung von Hochschul-Messestand-Besuchern, d.h. Messebesuchern (Zufallsauswahl), die den Hochschul-Messestand besucht und Kontakt zu Hochschul-

In der Vor-Messe-Phase wurden - abweichend von den Befragungen der Messe- und NachMesse-Phase - die Extrempole der Ratingskalen mit 1 = sehr gering, gar nicht wichtig bzw. trifft gar nicht zu bis $5=$ sehr hoch, sehr wichtig bzw. trifft sehr zu versehen. Vgl. Anhang III, Fragebogen 1: Teil 1 der Exponentenbefragung I in der Vor-Messe-Phase; Anhang III, Fragebogen 2: Teil 2 der Exponentenbefragung I in der Vor-Messe-Phase. Für die Auswertung der Daten erfolgte eine entsprechende Umkodierung, um eine Vergleichbarkeit mit den Ergebnissen der Messe- und Nach-Messe-Befragungen zu gewährleisten. Vgl. Anhang III, Fragebogen 3: Teil 1 der Exponentenbefragung II in der Nach-Messe-Phase; Anhang III, Fragebogen 4: Teil 2 der Exponentenbefragung II in der Nach-Messe-Phase; Anhang III, Fragebogen 5: Befragung der Hochschul-Messestand-Besucher in der Messe-Phase. Die im Anhang III abgebildeten Fragebögen wurden einer entsprechenden Anonymisierung unterzogen. 
Exponenten aufgenommen hatten. Neben der Erhebung soziodemographischer Daten stand dabei u.a. auch die Erfassung von Besucherzielsetzungen im Vordergrund der Befragung. ${ }^{115}$

Trotz der vergleichsweise umfangreichen Stichproben wird nicht der Anspruch einer statistischen Repräsentativität der Untersuchung erhoben. Dazu wäre sowohl ein Vergleich zwischen den befragten Exponenten und der Gesamtheit aller Hochschul-Exponenten als auch ein Vergleich zwischen den befragten Messebesuchern und der Gesamtheit aller Hochschul-Messestand-Besucher erforderlich gewesen. Diese Vergleiche waren jedoch nicht möglich, da weder über die Grundgesamtheitsstruktur der Hochschul-Exponenten noch über die Grundgesamtheitsstruktur der Hochschul-Messestand-Besucher Informationen vorliegen.

Die statistische Auswertung des erhobenen Datenmaterials erfolgte mit Hilfe des Statistikpakets SPSS (Superior Performing Software Systems). ${ }^{116}$ Zur Analyse der Daten kommen verschiedene uni-, bi- und multivariate Verfahren der strukturentdeckenden und -prüfenden Analyse zur Anwendung. ${ }^{117}$ In diesem Rahmen werden sowohl die Faktorenanalyse als auch die Cluster- sowie die Diskriminanzanalyse eingesetzt. Die Untersuchung von Mittelwertunterschieden erfolgt auf Basis des F- und Scheffé-Tests. ${ }^{118}$

Zur Zusammensetzung der Exponenten- und Messebesucher-Stichproben vgl. Anhang II, Zusammensetzung der Hochschul-Exponenten- und Hochschul-Messestand-Besucher-Stichproben.

116 Zur Anwendung kam die Programmversion SPSS v. 8.0. Zu den statistischen Prozeduren des SPSS-Programms vgl. Norius, M. J., SPSS Inc., SPSS for Windows: Advanced Statistics, Release 5, Chicago, III. 1992; derselbe, SPSS Inc., SPSS for Windows: Professional Statistics, Release 5, Chicago, III. 1992; Schubö, W. et al., SPSS: Handbuch der Programmversionen 4.0 und SPSS-X 3.0, Stuttgart, New York 1991, S. 282 ff.; Brosius, G., SPSS/PC+ Advanced Statistics and Tables - Einführung und praktische Beispiele, Hamburg u.a. 1989, S. $97 \mathrm{ff}$.

Während strukturprüfende Verfahren (z.B. Regressions-, Diskriminanz-, Kontingenz- und Conjoint-Analyse) auf die Überprüfung statistischer Zusammenhănge zwischen Variablen gerichtet sind, ist es Aufgabe der strukturentdeckenden Analyse (z.B. Faktoren- und Clusteranalyse), derartige Zusammenhänge aufzuspüren. Vgl. Backhaus, K. et al., Multivariate Analysemethoden: Eine anwendungsorientierte Einführung, 8. Aufl., Berlin u.a. 1996, S. XVIII. Allgemeine Verfahrensdarstellungen finden sich auch bei Meffert, $\mathrm{H}$., Marketingforschung und Käuferverhalten, a.a.O., S. 252 ff.; Fahrmeir, L., Häußler, W., Tutz, G., Diskriminanzanalyse, in: Fahrmeir, L., Hamerle, A. (Hrsg.), Multivariate statistische Verfahren, Berlin, NY 1984, S. $301 \mathrm{ff}$.

Der F-Test dient zur Globalbeurteilung von Mittelwertunterschieden. Vgl. Bleymüller, J., Gehlert, G., Gülicher, H., Statistik für Wirtschaftswissenschaftler, 10. Aufl., München 1996, S. $119 \mathrm{ff}$. Die Anwendung des als besonders konservativ geltenden Scheffé-Tests empfiehlt sich vor allem bei ungleichen Gruppengrößen. Vgl. Bauer, F., Datenanalyse mit SPSS, Berlin u.a. 1984 , S. 84 f. 


\section{B. Analyse hochschulspezifischer Messeziele und Messeziel- gruppen}

Messebeteiligungszielen ist als Ausgangspunkt des Managementprozesses von Messebeteiligungen ein besonderer Stellenwert einzuräumen. Grundsätzlich bringt der Zielbezug des Management von Hochschul-Messebeteiligungen den systematischen, strategisch intendierten Planungsprozeß zum Ausdruck, der über "ad hoc" ergriffene Einzelentscheidungen hinausgeht. Dabei haben die angestrebten hochschulspezifischen Messezielsetzungen sowohl hinsichtlich der Zielinhalte als auch des Zielgruppenbezugs dem öffentlich-rechtlichen Charakter der nichtkommerziellen Institution Hochschule in geeigneter Weise Rechnung zu tragen.

\section{Zielsystem von Hochschulen bei Messebeteiligungen}

Besondere Herausforderungen bei der Ableitung eines konsistenten Zielsystems für Messebeteiligungen im Hochschulbereich ergeben sich aus den hochschulspezifischen Rahmenbedingungen. Die Hochschulen sind in ein pluralistisches und komplexes, übergeordnetes Zielsystem eingebunden, wobei sie ihrerseits nicht über eine oberste Zielbildungsinstanz verfügen. ${ }^{1}$ Administrative und legislative Einflüsse führen zu einer Einengung der Autonomie bei der Festlegung von Messebeteiligungszielen der Hochschul-Exponenten. Messebeteiligungsziele kennzeichnen jene zukünftigen Zustände, die aus Sicht von Hochschul-Exponenten als erstrebenswert angesehen und durch entsprechende Handlungen erreicht werden sollen. ${ }^{2}$ Durch Vorgabe anzustrebender Zustände werden Messebeteiligungsziele zu konzeptionellen Orientierungs- bzw. Richtgrößen der Hochschulaktivitäten hinsichtlich einer Messebeteiligung. ${ }^{3}$

Vgl. Nietiedt, T., Kommunikationspolitik für Hochschulen: Analyse und Instrumentarium, a.a.O., S. 136.

Vgl. hierzu allgemeingültige Ausführungen bei Meffert, H., Unternehmensziele, in: Schöttle, K. M. (Hrsg.), Jahrbuch des Marketing, Essen 1971, S. 22 ff.; Heinen, E., Grundlagen betriebswirtschaftlicher Entscheidungen - Das Zielsystem der Unternehmung, 3. Aufl., Wiesbaden 1976, S. 49 ft.; Dinkelbach, W., Ziele, Zielvariablen und Zielfunktionen, in: DBW, 38. Jg., Heft 1, 1978, S. $51 \mathrm{ff}$.

3 Vgl. Amon, P., Messen + Ausstellungen: Messe-Ziele exakt formulieren und kontrollieren, in: Marketing Journal, 24. Jg., Heft 1, 1991, S. 56; Cavanaugh, S., Setting Objectives and Evaluating the Effectiveness of Trade Show Exhibits, in: JoM, Vol. 40, No. 4, 1976, S. $100 \mathrm{ff}$. 


\subsection{Zielsetzungen von Messebeteiligungen im Hochschulbereich}

\subsection{Hochschulspezifische Messeziele auf verschiedenen Ebenen des Hochschulbereichs}

Aus Hochschulperspektive sind bei einer Messebeteiligung nicht nur eigene Messebeteiligungsziele, sondern auch Ziele der ihr übergeordneten politischen Ebenen zu berücksichtigen. So sind insbesondere Ziele des Bundes und der Länder, die den Hochschulbereich durch Gesetze, Verordnungen und Verwaltungsakte regeln, ${ }^{4}$ darüber hinaus aber auch Ziele der einzelnen Kommunen bei der Messezielfestlegung der Hochschulen zu beachten. Dabei können Zielbeziehungen zwischen den Messebeteiligungszielen der einzelnen Ebenen (vertikale Zielbeziehungen) als auch zwischen den Messebeteiligungszielen auf der jeweiligen Ebene (horizontale Zielbeziehungen) bestehen. ${ }^{5}$ Die Vielzahl möglicher Zielsetzungen und deren Beziehungen untereinander läßt es notwendig erscheinen, die Darstellung hochschulspezifischer Messezielsetzungen auf den einzelnen Zielebenen aus Gründen eines noch handhabbaren Abstraktionsgrades auf wesentliche hochschulspezifische Messebeteiligungsziele auszurichten.

Bund, Länder und Kommunen können mit Hochschul-Messebeteiligungen nach Technologie-, Wirtschafts- und Standortförderungen streben. So können Kommunen bspw. das Ziel verfolgen, ihr Leistungspotential gegenüber ihren Landesregierungen zu präsentieren, um das Bild eines innovativen, dynamischen sowie gesicherten Standortes zu vermitteln. ${ }^{6}$ Die einzelnen Länder können Hochschul-Messebeteiligungen als Instrument zur länderbezogenen Wirtschaftsförderung heranziehen, da die Verfügbarkeit von Hochschul-Know-how einen wichtigen Faktor für die Bestandserhaltung und Neuansiedlung von Unternehmen in dem

Vgl. Gaugler, E., Hochschulen, a.a.O., Sp. 583.

5 Im allgemeinen können drei Formen von Zielbeziehungen unterschieden werden: Zielkomplementarität (die Zielerreichung des einen Ziels begünstigt die Erreichung eines anderen Ziels), Zielneutralität (es besteht keine Korrelation zwischen den Zielerreichungsgraden verschiedener Ziele) und Zielkonkurrenz (die Verfolgung des einen Ziels behindert die Erreichung eines anderen Ziels). Vgl. Meffert, H., Marketing - Grundlagen marktorientierter Unternehmensführung, a.a.O., S. 76.

6 Vgl. hierzu sowie im folgenden Budach, W., Einordnung der Messeteilnahme in das Hochschulmarketing, in: Budach, W. et al. (Hrsg.), Hochschulen und Messen: Praktische Hinweise für Messebeteiligungen durch Hochschulen, a.a.O., S. $17 \mathrm{ff}$. 
jeweiligen Land darstellt. Wenngleich die Hochschulpolitik in der Kompetenz der Länder liegt, darf die Ebene des Bundes im Zielsystem von Hochschul-Messebeteiligungen nicht vernachlässigt werden. Auf dieser Ebene können mit Hochschul-Messebeteiligungen insbesondere forschungspolitische Zielsetzungen verfolgt werden.

Bei der Festlegung von Messebeteiligungszielen aus Perspektive der Hochschulen sind die eigenen Marketing- und Kommunikationsziele ${ }^{7}$ zu berücksichtigen. Die hierarchisch übergeordneten Marketing- und Kommunikationsziele der Hochschule stellen den Orientierungsrahmen für Messebeteiligungsziele dar. Folglich werden Messebeteiligungsziele aus übergeordneten Marketing- bzw. Kommunikationszielen abgeleitet und besitzen damit keinen originären, sondern derivativen Charakter. Gemäß der zwei Transaktionsmärkte von Hochschulen kann bei Zielsetzungen von Hochschul-Exponenten zwischen beschaffungs- und leistungsgerichteten Messebeteiligungszielen ${ }^{8}$ unterschieden werden. $^{9}$

Zu den Marketing- und Kommunikationszielen von Hochschulen vgl. Wangen-Goss, M., Marketing für Universitäten: Möglichkeiten und Grenzen der Übertragbarkeit des MarketingGedankens auf den universitären Bereich, a.a.O., S. 53 ff.; Nietiedt, T., Kommunikationspolitik für Hochschulen: Analyse und Instrumentarium, a.a.O., S. $136 \mathrm{ff}$.

Oftmals sind die Überschneidungen der beschaffungs- und leistungsgerichteten Messebeteiligungsziele hoch. Dennoch sollen gemäß der ${ }_{n}$ getrennten“ Transaktionsprozesse auf den Teilmärkten die beiden Zielrichtungen separat analysiert werden. Vgl. Budach, W., Einordnung der Messeteilnahme in das Hochschulmarketing, a.a.O., S. $7 \mathrm{ff}$.

Die Auswahl der Einzelzielsetzungen von Hochschul-Exponenten wird auf Grundlage einer Analyse von in der Literatur aufgeführten Messebeteiligungszielen vorgenommen. Dabei erfolgt aus Gründen der Überschaubarkeit eine Fokussierung auf dominante, für den Hochschulbereich besonders relevante Messebeteiligungsziele. Die Auswahl der hochschulspezifischen Messezielsetzungen wird dabei unter besonderer Berücksichtigung der Zielsetzungen übergeordneter Ebenen des Hochschulbereichs sowie der Marketing- und Kommunikationsziele von Hochschulen auf Basis der Nennhäufigkeit jedes Einzelziels in der einschlägigen allgemeinen Messeliteratur vorgenommen. Im Hinblick auf eine umfassende Darstellung möglicher Messebeteiligungsziele in der allgemeinen Messeliteratur vgl. Selinski, H., Sperling U. A., Marketinginstrument Messe: Arbeitsbuch für Studium und Praxis, a.a.O., S. 108 f.; Fließ, S., Messeselektion - Entscheidungskriterien für Investitionsgüteranbieter, a.a.O., S. 12 f.; Schober, P. M., Messen + Ausstellungen: Teilnehmen oder wegbleiben? - Eine kleine Entscheidungs-Hilfe, in: Marketing Journal, 21. Jg., Heft 4, 1988, S. 402; Chapman, E. A., Exhibit Marketing: A Survival Guide for Managers, a.a.O., S. $57 \mathrm{ff}$.; Raffée, H., Messen als Herausforderung für die Marketing-Theorie, in: Deutsche Werbewissenschaftliche Gesellschaft e.V. (DWG) (Hrsg.), Messen als Marketinginstrument, a.a.O., S. 75 f.; Gräbener, W., Die Messepolitik als Marketinginstrument - dargestellt am Beispiel von Investitionsgüterproduzenten, a.a.O., S. 159 f.; Weishäupl, G., Die Messe als Kommunikationsmedium - unter besonderer Berücksichtigung der Öfentlichkeitsarbeit und Werbung einer Messegesellschaft, München 1980, S. $64 \mathrm{ff}$. sowie die an diesen Stellen jeweils zitierte Literatur. 
Mit auf den Beschaffungsmarkt gerichteten Messezielen können HochschulExponenten die Beschaffung von personellen Ressourcen sowie Finanz- und Sachmitteln anstreben. ${ }^{10}$ Im Hinblick auf die Personalbeschaffung verfolgen Hochschul-Exponenten mit Messebeteiligungen möglicherweise das Ziel, das Hochschulimage bzw. das Image des Instituts, Lehrstuhls und/oder Fachbereichs zu fördern, um damit den Zugang und Verbleib von Wissenschaftlern und Studierenden sowie Hochschulverwaltungsangestellten zu forcieren. Dabei können neben der Imageförderung ebenfalls Ziele wie Erhöhung des Bekanntheitsgrades der Hochschule bzw. des Instituts, Lehrstuhls und/oder Fachbereichs angestrebt werden.

Im Rahmen der Finanzmittelbeschaffung, deren Realisierung die Voraussetzung zur Beschaffung von Sachmitteln darstellt, kann eine Messebeteiligung auf die zusätzliche Einwerbung sowohl von öffentlichen Haushaltsmitteln als auch von öffentlichen sowie privaten bzw. gewerblichen Drittmitteln angelegt sein. Angesichts des Einflusses, den die Beurteilung einer Hochschule durch das zuständige Wissenschafts- und Wirtschaftsministerium auf die Beschaffung von Haushaltsmitteln besitzt, bieten Messebeteiligungen den Hochschul-Exponenten darüber hinaus die Möglichkeit, ihre Entwicklungspotentiale zu dokumentieren, die in Abhängigkeit von dem Erhalt zusätzlicher Etatmittel verwirklicht werden könnten. Von Bedeutung könnten dabei ebenfalls Darstellungen strukturpolitischer Aktivitäten sowie die in diesem Zusammenhang geplanten Projekte sein, da Wirtschaftsministerien Hochschulen und deren Standorte u.a. anhand strukturpolitisch orientierter Kriterien beurteilen. Dazu zählen bspw. Kooperationen mit regional naheliegenden Unternehmen, die „Hebelwirkungen“ auf zusätzliche Hochschulförderungen zur Folge haben können.

Ähnliche Kriterien finden bei der Vergabe von Drittmitteln aus Landeshaushalten, d.h. also öffentlichen Drittmitteln, Anwendung. In diesem Zusammenhang kann von Hochschul-Exponenten ebenfalls eine "Hebelwirkung“ in dem Sinne forciert werden, als daß eine Teilförderung (z.B. aus Landesmitteln) das Potential einer zusätzlichen Erschließung von Komplementärmitteln (z.B. aus Bundes-, EG- oder sonstigen Finanzquellen) bietet. Durch die Präsentation von bereits durchgeführ- 
ten Drittmittelprojekten auf Messen kann zudem eine Anschluß- oder zusätzliche Projekttörderung angestrebt werden. Neben der Dokumentation des Leistungspotentials bietet eine Messebeteiligung die Möglichkeit, Kontakt zu Vertretern entsprechender Institutionen aufzunehmen und damit die Beziehungen zu relevanten Institutionen des Hochschulbereichs aufzubauen bzw. zu pflegen.

Hinsichtlich der Beschaffung nicht-öffentlicher, d.h. also privater bzw. gewerblicher Drittmittel, können Hochschul-Exponenten mit Messebeteiligungen das Ziel verfolgen, ihre erbrachten wissenschaftlichen Leistungen, die idealerweise zu marktfähigen oder marktnahen Produkten bzw. Verfahren geführt haben, einem breiten Fachpublikum aus Industrie und Wissenschaft vorzustellen, um damit ihre Forschungspotentiale sowie Kooperationbereitschaft zu dokumentieren. Damit können Hochschul-Exponenten private bzw. gewerbliche Drittmittel für die Fortführung der bisherigen oder den Beginn neuer Forschungsprojekte einwerben, um notwendige sachliche und personelle Ressourcen für einen begrenzten Zeitraum zu decken. Für Hochschul-Exponenten eröffnet dies gleichzeitig die Möglichkeit, zusätzliche Finanzmittel bspw. aus Lizenzverträgen - sofern sich der HochschulExponent die Verwertungsrechte an der entsprechenden Entwicklung vorbehält für das präsentierte Exponat oder vorhandener, aber auf der Messe nicht präsentierter Entwicklungen einzuwerben. ${ }^{11}$

Die letztgenannten beschaffungsmarktgerichteten Ziele stehen in einem engen Zusammenhang mit den auf den Leistungsmarkt gerichteten Messezielen. So können Hochschul-Exponenten mit Messebeteiligungen auch Ziele verfolgen, die auf die Vermarktung von Forschung, Lehre und sonstigen Dienstleistungen gerichtet sind.

Exponenten des Hochschulbereichs können mit ihrer Exponatpräsentation auf Messen z.B. anstreben, die Breite, Tiefe, Qualität und Interdisziplinarität inrer Forschung herauszustellen. ${ }^{12}$ Dabei bieten sich in bezug auf die Grundlagenforschung Möglichkeiten, Anwendungsbezüge zu eruieren. Oftmals sind den Hochschul-Exponenten die Einsatzmöglichkeiten von Ergebnissen aus der Grundla-

11 Vgl. Krug, W., Kosten - Finanzierung - Erfolg: Spannungsfeld Erwartungen - Realität, in: Budach, W. et al. (Hrsg.), Hochschulen und Messen: Praktische Hinweise für Messebeteiligungen durch Hochschulen, a.a.O., S. 148.

12

Vgl. hierzu sowie im folgenden Budach, W., Einordnung der Messeteilnahme in das Hochschulmarketing, a.a.O., S. $10 \mathrm{ff}$. 
genforschung nicht bekannt, so daß die auf einer Messe geführten Gespräche mit Praktikern - aber auch eigene, durch die Messeatmosphäre stimulierte Nutzungsüberlegungen - wertvolle Anregungen zu praktischen Einsatzmöglichkeiten bieten können. Bei bereits transferfähigen Exponaten kann dabei eine Messebeteiligung zur Überprüfung der Marktreife von präsentierten Produkten und Leistungen beitragen. Auch kann durch eine Messebeteiligung die Kontaktaufnahme zu Kooperationspartnern angestrebt werden. Letztlich bieten sich Hochschul-Exponenten durch Exponatpräsentationen auf Messen Möglichkeiten, das Problemlösungspotential, die Themenvielfalt, die Kooperationsbereitschaft und -fähigkeit der Hochschule - sowohl gegenüber Unternehmen als auch gegenüber der Wissenschaft - darzustellen.

Die Darstellung von Hochschullehre, als weiteres mögliches leistungsmarktorientiertes Messeziel, bezieht sich u.a. auf den „Absatz der Hochschulabsolventen", der durch ein entsprechendes Hochschulimage forciert werden kann. Durch eine Messebeteiligung können Hochschul-Exponenten die Qualität inrer Hochschule sowie der an ihr ausgebildeten Studienabgänger präsentieren und damit den Berufseinstieg und die berufliche Karriere ihrer Absolventen positiv beeinflussen. In diesem Zusammenhang kann ebenfalls ein langfristig positiver Einfluß auf künftige Entwicklungen der jeweiligen Hochschulen angestrebt werden, da beruflich gut etablierte Absolventen wiederum Verbindungen für spätere Firmenkontakte oder Drittmittelprojekte herstellen können.

Im Hinblick auf die Vermarktung von sonstigen Dienstleistungen der Hochschulen können Hochschul-Exponenten mit ihrer Messebeteiligung die Zielsetzung verfolgen, die eigene Leistungskompetenz und das eigene Leistungspotential (z.B. in bezug auf klinische Leistungen oder Beraterleistungen) darzustellen. Nicht zuletzt kann eine Messebeteiligung in diesem Zusammenhang auch zur Informationsgewinnung (z.B. bzgl. wettbewerbsbezogener Entwicklungen) dienen, um ggf. Anregungen für die zukünftige Ausgestaltung der sonstigen Dienstleistungen zu erlangen.

Vor dem Hintergrund der aufgezeigten, von Hochschul-Exponenten verfolgten möglichen Messebeteiligungsziele ist davon auszugehen, daß zwischen den beschaffungs- und leistungsmarktgerichteten Messebeteiligungszielen Zielkom- 
plementaritäten bestehen. So wird sich bspw. das Image der Grundlagenforschung einer renommierten Hochschule positiv auf beschaffungsmarktgerichtete Messeziele, wie der Beschaffung von Finanz- und Sachmitteln, auswirken. ${ }^{13}$

Zwischen den vorrangig strukturpolitischen Zielsetzungen, die Bund, Länder und Kommunen mit Hochschul-Messebeteiligungen verfolgen, und den Messebeteiligungszielsetzungen der Hochschul-Exponenten lassen sich vielfältige - sowohl neutrale als auch konfliktäre, überwiegend jedoch komplementäre - Zielbeziehungen vermuten. Zielsetzungen des Bundes, der Länder und der Kommunen werden i.d.R. - wenn oftmals auch nur in indirekter Form - ebenfalls von Hochschul-Exponenten angestrebt. Zum einen sind Hochschulen z.T. dazu verpflichtet, wenn sie zur Finanzierung ihrer Messebeteiligung auf Etatmittel des Bundes bzw. der Länder oder auf kommunale bzw. regionale Finanzmittel zurückgreifen. Zum anderen werden Ziele wie Technologie-, Wirtschafts- und Standortförderung durch das Hochschulrahmengesetz vorgegeben. Bund- und länderbezogene sowie kommunale Zielsetzungen einer Hochschul-Messebeteiligung werden somit ebenfalls auf Hochschulebene angestrebt, wobei sie in den auf dieser Ebene verfolgten Messebeteiligungszielen implizit enthalten sind (vgl. Abb. 5). Vor diesem Hintergrund wird im folgenden die Untersuchung auf die Ebene der Hochschul-Exponenten fokussiert.

Die Vielfalt möglicher hochschulspezifischer Messezielsetzungen deutet darauf hin, daß im Rahmen der Messebeteiligung von Hochschulen i.d.R. mehrdimensionale Zielsysteme ${ }^{14}$ vorliegen. ${ }^{15}$ Hochschul-Exponenten werden nicht allen Zielsetzungen, auf die ihre Messebeteiligung ausgerichtet ist, den gleichen Stellenwert einräumen. Folglich ist zu überprüfen, inwieweit den einzelnen hochschulspezifi-

Empirische Untersuchungen belegen, daß auch im Rahmen der Messebeteiligung von kommerziellen Institutionen mehrdimensionale Zielkonzeptionen vorliegen. Vgl. stellvertr. Meffert, H., Ueding, R., Ziele und Nutzen von Messebeteiligungen, in: Ausstellungs- und Messeausschuss der Deutschen Wirtschaft e.V. (AUMA) (Hrsg.), AUMA Edition Nr. 4, Bergisch Gladbach 1996, S. 30 ff.; Spryß, W. M., Nerb, G., Reuter, J., Messeziele - Messeeffizienz, a.a.O., S. 10 f.; Täger, U. C., Ziegler, R., Die Bedeutung von Messen und Ausstellungen in der Bundesrepublik Deutschland für den Inlands- und Auslandsabsatz in ausgewählten Branchen, a.a.O., S. $51 \mathrm{f}$. 
schen Messebeteiligungszielen unterschiedliche Bedeutungsgewichte aus Exponentensicht beigemessen werden und ob sich ggf. eine eindeutige Prioritätenrangfolge identifizieren läßt.

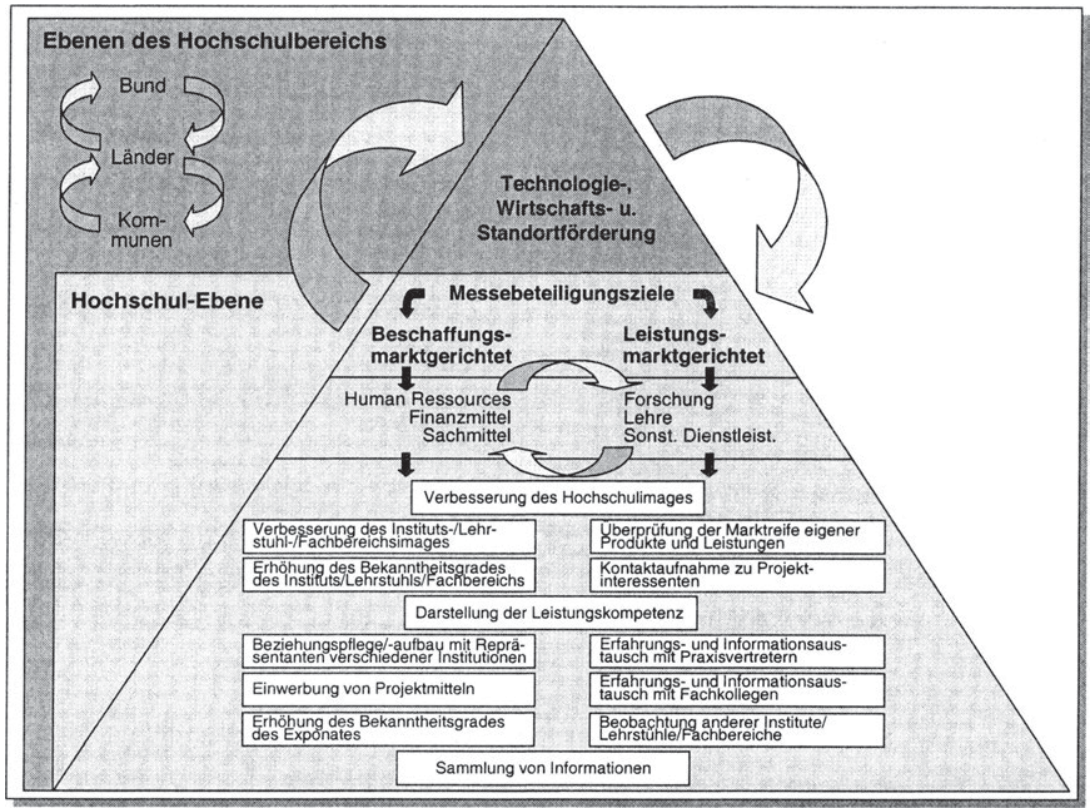

Abb. 5: Hochschulspezifische Messezielsetzungen auf verschiedenen Ebenen des Hochschulbereichs

\subsection{Empirische Analyse der Bedeutung hochschulspezifischer Messebe- teiligungsziele}

Die empirische Ermittlung der Bedeutungsgewichte von hochschulspezifischen Messebeteiligungszielen zeigt weniger eine eindeutige Prioritätenrangfolge der Zielsetzungen als vielmehr eine generell hohe Bedeutung der hochschulspezifischen Messebeteiligungsziele auf. ${ }^{16}$ Trotz des daraus resultierenden relativ zugrundeliegende empirische Untersuchung einbezogen. Vgl. Anhang III, Fragebogen 2: Teil 2 der Exponentenbefragung I in der Vor-Messe-Phase, Frage 7. 
linksschiefen Profils können abgestufte Bedeutungsrangfolgen innerhalb der generell als sehr wichtig bis wichtig eingestuften Messebeteiligungsziele aufgedeckt werden (vgl. Abb. 6). So bewerten Hochschul-Exponenten die Erhöhung des Bekanntheitsgrades des Exponates und der Institution des Exponenten, die Darstellung der Leistungskompetenz sowie den Erfahrungs- und Informationsaustausch mit Praxisvertretern mit Mittelwerten von 1,3 bzw. 1,4 als sehr wichtige Messebeteiligungsziele. Die geringen Standardabweichungen von 0,62 bis 0,71 belegen die überwiegende Einigkeit der Exponenten in dieser Bewertung. Darüber hinaus werden aus Sicht von Hochschul-Exponenten der Kontaktaufnahme zu Projektinteressenten sowie der Verbesserung des Instituts-, Lehrstuhl- und Fachbereichsimages mit Mittelwerten von 1,6 und 1,7 überdurchschnittliche Stellenwerte im Rahmen des Zielsystems von Hochschul-Messebeteiligungen zugeordnet. Den anderen aufgezeigten Messezielsetzungen werden demgegenüber insgesamt geringere Bedeutungen beigemessen, wenngleich diese Zielsetzungen mit Mittelwerten von 2,0 bis 2,7 im Rahmen von Hochschul-Messebeteiligungen nicht als unwichtig einzustufen sind.

\begin{tabular}{|c|c|c|c|c|c|}
\hline Hochschulspezifische Messebeteiligungsziele & $\begin{array}{l}\text { Stan- } \\
\text { dard- } \\
\text { abw. }\end{array}$ & sehr wicht & 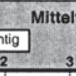 & & \begin{tabular}{|ll} 
\\
\end{tabular} \\
\hline Verbesserung des Hochschulimages & 0,94 & & 2,0 & & \\
\hline Verbesserung des Instituts-/Lehrstuhl-/Fachbereichsimages & 1,02 & . & 1,7 & & \\
\hline Erhöhung des Bekanntheitsgrades des Instit./Lehrst./Fachber. & 0,62 & - & 1,4 & & \\
\hline Erhöhung des Bekanntheitsgrades des Exponates & 0,71 & $\bullet 1,3$ & & & \\
\hline Darstellung der Leistungskompetenz & 0,62 & - 1 & 1,4 & & \\
\hline Sammlung von Informationen & 0,95 & & - 2 & 4 & \\
\hline Beziehungspflege/-aufbau mit Repräsent. verschied. Institutionen & 1,09 & & 2,1 & & \\
\hline Beobachtung anderer Institute/Lehrstühle/Fachbereiche & 0,98 & & $\bullet$ & 2,7 & \\
\hline Erfahrungs- und Informationsaustausch mit Praxisvertretern & 0,66 & $\bullet$ & 1,4 & & \\
\hline Erfahrungs- und Informationsaustausch mit Fachkollegen & 0,87 & & - 2, & 4 & \\
\hline Kontaktaufnahme zu Projektinteressenten & 1,04 & & 1,6 & & \\
\hline Überprüfung der Marktreife eigener Produkte und Leistungen & 1,10 & & - 2,2 & & \\
\hline Einwerbung von Projektmitteln & 1,23 & & 2,0 & & \\
\hline
\end{tabular}

\section{Abb. 6: Bedeutung hochschulspezifischer Messebeteiligungsziele}

Die aufgezeigte Priorisierung hochschulspezifischer Messebeteiligungsziele deutet auf zwei grundlegende Aspekte hin, die das Messeengagement von Hochschul-Exponenten besonders beeinflussen. Zum einen bildet das im Hochschulrahmengesetz verankerte Gebot, die Leistungen von Hochschulen gegenüber der 
sie alimentierenden Gesellschaft zu dokumentieren, einen wesentlichen Grund der Messebeteiligung. So erhält bspw. die Zielsetzung Darstellung der Leistungskompetenz eine der höchsten Prioritäten im Zielsystem von Hochschul-Messebeteiligungen. Zum anderen stellen knapper werdende Etatmittel, die eine Akquisition von zusätzlichen Finanzmitteln (u.a. durch Projektkooperationen) notwendig werden lassen, Bewegungsgründe einer Hochschul-Messebeteiligung dar. In diesem Zusammenhang ist Z.B. auf die hohe Bedeutung der Zielsetzung Kontaktaufnahme zu Projektinteressenten hinzuweisen.

Angesichts der Vielfältigkeit der aufgezeigten Messebeteiligungsziele stellt sich die Frage, inwieweit sich hochschulspezifische Einzelzielsetzungen systematisieren und auf eine geringere Anzahl übergeordneter Zieldimensionen verdichten lassen.

\subsection{Zieldimensionen von Hochschul-Messebeteiligungen}

\subsection{Systematisierung hochschulspezifischer Messebeteiligungsziele}

Zur Strukturierung von Hochschul-Messezielsetzungen können grundsätzlich verschiedene Systematisierungsansätze der Literatur herangezogen werden. So können hochschulspezifische Messebeteiligungsziele - wie übergeordnete Kommunikations- und Marketingziele - prinzipiell in ökonomische und psychographische Ziele unterteilt werden. ${ }^{17}$ Unter ökonomischen Messebeteiligungszielen werden solche Zielinhalte verstanden, die im wesentlichen monetäre Größen beinhalten. ${ }^{18}$ Dieser Kategorie gehört bspw. das Ziel Einwerbung von Projektmitteln an. Zu psychographischen Messebeteiligungszielen zählen dagegen solche Zielsetzungen, die auf mentale Prozesse des Messe- bzw. Hochschul-MessestandBesuchers ausgerichtet sind und sowohl affektiv-gefühlshafte als auch kognitivrationale Ergebnisse forcieren und damit letztlich beim Adressaten vom Expo-

17 Vgl. Meffert, H., Marketing - Grundlagen marktorientierter Unternehmensführung, a.a.O., S. 319 ff. In der Literatur werden zur Kennzeichnung psychographischer Zielsetzungen auch Begriffe wie „außerökonomische“ bzw. „kommunikative“ Ziele synonym verwendet. Vgl. stellvertr. Selinski, H., Sperling U. A., Marketinginstrument Messe: Arbeitsbuch für Studium und Praxis, a.a.O., S. 107.

Vgl. Behrens, K. C., Absatzwerbung, Wiesbaden 1963, S. $50 \mathrm{ff}$. 
nenten beabsichtigte Verhaltensweisen auslösen. ${ }^{19}$ In diese Zielkategorie kann z.B. das Streben nach Verbesserung des Hochschulimages sowie des Instituts-, Lehrstuhl- und Fachbereichsimages eingeordnet werden.

Bei der zuvor vorgenommenen Strukturierung von Messebeteiligungszielen wird lediglich ein allgemeiner Ansatz zur Systematisierung von Marketingzielen auf das Management von Messebeteiligungen übertragen. Aufgrund der unzureichenden Berücksichtigung von Messebesonderheiten bei derartigen Übertragungen entwickelte MEFFERT zur Erfassung messespezifischer Zielkonzeptionen von Ausstellern einen Systematisierungsansatz, der sich an den Funktionen von Mes$\operatorname{sen}^{20}$ orientiert und damit unmittelbar den Bezug zwischen der Funktionalebene einer Messeveranstaltung und der Zielebene eines einzelnen Ausstellers herstellt. MEFFERT unterscheidet die folgenden vier Zieldimensionen, denen messespezifische Einzelzielsetzungen zugeordnet werden können: ${ }^{21}$

$\begin{array}{ll}\text { Informationsziele } & \text { - Ziele der Informationsweitergabe } \\ & - \text { Ziele der Informationsbeschaffung } \\ & - \text { Ziele der Markterkundung } \\ \text { Motivationsziele } & - \text { mitarbeiterorientierte Ziele } \\ & - \text { veranstalterorientierte Ziele } \\ & - \text { besucherorientierte Ziele } \\ \text { Beeinflussungsziele } & - \text { Einstellungsziele } \\ & - \text { Bekanntheitsziele } \\ & - \text { Imageziele }\end{array}$

Verkaufsziele

- Verkaufsvorbereitungsziele

- Verkaufsdurchführungsziele

- Verkaufserhaltungsziele

Vgl. Meffert, H., Das System des Kommunikations-Mix, Münster 1979, S. 156.

Als zentrale Messefunktionen werden von MefFert die Informations-, Motivations-, Beeinflussungs- und Verkaufsfunktion identifiziert. Vgl. Meffert, H., Messen und Ausstellungen als Marketinginstrument, in: Goehrmann, K. E. (Hrsg.), Polit-Marketing auf Messen, Düsseldorf 1993, S. $10 \mathrm{f}$.

Vgl. Meffert, H., Messen und Ausstellungen als Marketinginstrument, a.a.O., S. 16 f. 
Nicht zuletzt die vierte Zieldimension Verkaufsziele verdeutlicht, daß sich diese Systematisierung weniger auf nicht-kommerzielle als vielmehr auf kommerzielle Messebeteiligungen bezieht. In diesem Zusammenhang ist zu überprüfen, inwieweit sich diese messefunktionsorientierten, für kommerzielle Messebeteiligungen geltenden Zieldimensionen in hochschulspezifischen Messebeteiligungszielen widerspiegeln oder inwieweit die Besonderheiten von Hochschul-Messebeteiligungen zu einer eigenen Systematisierung von hochschulspezifischen Messebeteiligungszielen führen.

\subsection{Empirische Ermittlung hochschulspezifischer Messezieldimensionen auf Basis einer Faktorenanalyse}

Zur Ermittlung hochschulspezifischer Messezieldimensionen ist eine Verdichtung der zur Kennzeichnung der verschiedenen, hochschulspezifischen Messebeteiligungszielsetzungen eingesetzten Variablen notwendig. Zur Erreichung dieser Datenreduktion bietet sich eine Faktorenanalyse an. ${ }^{22}$

Durch die Anwendung der Faktorenanalyse werden die Einzelzielsetzungen der Messebeteiligung von Hochschulen aufgrund vorliegender Interkorrelationen auf eine kleinere Anzahl von übergeordneten Zieldimensionen reduziert. Als Kriterium für die Anzahl der zu extrahierenden Faktoren soll dabei das Kaiser-Kriterium zugrunde gelegt werden, wonach solange zusätzliche Faktoren zu extrahieren sind, wie der Eigenwert ${ }^{23}$ des zusätzlichen Faktors größer eins ist. ${ }^{24}$ Nach diesem Kriterium sind aus den Einzelzielsetzungen der Messebeteiligung von Hochschulen fünf Faktoren zu extrahieren (vgl. Abb. 7). Mit diesen fünf Faktoren können insgesamt $75,6 \%$ der Gesamtvarianz erklärt werden, welches auf ein valides Ergebnis schließen läßt. ${ }^{25}$

Vgl. Backhaus, K. et al., Multivariate Analysemethoden: Eine anwendungsorientierte Einführung, a.a.O., S. 189 ff.; Bauer, F., Datenanalyse mit SPSS, a.a.O., S. $201 \mathrm{ff}$.

Der Eigenwert eines Faktors erklärt dessen Anteil an der erklärten Varianz aller Einzelvariablen.

Vgl. Meffert, H., Marketingforschung und Käuferverhalten, a.a.O., S. 264.

Nach dem Kaiser-Meyer-Olkin-Kriterium, das auf Basis der Anti-Image-Korrelationsmatrix eine Prüfgröße mit der Bezeichnung "measure of sampling (MSA)“ berechnet, wird mit einem Wert von MSA = 0,604 zwar nur eine "mittelmäßige“, aber dennoch hinreichende Eignung der Aus- 


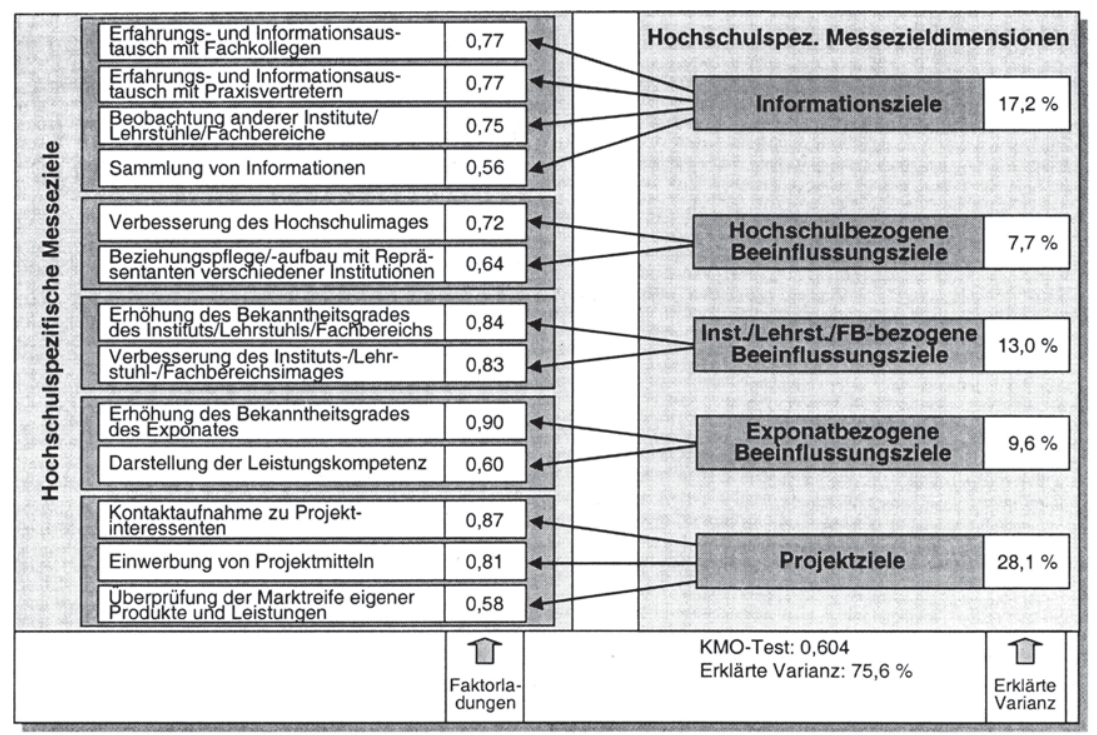

Abb. 7: Faktorenanalytische Ermittlung der Messezieldimensionen

Die Interpretation ${ }^{26}$ der ermittelten Faktoren bzw. Zieldimensionen weist teilweise inhaltliche Überschneidungen zu den von MEFFERT in der Literatur diskutierten Messebeteiligungs-Zieldimensionen kommerzieller Institutionen auf. So sind die in der Literatur aufgeführten Informations- und Beeinflussungsziele auch bei einer Hochschul-Messebeteiligung von besonderer Relevanz. Dabei sind in bezug auf Informationsziele neben der allgemeinen Informationserfassung persönliche Kontakte zur Informationsbeschaffung mit Vertretern aus Praxis und Wissenschaft anzuführen. So stehen hinter dem Faktor Informationsziele, der mit insgesamt $17,2 \%$ den zweitgrößten Erklärungsbeitrag der Gesamtvarianz liefert, insbesondere die Zielsetzungen Erfahrungs- und Informationsaustausch mit Fachkollegen sowie Praxisvertretern und Beobachtung anderer Institute/Lehrstühle/Fachbereiche. Das Ziel Sammlung von Informationen lädt ebenfalls, wenngleich in schwächerer Form, auf diese Dimension.

gangsvariablen zur Durchführung einer Faktorenanalyse erreicht. Vgl. Backhaus, K. et al., Multivariate Analysemethoden: Eine anwendungsorientierte Einführung, a.a.O., S. 206.

Zur Interpretationserleichterung wurde eine Varimax-Rotation durchgeführt. Zur Bedeutung von Rotationen im Rahmen von Faktorenanalysen vgl. Backhaus, K. et al., Multivariate Analysemethoden: Eine anwendungsorientierte Einführung, a.a.O., S. 229 ff. 
In Bezug auf die Beeinflussungsziele erscheint vor dem Hintergrund der dargestellten hochschulspezifischen Rahmenbedingungen bei Hochschul-Messebeteiligungen im Gegensatz zu Messebeteiligungen kommerzieller Institutionen eine Spezifizierung notwendig. Neben hochschulbezogenen Beeinflussungszielen sind auch Bekanntheits- und Imageziele auf Instituts-, Lehrstuhl- und Fachbereichsebene sowie die auf das Exponat bezogenen Beeinflussungsziele zu berücksichtigen. Der Faktor Hochschulbezogene Beeinflussungsziele, der mit 7,7 \% den geringsten Erklärungsbeitrag zu der Gesamtvarianz leistet, wird von dem Ziel Verbesserung des Hochschulimages, darüber hinaus aber auch - wenngleich mit geringerer Intensität - von der Zielsetzung Beziehungspflege/-aufbau mit Repräsentanten verschiedener Institutionen determiniert. Die hohen Faktorladungen der hinter dem Faktor Instituts-/Lehrstuhl-/Fachbereichsbezogene Beeinflussungsziele stehenden Zielsetzungen Erhöhung des Bekanntheitsgrades des Instituts/Lehrstuhls/Fachbereichs und Verbesserung des Instituts-/Lehrstuhls-/ Fachbereichsimages liegen annähernd auf gleichem Niveau. Dabei trägt dieser Faktor insgesamt 13,0 \% zur Erklärung der Gesamtvarianz bei, gefolgt von dem Faktor Exponatbezogene Beeinflussungsziele, der mit insgesamt $9,6 \%$ den viertgrößten Erklärungsbeitrag zur Gesamtvarianz leistet. Von den auf diesen Faktor ladenden Einzelzielsetzungen besitzt das Ziel Erhöhung des Bekanntheitsgrades des Exponates eine erheblich höhere Faktorladung als die Darstellung der Leistungskompetenz.

Im Hinblick auf die bei kommerziellen Messebeteiligungen verfolgten Verkaufsziele ist zu berücksichtigen, daß Messebeteiligungen von Hochschulen weniger auf den "Verkauf" präsentierter Exponate ausgerichtet sind. Vielmehr geht es bei Hochschul-Messebeteiligungen um die Anbahnung, Durchführung sowie Forttührung von Projekten bzw. Projektkooperationen sowie die Überprüfung der Marktreife von Projektentwicklungen. Somit ergibt sich mit den Projektzielen eine fünfte hochschulspezifische Messezieldimension. Dabei stehen hinter dieser Dimension neben dem Ziel der Überprüfung der Marktreife eigener Produkte und Leistungen insbesondere die Ziele Kontaktaufnahme zu Projektinteressenten und Einwerbung von Projektmitteln. Die Projektziele besitzen mit 28,1 \% den höchsten Erklärungsanteil an der Gesamtvarianz. 
Den hochschulspezifischen Zieldimensionen ${ }^{27}$ werden, wie die folgende Abbildung verdeutlicht, von Hochschul-Exponenten abgestufte Bedeutungsgewichte zugeordnet. ${ }^{28}$ Dabei messen die Hochschul-Exponenten insgesamt den Exponatbezogenen sowie den Instituts-/Lehrstuhl-/Fachbereichsbezogenen Beeinflussungszielen eindeutig die höchsten Prioritäten bei, gefolgt von Projekt- und Hochschulbezogenen Beeinflussungszielen. Die Bedeutung von Informationszielen wird demgegenüber vergleichsweise niedrig eingestuft. Diese Priorisierung verdeutlicht, daß im Rahmen einer Hochschul-Messebeteiligung vor allem diejenigen Zieldimensionen im Vordergrund stehen, die einen besonderen Beitrag zur profilierenden Darstellung der Exponenten leisten. Damit streben Exponenten mit ihrer Messebeteiligung vor allem danach, den vom Gesetzgeber erteilten Auftrag, die eigenen Leistungen gegenüber der Gesellschaft zu dokumentieren, Rechnung zu tragen. Zudem wird durch die vergleichsweise hohe Bewertung der Projektziele die Relevanz der Akquirierung zusätzlicher Finanzmittel als Zielsetzung einer Messebeteiligung bestätigt. ${ }^{29}$

Die sich in der Literatur in der Dimension Motivationsziele widerspiegelnden mitarbeiterbezogenen Ziele können i.d.R. erst dann erreicht werden, wenn die Ziele der anderen Zieldimensionen bereits realisiert sind. So kann bspw. eine Messebeteiligung, mit der keine der verfolgten Zielsetzungen auch nur annähernd erreicht wird, zur Demotivation der Mitarbeiter führen. Zur Vermeidung von Ergebnisverzerrungen - gerade bei Mißerfolgen früherer Messebeteiligungen - sind derartige Messezielsetzungen nicht explizit in die empirische Untersuchung aufgenommen worden, sondern wurden implizit in den anderen Zieldimensionen berücksichtigt. Somit kann die von MEFFERT aufgeführte Zieldimension Motivationsziele hier empirisch nicht abgeleitet werden.

Die Mittelwerte der Zieldimensionen ergeben sich aus dem arithmetischen Mittel der mittleren Gesamtpunktwerte. Der mittlere Gesamtpunktwert einer Person $\mathrm{g}$ in dem Faktor $\mathrm{h}$ ist dabei als Mittelwert der Ausprägungen der Person $\mathrm{g}$ in den Variablen, die den Faktor $\mathrm{h}$ messen, definiert als $y_{g h}=\left(\Sigma_{i \in h} x_{g i}\right) / m_{h}$. Dabei stellt $x_{g i}$ die Ausprägung der Person $g$ in der Variablen $i$ dar, während die Bezeichnung $m_{h}$ für die $Z$ ahl der Variablen $i$ steht, die den Faktor $h$ bilden. Anstelle der Faktorwerte liegt der Vorteil der mittleren Gesamtpunktwerte darin, daß diese Werte in der ursprünglichen Skala kodiert und interpretiert werden können und folglich Vergleiche zwischen Gesamtpunktwerten möglich sind. Vgl. Bacher, J., Clusteranalyse - Anwendungsorientierte Einführung, München 1994, S. 124 f.

Die Prioritätenränge der Einzelzielsetzungen sind ebenfalls in Abb. 8 aufgeführt. Die Einzelzielsetzungen, die von den Exponenten als gleichgewichtig eingestuft werden, erhalten den gleichen Prioritätenrang, wobei jeweils ein Buchstabe zur Differenzierung ergänzt wird. Für die dreizehn Einzelzielsetzungen ergeben sich letztlich neun Prioritätenränge. 


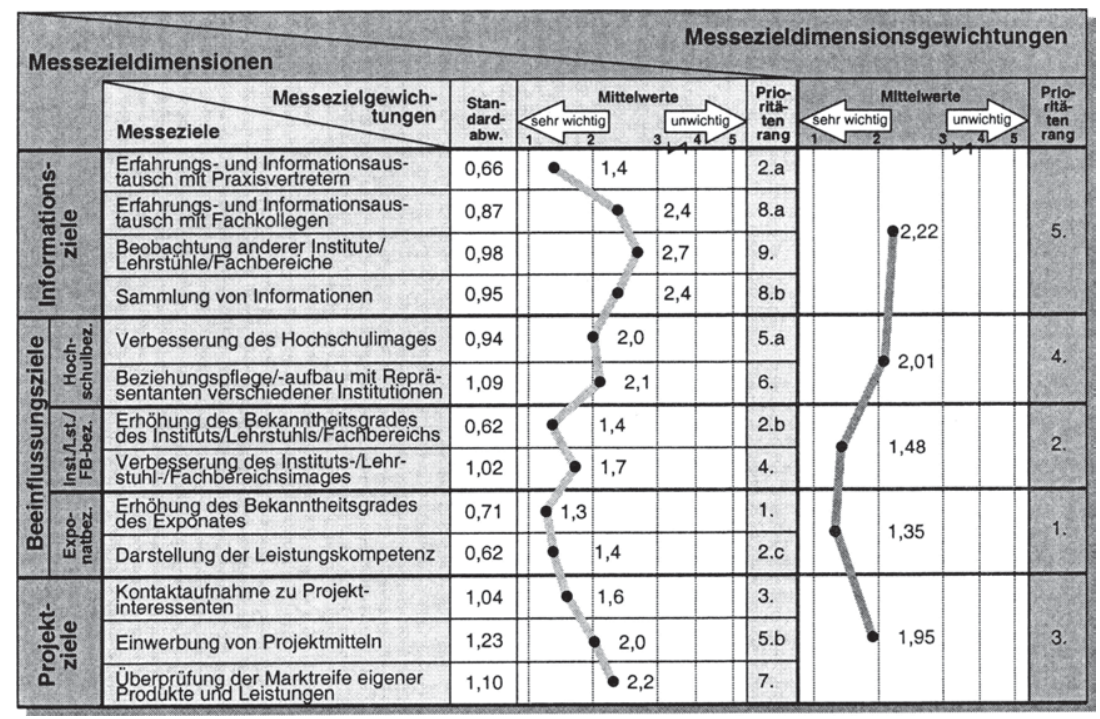

Abb. 8: Bedeutung hochschulspezifischer Messeziele und Messezieldimensionen

Trotz z.T. vorhandener Überschneidungen zu kommerziellen Messezieldimensionen läßt sich - so zeigen die vorangegangenen Ausführungen - im Rahmen von Hochschul-Messebeteiligungen ein Systematisierungsansatz mit fünf hochschulspezifischen Messezieldimensionen identifizieren. Angesichts der aufgezeigten Gewichtungen dieser Zieldimensionen ist davon auszugehen, daß sich auf Basis dieser Zielgewichtungen bestimmte Typen von Hochschul-Exponenten gegeneinander abgrenzen lassen, die sich in bezug auf ihre primär verfolgten Messezielsetzungen unterscheiden. Somit ist zu überprüfen, inwieweit sich hochschulspezifische Exponententypen bilden lassen, die mit ihrer Messebeteiligung ähnliche Zielsetzungen verfolgen. Dabei ist ebenfalls der Frage nachzugehen, ob sich diese ggf. ableitbaren Hochschul-Exponententypen angesichts der besonderen Rahmenbedingungen von Ausstellertypen im Bereich kommerzieller Messebeteiligungen unterscheiden. 


\subsection{Ableitung von Hochschul-Exponententypen auf Basis hochschulspe- zifischer Messezieldimensionen}

\subsection{Darstellung exponentenspezifischer Grundhaltungstypen}

Wenngleich die unterschiedliche Priorisierung von Messebeteiligungszielen das messespezifische Ausstellerverhalten determiniert und damit für das Messewesen insgesamt von besonderer Relevanz ist, hat sich erst in jüngster Vergangenheit UEDING unter der Zielsetzung, das messespezifische Verhalten kommerzieller Aussteller zu beschreiben und zu erklären, wissenschaftlich mit dieser Thematik auseinandergesetzt. ${ }^{30}$ Dabei bildeten die von den Exponenten verfolgten Messeziele eine zentrale Grundhaltungsdimension, ${ }^{31}$ die sich aus den sechs Zieldimensionen Informationsziele, Motivationsziele, Verkaufsziele, Kontaktziele, marktbezogene und anspruchsgruppenbezogene Beeinflussungsziele ${ }^{32}$ zusammensetzte. Auf dieser Basis identifizierte UEDING insgesamt fünf messespezifische Grundhaltungstypen: ${ }^{33}$

- Der Fachbesucherorientierte Hardseller verfolgt mit seiner Messebeteiligung insbesondere Verkaufsziele und räumt dieser Zieldimension insgesamt den höchsten Stellenwert ein. Dagegen werden von diesem Grundhaltungstyp die

Diese wissenschaftliche Auseinandersetzung basiert auf einer empirischen Untersuchung, die im Sommer 1995 in Zusammenarbeit mit dem Ausstellungs- und Messe-Ausschuss der Deutschen Wirtschaft e.V. (AUMA) durchgeführt wurde und die eine schriftliche Befragung von 602 Unternehmen umfaßt. Vgl. Ueding, R., Management von Messebeteiligungen: Identifikation und Erklärung messespezifischer Grundhaltungen auf der Basis einer empirischen Untersuchung, a.a.O. Vgl. hierzu auch Meffert, H., Ueding, R., Ziele und Nutzen von Messebeteiligungen, a.a.O.

Neben den Messebeteiligungszielen wurden ebenfalls die Grundhaltungsdimensionen „Messeselektion" und "messespezifische Positionierung" in der Untersuchung berücksichtigt, die jedoch bei Messebeteiligungen von Hochschul-Exponenten im Gegensatz zu Messebeteiligungen von kommerziellen Ausstellern untergeordnete Entscheidungsparameter bilden. Angesichts der überragenden Bedeutung hochschulspezifischer Messeziele für Hochschul-Messebeteiligungen bleiben diese Dimensionen zur Ableitung hochschulspezifischer Exponententypen unberücksichtigt.

Vgl. Ueding, R., Management von Messebeteiligungen: Identifikation und Erklärung messespezifischer Grundhaltungen auf der Basis einer empirischen Untersuchung, a.a.O., S. $50 \mathrm{ff}$. Zur ausführlichen Beschreibung der Grundhaltungsdimensionen vgl. ebenda, S. 41 ff.

Hierzu sowie zur ausführlichen Beschreibung der Grundhaltungstypen, die auf Grundlage einer Clusteranalyse abgeleitet wurden, vgl. Ueding, R., Management von Messebeteiligungen: Identifikation und Erklärung messespezifischer Grundhaltungen auf der Basis einer empirischen Untersuchung, a.a.O., S. $92 \mathrm{ff}$. 
anderen Zieldimensionen höchstens mit einer durchschnittlichen Wichtigkeit bewertet. Im Hinblick auf die Motivations- und anspruchsgruppenbezogenen Beeinflussungsziele sind unterdurchschnittliche Zielwichtigkeiten zu konstatieren.

- Der Kommunikationsorientierte Beziehungsmanager räumt gegenüber dem vorangegangenen Grundhaltungstyp den Verkaufszielen einen vergleichsweise untergeordneten Stellenwert ein. Für diesen Aussteller stehen vielmehr Kommunikationsziele, wie anspruchsgruppenbezogene Beeinflussungsziele, im Vordergrund des Messeengagements. Die höchste Bedeutung mißt dieser Grundhaltungstyp den Kontakt- und den marktbezogenen Beeinflussungszielen bei.

- Der Wettbewerbsorientierte Pflichtrepräsentant läßt sich durch eine im Vergleich zu dem Gesamtdurchschnitt geringere Zielorientierung charakterisieren. Nahezu jeder Zieldimension wird ein niedrigerer Stellenwert zugeordnet als von den übrigen Ausstellertypen. Die höchste Bedeutung besitzen mit Abstand die Kontaktziele sowie die marktbezogenen Beeinflussungsziele.

- Der Hybride Fachaussteller räumt dem Großteil der Zieldimensionen eher einen durchschnittlichen Stellenwert ein. Dennoch signalisieren diese Aussteller eine besondere Relevanz der Messebeteiligung in bezug auf die damit verbundenen Möglichkeiten der Mitarbeitermotivation bzw. innengerichteter Zielsetzungen.

- Der Ambitionierte Regionalaussteller läßt sich sowohl hinsichtlich der Wichtigkeit einzelner Zielsetzungen als auch bezogen auf die Bandbreite verfolgter Zielsetzungen durch eine überdurchschnittliche Zielorientierung charakterisieren. Mit Ausnahme der Verkaufsziele, die Fachbesucherorientierte Hardseller am höchsten priorisieren, wird jeder Zieldimension von dieser Ausstellergruppe die vergleichsweise höchste Zielpriorität eingeräumt. Ambitionierte Regionalaussteller präsentieren sich vorwiegend auf Messeveranstaltungen, bei denen nationales sowie regionales Fachpublikum vertreten ist und primär nationale Angebote ausgestellt werden.

Vor dem Hintergrund der bei kommerziellen Messebeteiligungen identifizierten Ausstellertypen ist zu prüfen, inwieweit sich die dargestellte Typologisierung kommerzieller Aussteller in der Typologisierung von Hochschul-Exponenten wider- 
spiegelt bzw. inwieweit die besonderen Rahmenbedingungen zur Herausbildung eigener, hochschulspezifischer Exponententypen führen.

\subsection{Clusteranalytische Bestimmung hochschulspezifischer Exponenten- typen und diskriminanzanalytische Überprüfung}

Als hochschulspezifische Exponententypen werden in der vorliegenden Arbeit homogene Gruppen von Hochschul-Exponenten bezeichnet, die sich anhand ihrer verfolgten Messebeteiligungszielsetzungen charakterisieren lassen. Können bei Hochschul-Exponenten identische Zielsetzungen nachgewiesen werden, so gehören sie zum gleichen Exponententyp. Zur Bildung derartiger in sich homogener, untereinander aber möglichst heterogener Gruppen von Untersuchungsobjekten anhand zugehöriger Merkmalsausprägungen stellt die Clusteranalyse ein geeignetes statistisches Instrument dar. ${ }^{34}$

In der vorliegenden Untersuchung wurde auf Basis der zuvor ermittelten, hochschulspezifischen Messezieldimensionen eine Clusteranalyse eingesetzt, wobei die Bestimmung von Hochschul-Exponententypen mit Hilfe eines dreistufigen Vorgehens erfolgte. ${ }^{35}$ Dabei kam zunächst das zur Gruppe der hierarchischagglomerativen Ansätze zählende Single-Linkage-Verfahren ${ }^{36}$ zur Anwendung, welches eine besondere Eignung zur Identifikation sogenannter Ausreißer ${ }^{37}$ auf-

Vgl. Bleymüller, J., Multivariate Analysen für Wirtschaftswissenschaftler, Manuskript, Münster 1987, S. $163 \mathrm{ff}$.

Bei Clusteranalysen können mit hierarchischen und partionierenden Verfahren zwei Hauptgruppen von Fusionierungsalgorithmen unterschieden werden. Hierarchische Verfahren weisen einen kontinuierlichen Fusionierungsproze $\beta$ auf, in dem entweder - ausgehend von der feinsten Partition (d.h. jedes Objekt bildet ein eigenes Cluster) - die Objekte schrittweise zusammengefaßt werden (agglomerative Verfahren), oder - ausgehend von der gröbsten Partition (d.h. alle Objekte bilden ein einziges Cluster) - die Gruppen schrittweise aufgeteilt werden (divisive Verfahren). Dagegen gehen partionierende Verfahren von einer bestimmten, vom Forscher vorgegebenen Gruppenstruktur aus und gruppieren die Objekte solange um, bis eine vorgegebene Zielfunktion ihr Optimum erreicht. Vgl. Steinhausen, D., Langer, K., Clusteranalyse: Einführung in Methoden und Verfahren der automatischen Klassifikation, Berlin, New York 1977, S. 69; Backhaus, K. et al., Multivariate Analysemethoden: Eine anwendungsorientierte Einführung, a.a.O., S. 281.

Beim Single-Linkage-Verfahren werden jeweils diejenigen Objekte mit der größten Ähnlichkeit bzw. der kleinsten Distanz zusammengefaßt. Vgl. Steinhausen, D., Langer, K., Clusteranalyse: Einführung in Methoden und Verfahren der automatischen Klassifikation, a.a.O., S. $77 \mathrm{f}$.

Ausreißer sind solche Objekte, die im Vergleich zu allen anderen zu klassifizierenden Objekten stark abweichende Merkmalsausprägungen aufweisen und daher keiner Gruppe sinnvoll zugewiesen werden können. Beläßt man diese Objekte in der Stichprobe, kann dies die Grup- 
weist. ${ }^{38}$ Auf diese Weise wurden die extremen Beurteilungen zweier Probanden von der weiteren Analyse ausgeschlossen. Damit verblieb insgesamt eine Stichprobe von 45 befragten Hochschul-Exponenten ${ }^{39}$ zur weiteren Typenbildung. In einem zweiten Schritt diente das in der Praxis oftmals zur Gruppierung eingesetzte Ward-Verfahren als Fusionierungsalgorithmus, um Anhaltspunkte über die optimale Clusteranzahl zu erhalten. ${ }^{40}$ Die Anzahl der Cluster wurde dabei mit dem Elbow-Kriterium ermittelt, welches auf der graphischen Darstellung der Fehlerquadratsumme im Verhältnis zur Anzahl der Cluster basiert. Diese wird in der nachfolgenden Abbildung aufgezeigt, wobei die Entwicklung der Fehlerquadratsumme für die letzten zehn Stufen des Fusionierungsprozesses abgebildet ist. Gemäß dem Elbow-Kriterium kann demnach sowohl eine 3-Cluster- als auch eine 7-Clusterlösung gewählt werden. Nicht zuletzt aufgrund des relativ geringen Stichprobenumfangs erschien die 3-Clusterlösung der 7-Clusterlösung überlegen. Damit führte die Analyse der Fehlerquadratsumme zu drei hochschulspezifischen Exponententypen bzw. zu drei Clustern, deren Centroide als Startpartition einer anschließend durchgeführten partitionierenden Clusteranalyse ${ }^{41}$ zur Optimierung der Clusterlösung diente. ${ }^{42}$

penbildung stark beeinträchtigen. Das Single-Linkage-Verfahren erlaubt aufgrund seines Fusionierungsalgorithmus die Identifikation von diesen Ausreißern. Vgl. Steinhausen, D., Langer, K., Clusteranalyse: Einführung in Methoden und Verfahren der automatischen Klassifikation, a.a.O., S. 78.

Vgl. Backhaus, K. et al., Multivariate Analysemethoden: Eine anwendungsorientierte Einführung, a.a.O., S. 290.

Bei den zwei Probanden, die aus der Analyse ausgeschlossen wurden, handelte es sich um Exponenten von Mehrbranchenmessen. Damit setzten sich die verbleibenden, in die Analyse einbezogenen 45 befragten Hochschul-Exponenten aus 30 Mehrbranchen- und 15 Fachmesse-Exponenten zusammen.

40 Zum Gegenstand und zur Beliebtheit des Ward-Verfahrens vgl. stellvertr. Bleymüller, J., Multivariate Analysen für Wirtschaftswissenschaftler, a.a.O., S. 176; Bergs, S., Optimalität bei Clusteranalysen, Experimente zur Bewertung numerischer Klassifikationsverfahren, Univ. Diss., Münster 1981, S. 96 f.

Im Gegensatz zu den hierarisch-agglomerativen Ansätzen ermöglichen partitionierende Cluster-Verfahren eine iterative Umgruppierung von Objekten, bis ein vorgegebenes Optimalitätskriterium erreicht ist. Damit eignen sich diese Verfahren besonders zur Optimierung von Clusterlösungen. Vgl. stellvertr. Bleymüller, J., Multivariate Analysen für Wirtschaftswissenschaftler, a.a.O., S. $181 \mathrm{ft}$.

Die im Rahmen dieser Analyse verwendete Kombination hierarchischer und partitionierender Verfahren kombiniert die Vorteile verschiedener Ansätze zur Clusteranalyse und kann infolgedessen als ein geeignetes Vorgehen zur Ermittlung trennscharfer Clusterlösungen angesehen werden. Zu der gewählten Vorgehensweise vgl. Perrey, J., Nutzenorientierte Marktsegmentierung: Ein integrativer Ansatz zum Zielgruppenmarketing im Verkehrsdienstleistungsbereich, 


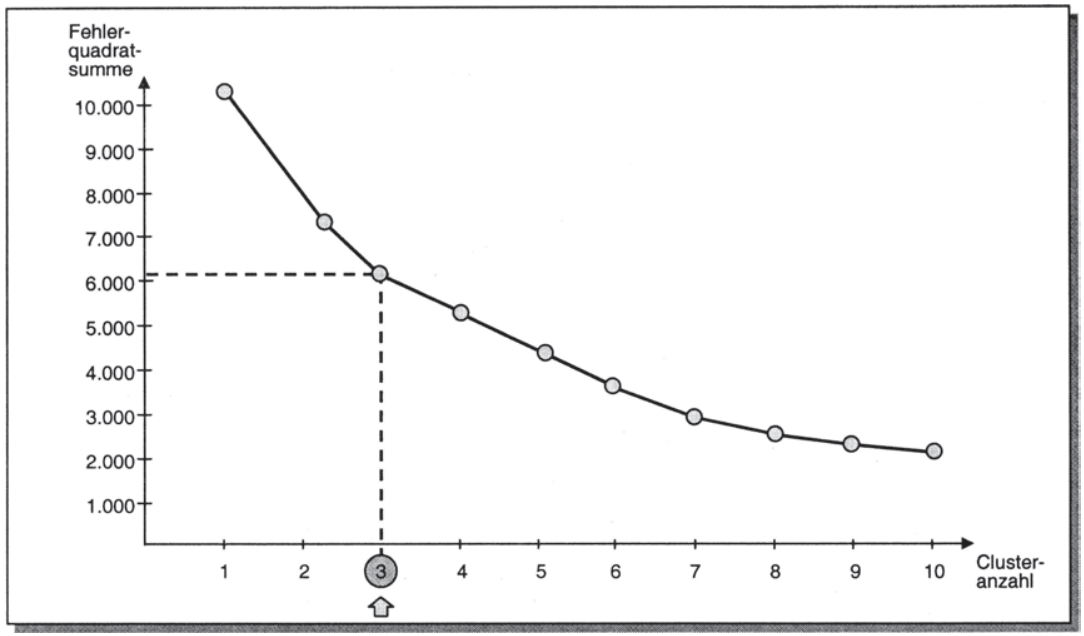

Abb. 9: Fusionierungsprozeß des Ward-Verfahrens und Bestimmung der Exponenten-Clusterlösung

Die ermittelte Lösung weist sehr unterschiedliche Clustergrößen auf. Während Cluster 2 als größtes Cluster 64,4\% $(n=29)$ der Hochschul-Exponenten umfaßt, gehören dem Cluster 3 als zweitgrößte Exponentengruppe lediglich 22,2\% $(n=10)$ und Cluster 1 als kleinster Gruppe nur noch 13,3\% $(n=6)$ der befragten Hochschul-Exponenten an. Die in Abb. 10 dargestellten Mittelwertabweichungen der hochschulspezifischen Messezieldimensionen deuten auf signifikante Unterschiede zwischen den identifizierten Exponententypen hin. Zur Überprüfung dieser Unterschiede wird als Testverfahren die einfaktorielle Varianzanalyse eingesetzt, die mit dem F-Wert ein Kriterium für die Globalbeurteilung von Mittelwertunterschieden bereitstellt. ${ }^{43}$

Wiesbaden 1998, S. 185 ff. Zu einer ähnlichen Vorgehensweise vgl. Green, P. E., Krieger, A. M., Alternative Approaches to Cluster-Based Market Segmentation, in: JoMRS, Vol. 37, No. 3, 1995, S. 225; Wöllenstein, S., Betriebstypenprofilierung in vertikalen Vertriebssystemen: Eine Analyse von Einflußfaktoren und Erfolgswirkungen auf der Grundlage eines Vertragshändlersystems im Automobilhandel, Frankfurt am Main 1996, S. $186 \mathrm{ff}$.

Vgl. Bleymüller, J., Gehlert, G., Gülicher, H., Statistik für Wirtschaftswissenschaftler, a.a.O., S. $119 \mathrm{ff}$. Mit dem F-Test kann geprüft werden, ob beim Vergleich der Mittelwerte mehrerer Gruppen die Differenz zwischen mindestens einem der Vergleichspaare signifikant ist. Vgl. Janssen, J., Laatz, W., Statistische Datenanalyse mit SPSS für Windows, 3. Aufl., Berlin u.a. 1999, S. 315. 


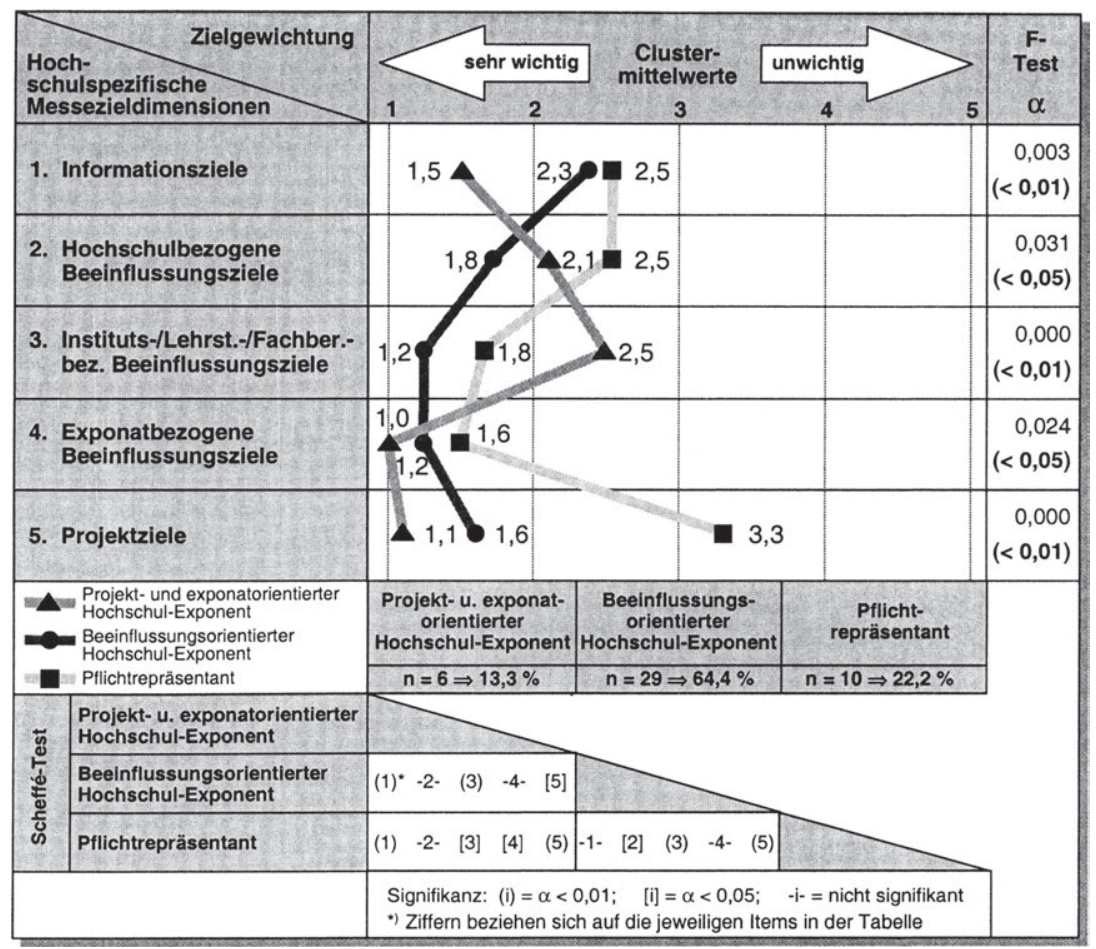

Abb. 10: Varianzanalytische Prüfung auf Bewertungsunterschiede bei Zieldimensionen zwischen hochschulspezifischen Exponententypen

Die Prüfmaße dieses Testverfahrens belegen die Existenz hoch signifikanter Unterschiede zwischen den ermittelten Exponententypen. Um die Differenzen zwischen den einzelnen Exponententypen aufzudecken, wurde überdies der Scheffé-Test $^{44}$ zur Überprüfung von Mittelwertunterschieden herangezogen. Dabei weisen die Prüfmaße dieses Testverfahrens ebenfalls auf signifikante Unterschiede zwischen den einzelnen Exponententypen hin. Lediglich in bezug

Der Scheffé-Test gilt als ein konservativer multipler Mittelwertvergleichstest. Er wird im vorliegenden Fall eingesetzt, weil hier nur solche Mittelwertunterschiede zwischen den Exponententypen aufgedeckt werden sollen, deren Differenzen einer strengen statistischen Interpretation genügen. Die Verwendung weniger konservativer Tests geht hingegen mit der Gefahr einer systematischen Überschätzung von kleinen Mittelwertunterschieden und dementsprechenden Fehlinterpretationen der Ergebnisse einher. Vgl. Diehl, J. M., Varianzanalyse, Frankfurt am Main 1977, S. $48 \mathrm{ff}$. 
auf vereinzelte Zieldimensionen können nicht signifikante Differenzen festgestellt werden (z.B. zwischen Hochschul- und Exponatbezogenen Beeinflussungszielen).

Auf Basis der clusterspezifischen Mittelwertausprägungen der hochschulspezifischen Messezieldimensionen können die empirisch ermittelten Hochschul-Exponententypen differenziert beschrieben werden.

Im Cluster der Projekt- und exponatorientierten Hochschul-Exponenten finden sich diejenigen Aussteller, die mit ihrer Messebeteiligung insbesondere Projektziele und Exponatbezogene Beeinflussungsziele verfolgen. Mit Mittelwerten von 1,0 bzw. 1,1 werden bei diesen Zieldimensionen die höchsten Werte realisiert. Auch räumen Projekt- und exponatorientierte Hochschul-Exponenten den Informationszielen im Vergleich zu den beiden anderen Exponententypen eine höhere Bedeutung ein (Mittelwert $=1,5$ ). Bei den anderen Zieldimensionen hingegen sind geringere Zielwichtigkeiten zu konstatieren (Hochschulbezogene Beeinflussungsziele: Mittelwert = 2,1 und Instituts-/Lehrstuhl-/Fachbereichsbezogene Beeinflussungsziele: Mittelwert =2,5). Die damit vorgenommenen Gewichtungen lassen auf eine im Rahmen der Messebeteiligung dieser Exponenten im Vordergrund stehende Akquisition von Drittmitteln schließen. Projekt- und exponatorientierte Hochschul-Exponenten gehören ausschließlich naturwissenschaftlichen sowie technisch-orientierten Fachbereichen an, ${ }^{45}$ die zum überwiegenden Teil aus Fachhochschulen $(66,7 \%)$ stammen. ${ }^{46}$ Der die Fachhochschulen kennzeichnende Praxisbezug wird durch die bei diesem Exponententyp im Vordergrund stehenden Messezielsetzungen deutlich. 83,3\% der Projekt- und exponatorientierten Hochschul-Exponenten stellen auf Mehrbranchenmessen aus, wohingegen nur $16,7 \%$ auf Fachmessen zu finden sind.

Das Cluster der Beeinflussungsorientierten Hochschul-Exponenten umfaßt solche Aussteller, bei denen Exponatbezogene, Instituts-/Lehrstuhl-/Fachbe-

Eine weitere fachbereichsspezifische Differenzierung erscheint aufgrund einer relativen Gleichverteilung über die jeweiligen Fachbereiche nicht sinnvoll. Vgl. Anhang II, Zusammensetzung der Hochschul-Exponenten- und Hochschul-Messestand-Besucher-Stichproben.

Zwischen den hochschulspezitischen Exponententypen lassen sich bzgl. ihrer soziodemographischen Ausprägungen keine signifikanten Unterschiede feststellen. Dies liegt aus Sicht der signifikanzprüfenden Verfahren insbesondere in dem relativ geringen Stichprobenumfang begründet. Dennoch werden zur Beschreibung der einzelnen Cluster ausgewählte, soziodemographische Ausprägungen herangezogen, da sie z.T. wichtige Informationen über die einzelnen Exponententypen liefern. 
reichsbezogene und Hochschulbezogene Beeinflussungsziele im Vordergrund des Messeengagements stehen (Mittelwert $=1,2$ bzw. 1,8). Den Projekt- (Mittelwert $=1,6$ ) und Informationszielen (Mittelwert $=2,3$ ) wird dagegen im Vergleich zu dem vorangegangenen Exponententyp eine niedrigere Bedeutung beigemessen. Folglich ist die Messebeteiligung dieser Exponenten vor allem auf die Präsentation eigener Aktivitäten gerichtet, um dem im Hochschulrahmengesetz verankerten Gebot, die Hochschulleistungen gegenüber der die Hochschulen alimentierenden Gesellschaft zu dokumentieren, entsprechend Rechnung zu tragen. Beeinflussungsorientierte Hochschul-Exponenten gehören überwiegend naturwissenschaftlichen sowie technisch-orientierten Fachbereichen ${ }^{47}$ an und stammen zu nahezu gleichen Teilen aus Universitäten (38,5\%) und Fachhochschulen $(42,3 \%)$. In bezug auf die Messetypauswahl des Beeinflussungsorientierten Hochschul-Exponenten läßt sich im Vergleich zu Projekt- und exponatorientierten Hochschul-Exponenten keine eindeutige Präferenz für bestimmte Messetypen feststellen, da dieser Exponententyp fast zu gleichen Anteilen auf Mehrbranchen$(55,2 \%)$ und Fachmessen $(44,8 \%)$ vertreten ist.

Die Pflichtrepräsentanten lassen sich im Vergleich zu den vorangegangenen Exponententypen durch eine geringere Zielorientierung charakterisieren. Bis auf die Instituts-/Lehrstuhl-/Fachbereichsbezogenen Beeinflussungsziele wird von diesem Exponententyp jeder Zieldimension ein niedrigerer Stellenwert zugeordnet als von den zwei zuvor dargestellten Exponentenclustern. Den höchsten Stellenwert besitzen mit Abstand die Exponatbezogenen und Institutsbezogenen Beeinflussungsziele (Mittelwert = 1,6 bzw. 1,8). Informationsziele, Hochschulbezogene Beeinflussungsziele sowie Projektziele nehmen mit Mittelwerten von 2,5 bzw. 3,3 eher mittlere Stellenwerte ein. Die geringere Zielorientierung dieser Exponentengruppe deutet darauf hin, daß sich diese Exponenten an einer Messe nicht aus eigener Initiative, sondern vielmehr aus der Pflicht ihres Status als öffentlichrechtliche, nicht-kommerzielle Institution heraus beteiligen. Pflichtrepräsentanten gehören zur einen Hälfte den Fachbereichen Maschinenbau (30\%) sowie Elektrotechnik $(20 \%)$ und zur anderen Hälfte überwiegend naturwissenschaftlichen

\footnotetext{
47 Auch bei diesem Exponententyp erscheint eine weitere fachbereichsspezifische Differenzierung aufgrund einer relativen Gleichverteilung über die jeweiligen Fachbereiche nicht sinnvoll. Vgl. Anhang II, Zusammensetzung der Hochschul-Exponenten- und Hochschul-MessestandBesucher-Stichproben.
} 
Fachbereichen ${ }^{48}$ an. Zu jeweils $30 \%$ stammen diese Hochschul-Exponenten aus Universitäten, Fach- sowie Gesamthochschulen. Anzutreffen sind Pflichtrepräsentanten vorwiegend auf Mehrbranchenmessen (90\%) und weniger auf Fachmessen (10\%).

Im Gegensatz zur Klassifizierung kommerzieller Exponenten lassen sich bei Hochschul-Messebeteiligungen somit nicht fünf, sondern drei Exponententypen identifizieren, wobei jedoch zwischen kommerziellen und hochschulspezifischen Exponententypen z.T. Ähnlichkeiten erkennbar sind. So weisen bspw. Projektund exponatorientierte Hochschul-Exponenten hinsichtlich ihrer Messezielbewertungen Ähnlichkeiten zu Fachbesucherorientierten Hardsellern auf. Von Projektund exponatorientierten Hochschul-Exponenten werden jedoch nicht Verkaufsziele, sondern projekt- und exponatbezogene Ziele in den Vordergrund des Messeengagements gestellt. Auch weisen Beeinflussungsorientierte Hochschul-Exponenten bzgl. ihrer Zielpriorisierungen Ähnlichkeiten zu Kommunikationsorientierten Beziehungsmanagern auf. Das gleiche gilt für Pflichtrepräsentanten, bei welchen ähnliche Messezielpriorisierungen wie bei den kommerziellen Wettbewerbsorientierten Pflichtrepräsentanten festgestellt werden können. ${ }^{49}$

Um die Güte der Clusterlösung einerseits statistisch zu überprüfen und andererseits die Bedeutung der Zieldimensionen für die Differenzierung der Exponententypen zu identifizieren, wird auf das Verfahren der Diskriminanzanalyse zurückgegriffen. ${ }^{50}$

Eine weitere fachbereichsspezifische Differenzierung erscheint auch bei diesem Exponententyp aufgrund einer relativen Gleichverteilung über die jeweiligen Fachbereiche nicht sinnvoll. Vgl. Anhang II, Zusammensetzung der Hochschul-Exponenten- und Hochschul-MessestandBesucher-Stichproben..

Der Hybride Fachaussteller konnte auf Basis der empirischen Untersuchung nicht identifiziert werden, da die mitarbeiterorientierten Zielsetzungen der Hochschul-Messebeteiligung implizit in den anderen hochschulspezifischen Messezieldimensionen ihren Niederschlag fanden. Ebenso gilt dies für den Ambitionierten Regionalaussteller, der vor allem eine geographischfokussierte Messeselektion verfolgt und damit dem Entscheidungstatbestand der Messeselektion einen besonderen Stellenwert beimißt. Die Messeselektion wird bei der Ableitung hochschulspezifischer Exponententypen aufgrund der überragenden Bedeutung der Messezielsetzungen für Hochschul-Exponenten vernachlässigt. Folglich konnte der Ambitionierte Regionalaussteller in der dieser Arbeit zugrundeliegenden Untersuchung nicht abgeleitet werden.

Die Diskriminanzanalyse, die zu den strukturprüfenden multivariaten Analyseverfahren zählt, bietet sich insbesondere zur Validierung der Ergebnisse von Clusteranalysen an. Vgl. Backhaus, K. et al., Multivariate Analysemethoden: Eine anwendungsorientierte Einführung, a.a.O., S. $90 \mathrm{ff}$. 
Die der eigentlichen Diskriminanzanalyse vorgeschaltete univariate Analyse zur Beurteilung der diskriminatorischen Bedeutung der Messezieldimensionen führte zunächst zu durchgehend signifikanten Werten $(\alpha<0,01)$. Damit konnte eine isolierte Trennfähigkeit aller Faktoren unterstellt werden. Im Rahmen der eigentlichen Diskriminanzanalyse wurden zwei Diskriminanzfunktionen ermittelt, die beide signifikant zur Trennung der Exponententypen beitragen $(\alpha<0,01)$. Die erste Funktion erklärt bereits $70 \%$ der Varianz bei einem geringen Wilks' Lambda von 0,100 . Die Einbeziehung der zweiten Funktion führte zu einem multivariaten Wilks' Lambda von $0,042 .{ }^{51}$ Anhand dieser Diskriminanzfunktionen können die in der Stichprobe befindlichen Exponenten von Hochschul-Messebeteiligungen klassifiziert, d.h. ihre Zugehörigkeit zu den drei Exponententypen geschätzt werden. Dabei signalisiert der Übereinstimmungsgrad von geschätzter und tatsächlicher Gruppenzugehörigkeit die Trennschärfe der Gruppenlösung ${ }^{52}$ (vgl. Tab. 2).

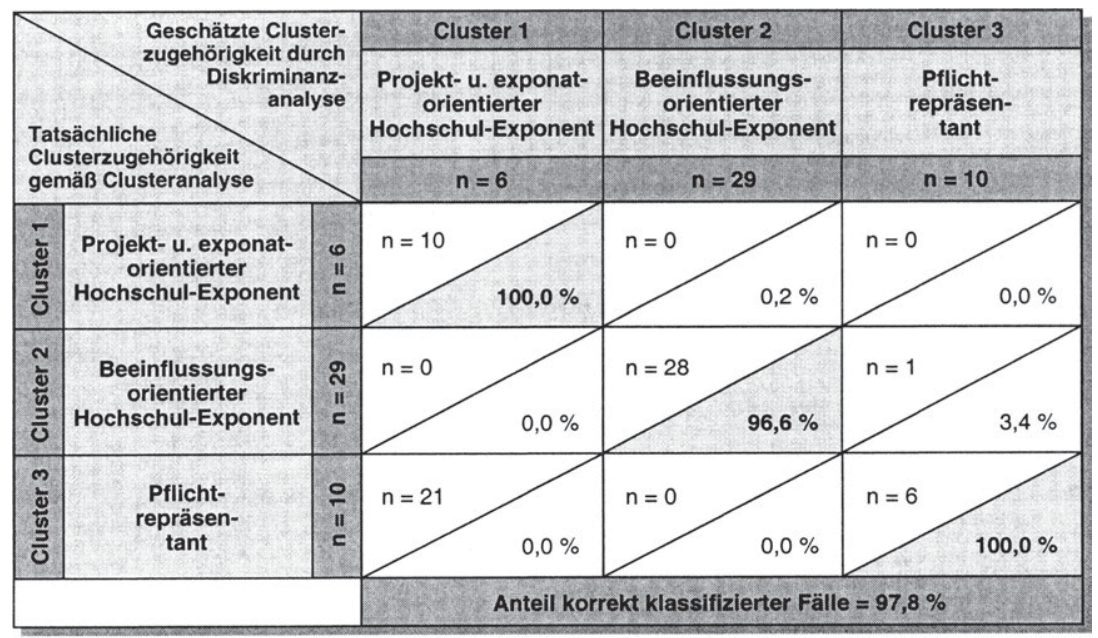

Tab. 2: Klassifizierungsmatrix der diskriminanzanalytischen Überprüfung der Exponenten-Clusterlösung

51 Während sich der kanonische Korrelationskoeffizient nach Einbeziehung der ersten Funktion auf einen Wert von 0,874 beläuft, weist dieser nach Berücksichtigung der zweiten Funktion schließlich einen Wert von 0,761 auf.

52 Vgl. Backhaus, K. et al., Multivariate Analysemethoden: Eine anwendungsorientierte Einführung, a.a.O., S. $115 \mathrm{f}$. 
Mit einem Anteil korrekter Klassifizierungen von 97,8 \% wird die Clusterlösung bestätigt. Zur Überprüfung der diskriminatorischen Bedeutung der einzelnen hochschulspezifischen Zieldimensionen können die mittleren Diskriminanzkoeffizienten herangezogen werden. ${ }^{53}$ Diese ermöglichen eine Aussage darüber, auf welche Zieldimensionen die Gruppenunterschiede in erster Linie zurückzuführen sind (vgl. Tab. 3).

\begin{tabular}{|l|c|c|c|}
\hline \multirow{2}{*}{$\begin{array}{l}\text { Zieldimensionen der } \\
\text { Messebeteiligung von } \\
\text { Hochschulen }\end{array}$} & $\begin{array}{c}\text { Mittlerer } \\
\text { Diskriminanz- } \\
\text { koeffizient }\end{array}$ & \multicolumn{2}{|c|}{$\begin{array}{c}\text { Relative Bedeutung des mittleren } \\
\text { Diskriminanzkoeffizienten }\end{array}$} \\
\cline { 3 - 4 } Informationsziele & 0,197 & 10,5 & Prozent \\
\hline $\begin{array}{l}\text { Hochschulbezogene } \\
\text { Beeinflussungsziele }\end{array}$ & 0,320 & 17,1 & $\mathbf{5}$ \\
\hline $\begin{array}{l}\text { Instituts-/Lehrstuhl-/Fachber-- } \\
\text { bezogene Beeinflussungsziele }\end{array}$ & 0,448 & 23,9 & $\mathbf{3}$ \\
\hline $\begin{array}{l}\text { Exponatbezogene } \\
\text { Beeinflussungsziele }\end{array}$ & 0,216 & 11,5 & $\mathbf{4}$ \\
\hline Projektziele & 0,694 & 37,0 & $\mathbf{1 .}$ \\
\hline$\sum$ & 1,875 & 100,0 & \\
\hline
\end{tabular}

Tab. 3: Diskriminanzanalytische Bedeutung der hochschulspezifischen Messezieldimensionen für Exponententypen

Die relative Bedeutung der Diskriminanzkoeffizienten zeigt, daß mit der Zielkategorie Projektziele (37,0\%) diejenige Zieldimension das wichtigste Trennkriterium darstellt, deren Stellenwert besonders deutlich zwischen den drei Exponententypen differenziert (vgl. Abb. 10). Daran anschließend tragen die Instituts-/Lehrstuhl-/Fachbereichsbezogenen Beeinflussungsziele $(23,9 \%)$ und die Hochschulbezogenen Beeinflussungsziele zur weiteren Clustertrennung bei. Sowohl Expo-

Aufgrund der in der vorliegenden Untersuchung ermittelten zwei Trenngraden, existieren für die Zielsetzungen auch jeweils zwei Diskriminanzkoeffizienten. Zur Ermittlung der Bedeutung einzelner Besucherzielsetzungen im Gesamtmodell sind die standardisierten Diskriminanzkoeffizienten mit den Eigenwertanteilen der beiden Trenngraden zu gewichten und zu addieren. Vgl. hierzu Backhaus, K. et al., Multivariate Analysemethoden: Eine anwendungsorientierte Einführung, a.a.O., S. 123 f. 
natbezogene Beeinflussungsziele (11,5\%) als auch Informationsziele $(10,5 \%)$ nehmen demgegenüber eine geringere Bedeutung zur Erklärung der Typenunterschiede ein. Dieses Ergebnis erscheint sehr plausibel, weil eine Messebeteiligung grundsätzlich von allen Hochschul-Exponenten zur Präsentation ihrer Exponate und auch zur Befriedigung vorhandener Informationsbedürfnisse genutzt wird. Somit sind es insbesondere spezifische Zielsetzungen, durch die sich einzelne Hochschul-Exponenten voneinander unterscheiden. Dazu zählen die auf die eigene Institution des Hochschulbereichs gerichteten Beeinflussungsziele (Hochschulbezogene sowie Instituts-/Lehrstuhl-/Fachbereichsbezogene Beeinflussungsziele) und insbesondere Projektziele, deren Verfolgung sehr spezifisch ausgerichtet ist.

Die in den vorangegangenen Kapiteln dargestellten hochschulspezifischen Messeziele bzw. Messezieldimensionen und die auf dieser Grundlage abgeleiteten hochschulspezifischen Exponententypen werden in der nachfolgenden Abbildung zusammenfassend dargestellt.

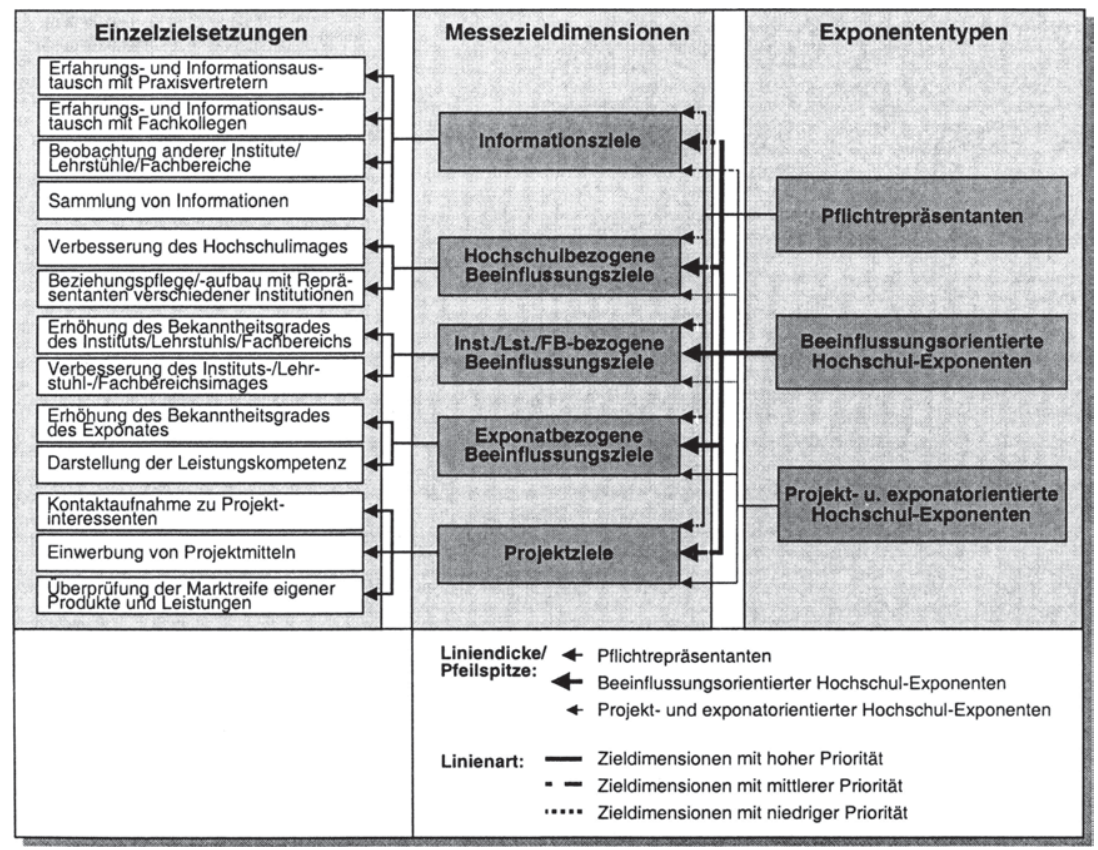

Abb. 11: Zieldimensionen und Exponententypen der Messebeteiligung von Hochschulen 
Die gewonnenen Erkenntnisse zu den hochschulspezifischen Messebeteiligungszielen legen die Grundlage zur Überprüfung des Erfolgs von Hochschul-Messebeteiligungen, zeigen darüber hinaus aber auch vielfältige Ansatzpunkte zur Ableitung von Implikationen für zukünftige Hochschul-Messebeteiligungen auf. In diesem Zusammenhang ist jedoch zu prüfen, inwieweit eine ausschließlich aus Exponentensicht vorgenommene und auf Grundlage exponententypspezifischer Messezielsetzungen abgeleitete Ausgestaltung von Hochschul-Messebeteiligungen ausreicht, um hochschulspezifische Zielgruppen zu erreichen.

\section{Zielgruppen der Messebeteiligung von Hochschulen}

Die unterschiedlichen Priorisierungen der einzelnen hochschulspezifischen Messezielsetzungen deuten darauf hin, daß mit der Verfolgung bestimmter Zielsetzungen möglicherweise die Erreichung ausgewählter hochschulspezifischer Anspruchsgruppen angestrebt wird. Damit ist zu prüfen, welche Anspruchsgruppen mit einer Hochschul-Messebeteiligung erreicht werden sollen und demzufolge als Zielgruppen einer Hochschul-Messebeteiligung fungieren.

\subsection{Anspruchsgruppen als Zielgruppen von Hochschul-Messebe- teiligungen}

\subsection{Systematisierung hochschulspezifischer Anspruchsgruppen}

In der Literatur existiert eine Vielzahl von mehr oder weniger differenzierten Ansätzen zur Systematisierung von Zielgruppen im Hochschulbereich. ${ }^{54}$ Dabei versuchen diese Systematisierungsansätze, die zahlreichen Anspruchsgruppen von Hochschulen in übergeordnete Zielgruppen zusammenzufassen.

54 Vgl. stellvertr. Heinzel, M., Anforderungen deutscher Unternehmen an betriebswirtschaftliche Hochschulabsolventen - Zur Marktorientierung von Hochschulen, Wiesbaden 1997, S. 38; Alewell, K., Wissenschaftsmarketing, a.a.O., Sp. 2779; Trogele, U., Strategisches Marketing für deutsche Universitäten: Die Anwendung von Marketing-Konzepten amerikanischer Hochschulen in deutschen Universitäten, a.a.O., S. 28; Kook, W., Einstellungen zur Universität, Frankfurt, Berlin, Cirencester 1981, S. 14; Alewell, K., Marketing-Management für Universitäten: Umweltbezogene Führung von Universitäten, a.a.O., S. 269. Zur allgemeinen Systematisierung von Zielgruppen im nicht-kommerziellen Bereich vgl. stellvertr. Kotler, P., Levy, S. J., Broadening the concept of Marketing, a.a.O., S. 12. 
Die bereits zuvor aufgegriffene Systematisierung nach internen und externen Anspruchsgruppen ${ }^{55}$ und die daraus resultierende Unterscheidung von zwei Zielgruppen erweisen sich vor dem Hintergrund der in den vorangegangenen Kapiteln dargestellten zahlreichen, auf verschiedene externe Anspruchsgruppen der Hochschule gerichteten Messezielsetzungen zur Ableitung hochschulspezifischer Messezielgruppen als nicht ausreichend.

An der Systematik der Zielgruppen für nicht-kommerzielle Institutionen von KOTLER und LEVY anknüpfend, ${ }^{56}$ können ebenfalls zwei Zielgruppen von Hochschulen unterschieden werden, wobei dieser Ansatz jedoch gegenüber dem vorangegangenen Ansatz interne Anspruchsgruppen vernachlässigt und externe Anspruchsgruppen differenzierter berücksichtigt. Dabei sind gemäß der zwei Teilmärkte von Hochschulen einerseits Zielgruppen des Beschaffungsmarktes und andererseits Zielgruppen des Leistungsmarktes zu nennen. Unter Zielgruppen des Beschaffungsmarktes werden „Lieferanten“ subsumiert, die Einsatzfaktoren (Human Ressources, Finanz- und Sachmittel) zur Erstellung von Hochschulleistungen zur Verfügung stellen. Dagegen setzen sich Zielgruppen des Leistungsmarktes aus "Abnehmern" von Hochschulleistungen (Forschung, Lehre und sonstige Dienstleistungen) zusammen, die sich wiederum in verschiedene Untergruppen unterteilen lassen. Eine Untergruppe bspw. umfaßt dabei aktuelle und potentielle Verwender, die als Austauschpartner Leistungen einer Hochschule in Anspruch nehmen. Als eine weitere Gruppe ist die aktive Öffentlichkeit anzuführen, die in einem engen Kontakt zur Hochschule steht (z.B. Ehemalige der Hochschule, Stiftungen und Politiker). Wenngleich gegenüber der zuvor dargestellten Systematisierung bei diesem Ansatz externe Anspruchsgruppen der Hochschulen differenzierter betrachtet werden, erscheint auch diese Unterteilung angesichts der zuvor dargestellten vielfältigen hochschulspezifischen Messezielsetzungen und nicht zuletzt auch aufgrund der Vernachlässigung interner Zielgruppen als nicht ausreichend.

Auf Grundlage eines differenzierten Strukturmodells, das Transaktionsbeziehungen einer Hochschule und ihres Umfeldes abbildet, leitet KooK insgesamt fünf

Vgl. Meffert, H., Kirchgeorg, M., Marktorientiertes Umweltmanagement, a.a.O., S. 95.

56 Vgl. hierzu sowie im folgenden Kotler, P., Levy, S. J., Broadening the concept of Marketing, a.a.O., S. $12 \mathrm{ff}$. 
Zielgruppen von Hochschulen $a b^{57}$ Dabei unterscheidet er zwischen InputÖffentlichkeit und interner Öffentlichkeit, Vermittler- und Verbraucherumwelt sowie der allgemeinen Öffentlichkeit. Zur Gruppe Input-Öffentlichkeit zählen insbesondere Wirtschaftsunternehmen und Zulieferer, die in kommerziellen Beziehungen zur Hochschule stehen. Darüber hinaus werden in diese Gruppe sowohl Förderer und Spender als auch Regierungsstellen des Bundes und der Länder, Rektorenkonferenzen sowie Wissenschafts- und Bundesrat, die steuernd, beratend und/oder anordnend in den Planungs- und Führungsprozeß von Hochschulen eingreifen, eingeordnet. Das wissenschaftliche und nicht-wissenschaftliche Personal bildet die Gruppe interne Öffentlichkeit. Massenmedien, Verlage, Bibliotheken sowie Fördergesellschaften und Ehemalige werden zur Zielgruppe VermittlerUmwelt zusammengefaßt, die Informationen über das Leistungsangebot von Hochschulen weitergibt. Demgegenüber werden unter Verbraucher-Umwelt die Gruppen subsumiert, die von Hochschulleistungen „profitieren“, wie aktuelle und potentielle Studierende sowie Wirtschaftsunternehmen. Die allgemeine Öffentlichkeit umfaßt schließlich Stadtbevölkerungen, Wettbewerber sowie Eltern von Studierenden und Schülern.

Der Ansatz von KoOK nimmt zwar gegenüber den vorangegangenen Systematisierungsansätzen eine differenziertere Betrachtung hochschulspezifischer Zielgruppen vor, weist jedoch sowohl in bezug auf die begriffliche Abgrenzung als auch hinsichtlich der inhaltlichen Bestimmung einzelner Zielgruppen Schwächen auf. So profitiert bspw. nicht nur die Verbraucher-Umwelt, sondern letztlich die gesamte Gesellschaft von den Leistungen einer Hochschule. Auch können Wirtschaftsunternehmen, die einerseits als Hochschullieferanten und andererseits als Hochschulleistungsempfänger fungieren, sowohl der Zielgruppe Input-Öffentlichkeit als auch der Zielgruppe Verbraucher-Umwelt zugeordnet werden. Diese beispielhaft angeführten Kritikpunkte weisen darauf hin, daß auch dieser Ansatz zur Systematisierung von Messezielgruppen als nicht geeignet einzustufen ist.

Wird dem Systematisierungsansatz von ALEWELL gefolgt, der aus der Vielzahl möglicher Gruppen von Hochschul-Transaktionspartnern sechs Segmente mit 
besonders bedeutsamen Hochschulbeziehungen herausgreift, können folgende sechs Zielgruppen unterschieden werden: ${ }^{58}$

Wissenschaftler und Nicht-Wissenschaftler, zu denen Kontakte als potentielle Leistungsträger von Hochschulen aufzunehmen und als aktive Leistungsträger Austauschbeziehungen zu pflegen sind.

- Andere Wissenschaftsinstitutionen, zu denen Transaktionsbeziehungen (Erkenntnis- und Leistungstransfer) in Form von Publikationen und/oder durch organisierte, vertragliche bzw. auf gemeinsamen Forschungsprojekten basierenden Kooperationen bestehen.

- (Potentielle) Studierende, zu denen Austauschbeziehungen aufzunehmen (Bestreben um Einschreibungen) bzw. zu pflegen (Halten der aktuellen Studierenden) sind.

Wirtschaftliche Unternehmen, die als Verwender von Hochschulleistungen (Forschungsergebnisse, Ausbildungsleistungen und sonstige Dienstleistungen) für eine Hochschule besonders relevant sind.

- Staatliche und Kommunale Institutionen, die bestimmte Leistungen von Hochschulen (z.B. Aus- und Fortbildungsleistungen für Lehrer, Verwaltungsbeamte, Juristen für Verwaltung und Rechtsprechung) in Anspruch nehmen.

Sonstige Öffentlichkeit, unter der alle Personen und Institutionen subsumiert werden, die in den vorangegangenen Zielgruppen nicht erfaßt werden.

Durch diese, von ALEWELL auf Grundlage von Wichtigkeiten vorhandener Austauschbeziehungen vorgenommene Systematisierung, ${ }^{59}$ wird eine differenzierte Betrachtung hochschulspezifischer Anspruchsgruppen ermöglicht. Dabei erweist sich dieser Ansatz sowohl in bezug auf die inhaltliche Bestimmung als auch im Hinblick auf die begriffliche Abgrenzung der einzelnen Zielgruppen gegenüber den zuvor diskutierten Systematisierungsansätzen als überlegen. Deshalb soll dem

Vgl. Alewell, K., Wissenschaftsmarketing, a.a.O., Sp. 2779 ff. Dabei stellt dieser Ansatz eine Weiterentwicklung des von Alewell im Jahre 1977 vertretenen Systematisierungsansatzes dar, in dem vier Zielgruppen von Hochschulen unterschieden wurden. Vgl. Alewell, K., MarketingManagement für Universitäten: Umweltbezogene Führung von Universitäten, a.a.O., S. 269 ff.

Vgl. Alewell, K., Wissenschaftsmarketing, a.a.O., Sp. 2781. 
Ansatz von ALEWELL in der vorliegenden Arbeit gefolgt werden, wobei er jedoch insofern eine Modifizierung erfährt, als daß zusätzlich eine für die Messebeteiligung sinnvolle Differenzierung in interne und externe Zielgruppen vorgenommen wird. $\mathrm{Zu}$ den internen Zielgruppen zählen die Gruppen, die an der Leistungserstellung einer Hochschule beteiligt sind und sich damit sowohl aus Wissenschaftlern als auch aus Nicht-Wissenschaftlern zusammensetzen. Diese internen Zielgruppen werden im folgenden nicht direkt betrachtet, da auch die innengerichteten Messezielsetzungen nur indirekt im dargestellten Zielsystem von Hochschul-Messebeteiligungen berücksichtigt wurden. Im Hinblick auf externe Zielgruppen können auf Grundlage hochschulspezifischer Messebeteiligungsziele in Anlehnung an ALEWELL fünf hochschulspezifische Messezielgruppen unterschieden werden (vgl. Abb. 12):

- Wissenschaft, d.h. Vertreter anderer wissenschaftlicher Institutionen (z.B. Professoren, wissenschaftliche Mitarbeiter), mit denen durch eine Messebeteiligung vor allem ein Informations- und Erfahrungsaustausch angestrebt wird.

- Studierende, d.h. aktuelle und potentielle Studierende, wobei bei letzteren durch die Präsentation innovativer Exponate u.a. das Ziel verfolgt wird, Interesse an der Hochschule zur Aufnahme eines Studiums zu wecken.

- Wirtschaft ${ }^{60}$, d.h. Vertreter wirtschaftlicher Unternehmen, zu denen durch eine Messebeteiligung insbesondere die Kontaktaufnahme angestrebt wird, um u.a. die Voraussetzungen für mögliche Projektkooperationen sowie zur Einwerbung von Projektmitteln zu schaffen.

- Staat, d.h. Vertreter von Behörden und sonstigen staatlichen Institutionen, denen durch eine Messebeteiligung u.a. das Leistungspotential der Hochschule präsentiert und damit letztlich die Einwerbung von zusätzlichen Etatmitteln forcient wird.

- Öffentlichkeit, d.h. Presse- und Medienvertreter, Privatpersonen sowie alle zuvor nicht erfaßten Institutionen und Personen, die - nicht zuletzt aufgrund

Der Begriff „Wirtschaft“ wird in vielfältiger Hinsicht verwendet, wobei darunter sehr unterschiedliche Aspekte subsumiert werden. Im folgenden soll der Begriff "Wirtschaft" ausschließlich als Bezeichnung für eine hochschulspezifische Zielgruppe i.S.d. aufgeführten Interpretation genutzt werden. 
des öffentlichen Auftrags der Hochschule - durch die Messebeteiligung über Aktivitäten der Hochschule informiert werden sollen.

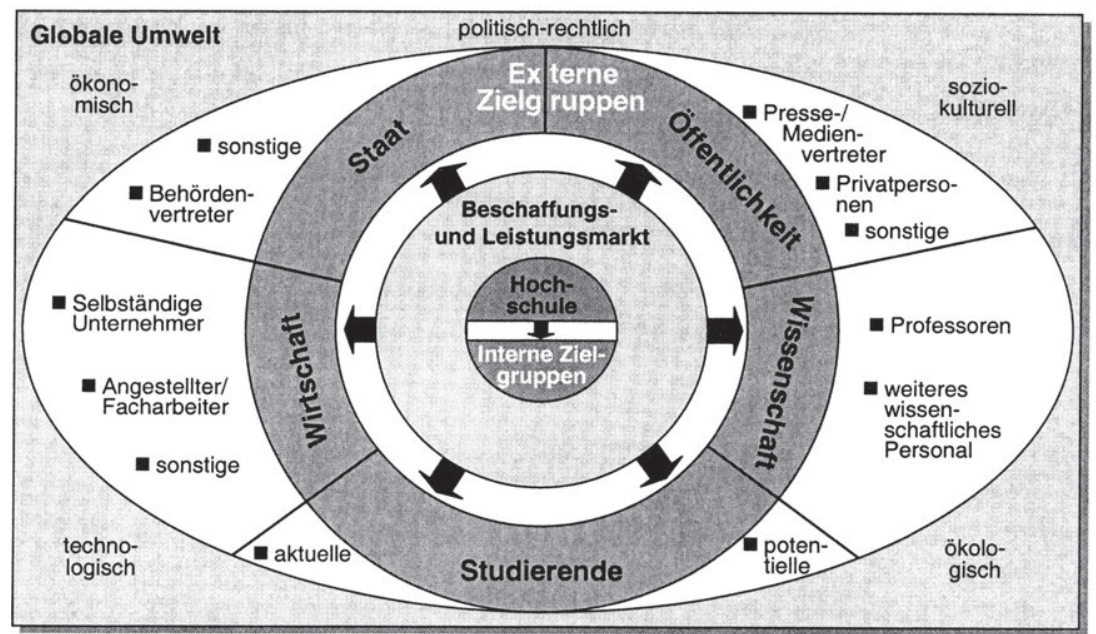

Abb. 12: Systematisierung von Anspruchsgruppen zur Ableitung hochschulspezifischer Messebeteiligungszielgruppen

Die Messezielsetzungen der Hochschul-Exponenten - so läßt sich aus der Beschreibung der einzelnen Messezielgruppen vermuten - sind voraussichtlich auf Kernzielgruppen gerichtet. Die nachfolgende Abbildung zeigt - auf Grundlage von Plausibilitätsüberlegungen - die auf einzelne Zielgruppen möglicherweise gerichteten Messebeteiligungsziele. Dabei wird deutlich, daß auf die Zielgruppe Wirtschaft die meisten hochschulspezifischen Messezielsetzungen fokussiert sind. In diesem Zusammenhang ist der Frage nachzugehen, inwieweit den hochschulspezifischen Zielgruppen gemäß den Messezielprioritäten unterschiedliche Stellenwerte beigemessen werden und ob sich gegebenenfalls exponententypspezifische Zielgruppenpriorisierungen identifizieren lassen. 


\begin{tabular}{|c|c|c|c|c|c|c|c|c|}
\hline \multicolumn{4}{|c|}{ Messezieldimensionen } & \multicolumn{5}{|c|}{ Messezielgruppen } \\
\hline & \begin{tabular}{|cc} 
Messezielgewich- \\
tungen
\end{tabular} & Mittelwer & unw & $\begin{array}{l}\text { Wis- } \\
\text { sen- } \\
\text { schaft }\end{array}$ & $\begin{array}{l}\text { Studie- } \\
\text { rende }\end{array}$ & $\begin{array}{l}\text { Wirt- } \\
\text { schaft }\end{array}$ & $\begin{array}{l}\begin{array}{l}\text { Öffent- } \\
\text { lich- } \\
\text { keit }\end{array} \\
\end{array}$ & Staat \\
\hline \multirow{4}{*}{ 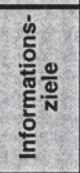 } & $\begin{array}{l}\text { Erfahrungs- und Informationsaus- } \\
\text { tausch mit Praxisvertretern }\end{array}$ & 1,4 & & - & - & ++ & - & - \\
\hline & $\begin{array}{l}\text { Erfahrungs-und Informationsaus- } \\
\text { tausch mit Fachkollegen }\end{array}$ & & 2,4 & ++ & - & - & - & - \\
\hline & $\begin{array}{l}\text { Beobachtung anderer Institute/ } \\
\text { Eehrstúhle/Fachbereiche }\end{array}$ & - & 2,7 & ++ & - & - & - & - \\
\hline & Sammlung von Informationen & - & 2,4 & ++ & + & ++ & + & + \\
\hline \multirow{2}{*}{ 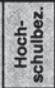 } & Verbesserung des Hochschulimages & 2,0 & & + & $++/+$ & + & ++ & ++ \\
\hline & $\begin{array}{l}\text { Beziehungspflege/-aufbau mit Reprä- } \\
\text { sentanten verschiedener Institutionen }\end{array}$ & 2,1 & & - & - & - & - & ++ \\
\hline \multirow{2}{*}{ 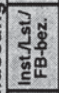 } & $\begin{array}{l}\text { Erhöhung des Bekanntheitsarades } \\
\text { des Instituts/Lehrstuhls/Fachbereichs }\end{array}$ & 1,4 & & + & $++/+$ & ++ & ++ & + \\
\hline & $\begin{array}{l}\text { Verbesserung d. Instituts-Lehrstuhl- } / \\
\text { Fachbereichsimages }\end{array}$ & 1,7 & & + & $++/+$ & ++ & ++ & + \\
\hline \multirow{2}{*}{ 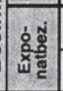 } & $\begin{array}{l}\text { Erhöhung des Bekanntheitsgrades } \\
\text { des Exponates }\end{array}$ & 1,3 & & + & $++/+$ & ++ & ++ & + \\
\hline & Darstellung der Leistungskompetenz & 1,4 & & + & $++/+$ & ++ & ++ & ++ \\
\hline \multirow{3}{*}{$\frac{\frac{1}{d}}{\frac{\pi}{0}} \frac{0}{\frac{1}{N}}$} & $\begin{array}{l}\text { Kontaktaufnahme zu Projekt- } \\
\text { interessenten }\end{array}$ & 1,6 & & + & - & ++ & - & $++/+$ \\
\hline & Einwerbung von Projektmitteln & 2,0 & & - & - & ++ & - & $++/+$ \\
\hline & $\begin{array}{l}\text { Überprüfung der Marktreife eigener } \\
\text { Produkte und Leistungen }\end{array}$ & - 2,2 & & - & - & ++ & - & + \\
\hline \multicolumn{9}{|c|}{$\begin{array}{l}++ \\
++ \text { Primär auf die entsprechende Zielgruppe gerichtete Messeziele } \\
++/+ \text { Teils primär und teils sekundär auf die entsprechende Zielgruppe ge } \\
+\quad=\text { Sekundär auf die entsprechende Zielgruppe gerichtete Messeziele } \\
-\quad=\text { Nicht auf die entsprechende Zielgruppe gerichtete Messeziele }\end{array}$} \\
\hline
\end{tabular}

Abb. 13: Hochschulspezifische Zielgruppen und die auf sie gerichteten hochschulspezifischen Messezielsetzungen

\subsection{Empirische Analyse der Bedeutung hochschulspezifischer Messe- zielgruppen}

Vor dem Hintergrund der vielfältigen Messezielsetzungen streben die HochschulExponenten mit ihrer Messebeteiligung die Erreichung unterschiedlicher Messezielgruppen an. Die Ergebnisse der Hochschul-Exponenten-Befragung zeigen (vgl. Abb. 14), daß klar abgestufte Bedeutungsgewichte für die einzelnen Zielgruppen existieren, die einerseits zu eindeutig priorisierten und andererseits $\mathrm{zu}$ weniger bedeutenden Messezielgruppen führen. 


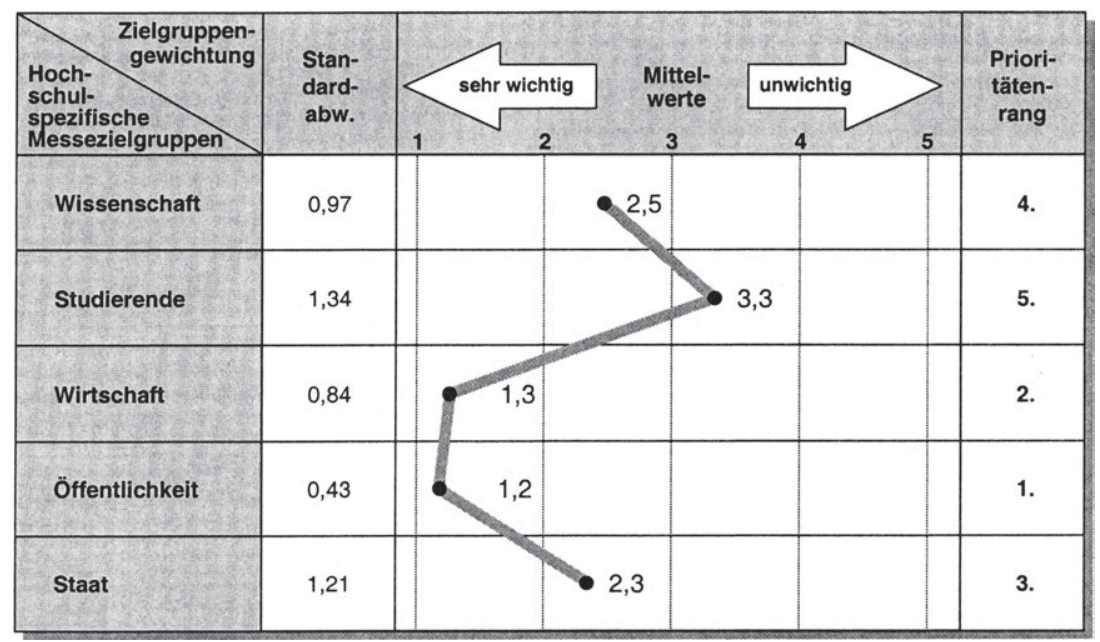

Abb. 14: Bedeutung hochschulspezifischer Messezielgruppen

Die Hochschulen streben mit ihrer Messebeteiligung insbesondere die Erreichung der Zielgruppen Öffentlichkeit und Wirtschaft an, die in der Prioritätenrangfolge mit einem deutlichen Abstand vor den anderen Zielgruppen eingestuft werden. Die wichtigste Zielgruppe stellt dabei die Öffentlichkeit ${ }^{61}$ dar, die mit einem Mittelwert von 1,2 die höchste Priorität erhält. Ausschlaggebend hierfür ist der vom Hochschulrahmengesetz festgelegte öffentliche Auftrag der Hochschulen und die damit zusammenhängende Rechtfertigungspflicht der Hochschulaktivitäten gegenüber der Öffentlichkeit, die sich insbesondere in den generell hoch priorisierten und auf die Zielgruppe der Öffentlichkeit gerichteten Beeinflussungszielen (z.B. Verbesserung des Hochschul- und Instituts-/Lehrstuhl-/Fachbereichsimages, Darstellung der Leistungskompetenz) widerspiegeln (vgl. Abb. 13).

Mit einem Mittelwert von 1,3 nimmt die Wirtschaft ${ }^{62}$ die zweithöchste Zielgruppenpriorität im Rahmen von Hochschul-Messebeteiligungen ein. Die geringe Stan-

61 Die Hochschul-Exponenten bewerteten nicht direkt die Zielgruppe Öffentlichkeit, sondern die sonstigen Zielgruppen, d.h. also diejenigen Personen und Gruppen, die nicht durch die anderen aufgeführten Zielgruppen erfaßt wurden. Vgl. Anhang III, Fragebogen 1: Teil 1 der Exponentenbefragung I in der Vor-Messe-Phase, Frage 5.

62

Im Rahmen der Befragungen gewichteten die Exponenten die Zielgruppe Praxisvertreter, die sich nur semantisch, nicht jedoch inhaltlich von der Zielgruppe Wirtschaftsvertreter differen- 
dardabweichung deutet in diesem Zusammenhang auf eine relativ homogene Priorisierung durch die Hochschul-Exponenten hin. Die Ursache für den hohen Stellenwert von Wirtschaftsvertretern als Messezielgruppe liegt vermutlich in den von Hochschul-Exponenten mit einer Messebeteiligung verfolgten und insgesamt hoch priorisierten Projektzielen begründet. ${ }^{63}$ Darüber hinaus determinieren die generell auf Wirtschaftsvertreter gerichteten Messezielsetzungen (z.B. Erfahrungs- und Informationsaustausch mit Praxisvertretern) die hohe Bedeutung dieser Zielgruppe. Als weiterer möglicher Grund der hohen Relevanz von Wirtschaftsvertretern für eine Hochschul-Messebeteiligung sind die sich den Hochschulen außerhalb von Messebeteiligungen nur begrenzt bietenden Möglichkeiten zur Kontaktaufnahme mit dieser Zielgruppe anzuführen.

Die Repräsentanten des Staates ${ }^{64}$ nehmen ebenfalls einen hohen, wenngleich im Vergleich zu den vorangegangenen Zielgruppen geringeren Prioritätsrang für eine Hochschul-Messebeteiligung ein (Mittelwert = 2,3). Diese Bedeutungsgewichtung kann wiederum auf die Stellenwerte der auf diese Zielgruppe gerichteten Messebeteiligungsziele zurückgeführt werden. Dabei bietet eine Messeteilnahme Hochschul-Exponenten die Möglichkeit, ihr Forschungspotential gegenüber Vertretern staatlicher Institutionen zu dokumentieren und damit nicht zuletzt auch die Einwerbung von Projektmitteln bzw. öffentlichen Drittmitteln zu forcieren. Dennoch existieren diesbezüglich auch weitere Gelegenheiten, z.B. im Rahmen von staatlichen Hochschul-Wettbewerbs- oder Hochschul-Projekt-Ausschreibungen, worin möglicherweise die Prioritätsabstufung zu den vorangegangenen hochschulspezifischen Zielgruppen begründet liegt.

Gleiches gilt für die Zielgruppe Wissenschaft ${ }^{65}$, zu der neben einer Messebeteiligung auch im Rahmen wissenschaftlicher Veranstaltungen, wie z.B. Fachkongresse und Symposien, eine Kontaktaufnahme möglich ist. Die eher mittlere

ziert. Vgl. Anhang III, Fragebogen 1: Teil 1 der Exponentenbefragung $\mathrm{I}$ in der Vor-MessePhase, Frage 5.

Insgesamt stufen $77,8 \%$ der Hochschul-Exponenten - Projekt- und exponatorientierte als auch Beeinflussungsorientierte Hochschul-Exponenten - Projektziele als besonders wichtig ein.

Befragt wurden die Exponenten zu der Gewichtung von Forschungsförderungsinstitutionen, die i.w.S. unter Einrichtungen des Staates zu subsumieren sind. Vgl. Anhang III, Fragebogen 1: Teil 1 der Exponentenbefragung I in der Vor-Messe-Phase, Frage 5.

Die Hochschul-Exponenten bewerteten nicht direkt die Zielgruppe Wissenschaft, sondern die Gruppe der Fachkollegen, welche die Wissenschaft adäquat repräsentiert. Vgl. Anhang III, Fragebogen 1: Teil 1 der Exponentenbefragung I in der Vor-Messe-Phase, Frage 5. 
Gewichtung von Wissenschaftsvertretern als Zielgruppe einer Hochschul-Messebeteiligung (Mittelwert $=2,5$ ) liegt vermutlich in den Stellenwerten der Messezielsetzungen begründet, die primär auf diese Zielgruppe gerichtet sind. So werden bspw. die primär auf Wissenschaftler gerichteten Zielsetzungen wie Erfahrungsund Informationsaustausch mit Fachkollegen, Beobachtung anderer Institute/Lehrstühle/Fachbereiche sowie Sammlung von Informationen mit Mittelwerten zwischen 2,4 und 2,7 gewichtet.

Die Zielgruppe Studierende nimmt schließlich den vergleichsweise geringsten Stellenwert im Rahmen von Hochschul-Messebeteiligungen ein. Innen kommt mit dem Mittelwert von 3,3 mit Abstand die geringste Bedeutung zu. Die Standardabweichung von 1,34 weist dabei jedoch auf Abweichungen der befragten Exponenten bzgl. dieser Bewertung hin. Während bei den meisten Hochschul-Exponenten - wahrscheinlich aufgrund einfacherer Möglichkeiten zur Ansprache von Studierenden - eindeutig die zuvor dargestellten Zielgruppen im Fokus der Messebeteiligung stehen, streben einige Exponenten durch die Präsentation von Forschungsentwicklungen auf Messen auch danach, die Kompetenz und Attraktivität inrer Ausbildungsstätte gegenüber Studierenden darzustellen bzw. zu erhöhen. Diese Hochschul-Exponenten messen Studierenden einen höheren Stellenwert bei, der wiederum durch die Gewichtungen der u.a. auf diese Zielgruppe gerichteten Messezielsetzungen (z.B. Verbesserung des Hochschul- und Instituts-/Lehrstuhl-/Fachbereichsimages, Erhöhung des Bekanntheitsgrades des Instituts/Lehrstuhls/Fachbereichs) determiniert wird.

Im Hinblick auf die Priorisierung von Studierenden als Messezielgruppe liefert die exponententypspezifische Betrachtung weitere Informationen (vgl. Abb. 15). Während Projekt- und exponatorientierte Hochschul-Exponenten Studierende mit einem Mittelwert von 4,0 als relativ unbedeutend für ihre Messebeteiligung einstufen, bewerten Pflichtrepräsentanten diese Zielgruppe mit einem Mittelwert von 2,6 für ihr Messeengagement als eher wichtig. Dennoch bilden auch bei diesem Exponententyp Studierende im Vergleich zu anderen Anspruchsgruppen die Zielgruppe mit der geringsten Bedeutung. Die unterschiedliche Bewertung von Studierenden als Messezielgruppe liegt dabei in der unterschiedlichen Zielstruktur der jeweiligen Exponententypen begründet. Letztlich sind nur in bezug auf diese Messezielgruppe auf einem Signifikanzniveau von 0,085 signifikante Unterschiede zwischen den Exponententypen zu verzeichnen. Zwar werden in Abhängigkeit der primär verfolgten Zielsetzungen bestimmte Zielgruppen (z.B. Wissenschaft und Staat) von den einzelnen Exponententypen leicht unterschiedlich bewertet, den- 
noch lassen sich hier keine signifikanten Unterschiede konstatieren. Damit wird die zuvor aufgestellte Vermutung, daß angesichts der signifikant unterschiedlichen Zielpriorisierungen ebenfalls unterschiedliche Gewichtungen der Messezielgruppen zu erwarten sind, widerlegt. Dieses Ergebnis deutet auf eine nicht konsistente, exponententypspezifische Planung von Messebeteiligungen hin und läßt die Ableitung von Implikationen für zukünftige Hochschul-Messebeteiligungen auf Basis der im Rahmen dieser Arbeit gewonnenen Erkenntnisse sinnvoll erscheinen.

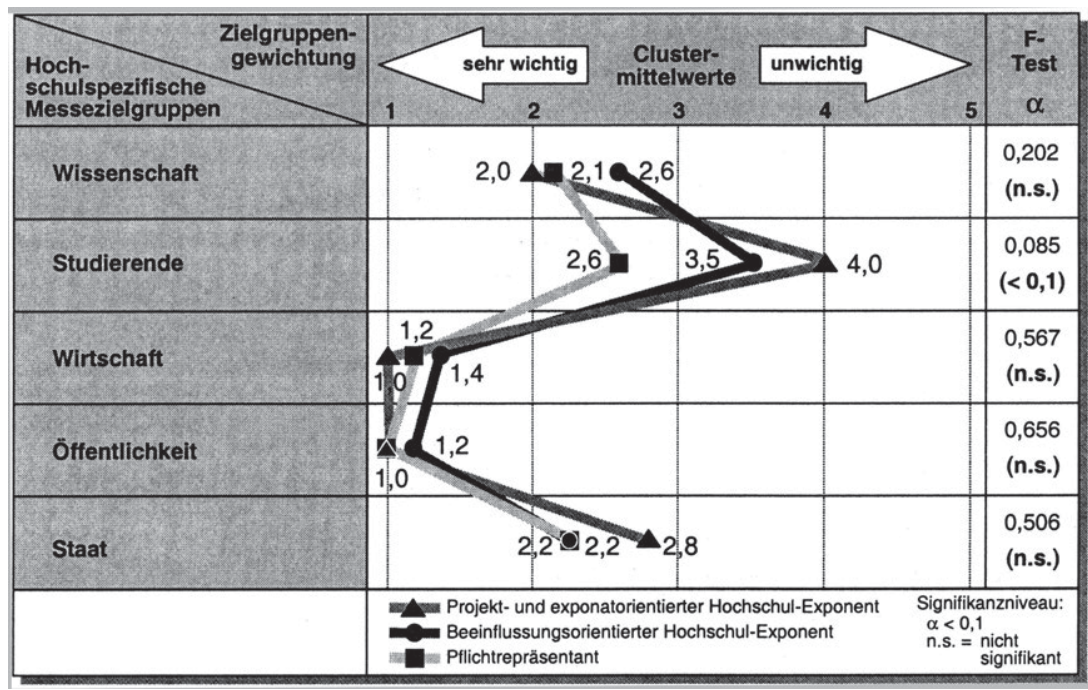

Abb. 15: Exponententypspezifische Bedeutung hochschulspezifischer Messezielgruppen

Nicht zuletzt aufgrund der sehr ähnlichen exponententypspezifischen Priorisierungen der Messezielgruppen stellt sich die Frage, inwieweit eine ausschließlich auf Grundlage von Austauschbeziehungen vorgenommene Systematisierung von hochschulspezifischen Anspruchsgruppen zur Systematisierung von Messezielgruppen ausreicht. 


\subsection{Ziele von Messebesuchern als komplementäres Zielgruppenkriterium für hochschulspezifische Messezielgruppen}

\subsection{Messebesucherziele als Determinante des Messebesucherverhaltens}

Eine Systematisierung von hochschulspezifischen Messezielgruppen nach der Relevanz ihrer Austauschbeziehungen reicht i.d.R. für die Ableitung hochschulspezifischer Ausgestaltungsformen nicht aus. Vielmehr ist eine zusätzliche Berücksichtigung des Verhaltens der Messebesucher bzw. der Hochschul-Messestand-Besucher notwendig, um sowohl verhaltensangepaßte Maßnahmen zu konzipieren als auch verhaltenssteuernd agieren zu können. Zur Ableitung hochschulspezifischer Messestrategien und -maßnahmen ist es demzufolge notwendig, das Verhalten von Messebesuchern zu analysieren und möglichst realitätsnah zu beschreiben. Trotz der allgemein hohen Bedeutung des Besucherverhaltens für Messebeteiligungen von Hochschul-Exponenten liegen bisher nur wenige empirische Untersuchungen in diesem Bereich vor.

In der Praxis werden bei den meisten Besucherbefragungen primär Strukturdaten, wie Branchenherkunft, Unternehmensgröße und Stellung im Unternehmen, erhoben. ${ }^{66}$ Darüber hinaus sind bei diesen Untersuchungen die Ausstellungsbereiche, denen sich die Messebesucher zuwenden, von Interesse. ${ }^{67}$ Werden derartige Befragungen sowohl für eine gesamte Messe als auch für einzelne Messestände durchgeführt, so liefert der Vergleich beider Ergebnisse Informationen für die jeweiligen Exponenten. ${ }^{68}$ Bei einer kritischen Betrachtung dieser Besuchererhebungen läßt sich feststellen, daß diese Untersuchungen wichtige Segmentierungskriterien - insbesondere verhaltenssteuernde Segmentierungskriterien - nicht berücksichtigen.

Vgl. Strothmann, K.-H., Im Verhalten unterscheiden sich Messe-Besucher beachtlich, in: Marketing Journal, 25. Jg., Heft 2, 1992, S. 164.

Vgl. Gesellschaft zur freiwilligen Kontrolle von Messe- und Ausstellungszahlen (FKM) (Hrsg.), Satzung und Regeln, Köln 1994. 
Vor diesem Hintergrund hat der SPIEgel-VerLag eine Studie durchgeführt, deren Ziel in der Klärung des Verhaltens von Messebesuchern liegt. ${ }^{69}$ Nach dieser Untersuchung, die sich grundsätzlich auf kommerzielle Messebeteiligungen bezieht, können vier Messebesuchertypen unterschieden werden, die sich durch grundlegende Eigenschaften und Verhaltensweisen differenzieren. ${ }^{70}$

- Intensive Messe-Nutzer, die sich gründlich auf ihren Messebesuch vorbereiten und Messen im Vergleich zu anderen Informationsquellen einen hohen Stellenwert einräumen. Ihre Aktivitäten reichen von Informationsbeschaffung, Kontaktpflege und Marktbeobachtung bis hin zu Preisvergleichen. Derartige Besucher halten sich mehrere Tage auf einer Messe auf und besuchen durchschnittlich 21 Aussteller.

- Punktuelle Messe-Nutzer, die sich gründlich auf ihren Messebesuch vorbereiten und von der Bedeutung einer Messe überzeugt sind. Dieser Besuchertyp ist auf der Suche nach innovativen Verfahren und damit nach gezielten Informationen. Er hält sich ein bis zwei Tage auf einer Messe auf, wobei er etwa zehn Aussteller besucht.

- Praxisorientierte Messe-Nutzer, die sich zwar nur oberflächlich auf ihren Messebesuch vorbereiten, Messen aber zur gezielten Information über technische Neuerungen, Systeme und Produkte nutzen. ${ }^{71}$ Diese Messebesucher halten sich i.d.R. nur einen Tag auf Messen auf und besuchen ebenfalls ca. zehn Aussteller.

- Messe-Bummler, die sich nur oberflächlich auf ihren Messebesuch vorbereiten und Messen primär als Instrument zur Marktbeobachtung nutzen. Dieser Besuchertyp hat i.d.R. keine Investitionsabsichten und läßt sich durch ein sehr

Vgl. Spiegel-Verlag (Hrsg.), Messen und Messebesucher in Deutschland, Hamburg 1992.

Die Typen des Messebesuchs-Verhaltens repräsentieren Besucher, die auf Bürokommunikations-/EDV-Messen in Erscheinung treten. Diese Messebesucher-Typen wurden mittels einer Clusteranalyse auf Grundlage einer reprăsentativen, mündlichen Befragung von 424 investitionsentscheidenden Fachleuten des verarbeitenden Gewerbes in den alten Bundesländern identifiziert. Vgl. Spiegel-Verlag (Hrsg.), Messen und Messebesucher in Deutschland, a.a.O., S. $1 \mathrm{ff}$.

Die Begriffswahl Praxisorientierter Messe-Nutzer erscheint hier im Gegensatz zu dem Intensiven Messe-Nutzer und dem Punktuellen Messe-Nutzer wenig zweckmäßig, da diese beiden Messebesuchertypen ebenfalls praxisorientiert sind. Aufgrund der Charakterisierung der Praxisorientierten Messe-Nutzer wäre die Bezeichnung Pragmatischer Messe-Nutzer zutreffender. 
eingeschränktes Informationsverhalten, d.h. es werden nur die vom Aussteller gestalteten und direkt vermittelten Informationen zur Kenntnis genommen, charakterisieren. Derartige Besucher "bummeln" oftmals nur einen Tag über eine Messe, um sich einen Überblick zu verschaffen. Dabei werden nicht mehr als fünf bis zehn Aussteller besucht, wobei ein Kontakt zum Standpersonal vermieden wird.

Die Beschreibung der einzelnen Messebesuchertypen zeigt, daß sich die einzelnen Gruppen insbesondere durch die verfolgten Zielsetzungen der Messebesucher, die wiederum das Messebesucherverhalten bestimmen, unterscheiden. Dabei können grundsätzlich Informations- und Kontaktziele angeführt werden. Bei hochschulspezifischen Messebesucherzielen erscheint in diesem Zusammenhang eine weitere Differenzierung notwendig. So können bei Informationszielen sowohl allgemeine Ziele, wie Sammlung von Informationen, als auch konkretere Zielsetzungen, wie Beobachtung von Entwicklungen der Wissenschaft, als Besucherzielsetzungen fungieren. Im Rahmen von Kontaktzielen kann zwischen dem Ziel der reinen Beziehungspflege oder dem Beziehungsaufbau mit Wissenschaftsvertretern und einer fokussiert verfolgten Kontaktaufnahme zu Projektanbietern differenziert werden. Darüber hinaus ist die zwischen den beiden grundsätzlichen Besucherzielkategorien einzuordnende Zielsetzung Erfahrungs- und Informationsaustausch mit Wissenschaftsvertretern anzuführen.

Die vor dem Hintergrund der SPIEgel-VerLAG-Studie aufgestellte These, daß das Verhalten der Hochschul-Messestand-Besucher durch die jeweils verfolgten Besucherzielsetzungen determiniert wird, bestätigt das Means-End-Konzept. Dieses Konzept wurde in der Sozialpsychologie entwickelt und geht auf Arbeiten von TOLMAN, der erstmals die Zielorientierung individuellen Verhaltens in den Mittelpunkt von Forschungsbemühungen stellte, zurück. ${ }^{72}$ Die Grundidee des MeansEnd-Konzeptes, welches hier auf Hochschul-Messebeteiligungen übertragen wird, besteht darin, daß ein Messebesucher im Rahmen eines Informationsverarbeitungsprozesses eine Vorstellung über die Eignung von Hochschul-ExponentenLeistungen („means“) zur Erfüllung bestimmter Messebesucherziele („end“) ent-

Vgl. Tolman, E., Purpose Behaviour in Animals and Men, New York 1932, S. 5 ff. 
wickelt. $^{73}$ Die Merkmale eines Hochschul-Messestands werden dabei als Mittel zur Zielerreichung aufgefaßt ${ }^{74}$ und verzahnen die Nutzenwahrnehmung von Hochschul-Messestand-Besuchern mit ihren übergeordneten Hochschul-MessestandBesuchszielen. ${ }^{75}$

Angesichts des Einflusses von Exponentenzielen auf die Ausgestaltung von Hochschul-Exponenten-Leistungen ist davon auszugehen, daß der Nutzen einer Hochschul-Messebeteiligung um so höher ausfällt, je mehr sich Exponentenzielsetzungen und Hochschul-Messestand-Besucherzielsetzungen entsprechen. Die nachfolgende Tabelle stellt die Messebeteiligungsziele von Hochschul-Exponenten einerseits und die Zielsetzungen von Hochschul-Messestand-Besuchern andererseits gegenüber. Dabei werden die zwischen diesen Zielsetzungen bestehende Zielbeziehungen dargestellt, die Ansatzpunkte zur Ableitung von Implikationen für zukünftige Hochschul-Messebeteiligungen aufzeigen. In diesem Zusammenhang stellt sich die Frage, inwieweit sich auf Grundlage der von den Hochschul-Messestand-Besuchern verfolgten Zielsetzungen Hochschul-Messestand-Besuchertypen bilden lassen.

Vgl. Herrmann, A., Nachfrageorientierte Produktgestaltung: Ein Ansatz auf Basis der ${ }_{\text {means }}$ end"-Theorie, Wiesbaden 1996, S. 7. Zur ausführlichen Darstellung der Means-End-Theorie vgl. darüber hinaus Olson, J. C., Theoretical Foundations of Means-End Chains, Working Paper 174, Pennsylvania State University, Pittsburgh, PA 1988; Olson, J. C., Reynolds, T. J., Understanding Consumer Cognitive Structures: Implications for Advertising Strategy, in: Percy, L., Woodside, A. (Ed.), Advertising and Consumer Psychology, Lexington, MA 1983, S. $77 \mathrm{ff.}$

Vgl. Gutschke, J., Produktpräferenzanalyse: Ein modelltheoretisches und methodisches Konzept zur Marktsimulation mittels Präferenzerfassungsmodellen, Berlin 1995, S. 36.

Vgl. Herrmann, A., Wertorientierte Produkt- und Werbegestaltung, in: Marketing ZFP, 18. Jg., Heft 3, 1996, S. 153. Dabei wird der Means-End-Theorie insbesondere deshalb eine hohe Aussagekraft zur Erklärung des Verhaltens von Nachfragern eingeräumt, da hier die Brücke zwischen den Nutzenerwartungen von Nachfragern bzw. Messestand-Besuchern und den zahlreichen die Wahrnehmung von Leistungen/Produkten beeinflussenden intrinsischen Antriebskräften wie Emotionen und Motiven geschaffen wird. 


\begin{tabular}{|c|c|c|c|c|c|c|}
\hline \multicolumn{2}{|c|}{$\begin{array}{|lr|}\begin{array}{l}\text { Hochschul- } \\
\text { Exponenten }\end{array} & \text { Hochschul-Messestand- } \\
\text { Besucher }\end{array}$} & \multicolumn{5}{|c|}{ Ziele zum Besuch eines Hochschul-Messestandes } \\
\hline $\begin{array}{l}\text { Messe- } \\
\text { ziel- } \\
\text { dimen- } \\
\text { sionen }\end{array}$ & $\begin{array}{l}\begin{array}{r}\text { Messebesuchsziele von } \\
\text { Hochschul-Messe- } \\
\text { stand-Besuchern }\end{array} \\
\begin{array}{l}\text { Messeeinzel- } \\
\text { zielsetzungen von } \\
\text { Hochschul-Exponenten }\end{array}\end{array}$ & $\begin{array}{l}\text { Sammlung } \\
\text { von Infor- } \\
\text { mationen }\end{array}$ & $\begin{array}{l}\text { Beobachtung } \\
\text { von Entwick- } \\
\text { lungen der } \\
\text { Wissenschaft }\end{array}$ & \begin{tabular}{|l|} 
Erfahrungs- u. \\
Informations- \\
austausch \\
mit Wis- \\
senschafts- \\
vertretern
\end{tabular} & \begin{tabular}{|l|} 
Beziehungs- \\
pflege oder \\
-aufbau mit \\
Wissen- \\
schafts- \\
vertretern \\
\end{tabular} & $\begin{array}{l}\text { Kontakt- } \\
\text { aufnah- } \\
\text { me zu } \\
\text { Projekt- } \\
\text { anbietern }\end{array}$ \\
\hline \multirow{4}{*}{ 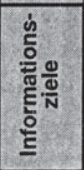 } & $\begin{array}{l}\text { Erfahrungs- und Informationsaus- } \\
\text { tausch mit Praxisvertretern }\end{array}$ & ++ & + & ++ & ++ & + \\
\hline & $\begin{array}{l}\text { Erfahrungs- und Informationsaus- } \\
\text { tausch mit Fachkollegen }\end{array}$ & ++ & + & ++ & ++ & + \\
\hline & $\begin{array}{l}\text { Beobachtung anderer Institute/ } \\
\text { ehrstunhle/Fachbereiche }\end{array}$ & + & 0 & 0 & 0 & 0 \\
\hline & Sammlung von Informationen & + & 0 & + & + & + \\
\hline \multirow{2}{*}{ 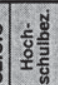 } & Verbesserung des Hochschulimages & + & + & + & + & + \\
\hline & $\begin{array}{l}\text { Beziehungspflege/-aufbau mit Reprä- } \\
\text { sentanten verschiedener Institutionen }\end{array}$ & + & 0 & ++ & + & + \\
\hline \multirow{2}{*}{ 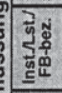 } & $\begin{array}{l}\text { Erhöhung des Bekanntheitsqrades } \\
\text { des Instituts/Lehrstuhls/Fachibereichs }\end{array}$ & + & + & + & + & + \\
\hline & $\begin{array}{l}\text { Verbesserung d. Instituts-/Lehrstuhl-/ } \\
\text { Fachbereichsimages }\end{array}$ & + & + & + & + & + \\
\hline \multirow{2}{*}{ 这究 } & $\begin{array}{l}\text { Erhöhung des Bekanntheitsgrades } \\
\text { des Exponates }\end{array}$ & + & + & + & + & + \\
\hline & Darstellung der Leistungskompetenz & ++ & + & + & + & + \\
\hline \multirow{3}{*}{$\frac{\frac{1}{0}}{\frac{0}{2}} \frac{0}{\frac{0}{N}}$} & $\begin{array}{l}\text { Kontaktaufnahme zu Projekt- } \\
\text { interessenten }\end{array}$ & + & + & + & + & ++ \\
\hline & Einwerbung von Projektmitteln & + & 0 & + & + & ++ \\
\hline & $\begin{array}{l}\text { Überprüfung der Marktreife eigener } \\
\text { Produkte und Leistungen }\end{array}$ & + & + & + & + & ++ \\
\hline \multicolumn{7}{|c|}{$\begin{array}{l}++=\text { Exponenten- und Besucherziele stehen komplementär zueir } \\
+=\text { Exponenten- und Besucherziele stehen bedingt komplemen } \\
0=\text { Exponenten- und Besucherziele stehen neutral zueinander }\end{array}$} \\
\hline
\end{tabular}

Tab. 4: Beziehungen zwischen Messebeteiligungszielen von Hochschul-Exponenten und Zielsetzungen von Hochschul-Messestand-Besuchern

\subsection{Clusteranalytische Bestimmung von Hochschul-Messestand- Besuchertypen und diskriminanzanalytische Überprüfung}

Als Messebesuchertypen auf Hochschul-Messeständen werden in der vorliegenden Arbeit homogene Gruppen von Hochschul-Messestand-Besuchern bezeichnet, die angesichts ihrer verfolgten Zielsetzungen ein ähnliches Verhalten aufweisen. Sind bei Hochschul-Messestand-Besuchern nahezu identische Zielsetzungen nachzuweisen, so bilden sie einen Besuchertyp. Zur Bildung derartiger, in sich homogener, untereinander aber möglichst heterogener Gruppen von Untersuchungsobjekten anhand zugehöriger Merkmalsausprägungen stellt - wie bei der Bildung der Exponententypen - die Clusteranalyse ein geeignetes statistisches Instrument dar. Die Bestimmung von Messebesuchertypen mit Hilfe der Clusteranalyse erfolgte dabei auf Basis von 1.218 befragten Messebesuchern, die zuvor einen Hochschul-Messestand besucht hatten. Aus dieser Stichprobe wurden 142 
Probanden aufgrund von missing values ausgeschlossen. Somit konnten zur Gruppenbildung 1.076 Probanden berücksichtigt werden.

Zur Ermittlung der Messebesuchertypen wird - wie bei der Ableitung der Exponententypen - im Rahmen der Clusteranalyse ein dreistufiges Vorgehen gewählt. Auf Grundlage des im ersten Schritt eingesetzten Single-Linkage-Verfahrens wurden die extremen Beurteilungen von 8 Probanden von der weiteren Analyse ausgeschlossen. Damit verblieb insgesamt eine Stichprobe von 1.068 Befragten zur weiteren Besuchertypenbildung. Das im zweiten Schritt eingesetzte Ward-Verfahren weist auf eine 3-Clusterlösung hin (vgl. Abb. 16). Die Centroiden dieser Cluster bzw. Besuchergruppen dienten wiederum als Startpartition einer anschlieBend durchgeführten partitionierenden Clusteranalyse zur Optimierung der Clusterlösung.

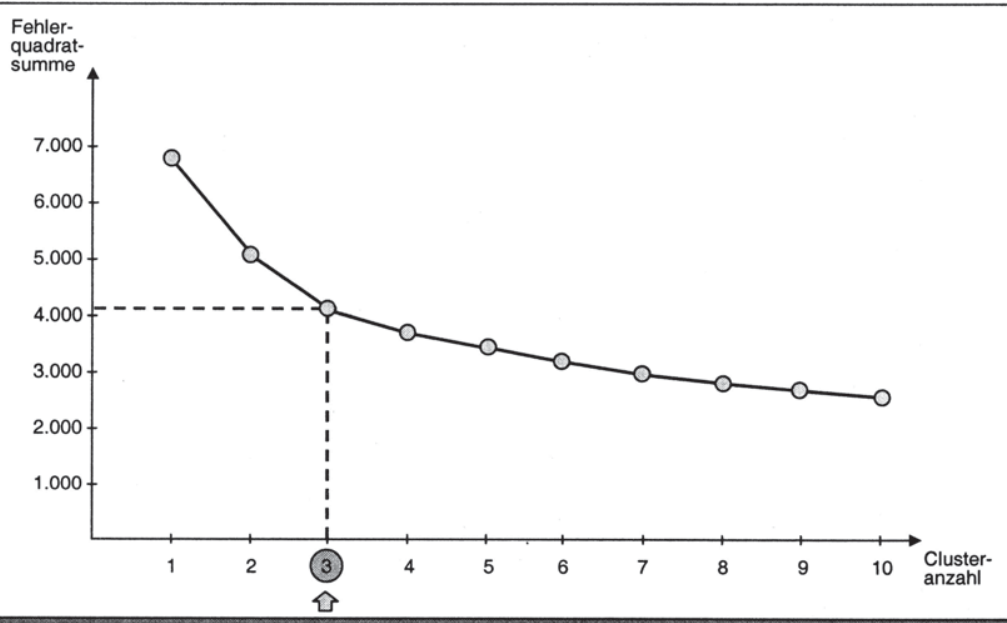

Abb. 16: Fusionierungsprozeß des Ward-Verfahrens und Varianzkriterium zur Bestimmung der Messestand-Besucher-Clusterlösung

Hervorzuheben sind bei der ermittelten Clusterlösung zunächst die relativ ähnlichen Gruppengrößen. Während Cluster 2 als größte Gruppe 40,6 \% $(n=433)$ der Hochschul-Messestand-Besucher umfaßt, gehören dem Cluster 1 als zweitgrößte Besuchergruppe $35,8 \%(n=381)$ und Cluster 3 als kleinste Gruppe immerhin noch 23,7\% $(n=254)$ der befragten Hochschul-Messestand-Besucher an. 
Darüber hinaus deuten die in Abb. 17 dargestellten Mittelwertabweichungen der Zielsetzungen von Hochschul-Messestand-Besuchern auf die Existenz signifikanter Unterschiede zwischen den identifizierten Besuchergruppen hin. Zur Überprüfung dieser Unterschiede wurde als Testverfahren wiederum die einfaktorielle Varianzanalyse eingesetzt. Darüber hinaus kam zur Überprüfung der Differenzen zwischen den einzelnen Besuchertypen abermals der Scheffé-Test zum Einsatz. Die Prüfmaße beider Testverfahren belegen signifikante Mittelwertunterschiede sowohl zwischen den Besuchertypen insgesamt als auch zwischen den einzelnen Besuchertypen. Lediglich bzgl. der Besucherzielsetzung Sammlung von Informationen ist zwischen zwei Besuchertypen kein signifikanter Unterschied feststellbar.

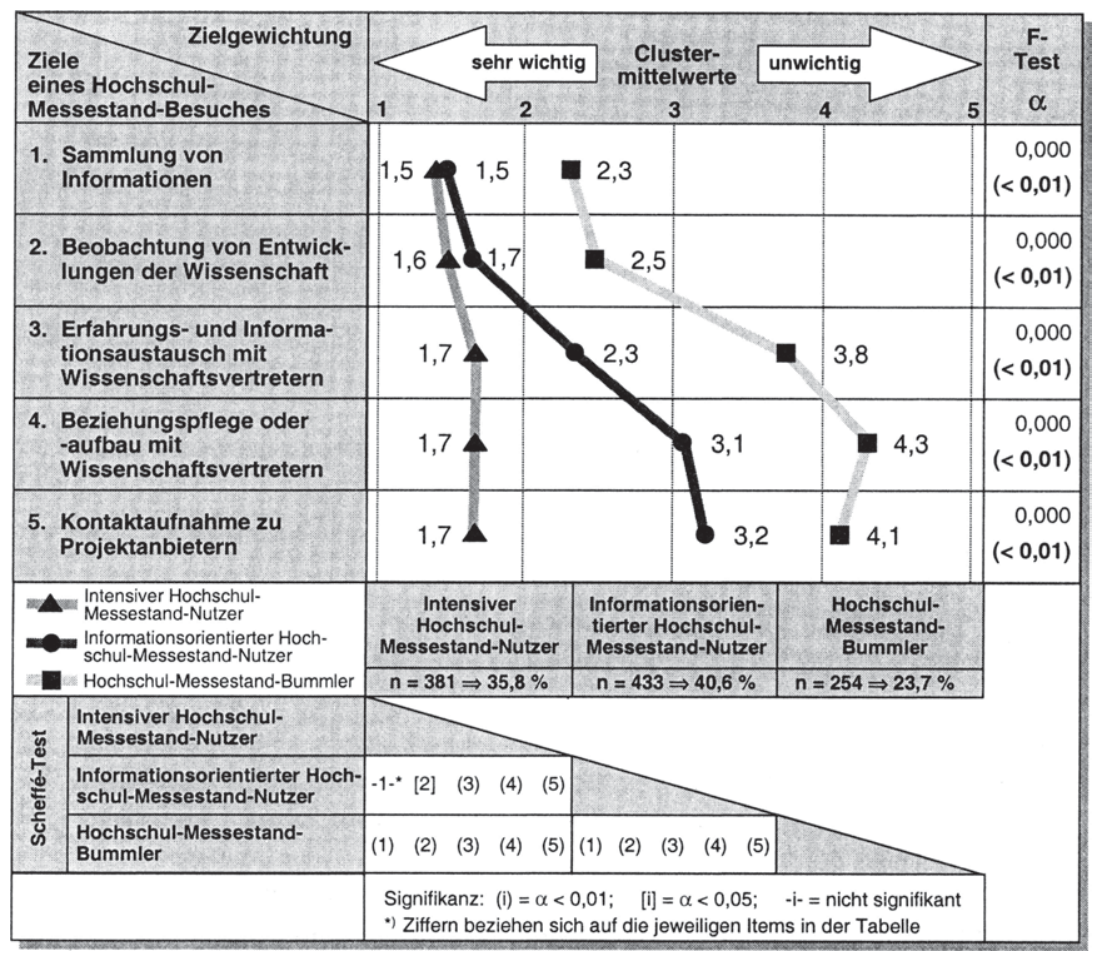

Abb. 17: Varianzanalytische Prüfung auf Bewertungsunterschiede bei Besucherzielsetzungen zwischen Hochschul-Messestand-Besuchertypen 
Auf Basis dieser konkreten Mittelwertausprägungen von Zielsetzungen eines Hochschul-Messestand-Besuchs können im folgenden die empirisch ermittelten Hochschul-Messestand-Besuchertypen differenzierter beschrieben werden.

Die Besuchergruppe der Intensiven Hochschul-Messestand-Nutzer umfaßt diejenigen Besucher, die mit inrem Hochschul-Messestand-Besuch sowohl Informations- als auch Kontaktziele verfolgen. So gewichtet diese Besuchergruppe die Sammlung von Informationen (Mittelwert $=1,5$ ) sowie die Beobachtung von Entwicklungen der Wissenschaft (Mittelwert $=1,6$ ) sehr hoch. Daneben messen sie den Kontaktzielen ebenfalls eine überdurchschnittlich hohe Bedeutung bei. In diesem Zusammenhang räumt dieser Besuchtertyp dem Erfahrungs- und Informationsaustausch mit Wissenschaftsvertretern, der Beziehungspflege und dem -aufbau mit Wissenschaftsvertretern sowie der Kontaktaufnahme zu Projektanbietern mit jeweils einem Mittelwert von 1,7 den gleichen Stellenwert ein. Die Zielsetzungen spiegeln sich dabei in dem Verhalten dieser Besuchergruppe wider, das sich vor allem in der Anzahl der auf dem Hochschul-Messestand geführten Gespräche (vgl. Tab. 5) niederschlägt. So führen Intensive Hochschul-Messestand-Nutzer im Vergleich zu den anderen Besuchertypen die meisten Gespräche auf einem Hochschul-Messestand. Mit $53 \%$ lag der Anteil dieses Besuchertyps auf der betrachteten Fachmesse höher als auf der untersuchten Mehrbranchenmesse. ${ }^{76}$

Die Informationsorientierten Hochschul-Messestand-Nutzer räumen den Informationszielen insgesamt den höchsten Stellenwert ein, so daß insbesondere die Sammlung von Informationen (Mittelwert $=1,5$ ) und die Beobachtung von Entwicklungen der Wissenschaft (Mittelwert $=1,7$ ) im Vordergrund eines HochschulMessestand-Besuchs stehen. Während für diese Besuchergruppe der Erfahrungsund Informationsaustausch mit Wissenschaftsvertretern noch von relativ hoher Bedeutung ist (Mittelwert = 2,3), wird der Beziehungspflege bzw. dem Beziehungsaufbau mit Wissenschaftsvertretern und der Kontaktaufnahme zu Projektanbietern mit Mittelwerten von 3,1 bzw. 3,2 eine geringere Bedeutung beigemessen. Ent-

Im Hinblick auf soziodemographische Aspekte lassen sich kaum signifikante Unterschiede zwischen den Hochschul-Messestand-Besuchertypen konstatieren. Grundsätzlich können diesbezüglich die Ausführungen zur Stichprobe der Hochschul-Messestand-Besucher herangezogen werden. Vgl. Anhang II, Zusammensetzung der Hochschul-Exponenten- und Hochschul-Messestand-Besucher-Stichproben. Nur in bezug auf eine anspruchsgruppenbezogene Betrachtung sind signifikante Unterschiede festzustellen, die aufgrund ihrer besonderen Relevanz in einem gesonderten Kapitel analysiert werden. Vgl. Kapitel B.2.3. 
sprechend seinen Zielsetzungen führt dieser Besuchertyp insgesamt weniger Gespräche mit Hochschul-Exponenten als Intensive Hochschul-Messestand-Nutzer. Wird die durchschnittliche Gesprächsanzahl zwischen Intensiven und Informationsorientierten Hochschul-Messestand-Nutzern verglichen, so sind - mit Ausnahme der Gespräche über mögliche Projektkooperationen - annähernd gleiche Werte festzustellen. Dies liegt darin begründet, daß Informationsorientierte Hochschul-Messestand-Nutzer zur Realisierung ihrer - wenngleich primär informationsorientierten - Zielsetzungen den Kontakt zu ausgewählten Exponenten auf dem Hochschul-Messestand forcieren. Informationsorientierte Hochschul-MessestandNutzer sind mit $62 \%$ auf der betrachteten Mehrbranchenmesse stärker vertreten als auf der untersuchten Fachmesse.

Die Hochschul-Messestand-Bummler messen den Zielsetzungen im Vergleich zu den vorangegangenen Besuchertypen insgesamt einen niedrigeren Stellenwert bei. Zwar priorisiert auch dieser Besuchertyp informationsbezogene Messebesucherziele mit Mittelwerten von 2,3 (Sammlung von Informationen) und 2,5 (Beobachtung von Entwicklungen der Wissenschaff), dennoch mißt er diesen Zielen im Vergleich zu den anderen zwei Besuchertypen insgesamt einen geringeren Stellenwert bei. Eine ähnliche Tendenz ist in bezug auf kontaktbezogene Messebesucherziele zu konstatieren. Dabei räumt der Hochschul-Messestand-Bummler diesen Zielen sogar einen relativ geringen Stellenwert ein. So stuft diese Besuchergruppe sowohl den Erfahrungs- und Informationsaustausch (Mittelwert $=3,8$ ) und die Beziehungspflege bzw. -aufbau mit Wissenschaftsvertretern (Mittelwert $=4,3$ ) als auch die Kontaktaufnahme zu Projektanbietern (Mittelwert $=4,1$ ) als eher unwichtig ein. Demzufolge führen Hochschul-Messestand-Bummler im Vergleich $\mathrm{zu}$ anderen Besuchertypen auf dem Hochschul-Messestand insgesamt die wenigsten Gespräche. Dieser Besuchertyp ist zu gleichen Anteilen auf den betrachteten Messetypen anzutreffen.

Die Analyse der drei ermittelten Hochschul-Messestand-Besuchertypen offenbart ausgeprägte Unterschiede im Hinblick auf die Zielsetzungen eines HochschulMessestand-Besuchs, die sich insbesondere auch in dem Gesprächsverhalten der Besuchertypen widerspiegelt ( $\mathrm{vgl}$. Tab. 5) und Ansatzpunkte zur Ableitung messebesuchertypspezifischer Messestrategien und -maßnahmen liefert. 


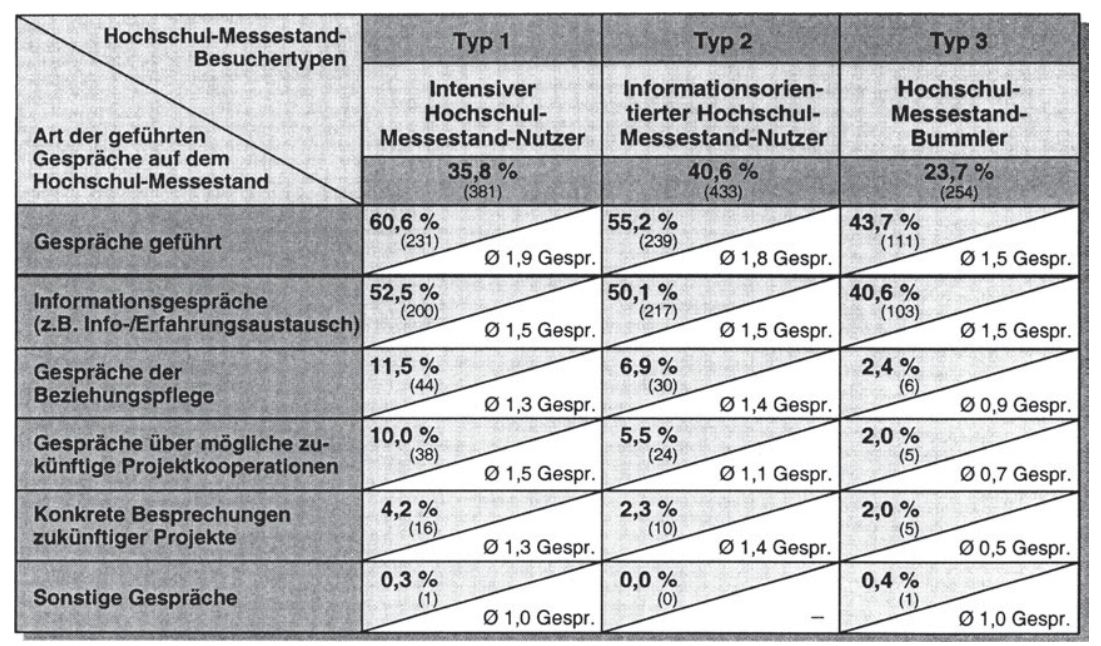

Tab. 5: Art und durchschnittliche Anzahl der geführten Gespräche von Hochschul-Messestand-Besuchertypen mit Hochschul-Exponenten ${ }^{77}$

Im Gegensatz zu kommerziellen Messebeteiligungen lassen sich somit nicht fünf Messebesuchertypen, sondern drei Besuchertypen auf Hochschul-Messeständen identifizieren. Zwischen kommerziellen und hochschulspezifischen Messestand-Besuchertypen sind jedoch z.T. Ähnlichkeiten feststellbar. So spiegeln sich in der Zielstruktur der Informationsorientierten Hochschul-MessestandNutzer die Zielstrukturen des Punktuellen Messe-Nutzers und des Praxisorientierten Messe-Nutzers wider, die beide die gezielte Informationssuche in den Vordergrund ihres Messebesuchs stellen. Die Intensiven Hochschul-MessestandNutzer sowie die Hochschul-Messestand-Bummler weisen im Hinblick auf ihre Zielstrukturen grundsätzlich Ähnlichkeiten zu den Intensiven Messe-Nutzern und den Messe-Bummlern auf.

Trotz der identifizierten, signifikanten Unterschiede zwischen den HochschulMessestand-Besuchertypen soll die Güte der Gruppenlösung einer statistischen Überprüfung unterzogen werden. Zu diesem Zweck wird - wie bei der Analyse der Exponententypen - auf das Verfahren der Diskriminanzanalyse zurückgegriffen. 
Die der eigentlichen Diskriminanzanalyse vorgeschaltete univariate Analyse zur Beurteilung der diskriminatorischen Bedeutung der Besucherziele führte zunächst zu durchgehend signifikanten Werten $(\alpha<0,01)$. Damit kann eine isolierte Trennfähigkeit aller Faktoren unterstellt werden. Im Rahmen der eigentlichen Diskriminanzanalyse konnten zwei Diskriminanzfunktionen ermittelt werden, die beide signifikant zur Trennung der Messestand-Besuchergruppen beitragen $(\alpha<0,01)$. Nach Einbeziehung der ersten Funktion wurden bei einem Wilks' Lambda von 0,158 bereits $97 \%$ der Varianz erklärt, während die Aufnahme der zweiten Funktion zu einem multivariaten Wilks' Lambda von 0,138 führte. ${ }^{78}$ Anhand dieser Diskriminanzfunktionen können die in der Stichprobe befindlichen Besucher von Hochschul-Messeständen klassifiziert, d.h. ihre Zugehörigkeit zu den drei Hochschul-Messestand-Besuchertypen geschätzt werden. Dabei signalisiert der Übereinstimmungsgrad von geschätzter und tatsächlicher Gruppenzugehörigkeit die Trennschärfe der Gruppenlösung (vgl. Tab. 6).

\begin{tabular}{|c|c|c|c|c|c|}
\hline \multirow{2}{*}{\multicolumn{3}{|c|}{$\begin{array}{r}\begin{array}{c}\text { Geschätzte Cluster- } \\
\text { zugehörigkeit durch } \\
\text { Diskriminanz- } \\
\text { analyse }\end{array} \\
\begin{array}{r}\text { analy } \\
\text { is }\end{array}\end{array}$}} & Cluster 1 & Cluster 2 & Cluster 3 \\
\hline & & & $\begin{array}{c}\text { Intensiver } \\
\text { Hochschul- } \\
\text { Messestand-Nutzer }\end{array}$ & $\begin{array}{l}\text { Informationsorien- } \\
\text { tierter Hochschul- } \\
\text { Messestand-Nutzer }\end{array}$ & $\begin{array}{l}\text { Hochschul- } \\
\text { Messestand- } \\
\text { Bummler }\end{array}$ \\
\hline \multicolumn{3}{|c|}{$\begin{array}{l}\text { Tatsächliche } \\
\text { Clusterzugehörigkeit } \\
\text { gemäß Clusteranalyse }\end{array}$} & $n=381$ & $n=433$ & $n=254$ \\
\hline$\frac{\frac{5}{d}}{\frac{5}{3}}$ & $\begin{array}{c}\text { Intensiver } \\
\text { Hochschul- } \\
\text { Messestand-Nutzer }\end{array}$ & $\begin{array}{l}\bar{\infty} \\
\text { II } \\
\text { C }\end{array}$ & & & \\
\hline$\frac{\sqrt{2}}{\frac{1}{3}}$ & $\begin{array}{l}\text { Informationsorien- } \\
\text { tierter Hochschul- } \\
\text { Messestand-Nutzer }\end{array}$ & 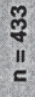 & & & \\
\hline$\frac{\frac{1}{5}}{\frac{5}{3}}$ & $\begin{array}{l}\text { Hochschul- } \\
\text { Messestand- } \\
\text { Bummler }\end{array}$ & $\begin{array}{l}\text { ปั่ } \\
\text { II } \\
\text { ᄃ }\end{array}$ & & & \\
\hline & & & & & \\
\hline
\end{tabular}

Tab. 6: Klassifizierungsmatrix der diskriminanzanalytischen Überprüfung der Besucher-Clusterlösung

Damit besitzt insbesondere die erste Diskriminanzfunktion eine extrem hohe Trennkraft. Während sich der kanonische Korrelationskoeffizient nach Einbeziehung der ersten Funktion auf einen Wert von 0,905 beläuft, weist dieser nach Berücksichtigung der zweiten Funktion einen Wert von 0,352 auf. 
Mit einem Anteil korrekter Klassifizierungen von 96,9\% wird die Clusterlösung bestätigt. Zur Überprüfung der diskriminatorischen Bedeutung der einzelnen Besucherzielsetzungen können die mittleren Diskriminanzkoeffizienten herangezogen werden. Diese ermöglichen eine Aussage darüber, auf welche Messebesucherziele die Gruppenunterschiede in erster Linie zurückzuführen sind (vgl. Tab. 7).

\begin{tabular}{|l|c|c|c|}
\hline \multirow{2}{*}{$\begin{array}{l}\text { Ziele eines Hochschul- } \\
\text { Messestand-Besuches }\end{array}$} & $\begin{array}{c}\text { Mittlerer } \\
\text { Diskriminanz- } \\
\text { koeffizient }\end{array}$ & \multicolumn{2}{|c|}{$\begin{array}{c}\text { Relative Bedeutung des mittleren } \\
\text { Diskriminanzkoeffizienten }\end{array}$} \\
\cline { 3 - 4 } $\begin{array}{l}\text { Sammlung von } \\
\text { Informationen }\end{array}$ & 0,192 & 8,9 & Rrozent \\
\hline $\begin{array}{l}\text { Beobachtung von Entwick- } \\
\text { lungen der Wissenschaft }\end{array}$ & 0,215 & 10,0 & $\mathbf{5}$ \\
\hline $\begin{array}{l}\text { Erfahrungs- u. Informationsaus- } \\
\text { tausch mit Wissenschaftsvertretern }\end{array}$ & 0,534 & 24,7 & $\mathbf{4}$ \\
\hline $\begin{array}{l}\text { Beziehungspflege od. -aufbau } \\
\text { mit Wissenschaftsvertretern }\end{array}$ & 0,608 & 28,1 & $\mathbf{3 .}$ \\
\hline $\begin{array}{l}\text { Kontaktaufnahme zu } \\
\text { Projektanbietern }\end{array}$ & 0,613 & 28,3 & $\mathbf{2 .}$ \\
\hline$\sum$ & 2,162 & 100,0 & $\mathbf{1 .}$ \\
\hline
\end{tabular}

Tab. 7: Diskriminanzanalytische Bedeutung der hochschulspezifischen Besucherzielsetzungen für Besuchertypen

Die relative Bedeutung der Diskriminanzkoeffizienten zeigt, daß mit der Kontaktaufnahme zu Projektanbietern (28,3\%), der Beziehungspflege oder dem Beziehungsaufbau mit Wissenschaftsvertretern $(28,1 \%)$ und dem Erfahrungs- und Informationsaustausch $(24,7 \%)$ insbesondere die kontaktbezogenen Besucherzielsetzungen zur Differenzierung der Besuchergruppen beitragen. Demgegenüber sind ausschließlich informationsbezogene Besucherzielsetzungen wie Beobachtung von Entwicklungen der Wissenschaft $(10,0 \%)$ und Sammlung von Informationen (8,9\%) von geringerer Bedeutung. Dieses Ergebnis erscheint plausibel, weil sich Messebesucher grundsätzlich aufgrund eines bestehenden Informationsbedürfnisses zu einem Messebesuch entscheiden. Liegt kein Informationsbedürfnis vor, so wird i.d.R. auch kein Messebesuch realisiert. Letztlich - so zeigen auch die vorangegangenen Ausführungen - unterscheiden sich die Messebesucher in ihrem, durch den Umfang und der Art des Informationsbedürfnisses bestimmten Verhalten, das sich insbesondere durch die Intensität ihres Hoch- 
schul-Messestand-Besuchs äußert und sich vor allem in ihren Kontaktquantitäten und -qualitäten mit Hochschul-Exponenten widerspiegelt.

\subsection{Integrierte Betrachtung von hochschulspezifischen Messeziel- gruppen und Hochschul-Messestand-Besuchertypen}

Die in die Messestand-Besuchertypenbildung einbezogenen, auf einem Hochschul-Messestand anwesenden Messebesucher sind - vor allem wegen der vielfältigen Möglichkeiten zur direkten Kontaktaufnahme von Hochschul-Exponenten mit Messebesuchern - für den Erfolg einer Hochschul-Messebeteiligung von besonderer Bedeutung. Dennoch dürfen Nicht-Messebesucher sowie auch Personen und Institutionen, die zwar auf Messen, aber nicht auf Hochschul-Messeständen vertreten sind, nicht unberücksichtigt bleiben, da sie ebenfalls hochschulspezifische Anspruchsgruppen bilden (vgl. Abb. 18). Wenngleich diese Gruppen nicht direkt auf Hochschul-Messeständen erreichbar sind, so können sie durch entsprechende Maßnahmen auf Messen bzw. mittels über den Messeveranstaltungsort hinausgehender Maßnahmen indirekt erreicht werden.

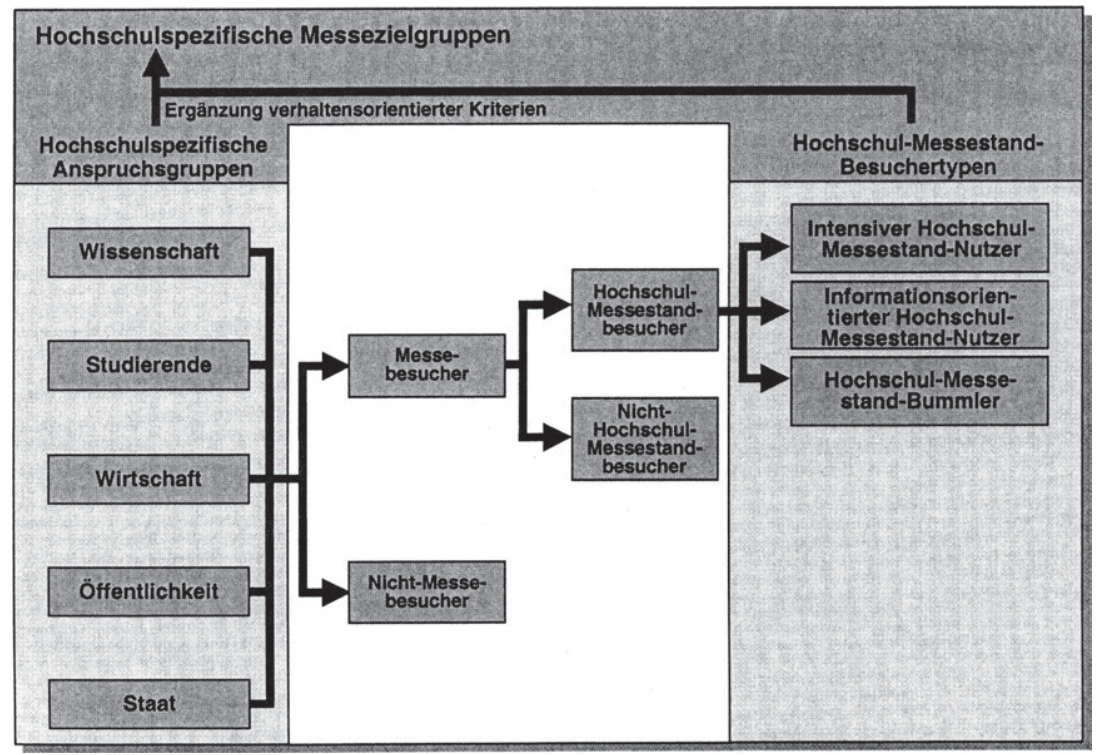

Abb. 18: Systematisierung hochschulspezifischer Messezielgruppen unter Berücksichtigung verhaltensorientierter Kriterien 
Im Rahmen der Betrachtung hochschulspezifischer Messezielgruppen ist - wie die vorangegangenen Ausführungen zeigen - die Bedeutung der Berücksichtigung verhaltensorientierter Kriterien hervorzuheben. Die auf Grundlage von Messebesucherzielsetzungen gebildeten Hochschul-Messestand-Besuchertypen zeichnen sich durch unterschiedliche Verhaltensweisen aus, die wiederum die Erreichung hochschulspezifischer Messebeteiligungszielsetzungen aus Exponentensicht beeinflussen. Angesichts der hohen Relevanz des Verhaltens von HochschulMessestand-Besuchern für Hochschul-Messebeteiligungen ist zu untersuchen, inwieweit sich die hochschulspezifischen Zielgruppen hinsichtlich ihres Verhaltens unterscheiden ( $v g l$. Tab. 8).

\begin{tabular}{|c|c|c|c|c|}
\hline \multirow{3}{*}{$\begin{array}{l}\text { Anspruchs- } \\
\text { gruppenbezo- } \\
\text { gene Hochschul- } \\
\text { Messezielguppen }\end{array}$} & Typ 1 & Typ 2 & Typ 3 & Signifik. \\
\hline & $\begin{array}{l}\text { Intensiver } \\
\text { Hochschul- } \\
\text { Messestand- } \\
\text { Nutzer }\end{array}$ & $\begin{array}{c}\text { Informationsorien- } \\
\text { tierter Hochschul- } \\
\text { Messestand- } \\
\text { Nutzer }\end{array}$ & $\begin{array}{l}\text { Hochschul- } \\
\text { Messestand- } \\
\text { Bummler }\end{array}$ & $\begin{array}{c}\chi^{2-}- \\
\text { Test }\end{array}$ \\
\hline & $\begin{array}{c}35,8 \% \\
(381)\end{array}$ & $\begin{array}{c}40,6 \% \\
(433)\end{array}$ & $\begin{array}{l}23,7 \% \\
(254)\end{array}$ & $\alpha$ \\
\hline Wissenschaft & $\begin{array}{r}40,7 \% \\
(50)\end{array}$ & $\begin{array}{r}49,6 \% \\
(61)\end{array}$ & $\begin{array}{r}9,8 \% \\
(12)\end{array}$ & $\begin{array}{r}0,000 \\
(<0,01)\end{array}$ \\
\hline Studierende & $\begin{array}{r}35,4 \% \\
(75)\end{array}$ & $\begin{array}{r}41,5 \% \\
(88)\end{array}$ & $\begin{array}{r}23,1 \% \\
(49)\end{array}$ & $\begin{array}{r}0,040 \\
(<0,05)\end{array}$ \\
\hline Wirtschaft & $\begin{array}{r}35,2 \% \\
(234)\end{array}$ & $\begin{array}{r}38,9 \% \\
(259)\end{array}$ & $\begin{array}{r}25,9 \% \\
(172)\end{array}$ & $\begin{array}{r}0,000 \\
(<0,01)\end{array}$ \\
\hline Öffentlichkeit & $\begin{array}{r}30,4 \% \\
(17)\end{array}$ & $\begin{array}{r}35,7 \% \\
(20)\end{array}$ & $\begin{array}{r}33,9 \% \\
(19)\end{array}$ & $\begin{array}{l}0,472 \\
\text { (n.s.) }\end{array}$ \\
\hline \multirow[t]{2}{*}{ Staat } & $\begin{array}{r}41,7 \% \\
(5)\end{array}$ & $\begin{array}{r}41,7 \% \\
(5)\end{array}$ & $\begin{array}{r}16,7 \% \\
\text { (2) }\end{array}$ & $\begin{array}{l}0,280 \\
\text { (n.s.) }\end{array}$ \\
\hline & $\begin{array}{l}\alpha<0,01 \quad(G r \\
\alpha<0,05 \quad(G \\
\text { n.s. } \quad(G\end{array}$ & $\begin{array}{l}\text { engrößen unterschei } \\
\text { engrößen unterschei } \\
\text { engrößen unterschei }\end{array}$ & $\begin{array}{l}\text { sich signifikant b } \\
\text { sich signifikant b } \\
\text { sich nicht signifik }\end{array}$ & $\begin{array}{l}\alpha<0,01) \\
\alpha<0,05) \\
\text { t) }\end{array}$ \\
\hline
\end{tabular}

Tab. 8: Zusammensetzung hochschulspezifischer Messezielgruppen nach Hochschul-Messestand-Besuchertypen ${ }^{79}$

79 Zur Prüfung der Signifikanz der Häufigkeitsverteilungen wurde auf den $\chi^{2}$-Test zurückgegriffen. Vgl. dazu Norius, M. J., SPSS für Windows: Anwenderhandbuch für das Base System 6.0, München 1994, S. $210 \mathrm{ff}$. 
Die Analyse der Zusammensetzung hochschulspezifischer Messezielgruppen nach Hochschul-Messestand-Besuchertypen zeigt in bezug auf die Häufigkeitsverteilungen z.T. signifikante Unterschiede auf. So sind hinsichtlich der Zielgruppen Wirtschaft, Wissenschaft und Studierende signifikante, dagegen bzgl. der Zielgruppen Öffentlichkeit und Staat keine signifikanten Unterschiede zu konstatieren. Werden in diese Betrachtung die Priorisierungen hochschulspezifischer Zielsetzungen aus Exponentensicht und deren Fokussierung auf die einzelnen Messezielgruppen einbezogen ( $\mathrm{vgl}$. Abb. 19), so lassen sich weitere Erkenntnisse gewinnen.

Die von Hochschul-Exponenten hoch priorisierte Zielgruppe Wirtschaft setzt sich fast zu gleichen Teilen aus Intensiven Hochschul-Messestand-Nutzern (35,2\%) und Informationsorientierten Hochschul-Messestand-Nutzern (38,9\%) zusammen, wohingegen Hochschul-Messestand-Bummler mit 25,9\% einen weitaus kleineren Anteil dieser Zielgruppe umfassen. Diese Aufteilung begünstigt die Erreichung der aus Sicht von Hochschul-Exponenten hoch priorisierten Messebeteiligungszielsetzungen. So ermöglicht der Besuch Intensiver Hochschul-Messestand-Nutzer den Hochschul-Exponenten die Kontaktaufnahme zu Projektinteressenten und die Erreichung von Projektzielen. Darüber hinaus bietet die Anwesenheit von Informationsorientierten Hochschul-Messestand-Nutzern die Möglichkeit zum Erfahrungsund Informationsaustausch mit Praxisvertretern, der wiederum zur Erreichung der verfolgten Informationsziele beitragen kann.

Im Hinblick auf die aus Exponentensicht zweitwichtigste Messezielgruppe Öffentlichkeit läßt sich gegenüber der Zielgruppe Wirtschaft eine gleichmäßigere besuchertypspezifische Häufigkeitsverteilung feststellen. Diese Zusammensetzung ist unter Berücksichtigung der Zielsetzungen, die u.a. durch die Ansprache dieser Zielgruppe erreicht werden können, als zielerreichungsfördernd einzustufen. So wird die Erreichung der von den Exponenten hoch priorisierten und auf die Öffentlichkeit gerichteten Instituts-/Lehrstuhl-/Fachbereichs- und Exponatbezogenen Beeinflussungsziele durch das entsprechende Verhalten der jeweiligen Hochschul-Messestand-Besuchertypen begünstigt. 


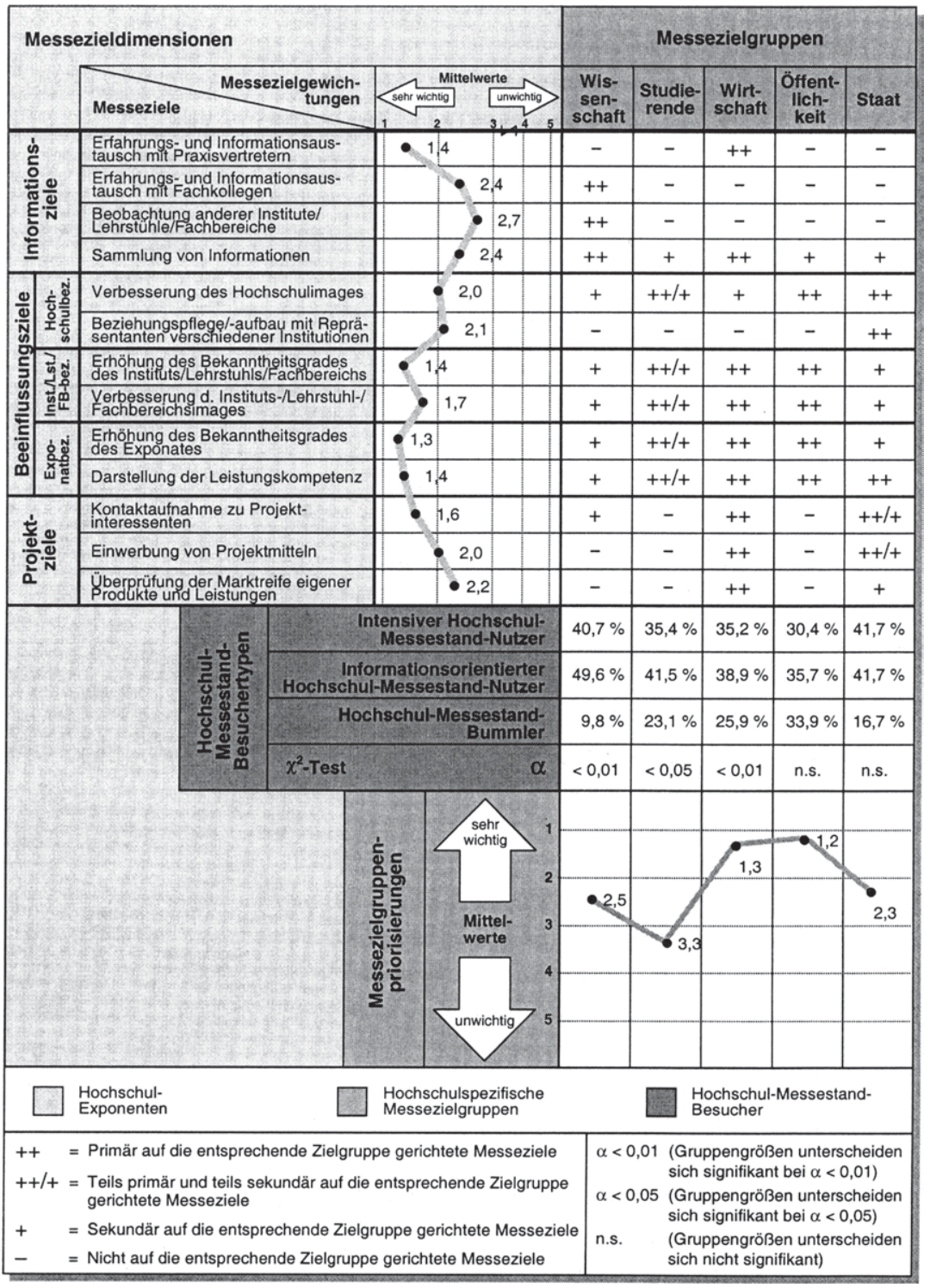

Abb. 19: Hochschulspezifische Messezielsetzungen und inr Fokus auf unterschiedliche Messezielgruppen unter Berücksichtigung verhaltensorientierter Kriterien 
Die im Vergleich zu den vorangegangenen Zielgruppen weniger hoch bewertete Messezielgruppe Staat zeigt trotz der differierenden Anteile der Messebesuchertypen (Intensiver und Informationsorientierter Hochschul-Messestand-Besucher jeweils 41,7\%; Hochschul-Messestand-Bummler 16,7\%) keine signifikanten Unterschiede bzgl. der Häufigkeitsverteilungen hochschulspezifischer Messestand-Besuchertypen auf. Dieses Ergebnis kann im geringen Stichprobenumfang der Zielgruppe begründet liegen. Die messebesuchertypspezifische Zusammensetzung der Zielgruppe Staat steht der Erreichung der von Hochschul-Exponenten verfolgten, u.a. durch die Ansprache dieser Zielgruppe zu realisierenden Zielsetzungen nicht entgegen, da letztlich die Erreichung von Zielsetzungen, wie Verbesserung des Hochschulimages, grundsätzlich nicht vom Verhalten der HochschulMessestand-Besucher abhängig ist.

Die aus Exponentensicht als viertwichtigste Messezielgruppe eingestuften Wissenschaftsvertreter, die hinsichtlich ihrer Verteilung auf Hochschul-MessestandBesucher signifikante Unterschiede aufweist, setzen sich zur Hälfte aus Informationsorientienten Hochschul-Messestand-Nutzern (49,6\%) zusammen. Die Intensiven Hochschul-Messestand-Nutzer nehmen mit 40,7 \% ebenfalls einen hohen, Hochschul-Messestand-Bummler dagegen mit 9,8\% einen relativ geringen Anteil ein. Die Erreichung der von Hochschul-Exponenten angestrebten, u.a. durch die Ansprache dieser Zielgruppe erreichbaren Informationsziele (z.B. Erfahrungs- und Informationsaustausch mit Fachkollegen), wird generell durch die Verhaltensweisen der den Messestand besuchenden Wissenschaftsvertreter begünstigt.

Die insgesamt für eine Messebeteiligung als weniger wichtig eingestufte Messezielgruppe Studierende weist - wie die Zielgruppen Wirtschaft und Wissenschaft ebenfalls signifikante Unterschiede in bezug auf die Häufigkeitsverteilung an Hochschul-Messestand-Besuchertypen auf, wobei in dieser Zielgruppe vermehrt Informationsorientierte Hochschul-Messestand-Nutzer (41,5\%) und Intensive Hochschul-Messestand-Nutzer $(35,4 \%)$ vertreten sind. Die Verhaltensweisen dieser Messebesuchertypen kommen den generellen Zielsetzungen der HochschulExponenten nicht entgegen, da letztlich zur Erreichung der primär verfolgten hochschulspezifischen Messezielsetzungen der knappe Faktor Zeit einer Messebeteiligung weniger für Studierende als vielmehr für hoch priorisierte Zielgruppen Wirtschaft und Öffentlichkeit zu verwenden ist.

Die Erreichung der von den Hochschul-Exponenten verfolgten Messebeteiligungszielsetzungen wird somit grundsätzlich durch das von den Besucherzielsetzungen determinierte Verhalten der Hochschul-Messestand-Besucher begünstigt (vgl. 
Abb. 20). Vor dem Hintergrund, daß der Erfolg einer Hochschul-Messebeteiligung insbesondere vom Messebeteiligungsnutzen i.S.d. Erreichung verfolgter Messebeteiligungsziele aus Exponentenperspektive abhängt, ist zu konstatieren, daß die Voraussetzungen zur Realisierung erfolgreicher Hochschul-Messebeteiligungen existieren. Inwieweit Hochschul-Messebeteiligungen letztlich zum Erfolg führen, wird im folgenden analysiert.

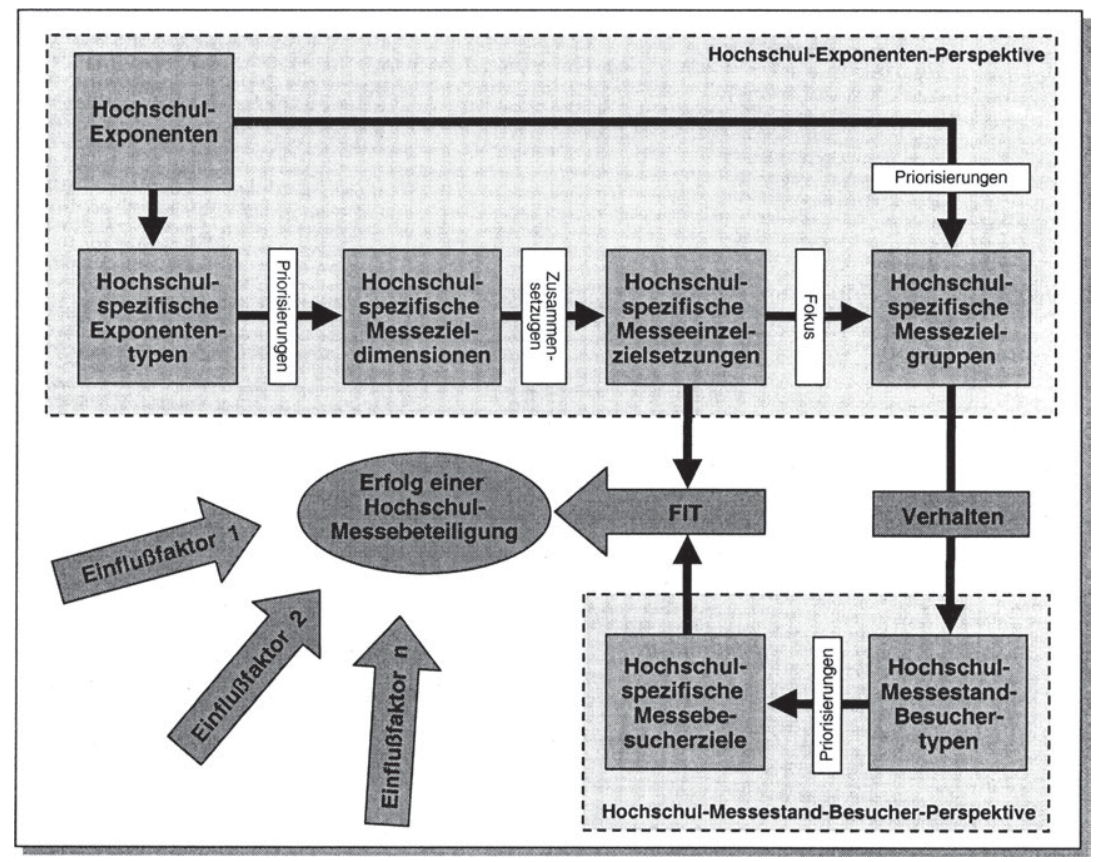

Abb. 20: Integration von Hochschul-Exponenten- und Hochschul-MessestandBesucher-Perspektive 


\section{Erfolgskontrolle der Messebeteiligung von Hochschulen}

Vor dem Hintergrund einer Verknappung öffentlicher Mittel für Hochschulen und der damit notwendig werdenden stärker ökonomischen Planungsorientierung innerhalb der Hochschulen nimmt die Betrachtung von Kosten-Nutzen-Relationen der eingesetzten Kommunikationsinstrumente des Hochschulmarketing und damit auch der kostenintensiven Messebeteiligungen ${ }^{1}$ einen zunehmenden Stellenwert ein. $^{2}$

\section{Unsicherheit über die Effizienz von Hochschul-Messebeteiligungen}

Der Messebeteiligungserfolg wird sowohl bei Hochschul-Messebeteiligungen als auch bei Messebeteiligungen im kommerziellen Bereich - wenn überhaupt - nur ansatzweise analysiert. Die Gründe hierfür liegen nach MORTSIEFER in der Vielzahl von Erfolgsgrößen, die mit einer Messebeteiligung verbunden sein können (z.B. ökonomischer vs. psychographischer Erfolg), in dem Wandel der meisten Messen von Order- zu Informationsmessen, der eine Erfolgsermittlung auf Basis ökonomischer Größen erschwert, Probleme der Kausalität und Zurechenbarkeit von Erfolgswirkungen bei Messebeteiligungen (da meist mehrere Messen beschickt und/oder auch andere Kommunikationsinstrumente vor und nach einer Messebeteiligung eingesetzt werden) sowie in der Wahl des Kontrollzeitpunkts bzw. -zeitraums (Problem der Bestimmung der über eine Messebeteiligung hinausgehenden Erfolgswirkungen). ${ }^{3}$ Damit wird die Messeerfolgskontrolle sowohl in der Praxis als auch in der Wissenschaft als ein wesentlicher Defizitbereich angesehen, ${ }^{4}$ was

Die Höhe der für die Messebeteiligung von den jeweiligen Hochschul-Exponenten aufzubringenden finanziellen Mittel ist im wesentlichen abhängig von der Auswahl des Messetyps, von der gewählten Messebeteiligungsform sowie von den eingesetzten phasenspezifischen Maßnahmen.

Vgl. Schoop, K., Sandt, B., Die Messeerfolgskontrolle, in: Tietz, B., Die Werbung - Handbuch der Kommunikations- und Werbewirtschaft, Bd. 2, Landsberg am Lech 1982, S. 1666.

Vgl. Stiege, J., Messebeteiligungen sollen gründlich bewertet werden, in: BddW, 24.04.1995, S. 1; Mortsiefer, J., Messen und Ausstellungen als Mittel der Absatzpolitik mittelständischer Herstellerbetriebe, a.a.O., S. 439 ff. Empirische Befunde finden sich zudem bei Täger, U. C., Ziegler, R., Die Bedeutung von Messen und Ausstellungen in der Bundesrepublik Deutschland für den Inlands- und Auslandsabsatz in ausgewählten Branchen, a.a.O., S. $63 \mathrm{ff}$.

4 Vgl. stellvertr. Bonoma, T. V., Get more out of your trade shows, a.a.O., S. 76; Gopalakrishna, S., Lilien, G. L., Williams, J. D. et al., Do Trade Shows Pay off?, a.a.O., S. 75 ff.; Raffée, H., 
letztlich dazu führt, daß die Effizienz von Messebeteiligungen bisher nur ansatzweise erfaßt wurde. ${ }^{5}$

Grundvoraussetzung einer erfolgreichen Hochschul-Messebeteiligung ist die Erzielung eines Nutzens i.S.d. Erreichung verfolgter Messebeteiligungsziele (erste notwendige Bedingung). Kann ein Messebeteiligungsnutzen erreicht und damit die Effektivität einer Messebeteiligung festgestellt werden, ist die Effizienz einer Messebeschickung zu überprüfen. Eine Hochschul-Messebeteiligung kann dann als effizient bezeichnet werden, wenn der erzielte Nutzen die entstandenen Messebeteiligungskosten übersteigt (zweite notwendige Bedingung) und aufgrund einer alternativen Mittelverwendung kein höherer Nutzen hätte erzielt werden können (hinreichende Bedingung). ${ }^{6}$ Im Rahmen der Effizienzbewertung von HochschulMessebeteiligungen wird damit nicht nur eine Beurteilung der Wirtschaftlichkeit des Messeengagements von Hochschul-Exponenten vorgenommen, sondern zudem eine optimale Budgetallokation innerhalb des Kommunikations-Mix der Hochschul-Exponenten angestrebt. ${ }^{7}$

Für die Effizienzbewertung von Hochschul-Messebeteiligungen bietet sich damit ein dreistufiges Vorgehen an:

- Ermittlung des Messebeteiligungsnutzens

(erste notwendige Bedingung)

- Analyse von Kosten-Nutzen-Relationen

(zweite notwendige Bedingung)

- Effizienzvergleiche mit geeigneten Vergleichsobjekten

(hinreichende Bedingung).

Die Ermittlung des Messebeteiligungsnutzens kann über den Erreichungsgrad der mit einer Messebeteiligung verfolgten Zielsetzungen erfolgen. Ob die ange-

Messen als Herausforderung für die Marketing-Theorie, a.a.O., S. 73 ff.; Sashi, C. M., Perretty, J., Do Trade Shows Provide Value?, in: IMM, Vol. 21, No. 3, 1993, S. $249 \mathrm{ff}$.

5

6

7
Vgl. Prüser, S., Messemarketing: Ein netzwerkorientierter Ansatz, Wiesbaden 1997, S. 86.

Vgl. hierzu sowie im folgenden Ueding, R., Management von Messebeteiligungen: Identifikation und Erklärung messespezifischer Grundhaltungen auf der Basis einer empirischen Untersuchung, a.a.O., S. $259 \mathrm{ff}$.

Die Einbeziehung des Opportunitätsansatzes in die Effizienzbewertung verdeutlicht, daß die Suche nach geeigneten Vergleichsobjekten eine wesentliche Aufgabe im Rahmen der Effizienzbewertung darstellt. 
strebten Zielsetzungen in ausreichendem Maße erfüllt werden, ist jedoch nur dann bestimmbar, wenn der Grad der angestrebten Zielerreichung von den HochschulExponenten zuvor festgelegt wurde. Unterbleibt eine Festlegung des ZielausmaBes, wird mit einer Messebeteiligung dann ein Nutzen erzielt, wenn durch die Messebeteiligung das angestrebte Ziel - unabhängig vom Erreichungsgrad - zumindest ansatzweise realisiert wird und ohne Messebeteiligung dieses Resultat nicht erzielt worden wäre. Der Messebeteiligungsnutzen steht oftmals bei der Erfolgsbetrachtung von Hochschul-Exponenten im Vordergrund, insbesondere dann, wenn erhebliche Teile der Messebeteiligungskosten von anderen Institutionen getragen werden. Damit kommt aus Sicht der Hochschul-Exponenten vor allem der Analyse des Nutzens im Rahmen der Messeerfolgskontrolle eine besondere Bedeutung zu. Dennoch dürfen die Messebeteiligungskosten bei der Ermittlung des Erfolgs von Hochschul-Messebeteiligungen nicht unberücksichtigt bleiben. Sowohl aus Perspektive der Finanzmittelgeber als auch aus Perspektive der Hochschul-Exponenten, die in der vorliegenden Arbeit im Vordergrund steht, ist der ermittelte Nutzen den jeweiligen Messekosten gegenüberzustellen.

Die Analyse von Kosten-Nutzen-Relationen zur Überprüfung der zweiten notwendigen Bedingung ist nur möglich, wenn Kosten und Nutzen einer HochschulMessebeteiligung die gleiche Maßeinheit aufweisen. Kosten unterliegen stets einer monetären Bewertung. Folglich können isolierte Aussagen über die Effizienz einer zu bewertenden Messebeteiligung nur dann getroffen werden, wenn die Nutzendimensionen sowohl quantifizierbar als auch unmittelbar monetär bewertbar sind. Diese Voraussetzungen erfüllen allerdings nur ökonomische Erfolgsdimensionen, wie bspw. Drittmittelvolumina von Projektaufträgen. Dabei sind jedoch i.d.R. die ökonomischen Wirkungen, die allein auf die Messebeteiligung zurückzuführen sind, nicht eindeutig quantifizierbar, da diese Wirkungen oftmals das Resultat vielfältiger Hochschulaktivitäten darstellen. Darüber hinaus ist eine isolierte monetäre Bewertung psychographischer Nutzendimensionen, wie Erhöhung des Bekanntheitsgrades oder Imageverbesserung des Instituts, des Lehrstuhls oder des Fachbereichs, nicht möglich.

Im Rahmen einer Effizienzbewertung von Hochschul-Messebeteiligungen auf Basis von Nutzendimensionen muß strenggenommen dann auf eine Überprüfung die notwendige Bedingung für die Effizienz verzichtet werden, wenn keine eindeutig zurechenbaren, ökonomischen Nutzendimensionen existieren. Folglich wird die Bildung von Vergleichskriterien notwendig, und der Suche nach geeigneten Vergleichsobjekten (hinreichende Bedingung) kommt für die Effizienzbewertung von 
Hochschul-Messebeteiligungen eine zentrale Bedeutung zu. In diesem Zusammenhang bieten sich sowohl intra-instrumentelle als auch inter-instrumentelle Vergleiche an.

Letztlich kann erst nach Prüfung der hinreichenden Bedingung die Effizienz von Hochschul-Messebeteiligungen beurteilt werden. Aussagen über ihren Erfolg hingegen sind bereits nach Abschluß der Überprüfung der beiden notwendigen Bedingungen möglich. Demnach erscheint es sinnvoll, zur Erfassung des Erfolgs von Hochschul-Messebeteiligungen eine zweistufige Erfolgskontrolle vorzunehmen. In diesem Zusammenhang bietet sich die von SCHOOP und SANDT vorgenommene Unterteilung der Messeerfolgskontrolle in die indirekte und direkte Erfolgskontrolle an. ${ }^{8}$ Während die indirekte Erfolgskontrolle den Erfolg als Zielerreichungsgrad der verfolgten Messebeteiligungsziele auffaßt und damit ausschließlich eine Nutzenbetrachtung vornimmt, wird im Rahmen der direkten Erfolgskontrolle der Erfolg i.S.v. Kosten-Nutzen-Relationen definiert.

\section{Indirekte Erfolgskontrolle von Hochschul-Messebeteiligungen}

Im Rahmen der indirekten Erfolgskontrolle von Hochschul-Messebeteiligungen gilt es, den Zielerreichungsgrad der verfolgten hochschulspezifischen Messebeteiligungsziele zu ermitteln. Folglich wird die indirekte Erfolgskontrolle auf Grundlage der Zielsetzungen durchgeführt, die Hochschul-Exponenten mit ihrer Messebeteiligung verfolgen.

\subsection{Messezielsetzungen als Grundlage zur indirekten Erfolgskontrolle von Hochschul-Messebeteiligungen}

Die Hochschul-Exponenten haben zur Ermittlung des indirekten Erfolgs bei der Festlegung von Messezielen Erfolgskriterien zu bestimmen, die das Ausmaß der Zielerreichung ihrer Messebeteiligung erfassen können. ${ }^{9}$ Zur Systematisierung bieten sich in diesem Zusammenhang die hochschulspezifischen Zieldimensionen

Vgl. Schoop, K., Sandt, B., Die Messeerfolgskontrolle, a.a.O., S. 1668.

9 Vgl. Berghäuser, B., So lassen sich Messen messen, in: Marketing Journal, 10. Jg., Heft 9, 1976, S. $61 \mathrm{ff}$.; Raffée, H., Messen als Herausforderung für die Marketing-Theorie, a.a.O., S. $79 \mathrm{f}$. Vgl. allgemein dazu Heinen, E., Grundlagen betriebswirtschaftlicher Entscheidungen Das Zielsystem der Unternehmung, a.a.O., S. 86 f. 
an, wobei sich den entsprechenden Einzelzielsetzungen ausgewählte Erfolgskriterien zuordnen lassen. ${ }^{10}$

\subsection{Erfolgskriterien zur Kontrolle von Informationszielen}

Zur Feststellung des Erreichungsgrads der von den Hochschul-Exponenten verfolgten Informationsziele sind verschiedenartige Erfolgskriterien heranzuziehen (vgl. Abb. 21). Aufschluß über die Erreichung des Ziels Erfahrungs- und Informationsaustausch mit Praxisvertretern können bspw. die Anzahl der Praxiskontakte und die mit dieser Zielgruppe geführten Gespräche geben. Ebenso kann die Erreichung des Ziels Erfahrungs- und Informationsaustausch mit Fachkollegen mittels der Anzahl der Kontakte zu Wissenschaftlern und der mit dieser Zielgruppe geführten Gespräche erfaßt werden. Darüber hinaus geben die Inhalte der mit den Praxisvertretern bzw. den Fachkollegen geführten Gespräche Anhaltspunkte über den jeweiligen Zielerreichungsgrad.

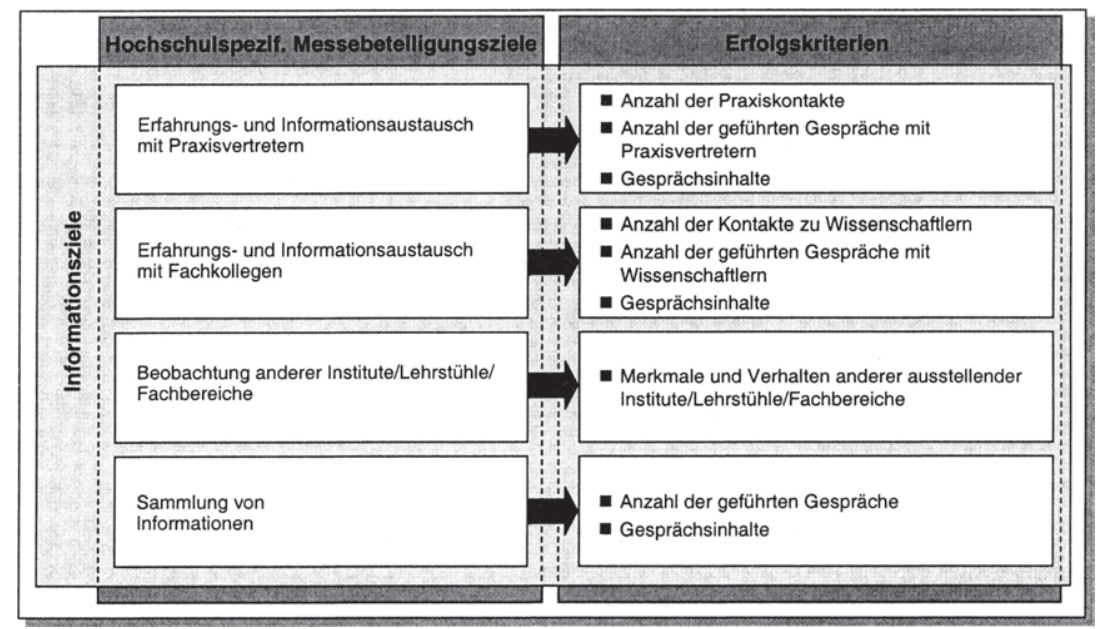

Abb. 21: Erfolgskriterien zur Kontrolle von Informationszielen

Zur Entwicklung von Erfolgskriterien vgl. Meffert, $H$., Messen und Ausstellungen als Marketinginstrument, a.a.O., S. 25; Amon, P., Messen + Ausstellungen: Messe-Ziele exakt formulieren und kontrollieren, a.a.O., S. $56 \mathrm{f}$. Die folgenden Ausführungen konzentrieren sich auf die primär relevanten Erfolgskriterien einer Hochschul-Messebeteiligung. 
Die Realisierung des Ziels Beobachtung anderer Institute/Lehrstühle/Fachbereiche ist daran zu messen, inwieweit es den Hochschul-Exponenten gelungen ist, die Merkmale und das Verhalten der ausstellenden Institute/Lehrstühle/Fachbereiche auf der Messe festzuhalten. Die Sammlung von Informationen hingegen ist über die Quantität (Gesprächsanzahl) und die Qualität (Gesprächsinhalte) der auf der Messe geführten Gespräche ermittelbar.

\subsection{Erfolgskriterien zur Kontrolle von Beeinflussungszielen}

Zur Kontrolle der mit einer Hochschul-Messebeteiligung verfolgten Beeinflussungsziele sind - wie bei der Erfolgskontrolle von Informationszielen - quantitative und qualitative Erfolgskriterien heranzuziehen. Gemäß der hochschulspezifischen Differenzierung zwischen Hochschulbezogenen, Instituts-/Lehrstuhl-/Fachbereichsbezogenen und Exponatbezogenen Beeinflussungszielen bietet sich eine entsprechende Systematisierung der Erfolgskriterien an (vgl. Abb. 22).

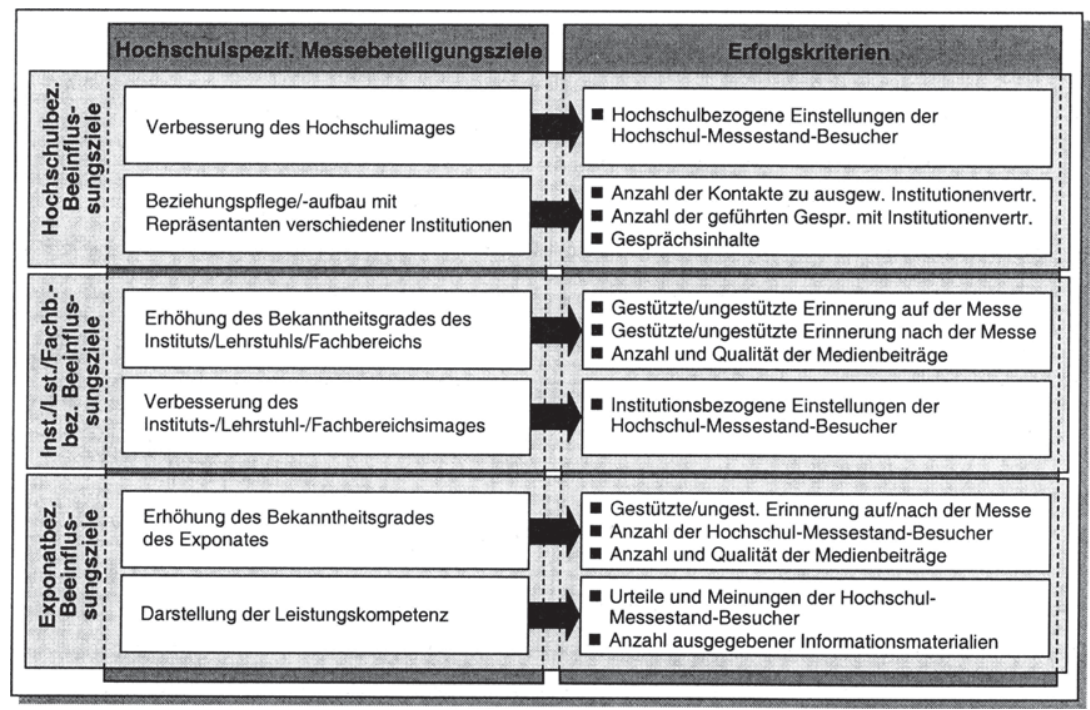

Abb. 22: Erfolgskriterien zur Kontrolle von Beeinflussungszielen

Die Erreichung Hochschulbezogener Beeinflussungsziele wie Verbesserung des Hochschulimages lassen sich über Einstellungen der Hochschul-MessestandBesucher zu der ausstellenden Hochschule erfassen. Demgegenüber ist die Realisierung des Ziels Beziehungspflege bzw. -aufbau mit Repräsentanten verschie- 
dener Institutionen weniger über Einstellungen als vielmehr über die Anzahl der Kontakte sowie die Quantität und Qualität der geführten Gespräche mit Vertretern dieser Institutionen kontrollierbar.

In bezug auf Instituts-/Lehrstuhl-/Fachbereichsbezogene Beeinflussungsziele kann die Erreichung der Zielsetzung Erhöhung des Bekanntheitsgrades des Instituts-/Lehrstuhl-/Fachbereichsimages über die Erinnerungen der Messebesucher, die sowohl gestützt als auch ungestützt in der Messe-Phase sowie in der NachMesse-Phase ermittelbar sind, erfaßt werden. Weiterhin geben Anzahl und Qualität der zur Messebeteiligung veröffentlichten Medienbeiträge Anhaltspunkte über die Bekanntheitsgraderhöhung der ausstellenden Institution. Die Realisierung des angestrebten Ziels Verbesserung des Instituts-/Lehrstuhl-/Fachbereichsimages ist wiederum über Einstellungen der Messestand-Besucher zur jeweils ausstellenden Institution überprüfbar.

Im Hinblick auf Exponatbezogene Beeinflussungsziele kann die Erreichung der Zielsetzung Erhöhung des Bekanntheitsgrades des Exponates ebenfalls mittels Erinnerungen der Messebesucher, die wiederum gestützt als auch ungestützt während und nach der Messe erhoben werden können, ermittelt werden. Darüber hinaus bieten sich als Anhaltspunkte für den Grad der Zielerreichung die Erhebung der Anzahl von Messebesuchern auf dem Messestand sowie der Quantität und Qualität der im Zusammenhang mit der Messebeteiligung zu dem Exponat veröffentlichten Medienbeiträge an. Die Erreichung der angestrebten Darstellung der Leistungskompetenz kann einerseits qualitativ über die Urteile und Meinungen der Hochschul-Messestand-Besucher erhoben, andererseits quantitativ über die Anzahl der ausgegebenen, exponatbezogenen Informationsmaterialien kontrolliert werden.

\subsection{Erfolgskriterien zur Kontrolle von Projektzielen}

Im Rahmen der Kontrolle von Projektzielen sind - wie bei der Kontrolle der vorangegangenen hochschulspezifischen Messebeteiligungsziele - ebenfalls sowohl quantitative als auch qualitative Erfolgskriterien von besonderer Bedeutung (vgl. Abb. 23). 


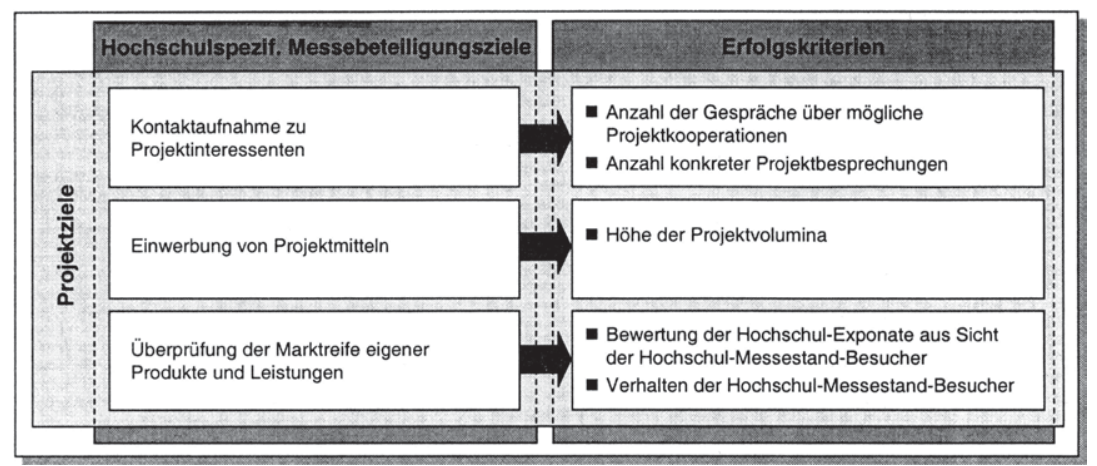

\section{Abb. 23: Erfolgskriterien zur Kontrolle von Projektzielen}

Die Kontaktaufnahme zu Projektinteressenten ist mittels quantitativer Kriterien erfaßbar. So geben sowohl die Anzahl der Gespräche über mögliche Projektkooperationen als auch die Anzahl der konkreten Projektbesprechungen Anhaltspunkte über die Erreichung des angestrebten Messebeteiligungsziels. Auch ist die Einwerbung von Projektmitteln durch quantitative Erfolgskriterien, wie Höhe der Projektvolumina, ermittelbar. Zur Kontrolle der Realisierung des Ziels Überprüfung der Marktreife eigener Produkte und Leistungen bedarf es hingegen qualitativer Erfolgskriterien. So kann die Zielerreichung einerseits über die Exponatbewertung der Hochschul-Messestand-Besuchern und andererseits über das Verhalten der Besucher auf dem Hochschul-Messestand, welches sich bspw. in der Anzahl und Qualität der geführten Gespräche widerspiegelt, erfaßt werden.

Eng mit der Auswahl der Erfolgskriterien verbunden ist die Wahl der Methoden zur indirekten Erfolgskontrolle. ${ }^{11}$ Dabei lassen sich einige Methoden zur Messung mehrerer Erfolgskriterien heranziehen, während andere Verfahren lediglich für bestimmte Fragestellungen eine besondere Eignung aufweisen.

11 Zum ersten Ansatz, der eine Verbindung zwischen Erfolgskriterien und Methoden der Erfolgskontrolle herstellt, vgl. Winnen, R., Beuster, A., Kontrolle des Messeerfolgs, in: Strothmann, K.-H., Busche, M. (Hrsg.), Handbuch Messemarketing, a.a.O., S. 375 f. 


\subsection{Analyse des indirekten Erfolgs von Hochschul-Messebeteiligungen durch Einsatz von Methoden zur Messeerfolgskontrolle}

Die indirekte Messeerfolgskontrolle umfaßt die systematische Gewinnung und Auswertung von spezifischen Daten zur Kontrolle des indirekten Erfolgs einer Hochschul-Messebeteiligung. ${ }^{12}$ Dabei ist die Messeerfolgskontrolle nicht nur in bezug auf ökonomische Kenngrößen (z.B. durch die Messebeteiligung realisierte Projektvolumina) zu interpretieren. Die Erreichung psychographischer Zielsetzungen kann mit Kontrollmethoden, die ausschließlich quantifizierbare Wirkungen von Hochschul-Messebeteiligungen überprüfen, nicht unmittelbar wiedergegeben werden. Vielmehr ist für die Überprüfung der Erreichung dieser Ziele der Einsatz qualitativer Verfahren der Erfolgskontrolle notwendig. Eine Übersicht über ausgewählte Methoden ${ }^{13}$ zur Messung der zuvor aus den hochschulspezifischen Messebeteiligungszielen abgeleiteten Erfolgskriterien vermittelt die folgende Abbildung. Aufgrund der ausgeprägten Komplexität von Messeerfolgskontrollen werden hier lediglich solche Methoden berücksichtigt, die in der Messepraxis die häufigste Verbreitung gefunden haben. ${ }^{14}$

Vgl. auch Winnen, R., Beuster, A., Kontrolle des Messeerfolgs, a.a.O., S. $368 \mathrm{ff}$.

Die Begriffe "Methoden“ und "Verfahren“ der Erfolgskontrolle werden im folgenden synonym verwendet.

Vgl. hierzu sowie im folgenden Ueding, R., Management von Messebeteiligungen: Identifikation und Erklärung messespezifischer Grundhaltungen auf der Basis einer empirischen Untersuchung, a.a.O., S. $116 \mathrm{ff}$. Die aufgeführten Methoden sind in der überwiegenden Mehrzahl den primärstatistischen Erhebungen zuzuordnen. Die messerelevanten Sekundärdaten umfassen meist allgemeine Rahmendaten, die wenig Aussagekraft zur Beurteilung des Messebeteiligungserfolgs eines einzelnen Exponenten besitzen. Vgl. hierzu auch Funke, K., Messeentscheidungen: Handlungsalternativen und Informationsbedarf, a.a.O., S. $290 \mathrm{ff}$. 


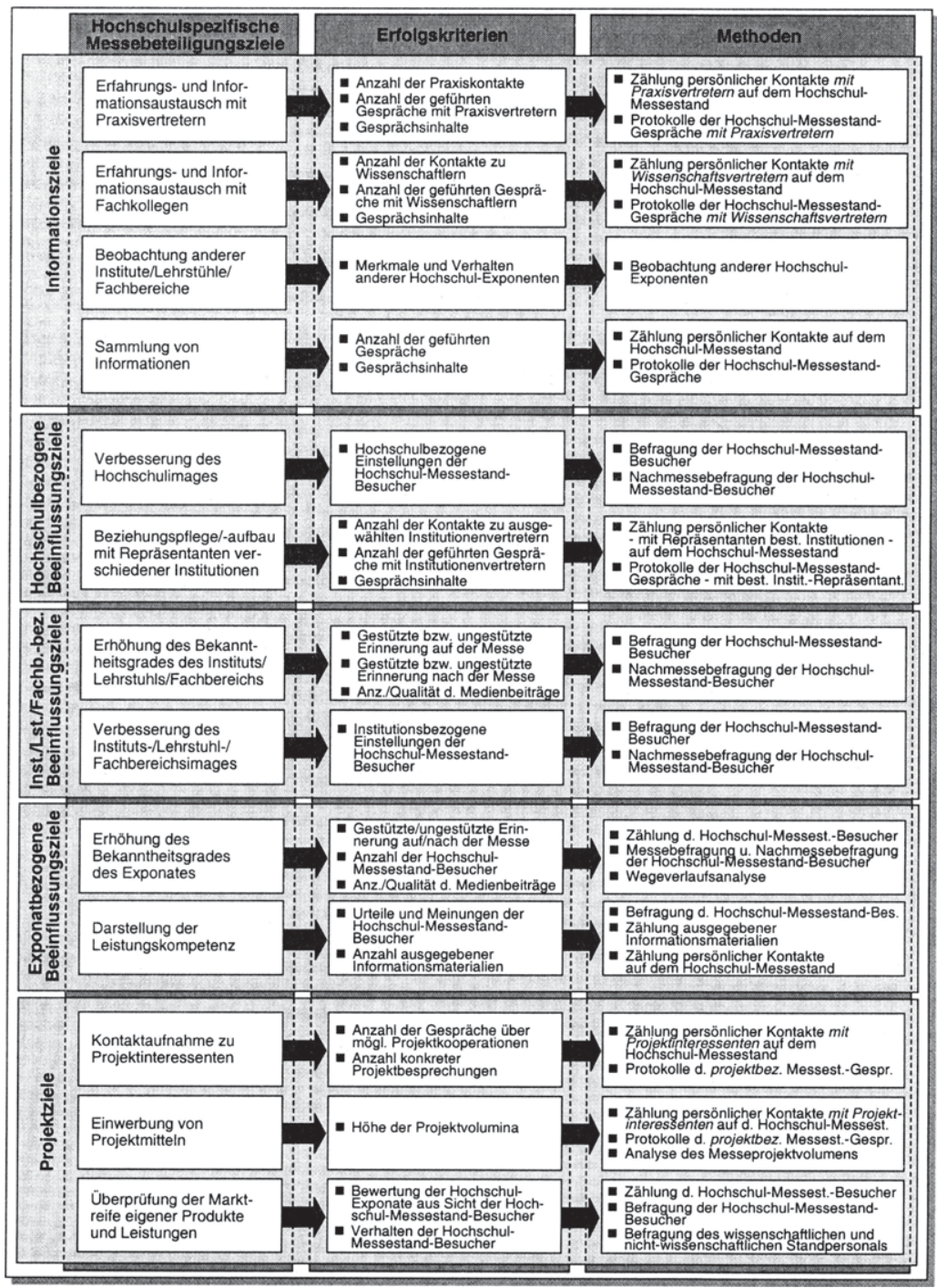

Abb. 24: Erfolgskriterien und Methoden zur Erfolgskontrolle von HochschulMessebeteiligungen 
Diese Methoden können den grundsätzlichen Erhebungsformen "Befragung", "Beobachtung" und „Experiment" zugeordnet werden, ${ }^{15}$ wobei die Wahl der Erhebungsform mit der Entscheidung über den Einsatz einer Methode zusammenfällt. Daraus folgen weitreichende Implikationen für den kontrollierenden HochschulExponenten, da die Erhebungsformen unterschiedliche Ansprüche an die Vorbereitung und Durchführung der Erfolgskontrolle stellen. Beobachtungen, wie einfache Zählungen, erfordern nur wenig marktforscherisches Know-how sowie nur relativ geringe finanzielle und personelle Ressourcen. Diese Methoden können demzufolge grundsätzlich von allen Hochschul-Exponenten problemlos angewendet werden. Dagegen bedarf eine Befragung eines entsprechenden fachlichen Know-hows sowie intensiver inhaltlicher Vorbereitungen und personeller Betreuung durch den kontrollierenden Hochschul-Exponenten. Folglich ist der Einsatz dieser Verfahren nur für solche Hochschul-Exponenten zu empfehlen, die einerseits entsprechende Marktforschungskenntnisse besitzen und andererseits über die notwendigen finanziellen sowie personellen Ressourcen verfügen.

Die Methoden zur indirekten Erfolgskontrolle können in bezug auf ihren zeitlichen Einsatz systematisiert und entsprechend dem „Phasenkonzept“ der Vor-Messe-, der Messe- oder der Nach-Messe-Phase zugeordnet werden. ${ }^{16}$ In der Vor-MessePhase können die Hochschul-Exponenten die indirekte Erfolgskontrolle zur Überprüfung ihres hochschulspezifischen Messebeteiligungskonzepts sowie zur Erfassung der Bedürfnisse hochschulspezifischer Messezielgruppen nutzen. Die indirekte Erfolgskontrolle in der Messe-Phase kann zeitnahe Informationen über die Notwendigkeit kurzfristiger Modifikationen des Messeengagements liefern. In der Nach-Messe-Phase finden schwerpunktmäßig Evaluierungen des gesamten Messebeteiligungserfolgs und damit Überprüfungen der Zielerreichungsgrade statt, die gesamthaft erst nach Beendigung der Messeveranstaltung beurteilt werden können.

Vgl. Berekoven, L., Eckert, W., Ellenrieder, P., Marktforschung - Methodische Grundlagen und praktische Anwendung, 6. Aufl., Wiesbaden 1993, S. 88 ff.; Meffert, H., Marketingforschung und Käuferverhalten, a.a.O., S. $182 \mathrm{ff}$.

16 Zur Verwendung eines Drei-Phasen-Modells vgl. Roloff, E., Messen und Medien: Ein sozialpsychologischer Ansatz zur Öffentlichkeitsarbeit, a.a.O., S. 215 ff.; Müller, K., Die Messepolitik im Marketing der handwerklichen Zulieferer, Göttingen 1985, S. 213 ff.; Roth, G. D., Messen und Ausstellungen verkaufswirksam planen und durchführen, a.a.O., S. $123 \mathrm{ff}$. 
In der nachfolgenden Tabelle sind ausgewählte Methoden zur indirekten Messeerfolgskontrolle einerseits nach ihrem methodischen Vorgehen und andererseits nach ihrem zeitlichen Einsatz systematisiert dargestellt.

\begin{tabular}{|c|c|c|c|}
\hline sse-Phasen & Beobachtung & Befragung & Experiment \\
\hline Vor-Messe-Phase & & $\begin{array}{l}\text { - Vorbefragung der } \\
\text { hochschulspezi- } \\
\text { fischen Messe- } \\
\text { zielgruppen }\end{array}$ & - Messekonzepttest \\
\hline Messe-Phase & $\begin{array}{l}\text { - Wegeverlaufsanalyse } \\
\text { auf dem Hochschul- } \\
\text { Messestand } \\
\text { - Zählung ausgege- } \\
\text { bener Informations- } \\
\text { materialien } \\
\text { - Zählung der Hoch- } \\
\text { schul-Messestand- } \\
\text { Besucher } \\
\text { - Zählung persönlicher } \\
\text { Kontakte auf dem } \\
\text { Hochschul-Messe- } \\
\text { stand } \\
\text { - Protokolle der Hoch- } \\
\text { schul-Messestand- } \\
\text { Gespräche }\end{array}$ & $\begin{array}{l}\text { - Befragung der } \\
\text { Hochschul-Messe- } \\
\text { stand-Besucher }\end{array}$ & \\
\hline Nach-Messe-Phase & $\begin{array}{l}\text { - Einladungsresonanz- } \\
\text { analyse } \\
\text { - Auswertung der } \\
\text { Veranstalter- } \\
\text { Besucherstatistiken } \\
\text { - Auswertung des } \\
\text { FKM-Tests } \\
\text { - Analyse des Messe- } \\
\text { projektvolumens } \\
\text { - Messebeteiligungs-Nu }\end{array}$ & $\begin{array}{l}\text { - Nachmessebefra- } \\
\text { gung der Hochschul- } \\
\text { Messestand- } \\
\text { Besucher } \\
\text { - Befragung des } \\
\text { wissenschaftlichen } \\
\text { und nicht- } \\
\text { wissenschaftlichen } \\
\text { Standpersonals } \\
\\
\text { zen-Index }\end{array}$ & \\
\hline
\end{tabular}

Tab. 9: Systematisierung der Methoden zur indirekten Erfolgskontrolle von Hochschul-Messebeteiligungen

Zur Ermittlung der Zielerreichung werden im folgenden die aufgeführten Methoden auf ihre Eignung zum Einsatz bei der Erfolgskontrolle von Hochschul-Messebeteiligungen überprüft. Dabei werden angesichts der begrenzten Hochschulbudgets insbesondere die zum Methodeneinsatz notwendigen finanziellen und personellen Ressourcen, die einerseits in bezug auf die Aneignung der entsprechenden Marktforschungskenntnisse und andererseits bei Anwendung des Verfahrens selbst anfallen, als Kriterien herangezogen. Erweisen sich die Methoden zum Einsatz im Hochschulbereich als geeignet, so liegt der Fokus der indirekten Messeerfolgskontrolle - nicht zuletzt aufgrund des expandierenden Messeange- 
bots und der damit einhergehenden Notwendigkeit einer verstärkten Messeselektion - insbesondere auf einer messetypspezifischen Betrachtung. Ziel dieser Analyse ist es zu erfassen, inwiefern die einzelnen, von den Hochschul-Exponenten verfolgten Zielsetzungen eher durch die Beteiligung an allgemeineren Messen oder spezifischeren Messen erreicht werden. Während zur Erfolgskontrolle von Hochschul-Messebeteiligungen an allgemeineren Messen die befragten Hochschul-Exponenten der Mehrbranchenmesse herangezogen werden, bilden die Befragungen der Fachmesse-Exponenten die Grundlage zur Darstellung des Messebeteiligungserfolgs an spezifischeren Messen. Darüber hinaus wird - unter der Voraussetzung, daß die einzelnen Methoden dazu geeignet sind - der indirekte Messebeteiligungserfolg für die zuvor abgeleiteten hochschulspezifischen Exponententypen analysiert, um zu ermitteln, inwieweit die einzelnen Exponententypen durch die Messebeteiligung die Erreichung ihrer primär verfolgten Zielsetzungen forcieren konnten.

\subsection{Einsatz von Methoden zur indirekten Messeerfolgskontrolle bzw. -prognose in der Vor-Messe-Phase}

Den Hochschul-Exponenten bieten sich im Vorfeld ihrer Messebeteiligung Verfahren an, die i.S.e. ex-ante-Analyse prognostischen Charakter aufweisen und damit eher der Erfolgsprognose und weniger der Erfolgskontrolle dienen. ${ }^{17}$ Dabei stehen den Hochschul-Exponenten grundsätzlich der Messekonzepttest sowie die Vorbefragung der Messezielgruppen als entsprechende Methoden zur Verfügung.

Der Einsatz eines Messekonzepttests ${ }^{18}$ liefert Hochschul-Exponenten Informationen über die Wirkungen einzelner Bestandteile ihres Messekonzepts. Durch die

Unter Prognosen werden Zukunftsaussagen mit einem gewissen Objektivitätsgrad verstanden. Sie umfassen Vorhersagen wahrscheinlicher oder möglicher Ereignisse bzw. Entwicklungen. Vgl. hierzu Meffert, H., Steffenhagen, H., Marketingprognosemodelle - Quantitative Modelle des Marketing, Stuttgart 1977, S. 5. Erfolgsprognosen werden hier somit als Instrument der Potentialabschätzung einzelner Gestaltungselemente bzw. Aktivităten im Zusammenhang mit Hochschul-Messebeteiligungen verstanden. Vgl. hierzu sowie im folgenden Ueding, R., Management von Messebeteiligungen: Identifikation und Erklärung messespezifischer Grundhaltungen auf der Basis einer empirischen Untersuchung, a.a.O., S. $122 \mathrm{ff}$.

Der Messekonzepttest weist vielfältige Ähnlichkeiten zu Labortestmärkten in der Werbewirkungsforschung auf, aus denen sich der Messekonzepttest entwickelt hat. Vgl. Hossinger, H.-P., Pretests in der Marktforschung - Die Validität von Pretestverfahren unter besonderer Berücksichtigung der Tachistoskopie, Würzburg, Wien 1982, S. 6 ff.; Rehorn, J., Markttests, 
systematische Variation der einzelnen Bestandteile des Messestands im Rahmen von experimentellen Untersuchungen können die jeweils wirkungsstärksten Gestaltungsalternativen für einzelne Messestandelemente (Plakate, Standbeschriftungen etc.) ermittelt werden. ${ }^{19}$ Im Mittelpunkt dieses Verfahrens stehen dabei die spontanen Reaktionen der hochschulspezifischen Zielgruppen als unmittelbare Konsequenz auf die Kommunikationsinstrumente am Messestand und die äußere Gestaltung des Messestands selbst sowie seiner Exponate. ${ }^{20}$ Damit bietet sich dieses Verfahren insbesondere zur Kontrolle bzw. Prognose der Erreichung verfolgter Beeinflussungsziele an.

Der zentrale Vorteil des Messekonzepttests liegt in der Aufdeckung von UrsacheWirkungs-Zusammenhängen, ohne dabei den Ablauf der Messebeteiligung zu tangieren. ${ }^{21}$ Als Nachteil dieser Methode ist jedoch ihre mangelhafte Repräsentativität anzuführen, da aufgrund von Wirtschaftlichkeitsaspekten häufig nur relativ kleine Stichproben realisiert werden können. ${ }^{22}$ Darüber hinaus stellt der Einsatz dieses experimentellen Verfahrens spezifische Anforderungen sowohl an die Kenntnisse der Hochschul-Exponenten im Bereich der Marktforschung als auch an finanzielle und personelle Ressourcen. Damit ist der Einsatz des Messekonzepttests zur Erfolgskontrolle bzw. -prognose von Hochschul-Messebeteiligungen eher weniger geeignet und wurde daher in der dieser Arbeit zugrundeliegenden Studie nicht eingesetzt.

Mit einer Vorbefragung der hochschulspezifischen Messezielgruppen können Hochschul-Exponenten im Vorfeld ihrer Messebeteiligung Informationen über die Zielgruppenpräsenz auf der Messe sowie die Zielgruppenvorstellungen und -erwartungen bzgl. des Messebesuchs ermitteln. ${ }^{23}$ Die hierbei gewonnenen Erkennt-

Neuwied 1977, S. 45 ff.; Rehorn, J., Labortestmarkt und Minitestmarkt - Alternativen zum Prägen von Marktchancen, in: asw, 20. Jg., Heft 9, 1977, S. 51-60.

Vgl. Gräbener, W., Die Messepolitik als Marketinginstrument - dargestellt am Beispiel von Investitionsgüterproduzenten, a.a.O., S. 325 ff.

Vgl. Funke, K., Messeentscheidungen - Handlungsalternativen und Informationsbedarf, a.a.O., S. 315.

21 Vgl. Doyle, P., Gidengil, B. Z., A Review of In-Store Experiments, in: JoR, Vol. 53, No. 2, 1977 , S. 47-62.

22 Vgl. Gluth, H., Keine schwarzen Messen - Erfolgskontrolle mit den Methoden der empirischen Sozialforschung, in: Beilage der FAZ, 23.01.1996, S. B7.

23

Vgl. o.V., Wirkungskontrollen für Messebeteiligungen, in: Fachverband Messe- und Ausstellungsbau e.V. (FAMAB) (Hrsg.), Jahresbericht '95, S. 21. 
nisse liefern den Hochschul-Exponenten wichtige Entscheidungshilfen zur Messeauswahl und zum Einsatz von Messebeteiligungsaktivitäten. Zudem bietet die direkte, meist telefonisch durchzuführende Vorbefragung von potentiellen Hochschul-Messestand-Besuchern die Möglichkeit, vielfältige Erkenntnisse über die Informationsbedürfnisse und das Informationsverhalten sowie das allgemeine Interesse der hochschulspezifischen Zielgruppen hinsichtlich der anstehenden Messe zu gewinnen. Somit kann diese Methode insbesondere zur Überprüfung einer möglichen Erreichung der von Hochschul-Exponenten mit einer Messebeteiligung angestrebten Informationsziele eingesetzt werden. ${ }^{24}$

Ein zentraler Vorteil dieser Methode liegt in der frühzeitigen Gewinnung relevanter Informationen über die Zielgruppen. ${ }^{25}$ Nachteilig ist der mit derartigen Befragungen verbundene, insbesondere im Hochschulbereich nicht vertretbare Aufwand für Exponenten. Vor diesem Hintergrund bietet sich die Vorbefragung der Messezielgruppen im Rahmen von Hochschul-Messebeteiligungen grundsätzlich nicht an.

\subsection{Einsatz von Methoden zur indirekten Messeerfolgskontrolle in der Messe-Phase}

Während einer Messeveranstaltung können Hochschul-Exponenten sowohl auf als auch außerhalb des Messegeländes Erhebungen durchführen. ${ }^{26}$ Grundsätzlich sind in dieser Phase vor allem die Erhebungsmethoden relevant, die auf dem eigenen Hochschul-Messestand durchgeführt werden. ${ }^{27}$

Vgl. Gelszus Messe-Marktforschung GmbH (Hrsg.), Mehr Erfolg auf Messen durch Messewirkungskontrolle, Dortmund 1994, S. 19.

Zur Notwendigkeit des Einsatzes von geeigneten Instrumenten zur frühzeitigen Erkennung bestimmter Entwicklungen vgl. Steger, U., Winter, M., Strategische Früherkennung zur Antizipation ökologisch motivierter Marktveränderungen, in: DBW, 56. Jg., Heft 5, 1996, S. 607 ff.; Liebl, F., Strategische Frühaufklärung, München 1996, S. 5 f.; Zurlino, F., Zukunftsorientierung von Industrieunternehmen durch strategische Früherkennung, München, Wien 1995.

Im kommerziellen Bereich finden insbesondere auf dem Messegelände durchgeführte Befragungen Anwendung. Vgl. hierzu sowie im folgenden Ueding, R., Management von Messebeteiligungen: Identifikation und Erklärung messespezifischer Grundhaltungen auf der Basis einer empirischen Untersuchung, a.a.O., S. $125 \mathrm{ft}$.

Begründet liegt dies nicht zuletzt auch in den nach geltenden Veranstaltungsbestimmungen oftmals nicht vorhandenen Rechten von ausstellerseitigen Erhebungen auf dem Messegelände. Vgl. Funke, K., Messeentscheidungen - Handlungsalternativen und Informationsbedarf, 
Mit der Wegeverlaufsanalyse auf dem Hochschul-Messestand ${ }^{28}$ bspw. können die Hochschul-Exponenten Informationen hinsichtlich der zukünftigen Ausgestaltung bzw. der kurzfristigen Modifikation des Hochschul-Messestands gewinnen. $\mathrm{Zu}$ diesem Zweck sind Besucherströme auf dem Hochschul-Messestand zu messen und das Besucherverhalten auf dem Hochschul-Messestand in räumlicher und zeitlicher Sicht zu erheben. Diese Methode ist bei einer möglichst großen Zahl von Hochschul-Messestand-Besuchern, die idealerweise keine Kenntnis von der Beobachtungssituation besitzen, durchzuführen. Die lückenlose Aufzeichnung der Besucherbewegungen auf dem Hochschul-Messestand ist die Voraussetzung dafür, Informationen über den Hauptwegeverlauf der Hochschul-MessestandBesucher, bevorzugte Haltepunkte sowie weniger frequentierte Bereiche des Hochschul-Messestands zu erhalten. Die Beobachtungen der Besucherströme durch Rechercheure kann dabei durch technische Hilfsmittel wie Videokameras ergänzt werden. ${ }^{29}$ Damit liefert die Wegeverlaufsanalyse wichtige Informationen über die Hauptbesucherströme, die Intensität der Nutzung von Ein- und Ausgängen sowie die Attraktivität einzelner Exponate. Folglich ist dieses Verfahren vor allem zur Überprüfung von Präsentations- bzw. Bekanntheitszielen hinsichtlich bestimmter Hochschul-Exponate bzw. präsentierter Forschungsergebnisse und damit zur Kontrolle von Beeinflussungszielen geeignet. ${ }^{30}$

Die Anwendung der Wegeverlaufsanalyse auf dem Hochschul-Messestand besitzt den Vorteil, daß der Beobachter den Einfluß von Architektur und Gestaltungselementen des Hochschul-Messestands auf das sichtbare Verhalten der HochschulMessestand-Besucher messen kann. Nachteilig ist jedoch die nicht vorhandene Möglichkeit, ausgewählte Hochschul-Messestand-Besucher hochschulspezifischen Messezielgruppen zuzuordnen. Zudem setzt die Anwendung der Wege-

a.a.O., S. 298 f. Aus diesem Grund werden im folgenden ausschließlich solche Methoden der Erfolgskontrolle von Messebeteiligungen dargestellt, die auf dem Messestand bzw. in seiner unmittelbaren Nähe eingesetzt werden können.

Synonym zur "Wegeverlaufsanalyse“ wird auch der Begriff "Wegeanalyse“ verwendet. Vgl. Groth, C., Die Wegeanalyse: Ein Instrument zur Verhaltensforschung bei Messeveranstaltungen und ähnlichen speziellen Märkten, in: GfK Mitteilungen zur Markt- und Absatzforschung, Heft 3, 1968, S. $87 \mathrm{ff}$.

Vgl. Dostal, G., Messen + Ausstellungen: So decken Sie Stärken und Schwächen Ihrer MesseBeteiligung auf, in: Marketing Journal, 20. Jg., Heft 2, 1987, S. $140 \mathrm{ff}$.

Vgl. Bachmeir, H. M., Marktforschung auf Messen, in: Meyer, P. W. (Hrsg.), Arbeitspapiere zur Schriftenreihe Schwerpunkt Marketing der Universität Augsburg, Bd. 23, Augsburg 1989, S. $25 \mathrm{f}$. 
verlaufsanalyse bei den Hochschul-Exponenten ein entsprechendes marktforscherisches Know-how und insbesondere auch umfangreichere finanzielle und personelle Ressourcen voraus, weshalb sich diese Methode zur indirekten Erfolgskontrolle von Hochschul-Messebeteiligungen nur bedingt eignet.

Einfacher durchzuführende Methoden für Hochschul-Exponenten zur indirekten Erfolgskontrolle ihrer Messebeteiligungen stellen Zählungen dar. ${ }^{31}$ Die Hochschul-Exponenten können z.B. mit Zählungen ausgegebener Informationsmaterialien Anhaltspunkte über das Verhalten und das konkrete Exponatinteresse der Besucher gewinnen. Dabei erheben die Hochschul-Exponenten die Anfangsund Endbestände aller Informationsmaterialien, die sie am Hochschul-Messestand für ihre Besucher zur Ausgabe bereithalten, sowie die entsprechende Zahl der entgegengenommenen Anforderungen von Informationsmaterialien. Auf Grundlage der erhobenen Daten lassen sich nicht nur Erkenntnisse über das schwerpunktmäßige Hochschul-Messestand-Besucherinteresse gewinnen. Darüber hinaus geben die Daten auch in begrenztem Umfang Auskunft über die Wahrnehmung des Exponatangebots am Hochschul-Messestand und dienen damit vor allem der Überprüfung von Informations- und Beeinflussungszielen. ${ }^{32}$ Zudem können sie Anhaltspunkte über die Förderung verfolgter Projektziele liefern.

Der zentrale Vorteil beim Einsatz von Zählungen ausgegebener Informationsmaterialien ist die einfache Durchführung am Hochschul-Messestand. Dagegen liegt der Nachteil in dem nicht nachvollziehbaren Zusammenhang zwischen mitgenommenen Informationsmaterialien und der Verwendung dieser Materialien sowie dem bestehenden Exponatinteresse bei den Hochschul-Messestand-Besuchern. ${ }^{33}$ Folglich kann die Zählung ausgegebener Informationsmaterialien lediglich als flankierendes Verfahren zur Erfolgskontrolle von Hochschul-Messebeteiligungen eingesetzt werden.

Zählungen umfassen beobachtende bzw. registrierende Verfahren, bei denen z.B. Häufigkeiten einzelner, genau gekennzeichneter Individualverhaltensweisen ermittelt werden. Die auf Basis derartiger Zählungen ermittelten Daten sind damit Reaktionsdaten, die in einem Vorgang erhoben und aggregiert werden. Vgl. Cunningham, M. T., White, J. G., The Role of Exhibitions in Industrial Marketing - An Evaluation of the International Machine Tool Exhibition, in: IMM, Vol. 2, No. 3, 1974, S. 238 f.

Vgl. Hagen, R., Erfolgskontrolle auf Verbrauchermessen - dargestellt am Beispiel der Analyse des Informationsstandes des Deutschen Bundestages, in: PuA, 10. Jg., Heft 7, 1983, S. 292 ff.

Vgl. Schönemann, K., Messen und Kataloge: Die Sache mit den Katalogen, in: Der Fremdenverkehr, Heft 2, 1989, S. 16. 
Die Hochschul-Exponenten auf den betrachteten Messen konnten eine Reihe von Informationsmaterialien an Messebesucher ausgeben (vgl. Tab. 10), wobei die Exponentenanteile mit ausgegebenen Informationsmaterialien insgesamt auf Fachmessen höher ausfällt als auf Mehrbranchenmessen. Die messetypspezifische Zählung abgegebener Informationsmaterialien zeigt hingegen eine auf Mehrbranchenmessen höhere Ausgabe an projekt- bzw. exponatbezogenen Informationsmaterialien. ${ }^{34}$ Auch konnten die Hochschul-Exponenten auf Mehrbranchenmessen mehr Image-Broschüren über die eigenen Institute/Lehrstühle/Fachbereiche sowie Hochschulen an Messebesucher abgeben. Demzufolge ist davon auszugehen, daß Hochschul-Exponenten der Mehrbranchenmesse angestrebte Exponatbezogene, Instituts-/Lehrstuhl-/Fachbereichsbezogene und Hochschulbezogene Beeinflussungsziele besser realisieren konnten als Fachmesse-Exponenten. Die Abgabe von Visitenkarten und sonstigen Informationsmaterialien (z.B. Forschungsberichten) fiel hingegen auf Fachmessen höher aus, was grundsätzlich auf eine potentiell bessere Erreichung von Informationszielen auf Fachmessen als auf Mehrbranchenmessen schließen läßt, da der Austausch von Visitenkarten oftmals Ausgangspunkt für einen über die Messe-Phase hinausgehenden Kontakt zwischen Messebesuchern und Hochschul-Exponenten bildet und damit letztlich die Basis für einen längerfristigen Erfahrungs- und Informationsaustausch legt. Durch diese Kontakte kann wiederum die Erreichung verfolgter Projektziele gefördert werden.

Im Gegensatz zur exponententypspezifischen Untersuchung, die auf Grundlage der in die Clusteranalyse einbezogenen 45 Hochschul-Exponenten ( 30 Mehrbranchenmesse- und 15 Fachmesse-Exponenten) durchgeführt wurde, erschien es für die messetypspezifische Betrachtung sinnvoll, alle befragten 47 Hochschul-Exponenten (32 Mehrbranchenmesse- und 15 Fachmesse-Exponenten) in die Analyse einzubeziehen, d.h. auch die zwei Exponenten zu berücksichtigen, die sich zur Einbeziehung in die Clusteranalyse als nicht geeignet enwiesen hatten. In diesen messetyp- bzw. exponententypspezifischen Analysen wurden - angesichts der aufgrund der relativ geringen Stichprobenumfänge i.d.R. nicht ermittelbaren signifikanten Unterschiede zwischen den Messen- bzw. Exponententypen - keine Signifikanzniveaus ausgewiesen. 


\begin{tabular}{|c|c|c|c|c|c|c|}
\hline \multirow[b]{2}{*}{$\begin{array}{l}\text { Aus- } \\
\text { gegebene } \\
\text { Informationsmaterialien }\end{array}$} & \multicolumn{2}{|c|}{ gesamt } & \multicolumn{2}{|c|}{ Mehrbranchenmesse } & \multicolumn{2}{|c|}{ Fachmesse } \\
\hline & $\begin{array}{l}\text { Exponen- } \\
\text { ten-Anteil } \\
\text { mit ausgeg. } \\
\text { Materialien }\end{array}$ & $\begin{array}{l}\varnothing \text {-Anzahl } \\
\text { an ausge- } \\
\text { gebenen } \\
\text { Materialien }\end{array}$ & $\begin{array}{l}\text { Exponen- } \\
\text { ten-Anteil } \\
\text { mit ausgeg. } \\
\text { Materialien }\end{array}$ & $\begin{array}{c}\varnothing \text {-Anzahl } \\
\text { an ausge- } \\
\text { gebenen } \\
\text { Materialien }\end{array}$ & $\begin{array}{l}\text { Exponen- } \\
\text { ten-Anteil } \\
\text { mit ausgeg. } \\
\text { Materialien }\end{array}$ & $\begin{array}{l}\text { Ø-Anzahl } \\
\text { an ausge- } \\
\text { gebenen } \\
\text { Materialien }\end{array}$ \\
\hline $\begin{array}{l}\text { Projekt-/Exponat- } \\
\text { beschreibungen }\end{array}$ & $75 \%(35)$ & 192 & $71 \%(23)$ & 230 & $80 \%(12)$ & 120 \\
\hline $\begin{array}{l}\text { Image-Broschüren des } \\
\text { Inst/Lehrst./Fachber. }\end{array}$ & $60 \%(28)$ & 74 & $56 \%(18)$ & 85 & $67 \%(10)$ & 55 \\
\hline $\begin{array}{l}\text { Image-Broschüre der } \\
\text { Hochschule }\end{array}$ & $51 \%(24)$ & 18 & $44 \%(14)$ & 20 & $67 \%(10)$ & 15 \\
\hline Visitenkarten & $79 \%(37)$ & 60 & $75 \%(24)$ & 54 & $87 \%(13)$ & 71 \\
\hline $\begin{array}{l}\text { Sonstiges } \\
\text { (Forschungsberichte etc.) }\end{array}$ & $36 \%(17)$ & 46 & $9 \% \quad(9)$ & 31 & $53 \% \quad(8)$ & 52 \\
\hline
\end{tabular}

Tab. 10: Messetypspezifische Betrachtung ausgegebener Informationsmaterialien im Rahmen von Hochschul-Messebeteiligungen

Die exponententypspezifische Zählung zeigt, daß die Ausgabe an Informationsmaterialien grundsätzlich gemäß der primär verfolgten Zielsetzungen ausfällt (vgl. Tab. 11). Der überwiegende Anteil der Projekt- und exponatorientierten Hochschul-Exponenten gab insbesondere Projekt- und Exponatbeschreibungen sowie Visitenkarten aus und forcierte damit die Realisierung angestrebter Projektziele. Der Großteil der Beeinflussungsorientierten Hochschul-Exponenten konnte die Erreichung ihrer primär verfolgten Messezielsetzungen vor allem durch die Ausgabe von Image-Broschüren des Instituts/Lehrstuhls/Fachbereichs sowie Projekt- und Exponatbeschreibungen fördern, die im Vergleich zu anderen hochschulspezifischen Exponententypen am höchsten ausfielen. Im Unterschied zu den zuvor betrachteten Hochschul-Exponententypen lag nicht nur der Anteil, sondern auch die durchschnittliche Anzahl der von Pflichtrepräsentanten ausgegebenen Informationsmaterialien deutlich niedriger. Hierin spiegelt sich die vergleichsweise geringe Zielorientierung dieses Exponententyps wider, die darin begründet liegt, daß das Messeengagement nicht aus eigener Initiative, sondern vielmehr aus der Pflicht des Status als öffentlich-rechtliche Institution heraus resultiert. 


\begin{tabular}{|c|c|c|c|c|c|c|}
\hline \multirow[b]{2}{*}{$\begin{array}{l}\text { Aus- } \\
\text { gegebene } \\
\text { Informationsmateriallen }\end{array}$} & \multicolumn{2}{|c|}{$\begin{array}{l}\text { Projekt-und exponat- } \\
\text { orientierte } \\
\text { Hochschul-Exponenten }\end{array}$} & \multicolumn{2}{|c|}{$\begin{array}{c}\text { Beeinflussungs- } \\
\text { orientierte } \\
\text { Hochschul-Exponenten }\end{array}$} & \multicolumn{2}{|c|}{$\begin{array}{l}\text { Pflicht- } \\
\text { repräsentanten }\end{array}$} \\
\hline & $\begin{array}{l}\text { Exponen- } \\
\text { ten-Anteil } \\
\text { mit ausgeg. } \\
\text { Materialien }\end{array}$ & $\begin{array}{l}\text { Ø-Anzahl } \\
\text { an ausge- } \\
\text { gebenen } \\
\text { Materialien }\end{array}$ & $\begin{array}{l}\text { Exponen- } \\
\text { ten-Anteil } \\
\text { mit ausgeg. } \\
\text { Materialien }\end{array}$ & $\begin{array}{l}\text { Ø-Anzahl } \\
\text { an ausge- } \\
\text { gebenen } \\
\text { Materialien }\end{array}$ & $\begin{array}{c}\text { Exponen- } \\
\text { ten-Anteil } \\
\text { mit ausgeg. } \\
\text { Materialien }\end{array}$ & $\begin{array}{l}\varnothing \text {-Anzahl } \\
\text { an ausge- } \\
\text { gebenen } \\
\text { Materialien }\end{array}$ \\
\hline $\begin{array}{l}\text { Projekt-/Exponat- } \\
\text { beschreibungen }\end{array}$ & $67 \%(4)$ & 213 & $79 \%(23)$ & 229 & $70 \%(7)$ & 69 \\
\hline $\begin{array}{l}\text { Image-Broschüren des } \\
\text { Inst/Lehrst/Fachber. }\end{array}$ & $33 \%(2)$ & 80 & $59 \%(17)$ & 107 & $30 \%(3)$ & 26 \\
\hline $\begin{array}{l}\text { Image-Broschüre der } \\
\text { Hochschule }\end{array}$ & $33 \%(2)$ & 55 & $31 \% \quad(9)$ & 16 & $30 \%(3)$ & 52 \\
\hline Visitenkarten & $67 \%(4)$ & 140 & $83 \%(24)$ & 60 & $70 \%(7)$ & 26 \\
\hline $\begin{array}{l}\text { Sonstiges } \\
\text { (Forschungsberichte etc.) }\end{array}$ & $67 \%(4)$ & 55 & $21 \% \quad(6)$ & 55 & $20 \%(2)$ & 10 \\
\hline
\end{tabular}

Tab. 11: Exponententypspezifische Betrachtung ausgegebener Informationsmaterialien im Rahmen von Hochschul-Messebeteiligungen

Die vorgenommene Zählung ausgegebener Informationsmaterialien liefert jedoch keine Informationen darüber, wie viele Messebesucher den Hochschul-Messestand auch tatsächlich besucht haben. Deshalb erscheint es unabdingbar, im Rahmen der indirekten Erfolgskontrolle von Hochschul-Messebeteiligungen zusätzlich die Anzahl der Hochschul-Messestand-Besucher zu erfassen.

Mit Zählungen der Hochschul-Messestand-Besucher können die HochschulExponenten den Grad des Besucherinteresses am Hochschul-Messestand und den auf inm präsentierten Exponaten ermitteln. ${ }^{35}$ Grundsätzlich werden alle Messebesucher, die den Hochschul-Messestand betreten, mittels beobachtender Verfahren in die Statistik aufgenommen. Die Zählung der Messestand-Besucher liefert Daten zur quantitativen Bestimmung der Besucherfrequenz auf dem Hochschul-Messestand während einzelner Messetage bzw. der gesamten Veranstaltungsdauer. Ausgewählte Zählpunkte werden mit entsprechendem Personal besetzt, das alle Hochschul-Messestand-Besucher über die gesamte Veranstal- 
tungsdauer der Messe erfaßt. ${ }^{36}$ In begrenztem Umfang läßt die Anwendung dieser Kontrollmethode Aussagen über die Erhöhung des Bekanntheitsgrades der Exponenten bzw. der ausgestellten Hochschul-Exponate zu und eignet sich damit zur Kontrolle von Beeinflussungszielen.

Die wesentlichen Vorteile der Zählungen von Hochschul-Messestand-Besuchern zur Erfolgskontrolle von Hochschul-Messebeteiligungen liegen in ihrer relativ einfachen und wenig kostenintensiven Durchführung. Dagegen sind als Nachteile die nicht selten auftretenden Doppelzählungen zu nennen, ${ }^{37}$ die zu einer höheren als der tatsächlichen Gesamtbesucherzahl des Hochschul-Messestands führen. Dennoch liefert der Einsatz von Hochschul-Messestand-Besucherzählungen Ausgangsinformationen zum Messebeteiligungserfolg und bietet sich grundsätzlich zur indirekten Erfolgsmessung von Hochschul-Messebeteiligungen an.

Insgesamt wurden die betrachteten Hochschul-Messestände von zahlreichen Messebesuchern aufgesucht. Auf der Mehrbranchenmesse - so zeigt die messetypspezifische Analyse - besuchten durchschnittlich 167 Hochschul-MessestandBesucher die einzelnen Hochschul-Exponenten. Demgegenüber fiel die Anzahl der Hochschul-Messestand-Besucher auf der Fachmesse mit durchschnittlich 104 Besuchern geringer aus. ${ }^{38}$ Diese Ergebnisse deuten tendenziell auf höhere Hochschul-Messestand-Besucherzahlen auf Mehrbranchenmessen hin, so daß Hochschul-Exponenten auf Mehrbranchenmessen einen generell höheren Bekanntheitsgrad und die Erreichung von Beeinflussungszielen eher realisieren können. Dabei geben die durchgeführten Zählungen jedoch keinerlei Auskunft darüber, mit welchen Messebesuchern die Hochschul-Exponenten auch tatsächlich in Kontakt getreten sind. Nicht zuletzt deshalb erscheint es hier wenig sinnvoll, eine expo-

Vgl. Gelszus, R. H., Messen + Ausstellungen: Vergessen Sie die Messe-Wirkungs-Kontrolle nicht, in: Marketing Journal, 18. Jg., Heft 2, 1985, S. 176. Anstelle von Personal können ebenfalls Kameras eingesetzt werden. Auch besteht die Möglichkeit, Standbesucher mit magnetischen Chipkarten auszustatten, die beim Betreten des Hochschul-Messestands einen Zählimpuls an einem „Computer-Counter" auslösen. Vgl. o.V., Erfolgskontrolle - Umstrittene Methode, in: FAMA intern, Heft 2, 1985, S. 12.

Doppelzählungen treten bspw. dann auf, wenn Hochschul-Messestand-Besucher den Messestand mehrmals aufsuchen, um einen bestimmten Hochschulvertreter zu sprechen.

Auch bei Berücksichtigung der unterschiedlichen Veranstaltungsdauer (Mehrbranchenmesse = 6 Messetage und Fachmesse $=5$ Messetage) ergibt sich tendenziell das gleiche Ergebnis. So haben auf der Mehrbranchenmesse pro Veranstaltungstag durchschnittlich 28 Messebesucher einen Hochschul-Exponenten besucht, während dies auf der Fachmesse durchschnittlich 21 Hochschul-Messestand-Besucher waren. 
nententypspezifische Betrachtung vorzunehmen. Vielmehr ist zunächst die Anzahl von persönlichen Kontakten auf den betrachteten Hochschul-Messeständen zu erfassen.

Durch Zählungen persönlicher Kontakte auf dem Hochschul-Messestand gewinnen die Hochschul-Exponenten Erkenntnisse über die Anzahl der Gespräche zwischen Hochschul-Exponenten und Hochschul-Messestand-Besuchern. Im Gegensatz zur Zählung der Hochschul-Messestand-Besucher beschränken sich diese Zählungen nur auf diejenigen Messestand-Besucher, mit denen die Hochschul-Exponenten ein persönliches Gespräch geführt haben. Neben der reinen Kontaktzählung kann die zeitliche Verteilung der Kontakte über den einzelnen Messetag bzw. über die gesamte Messe-Phase festgehalten werden. ${ }^{39}$ Folglich liefert der Einsatz dieser Methode nicht nur Informationen über die geführten Gespräche auf dem Hochschul-Messestand, sondern darüber hinaus ebenfalls Informationen für zukünftige Personaleinsatzplanungen auf dem Messestand. Zudem bietet sie die Basis für die standpersonalbezogene Bewertung von quantitativen Kontaktzielen. ${ }^{40}$ Folglich liefert der Einsatz dieser Kontrollmethode insbesondere Anhaltspunkte über die Erreichung von Informations- und Beeinflussungszielen.

Der Vorteil von Zählungen persönlicher Kontakte auf dem Hochschul-Messestand liegt in der einfachen Handhabung und dem relativ geringen Bedarf an finanziellen und personellen Ressourcen. Diesen stehen jedoch auch einige Nachteile gegenüber. So liefern die mit dieser Methode ermittelten quantitativen Kontaktzahlen den Hochschul-Exponenten weder Informationen über die Dauer und den Inhalt der realisierten Kontakte, noch über die Zielgruppenzugehörigkeit der Hochschul-Messestand-Besucher. Dennoch werden durch den Einsatz dieser Methode wichtige Informationen zum indirekten Erfolg von Hochschul-Messebeteiligung gewonnen.

Vgl. Krueger, J., Messen und Ausstellungen: Die Kontakt-Kosten sichtbar machen, in: Marketing Journal, 17. Jg., Heft 5, 1984, S. $484 \mathrm{ff}$. 
Die Zählung der persönlichen Kontakte auf den betrachteten Hochschul-Messeständen belegt durchschnittlich 123 geführte Gespräche zwischen HochschulExponenten und Messebesuchern. Die messetypspezifische Betrachtung zeigt durchschnittlich 140 geführte Gespräche auf Hochschul-Messeständen der Mehrbranchenmesse, hingegen nur 92 Gespräche auf Messeständen der Fachmesse. ${ }^{41}$ Somit kann davon ausgegangen werden, daß Hochschul-Exponenten auf Mehrbranchenmessen tendenziell eher ihre mit der Messebeteiligung verfolgten Informationsziele erreichen als Fachmesse-Exponenten, da persönliche Kontakte u.a. die Basis zum Informationsaustausch zwischen Exponenten und Messestand-Besuchern bilden. Dies gilt ebenfalls für Beeinflussungsziele, deren Erreichung die Hochschul-Exponenten durch entsprechende Kontakte auf der Messe unterstützen können.

Die exponententypspezifische Erhebung der auf dem Hochschul-Messestand geführten Gespräche belegt für Projekt- und exponatorientierte Hochschul-Exponenten durchschnittlich 166 und für Beeinflussungsorientierte Hochschul-Exponenten durchschnittlich 129 Gespräche. Diese Hochschul-Exponententypen konnten damit die Erreichung von Informations- und Beeinflussungszielen fördern. Pflichtrepräsentanten hingegen, die mit durchschnittlich 71 Gesprächen vergleichsweise wenig Gesprächskontakte zu Hochschul-Messestand-Besuchern herstellten, konnten diese Zielerreichung nicht in einem derartigen Ausmaß beeinflussen.

Die hier vorgenommene Zählung persönlicher Kontakte liefert zwar wichtige Anhaltspunkte zum indirekten Messebeteiligungserfolg von Hochschul-Exponenten, dennoch können die Ergebnisse isoliert betrachtet noch keine ausreichenden Informationen über die Erreichung von Informations- und Beeinflussungszielen geben. Zusätzlich ist der Einsatz von Methoden notwendig, die als qualitative Kriterien die Inhalte der Gespräche zwischen Hochschul-Exponenten und Hochschul-Messestand-Besuchern berücksichtigen. In diesem Zusammenhang bietet sich die Erstellung von Gesprächsprotokollen an.

41 Auch bei Berücksichtigung der unterschiedlichen Veranstaltungsdauer (Mehrbranchenmesse = 6 Messetage und Fachmesse = 5 Messetage) ergibt sich tendenziell das gleiche Ergebnis. So wurden auf der Mehrbranchenmesse pro Veranstaltungstag durchschnittlich 23 Gespräche und auf der Fachmesse durchschnittlich 18 Gespräche zwischen Hochschul-Exponenten und Hochschul-Messestand-Besuchern geführt. 
Mit der Anfertigung von Protokollen der Hochschul-Messestand-Gespräche können die von den Hochschul-Exponenten geführten Gespräche auf dem Hochschul-Messestand systematisch erfaßt, ${ }^{42}$ die Gesprächsinhalte festgehalten und wichtige Informationen über die Hochschul-Messestand-Besucher gewonnen werden. Gesprächsprotokolle ${ }^{43}$ ermöglichen damit die Überprüfung der Erreichung priorisierter Messezielgruppen. ${ }^{44}$ Zur Erfassung der Gespräche bieten sich Hochschul-Exponenten einerseits weitgehend formalisierte Gesprächsprotokolle an, die auf Grundlage eines streng strukturierten Vorgehens auf die Aufzeichnung einzelner Gespräche ausgerichtet sind. ${ }^{45}$ Andererseits können nicht formalisierte Gesprächsprotokolle eingesetzt werden, die damit keine spezifische Struktur aufweisen und häufig einen eher zusammenfassenden Charakter besitzen, so daß sie oftmals erst nach Ablauf eines Messetages angefertigt werden können.

Der Vorteil des Einsatzes von formalisierten Gesprächsprotokollen liegt in einer weitgehenden Unterstützung der Aufzeichnung relevanter Gesprächsaspekte, ${ }^{46}$ wobei die Hochschul-Exponenten die Möglichkeit haben, die Inhalte ihrer geführten Gespräche auf dem Hochschul-Messestand zu reflektieren und dabei aufgetretene Besonderheiten festzuhalten. Der generelle Nachteil der Anwendung von Gesprächsprotokollen besteht jedoch in ihrer Selektivität. Messebesucher, die zwar den Hochschul-Messestand besuchen und sich anhand ausgegebener Broschüren informieren, aber kein Gespräch mit den exponatbetreuenden Wissenschaftlern führen, werden mit dieser Methode nicht erfaßt. Damit bietet sich der

Hierzu wird der Gesprächsverlauf bzw. -inhalt jeweils nach Messetag und Uhrzeit dokumentiert. Vgl. Selinski, H., Messe- und Kongreßforschung (Teil II), in: PuA, 11. Jg., Heft 5, 1984, S. 214 f.

Gesprächsprotokolle werden z.T. auch als Berichtsbogen, Messekontaktbogen oder Kontakterfassungsbogen bezeichnet.

Vgl. Kayser, P., Messebeschickung und Durchführung: Auswahl der Messe, in: Budach, W. et al. (Hrsg.), Hochschulen und Messen: Praktische Hinweise für Messebeteiligungen durch Hochschulen, a.a.O., S. 71.

Zum grundsätzlichen Aufbau von formalisierten Gesprächsprotokollen vgl. Jaspersen, H., Mehr Messe-Transparenz: Hüls, in: Marketing Journal, 20. Jg., Heft 2, 1977, S. 169 ff. In erweiterter Form vgl. Meffert, H., Ueding, R., Ziele und Nutzen von Messebeteiligungen, a.a.O., S. $58 \mathrm{f}$.

Mit dem Einsatz von Personal Computern bei der Erstellung der Gesprächsprotokolle kann eine Effektivitätssteigerung dieser Methode erreicht werden, da die erfaßten Protokolle relativ schnell auswertbar sind, so daß ggf. noch während der Messe-Phase entsprechende Maßnahmen (z.B. Versendung von Informationsunterlagen) eingeleitet werden können. 
Einsatz dieser Methode als Ergänzung zu einer parallel durchgeführten Zählung von Hochschul-Messestand-Besuchern an.

Im Rahmen der vorliegenden Untersuchung wurden auf Basis von Protokollen die Inhalte der auf den Hochschul-Messeständen geführten Gespräche festgehalten. Dabei wurden folgende vier Gesprächsarten unterschieden:

- Informationsgespräche (z.B. Informations- und Erfahrungsaustausch mit Praxisvertretern und Fachkollegen),

- Gespräche der Beziehungspflege,

- Gespräche über mögliche zukünftige Projektkooperationen und

- konkrete Besprechung(en) zukünftiger Projekte.

In diesem Zusammenhang wurde ebenfalls erfaßt, welche Gespräche voraussichtlich die Basis für einen über die Messe-Phase hinausgehenden Kontakt zwischen Hochschul-Exponenten und Messebesucher legten.

Wie die folgende Tabelle zeigt, führten die Hochschul-Exponenten im Rahmen ihrer Messebeteiligung am häufigsten Informationsgespräche. Durch diese Gespräche wurde ein Erfahrungs- sowie Informationsaustausch realisiert und damit die Erreichung von Informationszielen gefördert. Auch wurden vor dem Hintergrund von Projektzielen viele Gespräche über mögliche zukünftige Projektkooperationen geführt. Diese nahmen zwar im Vergleich zu den Informationsgesprächen einen weitaus geringeren Anteil an den Messestandgesprächen ein, dafür wurde jedoch der Anteil an Gesprächspartnern höher eingeschätzt, zu denen die Exponenten in der Nach-Messe-Phase voraussichtlich ihren Kontakt intensivieren konnten. Eine ähnliche Struktur zeichnete sich bei Gesprächen zur Beziehungspflege ab. Werden die Priorisierungen der Einzelzielsetzungen einer Hochschul-Messebeteiligung mit in die Analyse einbezogen, so ist zu konstatieren, daß die primär verfolgten Ziele wie „Kontaktaufnahme zu Projektinteressenten“ und „Beziehungspflege bzw. -aufbau mit Repräsentanten verschiedener Institutionen" durch die auf dem Hochschul-Messestand geführten Gespräche zwischen Hochschul-Exponenten und Messebesuchern erreicht wurden. Die konkreten Besprechungen zukünftiger Projekte nahmen den geringsten Anteil an den geführten Gesprächen ein. Wurden jedoch Projektgespräche während der Messe geführt, so schätzten die Hochschul-Exponenten die Wahrscheinlichkeit, daß der Kontakt zu diesen Gesprächspartnern nach der Messe intensiviert würde, als hoch ein. Damit wurden für die Hochschul-Exponenten durch die Beteiligung an 
den betrachteten Messen die Voraussetzungen zur Erreichung ihrer verfolgten Projektziele geschaffen.

\begin{tabular}{|c|c|c|c|c|c|c|c|c|c|c|c|c|c|c|c|c|}
\hline \multirow{3}{*}{$\begin{array}{l}\text { Ge- } \\
\text { sprächsart }\end{array}$} & \multicolumn{8}{|c|}{$\begin{array}{l}\text { Anzahl der geführten Gespräche mit } \\
\text { den Messestandbesuchern }\end{array}$} & \multicolumn{8}{|c|}{$\begin{array}{l}\text { Anzahl der Gesprächspartner mit } \\
\text { voraussichtl. Nach-Messe-Kontakt }\end{array}$} \\
\hline & \multirow[b]{2}{*}{ k.A. } & \multirow{2}{*}{$\begin{array}{l}\text { Stan- } \\
\text { dard- } \\
\text { abw. }\end{array}$} & \multirow{2}{*}{$\begin{array}{l}\text { Mit- } \\
\text { tel- } \\
\text { wert } \\
\end{array}$} & \multicolumn{5}{|c|}{ Häufigkeiten } & \multirow[b]{2}{*}{ k.A. } & \multirow{2}{*}{$\begin{array}{l}\text { Stan- } \\
\text { dard- } \\
\text { abw. }\end{array}$} & \multirow{2}{*}{$\begin{array}{c}\text { Mit- } \\
\text { tel- } \\
\text { wert }\end{array}$} & \multicolumn{5}{|c|}{ Häufigkeiten } \\
\hline & & & & 0 & $1-9$ & $10-19$ & $20-49$ & 250 & & & & 0 & 1.4 & $5-10$ & $11-20$ & $>20$ \\
\hline $\begin{array}{l}\text { Informations- } \\
\text { gespräch }(e) \\
\text { z.B. Info--1 } \\
\text { Erfahrungs- } \\
\text { austausch) }\end{array}$ & $\begin{array}{c}15 \% \\
(7)\end{array}$ & 170,00 & 97 & $\begin{array}{c}5 \% \\
(2)\end{array}$ & $\begin{array}{c}10 \% \\
(4)\end{array}$ & \begin{tabular}{|c|}
$8 \%$ \\
$(3)$
\end{tabular} & $\begin{array}{c}28 \% \\
(11)\end{array}$ & $\begin{array}{l}50 \% \\
(20)\end{array}$ & $\begin{array}{c}40 \% \\
(19)\end{array}$ & 21,18 & 16 & $\begin{array}{c}11 \% \\
(3)\end{array}$ & $\begin{array}{c}14 \% \\
(4)\end{array}$ & $\begin{array}{c}36 \% \\
(10)\end{array}$ & $\begin{array}{c}18 \% \\
(5)\end{array}$ & $\begin{array}{c}21 \% \\
(6)\end{array}$ \\
\hline $\begin{array}{l}\text { Gespräch(e) } \\
\text { der } \\
\text { Beziehungs- } \\
\text { pflege }\end{array}$ & $\begin{array}{c}15 \% \\
(7)\end{array}$ & 18,55 & 10 & $\begin{array}{c}23 \% \\
(9)\end{array}$ & $\begin{array}{l}43 \% \\
(17)\end{array}$ & $\mid \begin{array}{c}18 \% \\
(7)\end{array}$ & $\begin{array}{c}13 \% \\
(5)\end{array}$ & $\begin{array}{c}5 \% \\
(2)\end{array}$ & $\begin{array}{c}45 \% \\
(21)\end{array}$ & 9,67 & 7 & $\begin{array}{c}23 \% \\
(6)\end{array}$ & $\begin{array}{c}31 \% \\
(8)\end{array}$ & $\begin{array}{c}27 \% \\
(7)\end{array}$ & $\begin{array}{c}8 \% \\
(2)\end{array}$ & $\begin{array}{c}12 \% \\
(3)\end{array}$ \\
\hline $\begin{array}{l}\text { Gespräch(e) } \\
\text { über mögliche } \\
\text { zukünftige } \\
\text { Projekt- } \\
\text { kooperationen }\end{array}$ & $\begin{array}{c}15 \% \\
(7)\end{array}$ & 16,46 & 13 & $\begin{array}{c}8 \% \\
(3)\end{array}$ & $\begin{array}{c}53 \% \\
(21)\end{array}$ & $\begin{array}{c}18 \% \\
(7)\end{array}$ & $\left\{\begin{array}{c}13 \% \\
(5)\end{array}\right.$ & $\begin{array}{c}10 \% \\
(4)\end{array}$ & $\begin{array}{r}38 \% \\
(18)\end{array}$ & 9,37 & 8 & $\begin{array}{c}3 \% \\
(1)\end{array}$ & $\begin{array}{c}52 \% \\
(15)\end{array}$ & $\begin{array}{c}24 \% \\
(7)\end{array}$ & $\begin{array}{c}7 \% \\
(2)\end{array}$ & $\begin{array}{c}14 \% \\
(4)\end{array}$ \\
\hline $\begin{array}{l}\text { konkrete } \\
\text { Bespre- } \\
\text { chung(en) } \\
\text { zukünftiger } \\
\text { Projekte } \\
\end{array}$ & $\begin{array}{c}21 \% \\
(10)\end{array}$ & 6,81 & 3 & $\begin{array}{l}43 \% \\
(16)\end{array}$ & $\begin{array}{c}49 \% \\
(18)\end{array}$ & $\begin{array}{c}8 \% \\
(3)\end{array}$ & - & - & $\begin{array}{c}45 \% \\
(21)\end{array}$ & 3,88 & 3 & $\begin{array}{l}39 \% \\
(10)\end{array}$ & $\begin{array}{c}35 \% \\
(9)\end{array}$ & $\begin{array}{c}23 \% \\
(6)\end{array}$ & $\begin{array}{l}4 \% \\
(1)\end{array}$ & - \\
\hline
\end{tabular}

Tab. 12: Kontaktqualität und -quantität der zwischen Hochschul-Exponenten und Hochschul-Messestand-Besuchern geführten Gespräche

Messetypspezifisch lag die durchschnittliche Anzahl der Informationsgespräche auf der Mehrbranchenmesse knapp doppelt so hoch wie auf der Fachmesse (vgl. Tab. 13). Dabei wurde jedoch der Anteil derjenigen Kontakte, die voraussichtlich nach der Messe intensiviert werden, von den Fachmesse-Exponenten weitaus höher eingeschätzt. Die durchschnittliche Anzahl der Gespräche über mögliche zukünftige Projektkooperationen fiel bei den Fachmesse-Exponenten höher aus als bei den Hochschul-Exponenten der Mehrbranchenmesse. Darüber hinaus schätzten die Fachmesse-Exponenten die Möglichkeiten der Intensivierung dieser Kontakte in der Nach-Messe-Phase deutlich höher ein. Bei den Gesprächen der Beziehungspflege können hinsichtlich der durchschnittlichen Anzahl der geführten Gespräche keine nennenswerten Unterschiede zwischen Beteiligungen an Mehrbranchen- und Fachmessen konstatiert werden, wenngleich auch hier der Anteil möglicher Folgekontakte in der Nach-Messe-Phase von Fachmesse-Exponenten höher eingestuft wurde. In bezug auf konkrete Besprechungen zukünftiger Projekte ließen sich ebenfalls keine hervorzuhebenden Unterschiede zwischen den beiden untersuchten Messetypen erkennen. Insgesamt ist aufgrund der auf Mehrbranchenmessen überwiegend geführten Informationsgespräche und der auf Fachmessen vermehrt geführten Gespräche über zukünftige Projektkooperationen 
davon auszugehen, daß Hochschul-Exponenten durch die Beteiligung an Mehrbranchenmessen besser die verfolgten Informationsziele erreichten als durch die Beteiligung an Fachmessen. Dagegen konnten Fachmesse-Exponenten durch ihre Messebeteiligung bessere Voraussetzungen zur Realisierung der verfolgten Projektziele schaffen.

\begin{tabular}{|c|c|c|c|c|c|c|c|c|c|c|c|c|c|c|c|c|}
\hline \multirow{3}{*}{$\begin{array}{l}\text { Ge- } \\
\text { sprächsart }\end{array}$} & \multicolumn{8}{|c|}{$\begin{array}{l}\text { Anzahl der geführten Gespräche mit } \\
\text { den Messestandbesuchern }\end{array}$} & \multicolumn{8}{|c|}{$\begin{array}{l}\text { Anzahl der Gesprächspartner mit } \\
\text { voraussichtl. Nach-Messe-Kontakt }\end{array}$} \\
\hline & \multirow[b]{2}{*}{ k.A. } & \multirow{2}{*}{$\begin{array}{l}\text { Stan- } \\
\text { dard- } \\
\text { abw. }\end{array}$} & \multirow{2}{*}{\begin{tabular}{|c|} 
Mit- \\
tel- \\
wert
\end{tabular}} & \multicolumn{5}{|c|}{ Häufigkeiten } & \multirow[b]{2}{*}{ k.A. } & \multirow{2}{*}{$\begin{array}{l}\text { Stan- } \\
\text { dard- } \\
\text { abw. }\end{array}$} & \multirow{2}{*}{\begin{tabular}{c|} 
Mit- \\
tel- \\
wert
\end{tabular}} & \multicolumn{5}{|c|}{ Häufigkeiten } \\
\hline & & & & 0 & $1-9$ & $10-19$ & $20-49$ & 250 & & & & 0 & 1.4 & 5-10 & $11-20$ & $>20$ \\
\hline \multirow{2}{*}{$\begin{array}{l}\text { Informations- } \\
\text { gespräch(e) } \\
\text { (z.B. Info.-1 } \\
\text { Erfahrungs- } \\
\text { austausch) }\end{array}$} & $\begin{array}{c}19 \% \\
(6)\end{array}$ & 208,03 & 115 & $\begin{array}{l}8 \% \\
(2)\end{array}$ & $\begin{array}{l}12 \% \\
(3)\end{array}$ & $\begin{array}{l}8 \% \\
(2)\end{array}$ & $\begin{array}{c}27 \% \\
(7)\end{array}$ & $\begin{array}{l}46 \% \\
(12)\end{array}$ & $\begin{array}{c}56 \% \\
(18)\end{array}$ & 26,50 & 14 & $\begin{array}{c}14 \% \\
(2)\end{array}$ & $\begin{array}{c}21 \% \\
(3)\end{array}$ & $\begin{array}{c}43 \% \\
(6)\end{array}$ & $\begin{array}{l}7 \% \\
(1)\end{array}$ & $\begin{array}{c}14 \% \\
(2)\end{array}$ \\
\hline & $\begin{array}{l}7 \% \\
(1)\end{array}$ & 39,66 & 63 & - & $\begin{array}{l}7 \% \\
(1)\end{array}$ & $\begin{array}{l}7 \% \\
(1)\end{array}$ & $\begin{array}{c}29 \% \\
(4)\end{array}$ & $\begin{array}{c}57 \% \\
(8)\end{array}$ & $\begin{array}{l}7 \% \\
(1)\end{array}$ & 14,71 & 19 & \begin{tabular}{|l|}
$7 \%$ \\
$(1)$ \\
\end{tabular} & $\begin{array}{l}7 \% \\
(1)\end{array}$ & $\begin{array}{c}29 \% \\
(4)\end{array}$ & $\begin{array}{c}29 \% \\
(4)\end{array}$ & $\begin{array}{c}29 \% \\
(4)\end{array}$ \\
\hline \multirow{2}{*}{$\begin{array}{l}\text { Gespräch(e) } \\
\text { der } \\
\text { Beziehungs- } \\
\text { pflege }\end{array}$} & $\begin{array}{c}19 \% \\
(6)\end{array}$ & 21,94 & 11 & $\begin{array}{c}31 \% \\
(8)\end{array}$ & $\begin{array}{c}42 \% \\
(11)\end{array}$ & $\begin{array}{c}12 \% \\
(3)\end{array}$ & $\begin{array}{c}8 \% \\
(2)\end{array}$ & \begin{tabular}{|c|}
$8 \%$ \\
$(2)$ \\
\end{tabular} & $\begin{array}{r}63 \% \\
(20)\end{array}$ & 8,43 & 5 & $\begin{array}{c}33 \% \\
(4)\end{array}$ & $\begin{array}{c}33 \% \\
(4)\end{array}$ & $\begin{array}{c}25 \% \\
(3)\end{array}$ & - & $\begin{array}{c}8 \% \\
(1)\end{array}$ \\
\hline & $\begin{array}{l}7 \% \\
(1)\end{array}$ & 10,27 & 10 & \begin{tabular}{l|}
$7 \%$ \\
$(1)$
\end{tabular} & $\begin{array}{c}43 \% \\
(6)\end{array}$ & $\begin{array}{c}29 \% \\
(4)\end{array}$ & $\begin{array}{c}21 \% \\
(3)\end{array}$ & - & \begin{tabular}{c|}
$7 \%$ \\
$(1)$
\end{tabular} & 10,47 & 10 & $\begin{array}{c}14 \% \\
(2)\end{array}$ & $\begin{array}{c}29 \% \\
(4)\end{array}$ & $\begin{array}{c}29 \% \\
(4)\end{array}$ & $\begin{array}{c}14 \% \\
(2)\end{array}$ & $\begin{array}{c}14 \% \\
(2)\end{array}$ \\
\hline \multirow{2}{*}{$\begin{array}{l}\text { Gespräch(e) } \\
\text { über mögliche } \\
\text { zukünftige } \\
\text { Projekt- } \\
\text { kooperationen }\end{array}$} & $\begin{array}{c}19 \% \\
(6)\end{array}$ & 14,95 & 11 & $\begin{array}{c}8 \% \\
(2)\end{array}$ & $\begin{array}{r}54 \% \\
(14) \\
\end{array}$ & $\begin{array}{c}23 \% \\
(6)\end{array}$ & $\begin{array}{c}8 \% \\
(2)\end{array}$ & $\begin{array}{l}8 \% \\
(2) \\
\end{array}$ & $\begin{array}{c}53 \% \\
(17) \\
\end{array}$ & 5,58 & 5 & $\begin{array}{c}0 \% \\
(0)\end{array}$ & $\begin{array}{c}80 \% \\
(12)\end{array}$ & $\begin{array}{c}13 \% \\
(2)\end{array}$ & - & $\begin{array}{c}7 \% \% \\
(1) \\
\end{array}$ \\
\hline & $\begin{array}{l}7 \% \\
(1)\end{array}$ & 19,19 & 16 & \begin{tabular}{|l|}
$7 \%$ \\
$(1)$
\end{tabular} & $\begin{array}{c}50 \% \\
(7)\end{array}$ & \begin{tabular}{|l|}
$7 \%$ \\
$(1)$
\end{tabular} & $\begin{array}{c}21 \% \\
(3)\end{array}$ & $\begin{array}{c}14 \% \\
(2)\end{array}$ & $\begin{array}{l}7 \% \\
(1)\end{array}$ & 11,25 & 12 & $\begin{array}{c}7 \% \\
(1) \\
\end{array}$ & \begin{tabular}{c|}
$21 \%$ \\
$(3)$
\end{tabular} & $\begin{array}{c}36 \% \\
(5)\end{array}$ & $\begin{array}{c}14 \% \\
(2)\end{array}$ & $\begin{array}{c}21 \% \\
(3)\end{array}$ \\
\hline \multirow{2}{*}{$\begin{array}{l}\text { konkrete } \\
\text { Bespre- } \\
\text { chung(en) } \\
\text { zukünftiger } \\
\text { Projekte }\end{array}$} & $\begin{array}{c}28 \% \\
(9)\end{array}$ & 8,35 & 3 & \begin{tabular}{|l|}
$44 \%$ \\
$(10)$
\end{tabular} & $\begin{array}{l}48 \% \\
(11)\end{array}$ & \begin{tabular}{|c|}
$8 \%$ \\
$(2)$ \\
\end{tabular} & - & - & \begin{tabular}{|l|}
$63 \%$ \\
$(20)$
\end{tabular} & 4,70 & 3 & $\begin{array}{c}33 \% \\
(4)\end{array}$ & $\begin{array}{c}50 \% \\
(6)\end{array}$ & \begin{tabular}{|c|}
$8 \%$ \\
$(1)$
\end{tabular} & \begin{tabular}{|l|}
$8 \%$ \\
$(1)$ \\
\end{tabular} & - \\
\hline & $\begin{array}{l}7 \% \\
(1)\end{array}$ & 3,19 & 3 & \begin{tabular}{|l|}
$43 \%$ \\
$(6)$
\end{tabular} & $\begin{array}{c}50 \% \\
(7)\end{array}$ & \begin{tabular}{|c|}
$87 \%$ \\
(1)
\end{tabular} & - & - & $\begin{array}{l}7 \% \\
(1)\end{array}$ & 3,19 & 3 & $\begin{array}{c}43 \% \\
(6)\end{array}$ & $\begin{array}{c}21 \% \\
(3)\end{array}$ & $\begin{array}{c}36 \% \\
(5)\end{array}$ & - & \\
\hline
\end{tabular}

Tab. 13: Messetypspezifischer Vergleich der Kontaktqualität und -quantität der geführten Hochschul-Messestand-Gespräche

Die exponententypspezifische Analyse zeigt für Projekt- und exponatorientierte Hochschul-Exponenten im Vergleich zu den anderen Exponententypen eine hohe Anzahl an realisierten projektbezogenen Gesprächen, bei denen die Wahrscheinlichkeiten zu einer Fortführung in der Nach-Messe-Phase als relativ hoch eingeschätzt wurde (vgl. Tab. 14). Dieses Ergebnis deutet auf eine intensive Nutzung der Messegespräche nicht nur zur Erreichung der hoch priorisierten Informationsziele, sondern auch i.S.d. angestrebten Projektziele hin. Somit konnten die Projekt- und exponatorientierten Hochschul-Exponenten mit den auf dem Messestand geführten Gesprächen die Erreichung ihrer primär verfolgten Messeziele fördern. Auch den Beeinflussungsorientierten Hochschul-Exponenten, die gegenüber den anderen Exponententypen im Durchschnitt die meisten Informationsgespräche führten, boten sich mit diesen Gesprächen Möglichkeiten zur Erreichung ihrer hoch priorisierten Messezielsetzungen. Demgegenüber realisierten die Pflichtrepräsentanten vergleichsweise wenig Hochschul-Messestand-Gespräche, worin 
sich die geringe Messebeteiligungsmotivation dieses Exponententyps widerspiegelt.

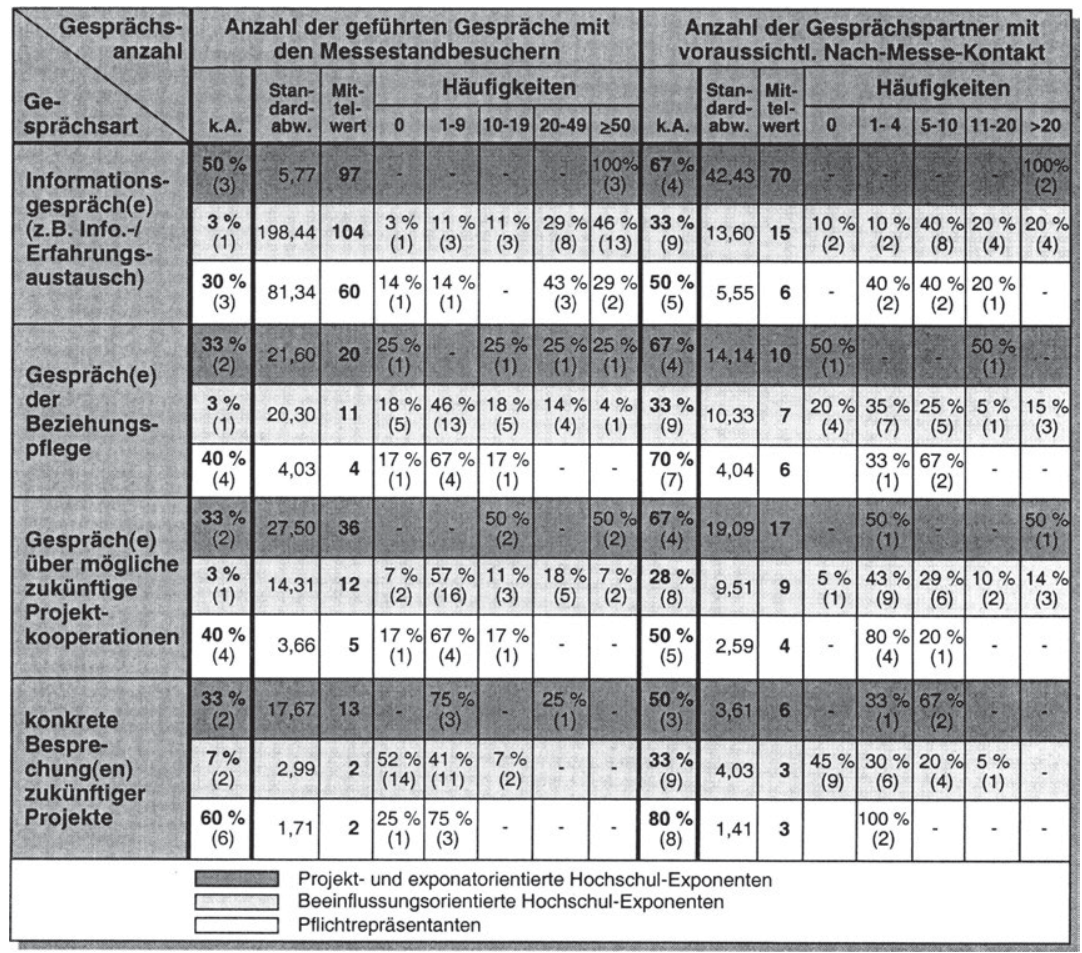

Tab. 14: Exponententypspezifischer Vergleich der Kontaktqualität und -quantität der geführten Hochschul-Messestand-Gespräche

Im Rahmen der indirekten Erfolgskontrolle von Hochschul-Messebeteiligungen sind nicht nur die Inhalte der geführten Gespräche von Relevanz, sondern auch die Gesprächspartner, mit denen Gespräche geführt wurden und deren Zugehörigkeit zu hochschulspezifischen Messezielgruppen. ${ }^{47}$ Wie Tab. 15 verdeut-

In der Messe-Phase wurden die Inhalte der Gespräche und die Zugehörigkeit der Gesprächspersonen zu den einzelnen hochschulspezifischen Zielgruppen getrennt erfaßt, so daß keine Informationen darüber vorliegen, mit welchen Zielgruppen welche Art von Gesprächen während der Messe geführt wurde. 
licht, nahmen die Hochschul-Exponenten im Rahmen ihrer Messebeteiligung zahlreiche Kontakte zu hochschulspezifischen Messezielgruppen auf. So wurden vor allem Gespräche mit den Zielgruppen Öffentlichkeit und Wirtschaft geführt und damit Kontakte zu den aus Sicht der Hochschul-Exponenten wichtigsten Messezielgruppen realisiert. Zudem bleibt - nach Einschätzung der Exponenten - der Kontakt zur Wirtschaft nach der Messe relativ häufig bestehen. Die weniger wichtige Zielgruppe der Studierenden nahm zu den Exponenten gleichwohl viele Kontakte auf, wobei sich diese Kontakte i.d.R. auf die Messe-Phase beschränkten. Diese Kontakte sind vor dem Hintergrund der von den Hochschul-Exponenten primär verfolgten Messezielsetzungen als wenig zielführend zu bewerten. Die Kontaktzahlen zu Wissenschaftsvertretern fielen gegenüber Kontakten mit Studierenden in der Messe-Phase geringer, die Intensivierungswahrscheinlichkeit in der Nach-Messe-Phase höher aus. So gingen die Hochschul-Exponenten davon aus, etwa die Hälfte der in der Messe-Phase geknüpften Kontakte in der Nach-MessePhase intensivieren zu können. Mit Vertretern des Staates wurden vergleichsweise wenig Kontakte aufgenommen. Begründen läßt sich dies vor allem mit der insgesamt relativ schwachen Vertretung dieser Zielgruppe auf den betrachteten Messen.

\begin{tabular}{|c|c|c|c|c|c|c|c|c|c|c|c|c|c|c|c|c|}
\hline \multirow{3}{*}{$\begin{array}{l}\text { Messe- } \\
\text { zielgruppe }\end{array}$} & \multirow[b]{3}{*}{ k.A. } & \multicolumn{7}{|c|}{$\begin{array}{l}\text { Anzahl der Kontakte bzw. } \\
\text { der geführten Gespräche }\end{array}$} & \multicolumn{8}{|c|}{$\begin{array}{l}\text { Anzahl der Kontakte, die voraussichtl. } \\
\text { nach der Messe intensiviert werden }\end{array}$} \\
\hline & & \multirow{2}{*}{$\begin{array}{l}\text { Stan- } \\
\text { dard- } \\
\text { abw. }\end{array}$} & \multirow{2}{*}{\begin{tabular}{|c|}
$\begin{array}{c}\text { Mit- } \\
\text { tel- } \\
\text { wert }\end{array}$ \\
\end{tabular}} & \multicolumn{5}{|c|}{ Häufigkeiten } & \multirow[b]{2}{*}{ k.A. } & \multirow{2}{*}{$\begin{array}{l}\text { Stan- } \\
\text { dard- } \\
\text { abw. }\end{array}$} & \multirow{2}{*}{\begin{tabular}{|l|} 
Mit- \\
tel- \\
wert \\
\end{tabular}} & \multicolumn{5}{|c|}{ Häufigkeiten } \\
\hline & & & & 0 & $1-9$ & $10-19$ & $20-49$ & 250 & & & & 0 & $1-4$ & $5-10$ & $11-20$ & $>20$ \\
\hline Wissenschaft & $\begin{array}{c}15 \% \\
(7)\end{array}$ & 8,57 & 11 & - & $\begin{array}{c}55 \% \\
(20)\end{array}$ & $\begin{array}{c}30 \% \\
(12)\end{array}$ & $\begin{array}{c}15 \% \\
(6)\end{array}$ & - & $\begin{array}{c}28 \% \\
(13)\end{array}$ & 5,41 & 5 & $\begin{array}{c}6 \% \\
(2)\end{array}$ & $\begin{array}{c}56 \% \\
(19)\end{array}$ & $\begin{array}{l}29 \% \\
(10)\end{array}$ & $\begin{array}{c}6 \% \\
(2)\end{array}$ & $\begin{array}{l}3 \% \\
(1)\end{array}$ \\
\hline Studierende & $\begin{array}{c}15 \% \\
(7)\end{array}$ & 30,57 & 27 & - & $\begin{array}{l}33 \% \\
(13)\end{array}$ & $\begin{array}{c}23 \% \\
(9)\end{array}$ & $\begin{array}{l}30 \% \\
(12)\end{array}$ & $\begin{array}{c}15 \% \\
(6)\end{array}$ & $\begin{array}{c}43 \% \\
(20)\end{array}$ & 7,37 & 3 & $\begin{array}{c}56 \% \\
(15)\end{array}$ & $\begin{array}{c}30 \% \\
(8)\end{array}$ & $\begin{array}{l}4 \% \\
(1)\end{array}$ & $\begin{array}{l}4 \% \\
(1)\end{array}$ & $\begin{array}{l}7 \% \\
(2)\end{array}$ \\
\hline Wirtschaft & $\begin{array}{c}17 \% \\
(8)\end{array}$ & 49,71 & 34 & $\begin{array}{l}5 \% \\
(2)\end{array}$ & $\begin{array}{c}23 \% \\
(9)\end{array}$ & $\begin{array}{c}23 \% \\
(9)\end{array}$ & $\begin{array}{c}26 \% \\
(10)\end{array}$ & $\begin{array}{c}23 \% \\
(9)\end{array}$ & $\begin{array}{c}30 \% \\
(14)\end{array}$ & 11,90 & 14 & $\begin{array}{c}3 \% \\
(1)\end{array}$ & $\begin{array}{c}27 \% \\
(9)\end{array}$ & $\begin{array}{c}24 \% \\
(8)\end{array}$ & $\begin{array}{c}18 \% \\
(6)\end{array}$ & $\begin{array}{c}27 \% \\
(9)\end{array}$ \\
\hline Öffentlichkeit & $\begin{array}{c}19 \% \\
(9)\end{array}$ & 48,75 & 59 & $\begin{array}{c}16 \% \\
(18)\end{array}$ & \begin{tabular}{|c|}
$49 \%$ \\
$(54)$
\end{tabular} & \begin{tabular}{|c|}
$15 \%$ \\
$(17)$
\end{tabular} & $\begin{array}{l}13 \% \\
(14)\end{array}$ & $\begin{array}{c}7 \% \\
(8)\end{array}$ & $\begin{array}{c}51 \% \\
(24)\end{array}$ & 1,25 & 5 & $\begin{array}{r}45 \% \\
(30)\end{array}$ & $\begin{array}{l}45 \% \\
(30)\end{array}$ & $\begin{array}{l}7 \% \\
(5)\end{array}$ & $\begin{array}{l}2 \% \\
\text { (1) }\end{array}$ & $\begin{array}{l}2 \% \\
\text { (1) }\end{array}$ \\
\hline Staat & $\begin{array}{c}19 \% \\
(9)\end{array}$ & 2,60 & 2 & $\begin{array}{r}42 \% \\
(16)\end{array}$ & \begin{tabular}{|c|}
$55 \%$ \\
$(21)$
\end{tabular} & $\begin{array}{c}3 \% \\
(1)\end{array}$ & - & - & $\begin{array}{c}43 \% \\
(20)\end{array}$ & 1,30 & 1 & $\begin{array}{c}57 \% \\
(15)\end{array}$ & $\begin{array}{c}41 \% \\
(11)\end{array}$ & $\begin{array}{l}4 \% \\
\text { (1) }\end{array}$ & - & - \\
\hline
\end{tabular}

Tab. 15: Analyse der Kontakte zwischen Hochschul-Exponenten und hochschulspezifischen Messezielgruppen 
Sowohl die Mehrbranchenmesse- als auch die Fachmesse-Exponenten - so zeigt die messetypspezifische Untersuchung - führten im Rahmen ihrer Messebeteiligung zahlreiche Gespräche mit den von innen hoch priorisierten Zielgruppen (vgl. Tab. 16). Dabei konnten Fachmesse-Exponenten mit Vertretern der Wirtschaft mehr Gespräche realisieren als die Exponenten der Mehrbranchenmesse. ${ }^{48}$ Die gleiche Tendenz war in bezug auf die Intensivierung der Kontakte in der NachMesse-Phase feststellbar. Hingegen wurden in der Messe-Phase auf der Mehrbranchenmesse mehr Gespräche mit der allgemeinen Öffentlichkeit geführt. Auch wenn die Fortführung dieser Kontakte in der Nach-Messe-Phase i.d.R. als unwahrscheinlich eingeschätzt wurde, konnten die Hochschul-Exponenten der Mehrbranchenmessen insgesamt ihre hoch priorisierte Zielgruppe Öffentlichkeit besser erreichen als die Fachmesse-Exponenten.

\begin{tabular}{|c|c|c|c|c|c|c|c|c|c|c|c|c|c|c|c|c|}
\hline \multirow{3}{*}{$\begin{array}{r}\begin{array}{r}\text { Kontakte/ } \\
\text { Gesprä- } \\
\text { che }\end{array} \\
\text { Messe- } \\
\text { zielgruppe }\end{array}$} & \multirow[b]{3}{*}{ k.A. } & \multicolumn{7}{|c|}{$\begin{array}{l}\text { Anzahl der Kontakte bzw. } \\
\text { der geführten Gespräche }\end{array}$} & \multicolumn{8}{|c|}{$\begin{array}{l}\text { Anzahl der Kontakte, die voraussichtl. } \\
\text { nach der Messe intensiviert werden }\end{array}$} \\
\hline & & \multirow{2}{*}{$\begin{array}{l}\text { Stan- } \\
\text { dard- } \\
\text { abw. }\end{array}$} & \multirow{2}{*}{$\begin{array}{l}\text { Mit- } \\
\text { tel- } \\
\text { wert }\end{array}$} & \multicolumn{5}{|c|}{ Häufigkeiten } & \multirow[b]{2}{*}{ k.A. } & \multirow{2}{*}{$\begin{array}{l}\text { Stan- } \\
\text { dard- } \\
\text { abw. }\end{array}$} & \multirow{2}{*}{\begin{tabular}{|c|} 
Mit- \\
tel- \\
wert
\end{tabular}} & \multicolumn{5}{|c|}{ Häufigkeiten } \\
\hline & & & & 0 & $1-9$ & $10-19$ & $20-49$ & 250 & & & & 0 & $1-4$ & $5-10$ & $11-20$ & $>20$ \\
\hline \multirow{2}{*}{ Wissenschaft } & $\begin{array}{c}19 \% \\
(6) \\
\end{array}$ & 7,11 & 10 & - & \begin{tabular}{c|c|}
$46 \%$ \\
$(12)$
\end{tabular} & $\begin{array}{c}31 \% \\
(8)\end{array}$ & $\begin{array}{c}15 \% \\
(4)\end{array}$ & - & $\begin{array}{l}38 \% \\
(12)\end{array}$ & 3,99 & 4 & \begin{tabular}{c|c|}
$10 \%$ \\
$(2)$ \\
\end{tabular} & \begin{tabular}{r|r|}
$55 \%$ \\
$(11)$ \\
\end{tabular} & \begin{tabular}{|c|}
$30 \%$ \\
$(6)$ \\
\end{tabular} & $\begin{array}{l}5 \% \\
(1) \\
\end{array}$ & - \\
\hline & $\begin{array}{l}7 \% \\
\text { (1) }\end{array}$ & 11,04 & 11 & - & $\begin{array}{c}57 \% \\
(8)\end{array}$ & $\begin{array}{c}27 \% \\
(4)^{2}\end{array}$ & $\begin{array}{c}14 \% \\
(2)\end{array}$ & - & $\begin{array}{l}7 \% \\
\text { (1) }\end{array}$ & 6,85 & 7 & - & $\begin{array}{c}57 \% \\
(8)\end{array}$ & $\begin{array}{c}29 \% \\
(4)\end{array}$ & $\begin{array}{l}7 \% \\
(1)\end{array}$ & $\begin{array}{l}7 \% \\
\text { (1) }\end{array}$ \\
\hline \multirow{2}{*}{ Studierende } & $\begin{array}{c}19 \% \\
(6)\end{array}$ & 29,53 & 29 & - & $\begin{array}{c}31 \% \\
(8)\end{array}$ & $\begin{array}{c}19 \% \\
(5)\end{array}$ & $\begin{array}{c}35 \% \\
(9)\end{array}$ & $\begin{array}{c}15 \% \\
(4)\end{array}$ & $\begin{array}{l}59 \% \\
(19) \\
\end{array}$ & 1,53 & 1 & $\begin{array}{c}22 \% \\
(7)\end{array}$ & $\begin{array}{c}39 \% \\
(5)\end{array}$ & $\begin{array}{l}3 \% \\
(1)\end{array}$ & - & - \\
\hline & $\begin{array}{l}7 \% \\
(1) \\
\end{array}$ & 33,22 & 24 & - & \begin{tabular}{|l|}
$36 \%$ \\
$(5)$
\end{tabular} & $29 \%$ & $21 \%$ & \begin{tabular}{|c|}
$14 \%$ \\
$(2)$ \\
\end{tabular} & $\begin{array}{l}7 \% \\
(1) \\
\end{array}$ & 9,81 & 5 & \begin{tabular}{|l|}
$57 \%$ \\
$(8)$ \\
\end{tabular} & \begin{tabular}{c|c|}
$21 \%$ \\
$(3)$
\end{tabular} & - & $\begin{array}{l}7 \% \\
(1) \\
\end{array}$ & $\begin{array}{l}14 \% \\
(2)\end{array}$ \\
\hline \multirow{2}{*}{ Wirtschaft } & $\begin{array}{c}22 \% \\
(7)^{2} \\
\end{array}$ & 59,44 & 34 & $\begin{array}{l}4 \% \\
(1) \\
\end{array}$ & $\begin{array}{c}32 \% \\
(8)\end{array}$ & $\begin{array}{c}28 \% \\
(7)\end{array}$ & $\begin{array}{c}20 \% \\
(5)^{2}\end{array}$ & $\begin{array}{c}16 \% \\
(4)\end{array}$ & $\begin{array}{l}41 \% \\
(13) \\
\end{array}$ & 11,83 & 11 & - & $\begin{array}{c}37 \% \\
(7)\end{array}$ & $\begin{array}{c}32 \% \\
(6) \\
\end{array}$ & $\begin{array}{c}11 \% \\
(2)\end{array}$ & $\begin{array}{c}21 \% \\
(4)^{2}\end{array}$ \\
\hline & $\begin{array}{l}7 \% \\
(1) \\
\end{array}$ & 26,37 & 36 & $\begin{array}{l}7 \% \\
\text { (1) } \\
\end{array}$ & $\begin{array}{l}7 \% \\
(1)\end{array}$ & $\begin{array}{c}14 \% \\
(2)\end{array}$ & $\begin{array}{c}36 \% \\
(5)\end{array}$ & $\begin{array}{c}36 \% \\
(5) \\
\end{array}$ & $\begin{array}{l}7 \% \\
(17) \\
\end{array}$ & 11,71 & 17 & $\begin{array}{l}7 \% \\
(1) \\
\end{array}$ & $\begin{array}{c}14 \% \\
(2)\end{array}$ & $\begin{array}{c}14 \% \\
(2)\end{array}$ & $\begin{array}{c}29 \% \\
(4)\end{array}$ & $\begin{array}{c}36 \% \\
(5) \\
\end{array}$ \\
\hline \multirow{2}{*}{ Öffentlichkeit } & $\begin{array}{c}19 \% \\
(6)\end{array}$ & 64,23 & 92 & $\begin{array}{l}19 \% \\
(15)\end{array}$ & $\begin{array}{l}44 \% \\
(34) \\
\end{array}$ & $\begin{array}{l}15 \% \\
(12)\end{array}$ & $\begin{array}{c}12 \% \\
(9)\end{array}$ & $9 \%$ & $\begin{array}{l}66 \% \\
(21)\end{array}$ & 1,64 & 4 & $\begin{array}{l}42 \% \\
(14) \\
\end{array}$ & $\begin{array}{l}52 \% \\
(17) \\
\end{array}$ & $\begin{array}{l}6 \% \\
(2) \\
\end{array}$ & - & - \\
\hline & $\begin{array}{c}20 \% \\
(3)\end{array}$ & 9,56 & 24 & $\begin{array}{l}9 \% \\
3) \\
\end{array}$ & \begin{tabular}{l|}
$59 \%$ \\
$(20)$ \\
\end{tabular} & $\begin{array}{c}15 \% \\
(5)\end{array}$ & $\begin{array}{c}15 \% \\
(5)\end{array}$ & \begin{tabular}{|l|}
$3 \%$ \\
$(1)$ \\
\end{tabular} & $\begin{array}{c}20 \% \\
(3)\end{array}$ & 1,98 & 7 & \begin{tabular}{|l|}
$47 \%$ \\
$16)$ \\
\end{tabular} & $\begin{array}{l}38 \% \\
(13) \\
\end{array}$ & $\begin{array}{l}9 \% \\
(3)\end{array}$ & $\begin{array}{l}3 \% \\
(1) \\
\end{array}$ & $\begin{array}{l}3 \% \\
\text { (1) } \\
\end{array}$ \\
\hline \multirow{2}{*}{ Staat } & $\begin{array}{c}25 \% \\
(8)\end{array}$ & 2,34 & 2 & $\begin{array}{c}33 \% \\
(8) \\
\end{array}$ & $\begin{array}{l}67 \% \\
(16) \\
\end{array}$ & - & - & - & $\begin{array}{l}59 \% \\
(19)\end{array}$ & 1,14 & 1 & \begin{tabular}{|c|}
$39 \%$ \\
$(5)$
\end{tabular} & \begin{tabular}{c|}
$62 \%$ \\
$(8)$
\end{tabular} & $\begin{array}{l}0 \% \\
(0)\end{array}$ & - & - \\
\hline & $\begin{array}{l}7 \% \\
(1)\end{array}$ & 3,07 & 2 & $\begin{array}{l}57 \% \\
(8)\end{array}$ & $\begin{array}{c}36 \% \\
(5)\end{array}$ & $\begin{array}{l}7 \% \\
\text { (1) }\end{array}$ & - & - & $\begin{array}{l}7 \% \\
(1)\end{array}$ & 1,44 & 1 & \begin{tabular}{r|}
$71 \%$ \\
$(10)$ \\
\end{tabular} & $\begin{aligned} 22 \% \\
(3)\end{aligned}$ & $\begin{array}{l}7 \% \\
\text { (1) }\end{array}$ & - & - \\
\hline & & $\mathrm{N}$ & & & & & & & & & & & & & & \\
\hline
\end{tabular}

Tab. 16: Messetypspezifische Analyse der Kontakte zwischen Hochschul-Exponenten und hochschulspezifischen Messezielgruppen

48 Fachmesse-Exponenten realisierten mehr Praxiskontakte in der Messe-Phase, obwohl hier weniger Messezeit zur Verfügung stand. Während die betrachtete Mehrbranchenmesse an sechs Messetagen jeweils neun Stunden zu besuchen war, lag die Öffnungsdauer der betrachteten Fachmesse bei fünf Messetagen mit jeweils acht Stunden. 
Die exponententypspezifische Analyse belegt, daß hochschulspezifische Exponententypen insbesondere Gespräche mit den Zielgruppen führten, mit denen eine Kontaktaufnahme zur Erreichung ihrer hoch priorisierten Messeziele notwendig erschien (vgl. Tab. 17). So führten Projekt- und exponatorientierte HochschulExponenten am häufigsten Gespräche mit Wirtschaftsvertretern. Dabei schätzten diese Exponenten eine Fortführung der Kontakte in der Nach-Messe-Phase als vergleichsweise hoch ein, welches auf eine grundsätzliche Erreichung verfolgter Projektziele schließen läßt. Beeinflussungsorientierte Hochschul-Exponenten realisierten die meisten Gespräche mit der Zielgruppe Öffentlichkeit. Damit konnte dieser Exponententyp seine primär verfolgten Beeinflussungsziele entsprechend fördern. Werden die Messegespräche der Pflichtrepräsentanten betrachtet, so ist im Vergleich zu den anderen Hochschul-Exponententypen eine hohe Anzahl an Gesprächen mit Vertretern des Staates zu konstatieren. Auch wenn die zuvor dargestellten Ergebnisse auf ein wenig engagiertes Messebeteiligungsverhalten des Pflichtrepräsentanten hinweisen, so drückt sich das Motiv seiner Messebeteiligung zumindest in seinem zielgruppenspezifischen Gesprächsverhalten aus. Gerade diese Kontakte bieten Pflichtrepräsentanten die Möglichkeit dem Staat zu dokumentierten, daß sie ihrer Pflicht als öffentlich-rechtliche Institution entsprechend nachkommen.

Auch wenn die von den einzelnen Hochschul-Exponenten mit den jeweiligen Messezielgruppen geführten Gespräche gemäß der primär verfolgten Zielsetzungen realisiert wurden, so spiegelt das Gesprächsverhalten der Exponenten nicht die exponententypspezifischen Zielgruppenpriorisierungen wider. Dieses Ergebnis ist aufgrund der empirisch ermittelten, relativ geringen Unterschiede zwischen den exponententypspezifischen Zielgruppengewichtungen wenig überraschend. Obwohl diese Zielgruppenpriorisierungen auf nicht konsistente, exponententypspezifische Planungen von Messebeteiligungen schließen lassen, so ist angesichts des zielgruppenbezogenen Gesprächsverhaltens der Exponententypen von einer exponententypspezifischen Durchführung von Messebeteiligungen auszugehen. 


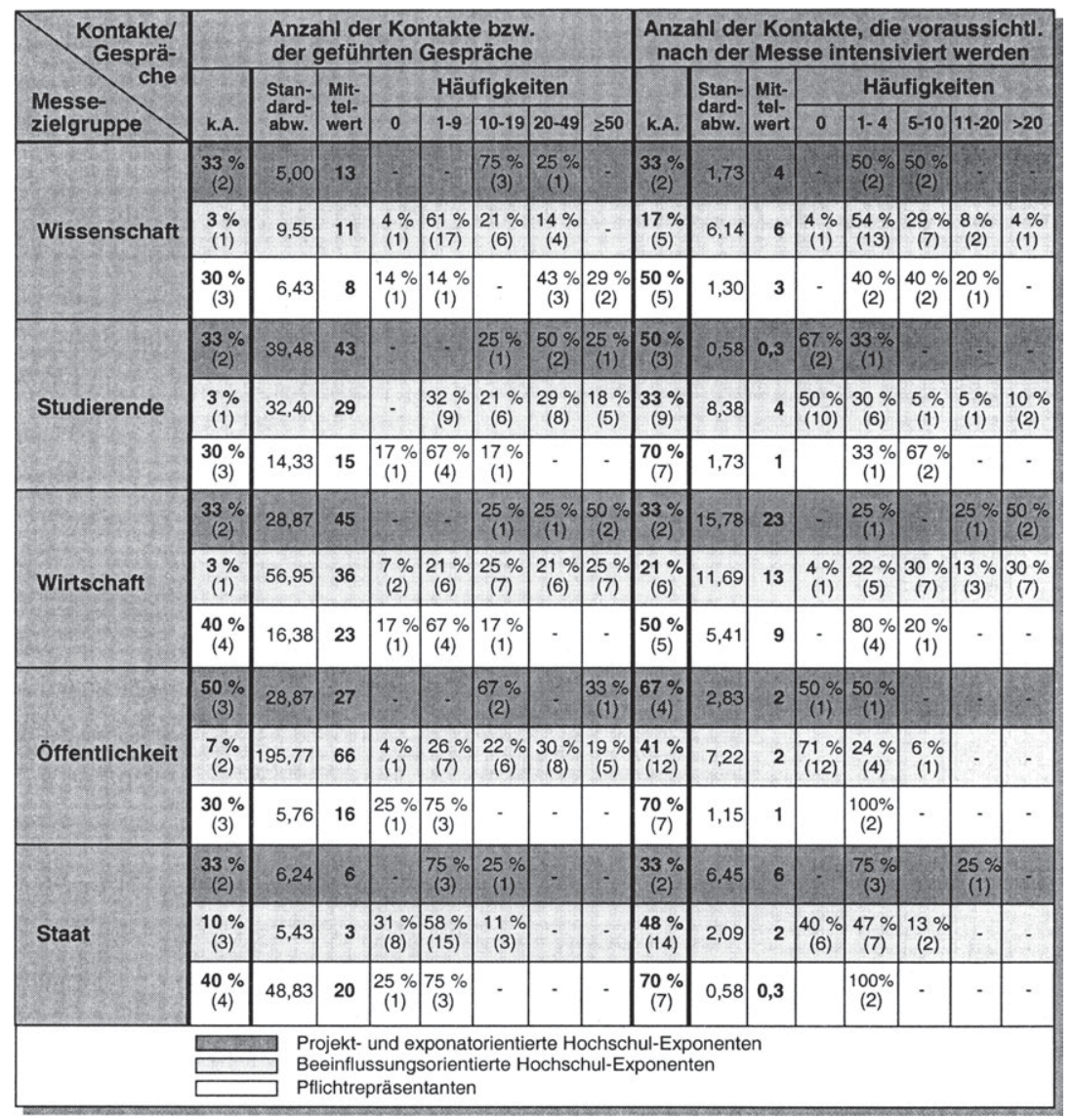

Tab. 17: Analyse der Kontakte zwischen Hochschul-Exponententypen und hochschulspezifischen Messezielgruppen

Neben Beobachtungen, zu denen die vorangegangenen Methoden i.w.S. zu zählen sind, können in der Messe-Phase zur Messeerfolgskontrolle auch Befragungen zum Einsatz kommen. Befragungen während einer Messeveranstaltung umfassen dabei z.B. die Ermittlung von Hochschul-Messestand-Besucherstrukturen und die Beurteilung des Hochschul-Messestands im Hinblick auf das wahrgenommene Informationsangebot, die Verständlichkeit der Informationen, die Aus- 
wahl der Exponate, die Qualität der Kontakte mit dem Standpersonal, seine Freundlichkeit und die Attraktivität des Hochschul-Messestands insgesamt. ${ }^{49}$

Mit der Befragung von Hochschul-Messestand-Besuchern steht den Hochschul-Exponenten ein flexibles Verfahren zur Kontrolle des indirekten Erfolgs ihrer Messebeteiligung zur Verfügung. Dabei kann diese Methode sowohl isoliert als auch in Kombination mit den zuvor aufgeführten beobachtenden Methoden eingesetzt werden. Diese Flexibilität besitzt die Befragung für Hochschul-Exponenten deshalb, weil sowohl der Kreis der Befragten als auch die Befragungsinhalte an individuelle Zielsetzungen der Hochschul-Exponenten angepaßt werden können. ${ }^{50}$ Steht die Erfassung der Besucherstruktur des Hochschul-Messestands im Vordergrund der Erhebung, kann in diesem Zusammenhang auch von Besucherstrukturbefragungen gesprochen werden. Bei Intensivbefragungen von Messebesuchern liegt hingegen der Schwerpunkt auf der Behandlung komplexer Themen. ${ }^{51}$ Die verschiedenen Formen der Befragungen von Hochschul-MessestandBesuchern stellen für die Hochschul-Exponenten zentrale Informationsquellen zur Überprüfung ihrer Zielgruppen- und Messezielerreichung insbesondere hinsichtlich psychographischer Zielsetzungen - wie Beeinflussungsziele - dar. Zudem können im Rahmen von Besucherbefragungen Anhaltspunkte einer Realisierung von Projektzielen gewonnen werden.

Der Einsatz von Befragungen weist den Vorteil auf, nicht beobachtbare Reaktionen der Hochschul-Messestand-Besucher auf bestimmte Maßnahmen des Hochschul-Exponenten erheben zu können. So ist es möglich, Informationen über die Wirkung von messebezogenen Maßnahmen hinsichtlich der Einstellungen und

Vgl. Gelszus, R., Messen + Ausstellungen: Vergessen Sie die Messe-Wirkungs-Kontrolle nicht, a.a.O., S. $172 \mathrm{ff}$.

Vgl. Rost, D., Maßstäbe zur Beurteilung der Effizienz von Messen, in: VDI-Nachrichten, Nr. 115, 1973, S. $333 \mathrm{ff}$.

Während bei Besucherstrukturbefragungen die Auswahl der Auskunftspersonen nach dem Zufallsprinzip aus allen Messestand-Besuchern erfolgt, wird bei der Besucherintensivbefragung eine Selektion der Auskunftspersonen nach Zielgruppen vorgenommen. Dabei werden nur solche Messestand-Besucher um ein weiterführendes Interview gebeten, die sich nach einigen Fragen als Mitglied der Messezielgruppe erweisen. Zu den wesentlichen Unterschieden der beiden Befragungsarten vgl. Gelszus Messe-Marktforschung GmbH (Hrsg.), Mehr Erfolg auf Messen - Wie Sie Ihren Erfolg messen und neue Potentiale entdecken, a.a.O., S. 9 ff. 
Meinungen der Hochschul-Messestand-Besucher zu erheben. ${ }^{52}$ Dennoch stehen diesen Aspekten Nachteile gegenüber, die sich generell für Befragungen anführen lassen. ${ }^{53}$ So müßte zur weitgehend repräsentativen Befragung streng genommen die Grundgesamtheit der Hochschul-Messestand-Besucher vor der Messeveranstaltung bekannt sein. Diese kann jedoch zahlenmäßig erst nach Beendigung der Messe erfaßt werden. Trotz dieser zu berücksichtigenden Einschränkungen ist der Einsatz von Besucherbefragungen aufgrund ihres hohen Informationsgehaltes - wenngleich deren Einsatz nicht zu unterschätzender finanzieller und personeller Ressourcen bedarf - für die Hochschul-Exponenten empfehlenswert. Demzufolge wurde sie in der vorliegenden Untersuchung ergänzend zu den zuvor durchgeführten Erfolgskontrollmethoden eingesetzt. Dabei beschränkten sich die Analysen auf eine messetypspezifische Betrachtung, da eine exponententypspezifische Befragung von Hochschul-Messestand-Besuchern mit nicht vertretbarem Aufwand verbunden gewesen wäre. Im Rahmen der Befragungen standen vor allem die Besucherstruktur und die Ziele eines Hochschul-MessestandBesuchs sowie die Attraktivität der präsentierten Exponate im Vordergrund.

Insbesondere die Gegenüberstellung der im Rahmen der Besucherbefragung erhobenen Hochschul-Messestand-Besucherstruktur mit der aus Sicht der Hochschul-Exponenten vorgenommenen Priorisierung ihrer Messezielgruppen liefert wichtige Erkenntnisse. Wie die folgende Abbildung verdeutlicht, ist die hoch priorisierte Messezielgruppe Wirtschaft auf Hochschul-Messeständen überproportional stark vertreten und somit durch eine Messebeteiligung grundsätzlich gut erreichbar. Der Anteil der ebenfalls hoch priorisierten Zielgruppe Öffentlichkeit hingegen fällt mit $5 \%$ relativ gering aus, wenngleich mit dieser Zielgruppe - so belegen die vorangegangenen Ausführungen - trotzdem vergleichsweise viele Gespräche realisiert werden konnten. Die als wichtig eingestufte Messezielgruppe Staat ist dagegen auf den betrachteten Hochschul-Messeständen kaum anzu-

Vgl. Funke, K., Messeentscheidungen - Handlungsalternativen und Informationsbedarf, a.a.O., S. $303 \mathrm{f}$.

Die mittels einer Befragung von Hochschul-Messestand-Besuchern gewonnenen Informationen können systematischen Fehlern unterliegen. Diese liegen bspw. dann vor, wenn die Interviewer unbewußt Einfluß auf die Beantwortung der Fragen nehmen oder die Struktur der auskunftswilligen nicht mit derjenigen der nicht-auskunftswilligen Hochschul-Messestand-Besucher übereinstimmt. Dies führt nicht selten zu befragungsbedingten Fehlinterpretationen der Daten. Zu den allgemeinen Risiken von Befragungen vgl. Böhler, H., Marktforschung, Stuttgart u.a. 1985, S. 121 ff.; Hammann, P., Erichson, B., Marktforschung, Stuttgart, New York 1978, S. $30 \mathrm{ff}$. 
treffen ( $1 \%$ der Hochschul-Messestand-Besucher). Diese Zielgruppe - sowie auch die Zielgruppe der Wissenschaftsvertreter - ist jedoch generell unter den Messebesuchern nur relativ schwach vertreten. Die Zielgruppe der Studierenden, die im Vergleich zu anderen hochschulspezifischen Messezielgruppen einen untergeordneten Stellenwert einnimmt, hält sich dagegen auf den betrachteten HochschulMesseständen relativ häufig auf.

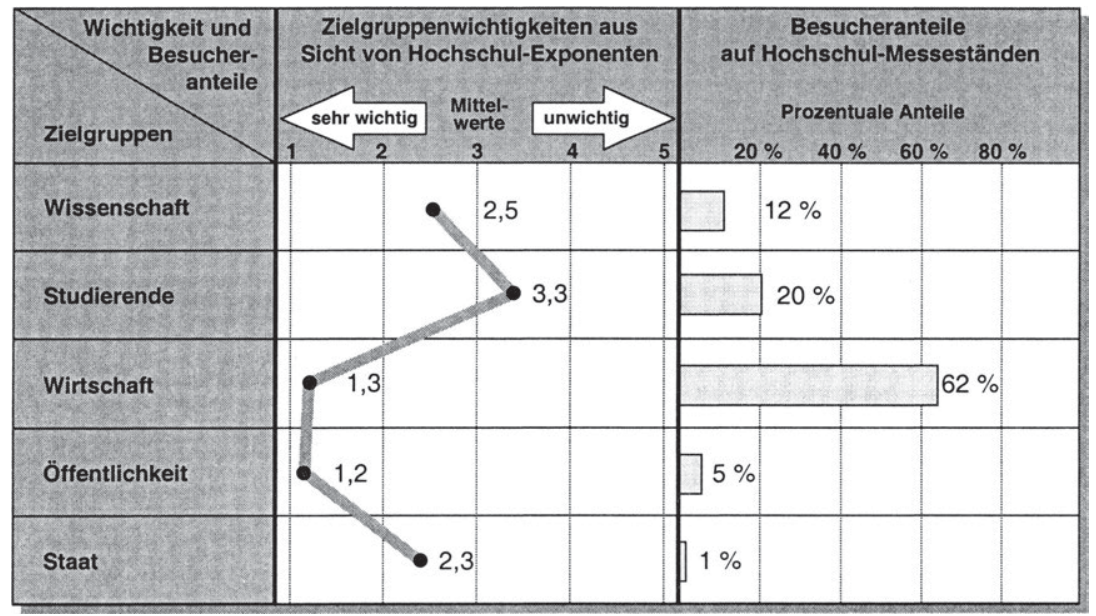

Abb. 25: Wichtigkeiten und Besucheranteile hochschulspezifischer Messezielgruppen

Bei dem messetypspezifischen Vergleich stimmt die Zielgruppenpriorisierung der Hochschul-Exponenten mit der mengenmäßigen Verteilung der Zielgruppen in den jeweiligen Besucherstichproben überein ( $v g l$. Abb. 26). So ist bspw. der Besucheranteil an Vertretern aus der Wirtschaft auf Hochschul-Messeständen der Fachmesse im Vergleich zur Mehrbranchenmesse höher, wobei die FachmesseExponenten dieser Zielgruppe auch einen höheren Stellenwert beimessen. Bei der Zielgruppe der Studierenden stimmt die Priorisierung mit der tatsächlichen Besucherstruktur ebenfalls überein. 


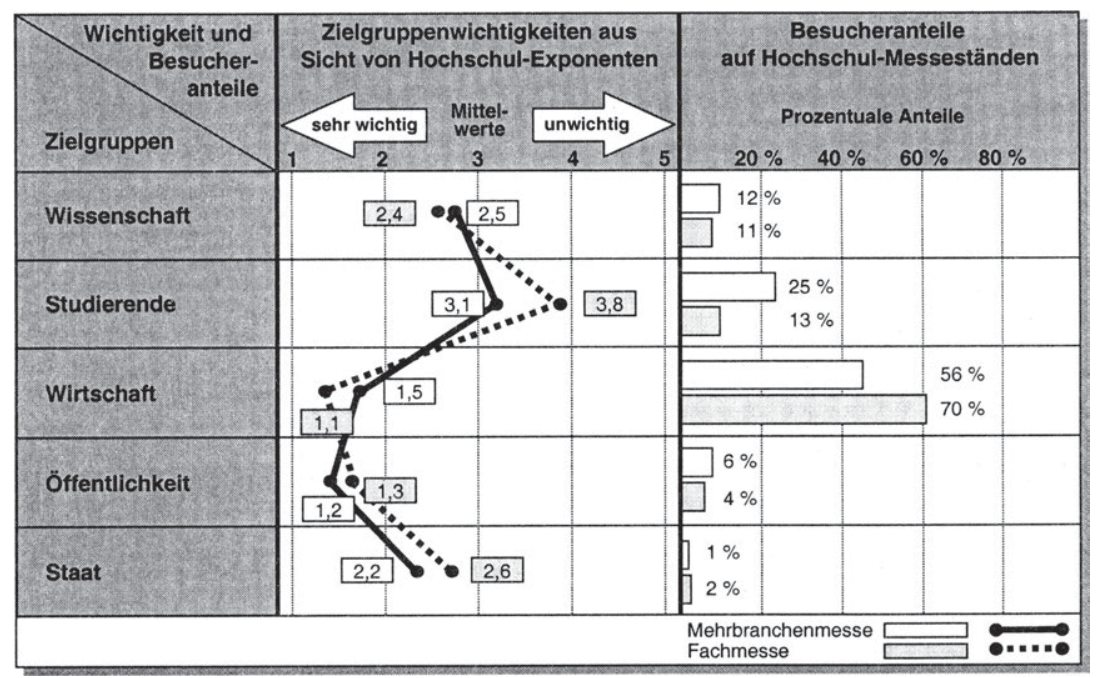

Abb. 26: Messetypspezifische Betrachtung von Wichtigkeiten und Besucheranteilen hochschulspezifischer Messezielgruppen

Für die Zielgruppe Wirtschaftsvertreter, die einerseits mit $62 \%$ den größten Anteil an den Besuchern einnimmt und der andererseits für die Erreichung von Projektzielen eine besondere Bedeutung zukommt, erscheint eine separate Analyse sinnvoll. Von den auf den Hochschul-Messeständen befragten Wirtschaftsvertretern verfügten $38 \%$ über mitentscheidende und $27 \%$ über ausschlaggebende Entscheidungskompetenz in ihren Unternehmen (vgl. Tab. 18). Dabei fielen die Anteile auf den betrachteten Messen in etwa gleich hoch aus. Beratende Entscheidungskompetenz besaßen etwa $26 \%$ der befragten Wirtschaftsvertreter, wohingegen nur $9 \%$ der Wirtschaftsvertreter (Mehrbranchenmesse: $12 \%$; Fachmesse: $5 \%$ ) angaben, über keine Entscheidungskompetenzen in ihrem Unternehmen zu verfügen. Im Hinblick auf die berufliche Position setzten sich die befragten Wirtschaftsvertreter vor allem aus Interessenten zusammen, die sich in beruflichen Positionen wie Angestellte und Facharbeiter (37\%) sowie Prokuristen, Direktoren, Abteilungsleiter und Angestellte mit Leitungsfunktion (33\%) befanden, gefolgt von selbständigen Unternehmern, Teilhabern und Freiberuflern (17\%). Dies galt messeübergreifend sowohl bezogen auf die Hochschul-MessestandBesucherstrukturen als auch bezogen auf die Gesamtbesucherstrukturen der jeweiligen Messen. Dagegen waren unter den Hochschul-Messestand-Besuchern die Auszubildenden und Praktikanten im Vergleich zu den Messebesuchern generell unterproportional vertreten. Letztlich wurden vor dem Hintergrund der Ent- 
scheidungskompetenzen und beruflichen Positionen der befragten, den Hochschul-Messestand besuchenden Wirtschaftsvertreter für Hochschul-Exponenten sowohl auf Fach- als auch auf Mehrbranchenmessen die Voraussetzungen geschaffen, angestrebte Projektziele zu erreichen.

\begin{tabular}{|c|c|c|c|c|}
\hline \multicolumn{2}{|c|}{$\begin{array}{l}\text { Entscheidungskompetenz/ } \\
\text { Berufliche Position im Unternehmen }\end{array}$} & & $\begin{array}{l}\text { Mehr- } \\
\text { branchen- } \\
\text { messe }\end{array}$ & Fachmesse \\
\hline \multirow{4}{*}{ 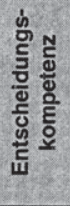 } & ausschlaggebend & $27 \% \quad(212)$ & $26 \%(104)$ & $28 \%(108)$ \\
\hline & mitentscheidend & $38 \% \quad(299)$ & $37 \%(144)$ & $40 \%(155)$ \\
\hline & beratend & $26 \% \quad(204)$ & $26 \%(101)$ & $27 \%(103)$ \\
\hline & keine & $9 \% \quad(66)$ & $12 \% \quad(46)$ & $5 \% \quad(20)$ \\
\hline \multirow{6}{*}{ 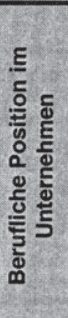 } & $\begin{array}{l}\text { Selbständiger Unternehmer, } \\
\text { Teilhaber, freier Beruf o.ä. }\end{array}$ & $17 \% \quad(129)$ & $17 \% \quad(68)$ & $16 \%(61)$ \\
\hline & $\begin{array}{l}\text { Geschäftsführer, Vorstands- } \\
\text { mitglied, Behördenleiter o.ä. }\end{array}$ & $9 \% \quad(71)$ & $8 \% \quad(33)$ & $10 \% \quad(38)$ \\
\hline & $\begin{array}{l}\text { Prokurist, Direktor, Abteilungslei- } \\
\text { ter, Angest. mit Leitungsfkt. o.ä. }\end{array}$ & $33 \% \quad(257)$ & $36 \%(139)$ & $30 \%(118)$ \\
\hline & $\begin{array}{l}\text { Angestellter, } \\
\text { Facharbeiter o.ä. }\end{array}$ & $37 \% \quad(285)$ & $34 \%(133)$ & $39 \%(152)$ \\
\hline & $\begin{array}{l}\text { Auszubildender, } \\
\text { Praktikant o.ä. }\end{array}$ & $2 \% \quad(18)$ & $4 \% \quad(15)$ & $1 \% \quad(3)$ \\
\hline & Sonstige & $3 \% \quad(21)$ & $1 \% \quad(4)$ & $4 \% \quad(17)$ \\
\hline
\end{tabular}

Tab. 18: Wirtschaftsvertreter auf den Hochschul-Messeständen und ihre Entscheidungskompetenz innerhalb der Unternehmen

Die Befragung zur Attraktivität präsentierter Hochschul-Exponate lieferte die in der nachfolgenden Abbildung aufgezeigten Ergebnisse. Zahlreiche HochschulMessestand-Besucher beurteilten die dargestellten Exponate als interessant (Mittelwert 1,8). Wie die Standardabweichung von 0,74 verdeutlicht, ist sich ein Großteil der Messestand-Besucher in dieser Einschätzung einig. Des weiteren wurden die präsentierten Hochschul-Exponate als innovativ (Mittelwert 2,0), praxisrelevant (Mittelwert 2,1) und wissenschaftlich anspruchsvoll (Mittelwert 2,2) eingestuft. 


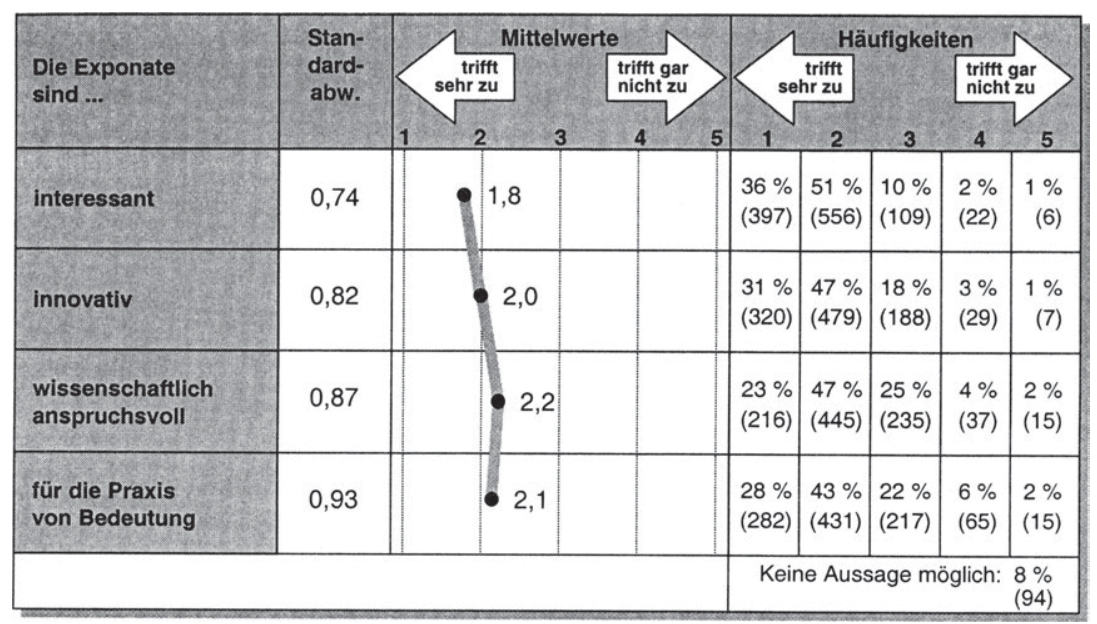

Abb. 27: Beurteilung der von den Hochschul-Exponenten präsentierten Exponate aus Sicht der Hochschul-Messestand-Besucher

Messetypspezifisch ergeben sich signifikante Unterschiede zwischen den Exponatbewertungen der Mehrbranchen- und Fachmessebesucher (vgl. Abb. 28). So bewerteten die Hochschul-Messestand-Besucher der Mehrbranchenmesse die präsentierten Hochschul-Exponate als weitaus interessanter und innovativer sowie wissenschaftlich anspruchsvoller als die Befragten der Mehrbranchenmesse. Dagegen wurde den Hochschul-Exponaten von den Hochschul-MessestandBesuchern der Fachmesse eine signifikant höhere Bedeutung für die Praxis beigemessen. Letztlich konnten somit auf beiden Messen Exponatbezogene Beeinflussungsziele realisiert werden. Dies kann wiederum positiven Einfluß auf Instituts-/Lehrstuhl-/Fachbereichs- sowie Hochschulbezogene Beeinflussungsziele nehmen. 


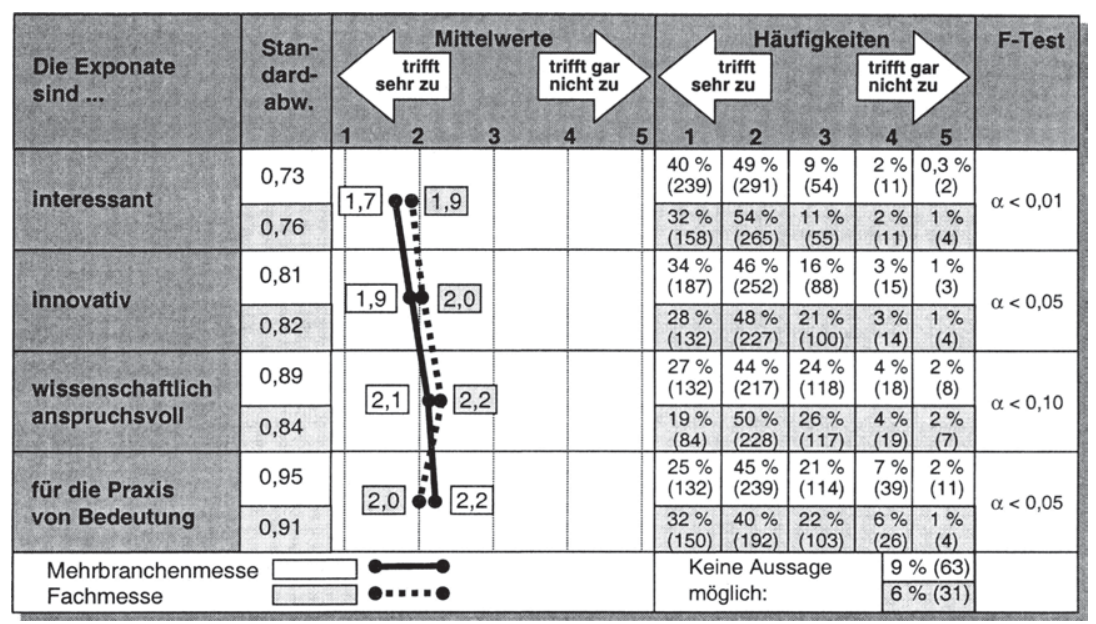

Abb. 28: Messetypspezifische Beurteilung der von den Hochschul-Exponenten präsentierten Exponate aus Sicht der Hochschul-Messestand-Besucher

Die Erreichung der von den Hochschul-Exponenten verfolgten Zielsetzungen kann erst in der Nach-Messe-Phase abschließend geklärt werden. Dies gilt insbesondere für die Kontrolle des ökonomischen Erfolgs von Hochschul-Messebeteiligungen (z.B. die Realisierung von Projektvolumina). Darüber hinaus ist es für Hochschul-Exponenten von großer Bedeutung, Informationen über die Dauerhaftigkeit psychographischer Wirkungen der Messebeteiligung bei den Hochschul-Messestand-Besuchern zu erhalten. Deshalb ist zur indirekten Erfolgskontrolle von Hochschul-Messebeteiligungen neben den vorangegangenen, in der MessePhase einzusetzenden Methoden der zusätzliche Einsatz entsprechender Verfahren in der Nach-Messe-Phase unabdingbar.

\subsection{Einsatz von Methoden zur indirekten Messeerfolgskontrolle in der Nach-Messe-Phase}

Als eine Methode zur indirekten Messeerfolgskontrolle in der Nach-Messe-Phase können von Hochschul-Exponenten Einladungsresonanzanalysen eingesetzt werden. Damit lassen sich neben der Überprüfung der Resonanz auf Einladun- 
gen, die an wichtige hochschulspezifische Messezielgruppen versandt wurden, auch mögliche Defizite der Einladungsaktion analysieren. ${ }^{54}$

Der Vorteil von Einladungsresonanzanalysen liegt insbesondere in dem Einsatz der mit ihr gewonnenen Ergebnisse zur gezielten Nach-Messe-Kontaktintensivierung mit eingeladenen Messezielgruppen. Diejenigen Zielgruppenmitglieder, die auf dem Hochschul-Messestand erschienen sind, können aufbauend auf den Ergebnissen der auf dem Hochschul-Messestand geführten Gespräche kontaktiert werden. Bei Zielgruppenvertretern, die trotz einer Einladung den Hochschul-Messestand nicht besucht haben, bietet diese Methode Möglichkeiten zur Erfassung der Gründe des Fernbleibens. ${ }^{55}$ Der Nachteil liegt dagegen in der Notwendigkeit der Einzelerhebung der ausgesandten Einladungen und der dieser Einladung gefolgten Hochschul-Messestand-Besucher. Die in der vorliegenden Untersuchung einbezogenen Hochschul-Exponenten haben i.d.R. diese Einzelerhebungen nicht vorgenommen. Somit konnten Einladungsresonanzanalysen im Rahmen der dieser Arbeit zugrundeliegenden Studie nicht eingesetzt werden, wenngleich diese Methode zur Erfolgskontrolle von Hochschul-Messebeteiligungen grundsätzlich geeignet erscheint.

Die Auswertung der Veranstalter-Besucherstatistiken ${ }^{56}$ ermöglicht den Hochschul-Exponenten Vergleiche der Besucherstruktur einer Messeveranstaltung mit der Struktur hochschulspezifischer Messezielgruppen. ${ }^{57}$ Informationen über die Besucherstruktur der Messeveranstaltung ${ }^{58}$ geben dem Hochschul-Exponenten

Vgl. Selinski, H., Sperling U. A., Marketinginstrument Messe: Arbeitsbuch für Studium und Praxis, a.a.O., S. 236.

Zudem können sich den Hochschul-Exponenten durch Abgabe eines Messeberichts interessante Anknüpfungspunkte zur Initiierung bzw. Weiterführung des Dialogs mit entsprechenden Anspruchs- bzw. Messezielgruppen ergeben.

Messegesellschaften bemühen sich häufig um die Erhebung von Besucherdaten ihrer Veranstaltungen. Im Rahmen von Besucheranalysen der Messegesellschaften werden die Messebesucher u.a. zu ihren Messeeindrücken befragt. Vgl. Gelszus Messe-Marktforschung GmbH (Hrsg.), Mehr Erfolg auf Messen - Wie Sie Ihren Erfolg messen und neue Potentiale entdecken, a.a.O., S. 5.

Über die Strukturmerkmale der Besucher, die bei derartigen Besucherstatistiken erhoben werden, entscheiden die Messegesellschaften. Oftmals werden regionale Herkunft, Geschlecht, Alter und Beruf sowie Besuchsmotive der Befragten erfaßt. Durch Hochrechnungen der Stichprobe auf die gesamte Veranstaltung können sowohl quantitative als auch qualitative "Strukturdaten“ der Messeveranstaltung ermittelt werden. Die Deutsche Messe AG Hannover bspw. veröffentlicht in regelmäßigen Abständen die Ergebnisse der Besucherbefragungen ausge- 
die Möglichkeit zur Überprüfung des Anteils seiner Messezielgruppen an der Gesamtbesucherzahl einer Messeveranstaltung. Damit gewinnt er einen groben Anhaltspunkt zur Beurteilung der zielgruppenspezifischen Reichweite der Messe. ${ }^{59}$

Als Vorteil einer Auswertung von Veranstalter-Besucherstatistiken ist der Vergleich allgemeiner und zielgruppenspezifischer Besucherstrukturen anzuführen. Die Zahlen beider Auswertungen weisen - zukünftig gleichbleibende Besucherstrukturen vorausgesetzt - auch prognostischen Charakter auf. Nachteilig in bezug auf die Besucherstatistiken der Messegesellschaften ist, daß diese nicht für jede Veranstaltung vorliegen oder den Hochschul-Exponenten nicht zur Verfügung gestellt werden. Darüber hinaus legt jeder Veranstalter die Befragungsinhalte individuell fest. Dies erschwert nicht nur die Vergleichbarkeit der Leistungsdaten verschiedener Messeveranstaltungen, ${ }^{60}$ sondern induziert für den einzelnen Hochschul-Exponenten erheblichen Aufwand, da seine eigenen Erhebungen an wechselnde Erhebungsstandards der Messegesellschaften angepaßt werden müssen, um jeweils die Vergleichbarkeit der individuellen Daten mit den Gesamtergebnissen zu gewährleisten. Aufgrund der aufgezeigten Problematiken erscheint es sinnvoll, im Rahmen der indirekten Erfolgskontrolle von Hochschul-Messebeteiligungen objektivere Besucherstrukturstatistiken - wie FKM-Besucherstrukturtests ${ }^{61}$ - heranzuziehen.

wählter Veranstaltungen in den Messe-Nachrichten Hannover sowie in gebundenen Publikationen. Vgl. z.B. Deutsche Messe AG (Hrsg.), Das transparente Medium - Aussteller- und Besucher-Analysen Hannover Messe '94, Hannover 1994.

Der Begriff der Reichweite stellt die Messeveranstaltung in eine Analogie zum Bereich der Print- und elektronischen (Radio, TV) Medien. Mit dem Begriff Reichweite sind im Zusammenhang mit diesen Medien Ermittlungen des Nutzeranteils eines bestimmten Mediums an einer fest umrissenen Grundgesamtheit verbunden, die im Rahmen von Messeveranstaltungen noch nicht entwickelt wurden. Vgl. hierzu Strothmann, K.-H., Messeforschung, in: Deutsche Werbewissenschaftliche Gesellschaft e.V. (DWG) (Hrsg.), Messen als Marketinginstrument, a.a.O., S. 104.

Insgesamt existieren noch keine allgemeingültigen Standards zur Erhebung von Besucherstrukturen. Zur Auseinandersetzung mit dieser Problematik vgl. Merbold, C., Strukturerhebungen noch die Ausnahme - Transparenz läßt weiter sehr zu wünschen übrig, in: HB, Nr. 235, 07.12.1983, S. 17; Schwenzner, J. M., Messen und Ausstellungen im Kreuzfeuer der Begriffe Reichweitenanalyse und Messeerfolgskontrolle, in: GfK-Mitteilungen zur Markt- und Absatzforschung, 18. Jg., Heft 1, 1972, S. $10 \mathrm{ff}$.

Insbesondere die Kritik an der Auswertung der Besucherstatistiken des Veranstalters im Hinblick auf die Datenvergleichbarkeit hat zur Entwicklung des standardisierten Verfahrens der Besucherstrukturermittlung in Form des FKM-Besucherstrukturtests beigetragen. 
Bei den FKM-Besucherstrukturtests handelt es sich um ein von der Gesellschaft zur freiwilligen Kontrolle von Messe- und Ausstellungszahlen (FKM) entwickeltes Konzept zur Ermittlung von Messebesucherstrukturdaten, das auf allen Messeveranstaltungen zur Anwendung kommen kann und dessen korrekte Durchführung durch einen Wirtschaftsprüfer kontrolliert wird. ${ }^{62}$ Mit der Auswertung des FKM-Besucherstrukturtests ${ }^{63}$ erhalten die Hochschul-Exponenten eine Datengrundlage sowohl für Entscheidungen über ihre Messeauswahl als auch für Vergleiche der Besucherschaft mit der erreichten (ex-post) bzw. anvisierten (ex-ante) Zielgruppe auf dem Hochschul-Messestand. ${ }^{64}$ Insgesamt erlauben die Ergebnisse der FKM-geprüften Besucheranalysen den Vergleich verschiedener Messeveranstaltungen hinsichtlich ihrer Besucherschaft und häufig auch deren Verhalten und Motive.

Ein wesentlicher Vorteil der Auswertung des FKM-Besucherstrukturtests (ex-post) liegt für Hochschul-Exponenten in der Möglichkeit, auf Basis der Besucherstrukturkenntnisse (ex-ante) Anhaltspunkte für die zukünftige Ausgestaltung ihrer Messebeteiligung im Hinblick auf die Festlegung inrer Messebeteiligungsziele und -aktivitäten zu gewinnen und zur Förderung der Zielerreichung entsprechend umsetzen zu können. Ein im Zusammenhang mit dieser Methode anzuführender Nachteil besteht darin, daß die Besucherstrukturen zahlreicher Messeveranstaltungen nicht nach diesem standardisierten Verfahren erfaßt werden. ${ }^{65}$ Auch darf

$\mathrm{Zu}$ den genauen Durchführungsvorschriften vgl. Gesellschaft zur freiwilligen Kontrolle von Messe- und Ausstellungszahlen (FKM) (Hrsg.), Satzung und Regein, a.a.O., S. 20 ff. Ziel des FKM-Besucherstrukturtests ist die Vergleichbarkeit der auf verschiedenen Messeveranstaltungen ermittelten Daten zu Merkmalen und Verhalten der Besucher. Die FKM schreibt hierfür die zu erhebenden Strukturmerkmale vor. So sind im Rahmen von Fachbesucherstrukturtests die folgenden Informationen abzufragen: Regionale Herkunft (Inland: Bundesland; Ausland: Staatszugehörigkeit) Wirtschaftszweig, Betriebsgröße, berufliche Stellung, Entscheidungskompetenz, Aufgabenbereich im Unternehmen, Häufigkeit des Messebesuchs sowie Aufenthaltsdauer.

Veröffentlicht werden die von der FKM vorgeschriebenen Besucherstrukturdaten der untersuchten Messen in den vom AUMA jährlich herausgegebenen Handbüchern "Messeplatz Deutschland" und "Regional". Auch die FKM veröffentlicht diese Daten in ihrem Jahresbericht. Vgl. Gesellschaft zur freiwilligen Kontrolle von Messe- und Ausstellungszahlen (FKM) (Hrsg.), Bericht 1995, Köln 1996, S. 8 ff.; Ausstellungs- und Messe-Ausschuss der Deutschen Wirtschaft e.V. (AUMA) (Hrsg.), Handbuch Messeplatz Deutschland 1999, a.a.O., S. 7 ff.

Folglich kann diese Methode sowohl in der Nach-Messe- als auch in der Vor-Messe-Phase eingesetzt werden. Aufgrund des überwiegenden Einsatzes von Auswertungen des FKMBesucherstrukturtests nach Ablauf der Messeveranstaltungen wurde hier die Einordnung in die Nach-Messe-Phase gewählt.

Vgl. Rost, D., Messetransparenz - ein Imperativ, in: asw, 27. Jg., Heft 8, 1984, S. 30 ff. 
das Prognosepotential der Besucherstrukturdaten nicht überschätzt werden. Angesichts der Dynamik des Messemarktes, auf dem ständig neue Messeveranstaltungen entstehen, vorhandene Messekonzepte verändert oder bislang integrierte Bereiche von Messeveranstaltungen zu eigenständigen Messen weiterentwickelt werden, erscheint die Relevanz vergangenheitsorientierter Daten für zukunftsorientierte Messebeteiligungsentscheidungen in vielen Fällen zunehmend fraglich. ${ }^{66}$ Somit eignet sich die Auswertung des FKM-Besucherstrukturtests i.S.d. Vergleichs mit der eigenen Hochschul-Messestand-Besucherstruktur und der damit deutlich werdenden Ausschöpfung der Erreichung von Messezielgruppen - im Rahmen von Hochschul-Messebeteiligungen zur Kontrolle gut, zur Prognose dagegen kaum.

Die Auswertung des FKM-Besucherstrukturtests zur Kontrolle der HochschulMessebeteiligungen auf den betrachteten Messen zeigt wichtige messetypspezifische Besonderheiten auf. So deutet der Vergleich zwischen der für die Hochschul-Messestände erhobenen Besucherstruktur und der den FKM-Besucherstrukturtest widerspiegelnde Gesamtbesucherstruktur grundsätzlich für die Mehrbranchenmesse auf eine unterproportionale Präsenz der Wirtschaft und gleichzeitig überproportionale Präsenz an Studierenden (vgl. Abb. 29) hin. Die gleiche Tendenz ist in abgeschwächter Form für die Fachmesse zu konstatieren. Die überproportionale Vertretung von Studierenden sowie auch von Wissenschaftlern auf dem Hochschul-Messestand, die zumindest für die Mehrbranchenmesse belegt wird, liegt in der Konzeption eines Hochschul-Messestands begründet. So ist ein überaus hohes Interesse an Hochschul-Exponaten für Wissenschaftler und Studierende offensichtlich. 


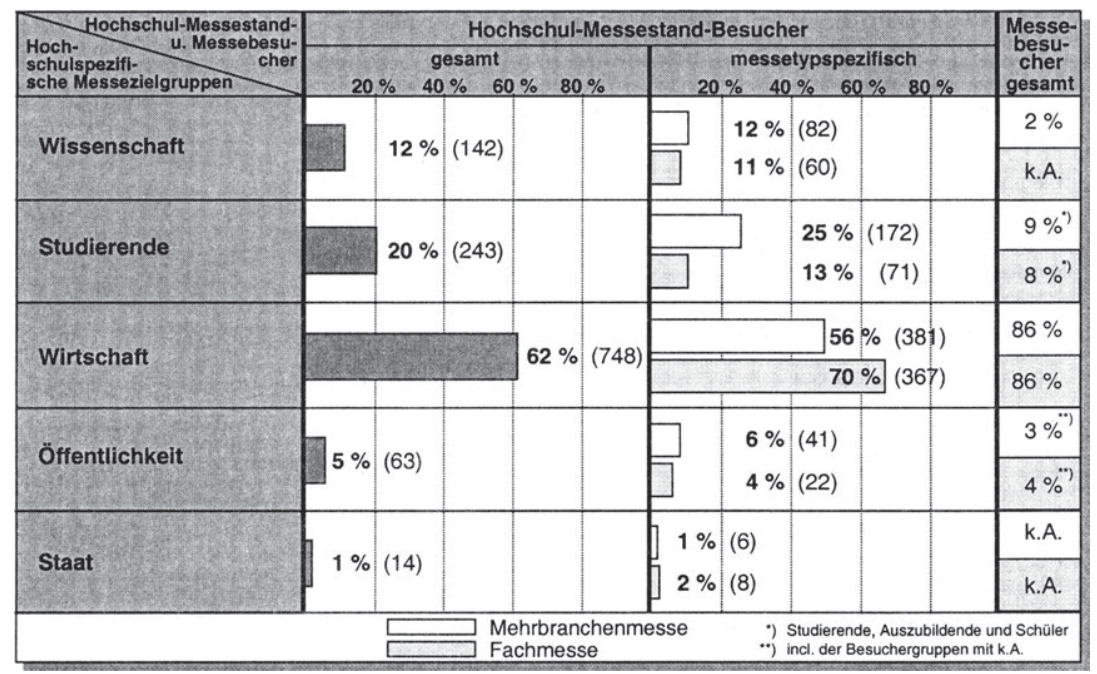

Abb. 29: Vergleich der Hochschul-Messestand-Besucherstruktur mit der Gesamtbesucherstruktur der betrachteten Messen

Die erhobenen Besucherstrukturen geben somit Auskunft über die Erreichung hochschulspezifischer Zielgruppen im Rahmen von Hochschul-Messebeteiligungen, darüber hinaus jedoch keinerlei Anhaltspunkte zur Wirksamkeit des Messeengagements bei den jeweiligen Zielgruppen. Zur Gewinnung derartiger Informationen bedarf es an Nach-Messebefragungen von Hochschul-MessestandBesuchern. Durch derartige Befragungen können Hochschul-Exponenten neben Erkenntnissen über die mittel- und längerfristige Wirksamkeit ihres Messeengagements bei hochschulspezifischen Zielgruppen auch ein reflektiertes Gesamtbild inrer Messebeteiligung gewinnen. ${ }^{67}$ Dabei bieten sich derartige Befragungen, die häufig telefonisch durchgeführt werden, insbesondere zur Kontrolle der Erreichung von Beeinflussungs- und Projektzielen an.

Die vielfältigen optischen und akustischen Reize auf Messen sowie die hohe Anzahl der geführten Gespräche bewirken bei Hochschul-Messestand-Besuchern, daß viele der auf der Messe bzw. auf dem Hochschul-Messestand aufgenommenen Informationen nur kurzfristig präsent bleiben. Erst einige Zeit nach dem Messebesuch konsolidiert sich der Gesamteindruck der Messeveranstaltung, wobei die als wichtig empfundenen Informationen im Gedächtnis verhaftet bleiben. Vgl. Funke, K., Messeentscheidungen - Handlungsalternativen und Informationsbedarf, a.a.O., S. $308 \mathrm{ff}$. 
Der zentrale Vorteil einer Nachbefragung von Hochschul-Messestand-Besuchern liegt in der Durchführung der Befragung zu einem Zeitpunkt, zu dem bei den Hochschul-Messestand-Besuchern kurzfristige Eindrücke häufig in den Hintergrund und längerfristige Eindrücke klarer in den Vordergrund getreten sind. Hochschul-Exponenten können damit u.a. Messeaktivitäten identifizieren, mit denen längerfristige Eindrücke bei den Hochschul-Messestand-Besuchern erzielt werden. ${ }^{68}$ Nachteile von Nach-Messebefragungen liegen in den zusätzlich entstehenden Kosten, die vor allem aus dem organisatorischen Doppelaufwand (Erfassung der erforderlichen Adressen während der Messe und Durchführung der Befragung nach der Messe) und den für die Durchführung benötigten Personalressourcen resultieren. Damit erscheint die Anwendung dieser Methode zur indirekten Erfolgskontrolle von Hochschul-Messebeteiligungen eher nicht geeignet.

Nicht zuletzt sollte im Rahmen von Befragungen das wissenschaftliche und nicht-wissenschaftliche Standpersonal Möglichkeiten zur Bewertung der Messebeteiligung und zu Verbesserungsvorschlägen erhalten, um seine Erfahrungen als Bindeglied zwischen Hochschul-Exponenten und Messebesuchern wiederzugeben und die gewonnenen Informationen für die Organisation und Durchführung der nächsten Messebeteiligung nutzen zu können. ${ }^{69}$ Darüber hinaus können durch den Einsatz dieses Verfahrens Informationen über den Erreichungsgrad verfolgter Messebeteiligungsziele gewonnen werden.

Dem Vorteil von Standpersonalbefragungen, relevante Informationen für zukünftige Messebeteiligungen ${ }^{70}$ zu gewinnen, steht jedoch ein zentraler Nachteil

Vgl. Funke, K., Messeentscheidungen - Handlungsalternativen und Informationsbedarf, a.a.O., S. 308.

Befragungen des Standpersonals werden oftmals in kurzen Einzelinterviews oder Gruppengesprächen durchgeführt. In der Literatur werden z.T. auch anonyme schriftliche Befragungen der Standmitarbeiter vorgeschlagen, um den Informationsnutzen der Erhebung für die jeweiligen Exponenten zu erhöhen. Vgl. Selinski, H., Sperling U. A., Marketinginstrument Messe: Arbeitsbuch für Studium und Praxis, a.a.O., S. 224.

Die Ergebnisse liefern nicht nur Anhaltspunkte für eine gezielte Nacharbeit der HochschulMessebeteiligung, sondern können auch i.S. einer Tätigkeitskontrolle genutzt werden, um korrigierend in den Ablauf zukünftiger Messebeschickungen einzugreifen. Vgl. Berghäuser, B., Messen + Ausstellungen: Entscheidungshilfen für die ${ }_{n}$ richtige ${ }^{*}$ Beteiligung, in: Marketing Journal, 16. Jg., Heft 2, 1983, S. 168. Diese Methode bietet zudem die Möglichkeit der Kontrolle von Motivationszielen, die ggf. bezüglich der Mitarbeiter formuliert wurden. Vgl. Leicher, R., Die Manöverkritik. Unerläßlicher Bestandteil der Messenacharbeit, in: Ausstellungs- und MesseAusschuss der Deutschen Wirtschaft e.V. (AUMA) (Hrsg.), Literatursammlung des AUMA, Köln O.J., S. 79. 
gegenüber. Dieser liegt in der Erhebung von ausschließlich subjektiven Eindrücken der Mitarbeiter, die keine konkrete Überprüfung der Erreichung von anspruchsgruppenorientierten Messebeteiligungszielen zuläßt. Vor diesem Hintergrund sollten derartige Befragungen allenfalls als flankierendes Instrument zur Erfolgskontrolle von Hochschul-Messebeteiligungen dienen, wenngleich sie aufgrund ihres hohen Informationscharakters und des geringen Durchführungsaufwands prinzipiell bei allen Hochschul-Messebeteiligungen eingesetzt werden sollten.

Im Rahmen der Befragung des Standpersonals, welches bei den analysierten Hochschul-Messebeteiligungen überwiegend die Hochschul-Exponenten selbst darstellten, lag der Fokus insbesondere auf der Zufriedenheit mit der betreffenden Messebeteiligung. Diese kann als ein Indikator für die subjektive Erreichung der verfolgten Messezielsetzungen angesehen werden. Mit der Messebeteiligung auf den betrachteten Messen waren - im Sinne eines Globalurteils - weit über die Hälfte der befragten Hochschul-Exponenten sehr zufrieden bis zufrieden ${ }^{71}$ (vgl. Abb. 30). Viele dieser Exponenten begründeten ihre Zufriedenheit insbesondere mit den durch die Messebeteiligung geknüpften Kontakten, die aus Sicht der Hochschul-Exponenten sowohl qualitativ als auch quantitativ außerordentlich positiv ausfielen. Darüber hinaus wurde in diesem Zusammenhang die Steigerung des exponenten- sowie exponatbezogenen Bekanntheitsgrades angegeben. Werden in die Betrachtung die Bedeutungsgewichte der einzelnen Zielsetzungen einbezogen, so ist festzustellen, daß insbesondere die priorisierten Zielsetzungen von den befragten Hochschul-Exponenten explizit zur Zufriedenheitsbegründung herangezogen wurden. Dies läßt auf die Erreichung dieser mit der Messebeteiligung verfolgten hochschulspezifischen Ziele schließen. 


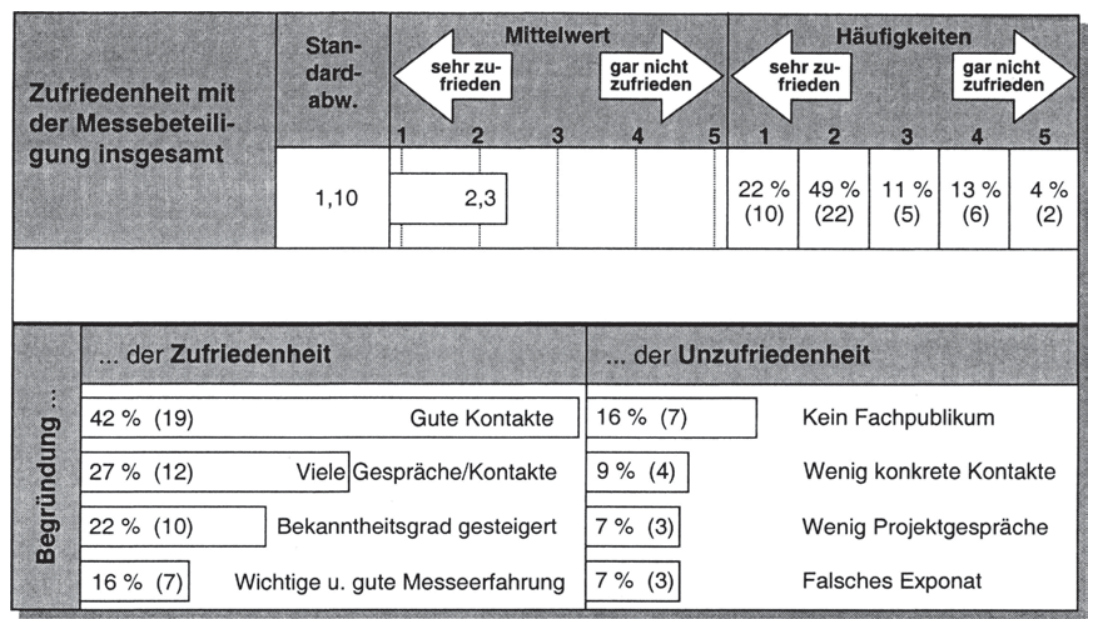

Abb. 30: Zufriedenheit der Hochschul-Exponenten mit der Messebeteiligung

Die Fachmesse-Exponenten - so belegt die messetypspezifische Analyse - waren mit ihrer Messebeteiligung im Vergleich zu Hochschul-Exponenten der Mehrbranchenmesse zufriedener (vgl. Abb. 31). Der hohe Mittelwert von 1,9 (vs. 2,5 bei den Exponenten der Mehrbranchenmesse) bei einer moderaten Standardabweichung von 0,80 (vs. 1,20 bei den Exponenten der Mehrbranchenmesse) bestätigt die höhere Zufriedenheit der Fachmesse-Exponenten, wenngleich immerhin $63 \%$ der befragten Mehrbranchenmesse-Exponenten mit ihrer Messebeteiligung ebenfalls zufrieden waren. Sowohl auf der Fachmesse als auch auf der Mehrbranchenmesse wurden von den Hochschul-Exponenten zur Zufriedenheitsbegründung vor allem gute Kontakte zu Messestand-Besuchern angeführt. Auf Grundlage der aufgezeigten Ergebnisse kann insgesamt von einer höheren Zielerreichung durch eine Hochschul-Messebeteiligung auf Fachmessen als auf Mehrbranchenmessen ausgegangen werden. 


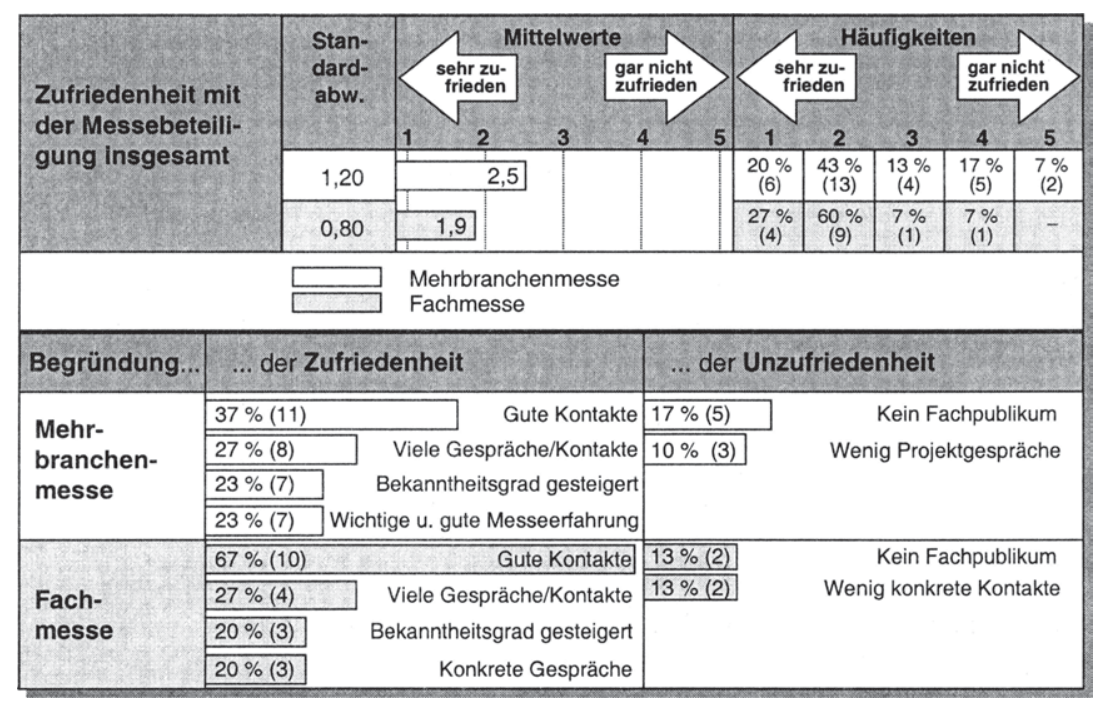

Abb. 31: Messetypspezifische Zufriedenheit der Hochschul-Exponenten mit der Messebeteiligung

Die exponententypspezifische Betrachtung zeigt für Projekt- und exponatorientierte Hochschul-Exponenten den höchsten Zufriedenheitsgrad auf (vgl. Abb. 32). Keiner der Befragten gab an, mit der Messebeteiligung unzufrieden zu sein. Diese Ergebnisse lassen auf eine hohe Zielerreichung der primär verfolgten Projekt- und exponatbezogenen Beeinflussungsziele schließen. Bestätigt wird diese Vermutung durch die Zufriedenheitsbegründungen dieser Hochschul-Exponenten, bei denen eine hohe Anzahl geführter Gespräche sowie realisierter Kontakte angeführt wurden. Auch die Beeinflussungsorientierten Hochschul-Exponenten gaben eine hohe Zufriedenheit mit der durchgeführten Messebeteiligung an, welches wiederum auf einen hohen Zielerreichungsgrad der von innen hoch priorisierten Beeinflussungsziele hindeutet. Diese Exponenten gaben als Gründe ihrer Zufriedenheit neben der Quantität und Qualität der realisierten Messekontakte die Steigerung des Bekanntheitsgrades ihres Exponates sowie inres Instituts/Lehrstuhls/Fachbereichs an. Pflichtrepräsentanten hingegen waren mit ihren Messebeteiligungen weniger zufrieden. Häufigste Begründung für diese Unzufriedenheit stellt die geringe Anzahl von Projektgesprächen auf der Messe dar. Diese Begründung steht im Widerspruch zu der von diesen Exponenten vorgenommenen Priorisierung inrer verfolgten Zielsetzungen, da sie die Projektziele für ihre Messebeteiligung als weniger wichtig einstuften. Somit spiegelt sich das nicht auf eigener 
Initiative basierende Messeengagement nicht nur in einem gering motivierten, sondern auch in einem „hybriden“ Hochschul-Exponenten wider.

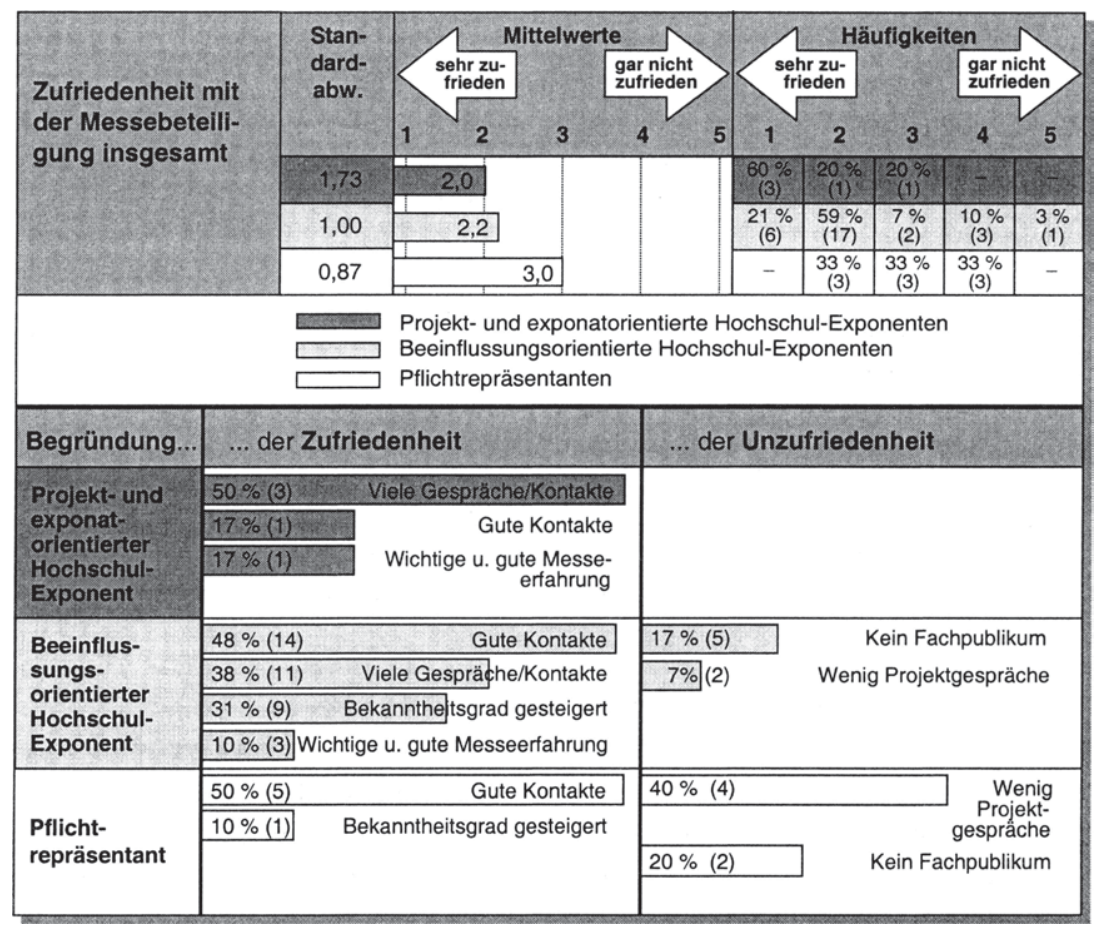

\section{Abb. 32: Exponententypspezifische Zufriedenheit mit der Messebeteiligung}

Neben der Zufriedenheit als Indikator für die subjektive Erreichung verfolgter Messebeteiligungsziele kann ebenfalls eine Reflexion der Zielerreichung aus Sicht der Exponenten mittels eines Messebeteiligungs-Nutzen-Index vorgenommen werden. ${ }^{72}$ Angesichts der vielfältigen, von Hochschul-Exponenten parallel verfolgten Messezielsetzungen hängt die relative Beurteilung des Messeerfolgs von der jeweiligen Zielpriorität ab. Eine Gesamtbeurteilung des indirekten Erfolgs einer Messebeteiligung entsteht dabei durch Aggregation der Zielerreichungsgrade spezifischer Grundhaltungen auf der Basis einer empirischen Untersuchung, a.a.O., S. 263 f. 
verfolgter Einzelzielsetzungen unter Berücksichtigung der jeweiligen Gewichtung. Zu diesem Zweck sind zunächst die individuellen Zielerreichungsgrade der Einzelzielsetzungen zu erfassen, sodann mit den jeweiligen Prioritäten zu gewichten, um sie schließlich irı einen Messebeteiligungs-Nutzen-Index zu überführen.

Der Vorteil des Messebeteiligungs-Nutzen-Index liegt in einer Quantifizierung der Zielerreichung, die den Hochschul-Exponenten im Zeitablauf wichtige Informationen über die Erfolgsentwicklung ihres Messeengagements liefern können. Als Nachteil ist demgegenüber anzuführen, daß es sich hierbei um eine subjektive Erfassung des indirekten Messebeteiligungserfolgs handelt. Damit ist dieses Instrument eher flankierend zu objektiven Verfahren der indirekten Messeerfolgskontrolle einzusetzen.

Im Rahmen der vorliegenden Untersuchung wurden für die Hochschul-Exponenten auf den betrachteten Messen derartige Nutzen-Indizes ermittelt. ${ }^{73}$ Den Ausgangspunkt bildete dabei die messetypspezifische Erfassung der von den einzelnen Hochschul-Exponenten verfolgten Einzelzielsetzungen. Für diese Zielsetzungen wurden messetypspezifisch die jeweiligen Zielerreichungsgrade ermittelt und mit den zugehörigen messetypspezifischen Zielbedeutungen gewichtet. ${ }^{74}$ Die resultierenden Nutzenbeiträge wurden schließlich zu einem MessebeteiligungsNutzen-Index aggregiert. Die Berechnungen zeigen, daß die Fachmesse-Exponenten im Rahmen ihrer Messebeteiligung höhere Nutzenwerte erreichten als die Hochschul-Exponenten der Mehrbranchenmesse (vgl. Abb. 33). Dabei konnten für die Fachmesse-Exponenten überdurchschnittliche und die Hochschul-Exponenten der Mehrbranchenmesse unterdurchschnittliche Nutzen-Indizes ermittelt werden,

Die Hochschul-Exponenten wurden in der Nach-Messe-Phase zu der Erreichung ihrer mit der Messebeteiligung verfolgten Zielsetzungen befragt. Diese Daten sowie die aus Exponentensicht vorgenommenen Messezielgewichtungen bilden die Grundlage zur Berechnung des Messebeteiligungs-Nutzen-Index.

Zu diesem Zweck wurde eine zur Bewertung von Nutzen-Indizes notwendige Umkodierung der Bewertungen vorgenommen. So wurde die Zielerreichung, die entgegen der in der empirischen Untersuchung generell verwendeten 5er-Skala aut einer 4er-Skala erfaßt wurde, um ein Ausweichen der Befragten auf einen Mittelwert zu vermeiden, wie folgt umkodiert: 1 = realisiert bis $4=$ nicht realisiert. Vgl. Anhang III, Fragebogen 4: Teil 2 der Exponentenbefragung II in der Nach-Messe-Phase. Die mit einer 5er-Skala bewerteten Einzelzielsetzungen konnten in ihrer originär ermittelten Kodierung $1=$ unwichtig und $5=$ sehr wichtig übernommen werden. Vgl. Anhang III, Fragebogen 2: Teil 2 der Exponentenbefragung I in der Vor-Messe-Phase. Damit läßt sich für jedes der priorisierten Ziele ein Nutzen-Index mit einem maximalen Wert von 20 ermitteln. Bezogen auf alle dreizehn Einzelzielsetzungen kann somit ein maximaler Nutzenwert in Höhe von 260 für eine Messebeteiligung ermittelt werden. 
wobei beide Nutzenwerte relativ nah bei dem gesamt ermittelten Nutzen-Mittelwert liegen.

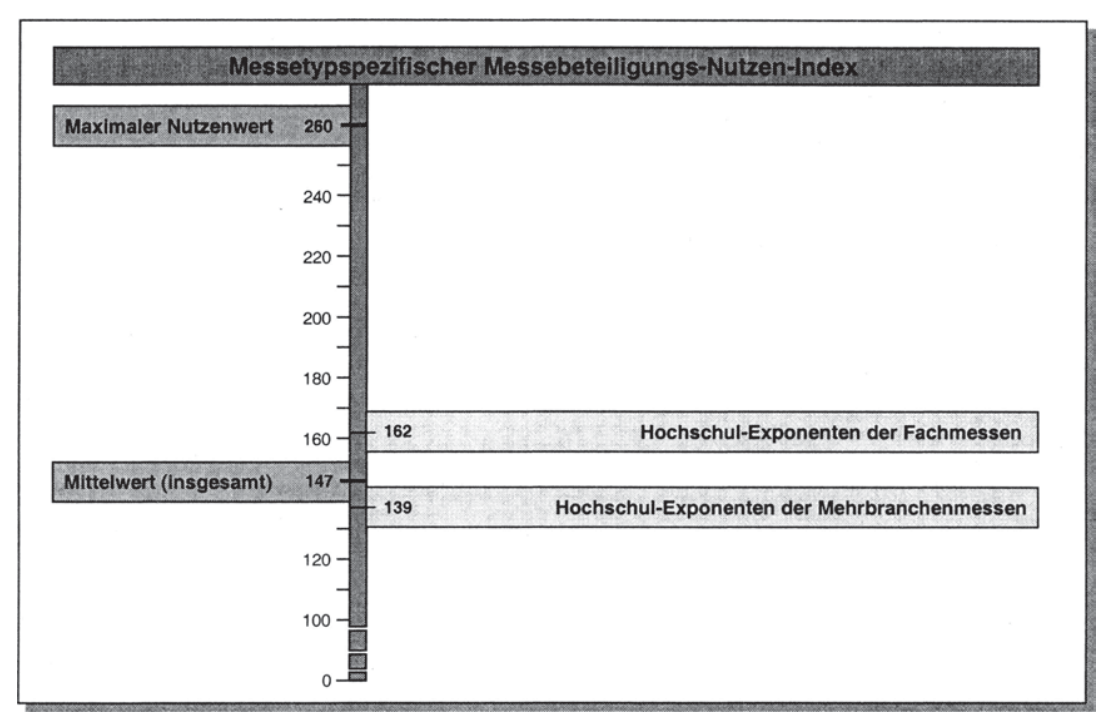

Abb. 33: Messetypspezifische Messebeteiligungs-Nutzen-Indizes

Angesichts der von den hochschulspezifischen Exponententypen vorgenommenen unterschiedlichen Zielgewichtungen erscheint die Bildung exponententypspezifischer Nutzen-Indizes sinnvoll. Hierzu wurden ausgehend von den Einzelzielsetzungen der jeweiligen Hochschul-Exponententypen die exponententypspezifischen Zielerreichungsgrade ermittelt und mit den zugehörigen exponententypspezifischen Zielbedeutungen gewichtet. Die resultierenden Nutzenbeiträge wurden schließlich zu exponententypspezifischen Messebeteiligungs-Nutzen-Indizes aggregiert, die in der nachfolgenden Abbildung wiedergegeben werden. 


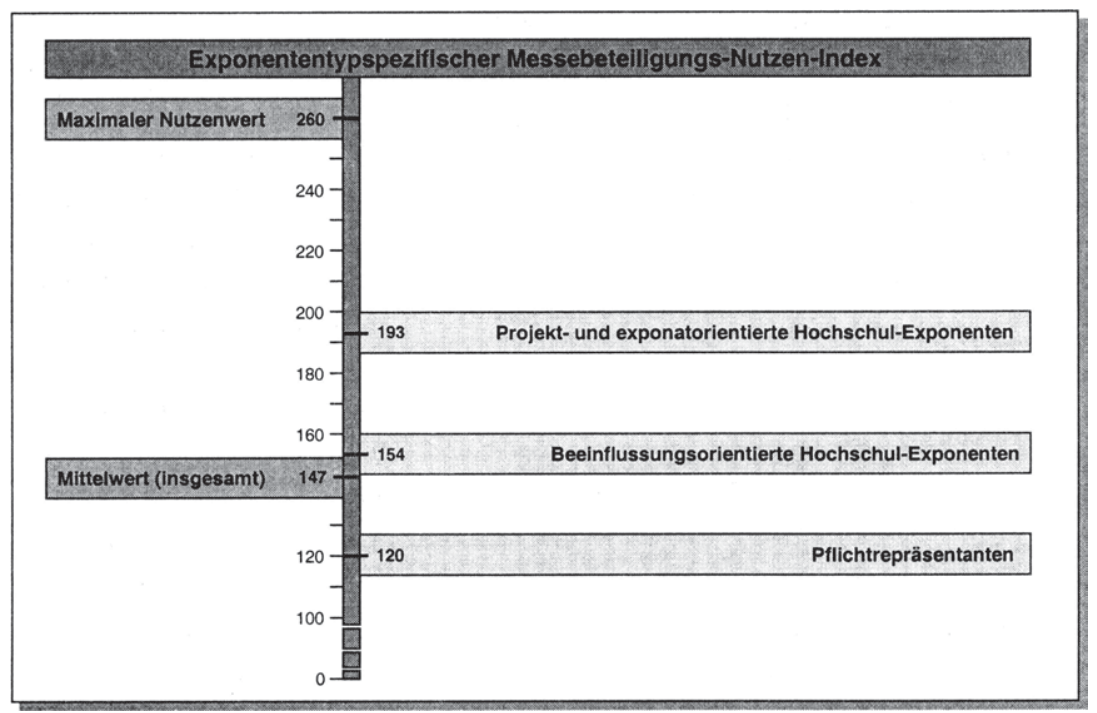

Abb. 34: Exponententypspezifische Messebeteiligungs-Nutzen-Indizes

Die exponententypspezifischen Berechnungen belegen die höchsten Nutzenwerte für Projekt- und exponatorientierte Hochschul-Exponenten. Im Vergleich dazu schätzten die Beeinflussungsorientierten Hochschul-Exponenten ihre Messebeteiligung als weniger nutzenstiftend ein. Dennoch erzielten sie im Vergleich zum Nutzenwert über alle Hochschul-Exponenten (Mittelwert - insgesamt) einen überdurchschnittlichen Nutzenwert. Im Gegensatz dazu bewerteten die Pflichtrepräsentanten den Nutzen ihrer Messebeteiligung sehr vorsichtig und stuften Messebeteiligungen insgesamt als wenig nutzenstiftend ein. Diese Ergebnisse belegen nochmals die bereits zuvor gewonnenen Erkenntnisse anderer Methoden der indirekten Erfolgskontrolle.

Bei den bisher dargestellten Methoden zur indirekten Erfolgskontrolle von Hochschul-Messebeteiligungen wurden bisher keine ökonomischen Größen berücksichtigt. Angesichts der von einem Großteil der Hochschul-Exponenten im Rahmen ihrer Messebeteiligung hoch priorisierten Projektziele ${ }^{75}$ erscheint es jedoch ziele als wichtig. Auf einer Skala von $1=$ sehr wichtig bis $5=$ unwichtig bewerteten diese Expo- 
unabdingbar, das Volumen der durch die Messebeteiligung angebahnten Projekte zu ermitteln. Dabei kann sich das Volumen sowohl auf die Anzahl als auch auf die monetäre Bewertung der angebahnten Projekte beziehen. Im Rahmen der Analyse des Messeprojektvolumens werden nicht nur alle unmittelbar auf der Messeveranstaltung abgeschlossenen Projektverträge, sondern auch die mittelbaren Messeaufträge, die maßgeblich auf der Messe initiiert wurden, aber erst später realisiert werden konnten, betrachtet. ${ }^{76}$ Damit steht beim Einsatz dieser Methode insbesondere die Überprüfung der Erreichung von Projektzielen im Vordergrund.

Dem Vorteil der unmittelbaren Überprüfung des auf die Messebeteiligung zurückzuführenden Projektvolumens stehen jedoch auch Nachteile gegenüber, welche sich insbesondere in der Zurechnungsproblematik widerspiegeln. Zum einen ist nicht sicher, ob die auf der Messe abgeschlossenen bzw. vorbereiteten Projektverträge sowie die erhaltenen Aufträge tatsächlich den Messeaktivitäten zurechenbar oder ursächlich auf zeitlich vor der Messe liegende Aktivitäten der Hochschul-Exponenten zurückzuführen sind. Zum anderen stellt sich die Abgrenzungsproblematik des Zeitintervalls in der Nach-Messe-Phase, in dem die eingehenden Projektaufträge noch den Auswirkungen der Messebeteiligung zuzuschreiben sind. ${ }^{77}$ Weiterhin ist aufgrund der Nicht-Berücksichtigung des psychographischen Erfolgs, der im Rahmen der Messebeteiligung aller Hochschul-Exponenten einen besonderen Stellenwert einnimmt, die ausschließliche Analyse des Projektvolumens zur indirekten Erfolgskontrolle von Hochschul-Messebeteiligungen nicht ausreichend. ${ }^{78}$ Vielmehr sollte dieses Verfahren, nicht zuletzt auch aufgrund der

nententypen die Projektziele mit den Werten 1,1 (Projekt- und exponatorientierte HochschulExponenten) sowie 1,6 (Beeinflussungsorientierte Hochschul-Exponenten).

Im Rahmen der Messebeteiligungen im kommerziellen Bereich werden anstelle der Projektvolumina die Messeumsätze analysiert. Vgl. Blohm, H., Messen und Ausstellungen als Mittel der Absatzförderung in der Schweizerischen Maschinenindustrie, Freiburg, Winterthur 1960, S. 126.

Eine umfassende Möglichkeit zur Berechnung des Messe- und besonders des messeinduzierten Nachmesseumsatzes bzW. -projektvolumens stellt FUNKE mit dem "modellgestützten Verfahren zur Ermittlung auftragsbezogener Messewirkungen“ vor. Vgl. Funke, K., Messeentscheidungen - Handlungsalternativen und Informationsbedarf, a.a.O., S. $354 \mathrm{ff}$.

Daß das Kriterium "Messeumsatz" als alleiniger Erfolgsindikator für kommerzielle Messebeteiligungen nur wenig repräsentativ ist, wurde schon früh aufgrund des Wandels der Messen zu informations- und kommunikationsorientierten Veranstaltungen erkannt und führt daher im kommerziellen Bereich zu einer eher kritischen Beurteilung dieses Erfolgskriteriums. Vgl. Schwenzner, J. M., Andersen, E., Der Messe-Test, in: Wagner, K. (Hrsg.), Allgemeines Statistisches Archiv, Bd. 39, München 1955, S. 25 ff. 
nur geringen finanziellen und personellen Anforderungen, immer flankierend zu weiteren, auch qualitativ ausgerichteten Verfahren eingesetzt werden.

Aufgrund des oftmals nach der Messebeteiligung noch nicht quantifizierbaren monetären Projektvolumens wurde die Analyse des Messeprojektvolumens auf die Anzahl der durch das Messeengagement initiierten Kontakte fokussiert. Dabei ist zu berücksichtigen, daß bezogen auf den Zeitpunkt der Erfassung - fünf bzw. sechs Monate nach der Messe-Phase - noch viele Kontakte als Informationsgespräche zu klassifizieren sind, die jedoch potentielle Kontakte für zukünftige Projekte darstellen. Darüber hinaus können viele Projektgespräche bezüglich ihrer zukünftigen Entwicklung noch nicht abschließend beurteilt werden. Die nachfolgenden Ausführungen sind daher als Tendenzen zu verstehen und weniger als Dokumentation erzielter Projekterfolge.

Insgesamt konnte, wie die folgende Abbildung verdeutlicht, knapp ein Drittel der befragten Exponenten auf Grundlage ihrer Messebeteiligung noch keine Projektabschlüsse verzeichnen. Knapp zwei Drittel der Befragten gaben an, aufgrund ihrer Messebeteiligung konkrete Projektgespräche geführt zu haben. Diese Gespräche legen grundsätzlich die Basis für zukünftige Projektabschlüsse, deren Anteil - bezogen auf den Zeitpunkt der Befragung - immerhin bereits bei $13 \%$ lag.

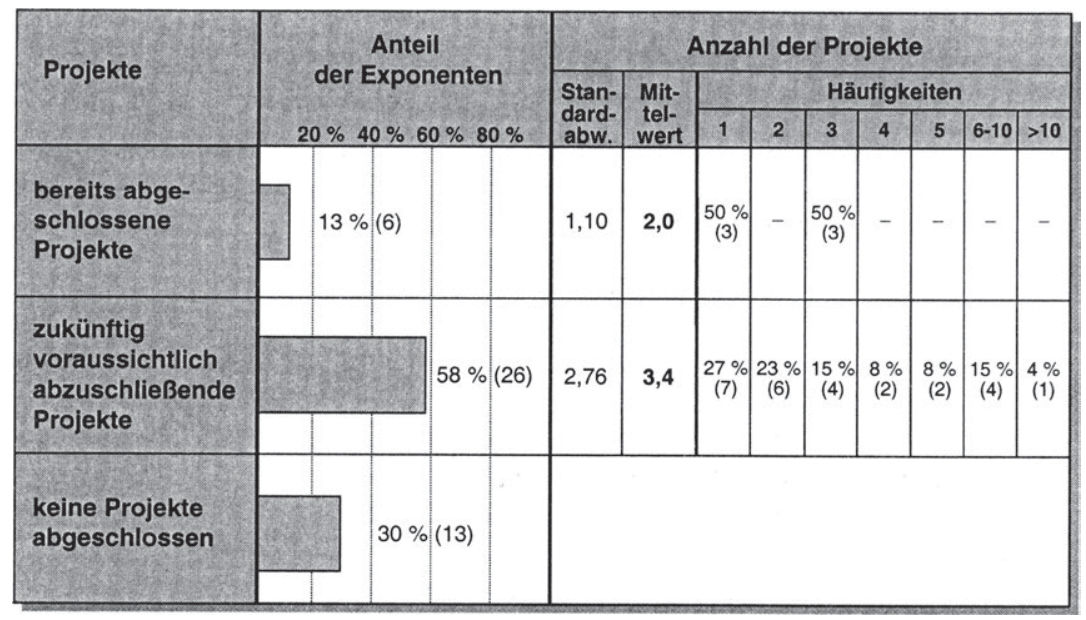

Abb. 35: Angebahnte Projekte aufgrund des von Hochschul-Exponenten realisierten Messeengagements 
Messetypspezifisch betrachtet fiel der Anteil der Hochschul-Exponenten, die bis zu dem Zeitpunkt der Befragung keine Projekte abschließen konnten, für die Mehrbranchenmesse höher aus als für die betrachtete Fachmesse (vgl. Abb. 36). Auch lag die höhere Wahrscheinlichkeit, voraussichtlich in Zukunft Projekte abzuschließen, bei den Fachmesse-Exponenten. Dagegen ließen sich in bezug auf bereits abgeschlossene Projekte keinerlei Unterschiede zwischen HochschulExponenten der Mehrbranchenmesse und Fachmesse-Exponenten feststellen. Insgesamt deuten die Ergebnisse auf eine potentiell bessere Erreichung von Projektzielen durch Beschickungen von Fachmessen hin.

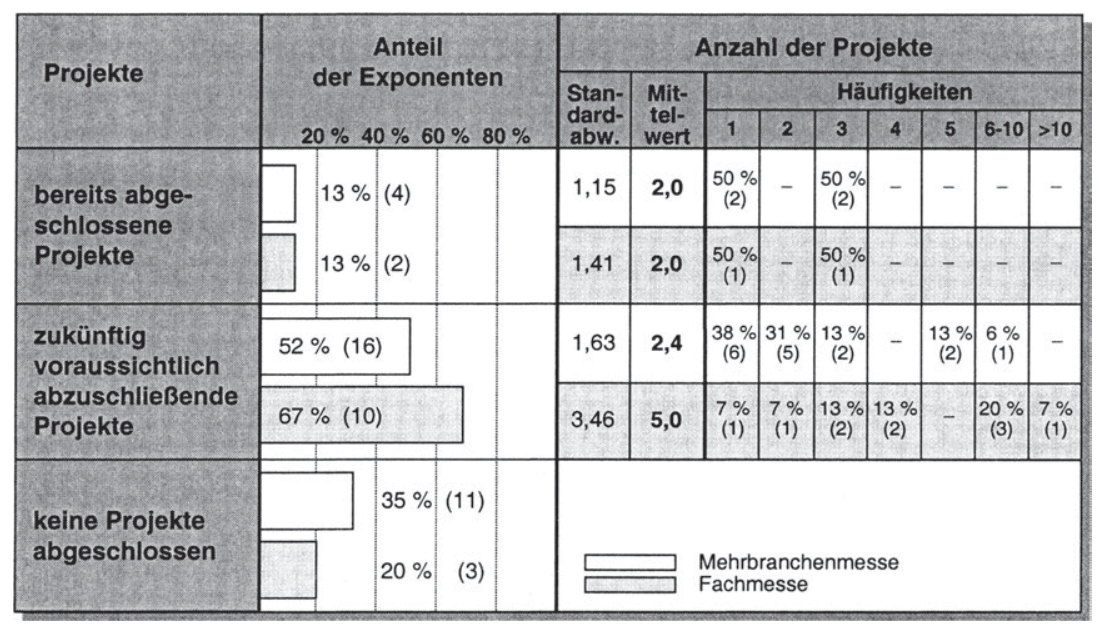

Abb. 36: Messetypspezifisch angebahnte Projekte aufgrund des von HochschulExponenten realisierten Messeengagements

Die exponententypspezifische Analyse zeigt, daß insbesondere Projekt- und exponatorientierte Hochschul-Exponenten durch ihre Messebeteiligung Projekte anbahnen konnten (vgl. Abb. 37). Zwar sah sich zum Zeitpunkt der Befragung erst ein Exponent in der Lage, ein bereits abgeschlossenes Projekt vorzuweisen, jedoch gingen die anderen Exponenten dieser Gruppe davon aus, zukünftig Projekte auf Grundlage von auf der Messe initiierten Kontakten abschließen zu können. Somit ließen sich für die Projekt- und exponatorientierten Hochschul-Exponenten durch die Messebeteiligung ihre hoch priorisierten Projektziele realisieren. Im Rahmen der betrachteten Messebeteiligungen boten sich ebenfalls Beeinflussungsorientierten Hochschul-Exponenten Möglichkeiten, Projekte anzubahnen. Zum Befragungszeitpunkt hatten bereits $14 \%$ der Befragten ein bis drei Projekte 
abgeschlossen. $70 \%$ der interviewten Exponenten gaben an, zukünftig - häufig sogar mehrere - Projekte abschließen zu können. Folglich konnten auch Beeinflussungsorientierte Hochschul-Exponenten ihre zwar nicht primär verfolgten, aber dennoch als sehr wichtig eingestuften Projektziele realisieren. Der Anteil derjenigen, die im Rahmen ihrer Messebeteiligung keine Projekte abschließen bzw. vergleichsweise wenig Projekte anbahnen konnten, fällt bei den Pflichtrepräsentanten am höchsten aus. Dabei ist zu berücksichtigen, daß diese Exponentengruppe Projektzielen als Messebeteiligungsziele eine relativ geringe Bedeutung beigemessen hat.

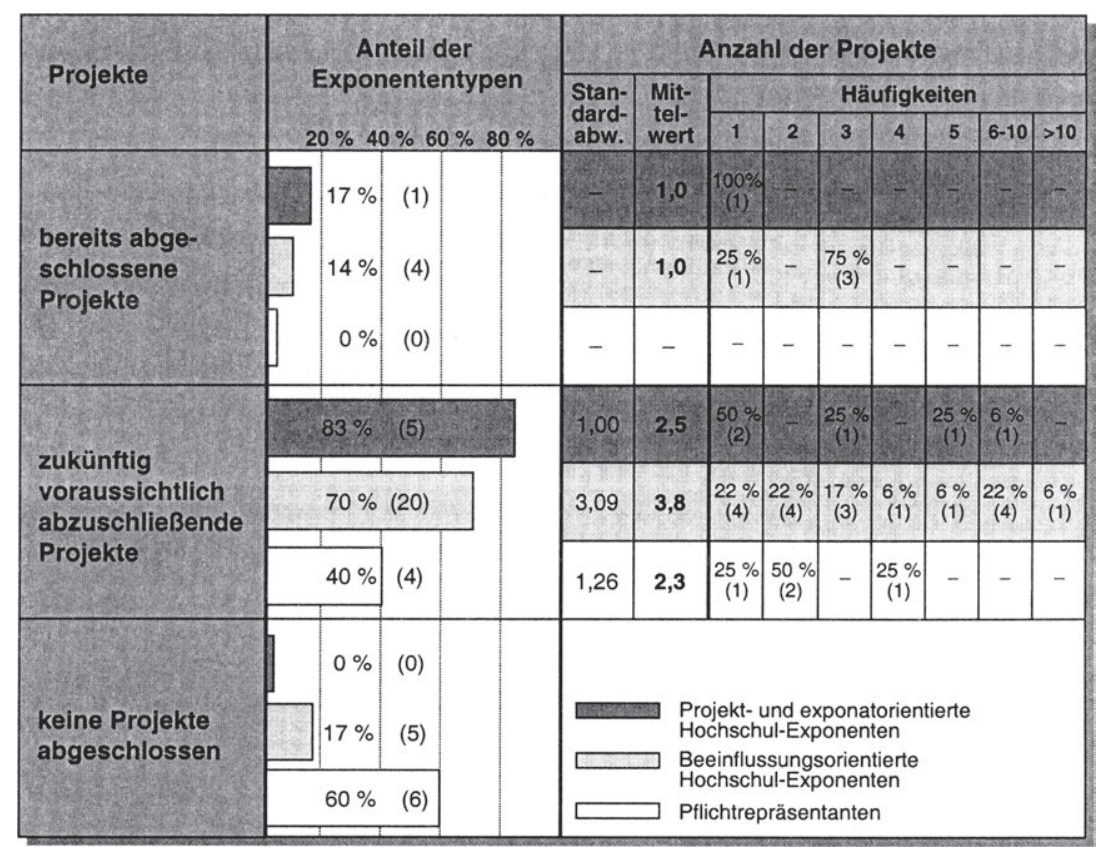

Abb. 37: Exponententypspezifisch angebahnte Projekte aufgrund des von Hochschul-Exponenten realisierten Messeengagements

Nicht die ausschließliche Betrachtung des Messeprojektvolumens, sondern die Beziehung zwischen Projektvolumen und der durch die Messebeteiligung entstandenen Kosten liefern Informationen über den direkten Erfolg des Messeengagements. Derartige Kosten-Nutzen-Relationen werden im Rahmen der direkten Erfolgskontrolle ermittelt, wobei die Analyse der Kosten von Hochschul-Messebeteiligungen eine notwendige Voraussetzung darstellt. 


\section{Direkte Erfolgskontrolle von Hochschul-Messebeteiligungen}

\subsection{Kostenanalyse als Voraussetzung zur Ermittlung des direkten Erfolgs von Hochschul-Messebeteiligungen}

Die Kostenstruktur von Hochschul-Messebeteiligungen wurde bisher noch nicht repräsentativ erhoben. Im kommerziellen Bereich führte der AUMA im Jahre 1994 erstmalig eine Studie durch, welche die Messekosten der Exponenten kommerzieller Institutionen untersucht. ${ }^{79}$ Sie beinhaltet die Analyse der Höhe und Struktur von Messebeteiligungskosten deutscher Aussteller auf internationalen und regionalen Messeveranstaltungen. Gemäß dieser Studie entstehen für die Bereiche Standbau, -ausstattung und -gestaltung die höchsten anteilsmäßigen Kosten, gefolgt von den Bereichen Personal- und Reisekosten, Standservice und Kommunikationskosten, Standmiete, Standunterhaltungskosten, Transportkosten und "Sonstigen Kosten". ${ }^{80}$ Die Kostenstruktur von Hochschul-Messebeteiligungen ist grundsätzlich nicht mit dieser Kostenstruktur kommerzieller Messebeteiligungen vergleichbar. Zum einen ergeben sich durch die Auswahl der Messebeteiligungsform erhebliche Kostenstrukturunterschiede sowohl zwischen kommerziellen Messebeteiligungen und Hochschul-Messebeteiligungen als auch zwischen verschiedenen Hochschul-Messebeteiligungen. Zum anderen räumen einzelne Messegesellschaften Exponenten des Hochschulbereichs Sonderkonditionen für Standflächenmiete, Standbau, Verbrauchsgüter und Dienstleistungen vor Ort ein, die zu Kostenstrukturdifferenzen zwischen Messebeteiligungen kommerzieller

Diese Primärerhebung wurde von Mai bis September 1993 durchgeführt, wobei insgesamt 1.256 Kostenbögen erfaßt wurden. Vgl. Ausstellungs- und Messe-Ausschuss der Deutschen Wirtschaft e.V. (AUMA) (Hrsg.), Die Messekosten der deutschen Aussteller - Die Aufwendungen deutscher Unternehmen für Beteiligungen an Messen und Ausstellungen in Deutschland, Köln 1994.

Konkret liefert die Studie folgende Ergebnisse: Der Bereich Standbau, -ausstattung und -gestaltung nimmt mit $39 \%$ den größten Anteil der Messebeteiligungskosten ein, gefolgt von den Bereichen Personal- und Reisekosten $(21 \%)$, Standservice inklusive der Kommunikationskosten $(12 \%)$, Standmiete $(15 \%)$, Standunterhaltungskosten $(5 \%)$, Transportkosten $(3 \%)$ und "Sonstige Kosten" (5\%). Vgl. Ausstellungs- und Messe-Ausschuss der Deutschen Wirtschaft e.V. (AUMA) (Hrsg.), Die Messekosten der deutschen Aussteller - Die Aufwendungen deutscher Unternehmen für Beteiligungen an Messen und Ausstellungen in Deutschland, a.a.O., S. 66. 
Aussteller und Hochschul-Exponenten führen. ${ }^{81}$ Mit diesen Sonderkonditionen, die teils individuell ausgehandelt, teils jedem Hochschul-Exponenten eingeräumt werden, subventionieren Messegesellschaften oftmals die Messebeteiligungen von Hochschulen.

Die Kosten, die im Rahmen von Hochschul-Messebeteiligungen entstehen, werden von vielfältigen Einflußfaktoren determiniert. Diese Einflußfaktoren liegen einerseits auf der strategischen Entscheidungsebene (z.B. ausgewählter Messetyp, gewählte Messebeteiligungsform) und andererseits auf der operativen Entscheidungsebene (z.B. Ausgestaltung des Messestands, Präsentationsform des Exponates). Grundsätzlich lassen sich die im Rahmen einer Hochschul-Messebeteiligung anfallenden Kosten wie folgt unterteilen: ${ }^{82}$

- Standmiete und Nebenkosten des Hochschul-Messestands,

- Kosten für Bau, Ausstattung und Gestaltung des Hochschul-Messestands,

- Exponatkosten,

- Kosten für wissenschaftliches sowie nicht-wissenschaftliches HochschulMessestandpersonal und

messespezifische Kommunikationskosten.

Standmiete und Nebenkosten des Hochschul-Messestands umfassen alle Gebühren, die an die Messegesellschaften zu entrichten sind (z.B. Miet- und Anmeldegebühren) sowie alle Kosten zur Standversorgung (z.B. Anschluß und Verbrauch von Strom, Wasser, Gas, Telefon). ${ }^{83}$ Messetypspezifisch betrachtet liegen diese Kosten auf Fachmessen niedriger als auf allgemeinen Messen. Wählt

Vgl. Krug, W., Kosten - Finanzierung - Erfolg: Kosten, in: Budach, W. et al. (Hrsg.), Hochschulen und Messen: Praktische Hinweise für Messebeteiligungen durch Hochschulen, a.a.O., S. 122.

In der allgemeinen Messeliteratur sind unterschiedliche Unterteilungen in bezug auf die Kosten einer Messebeteiligung zu finden. Während bspw. SELINSKI und SPERLING vier Kostenbereiche unterscheiden, differenzieren WINNEN und BEUSTER zwischen sieben und der AUMA zwischen sechs Kostenbereichen. Vgl. Selinski, H., Sperling U. A., Marketinginstrument Messe: Arbeitsbuch für Studium und Praxis, a.a.O., S. 204 f.; Winnen, R., Beuster, A., Kontrolle des Messeerfolgs, a.a.O., S. 373; Ausstellungs- und Messe-Ausschuss der Deutschen Wirtschaft e.V. (AUMA) (Hrsg.), Die Messekosten der deutschen Aussteller - Die Aufwendungen deutscher Unternehmen für Beteiligungen an Messen und Ausstellungen in Deutschland, Köln 1994, S. $17 \mathrm{ff}$. Auf Grundlage dieser im kommerziellen Bereich existierenden Kostenunterteilungen wird im folgenden eine Unterteilung gewählt, die - unter Berücksichtigung von hochschulspezifischen Aspekten - für Hochschul-Messebeteiligungen geeignet erscheint.

Vgl. Winnen, R., Beuster, A., Kontrolle des Messeerfolgs, a.a.O., S. 373. 
der Hochschul-Exponent als Messebeteiligungsform einen eigenen Messestand, sind dessen Kosten in voller Höhe von inm selbst zu tragen. Tritt er dagegen zusammen mit anderen Hochschul-Exponenten auf einem WissenschaftsGemeinschaftsstand auf, so werden die Kosten zwischen den sich auf dem Gemeinschaftsstand präsentierenden Hochschul-Exponenten aufgeteilt. Bei Wirtschafts-, Regionen- und Landes-Gemeinschaftsständen wird oftmals der Großteil dieser Kosten von den entsprechenden Standausrichtern getragen.

Die Kosten für Bau, Ausstattung und Gestaltung des Hochschul-Messestands umfassen die mit der direkten Messestanddarstellung entstehenden Kosten. Die Höhe dieser Kosten hängt weniger vom zu beschickenden Messetyp als vielmehr von der gewählten Messebeteiligungsform ab, wobei auf hochschuleigenen Messeständen i.d.R. höhere Kosten von den Hochschul-Exponenten zu tragen sind als auf Gemeinschaftsständen. Die Kostenhöhe hochschuleigener Messestände wird wiederum dadurch bestimmt, ob ein Systemstand gemietet, ein eigener Systemstand genutzt oder die Anfertigung eines individuellen Messestands notwendig wird. ${ }^{84}$ Die von den Hochschul-Exponenten auf Gemeinschaftsständen zu tragenden Kosten sind davon abhängig, inwieweit der Gemeinschaftsstand-Ausrichter die Ausstattung des für den Hochschul-Exponenten vorgesehenen Standplatzes und dessen entsprechende Gestaltung übernimmt.

Die Exponatkosten werden im wesentlichen davon determiniert, inwieweit zur Darstellung der zu präsentierenden Forschungs- und Entwicklungsergebnisse die Anfertigung besonderer Vorführmodelle erforderlich ist (bspw. Entwicklungen im Bereich der Atomphysik) oder inwieweit sich das Forschungsergebnis selbst zur Präsentation als Exponat anbietet (bspw. Entwicklung eines Roboters). Zudem werden zu den Exponatkosten die Transportkosten (Hin- und Rücktransport des Exponates zur Messeveranstaltung) gezählt, ${ }^{85}$ die wiederum von der Größe und Beschaffenheit des Exponates beeinflußt werden. Die Höhe der Exponatkosten, die von den Hochschul-Exponenten selbst - unabhängig von der gewählten Messebeteiligungsform - zu tragen sind, ist messetypunabhängig.

Vgl. Ausstellungs- und Messe-Ausschuss der Deutschen Wirtschaft e.V. (AUMA) (Hrsg.), Erfolgreiche Messebeteiligung - Made in Germany, Köln 1993, S. 40. 
$\mathrm{Zu}$ den Kosten für wissenschaftliches und nicht-wissenschaftliches Hochschulpersonal zählen sowohl Kosten, die während der eigentlichen Messeveranstaltung entstehen (z.B. Reisekosten), als auch Kosten, die für Vorbereitung, Organisation und Nachbereitung der Messebeteiligung anfallen, ${ }^{86}$ wobei letztere insbesondere durch die gewählte Messebeteiligungsform bestimmt werden. Bei eigenen Hochschul-Messeständen haben die Hochschul-Exponenten i.d.R. höhere Kosten zu tragen als bei der Präsentation auf Gemeinschaftsständen. Die Wahl des Messetyps hingegen wirkt sich nicht auf die Kosten für wissenschaftliches und nicht-wissenschaftliches Hochschulpersonal aus.

Unter den messespezifischen Kommunikationskosten werden Kosten subsumiert, die für im Rahmen der Messebeteiligung eingesetzte Kommunikationsmaßnahmen anfallen. Dazu zählen bspw. Kosten, die für Pressearbeit (z.B. Kosten für Presseinfos, Pressemappen) oder für Aktionen am Hochschul-Messestand bzw. Gemeinschaftsstand (z.B. Kosten für bestimmte Darbietungen) entstehen. ${ }^{87}$ Die Höhe der Kommunikationskosten ist unabhängig vom zu beschickenden Messetyp, kann aber in Abhängigkeit der gewählten Messebeteiligungsform stark variieren. Während die Hochschul-Exponenten mit eigenen Messeständen selbst - z.T. unterstützt durch die Pressestellen der Hochschulen - die Kosten ihrer Kommunikationsmaßnahmen zu tragen haben, können die sich an Gemeinschaftsständen beteiligenden Hochschul-Exponenten oftmals von Kommunikationsmaßnahmen der Gemeinschaftsstand-Ausrichter profitieren.

Die vorangegangenen Ausführungen zeigen, daß Kosten, welche HochschulExponenten im Rahmen ihrer Messebeteiligungen zu tragen haben, insbesondere von der gewählten Messebeteiligungsform abhängen. Die Kostenbelastungen, die mit einzelnen Messebeteiligungsformen für die Hochschul-Exponenten verbunden sind, werden zusammenfassend in Tab. 19 dargestellt. Eine exponententypspezifische Kostenbetrachtung ist demgegenüber nicht erforderlich, da die Kosten unabhängig vom jeweiligen Exponententyp anfallen. Aussagen über messetypspezifische Unterschiede setzen hingegen eine empirische Kostenanalyse voraus.

Vgl. Ausstellungs- und Messe-Ausschuss der Deutschen Wirtschaft e.V. (AUMA) (Hrsg.), Erfolgreiche Messebeteiligung - Made in Germany, a.a.O., S. 40.

87

Vgl. Winnen, R., Beuster, A., Kontrolle des Messeerfolgs, a.a.O., S. 373. 


\begin{tabular}{|c|c|c|c|c|c|}
\hline $\begin{array}{l}\text { Mosten } \\
\text { von Hochschul- } \\
\text { Messebeteiligungen } \\
\text { Mesteiligungs- } \\
\text { form }\end{array}$ & $\begin{array}{l}\text { Hochschul- } \\
\text { Einzel- } \\
\text { Messestand }\end{array}$ & $\begin{array}{c}\text { Wissensch-- } \\
\text { Gemein- } \\
\text { schaftsstand }\end{array}$ & $\begin{array}{c}\text { Wirtschafts- } \\
\text { Gemein- } \\
\text { schaftsstand }\end{array}$ & $\begin{array}{c}\text { Regionen- } \\
\text { Gemein- } \\
\text { schaftsstand }\end{array}$ & $\begin{array}{c}\text { Landes- } \\
\text { Gemein- } \\
\text { schaftsstand }\end{array}$ \\
\hline $\begin{array}{l}\text { Standmiete und Neben- } \\
\text { kosten des Hochschul- } \\
\text { Messestandes }\end{array}$ & -- & 0 & + & + & + \\
\hline $\begin{array}{l}\text { Kosten f. Bau, Ausstattung } \\
\text { u. Gestaltung d. Hochschul- } \\
\text { Messestandes }\end{array}$ & -- & 0 & ++ & ++ & ++ \\
\hline Exponatkosten & - & - & - & - & - \\
\hline $\begin{array}{l}\text { Kosten für wissenschaftl. } \\
\text { und nicht-wissenschaftl. } \\
\text { Hochschulpersonal }\end{array}$ & - & - & - & - & - \\
\hline \multirow[t]{2}{*}{ Kommunikationskosten } & -- & 一 & 0 & 0 & 0 \\
\hline & \multicolumn{5}{|c|}{$\begin{array}{ll}++ & =\text { niedrige Kostenbelastung für den einzelnen Hochschul-Exponenten } \\
+ & =\text { mittlere bis niedrige Kostenbelastung für den einzelnen Hochschul-Exponenten } \\
0 & =\text { mittlere Kostenbelastung für den einzelnen Hochschul-Exponenten } \\
- & =\text { hohe bis mittlere Kostenbelastung für den einzelnen Hochschul-Exponenten } \\
-- & =\text { hohe Kostenbelastung für den einzelnen Hochschul-Exponenten }\end{array}$} \\
\hline
\end{tabular}

Tab. 19: Kostenbelastungen für Hochschul-Exponenten in Abhängigkeit der hochschulspezifischen Messebeteiligungsform

Die empirische Analyse der Kostenstruktur der dieser Untersuchung zugrundeliegenden Hochschul-Messebeteiligungen (vgl. Tab. 20) ${ }^{88}$ zeigt, daß Standmiete inklusive Nebenkosten, Kosten für Bau, Ausstattung und Gestaltung des Hochschul-Messestandes sowie Exponatkosten die größten Anteile an den Gesamtkosten einer Hochschul-Messebeteiligung einnehmen. Hingegen umfassen die messebezogenen Personal- und Kommunikationskosten einen vergleichsweise gerin-

Auf vergleichbaren Unternehmensmesseständen wurden im Jahre 1992 Messebeteiligungskosten auf Mehrbranchenmessen in Höhe von DM 1.488,- pro qm und auf Fachmessen in Höhe von DM 764,- pro qm ermittelt. Vgl. Ausstellungs- und Messe-Ausschuss der Deutschen Wirtschaft e.V. (AUMA) (Hrsg.), Die Messekosten der deutschen Aussteller - Aufwendungen deutscher Unternehmen für Beteiligungen an Messen und Ausstellungen in Deutschland, a.a.O. Empirische Untersuchungen zur Erfassung des hier relevanten Kostensteigerungsfaktors wurden bisher nicht durchgeführt. Nach Expertenauffassungen liegt die Steigerung der Messekosten bezogen auf den deutschen Messemarkt bei durchschnittlich $2 \%$ pro Jahr. Unter Berücksichtigung der Kostensteigerung von $2 \%$ pro Jahr ergeben sich bezogen auf das Messejahr 1998 für vergleichbare Unternehmensmessestände auf Mehrbranchenmessen Messebeteiligungskosten von DM 1.676,- pro qm. Damit liegen diese Kosten um DM 115,- pro qm höher als die Kosten der betrachteten Hochschul-Messestände. Nach Berücksichtigung der Kostensteigerung ergeben sich bezogen auf das Messejahr 1998 für vergleichbare Unternehmensmessestände auf Fachmessen Gesamtkosten in Höhe von DM 810,- pro qm. Damit liegen diese Kosten um DM 213,- pro qm niedriger als die Kosten der betrachteten Hochschul-Messestände. 
gen Anteil an den Gesamtkosten. Insgesamt fallen die Hochschul-Messebeteiligungskosten auf Mehrbranchenmessen höher aus als auf Fachmessen. Diese Kostendifferenz kann insbesondere auf messetypspezifische Standmieten und Nebenkosten zurückgeführt werden. Vor diesem Hintergrund ist bei der Analyse des direkten Messeerfolgs, dessen Ermittlung auf Basis von Kosten-Nutzen-Relationen vorgenommen wird, der Fokus insbesondere auf die messetypspezifische Betrachtung zu legen.

\begin{tabular}{|c|c|c|c|c|}
\hline \multirow{2}{*}{$\begin{array}{l}\text { Kosten } \\
\text { von Hochschul- } \\
\text { Messebeteiligungen }\end{array}$} & \multicolumn{2}{|c|}{ Mehrbranchenmesse } & \multicolumn{2}{|c|}{ Fachmesse } \\
\hline & $\mathrm{DM} / \mathrm{qm}$ & Anteil & $\mathrm{DM} / \mathrm{qm}$ & Anteil \\
\hline $\begin{array}{l}\text { Standmiete und Neben- } \\
\text { kosten des Hochschul- } \\
\text { Messestandes }\end{array}$ & $535,-$ & $34 \%$ & $241,-$ & $23 \%$ \\
\hline $\begin{array}{l}\text { Kosten f. Bau, Ausstattung } \\
\text { u. Gestaltung d. Hochschul- } \\
\text { Messestandes }\end{array}$ & $370,-$ & $24 \%$ & $364,-$ & $35 \%$ \\
\hline Exponatkosten & $422,-$ & $27 \%$ & $370,-$ & $35 \%$ \\
\hline $\begin{array}{l}\text { Kosten für wissenschaftl. } \\
\text { und nicht-wissenschaftl. } \\
\text { Hochschulpersonal }\end{array}$ & $148,-$ & $9 \%$ & $35,-$ & $3 \%$ \\
\hline Kommunikationskosten & $86,-$ & $6 \%$ & $44,-$ & $4 \%$ \\
\hline Gesamtkosten & $1.561,-$ & $100 \%$ & 1.054,- & $100 \%$ \\
\hline
\end{tabular}

Tab. 20: Kostenstruktur der Messebeteiligung von Hochschulen

\subsection{Analyse des direkten Erfolgs von Hochschul-Messebeteiligungen auf Grundlage von Kosten-Nutzen-Relationen}

Die Methoden der direkten Erfolgskontrolle können grundsätzlich erst in der NachMesse-Phase zum Einsatz kommen, da in dieser Phase die zur Bildung von Kosten-Nutzen-Relationen erforderlichen Daten vorliegen. Zur Erfassung des direkten Erfolgs werden häufig Kennziffernanalysen und Messekontaktwertrechnungen eingesetzt. 


\subsection{Einsatz von Kennziffernanalysen}

Durch den Einsatz von Kennziffernanalysen, auch Kennzahlenanalysen genannt, erhalten Hochschul-Exponenten die Möglichkeit, die Beteiligungen an verschiedenen Messeveranstaltungen mit alternativen Marketinginstrumenten hinsichtlich ihrer Kosten-Nutzen-Relationen zu vergleichen. ${ }^{89}$ Neben ökonomischen Größen wie Projektvolumina werden bei der Kennziffernanalyse oftmals auch psychographische Größen herangezogen. Auf Grundlage von Projektvolumina gebildete Messekoeffizienten stellen ein Maß für die Wirtschaftlichkeit von Projektbemühungen am Hochschul-Messestand dar. Derartige Koeffizienten können die Hochschul-Exponenten aus dem Verhältnis ihres Messe- und Nachmesseprojektvolumens zu den angefallenen Messekosten bilden. Dabei können die Koeffizienten auf eine einzelne Messeveranstaltung oder auf eine bestimmte Zeitspanne bezogen werden. ${ }^{90}$ In Analogie zu dem aus der klassischen Werbung stammenden Tausenderkontaktpreis können ebenfalls messespezifische Kennzahlen, sog. Kontaktkosten, gebildet werden. ${ }^{91}$ Dabei ist prinzipiell die Ermittlung von Kennzahlen mit Zielgruppenbezug möglich. ${ }^{92}$ Auch bieten sich Hochschul-Exponenten Berechnungen weiterer Kennzahlen, die sich bspw. aus der Gegenüberstellung von Messekosten zur Anzahl der Gesprächskontakte, zur Quadratmeterzahl des Messestands oder zur Zahl der Standmitarbeiter ergeben.

Um die Vorteile von Kennziffernanalysen zu nutzen, sind von den HochschulExponenten einige Voraussetzungen zu erfüllen. Neben der korrekten Ermittlung

Vgl. Haeberle, K. E., Erfolg auf Messen und Ausstellungen: Handbuch für Teilnahme, Organisation, Gestaltung, Technik, a.a.O., S. 271; Wedel, P. Graf von, Messen - vom Markt zum Marketing, Frankfurt 1977, S. 47.

Hochschul-Exponenten können z.B. veranstaltungsbezogene Messekoeffizienten (= Wert der Abschlüsse auf einer Messe/Kosten der Messebeteiligung) oder zeitbezogene Messekoeffizienten (= Zwei-Jahresprojektvolumen des Hochschul-Exponenten/jährliche Kosten der Messebeteiligungen) bilden. Vgl. Blohm, H., Messen und Ausstellungen als Mittel der Absatzförderung in der Schweizerischen Maschinenindustrie, a.a.O., S. 135.

91 So können bspw. Hochschul-Messestand-Kontaktpreise (= Messekosten/Anzahl der Kontakte am Hochschul-Messestand) oder Messebesucherpreis (= Messekosten/Messebesucher) ermittelt werden. Vgl. Cunningham, M. T., White, J. G., The Role of Exhibitions in Industrial Marketing - An Evaluation of the International Machine Tool Exhibition, a.a.O., S. 239.

Als Beispiele können hierzu Fachbesucherpreise (= Messekosten/Fachbesucher) oder Zielgruppenpreise (= Messekosten/Zielgruppe) angeführt werden. Vgl. Backhaus, K., Messen als Institutionen der Informationspolitik, a.a.O., S. 91. Vgl. hierzu auch Naumann, C., Ist es eine Messe wert? - Den Messeerfolg organisieren und kontrollieren, in: BddW, Nr. 170, 06.09.1982, S. 7 . 
relevanter Größen (Projektvolumen, Messekosten, Kontakte etc.) ist eine über mehrere Messen und Veranstaltungstermine hinweg konstante und konsequente Anwendung einheitlicher Methoden der Datenermittlung notwendig, um auf diese Weise eine Vergleichbarkeit der Ergebnisse der Kennziffernanalyse zu gewährleisten. Als ein Nachteil von Kennziffernanalysen kann die oftmals mangelnde Berücksichtigung von unterschiedlichen Zielgruppenstrukturen angeführt werden. Des weiteren erfolgt bei der Interpretation der Kennzahlen i.d.R. keine Berücksichtigung qualitativer Aspekte der Begegnungen zwischen Hochschul-Exponenten und Messebesuchern. ${ }^{93}$

Im Rahmen der betrachteten Hochschul-Messebeteiligungen wurde die Qualität der Begegnungen zwischen Exponenten und Hochschul-Messestand-Besuchern insofern berücksichtigt, als daß eine Differenzierung zwischen Messebesucher-, Messestandkontakt- und Messestandgesprächspreisen vorgenommen wurde (vgl. Tab. 21). Der Messebesucherpreis, bei dem die gesamten Messekosten ${ }^{94}$, die im Rahmen der Messebeteiligung anfallen, auf die Anzahl der Messebesucher bezogen wurden, lag auf der Mehrbranchenmesse mit DM 2,- weit unter dem Messebesucherpreis der betrachteten Fachmesse, der mit DM 7,- vergleichsweise hoch ausfiel. Auch der Messestandkontaktpreis, der die gesamten Messekosten auf die Anzahl der Messestandkontakte bezieht, überstieg auf der Fachmesse mit DM 146,- den Messestandkontaktpreis der Mehrbranchenmesse (DM 112,- pro Messestandkontakt). Wie der Messebesucher- und der Messestandkontaktpreis lag der Messestandgesprächspreis, der die Kosten auf die Anzahl der auf dem Gemeinschaftsstand geführten Gespräche bezieht, auf der Fachmesse mit DM 156,- höher als der Gesprächspreis von DM 133,- auf der Mehrbranchenmesse. Folglich sind aus Sicht der aufgeführten Kennziffernanalysen HochschulMessebeteiligungen auf Mehrbranchenmessen den Messebeteiligungen an Fachmessen überlegen.

Vgl. Backhaus, K., Messen als Institutionen der Informationspolitik, a.a.O., S. 91. Kosten als auch die Kosten, die ggf. von finanzmittelunterstützenden Institutionen übernommen werden. 


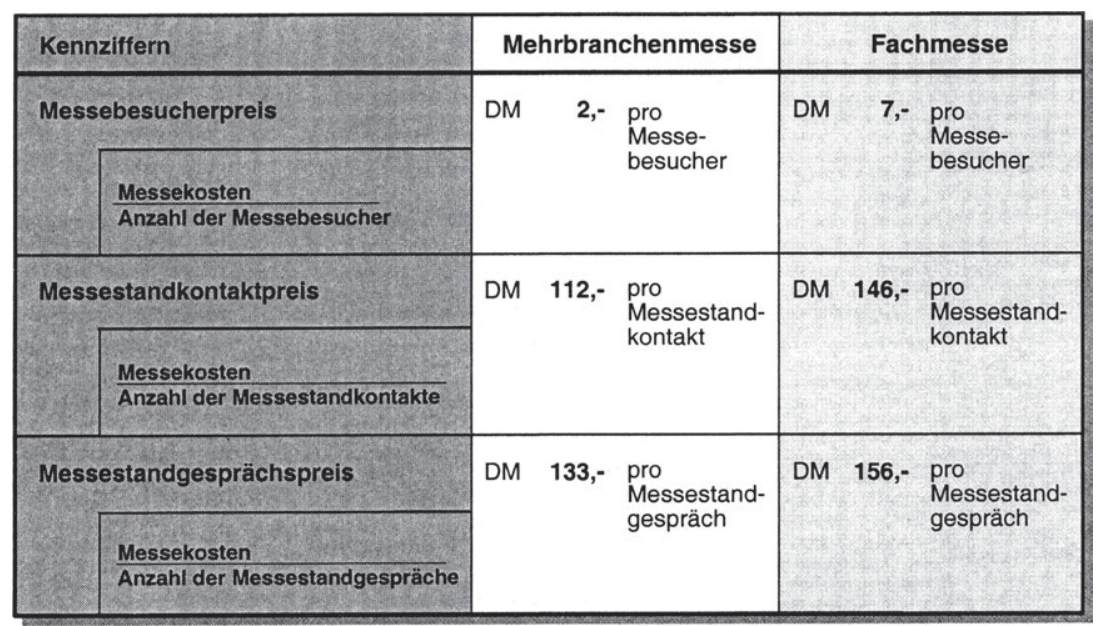

Tab. 21: Kennziffern der Messebeteiligung von Hochschulen

Im Rahmen von Kosten-Nutzen-Analysen sind nicht nur potentielle und reale Kontakte auf Messen, sondern darüber hinaus auch etwaige Kontakte durch die zu den jeweiligen Hochschul-Messebeteiligungen erschienenen Medienbeiträgen zu berücksichtigen. So wurden zu den Hochschul-Messebeteiligungen auf der Mehrbranchenmesse insgesamt 38 TV-Beiträge und 24 Hörfunk-Beiträge ausgestrahlt sowie 157 Presseartikel verfaßt (vgl. Tab. 22). Werden nur die erhobenen Reichweiten - nicht von allen Medien liegen Reichweiten vor - auf die Kosten der jeweiligen Messebeteiligung bezogen, so wird ein Tausenderkontaktpreis von DM 17,- und damit ein Kontaktpreis pro erreichter Person von ca. DM 0,02 realisiert. Im Rahmen der Fachmessebeteiligung, zu der 5 TV-, 11 Hörfunk- und 87 Presse-Beiträge verfaßt wurden, liegt der Tausenderkontaktpreis mit DM 13,- bzw. der Kontaktpreis pro erreichte Person mit ca. DM 0,01 noch etwas niedriger. Angesichts der nur begrenzt möglichen Berücksichtigung der Reichweiten ist von insgesamt noch besseren Kosten-Nutzen-Relationen auszugehen. ${ }^{95}$

Auf eine exponententypspezifische Betrachtung muß bei der hier vorgenommenen Kennziffernanalyse sowie bei der folgenden Messekontaktwertrechnung verzichtet werden, da keine verläßlichen Informationen über exponententypspezifische Kosten vorliegen. Vor dem Hintergrund der zuvor aufgezeigten Kostenstruktur ist jedoch davon auszugehen, daß - nicht zuletzt aufgrund der determinierenden messetypspezifischen Kostenanteile - grundsätzlich relativ geringe exponententypspezifische Kostenunterschiede existieren. 


\begin{tabular}{|l|c|c|c|c|c|c|}
\hline \multirow{2}{*}{ Reichweiten } & \multicolumn{3}{|c|}{ Mehrbranchenmesse } & \multicolumn{3}{c|}{ Fachmesse } \\
\cline { 2 - 7 } & $\begin{array}{c}\text { Beiträge } \\
\text { insgesamt }\end{array}$ & $\begin{array}{c}\text { Beiträge } \\
\text { mit erhobener } \\
\text { Reichweite }\end{array}$ & $\begin{array}{c}\text { erhobene } \\
\text { Reichweite }\end{array}$ & $\begin{array}{c}\text { Beiträge } \\
\text { insgesamt }\end{array}$ & $\begin{array}{c}\text { Beiträge } \\
\text { mit erhobener } \\
\text { Reichweite }\end{array}$ & $\begin{array}{c}\text { erhobene } \\
\text { Reichweite }\end{array}$ \\
\hline $\begin{array}{l}\text { TV } \\
\text { (Zuschauer ab 14 J.) }\end{array}$ & 38 & 29 & 24,03 Mio. & 5 & 3 & 0,45 Mio. \\
\hline $\begin{array}{l}\text { Hörfunk } \\
\text { (Hörer ab 14 J.) }\end{array}$ & 24 & 7 & 2,75 Mio. & 11 & 6 & 1,86 Mio. \\
\hline $\begin{array}{l}\text { Presse } \\
\text { Insgesamt }\end{array}$ & 219 & 99 & 35,37 Mio. & 103 & 62 & 17,07 Mio. \\
\hline
\end{tabular}

Tab. 22: Medienbeiträge zu Hochschul-Messebeteiligungen

Den aufgezeigten Defiziten von Kennziffernanalysen kann durch die Anwendung von Messekontaktwertrechnungen entgegengewirkt werden.

\subsection{Einsatz von Messekontaktwertrechnungen}

Mit dem Einsatz von Messekontaktwertrechnungen ${ }^{96}$ können Hochschul-Exponenten alle Besucherkontakte auf dem Hochschul-Messestand nicht nur quantitativ, sondern auch qualitativ erfassen. Neben einer zahlenmäßigen Aufnahme der Besucherkontakte auf dem Hochschul-Messestand ${ }^{97}$ bietet diese Methode den Hochschul-Exponenten eine qualitative Unterscheidung der Messestand-Besucher nach vier Kriterien: Dauer der Beziehung zum Hochschul-Exponenten (neu, alt),

Die Messekontaktwertrechnung, auch Messekontaktbewertung genannt, wurde insbesondere aufgrund der zuvor angeführten Kritik der Kennziffernanalyse, daß bei der Interpretation der Kennzahlen keine Aspekte der Begegnungen zwischen Ausstellern und Nachfragern berücksichtigt werden, entwickelt.

Problematisch sind in diesem Zusammenhang die aus Sicht der einzelnen Exponenten unterschiedlich vorgenommenen Definitionen von Messestand-Kontakten. Zahlreiche Exponenten definieren einen Kontakt zu Messebesuchern dann als Messestand-Kontakt, wenn sie mit diesem Besucher ein längeres Gespräch geführt haben, andere Aussteller dagegen zählen zu den Messestand-Kontakten auch Prospektanfragen, und wiederum andere Aussteller definieren bereits einen einfachen Besuch des Messestands ohne Gespräch und ohne Prospektanfrage als einen Messestand-Kontakt. 
Herkunft (Inland, Ausland), Kompetenz (Geschäftsleitung, Mitarbeiter), projektbezogenes Verhalten (mit Projektauftrag, ohne Projektauftrag). ${ }^{98}$ Hochschul-Exponenten können dabei die einzelnen Hochschul-Messestand-Besucher oder -Besuchergruppen mit sogenannten Kontaktwertpunkten bewerten. Diese Gewichtungen erfolgen unter Berücksichtigung der jeweils durch die Hochschul-Exponenten primär mit der Messebeteiligung anvisierten hochschulspezifischen Zielgruppen. Zur Erfassung der Kontakte auf dem Hochschul-Messestand bietet sich die Verwendung von Gesprächsprotokollen an. Die geführten Gespräche können von den Hochschul-Exponenten mit Hilfe der vorab festgelegten Kontaktwertpunkte gewichtet werden. Der Vergleich der ermittelten Daten mit den Soll-Vorstellungen sowie die Betrachtung von Vergleichsdaten mehrerer Messeveranstaltungen erlauben Aussagen über die Rentabilität der Messebeteiligungen, wenn den Kontaktwerten die Messekosten gegenübergestellt werden..$^{99}$ Möglich sind auch Vergleiche mit den von der Gesellschaft zur freiwilligen Kontrolle von Messe- und Ausstellungszahlen (FKM) erhobenen Daten zur Besucherstrukturanalyse. Diese Vergleiche liefern den Hochschul-Exponenten Erkenntnisse darüber, ob es gelungen ist, die angestrebte Zielgruppe auf der Messe effizient zu erreichen.

Dem Vorteil, Erkenntnisse über die Effizienz der Zielgruppenerreichung und die Rentabilität der Messebeteiligungen zu gewinnen, steht als zentraler Nachteil die mangelnde Berücksichtigung unterschiedlicher Projektvolumina und Ergebnisse der Nach-Messe-Aktivitäten gegenüber. Dennoch bietet sich mit der Messebewertung den Hochschul-Exponenten eine flexible Methode zur Überprüfung der Erreichung hochschulspezifischer Kontaktziele, die darüber hinaus einen einfachen und nachvollziehbaren Aufbau aufweist. Somit bietet sich die Messekontaktwertrechnung zum Einsatz im Rahmen der direkten Erfolgskontrolle von Hochschul-Messebeteiligungen an. Zu berücksichtigen ist jedoch, daß die Messekontaktwertrechnung sowie auch die Kennziffernanalyse auf Ergebnisse der indirekten Erfolgskontrolle (Z.B. Kontaktzählung, Gesprächsprotokoll) zurückgreifen muß, die damit zum integrativen Bestandteil dieses Verfahrens werden.

Vgl. Naumann, C., Was war die letzte Messe wert? - Kontaktwertmessung nach Punkten, in: $\mathrm{m}+\mathrm{a}$ report, 55. Jg., Heft 1, 1974, S. $15 \mathrm{ff}$.

99 Vgl. Naumann, C., Was war die letzte Messe wert? - Kontaktwertmessung nach Punkten, a.a.O., S. 17. 
Im Rahmen der vorliegenden Untersuchung wurde eine zielgruppen- und gesprächsbezogene Messekontaktwertrechnung durchgeführt. Bei der zielgruppenbezogenen Messekontaktwertrechnung lag der ermittelte Messestandkontaktpreis für Kontakte mit Praxisvertretern auf der Mehrbranchenmesse mit DM 200,annähernd auf gleichem Niveau mit dem der Fachmesse (DM 209,- pro Kontakt). Bei der gesprächsbezogenen Messekontaktwertrechnung konnten hingegen unterschiedliche Tendenzen festgestellt werden. So konnten auf Fachmessen mit DM 779,- niedrigere Gesprächspreise für auf dem Hochschul-Messestand geführte Gespräche über zukünftige Projektkooperationen erhoben werden als auf Mehrbranchenmessen (DM 1.330,- pro Gespräch). Der Gesprächspreis für Informationsgespräche fiel demgegenüber zugunsten der Mehrbranchenmesse aus (DM 162,- vs. DM 226,- pro Gespräch auf Fachmessen).

\subsection{Zentrale Ergebnisse der Erfolgskontrolle von Hochschul-Messe- beteiligungen}

Die Ergebnisse der verschiedenartigen, zum Einsatz im Hochschulbereich geeigneten Methoden zur Erfolgskontrolle von Hochschul-Messebeteiligungen zeigen, daß die befragten Hochschul-Exponenten ihre primär verfolgten Messezielsetzungen erreichen konnten. Darüber hinaus liefern die ermittelten Ergebnisse vielfältige Erkenntnisse sowohl zum messetypspezifischen als auch zum exponententypspezifischen Vergleich von Hochschul-Messebeteiligungen.

Die folgende Tabelle faßt die zentralen messetypspezifischen Ergebnisse zusammen. Dabei wird dargestellt, welche Methoden sich zur Kontrolle der Erreichung welcher Messeziele bzw. -zielgruppen anbieten und ob die durch den Einsatz dieser Methode gewonnenen Ergebnisse zugunsten von Fach- oder Mehrbranchenmessen ausfallen. 


\begin{tabular}{|c|c|c|c|c|c|c|c|c|c|c|c|c|}
\hline \multicolumn{2}{|r|}{ Direkte Erfolgskontrolle } & \multicolumn{10}{|c|}{ Nutzen von Hochschul-Messebeteiligungen } & \multirow[b]{3}{*}{$\begin{array}{l}\text { Kosten } \\
\text { Nut- } \\
\text { zen- } \\
\text { Rela- } \\
\text { tionen }\end{array}$} \\
\hline \multirow{2}{*}{\multicolumn{2}{|c|}{$\begin{array}{l}\text { Elngesetzte } \\
\text { Methoden zur } \\
\text { Messeerfolgskontrolle }\end{array}$}} & \multicolumn{5}{|c|}{ Hochschulspezif. Messezieldimensionen } & \multicolumn{5}{|c|}{ Hochschulspezif. Messezielgruppen } & \\
\hline & & $\begin{array}{l}\text { Infor- } \\
\text { ma- } \\
\text { tions- } \\
\text { ziele }\end{array}$ & $\begin{array}{l}\text { Hoch- } \\
\text { schul- } \\
\text { bez. } \\
\text { Beainfl- } \\
\text { ziele }\end{array}$ & \begin{tabular}{|c|} 
Inst._. \\
Lehrst.J \\
Fachb. \\
bez. \\
Beeinti-. \\
ziele
\end{tabular} & $\begin{array}{c}\text { Expo- } \\
\text { natbez. } \\
\text { Beainfl- } \\
\text { zielo }\end{array}$ & $\begin{array}{l}\text { Pro- } \\
\text { lekt- } \\
\text { zlele }\end{array}$ & $\begin{array}{l}\text { Wis- } \\
\text { sen- } \\
\text { schaft }\end{array}$ & $\begin{array}{l}\text { Stu- } \\
\text { die- } \\
\text { rende }\end{array}$ & $\begin{array}{l}\text { Wirt- } \\
\text { schaft }\end{array}$ & $\begin{array}{l}\text { Offent- } \\
\text { lich- } \\
\text { kelt }\end{array}$ & Staat & \\
\hline \multirow{5}{*}{$\begin{array}{l}\text { \& } \\
\frac{8}{2} \\
\frac{8}{\$} \\
\text { \& } \\
\frac{1}{2}\end{array}$} & $\begin{array}{l}\text { Zählung ausgegebener } \\
\text { Informatlons-Materialien }\end{array}$ & FM & MM & MM & MM & & & & & & & \\
\hline & $\begin{array}{l}\text { Zahlung der Hochschul- } \\
\text { Messestand-Besucher }\end{array}$ & & MM & MM & MM & & & & & & & \\
\hline & $\begin{array}{l}\text { Zählung persönlicher } \\
\text { Kontakte auf dem } \\
\text { Hochschul-Messestand }\end{array}$ & MM & MM & MM & MM & & & & & & & \\
\hline & $\begin{array}{l}\text { Protokolle der Hochschul- } \\
\text { Messestand-Gespräche }\end{array}$ & MM & & & & FM & FM & MM & FM & MM & $\begin{array}{l}\text { FM } \\
\text { MM }\end{array}$ & \\
\hline & $\begin{array}{l}\text { Befragung der Hochschul- } \\
\text { Messestand-Besucher }\end{array}$ & & $\begin{array}{l}\text { FM } \\
\text { MM }\end{array}$ & $\begin{array}{l}\text { FM } \\
\text { MM }\end{array}$ & $\begin{array}{l}\text { FM } \\
\text { MM }\end{array}$ & $\begin{array}{l}F M \\
\text { MM }\end{array}$ & $\begin{array}{l}\mathrm{FM} \\
\mathrm{MM}\end{array}$ & MM & $\begin{array}{l}\text { FM } \\
\text { MM }\end{array}$ & $\begin{array}{l}F M \\
M M\end{array}$ & $\begin{array}{l}\text { FMI } \\
\text { MM }\end{array}$ & \\
\hline \multirow{6}{*}{$\begin{array}{l}\frac{\$}{2} \\
\frac{2}{2} \\
\frac{2}{\delta} \\
\frac{8}{2} \\
\frac{8}{2} \\
\frac{1}{0} \\
\frac{\pi}{2}\end{array}$} & $\begin{array}{l}\text { Auswertung des } \\
\text { FKM-Tests }\end{array}$ & & & & & & $\begin{array}{l}\mathrm{FM} \\
\mathrm{MM}\end{array}$ & MM & $\begin{array}{l}F M \\
M M\end{array}$ & $\begin{array}{l}F M \\
M M\end{array}$ & $\begin{array}{l}\text { FM } \\
\text { MM }\end{array}$ & \\
\hline & $\begin{array}{l}\text { Betragung des wissen- } \\
\text { schaft. u, nicht-wissen- } \\
\text { schaftl. Standpersonals }\end{array}$ & FM & FM & FM & FM & FM & & & & & & \\
\hline & $\begin{array}{l}\text { Messebetelligungs- } \\
\text { Nutzen-Index }\end{array}$ & FM & FM & FM & FM & FM & & & & & & \\
\hline & $\begin{array}{l}\text { Analyse des Messe- } \\
\text { projektvolumens }\end{array}$ & & & & & FM & & & & & & \\
\hline & $\begin{array}{l}\text { Kennziffern- } \\
\text { analysen }\end{array}$ & & & & & & & & & & & $\begin{array}{l}\mathrm{FM} \\
\mathrm{MM}\end{array}$ \\
\hline & $\begin{array}{l}\text { Messekontakt- } \\
\text { wertrochnung }\end{array}$ & 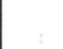 & & & & & & & & & & $\begin{array}{l}\mathrm{FM} \\
\mathrm{MM}\end{array}$ \\
\hline & $\begin{array}{l}\text { Betreffende Methode } \\
\text { Kontrolle der jeweilig } \\
\text { spezifischen Messez } \\
\text { bzw. Messezielgrupp }\end{array}$ & $\begin{array}{l}\text { bietet sich } \\
\text { en hochsc } \\
\text { ielerreichu } \\
\text { enerreichy }\end{array}$ & & & 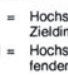 & $\begin{array}{l}\text { hul-Mess } \\
\text { nsionen } \\
\text { hul-Mess } \\
\text { Zieldime }\end{array}$ & $\begin{array}{l}\text { eteiligung } \\
\text { w. Zielgn } \\
\text { eteiligung } \\
\text { onen bzw. }\end{array}$ & $\begin{array}{l}n \text { Fachn } \\
\text { pe ertolg } \\
\text { n Mehrt } \\
\text { Kielgrupp }\end{array}$ & $\begin{array}{l}\text { sen bzgl, o } \\
\text { cher als } \mathrm{M} \\
\text { chenmess } \\
\text { irolgreiche }\end{array}$ & $\begin{array}{l}\text { Erreichy } \\
\text { hrobranche } \\
\text { bzgl. der } \\
\text { als Fachn }\end{array}$ & $\begin{array}{l}\text { der betre } \\
\text { eesse-Be: } \\
\text { reichung } \\
\text { sse-Besc }\end{array}$ & $\begin{array}{l}\text { Henden } \\
\text { chickung } \\
\text { ler betret- } \\
\text { lickung }\end{array}$ \\
\hline
\end{tabular}

Tab. 23: Methoden zur Erfolgskontrolle von Hochschul-Messebeteiligungen und deren messetypspezifische Ergebnisse

Die in der Messe-Phase eingesetzten Methoden zur indirekten Messeerfolgskontrolle liefern - so zeigt die messetypspezifische Analyse - grundsätzlich bessere Ergebnisse für Hochschul-Messebeteiligungen auf Mehrbranchenmessen. Hingegen zeigen die Ergebnisse der in der Nach-Messe-Phase eingesetzten Methoden zur indirekten Messeerfolgskontrolle eine Überlegenheit von Hochschul-Messebeteiligungen auf Fachmessen. Diese Ergebnisse lassen vermuten, daß Messebeteiligungen an Mehrbranchenmessen besser geeignet sind, kurzfristige indirekte Messeerfolge zu realisieren, während mit Fachmessebeteiligungen eher langfristige indirekte Messeerfolge erzielt werden. Differenziertere Ergebnisse lassen sich durch die Analyse der Erreichungsgrade angestrebter Messeziele- und zielgruppen gewinnen. Deshalb sollen in einer abschließenden Übersicht auf Basis der zuvor dargestellten empirischen Ergebnisse die Erreichung hochschulspezifi- 
scher Messeziele und Messezielgruppen - differenziert nach Messetypen und Exponententypen - dargestellt werden (vgl. Tab. 24).

\begin{tabular}{|c|c|c|c|c|c|c|c|c|c|c|c|}
\hline \multirow[b]{3}{*}{$\begin{array}{l}\text { Hochschul- } \\
\text { Messebetell gungen } \\
\text { (allg Jmessetypspezif } J \\
\text { exponententypspezif. }\end{array}$} & \multicolumn{10}{|c|}{ Nutzen von Hochschul-Messebeteiligungen } & \multirow[b]{3}{*}{$\begin{array}{l}\text { Kostan- } \\
\text { Nut- } \\
\text { zen- } \\
\text { Rela- } \\
\text { tlonen }\end{array}$} \\
\hline & \multicolumn{5}{|c|}{ Hochschulspezif. Messezieldimensionen } & \multicolumn{5}{|c|}{ Hochschulspezif. Messezielgruppen } & \\
\hline & $\begin{array}{l}\text { Infor- } \\
\text { ma- } \\
\text { tlons: } \\
\text { ziele }\end{array}$ & 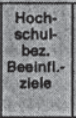 & \begin{tabular}{|l|} 
Inst.-. \\
Lehrst/J \\
Fachb. \\
bez. \\
Beeint.- \\
ziele \\
\end{tabular} & 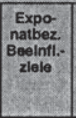 & $\begin{array}{l}\text { Pro } \\
\text { lekt- } \\
\text { ziele }\end{array}$ & $\begin{array}{l}\text { Wis- } \\
\text { sen- } \\
\text { schaft }\end{array}$ & $\begin{array}{l}\text { Stu- } \\
\text { die- } \\
\text { rende }\end{array}$ & $\begin{array}{l}\text { Wirt- } \\
\text { schaft }\end{array}$ & $\begin{array}{l}\text { Oftent- } \\
\text { Hich- } \\
\text { kait }\end{array}$ & Staat & \\
\hline $\begin{array}{l}\text { Hochschut-Messobetelligung } \\
\text { (allgemein) }\end{array}$ & + & + & + & + & + & 0 & + & ++ & + & - & $+/-$ \\
\hline \multicolumn{12}{|l|}{ Messetypspezifische Analyse } \\
\hline $\begin{array}{l}\text { Hochschul-Messebetelligung } \\
\text { an Mehrbranchenmessen }\end{array}$ & + & ++ & ++ & ++ & + & 0 & + & ++ & ++ & - & $+/-$ \\
\hline $\begin{array}{l}\text { Hochschul-Messebetelligung } \\
\text { an Fachmessen }\end{array}$ & ++ & + & + & + & ++ & 0 & + & ++ & + & - & $+/-$ \\
\hline \multicolumn{12}{|c|}{ Exponententypspezifische Analyse } \\
\hline $\begin{array}{l}\text { Prolekt-und exponatorlentierte } \\
\text { Hochschul-Exponenten }\end{array}$ & + & + & + & ++ & ++ & 0 & ++ & ++ & + & - & $+/-$ \\
\hline $\begin{array}{l}\text { Beelnflussungsorlentierte } \\
\text { Hochschut-Exponenten }\end{array}$ & + & + & ++ & ++ & + & 0 & + & ++ & ++ & -- & $+1-$ \\
\hline Pflichtreprăsentanten & 0 & 0 & 0 & 0 & - & - & 0 & + & 0 & + & $+1-$ \\
\hline \multicolumn{9}{|c|}{ 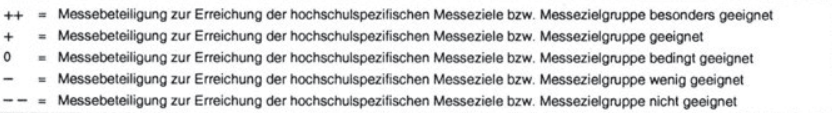 } & \multicolumn{3}{|c|}{$\begin{array}{l}\text { Kosten-Nutzen- } \\
\text { Relation stark } \\
\text { abhängig von der } \\
\text { jeweiligen } \\
\text { Bezugsbasis }\end{array}$} \\
\hline
\end{tabular}

Tab. 24: Messetypspezifische und exponententypspezifische Analyse des Erfolgs von Hochschul-Messebeteiligungen

Messetypspezifisch betrachtet können die Messeziele Informations- und Projektziele besser durch eine Beteiligung an Fachmessen als an Mehrbranchenmessen erreicht werden. Hingegen ist von einer tendenziell höheren Realisierung von Beeinflussungszielen auf Mehrbranchenmessen als auf Fachmessen auszugehen. Die Analyse der Erreichung hochschulspezifischer Messezielgruppen belegt, daß sowohl Exponenten der Fach- als auch der Mehrbranchenmesse Kontakt zu den hoch priorisierten Wirtschaftsvertretern aufnehmen konnten. Die ebenfalls als wichtig eingestufte Zielgruppe Öffentlichkeit konnte auf der Mehrbranchenmesse häufiger kontaktiert werden als auf der Fachmesse, wenngleich auch hier zahlreiche Kontakte zu dieser Zielgruppe realisiert wurden. Die im Vergleich zur Wirtschaft und Öffentlichkeit als weniger wichtig eingestuften Messezielgruppen Wissenschaftler und Studierende nahmen auf beiden Messen zahlreiche Kontakte zu den Hochschul-Exponenten auf, wobei die Kontakthäufigkeit zu 
Studierenden höher ausfiel. Zum Staat wurden insgesamt relative wenig Kontakte hergestellt. Die Kosten-Nutzen-Analysen lieferten keine Erkenntnisse zur generellen Überlegenheit bzgl. der Mehrbranchen- oder Fachmesse, da die ermittelten Kosten-Nutzen-Relationen in Abhängigkeit der gewählten Bezugsbasis teils zugunsten von Fachmessen, teils zugunsten von Mehrbranchenmessen ausfielen.

Die exponententypspezifische Analyse des Messebeteiligungserfolgs zeigt, daß sowohl Projekt- und exponatorientierte Hochschul-Exponenten als auch Beeinflussungsorientierte Hochschul-Exponenten mit ihrer Messebeteiligung die Erreichung inrer primär verfolgten Zielsetzungen forcieren konnten. So realisierten erstere mit ihrem Messeengagement vor allem Projekt- und Exponatbezogene Messezielsetzungen und letztere insbesondere Instituts-/Lehrstuhl-/Fachbereichsbezogene und Exponatbezogene Beeinflussungsziele. Zudem konnten diese Hochschul-Exponententypen zahlreiche Kontakte zu ihren hoch priorisierten Messezielgruppen herstellen. Demzufolge lassen sich für Projekt- und exponatorientierte sowie Beeinflussungsorientierte Hochschul-Exponenten Messebeteiligungserfolge konstatieren. Die Pflichtrepräsentanten nutzten ihre Messebeteiligung hingegen weniger, um hochschulspezifische Messeziele zu erreichen. Zwar konnten diese Exponententypen Kontakte zu ihren primär verfolgten Zielgruppen - insbesondere Staat - realisieren, dennoch kann insgesamt ihre Messebeteiligung im Vergleich zu den Messebeteiligungen der anderen Hochschul-Exponententypen als wenig erfolgreich eingestuft werden.

Die vorangegangenen Ausführungen zeigen, daß der Erfolg von Hochschul-Messebeteiligungen insbesondere von den primär verfolgten Zielsetzungen der jeweiligen Hochschul-Exponenten abhängt. Eine vor dem Hintergrund von Projekt-, Beeinflussungs- und Informationszielen durchgeführte Messebeteiligung kann grundsätzlich zum Erfolg für den jeweiligen Hochschul-Exponenten führen. Damit sind die notwendigen Bedingungen im Rahmen der Effizienzbetrachtung von Hochschul-Messebeteiligungen erfüllt. Zur Effizienzbeurteilung besteht jedoch darüber hinaus die Notwendigkeit, die hinreichende Bedingung zu prüfen. Der Suche nach geeigneten Vergleichsobjekten kommt dabei eine zentrale Bedeutung zu. Im Hinblick auf die Vergleichsobjekte kann zwischen intra- und inter-instrumentellen Vergleichen unterschieden werden. Während bei den intra-instrumentellen Vergleichen andere Messebeteiligungen als Vergleichsobjekte fungieren, werden bei inter-instrumentellen Vergleichen andere Kommunikationsmaßnahmen des Hochschulmarketing-Mix als Vergleichsobjekte herangezogen. 


\section{Effizienzvergleiche von Hochschul-Messebeteiligungen mit ausge- wählten Vergleichsobjekten}

\subsection{Intra-instrumentelle Vergleiche zwischen verschiedenen Messebe- teiligungen}

Bei intra-instrumentellen Vergleichen können einerseits hochschuleigene Messebeteiligungen einbezogen werden, wobei entweder Messebeteiligungen an identischen Messen aus zeitlich vorgelagerten Perioden (Zeitvergleiche) oder Beteiligungen an verschiedenen Messeveranstaltungen bzw. -typen (Veranstaltungsvergleiche) als Vergleichsobjekte i.S.v. Benchmarks dienen können. Andererseits können Messebeteiligungen anderer Institutionen als Vergleich herangezogen werden, wobei sowohl Messebeteiligungen anderer Hochschulen (Hochschulvergleiche) als auch Messebeteiligungen von Unternehmen (Unternehmensvergleiche) als Vergleichsobjekte i.S.v. Benchmarks denkbar sind (vgl. Abb. 38).

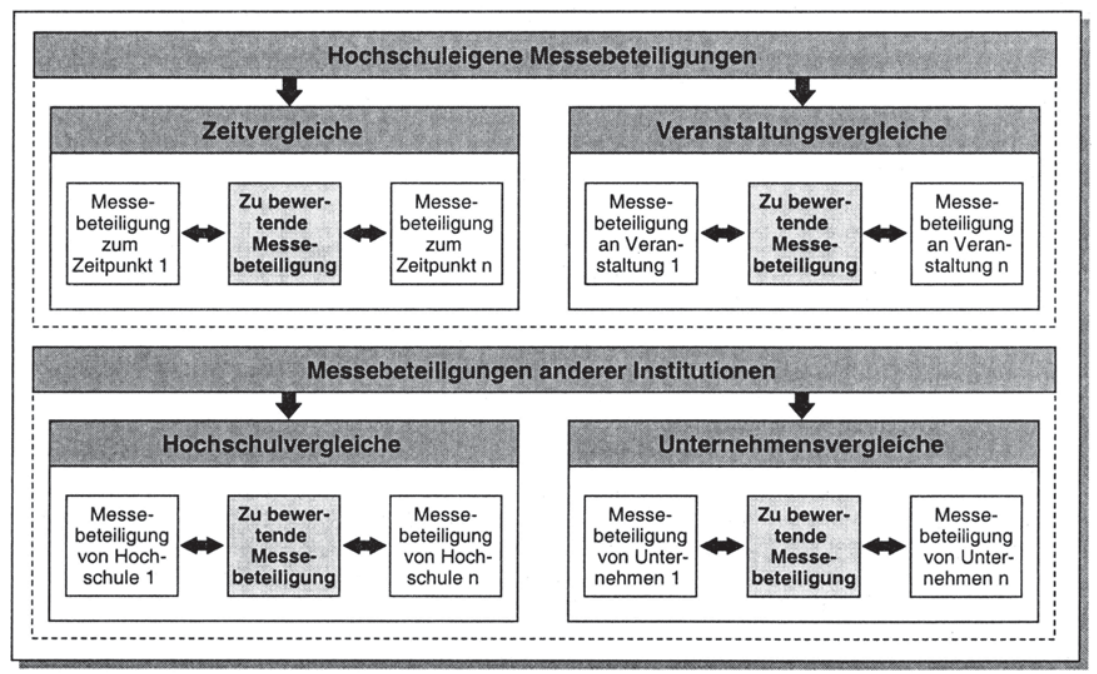

Abb. 38: Intra-instrumentelle Vergleiche zwischen verschiedenen Messebeteiligungen 
Insbesondere bei Hochschul- bzw. Unternehmensvergleichen stellen jedoch die oftmals unzureichende Datenbasis sowie die Notwendigkeit einer weitestgehend kongruenten Hochschul- bzw. Unternehmensstruktur und Umweltsituation implizite Probleme dar. ${ }^{100}$ Weitere Schwierigkeiten treten auf, wenn bspw. Messekontaktkosten $^{101}$ als ein mögliches Vergleichskriterium im Rahmen des intra-instrumentellen Vergleichs herangezogen werden. So werden nicht nur aufgrund der von Messe zu Messe unterschiedlich anfallenden Kosten, sondern auch aufgrund der Zurechnungsproblematik messebezogener Kosten sowie der unterschiedlichen Definition eines Messestandkontakts von den einzelnen Hochschul-Exponenten unterschiedliche Messestandkontaktkosten bzw. Messestandkontaktpreise realisiert. $^{102}$

Im Rahmen von Unternehmensvergleichen ${ }^{103}$ konnten Messestandkontaktpreise von Unternehmen auf Fachmessen bei Messeständen der Automobilindustrie zwischen DM 2,- und DM 12,- ${ }^{104}$ und bei Messeständen der Elektroindustrie zwischen DM 16,- und DM 24,- ermittelt werden. ${ }^{105}$ Dagegen waren bei Messeständen der Maschinenbauindustrie mit über DM 200,- pro Messestandkontakt deutlich höhere

Vgl. Mellerowicz, K., Kontrolle in der Absatzwirtschaft, in: Tietz, B. (Hrsg.), HWA-Handwörterbuch der Absatzwirtschaft, Stuttgart 1974, Sp. $1104 \mathrm{ff}$.

Die Messekontaktkosten, bei denen die gesamten Messebeteiligungskosten eines HochschulExponenten auf die Anzahl der Messekontakte bezogen werden, geben die Kosten für die Erreichung eines Messekontakts an.

Vgl. Naumann, C., Erfolgreich auf der Messe: Handbuch für die Beteiligung an Messen und Ausstellungen, a.a.O., S. 165 ff.; Bruhn, M., Kommunikationspolitik, München 1997, S. 768; Jaspersen, H., Messen beschicken oder Anzeigen schalten, in: Marketing Journal, 11. Jg., Heft 5, 1978, S. 461.

Obwohl Hochschul-Messebeteiligungen mit Unternehmens-Messebeteiligungen aufgrund hochschulspezifischer Rahmenbedingungen grundsätzlich nicht miteinander vergleichbar sind, soll im folgenden auf Vergleichsgrößen kommerzieller Messebeteiligungen zurückgegriffen werden, da für Messebeteiligungen von nicht-kommerziellen Institutionen, die sich hier als Vergleichsobjekte prinzipiell eher anbieten, keine konkreten Vergleichskriterien vorliegen. Für Unternehmens-Messebeteiligungen hingegen liegen Kriterien in Form von Messestandkontaktkosten vor.

So lag bspw. 1998 der Messestandkontaktpreis bei einem deutschen Automobilhersteller auf der Motor Show in Essen bei knapp DM 2,- und auf der AMI Leipzig bei knapp DM 12,-. Dabei ist zu berücksichtigen, daß der Automobilhersteller keine direkten Messestandkontakte erhoben hat, sondern davon ausging, daß alle Messebesucher auf seinen Messestand kamen und demzufolge in Kontakt mit inm traten.

Ein japanisches Unternehmen der Unterhaltungselektronik realisierte bspw. auf der CeBIT '98 einen Messestandkontaktpreis von DM 16,- und auf der CeBIT home '98 einen MessestandKontakt-Preis von DM 24,-- In diese Analyse wurden alle Messebesucher einbezogen. 
Messestandkontaktpreise feststellbar. ${ }^{106}$ Im Rahmen der betrachteten HochschulMessebeteiligungen lagen die Messestandkontaktpreise, wie die Ergebnisse der Kennziffernanalyse zeigten, tendenziell in höheren Bereichen, wenngleich sie nicht die Höhe der Maschinenbauindustrie erreichten. ${ }^{107}$ Die aufgeführten Messestandkontaktpreise basieren jedoch weder auf einheitlichen MessestandkontaktDefinitionen noch auf vergleichbaren Unternehmens- bzw. Hochschulstrukturen und Umweltsituationen. Folglich sind diese Kennziffern als solche nur schwer vergleichbar und können damit allenfalls erste Ansatzpunkte für weitere Vergleiche bieten.

\subsection{Inter-instrumentelle Vergleiche zwischen Messebeteiligungen und alternativen Kommunikationsinstrumenten}

Neben intra-instrumentellen Vergleichen auf Basis alternativer Hochschul- oder Unternehmens-Messebeteiligungen sind im Rahmen einer Effizienzbewertung von Hochschul-Messebeteiligungen ebenfalls inter-instrumentelle Vergleiche durchzuführen. Bei derartigen Vergleichen werden andere Kommunikationsmaßnahmen des Hochschulmarketing-Mix als Vergleichsobjekte herangezogen (vgl. Abb. 39).

So wurden die Messestandkontaktpreise eines deutschen Maschinenbau-Unternehmens auf verschiedenen Fachmessen (z.B. EMO) mit mehr als DM 200,- beziffert. Das Unternehmen ging dabei davon aus, daß alle Messebesucher auf seinen Messestand kamen und demzufolge in Kontakt mit inm traten. Die Kontakte auf dem Messestand wurden bei diesem Unternehmen durch entsprechende elektronische Aufzeichnungen exakt erfaßt.

\footnotetext{
Im Rahmen der dieser Untersuchung zugrundeliegenden Hochschul-Messebeteiligung konnten Hochschul-Messestandkontaktkosten zwischen DM 112,- (Fachmesse) und DM 146,(Mehrbranchenmesse) ermittelt werden. Als Messestandkontakt wurde dabei die Interaktion zwischen Exponent und Messebesucher auf dem Hochschul-Messestand definiert, wobei diese unterschiedliche Intensitätsgrade - angefangen von Informationsgespräche bis hin zu konkreten Besprechungen zukünftiger Projekte - annehmen kann.
} 


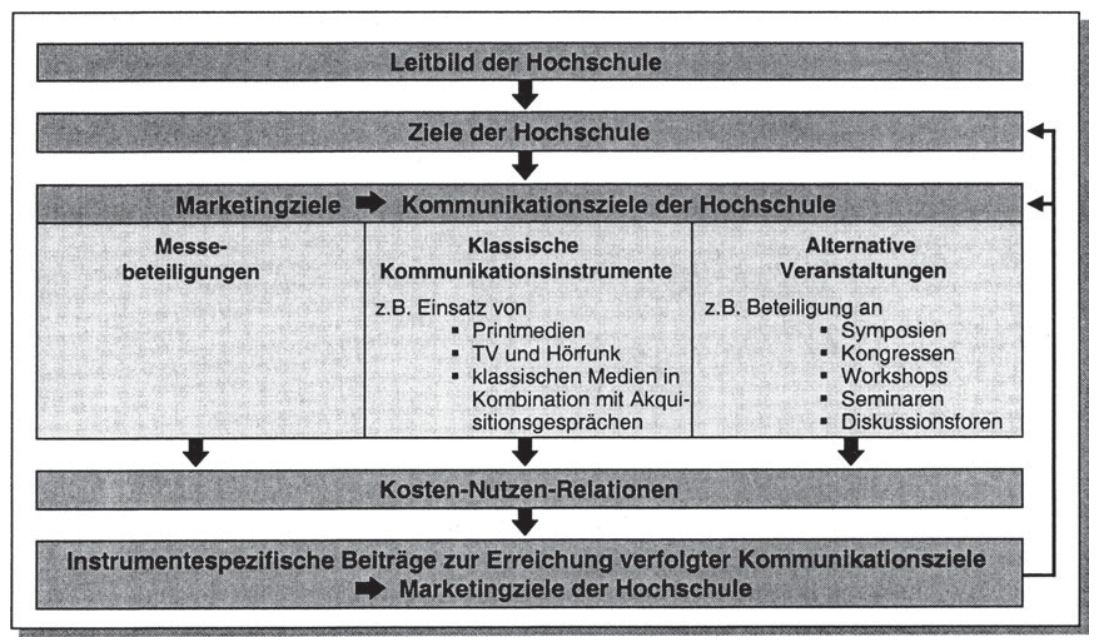

Abb. 39: Inter-instrumentelle Vergleiche zwischen Messebeteiligungen und alternativen Kommunikationsinstrumenten

Dienen wiederum Kontaktkosten als mögliches Vergleichskriterium, so können bei klassischen Kommunikationsinstrumenten, wie dem Printmedienbereich, für fachspezifische, hochschulrelevante Titel (z.B. VDI Nachrichten, Elektronische Zeitschrift, Angewandte Chemie) Tausenderkontaktkosten ${ }^{108}$ zwischen DM 230,und DM 1.220,- ermittelt werden. Dagegen werden im TV- und Hörfunkbereich bspw. Tausenderkontaktkosten zwischen DM 4,- und DM 117,- realisiert. ${ }^{109}$ Die sehr unterschiedliche Höhe der Tausenderkontaktkosten liegt dabei in der zielgruppenspezifischen Gewichtung begründet. Ein Vergleich der angeführten, nicht zielgruppenspezifischen Messestandkontaktpreise bzw. -kosten mit den Kontaktkosten klassischer Kommunikationsinstrumente deutet darauf hin, daß die Kon-

Die Tausenderkontaktkosten stehen hier als Synonym für den in der Literatur oftmals verwendeten Begriff Tausenderkontaktpreis. Der Tausenderkontaktpreis, der auch Tausenderpreis genannt wird, gibt die Kosten für 1.000 Werbeträgerkontakte an. Er ergibt sich aus dem Quotienten von Belegungskosten und erreichbaren Lesern multipliziert mit 1.000. Vgl. Meffert, $H$., Marketing - Grundlagen marktorientierter Unternehmensführung, a.a.O., S. $757 \mathrm{ff}$.

Vgl. Axel Springer Verlag (Hrsg.), Verbraucher-Analyse 97/3, Hamburg 1997 sowie die Auswertung weiterer print- und hörfunkspezifischer Sekundärstatistiken. Die Tausenderkontaktkosten sind jeweils von vielfältigen Faktoren abhängig. Während bspw. die Kontaktkosten bei Printmedien u.a. abhängig sind von der Anzeigengröße, können die Kontaktkosten im TV- und Hörfunkbereich u.a. in Abhängigkeit von der Tageszeit stark variieren. 
taktkosten auf Messen i.d.R. über den Kontaktkosten klassischer Kommunikationsinstrumente liegen.

Ein inter-instrumenteller Vergleich auf Basis von Kontaktkosten ist jedoch nicht ausreichend. Vielmehr sind derartige Vergleiche durch die jeweiligen Kontaktqualitäten zu ergänzen. So sind Messebeteiligungen den anderen Kommunikationsinstrumenten insbesondere hinsichtlich

- der persönlichen Kontakte,

- der Interaktivität zwischen Hochschul-Exponenten und Hochschul-Messestand-Besuchern,

- der guten Möglichkeiten zur Besichtigung sowie Erklärung von wissenschaftlichen Exponaten,

- der Erlebniswerte für Hochschul-Exponenten und Messebesucher sowie

- des hohen Informationsnutzens aufgrund der unmittelbaren und i.d.R. umfassenden Möglichkeit zu Konkurrenzvergleichen

überlegen. ${ }^{110}$ Werden im Rahmen des inter-instrumentellen Vergleichs sowohl quantitative als auch qualitative, i.d.R. nicht-monetär-bewertbare Aspekte mit in die Betrachtung einbezogen, so können weitere Erkenntnisse gewonnen werden.

Setzen Hochschulen bspw. klassische Medien in Kombination mit Akquisitionsgesprächen anstelle von Messebeteiligungen ein, sind dabei mit Messekontakten vergleichbare persönliche Kontakte zu realisieren. Die Kosten des persönlichen Kontakts liegen i.d.R. auf dem Niveau der Messestandkontaktkosten einer Hochschul-Messebeteiligung. ${ }^{111}$ Somit ergibt sich bei zusätzlicher Berücksichtigung der Kosten für den flankierenden Einsatz klassischer Medien ein Kostennachteil gegenüber einer Messebeteiligung (quantitativer Vergleich). Darüber hinaus können derartige Instrumentekombinationen nur selten die Möglichkeit einer physischen Besichtigung der Forschungsergebnisse und des Vergleichs verschiedener Exponate bieten (qualitativer Vergleich). Aus diesen Gründen ist die alleinige persönliche Kommunikation mit flankierendem Einsatz klassi-

110 Vgl. Backhaus, K., Messen als Institutionen der Informationspolitik, a.a.O., S. 91 ff.; Rost, D., Messen als Kommunikations-Problem, a.a.O., S. $35 \mathrm{ff}$.

111 Experten schätzen die Kosten der Akquisitionsgespräche auf DM 100,- bis DM 150,- pro Kontakt. 
scher Medien zur Erreichung der von den Hochschul-Exponenten verfolgten Zielsetzungen weniger geeignet als eine Messebeteiligung und stellt demzufolge keine adäquate Alternative dar.

Unter Berücksichtigung der Messekontaktqualitäten kommen grundsätzlich als generelle Alternativen einer Hochschul-Messebeteiligung nur solche Instrumente in Frage, die zur Initierung und Intensivierung eines fachlich orientierten Dialogs zwischen den Hochschul-Exponenten und den relevanten Zielgruppen beitragen. In diesem Zusammenhang bietet sich z.B. die Nutzung von interaktiven Medien (z.B. Internet) an. Weitere mögliche Alternativen sind Beteiligungen an Veranstaltungen wie Symposien, Kongressen, Workshops, Seminaren und Diskussionsforen, die meist vom finanziellen Ressourcenbedarf mit Messebeteiligungen vergleichbar sind (quantitativer Vergleich). Diese Veranstaltungen eröffnen den Hochschul-Exponenten die Möglichkeit, die anwesenden Teilnehmer über Qualität, Aktualität und wissenschaftlichen Stellenwert ihrer Forschungsprojekte zu informieren und damit ihre Leistungskompetenz darzustellen. Positive Bekanntheitsgrad- und Imagewirkungen sind ebenfalls realisierbar. Darüber hinaus ist auf solchen Veranstaltungen insbesondere Gelegenheit zum Erfahrungs- und Informationsaustausch zwischen Wissenschaftlern und Wirtschaftsvertretern gegeben (qualitativer Vergleich).

In diesem Zusammenhang stellt sich jedoch die Frage, inwieweit die von den Hochschul-Exponenten hoch priorisierten Zielgruppen durch derartige Veranstaltungen auch tatsächlich erreicht werden können. Die Teilnahme an Symposien, Kongressen, Workshops, Seminaren und Diskussionsforen ist mit der Gefahr einer begrenzten Resonanz von Wirtschaftsvertretern verbunden. Wirtschaftsvertreter richten ihre Terminplanung an einem attraktiven, sowohl wissenschaftlich als auch praxisorientierten Veranstaltungsangebot aus. Diese Zielgruppen werden auf Veranstaltungen, auf denen ausschließlich Hochschulvertreter ihre wissenschaftlichen Forschungsprojekte in Vorträgen und Diskussionen darstellen, nur bedingt erreicht. Somit ist bei inter-instrumentellen Vergleichen die Betrachtung der Zielgruppen von zentraler Bedeutung. Folglich ist an ein potentielles Vergleichsobjekt die (idealtypische) Anforderung der Zielgruppenkongruenz zu stellen. Nur wenn bei identischen Zielgruppen identische Wirkungen möglich sind, ist eine adäquate Vergleichsbasis gegeben.

Zusammenfassend ist vor dem Hintergrund der vorangegangenen Ausführungen zu konstatieren, daß Hochschul-Messebeteiligungen grundsätzlich ein effizientes Instrument zur Realisierung der von Hochschul-Exponenten angestrebten hoch- 
schulspezifischen Messezielsetzungen darstellen. Letztlich ist die Entscheidung zur Messebeteiligung auf Grundlage der primär verfolgten Messezielsetzungen zu treffen. In der folgenden Tabelle ist die Eignung verschiedener inter- und intrainstrumenteller Vergleichsobjekte zur Erreichung hochschulspezifischer Messeziele und -zielgruppen dargestellt.

\begin{tabular}{|c|c|c|c|c|c|c|c|c|c|c|c|c|c|}
\hline \multicolumn{3}{|c|}{ Kostenbetrachtung } & \multicolumn{10}{|c|}{ t) } & \multirow[b]{3}{*}{ Kosten } \\
\hline \multirow{2}{*}{\multicolumn{3}{|c|}{$\begin{array}{l}\text { Intra- und Inter- } \\
\text { instrumentelle Vergleiche }\end{array}$}} & \multicolumn{5}{|c|}{ Hochschulspezif. Messezieldimensionen } & \multicolumn{5}{|c|}{ Hochschulspezif. Messezielgruppen } & \\
\hline & & & $\begin{array}{l}\text { Infor- } \\
\text { ma- } \\
\text { tions- } \\
\text { ziele }\end{array}$ & \begin{tabular}{|c|} 
Hoch- \\
schul- \\
bez. \\
Beeintl- \\
zlele
\end{tabular} & \begin{tabular}{|c|} 
Inst.-1 \\
Lohrst' \\
Fachb. \\
Bez. \\
Beeinfl.- \\
ziele
\end{tabular} & \begin{tabular}{|c|}
$\begin{array}{c}\text { Expo- } \\
\text { natbez. } \\
\text { Beeinfl. }\end{array}$ \\
\end{tabular} & $\begin{array}{l}\text { Pro- } \\
\text { lekt- } \\
\text { ziele }\end{array}$ & $\begin{array}{l}\text { Wls- } \\
\text { sen- } \\
\text { schaft }\end{array}$ & $\begin{array}{l}\text { Stu- } \\
\text { dle- } \\
\text { rende }\end{array}$ & $\begin{array}{l}\text { Wirt- } \\
\text { schaft }\end{array}$ & $\begin{array}{l}\text { Offent- } \\
\text { Ilch- } \\
\text { kelt }\end{array}$ & Staat & \\
\hline \multirow{2}{*}{ 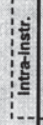 } & \multirow{2}{*}{ 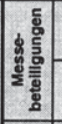 } & $\begin{array}{l}\text { Mehrbranchen- } \\
\text { messe }\end{array}$ & ++ & ++ & ++ & ++ & + & + & + & ++ & ++ & + & $--1-$ \\
\hline & & Fachmesse & ++ & + & + & + & ++ & + & + & ++ & + & + & $--1-$ \\
\hline \multirow{4}{*}{ 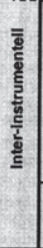 } & \multirow{3}{*}{ 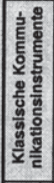 } & Printmedien & -- & ++ & ++ & ++ & -- & + & + & + & + & + & $+1-$ \\
\hline & & TV und Hörfunk & -- & ++ & ++ & ++ & -- & + & + & + & + & + & $+1-$ \\
\hline & & $\begin{array}{l}\text { Klassische Medien } \\
\text { \& Akquistions. } \\
\text { gespräche }\end{array}$ & ++ & + & + & + & ++ & - & - & ++ & 0 & 0 & $-1-$ \\
\hline & 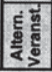 & $\begin{array}{l}\text { Symposien, Kon- } \\
\text { gresse, Workshops, } \\
\text { Seminare etc. }\end{array}$ & ++ & ++ & ++ & ++ & -- & + & + & -- & 0 & 0 & $-1-$ \\
\hline \multicolumn{10}{|c|}{$\begin{aligned} &++=\text { zur Erreichung des Messeziels bzw. der Messezielgruppe geeignet } \\
&+\quad=\text { zur Erreichung des Messeziels bzw. der Messezielgruppe geeignet bis bedingt geeignet } \\
& \text { (stark abhāngig von gewăhlter Instrumentausprägung) }\end{aligned}$} & $\begin{array}{l}+1- \\
-1- \\
--1-\end{array}$ & \multicolumn{3}{|c|}{$\begin{array}{l}=\text { mittiere Kosten } \\
=\text { relativ hohe Kosten } \\
=\text { hohe Kosten }\end{array}$} \\
\hline
\end{tabular}

Tab. 25: Eignung ausgewählter inter- und intra-instrumenteller Vergleichsobjekte zur Erreichung hochschulspezifischer Messeziele und -zielgruppen

Die in diesem Rahmen gewonnenen Erkenntnisse zeigen eine Vielzahl von Ansatzpunkten zur Ableitung von Implikationen für zukünftige Hochschul-Messebeteiligungen auf. In diesem Zusammenhang können sowohl Empfehlungen für Hochschul-Exponenten als auch für Finanzmittelgeber von Hochschul-Messebeteiligungen gegeben werden. 


\title{
D. Implikationen für die Messebeteiligung von Hochschulen
}

\author{
1. Implikationen für Hochschul-Exponenten
}

\subsection{Entscheidungen über die Beteiligung an Messen}

Eine strategisch geplante, auf die verfolgten Zielsetzungen der Hochschule abgestimmte Messebeteiligung bildet eine unabdingbare Voraussetzung zum erfolgreichen Messeengagement. Die Ergebnisse der vorangegangenen Messeerfolgskontrolle zeigen, daß der Erfolg einer Messebeteiligung insbesondere auch von der Auswahl des Messetyps bestimmt wird.

\subsection{Auswahl des Messetyps}

Je nach verfolgten Zielsetzungen ist der zu beschickende Messetyp von den jeweiligen Hochschul-Exponenten, d.h. exponententypspezifisch, auszuwählen. Stehen primär beeinflussungsorientierte Ziele im Vordergrund der HochschulMessebeteiligung, so bieten sich für diese Hochschul-Exponenten breiter angelegte Messen zur Messebeschickung an. Folglich erreichen Beeinflussungsorientierte Hochschul-Exponenten ihre hoch priorisierten Beeinflussungsziele eher durch Beteiligungen an Mehrbranchenmessen als durch Beschickungen von Fachmessen.

Wird der Öffentlichkeitsaspekt im Rahmen der Hochschul-Messebeteiligung weniger, der Transferaspekt dagegen stärker gewichtet, so sind gegenüber Mehrbranchenmessen oder auch Universalmessen fokussiertere Formen von Messen vorzuziehen. So können Projekt- und exponatorientierte Hochschul-Exponenten ihre im Vordergrund des Messeengagements stehenden Projektziele weniger auf Mehrbranchenmessen, sondern vielmehr auf Fachmessen realisieren. Die Fachmessen - sowie auch Verbundmessen - bieten diesen Exponententypen Möglichkeiten, sich als Technologie- und Know-how-Anbieter den potentiellen Nachfragern zu präsentieren und transferorientierte Fachgespräche mit sach- und entscheidungskompetenten Fachbesuchern zu führen. Damit erhalten HochschulExponenten auf Fachmessen Gelegenheiten, im Umfeld der jeweiligen Fachaussteller und Fachbesucher eine transferorientierte Kommunikation zu führen.

Für Pflichtrepräsentanten hingegen, die sich im Vergleich zu den anderen hochschulspezifischen Exponententypen als weniger zielorientiert charakterisieren las- 
sen, erscheint wiederum eine Teilnahme an allgemeineren Messen sinnvoll. Dieser Exponententyp, der sich vor allem aus der Erfordernis des öffentlich-rechtlichen Charakters der nicht-kommerziellen Institution Hochschule heraus an Messen beteiligt, wird diese Bestrebungen weniger auf thematisch eng fokussierten, sondern eher auf thematisch breit angelegten Messen realisieren können. Demzufolge sind für inn primär Mehrbranchenmessen sowie Universalmessen relevant.

In der nachfolgenden Tabelle wird das für eine Messebeteiligung im Hochschulbereich besonders relevante Entscheidungsspektrum bzgl. der Messeauswahl dargestellt. Dabei sind die für die einzelnen Exponententypen primär relevanten Entscheidungsfelder, die sich aus den jeweiligen Messezielsetzungen ergeben, entsprechend gekennzeichnet.

\begin{tabular}{|c|c|c|c|}
\hline $\begin{array}{l}\text { Hochschulspezifische } \\
\text { Ange- } \\
\text { botsorien- } \\
\text { tierte Messetypenten }\end{array}$ & $\begin{array}{c}\begin{array}{c}\text { Projekt- u. expo- } \\
\text { natorientierter } \\
\text { Hochschul-Exponent }\end{array} \\
\end{array}$ & $\begin{array}{c}\begin{array}{c}\text { Beeinflussungs- } \\
\text { orientierter }\end{array} \\
\text { Hochschul-Exponent }\end{array}$ & $\begin{array}{l}\text { Pflicht- } \\
\text { repräsen- } \\
\text { tant }\end{array}$ \\
\hline Fachmessen & ++ & 0 & - \\
\hline Verbundmessen & + & 0 & - \\
\hline $\begin{array}{l}\text { Branchen-bzw. } \\
\text { Mehrbranchenmessen }\end{array}$ & 0 & + & + \\
\hline Universalmessen & - & ++ & ++ \\
\hline \multicolumn{4}{|c|}{ 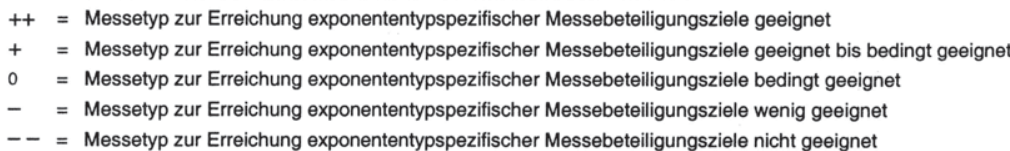 } \\
\hline
\end{tabular}

Tab. 26: Eignung verschiedener, nach Angebotsbreite klassifizierter Messetypen zur Erreichung exponententypspezifischer Messezielsetzungen

Die Auswahl des Messetyps ist insbesondere auch von den auf den jeweiligen Messen vertretenen Messezielgruppen abhängig, da die Realisierung verfolgter Messezielsetzungen im wesentlichen durch die Erreichung der entsprechenden hochschulspezifischen Messezielgruppen bestimmt wird. Grundsätzlich läßt sich die Zusammensetzung der Messebesucher aus den bereits angeführten Besucherstrukturanalysen ermitteln. Wie der Vergleich der Besucherstrukturen der betrachteten Mehrbranchen- und Fachmesse zeigte, läßt insbesondere eine nach der Angebotsbreite vorgenommene Klassifizierung von Messetypen wesent- 
liche Unterschiede im Hinblick auf die Anteile der auf der jeweiligen Messe vertretenen hochschulspezifischen Messezielgruppen erkennen. Zur Erreichung von Wirtschaftsvertretern können grundsätzlich alle klassischen Messetypen beschickt werden. Auch die Vertreter des Staates sind sowohl auf allgemeineren als auch auf thematisch eng fokussierten Messetypen anzutreffen, wohingegen die Anspruchsgruppe Öffentlichkeit eher durch die Messebeteiligung an Mehrbranchen- sowie Universalmessen erreicht wird. Zwar sind Wissenschaftler und Studierende - wie die vorliegende Untersuchung belegt - auch auf klassischen Fachmessen sowie auf Mehrbranchenmessen vertreten, eine bessere Zielgruppenansprache kann jedoch durch die Beschickung von hochschulspezifischen Fachmessen wie Wissenschafts- und Bildungsmessen erzielt werden. Die folgende Tabelle stellt die auf den jeweiligen Messetypen primär erreichbaren hochschulspezifischen Messezielgruppen dar. Dabei beschränkt sich die Zuordnung auf die in diesem Zusammenhang im Vordergrund stehende Klassifizierungsdimension Angebotsbreite, wobei in bezug auf Fachmessen eine differenzierte Betrachtung nach hochschulspezifischen Messetypen, wie Wissenschafts- und Bildungsmessen, sowie nach klassischen Fachmessen erfolgt.

\begin{tabular}{|c|c|c|c|c|c|c|}
\hline \multicolumn{2}{|c|}{$\begin{array}{l}\text { Hochschulspezifische } \\
\begin{array}{l}\text { Ange- } \\
\text { Motssezien- } \\
\text { botsien- } \\
\text { tierte Messetypen }\end{array}\end{array}$} & \multirow{2}{*}{$\begin{array}{l}\text { Wissen- } \\
\text { schaft } \\
++\end{array}$} & \multirow{2}{*}{$\begin{array}{c}\begin{array}{c}\text { Studie- } \\
\text { rende }\end{array} \\
++\end{array}$} & \multirow{2}{*}{$\begin{array}{c}\begin{array}{c}\text { Wirt- } \\
\text { schaft }\end{array} \\
-\end{array}$} & \multirow{2}{*}{$\begin{array}{c}\text { Öffent- } \\
\text { lichkeit }\end{array}$} & \multirow{2}{*}{ Staat } \\
\hline \multirow{3}{*}{$\begin{array}{l}\text { Fach- } \\
\text { mes- } \\
\text { sen }\end{array}$} & $\begin{array}{l}\text { Wissenschafts- } \\
\text { messen }\end{array}$ & & & & & \\
\hline & $\begin{array}{l}\text { Bildungs- } \\
\text { messen }\end{array}$ & ++ & ++ & -- & 0 & 0 \\
\hline & $\begin{array}{l}\text { Klassische } \\
\text { Fachmessen }\end{array}$ & + & 0 & ++ & 0 & + \\
\hline \multicolumn{2}{|c|}{ Verbundmessen } & + & 0 & ++ & + & + \\
\hline \multicolumn{2}{|c|}{$\begin{array}{l}\text { Branchen- bzw. } \\
\text { Mehrbranchenmessen }\end{array}$} & + & + & ++ & ++ & ++ \\
\hline \multicolumn{2}{|c|}{ Universalmessen } & + & + & ++ & ++ & ++ \\
\hline \multicolumn{7}{|c|}{$\begin{array}{ll}++ & =\text { Messetyp zur Erreichung der hochschulspezifischen Messezielgruppe geeignet } \\
+ & =\text { Messetyp zur Erreichung der hochschulspezifischen Messezielgruppe geeignet bis bedingt geeignet } \\
0 & =\text { Messetyp zur Erreichung der hochschulspezifischen Messezielgruppe bedingt geeignet } \\
- & =\text { Messetyp zur Erreichung der hochschulspezifischen Messezielgruppe wenig geeignet } \\
-- & =\text { Messetyp zur Erreichung der hochschulspezifischen Messezielgruppe nicht geeignet }\end{array}$} \\
\hline
\end{tabular}

Tab. 27: Eignung verschiedener, nach Angebotsbreite klassifizierter Messetypen zur Erreichung hochschulspezifischer Messezielgruppen 
Wird die hochschulspezifische Messezielgruppenbetrachtung um verhaltensorientierte Messebesucherkriterien ergänzt, so können grundsätzlich die zuvor abgeleiteten Empfehlungen zur exponententypspezifischen Messeauswahl bestätigt werden. In Abhängigkeit von der Angebotsbreite sind einzelne Hochschul-Messestand-Besuchertypen auf einigen Messetypen stärker und auf anderen dagegen schwächer vertreten (vgl. Tab. 28). Wie die Analyse der Hochschul-Messestand-Besuchertypen zeigte, sind Intensive Hochschul-Messestand-Nutzer eher auf angebotsspezialisierten Messen anzutreffen, weil gerade diese Messen gute Voraussetzungen zur Realisierung ihrer mit dem Hochschul-Messestand-Besuch primär verfolgten Informations- und Kontaktziele bieten. Folglich sollten Projektund exponatorientierte Hochschul-Exponenten, bei denen zur Erreichung ihrer verfolgten Projektziele die Kontaktaufnahme zu Messebesuchern unabdingbar ist, sich eher auf spezialisierteren Messen präsentieren.

Informationsorientierte Hochschul-Messestand-Nutzer hingegen sind tendenziell eher auf thematisch breiter angelegten Messen und weniger auf spezialisierten Fachmessen vertreten. Dieser Messebesuchertyp ist insbesondere zur Erreichung der von Beeinflussungsorientierten Hochschul-Exponenten primär verfolgten Messezielsetzungen von besonderer Bedeutung, da letztlich ein Informationsorientierter Hochschul-Messestand-Nutzer zur Aufnahme der zur Beeinflussung relevanten Informationen sehr offen ist. Somit wird die zuvor abgeleitete Empfehlung für Beeinflussungsorientierte Hochschul-Exponenten, sich an allgemeineren Messen zu beteiligen, unter messebesuchertypspezifischen Aspekten bestätigt.

Hochschul-Messestand-Bummler sind sowohl auf breiter angelegten als auch auf enger fokussierten Messen anzutreffen. Dieser Hochschul-Messestand-Besuchertyp stellt i.w.S. den Gegenpart zu den Pflichtrepräsentanten auf der Exponentenseite dar. Angesichts der nicht unbedeutenden Relevanz von Beeinflussungszielen sind für Pflichtrepräsentanten insbesondere Kontakte zu Informationsorientierten Hochschul-Messestand-Besuchern notwendig. Folglich kann auch unter messebesuchertypspezifischer Perspektive für Pflichtrepräsentanten die Beschickung von allgemeineren Messen empfohlen werden. 


\begin{tabular}{|c|c|c|c|}
\hline $\begin{array}{l}\begin{array}{l}\text { Ange- } \\
\text { Anschul-Messe- } \\
\text { botsorien- } \\
\text { tierte Messetypen } \\
\text { stand-Besu- } \\
\text { chertypen }\end{array} \\
\end{array}$ & $\begin{array}{c}\text { Intensiver } \\
\text { Hochschul- } \\
\text { Messestand-Nutzer }\end{array}$ & $\begin{array}{l}\text { Informationsorien- } \\
\text { tierter Hochschul- } \\
\text { Messestand-Nutzer }\end{array}$ & $\begin{array}{l}\text { Hochschul- } \\
\text { Messestand- } \\
\text { Bummler }\end{array}$ \\
\hline Fachmessen & ++ & 一 & + \\
\hline Verbundmessen & + & 0 & + \\
\hline $\begin{array}{l}\text { Branchen- bzw. } \\
\text { Mehrbranchenmessen }\end{array}$ & 0 & + & + \\
\hline Universalmessen & 一 & ++ & + \\
\hline \multicolumn{4}{|c|}{$\begin{array}{l}++=\text { Messetyp zur Erreichung des Hochschul-Messestand-Besuchertyps geeignet } \\
+\quad=\text { Messetyp zur Erreichung des Hochschul-Messestand-Besuchertyps geeignet bis bedingt geeignet } \\
0 \quad=\text { Messetyp zur Erreichung des Hochschul-Messestand-Besuchertyps bedingt geeignet } \\
-\quad=\text { Messetyp zur Erreichung des Hochschul-Messestand-Besuchertyps wenig geeignet } \\
--=\text { Messetyp zur Erreichung des Hochschul-Messestand-Besuchertyps nicht geeignet }\end{array}$} \\
\hline
\end{tabular}

Tab. 28: Eignung verschiedener, nach Angebotsbreite klassifizierter Messetypen zur Erreichung von Hochschul-Messestand-Besuchertypen

Ist der zu beschickende Messetyp ausgewählt, haben Hochschul-Exponenten Entscheidungen darüber zu treffen, in welcher Form sie sich auf den einzelnen Messen präsentieren.

\subsection{Auswahl hochschulspezifischer Messebeteiligungsformen}

Die Auswahl einer Messebeteiligungsform wird nicht nur durch den mit der jeweiligen Form verbundenen und von dem jeweiligen Hochschul-Exponenten selbst zu tragenden Aufwand beeinflußt, sondern ist insbesondere auch von den priorisierten Messezielsetzungen abhängig und damit exponententypspezifisch zu treffen (vgl. Tab. 29).

Projekt- und exponatbezogene Hochschul-Exponenten werden die im Vordergrund ihres Messeengagements stehenden projekt- und exponatbezogenen Zielsetzungen vor allem durch eine gemeinsame Messeteilnahme mit Wirtschaftspartnern forcieren können. Folglich bietet sich für diesen Exponententyp insbesondere die Exponatpräsentation auf Wirtschafts-Gemeinschaftsständen an. Dies nicht zuletzt aufgrund der somit realisierbaren Dokumentation des eigenen Kooperationspotentials gegenüber weiteren Wirtschaftsvertretern. Darüber hinaus können Präsentationen auf eigenen Hochschul-Messeständen sowie auf Gemeinschaftsständen zusammen mit anderen wissenschaftlichen und/oder regionalen Institu- 
tionen diesem Exponententyp gute Voraussetzungen zur Erreichung seiner primär verfolgten Messebeteiligungsziele bieten.

Beeinflussungsorientierten Hochschul-Exponenten eröffnen sich Erfolgschancen zur Realisierung ihrer exponat- und institutionenbezogenen Beeinflussungsziele vor allem auf eigenen Hochschul-Messeständen und Gemeinschaftsständen mit anderen wissenschaftlichen Institutionen. Die Präsentation auf Wirtschafts-, Regionen- und Landes-Gemeinschaftsständen kann diesem Exponententyp zwar ebenfalls Möglichkeiten zur Realisierung seiner verfolgten Zielsetzungen bieten, dennoch besteht auf derartigen Gemeinschaftsständen die Gefahr, die eigene Profilierung aufgrund der im Vordergrund stehenden Darstellung des Messestandausrichters nur begrenzt realisieren zu können.

Pflichtrepräsentanten hingegen erhalten gerade durch die Teilnahme an LandesGemeinschaftsständen die Gelegenheit, den verfolgten Motiven ihrer Messebeteiligung, die insbesondere aus dem Status als öffentlich-rechtliche, nicht-kommerzielle Institution resultiert, nachzukommen. Derartige Gemeinschaftsstände bieten Pflichtrepräsentanten die Chance, ihre Leistungen mit relativ geringem Aufwand der Gesellschaft und Vertretern des Staates darzustellen.

\begin{tabular}{|c|c|c|c|}
\hline $\begin{array}{l}\text { Hochschulspezifische } \\
\text { Exponenten- } \\
\text { typen }\end{array}$ & $\begin{array}{c}\text { Projekt- u. exponat- } \\
\text { orientierter } \\
\text { Hochschul-Exponent }\end{array}$ & $\begin{array}{c}\begin{array}{c}\text { Beeinflussungs- } \\
\text { orientierter } \\
\text { Hochschul-Exponent }\end{array} \\
\end{array}$ & $\begin{array}{l}\text { Pflicht- } \\
\text { repräsen- } \\
\text { tant }\end{array}$ \\
\hline $\begin{array}{l}\text { Hochschul- } \\
\text { Einzel-Messestand }\end{array}$ & + & ++ & 0 \\
\hline $\begin{array}{l}\text { Wissenschafts- } \\
\text { Gemeinschaftsstand }\end{array}$ & + & ++ & 0 \\
\hline $\begin{array}{l}\text { Wirtschafts- } \\
\text { Gemeinschaftsstand }\end{array}$ & ++ & + & -- \\
\hline $\begin{array}{l}\text { Regionen- } \\
\text { Gemeinschaftsstand }\end{array}$ & + & + & - \\
\hline $\begin{array}{l}\text { Landes- } \\
\text { Gemeinschaftsstand }\end{array}$ & + & + & ++ \\
\hline \multicolumn{4}{|c|}{ 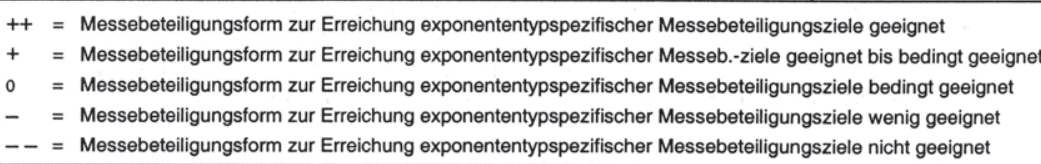 } \\
\hline
\end{tabular}

Tab. 29: Hochschulspezifische Messebeteiligungsformen und ihre Eignung für Hochschul-Exponententypen 
Zielgruppenspezifisch betrachtet ist von einer unterschiedlich starken Frequentierung der hochschulspezifischen Messezielgruppen auf den verschiedenen Messeständen auszugehen ( $\mathrm{vgl}$. Tab. 30). Wissenschaftler bspw. werden voraussichtlich eher auf Hochschul-Einzel-Messeständen und Wissenschafts-Gemeinschaftsständen anzutreffen sein, da sie als Messebesucher auch aus wettbewerbsorientiertem Interesse heraus solche Messestände aufsuchen. Dagegen besuchen sie vermutlich weniger Wirtschafts-Gemeinschaftsstände. Auf diesen Messeständen werden hingegen Wirtschaftsvertreter überproportional häufig vertreten sein. Sollen mit der Messebeteiligung insbesondere auch Vertreter des Staates erreicht werden, so ist eine Beteiligung an Landes-Gemeinschaftsständen empfehlenswert. Diese häufig von Ministerien ausgerichteten Gemeinschaftsstände werden i.d.R. von den zuständigen Ministern und/oder auch von weiteren Staatsvertretern aufgesucht. In bezug auf die Messestand-Besuchertypen ist von einer eher gleichmäßigen Verteilung auf den verschiedenen Messeständen auszugehen.

\begin{tabular}{|c|c|c|c|c|c|}
\hline $\begin{array}{l}\text { Hochschulspezifische } \\
\begin{array}{l}\text { Messeziel- } \\
\text { gruppen } \\
\text { Messe- } \\
\text { beteiligungsform }\end{array}\end{array}$ & $\begin{array}{l}\text { Wissen- } \\
\text { schaft }\end{array}$ & $\begin{array}{l}\text { Studie- } \\
\text { rende }\end{array}$ & $\begin{array}{l}\text { Wirt- } \\
\text { schaft }\end{array}$ & $\begin{array}{l}\text { Öffent- } \\
\text { lichkeit }\end{array}$ & Staat \\
\hline $\begin{array}{l}\text { Hochschul- } \\
\text { Einzel-Messestand }\end{array}$ & ++ & ++ & -- & + & 0 \\
\hline $\begin{array}{l}\text { Wissenschafts- } \\
\text { Gemeinschaftsstand }\end{array}$ & ++ & ++ & - & + & 0 \\
\hline $\begin{array}{l}\text { Wirtschafts- } \\
\text { Gemeinschaftsstand }\end{array}$ & - & - & ++ & + & - \\
\hline $\begin{array}{l}\text { Regionen- } \\
\text { Gemeinschaftsstand }\end{array}$ & 0 & 0 & + & ++ & + \\
\hline $\begin{array}{l}\text { Landes- } \\
\text { Gemeinschaftsstand }\end{array}$ & ++ & ++ & + & ++ & ++ \\
\hline \multicolumn{6}{|c|}{$\begin{array}{ll}++ & =\text { Messebeteiligungsform zur Erreichung der hochschulspezifischen Messezielgruppe geeignet } \\
+ & =\text { Messebeteiligungsform zur Erreichung der hochschulspezifischen Messezielgr. geeignet bis bedingt geeignet } \\
0 & =\text { Messebeteiligungsform zur Erreichung der hochschulspezifischen Messezielgruppe bedingt geeignet } \\
- & =\text { Messebeteiligungsform zur Erreichung der hochschulspezifischen Messezielgruppe wenig geeignet } \\
-- & =\text { Messebeteiligungsform zur Erreichung der hochschulspezifischen Messezielgruppe nicht geeignet }\end{array}$} \\
\hline
\end{tabular}

Tab. 30: Hochschulspezifische Messebetelligungsformen und Ihre Elgnung zur Erreichung hochschulspezifischer Messezielgruppen

Neben den auf Grundlage der durchgeführten Erfolgskontrolle von HochschulMessebeteiligungen abgeleiteten strategischen Empfehlungen konnten im Rah- 
men der bisherigen Ausführungen ebenfalls zahlreiche Ansatzpunkte zur operativen Gestaltung von Hochschul-Messebeteiligungen identifiziert werden. Auf diese Entscheidungstatbestände wird im folgenden eingegangen, wobei die Ergebnisse der durchgeführten Messeerfolgskontrolle implizit berücksichtigt werden. Die Empfehlungen für die zukünftige Ausgestaltung von Hochschul-Messebeteiligungen werden hinsichtlich des zeitlichen Einsatzes der einzelnen Aktivitäten unter besonderer Berücksichtigung der in der vorliegenden Arbeit identifizierten hochschulspezifischen Messezieldimensionen abgeleitet.

\subsection{Entscheidungen über die Gestaltung von Hochschul-Messebeteili- gungen}

Die zur Gestaltung des Messeengagements eingesetzten Maßnahmen können wie auch die Methoden zur Messeerfolgskontrolle - auf Basis des „Phasenkonzepts“ nach der Vor-Messe-, der Messe- und der Nach-Messe-Phase strukturiert werden. Dieser Ansatz bietet Anhaltspunkte zur Auswahl und zeitgerechten Umsetzung einzelner Maßnahmen. ${ }^{1}$ Innerhalb jeder Phase können HochschulExponenten spezifische Maßnahmen im Sinne ihrer primär verfolgten Zielsetzungen einsetzen. Dabei sind vor allem diejenigen Maßnahmen von hoher Relevanz, die eine messebesucherorientierte Fokussierung aufweisen ${ }^{2}$ und damit einen

Derartige Ansätze im Rahmen von Messebeteiligungen befassen sich mit der Einordnung und Beschreibung von messebezogenen Maßnahmen nach ihrem zeitlichen Einsatz. Diese Betrachtung geht auf eine lange Tradition zurück. Vgl. hierzu Reuther, K. H., Die betriebswirtschaftliche Bedeutung der Messen und Ausstellungen für die Konsumgüterindustrie, München 1956, S. 75 ff. sowie S. 168 ff.; Blohm, H., Messen und Ausstellungen als Mittel der Absatzförderung in der Schweizerischen Maschinenindustrie, a.a.O., S. 76 ff.; Lohmaier, P., Die Messe Bedeutung und Problematik aus der Sicht der Aussteller, in: Arbeitspapier des Instituts für Absatzwirtschaft der Universität Augsburg Nr. 1/85, Augsburg 1985, S. 28 ff. Die zentrale Kritik an dieser Sichtweise richtet sich insbesondere auf Abgrenzungs- bzw. Zuordnungsprobleme, wenn einzelne Aktivitäten in mehreren Phasen gleichermaßen Relevanz besitzen. Zur Kritik an chronologischen Ansätzen vgl. Funke, K., Ausstelleraktivitäten auf Messen: Eine kritische Analyse einschlägiger Darstellungen und Systematisierungsansätze in der Literatur, in: Arbeitsbericht Nr. 03/86 des Instituts für Wirtschaftswissenschaften der RWTH Aachen, Aachen 1986, S. $14 \mathrm{f}$. Diese Kritik ist jedoch insofern zu relativieren, als chronologische Ansätze hinsichtlich der Planung von Messemaßnahmen zumindest in der Lage sind, das Planungsproblem nach der Zeitdimension zu strukturieren. Damit stellen derartige Ansätze ein geeignetes Instrument zur Auswahl und zeitgerechten Umsetzung der einzelnen Messemaßnahmen dar.

Zur Notwendigkeit einer besucherorientierten Maßnahmengestaltung im Rahmen von Messebeteiligungen vgl. Funke, K., Ausstelleraktivitäten auf Messen: Eine kritische Analyse einschlägiger Darstellungen und Systematisierungsansätze in der Literatur, a.a.O., S. 4 ff. FUNKE 
wesentlichen Beitrag zur Erreichung hochschulspezifischer Messebeteiligungsziele leisten.

\subsection{Maßnahmen in der Vor-Messe-Phase}

Der Erfolg einer Messebeteiligung wird von den Hochschul-Exponenten u.a. durch die Bewältigung der in der Vor-Messe-Phase anfallenden Aufgaben determiniert. Dazu zählen insbesondere die Auswahl des Exponates bzw. der auszustellenden Forschungsergebnisse und deren Präsentationsform, die Standgestaltung, die Bekanntmachung der eigenen Messebeteiligung bei hochschulspezifischen Zielgruppen und die Auswahl sowie Qualifizierung des exponatbetreuenden Standpersonals.

Die Exponatauswahl ${ }^{3}$ im Rahmen einer Hochschul-Messebeteiligung kann anhand vielfältiger Kriterien vorgenommen werden, ${ }^{4}$ wobei vor dem Hintergrund der jeweils verfolgten Zielsetzungen vor allem die Transfereignung und die Öffentlichkeitswirksamkeit des Exponates von besonderer Bedeutung sind. Im Hinblick auf die Transfereignung eines Exponates ist zu überprüfen, welches Transferpotential die zur Auswahl stehenden Exponate bieten und wie hoch die jeweiligen Chancen zur kommerziellen Nutzung eingeschätzt werden. Dies ist zur Realisierung angestrebter Projektziele von besonderer Bedeutung, da ein nicht zum Transfer geeignetes Exponat die Anbahnung von Projektkooperationen und damit die Erreichung von Projektzielen eher negativ beeinflussen wird. Ist die Messebeteiligung auf die Erreichung von Beeinflussungszielen fokussiert, so sollte die Auswahl des Exponates vorwiegend unter Kriterien der Öffentlichkeitswirksamkeit erfolgen, da die Publikumswirksamkeit eines Exponates die Erreichung verfolgter Beeinflussungsziele fördern kann. Neben der Transfereignung und Öffentlichkeitswirksamkeit sind bei der Exponatauswahl weitere Kriterien, wie Aktualität und wissenschaftlicher Stellenwert des Ausstellungsobjektes, zu berücksichtigen. Zudem sollte ein Hochschul-Exponat einen exemplarischen Charakter für den

nimmt dabei insbesondere eine Abgrenzung zu solchen Aktivitäten vor, die primär an organisations- und ablauftechnischen Fragestellungen orientiert sind.

3

Vgl. Funke, K., Messeentscheidungen - Handlungsalternativen und Informationsbedarf, a.a.O., S. $101 \mathrm{ff}$.

4 Vgl. hierzu sowie im folgenden Stracke, F., Messebeschickung und Durchführung: Exponatauswahl, in: Budach, W. et al. (Hrsg.), Hochschulen und Messen: Praktische Hinweise für Messebeteiligungen durch Hochschulen, a.a.O., S. $78 \mathrm{ff}$. 
jeweiligen Exponenten, für die Schwerpunktbildung einer Hochschule oder für eine besondere Fachkompetenz eines Landes bzw. eines Bundeslandes besitzen. Darüber hinaus kann unter dem Aspekt einer verbesserten Zielgruppenansprache die Festlegung thematischer Schwerpunkte - z.B. bei der Beschickung von Fachmessen - sinnvoll sein. Auf allgemeineren Messen können grundsätzlich alle aus dem Forschungsspektrum einer Hochschule resultierenden Projekte präsentiert werden.

Wie die Auswahl des Exponates hängt auch seine Präsentationsform ${ }^{5}$ wesentlich von den im Rahmen einer Messebeteiligung im Vordergrund stehenden Zielsetzungen ab. Stehen Hochschulbezogene oder Instituts-/Lehrstuhl-/Fachbereichsbezogene Beeinflussungsziele im Vordergrund des Messeengagements, sollte der Fokus insbesondere auf dem wissenschaftlichen Anspruch des Exponates liegen. Werden hingegen primär Projektziele verfolgt, so ist die Praxisrelevanz des Exponates durch die Präsentationsform hervorzuheben. Neben den im Vordergrund der Messebeteiligung stehenden Zielsetzungen hängt die Auswahl der Präsentationsform ebenfalls vom zu beschickenden Messetyp ab. Während bspw. eine Beschickung von Mehrbranchenmessen eine eher breit gefächerte Hochschul-Leistungspräsentation für ein unspezifisches Messepublikum erfordert, ist auf Fachmessen eine thematisch fokussiertere Darstellung vorzuziehen. Dabei ist für die Präsentation eines Exponates die tatsächliche Demonstration (nach dem Motto: „Forschung zum Anfassen“) zu bevorzugen. Als Ersatz kann ein Dummy, eine Simulation, eine Diashow oder eine Kombination aus allen Elementen herangezogen werden, wenn Aufwand und zu erwartende Risiken bei den meist als Unikat geltenden Hochschul-Exponaten zu hoch erscheinen. ${ }^{6}$ Letztlich ist jedoch eine Präsentationsform zu realisieren, durch welche die Attraktivität des Exponates und damit der Aufmerksamkeitswert der Messebeteiligung insgesamt gesteigert werden können. Dazu können optische und akustische Effekte in hohem Maße beitragen, wobei diese auf das wissenschaftliche Exponat abgestimmt sein sollten. Besonders erfolgsversprechende Präsentationsformen sind dabei Darstellungen, die Messebesucher in die Demonstration mit einbeziehen.

Vgl. Funke, K., Messeentscheidungen - Handlungsalternativen und Informationsbedarf, a.a.O., S. $104 \mathrm{ff}$.

6

Vgl. hierzu sowie im folgenden Stracke, F., Messebeschickung und Durchführung: Präsentationsformen, in: Budach, W. et al. (Hrsg.), Hochschulen und Messen: Praktische Hinweise für Messebeteiligungen durch Hochschulen, a.a.O., S. 89. 
Damit können die Hochschul-Exponenten vor allem die Realisierung von Beeinflussungszielen forcieren.

Neben der ansprechenden Exponatdarstellung sollte die Aufmerksamkeit von Messebesuchern für das Hochschul-Exponat zusätzlich durch eine attraktive Standgestaltung ${ }^{7}$ unterstützt werden. Dabei sollte die Standgestaltung, die viele Möglichkeiten zur visuellen und emotionalen Beeinflussung der Messebesucher bietet, ${ }^{8}$ in Abstimmung mit den auszustellenden Hochschul-Exponaten sowie der jeweiligen Messebeteiligungsform erfolgen. Je nach angestrebten Zielsetzungen der Hochschul-Exponenten sind im Rahmen der Standgestaltung Hochschulbezogene, Instituts-/Lehrstuhl-/Fachbereichs- oder Exponatbezogene Aspekte besonders hervorzuheben, um die Erreichung der jeweils verfolgten Beeinflussungsziele entsprechend unterstützen zu können.

Im Rahmen der Bekanntmachung der Messebeteiligung gilt es, insbesondere diejenigen hochschulspezifischen Zielgruppen von der Messebeteiligung zu informieren, deren Erreichung die Hochschul-Exponenten mit ihrer Messebeteiligung anstreben. Um die Aufmerksamkeit auf die Messebeteiligung zu lenken, sind in der Vor-Messe-Phase schwerpunktmäßig Messeinformations- bzw. Messeeinladungsaktionen durchzuführen. ${ }^{9}$ Dabei können in Abhängigkeit von der Zielgruppenstruktur persönliche und/oder unpersönliche Kommunikationsinstrumente zum Einsatz kommen.

Zu Instrumenten der unpersönlichen Kommunikation gehören grundsätzlich alle Aktivitäten der Vor-Messe-Werbung ${ }^{10}$ in Massenmedien ${ }^{11}$ und hochschulspezifischen Veröffentlichungen. Dabei sind im Rahmen der Öffentlichkeitsarbeit Messevorberichte für die hochschulinterne, regionale und überregionale sowie ggf.

Vgl. Funke, K., Messeentscheidungen - Handlungsalternativen und Informationsbedarf, a.a.O., S. $189 \mathrm{ff}$.

8 Vgl. Clausen, E., Mehr Erfolg auf Messen, Landsberg am Lech 1997, S. 105.

9 Vgl. Küng, P., Der Einsatz des Verkaufs an Messen, in: VM international, Heft 3, 1990, S. 47.

10 Die Vor-Messe-Werbung zielt dabei auf eine Steuerung des Verhaltens aktueller und potentieller Exponat-Interessenten ab. Ihre Hauptaufgabe besteht darin, für die Messebeteiligung einen möglichst hohen Bekanntheitsgrad zu erzeugen. Vgl. Becker, J., Marketing-Konzeption Grundlagen des strategischen Marketing-Managements, 6. Aufl., München 1998, S. 538 ff.

Vgl. Selinski, H., Sperling U. A., Marketinginstrument Messe: Arbeitsbuch für Studium und Praxis, a.a.O., S. 179 ff.; Mortsiefer, J., Messen und Ausstellungen als Mittel der Absatzpolitik mittelständischer Herstellerbetriebe, a.a.O., S. $401 \mathrm{f}$. 
auch für die internationale Presse zu verfassen. Im Rahmen von Pressemitteilungen können in der Vor-Messe- sowie auch in der Messe- und Nach-Messe-Phase fachliche Informationen über die Exponate im Mittelpunkt stehen. ${ }^{12}$ Darüber hinaus können allgemeine Informationen, wie Angaben über die Hochschule oder des Instituts/Lehrstuhls/Fachbereichs, je nach verfolgten Zielsetzungen und je nach Interesse der einzelnen Ebenen des Hochschulbereichs in den verschiedenen Medien unterschiedlich stark gewichtet werden. In diesem Zusammenhang kann die grundsätzliche Bereitschaft der jeweiligen Hochschule oder des Landes, sich mit Kooperationsangeboten um eine Verbesserung der Kontakte zwischen Wissenschaft und Wirtschaft zu bemühen, dargestellt werden. Zudem ist die Messebeteiligung in die allgemeine Öffentlichkeitsarbeit der einzelnen Hochschule einzubinden. Bei einer arbeitsteiligen Pressearbeit von Hochschul-Exponenten und Pressestellen der jeweiligen Hochschulen sowie des zuständigen Ministeriums (bei Landes-Gemeinschaftsständen) ist die Pressearbeit zwischen diesen Institutionen zu koordinieren. Zur persönlichen Kommunikation mit hochschulspezifischen Zielgruppen können Verschickungen persönlicher Einladungen (Direct Mail) durchgeführt sowie konkrete Termine mit hoch priorisierten Messezielgruppen abgesprochen werden. ${ }^{13}$ Gerade diese persönlichen Kommunikationsmaßnahmen bieten den Hochschul-Exponenten vielfältige Möglichkeiten zum Erfahrungs- und Informationsaustausch mit diesen Zielgruppen und fördern damit insbesondere die Erreichung angestrebter Informationsziele. Zudem können diese Maßnahmen zur Forcierung verfolgter Projektziele beitragen.

In der Vor-Messe-Phase kommt der Auswahl und Qualifizierung des exponatbetreuenden Standpersonals ${ }^{14}$ aufgrund der auf der Messe nur zeitlich begrenzten Möglichkeit der direkten Kommunikation mit hochschulspezifischen Messebe-

Vgl. hierzu sowie im folgenden Stracke, F., Messebeschickung und Durchführung: Begleitende Pressearbeit, in: Budach, W. et al. (Hrsg.), Hochschulen und Messen: Praktische Hinweise für Messebeteiligungen durch Hochschulen, a.a.O., S. $98 \mathrm{ff}$.

Vgl. Schmitz, U., Messepolitik eines Unternehmens der Investitionsgüterindustrie, in: Strothmann, K.-H., Busche, M. (Hrsg.), Handbuch Messemarketing, a.a.O., S. 393; Zahner, W., Directmail - Sein Einsatz im Marketing, Bern 1991, S. 18 ff.; Kohle, G. U., Von der "MesseEinladung" zur Einladung mit Konzept, in: Marketing Journal, 24. Jg., Heft 5, 1991, S. 460 ff.; Pidun, R., Messen und Ausstellungen: Messe-Promotion - einfach faszinierend und glaubwürdig, in: Marketing Journal, 23. Jg., Heft 1, 1990, S. 70 f.

14 Vgl. Selinski, H., Sperling U. A., Marketinginstrument Messe: Arbeitsbuch für Studium und Praxis, a.a.O., S. $118 \mathrm{f}$. 
suchern eine hohe Bedeutung zu. ${ }^{15} \mathrm{Um}$ die Realisierung der angestrebten Zielsetzungen zu fördern, ist das exponatbetreuende Standpersonal in der VorMesse-Phase über Ziele und Zielgruppen der Messebeteiligung aufzuklären. Dabei hat das Standpersonal eine entsprechende Fachkompetenz aufzuweisen, um das Exponat entsprechend den Erwartungen des Messebesuchers erklären, den Projektstatus in Relation zu anderen wissenschaftlichen, sowohl nationalen als auch internationalen Arbeiten erläutern und den Stellenwert des Projektes im Forschungsgebiet der eigenen Forschungseinrichtung und Hochschule einordnen zu können. ${ }^{16}$ Des weiteren sind im Hinblick auf Verhaltens $-{ }^{17}$ und inhaltliche Argumentationsaspekte ${ }^{18}$ messevorbereitende Schulungen bzw. Gesprächsführungsseminare durchzuführen. In diesen Seminaren ist dem Standpersonal bspw. zu vermitteln, wie Gespräche mit Vertretern wichtiger Messezielgruppen (z.B. Wirtschaftsvertreter) i.S.d. verfolgten Zielsetzungen intensiviert, hingegen Gespräche mit weniger wichtigen Zielgruppenvertretern (z.B. Studierende) „elegant" beendet werden können.

Die Maßnahmen, durch deren Einsatz in der Vor-Messe-Phase die Erreichung bestimmter hochschulspezifischer Messezielsetzungen und die Ansprache von Messezielgruppen forciert werden können, sind in der nachfolgenden Tabelle zusammenfassend dargestellt.

Vgl. o.V., Mitarbeiterschulung - Hymne in der Halle: Unternehmen bereiten sich mit ungewöhnlichen Methoden auf den Messeauftritt vor, in: Wirtschaftswoche, 49. Jg., Heft 1/2, 1995, S. 70 ff.; Mortsiefer, J., Die Vorbereitung entscheidet über den Erfolg - Planung und Vorbereitung einer Messebeteiligung, in: Küffner, G., Mortsiefer J. (Hrsg.), Messeplatz Europa: Messen als Bestandteil des betrieblichen Marketings, a.a.O., S. $59 \mathrm{ff}$.

Vgl. Stracke, F., Messebeschickung und Durchführung: Standbesatzung/Personelle Präsentation, in: Budach, W. et al. (Hrsg.), Hochschulen und Messen: Praktische Hinweise für Messebeteiligungen durch Hochschulen, a.a.O., S. $94 \mathrm{f}$.

Vgl. Spryß, W. M., Für Standmitarbeiter gibt es viele Stolpersteine, in: Beilage der FAZ, Nr. 20, 25.01.1994, S. 8; Strothmann, K.-H., Suchen Sie das Gesprăch mit dem Standbesucher, in: Marketing Journal, 22. Jg., Heft 4, 1989, S. 388 f.; Aries, L. A., Bereiten Sie Ihr Standpersonal intensiv auf das Messe-Verkaufsgespräch vor, in: Marketing Journal, 19. Jg., Heft 4, 1986, S. $365 \mathrm{ff}$.

Vgl. Wagner, G., Innovatives Messe-Team-Training, in: Beilage der FAZ, Nr. 20, 25.01.1994, S. 5; Mortsiefer, J., Informationen sammeln und auswerten - Die Abwicklung einer Messebeteiligung, in: Küffner, G., Mortsiefer, J. (Hrsg.), Messeplatz Europa: Messen als Bestandteil des betrieblichen Marketings, a.a.O., S. 62 f. 


\begin{tabular}{|c|c|c|c|c|c|c|c|}
\hline \multirow{3}{*}{\multicolumn{2}{|c|}{ Maßnahmen in der Vor-Messe-Phase }} & \multicolumn{5}{|c|}{ Messezieldimensionen } & \multirow{3}{*}{$\begin{array}{l}\text { Mes- } \\
\text { se- } \\
\text { ziel- } \\
\text { grup- } \\
\text { pen }\end{array}$} \\
\hline & & \multirow{2}{*}{\begin{tabular}{|c|} 
Infor- \\
ma- \\
tions- \\
ziele \\
\end{tabular}} & \multicolumn{3}{|c|}{$\begin{array}{l}\text { Beeinflussungs- } \\
\text { ziele }\end{array}$} & \multirow{2}{*}{$\begin{array}{l}\text { Pro- } \\
\text { jekt- } \\
\text { ziele }\end{array}$} & \\
\hline & & & $\begin{array}{l}\text { Hoch- } \\
\text { schul- } \\
\text { bez. }\end{array}$ & $\begin{array}{c}\text { Inst.J } \\
\text { Lst.J } \\
\text { FB- } \\
\text { bez. }\end{array}$ & $\begin{array}{l}\text { Expo- } \\
\text { nat- } \\
\text { bez. }\end{array}$ & & \\
\hline \multicolumn{7}{|c|}{ Auswahl des Exponates und Präsentationsform } & 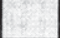 \\
\hline & Auswahl nach Qualität, Aktualität und wissenschaftlichem Stellenwert & 0 & ++ & ++ & ++ & ++ & ++ \\
\hline & Auswahl nach exemplar. Charakter für die Aktivitäten des Exponenten & 0 & ++ & ++ & ++ & ++ & ++ \\
\hline & Auswahl nach Transfereignung & 0 & 0 & 0 & 0 & ++ & ++ \\
\hline & Auswahl nach Öffentlichkeitswirksamkeit und Attraktivität & 0 & ++ & ++ & ++ & + & ++ \\
\hline \multicolumn{2}{|c|}{ Standgestaltung } & & & & & & \\
\hline & Nutzung visueller u. emot. Möglichkeiten in Exponat-Abstimmung (Fit) & - & ++ & ++ & ++ & + & ++ \\
\hline & Gestaltung in Abhängigkeit der Messebeteiligungsform & - & ++ & ++ & ++ & + & ++ \\
\hline \multicolumn{2}{|r|}{ Bekanntmachung der Messebeteiligung } & & & & & & \\
\hline & Abfassung v. Messevorber. für uni-int./(über-)regionale/internat. Presse & 0 & ++ & ++ & ++ & + & ++ \\
\hline & Vorankündigung der Messebeteiligung im Internet & 0 & ++ & ++ & ++ & + & ++ \\
\hline & Eintrag im Messekatalog & 0 & ++ & ++ & ++ & + & ++ \\
\hline & Verschickung persönlicher Einladungen (Direct Mail) & ++ & + & + & + & ++ & ++ \\
\hline & Direkte Terminabsprachen mit aktuellen u. potent. Kooperationspartnern & ++ & 0 & 0 & 0 & ++ & ++ \\
\hline \multicolumn{2}{|r|}{ Auswahl und Qualifizierung d. exponatbetreuenden Standpersonals } & & & & & & \\
\hline & Sicherstellung der entsprechenden wissenschaftlichen Qualifikation & ++ & + & + & + & ++ & + \\
\hline & Vermittlung angestrebter Ziele und Zielgruppen & ++ & + & + & + & ++ & ++ \\
\hline & Training von Verhalten und Gesprächsführung & ++ & + & + & + & ++ & ++ \\
\hline \multicolumn{8}{|c|}{$\begin{array}{l}++\quad \text { Maßnahme kann die Erreichung der Messeziele bzw. Messezielgruppen besonders beeinflussen } \\
+\quad=\text { Maßnahme kann die Erreichung der Messeziele bzw. Messezielgruppen beeinflussen } \\
0 \quad=\text { Maßnahme kann die Erreichung der Messeziele bzw. Messezielgruppen bedingt beeinflussen } \\
-\quad=\text { Maßnahme kann die Erreichung der Messeziele bzw. Messezielgruppen wenig beeinflussen } \\
--\quad=\text { Maßnahme kann die Erreichung der Messeziele bzw. Messezielgruppen nicht beeinflussen }\end{array}$} \\
\hline
\end{tabular}

Tab. 31: Maßnahmen in der Vor-Messe-Phase und deren Einflußmöglichkeiten auf die Erreichung hochschulspezifischer Messeziele und -zielgruppen

In Abhängigkeit von den Zielsetzungen, die die hochschulspezifischen Exponententypen mit ihrer Messebeteiligung verfolgen, sind die entsprechenden VorMesse-Aktivitäten typenspezifisch einzusetzen. So ist im Rahmen der Exponatauswahl bspw. die Überprüfung der Transfereignung insbesondere für Projektund exponatorientierte Hochschul-Exponenten von besonderer Bedeutung, da ein nicht zum Transfer geeignetes Exponat die Realisierung von Projektzielen eher negativ beeinflussen wird. Hingegen haben Beeinflussungsorientierte HochschulExponenten bei der Exponatauswahl vor allem auf die Öffentlichkeitswirksamkeit ihres Exponates zu achten, da diese die Erreichung von Beeinflussungszielen unterstützen kann. Dennoch dürfen die einzelnen Exponententypen sich nicht nur auf die Maßnahmen fokussieren, die zur Erreichung ihrer primär verfolgten Messezielsetzungen beitragen. Darüber hinaus sollten auch die primär auf andere 
Messezieldimensionen gerichteten Maßnahmen eingesetzt werden. Letztlich führt erst das auf die Zielsetzungen des jeweiligen Hochschul-Exponenten abgestimmte Zusammenspiel aller Maßnahmen der Vor-Messe-Phase zu einer adäquaten Messevorbereitung und legt damit die Basis für eine erfolgreiche Messebeteiligung.

\subsection{Maßnahmen in der Messe-Phase}

Ausgangspunkt einer zielorientierten Hochschul-Messebeteiligung ist der Kontakt zu den hochschulspezifischen Zielgruppen auf dem eigenen Messestand..$^{19}$ In diesem Zusammenhang ist die Akquisition der Messebesucher zur Intensivierung des Dialogs mit ausgewählten Zielgruppen sowie die Aktivierung des anonymen Hochschul-Messestand-Publikums von besonderer Bedeutung. ${ }^{20}$

Während die Kommunikationsarbeit der Messeveranstalter vor allem darauf gerichtet ist, Exponenten und Messebesuchern ein Forum der Begegnung zur Verfügung zu stellen, ${ }^{21}$ sind die Aktivitäten der Hochschul-Exponenten während der Messe darauf auszurichten, die Messebesucher zum eigenen Messestand zu leiten. Die zur Akquisition notwendige Überbrückung der Wahrnehmungsbarriere bei den Messebesuchern sowie die Lenkung ihrer Aufmerksamkeit und ihres Interesses auf den Messestand bilden dabei die wesentlichen Herausforderungen. ${ }^{22}$

Für Messebesucher stellen Messegesellschaften i.d.R. computergestützte MesseInformationssysteme zur Verfügung. ${ }^{23}$ Diese Informations-Terminals erleichtern dem Besucher die Orientierung auf dem Messegelände und vermitteln einen Überblick über das Ausstellungsprogramm. Beteiligen sich Hochschul-Exponenten

Vgl. hierzu auch die Ergebnisse der Arbeiten von Mortsiefer, J., Messen und Ausstellungen als Mittel der Absatzpolitik mittelstăndischer Herstellerbetriebe, a.a.O., S. 322 ff.; Tăger, U. C., Ziegler, R., Die Bedeutung von Messen und Ausstellungen in der Bundesrepublik Deutschland für den Inlands- und Auslandsabsatz in ausgewählten Branchen, a.a.O., S. 30 und S. 52 f.

Vgl. Ueding, R., Management von Messebeteiligungen: Identifikation und Erklärung messespezifischer Grundhaltungen auf der Basis einer empirischen Untersuchung, a.a.O., S. 110.

Vgl. Roloff, E., Messen und Medien: Ein sozialpsychologischer Ansatz zur Öfentlichkeitsarbeit, a.a.O., S. 46 f.; Selinski, H., Messe- und Kongreßmarketing, Berlin 1983, S. 60 f.

Vgl. Selinski, H., Sperling, U. A., Marketinginstrument Messe: Arbeitsbuch für Studium und Praxis, a.a.O., S. 185. 
an Gemeinschaftsständen, so sollten sie sowohl zusammen mit dem Gemeinschaftsstand als auch separat als einzelner Exponent in diesem Informationssystem erfaßt und für die Messebesucher aufgeführt werden. Informationssysteme bieten zudem den Hochschul-Exponenten die Möglichkeit der werblichen Nutzung. Generell hängt jedoch der Einsatz „werbender“ Aktivitäten, wie Anzeigen und die Nutzung von Außenwerbeflächen ${ }^{24}$, von der Höhe des dem Hochschul-Exponenten zur Verfügung stehenden Messebeteiligungsbudgets ab. Durch Werbeaktionen an Standorten in der Nähe des eigenen Messestands können Hochschul-Exponenten eine erhöhte Aufmerksamkeit hinsichtlich der eigenen Messebeteiligung erzielen und Interesse an einem Besuch des HochschulMessestands wecken (z.B. Verteilung von Handzetteln mit Informationen über das Hochschul-Exponat sowie einer Wegbeschreibung zum Messestand etc.). ${ }^{25}$ Darüber hinaus nimmt zur Besucherakquisition auch die Pressearbeit, die anknüpfend an die Pressearbeit der Vor-Messe-Phase durchzuführen ist, einen hohen Stellenwert ein. Neben dem Verfassen von Messezwischenberichten für die hochschulinterne, regionale, überregionale oder auch internationale Presse sind hier insbesondere Pressegespräche mit Journalisten auf dem Hochschul-Messestand hervorzuheben. ${ }^{26}$ Diese können - wie die Pressearbeit insgesamt - zur Erreichung der verfolgten Beeinflussungsziele einen wesentlichen Beitrag leisten, da derartige Berichte eine hohe Wirkung bei den hochschulspezifischen Messezielgruppen auslösen können.

Auf dem Hochschul-Messestand sollte neben den Gesprächen mit Journalisten eine Intensivierung des Dialogs mit den hochschulspezifischen Zielgruppen erfolgen. Dabei ist in den persönlichen Gesprächen auf die Zielsetzungen der Hochschul-Messestand-Besucher einzugehen. Die Dialogbereitschaft der Messestand-Besucher kann dabei durch die Präsenz von Leitungspersönlichkeiten

Unter Außenwerbung ist hier allgemein die großflächige Belegung von standexternen Werbeflächen zu verstehen, die i.d.R. in den Messestädten bzw. an Verkehrsknotenpunkten in der Nähe des Messegeländes zu finden sind. Vgl. Selinski, H., Sperling, U. A., Marketinginstrument Messe: Arbeitsbuch für Studium und Praxis, a.a.O., S. 187. Außenwerbeflächen stehen z.T. im Umfeld und auf dem Gelände der Messeveranstaltung selbst zur Vertügung und ermöglichen den ausstellenden Hochschulen, auf die eigene Messebeteiligung hinzuweisen.

Vgl. Selinski, H., Sperling, U. A., Marketinginstrument Messe: Arbeitsbuch für Studium und Praxis, a.a.O., S. 187.

Vgl. Stracke, F., Messebeschickung und Durchführung: Begleitende Pressearbeit, a.a.O., S. 102. 
der Hochschul-Exponenten, wie Institutsdirektoren, Lehrstuhlinhabern oder Fachbereichsleitern, erhöht werden. Die Anwesenheit derartiger Leitungspersönlichkeiten und ihre Bereitschaft zum Dialog mit Hochschul-Messestand-Besuchern signalisiert nicht nur die Offenheit des Hochschul-Exponenten zum Austausch mit Dritten, sondern sie erhöht auch den Stellenwert und die Kompetenzanmutung der Kommunikation aus Sicht der Gesprächspartner. ${ }^{27}$ Zudem können zur Initiierung und Intensivierung eines fachlich orientierten Dialogs mit relevanten Zielgruppen Begleitveranstaltungen, wie Podiumsdiskussionen, Fachvorträge oder Pressekonferenzen, beitragen. Diese Veranstaltungen, die primär der konzentrierten Informationsvermittlung dienen, weisen meist einen besonderen Ereignischarakter auf. Bei Vertretern der verschiedenen Zielgruppen führt dies oftmals zur Verstärkung der positiven Eindrücke am Messestand ${ }^{28}$ und kann folglich zur Realisierung von Beeinflussungszielen einen wesentlichen Beitrag leisten.

Zur Wirkungserhöhung der Messepräsentation sind darüber hinaus Maßnahmen zur Aktivierung ${ }^{29}$ des anonymen Hochschul-Messestand-Publikums unerläßlich. Nur aktivierte Besucher bringen den Messe-Botschaften des HochschulExponenten die notwendige Aufmerksamkeit entgegen, um diese bewußt wahrzunehmen und gedanklich zu verarbeiten. Dies ist von besonderer Bedeutung, da Messebesuchern die zentralen Botschaften des Exponates sowie des HochschulExponenten im Wettbewerb der Messekommunikation möglichst lange im Gedächtnis verhaftet bleiben sollen, um damit die Erreichung von Beeinflussungszielen langfristig zu sichern.

Über die notwendige Informationsvermittlung hinaus sollte daher auch ein emotional geprägtes Hochschul-Messestand-Erlebnis in Form eines Events ${ }^{30}$ für die

Vgl. Roth, G. D., Messen und Ausstellungen verkaufswirksam planen und durchführen, a.a.O., S. $139 \mathrm{ff}$.

Zu den Ausgestaltungsmöglichkeiten von Begleitveranstaltungen bei Messen vgl. Selinski, $\mathrm{H}$., Begleitveranstaltungen von Messen, in: Strothmann, K.-H., Busche, M. (Hrsg.), Handbuch Messemarketing, a.a.O., S. 485 ff. Zu den Einsatzbereichen und Integrationsmöglichkeiten vgl. Strothmann, K.-H., Verbundveranstaltungen des Messe- und Kongresswesens im Investitionsgüter-Marketing, in: Rost, D., Strothmann, K.-H. (Hrsg.), Handbuch Werbung für Investitionsgüter, Wiesbaden 1983, S. $393 \mathrm{ft}$.

Nach Kroeber-Riel ist Aktivierung eine Grunddimension aller Antriebsprozesse. Zur Aktivierung vgl. Kroeber-Riel, W., Konsumentenverhalten, 6. Aufl., München 1996, S. 58 ff.

Zu den Grundlagen des Event-Marketing vgl. Nickel, H., Event-Marketing: Grundlagen und Erfolgsbeispiele, München 1998; Seitel, K., Erlebniswelt schaffen, in: Direkt Marketing, 32. Jg., 
Messebesucher erzeugt werden. ${ }^{31}$ Für die Umsetzung sind - unter Berücksichtigung des wissenschaftlichen Rahmens - „Exponatshows“ (Exponat in Aktion) ebenso geeignet wie Video- oder Filmvorführungen. Statt eines einfachen Kommunikationstransfers werden vom Hochschul-Exponenten damit Information und Unterhaltung gemeinsam angeboten. Dabei ist aufgrund der multisensorischen Anspracheform von einer erhöhten Aktivierung der Anwesenden auszugehen. ${ }^{32}$ Zudem sollte eine Kontaktaufnahme zum anonymen Messestand-Publikum durch eine aktive Ansprache derjenigen Besucher erfolgen, die ein Interesse an den ausgestellten hochschulspezifischen Exponaten signalisieren, da diese Kontakte oftmals die Basis zur Erreichung angestrebter Projektziele legen.

In der nachfolgenden Tabelle werden zusammenfassend die Maßnahmen dargestellt, die anknüpfend an die Aktivitäten der Vor-Messe-Phase im Rahmen der Messebeteiligung von Hochschulen empfehlenswert erscheinen, um die Erreichung der mit einer Messebeteiligung verfolgten Ziele und die Ansprache entsprechender Messezielgruppen zu fördern.

Heft 5, 1996, S. 14 ff.; Kinnebrock, W., Integriertes Event-Marketing: Vom Marketing-Erleben zum Erlebnis-Marketing, Wiesbaden 1993.

Vgl. Selinski, H., Sperling, U. A., Marketinginstrument Messe: Arbeitsbuch für Studium und Praxis, a.a.O., S. $193 \mathrm{f}$.

Vgl. Ueding, R., Management von Messebeteiligungen: Identifikation und Erklärung messespezifischer Grundhaltungen auf der Basis einer empirischen Untersuchung, a.a.O., S. 112 f. 


\begin{tabular}{|c|c|c|c|c|c|c|c|}
\hline \multirow{3}{*}{\multicolumn{2}{|c|}{ Maßnahmen In der Messe-Phase }} & \multicolumn{5}{|c|}{ Messezieldimensionen } & \multirow{3}{*}{$\begin{array}{l}\text { Mes- } \\
\text { se- } \\
\text { ziel- } \\
\text { grup- } \\
\text { pen }\end{array}$} \\
\hline & & \multirow{2}{*}{\begin{tabular}{|c|} 
Infor- \\
ma- \\
tions- \\
ziele \\
\end{tabular}} & \multicolumn{3}{|c|}{$\begin{array}{l}\text { Beeinflussungs- } \\
\text { ziele }\end{array}$} & \multirow{2}{*}{$\begin{array}{l}\text { Pro- } \\
\text { jekt- } \\
\text { ziele }\end{array}$} & \\
\hline & & & $\begin{array}{l}\text { Hoch- } \\
\text { schul- } \\
\text { bez. }\end{array}$ & \begin{tabular}{|l|} 
Inst \\
LstJ \\
FB- \\
bez. \\
\end{tabular} & $\begin{array}{l}\text { Expo- } \\
\text { nat- } \\
\text { bez. }\end{array}$ & & \\
\hline \multicolumn{8}{|c|}{ Akquisition von Messebesuchern } \\
\hline & Nutzung des Messe-Informationssystems für Besucher & - & + & + & + & + & + \\
\hline & Messebegleitende kommunikative Maßnahmen & - & ++ & ++ & ++ & + & ++ \\
\hline & Aufmerksamkeitserzeugende Aktionen in Hochschul-Messestand-Nähe & - & ++ & ++ & ++ & + & + \\
\hline & Pressearbeit & + & ++ & ++ & ++ & + & + \\
\hline \multicolumn{7}{|c|}{ Intensivierung des Dialogs mit priorisierten Zielgruppen } & \\
\hline & persönliche Gespräche & ++ & ++ & ++ & ++ & ++ & ++ \\
\hline & Präsenz von Leitungspersönlichkeiten der Exponenten & + & ++ & ++ & ++ & ++ & ++ \\
\hline & Teilnahme an Messebegleitveranstaltungen & ++ & ++ & ++ & ++ & + & + \\
\hline \multicolumn{7}{|c|}{ Aktivierung des anonymen Hochschul-Messestand-Pubikums } & \\
\hline tit & "Exponatshows" & -- & ++ & ++ & ++ & + & + \\
\hline & Video- oder Filmvorführungen & - & ++ & ++ & ++ & + & + \\
\hline & Aktive Ansprache interessierter Hochschul-Messestand-Besucher & ++ & ++ & ++ & ++ & ++ & ++ \\
\hline \multicolumn{8}{|c|}{$\begin{aligned}++ & =\text { Maßnahme kann die Erreichung der Messeziele bzw. Messezielgruppen besonders beeinflussen } \\
+ & =\text { Maßnahme kann die Erreichung der Messeziele bzw. Messezielgruppen beeinflussen } \\
0 & =\text { Maßnahme kann die Erreichung der Messeziele bzw. Messezielgruppen bedingt beeinflussen } \\
- & =\text { Maßnahme kann die Erreichung der Messeziele bzw. Messezielgruppen wenig beeinflussen } \\
-- & =\text { Maßnahme kann die Erreichung der Messeziele bzw. Messezielgruppen nicht beeinflussen }\end{aligned}$} \\
\hline
\end{tabular}

Tab. 32: Maßnahmen in der Messe-Phase und deren Einflußmöglichkeiten auf die Erreichung hochschulspezifischer Messeziele und -zielgruppen

Wie bei den messevorbereitenden Aktivitäten sind auch die Maßnahmen der Messe-Phase in Abhängigkeit von den mit der Messebeteiligung primär verfolgten Messezielsetzungen fokussiert einzusetzen. Folglich ist von den einzelnen hochschulspezifischen Exponententypen der Schwerpunkt der Messe-Aktivitäten in Abhängigkeit der jeweils im Vordergrund der Messebeteiligung stehenden Zielsetzung zu planen. Projekt- und exponatbezogene Hochschul-Exponenten sollten z.B. im Rahmen ihres Messeengagements verstärkt Maßnahmen zur Intensivierung persönlicher Gespräche mit Projektinteressenten einsetzen, um die Erreichung von Projektzielen zu unterstützen. Die Beeinflussungsorientierten Hochschul-Exponenten sollten hingegen zur Erreichung ihrer primär verfolgten Beeinflussungsziele insbesondere den pressebezogenen Maßnahmen eine besondere Aufmerksamkeit widmen. Letztlich ist aber auch in dieser Phase das Zusammenspiel aller Messeaktivitäten für eine erfolgreiche Messebeteiligung erforderlich. Demzufolge sind auch die Messeaktivitäten, die nicht primär auf die im Vordergrund des Messeengagements stehenden Messebeteiligungsziele gerichtet sind, mit entsprechend notwendiger Intensität einzusetzen. 


\subsection{Maßnahmen in der Nach-Messe-Phase}

Im Rahmen der Nachbereitung von Hochschul-Messebeteiligungen in der Nach-Messe-Phase sind neben operativ notwendigen Aktivitäten (z.B. Rücktransport der Exponate) vor allem Maßnahmen zur Nachbereitung des Messegeschehens durchzuführen. In diesem Zusammenhang nehmen für Hochschul-Exponenten Instrumente der Kommunikation einen besonderen Stellenwert ein, wobei sich einerseits eine extern-gerichtete, zielgruppenspezifische Kommunikation und andererseits eine intern-gerichtete, mitarbeiterbezogene Kommunikation unterscheiden lassen.

Diese nachbereitenden Aktivitäten der Hochschul-Messebeteiligung sind insbesondere auf die unterschiedlichen Anforderungen der hochschulspezifischen Messezielgruppen auszurichten. Von zentraler Bedeutung ist dabei die systematische Weiterverfolgung der auf der Messe geknüpften Kontakte zu aktuellen und potentiellen Kooperationspartnern. ${ }^{33}$ Im Mittelpunkt stehen hier die Bezugnahme auf konkrete Gesprächsinhalte bzw. die Weiterführung des Dialogs mit den Gesprächspartnern zum Aufbau langfristiger Kooperationsbeziehungen. Das Engagement der Hochschul-Exponenten sollte sich dabei nach den jeweiligen Kontaktqualitäten richten. Diese ergeben sich meist aus wissenschaftlichen Kooperationspotentialen sowie aus Möglichkeiten gemeinsamer Forschungsprojekte. Die im Zusammenhang mit der Realisierung von Projektzielen ggf. erforderliche administrative Unterstützung bis hin zu notwendigen Vertragsgestaltungen und Projektbetreuungen kann durch die Hochschul-Transferstelle erfolgen. ${ }^{34}$

Die Nachbereitung darf jedoch nicht auf die Weiterverfolgung der direkten Messekontakte beschränkt bleiben. Auch diejenigen hochschulspezifischen Zielgruppen, die den Hochschul-Exponenten auf der Messe nicht besucht haben, deren Erreichung aber durch die Messebeteiligung angestrebt wurde (z.B. Vertreter des Staates bzw. des Ministeriums), sind in der Nach-Messe-Phase in bezug auf eigene Messeergebnisse zu kontaktieren. Ein kommunikativ ausge-

Vgl. Naumann, C., Die Arbeit nach der Arbeit - Messe-Nachbereitung und Erfolgskontrolle, in: Küffner, G., Mortsiefer, J. (Hrsg.), Messeplatz Europa: Messen als Bestandteil des betrieblichen Marketings, a.a.O., S. $75 \mathrm{ff}$.

Vgl. Stracke, F., Messebeschickung und Durchführung: Nachbereitung, in: Budach, W. et al. (Hrsg.), Hochschulen und Messen: Praktische Hinweise für Messebeteiligungen durch Hochschulen, a.a.O., S. 106. 
richteter Messebericht des Hochschul-Exponenten kann sowohl zur Profilierung der eigenen Institution beitragen als auch zu direkten Kooperationsbeziehungen nach der Messeveranstaltung führen ${ }^{35}$ und damit die Erreichung angestrebter Beeinflussungs- und Projektziele fördern.

Unmittelbar nach der Messe sollten im Rahmen der Öffentlichkeitsarbeit Messeberichte verfaßt und der Presse zur Verfügung gestellt werden. Diese Berichte sollten den Ablauf und die Ergebnisse der durchgeführten Messebeteiligung des Hochschul-Exponenten umfassen. Die Adressaten ergeben sich, ebenso wie die Form und die inhaltliche Schwerpunktsetzung, aus der in den vorangegangenen Phasen getätigten Pressearbeit und der Resonanz darauf. Auch wenn die Abfassung solcher Presseberichte kurz nach der Messe aufgrund oftmals noch nicht nachweisbarer Ergebnisse problematisch ist, ${ }^{36}$ sollten Hochschul-Exponenten durch eine gezielte Ansprache von Pressevertretern und Bereithaltung von geeigneten Informationen zur Messebeteiligung die Presseberichterstattung unterstützen. Derartige Berichterstattungen erzeugen i.d.R. eine hohe Wirkung bei den hochschulspezifischen Zielgruppen, da in dieser Phase das allgemeine Interesse an der gerade beendeten Messeveranstaltung noch vergleichsweise hoch ist. ${ }^{37}$

Die abschließende Pressearbeit kann in Abhängigkeit der gewählten Messebeteiligungsform einen unterschiedlichen Intensitätsgrad für einzelne Hochschul-Exponenten annehmen. So wird bei der Präsentation auf Landes-Gemeinschaftsständen meist eine intensive Pressearbeit vom Organisator des Messestands (z.B. Landesministerium) vorgenommen. Dennoch besitzt die Pressearbeit solcher Institutionen in Relation zur Pressearbeit der einzelnen Hochschul-Exponenten weniger ersetzenden als vielmehr flankierenden Charakter. Zur Realisierung der von den Hochschul-Exponenten verfolgten Hochschulbezogenen, Instituts-/Lehrstuhl-/Fachbereichsbezogenen oder Exponatbezogenen Beeinflussungsziele ist

Vgl. Naumann, C., Die Arbeit nach der Arbeit - Messe-Nachbereitung und Erfolgskontrolle, a.a.O., S. 81 .

Vgl. Stracke, F., Messebeschickung und Durchführung: Begleitende Pressearbeit, a.a.O., S. 102.

Vgl. Wenge, H. U., Müller, A., Das Management der Messe-Beteiligungen, in: Berndt, R., Hermanns, A. (Hrsg.), Handbuch Marketing-Kommunikation: Strategien - Instrumente - Perspektiven, a.a.O., S. 742 f.; Mortsiefer, J., Informationen sammeln und auswerten - Die Abwicklung einer Messebeteiligung, a.a.O., S. 67. 
eine eigene Pressearbeit unabdingbar, da in dieser die eigenen Zielsetzungen entsprechend berücksichtigt werden können.

Letztlich sollten auch die eigenen Mitarbeiter ${ }^{38}$ im Rahmen einer mitarbeiterbezogenen Kommunikation mittels interner Messeberichte über die Ergebnisse der Messebeteiligung informiert werden. ${ }^{39}$ Den an der Messebeteiligung involvierten wissenschaftlichen sowie auch nicht-wissenschaftlichen Mitarbeitern bieten derartige Berichte eine geeignete Feedback-Möglichkeit. Den nicht auf der Messe anwesenden Mitarbeitern liefern sie relevante Informationen über die Messebeteiligung. Neben der Informationsfunktion weisen interne Messeberichte, die über den Verlauf und das Ergebnis der eigenen Messebeteiligung Auskunft geben, auch vielfältige Möglichkeiten zur Motivation der Mitarbeiter auf. Dazu ist jedoch eine hinreichende Diffusion der Messeberichte in den einzelnen Hochschul-Institutionen erforderlich. Im Rahmen der mitarbeiterbezogenen Kommunikation ist darüber hinaus die persönliche Kommunikation in Form von persönlichen Gesprächen zu forcieren.

In der nachfolgenden Tabelle werden die Maßnahmen, welche die HochschulExponenten zur Aufbereitung des Messegeschehens in der Nach-Messe-Phase einsetzen können, und die durch sie primär tangierbaren Messezieldimensionen zusammenfassend dargestellt.

Im Rahmen der empirischen Untersuchung wurden die mitarbeiterbezogenen Messeziele nicht explizit, sondern nur implizit durch die anderen Messezieldimensionen berücksichtigt. Aufgrund ihrer gerade im Rahmen der Nach-Messe-Phase hervorzuhebenden Relevanz werden sie hier explizit mit aufgeführt.

Vgl. Spryß, W. M., Messen + Ausstellungen: Mit dem "Messe-Diagramm" vorbereiten - von den Zielen bis zum Controlling, in: Marketing Journal, 20. Jg., Heft 4, 1987, S. 370; Dostal, G., Messen + Ausstellungen: So decken Sie die Stärken und Schwächen Ihrer Messe-Beteiligung auf, a.a.O., S. $140 \mathrm{ff}$. 


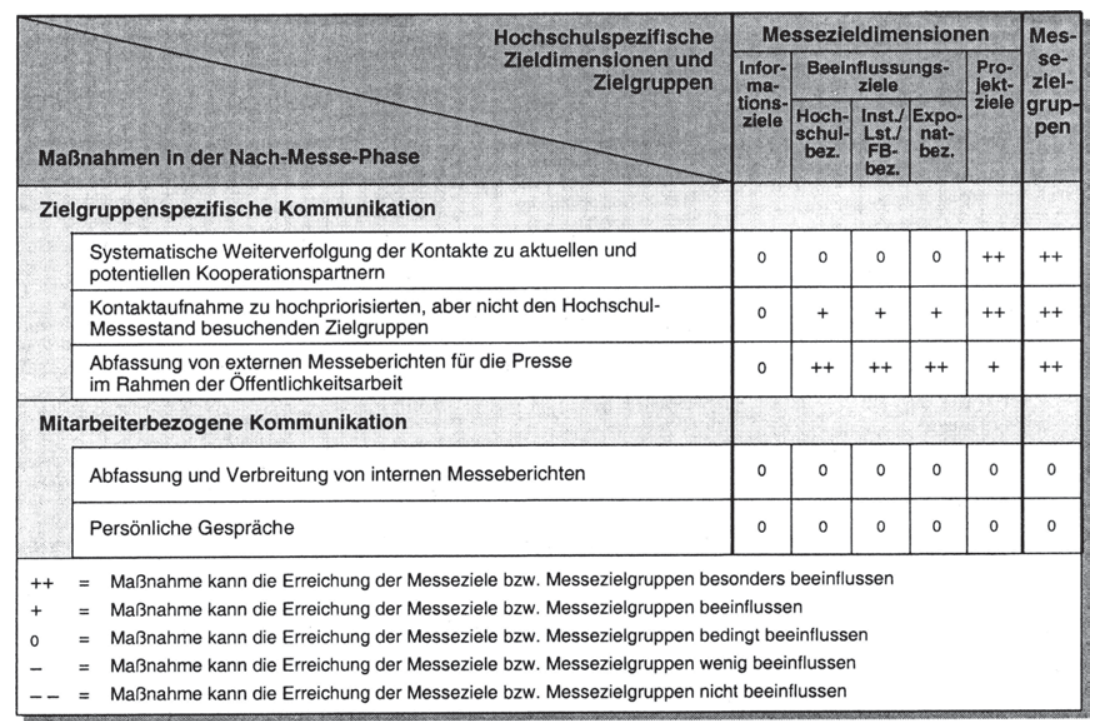

Tab. 33: Maßnahmen in der Nach-Messe-Phase und deren Einflußmöglichkeiten auf die Erreichung hochschulspezifischer Messeziele und -zielgruppen

Auch in der Nach-Messe-Phase sind in Abhängigkeit von den Zielsetzungen, die im Vordergrund der Hochschul-Messebeteiligung stehen, die entsprechenden Aktivitäten von den jeweiligen Exponententypen gezielt durchzuführen. Damit ergeben sich auch für die hochschulspezifischen Exponententypen entsprechende Implikationen bzgl. des verstärkten Einsatzes derjenigen Aktivitäten, die vor allem auf die primär verfolgten Messeziele gerichtet sind. So sollten bspw. Projekt- und exponatorientierte Hochschul-Exponenten systematisch die Kontakte zu aktuellen und potentiellen Kooperationspartnern forcieren, während Beeinflussungsorientierte Hochschul-Exponenten auch in der Nach-Messe-Phase ihren Schwerpunkt eher auf pressebezogene Aktivitäten setzen sollten.

Letztlich wird jedoch erst das Zusammenspiel aller in der Vor-Messe-, Messe- und Nach-Messe-Phase eingesetzten Maßnahmen i.S.e. integrierten Messedurchführung zum Erfolg einer Hochschul-Messebeteiligung führen. Neben dem gezielten und abgestimmten Einsatz der Messebeteiligungsmaßnahmen kommt auch der Integration von Messebeteiligungen in das Hochschul-Marketing eine besondere Bedeutung zu. 


\subsection{Implikationen für die Integration von Messebeteiligungen in das Hochschulmarketing}

Der Erfolg einer Messebeteiligung ist von ihrer Integration in das Hochschulmarketing abhängig. Der in diesem Zusammenhang auftretende Koordinationsbedarf im Hochschulmarketing bezieht sich sowohl auf die Koordination des Marketing mit den anderen hochschulspezifischen Funktionsbereichen als auch auf die Abstimmung aller Aktivitäten innerhalb des Hochschulmarketing i.S.d. Koordination der einzelnen Marketing-Mix-Instrumente. ${ }^{40}$

Eine wirkungsvolle Koordination erfordert zunächst eine Analyse der Interdependenzen innerhalb und zwischen den Instrumentalbereichen des Hochschulmarketing sowie eine Entscheidung über die organisatorische Verankerung des Marketing innerhalb der Hochschule. In einem weiteren Schritt sind die aufeinander abgestimmten Marketingentscheidungen, bei denen Messebeteiligungen entsprechend zu berücksichtigen sind, zu implementieren, d.h. weiter zu konkretisieren und in der Hochschulorganisation durchzusetzen. Abschließend sind die bei der Implementierung auftretenden Probleme sowie die Wirkungen der Marketingaktivitäten im Rahmen eines laufenden Prozesses i.S.e. Marketing-Controlling zu überwachen. Somit sind Messebeteiligungen sowohl im Planungs- und Koordinationsprozeß als auch im Kontrollprozeß des Hochschulmarketing zu berücksichtigen und entsprechend zu integrieren.

Von zentraler Bedeutung ist in diesem Zusammenhang die Entwicklung eines integrierten Kommunikationskonzepts, das der Hochschule über die Abstimmung von Messebeteiligungen mit weiteren kommunikativen Hochschulaktivitäten in einem langfristig angelegten Prozeß zu einer individuellen Corporate Identity verhilft. Unter Corporate Identity wird dabei im weitesten Sinne die "Identität einer Hochschule" subsumiert, die sich im Verhalten, der Kommunikation und dem Erscheinungsbild einer Hochschule ausdrückt ${ }^{41}$ und damit auch durch eine Mes-

Vgl. hierzu sowie im folgenden Meffert, H., Marketing - Grundlagen marktorientierter Unternehmensführung, a.a.O., S. $881 \mathrm{ff}$.

Vgl. Meffert, H., Burmann, C., Identitätsorientierte Markenführung - Grundlagen für das Management von Markenportfolios, in: Meffert, H., Wagner, H., Backhaus, K. (Hrsg.), Arbeitspapier Nr. 100 der Wissenschaftlichen Gesellschaft für Marketing und Unternehmensführung e.V., Münster 1996, S. 23 ff.; Birkigt, K., Stadler, M. M., Funck, H. J. (Hrsg.), Corporate Identity: Grundlagen, Funktionen, Fallbeispiele, 5. Aufl., Landsberg am Lech 1993, S. 20 ff. 
sebeteiligung geprägt werden kann. Die Corporate Identity reflektiert den gegenwärtigen Zustand einer Hochschule, ihre Tradition, die bisherige Hochschulpolitik sowie die Einstellungen des wissenschaftlichen und nicht-wissenschaftlichen Hochschulpersonals. Die Elemente der Hochschulidentität strahlen kontinuierlich sowohl nach innen (auf wissenschaftliches und nicht-wissenschaftliches Hochschulpersonal) als auch nach außen (auf die hochschulspezifische Umwelt) ab und prägen bei den hochschulspezifischen Zielgruppen ein spezifisches Image als (mehr oder weniger genaues) Abbild der Identität. ${ }^{42}$ Gerade in bezug auf die außengerichtete Darstellung bieten Messebeteiligungen den Hochschulen durch inre vielfältigen Ausgestaltungsmöglichkeiten zahlreiche Ansatzpunkte, die Wahrnehmung der Hochschule aus Sicht der Zielgruppen zu beeinflussen. So kann bspw. eine Hochschule durch die Präsentation von Entwicklungen aus ihrer Grundlagenforschung das Image einer "Klassischen Hochschule“ forcieren, wohingegen die Darstellung eines in Kooperation mit der Wirtschaft entwickelten Exponates das Hochschulimage eher in Richtung einer "Transferorientierten Hochschule" lenkt.

Im Hinblick auf die Identität einer Hochschule ist die Wechselseitigkeit zwischen Selbstbild und Fremdbild der Hochschule besonders hervorzuheben. Während einerseits das Selbstbild innerhalb der Hochschule entsteht, von verschiedenen hochschulspezifischen Komponenten (z.B. Trägerschaft und wissenschaftlicher Auftrag der Hochschule) geprägt und insbesondere vom Hochschulmanagement beeinflußbar ist, formt sich andererseits ein Fremdbild der Hochschulidentität bei verschiedenen Anspruchsgruppen, welches sich letztlich im Image der einzelnen Hochschule widerspiegelt (vgl. Abb. 40). ${ }^{43}$ Die Hochschulidentität entsteht und verändert sich im dauerhaften Austausch mit internen und externen Anspruchsgruppen der Hochschule, wobei externe Beziehungen zu hochschulspezifischen Anspruchsgruppen im Mittelpunkt stehen. Damit bieten Messebeteiligungen durch die Kontaktaufnahme zu Messezielgruppen, in denen sich die hochschulspezifischen Anspruchsgruppen widerspiegeln, zahlreiche Ansatzpunkte zur Beeinflussung des Hochschulimage im angestrebten Sinne. Hierbei sind jedoch

Vgl. Meffert, H., Marketing - Grundlagen marktorientierter Unternehmensführung, a.a.O., S. $68 \mathrm{f}$.

43 Vgl. hierzu sowie im folgenden Meffert, H., Burmann, C., Identitätsorientierte Markenführung Grundlagen für das Management von Markenportfolios, a.a.O., S. $34 \mathrm{ff}$. 
nicht nur die auf der Messe realisierten Kontakte, sondern insbesondere auch der über die Messe-Phase hinausgehende Austausch mit diesen Zielgruppen von besonderer Bedeutung. Derartige Kontakte ermöglichen den Hochschul-Exponenten, sich gegenüber Wettbewerbern zu differenzieren und eine angestrebte strategische Positionierung entsprechend zu forcieren.

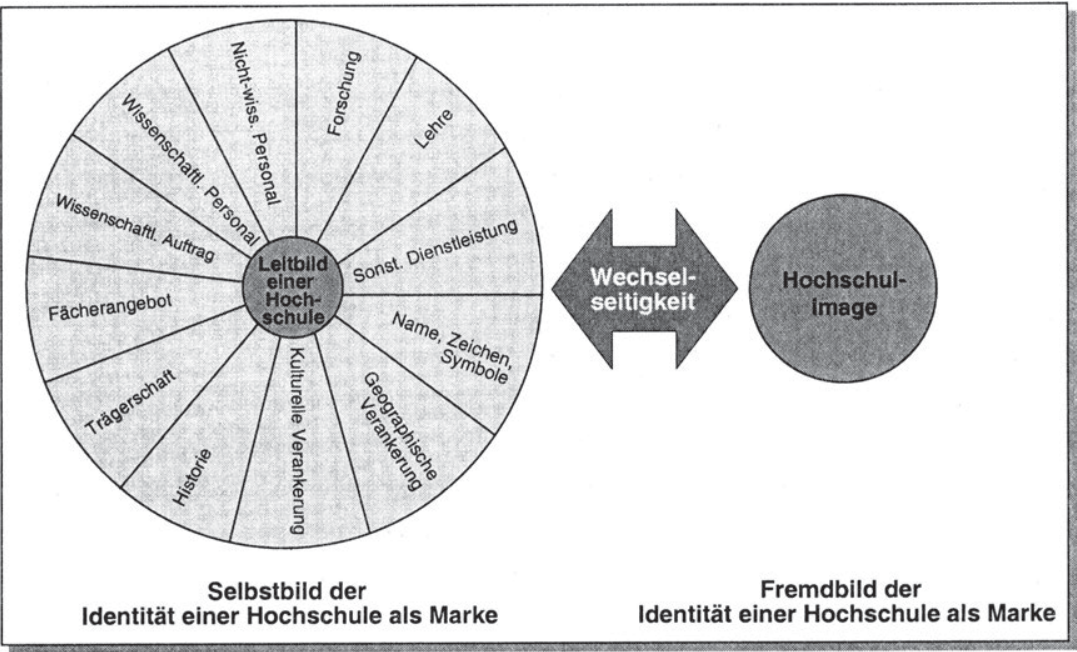

Abb. 40: Identität einer Hochschule als Marke

(Quelle: In Anlehnung an Meffert, H., Burmann, C., Identitätsorientierte Markenführung - Grundlagen für das Management von Markenportfolios, a.a.O., S. 35.)

Stärke und Prägnanz der Hochschulidentität sind vor allem vom Grad der Übereinstimmung zwischen Selbst- und Fremdbild abhängig. ${ }^{44}$ Nur bei einer weitgehenden Übereinstimmung kann eine Hochschule als Marke - i.S.e. in der Psyche der hochschulspezifischen Anspruchsgruppen verankertes, unverwechselbares Vorstellungsbild von einer Hochschule ${ }^{45}$ - aufgebaut sowie erfolgreich auf

Vgl. hierzu sowie im folgenden Meffert, H., Burmann, C., Identitätsorientierte Markenführung Grundlagen für das Management von Markenportfolios, a.a.O., S. $38 \mathrm{ff}$.

Vgl. Meffert, H., Marketing - Grundlagen marktorientierter Unternehmensführung, a.a.O., S. 785. Hier wird das Markenverständnis des identitätsorientierten Ansatzes der Markenführung von MEFFERT und BURMANN zugrunde gelegt. Im Vergleich zu anderen Ansätzen der Markenführung liegen die zentralen Merkmale dieses Ansatzes in der Erweiterung der außengerichteten Perspektive um die innengerichtete Sichtweise. Im Vordergrund dieses Ansatzes steht die Wechselseitigkeit von Image und Identităt einer Marke. Vgl. Meffert, H., Burmann, C., 
Basis ihrer Identität geführt werden. ${ }^{46}$ Hochschul-Messebeteiligungen können in diesem Zusammenhang positiven Einfluß auf die Stärke und Prägnanz der ausstellenden Hochschule nehmen. Voraussetzung hierfür ist die Orientierung der Messebeteiligungsziele an der angestrebten, klar definierten Hochschulidentität, da die Schaffung eines präzisen Vorstellungsbildes der Hochschule bei ihren Zielgruppen nach exakten Formulierungen des gewünschten Vorstellungsbildes verlangt, dessen Qualität den Grad der Identifizierbarkeit und Profilierung bestimmt.

Die Herausbildung einer hochschulspezifischen Identität stellt somit hohe Anforderungen nicht nur an das Hochschulmanagement, sondern an die Mitglieder einer Hochschule. ${ }^{47}$ Die Entfaltung eines über die Fachbereiche, Institute und Lehrstühle hinausgehenden Identitätsbewußtseins, das auch Studierende mit einbezieht, dürfte bei den Mitgliedern der unterschiedlichen Hochschulebenen stark differenzieren. In diesem Zusammenhang stellt sich die Frage, ob eine auf Pluralismus, Meinungsvielfalt und kontroverse Diskussion angelegte Organisation wie die Hochschule in allen Bereichen widerspruchsfreies Verhalten zum obersten Prinzip machen sollte. Grundsätzlich sollte der pluralistische Meinungsbildungsprozeß von Lehrenden und Lernenden eher weiter gefördert werden, da er zur internen Identifikation der Beteiligten beiträgt und die Sekundärwirkung einer positiven Außendarstellung durch die Betroffenen zur Folge hat, welche letztlich i.S.e. Corporate Identity liegt. ${ }^{48}$

Eine starke Hochschulidentität, die einen abgestimmten Einsatz von Hochschul-Messebeteiligungen mit allen weiteren Marketing-Mix-Instrumenten voraussetzt, verschafft Glaubwürdigkeit und stellt damit die notwendige Bedingung zur Entstehung von Vertrauen dar. Die hinreichende Bedingung zur Vertrauensbil-

Identitätsorientierte Markenführung - Grundlagen für das Management von Markenportfolios, a.a.O., S. 14. Zu weiteren Ansätzen der identitätsorientierten Markenführung vgl. Aaker, D. A., Building Strong Brands, New York u.a. 1996; Kapferer, J.-N., Die Marke - Kapital der Unternehmung, Landsberg am Lech 1992.

Hochschulmarken können den Dienstleistungsmarken zugeordnet werden, denn Hochschulen stellen mit Forschung, Lehre und sonstigen Dienstleistungen klassische Anbieter von Dienstleistungen dar.

Vgl. hierzu sowie im folgenden Wangen-Goss, M., Marketing für Universitäten: Möglichkeiten und Grenzen der Übertragbarkeit des Marketing-Gedankens auf den universitären Bereich, a.a.O., S. $246 \mathrm{f}$.

Vgl. Wangen-Goss, M., Marketing für Universităten: Möglichkeiten und Grenzen der Übertragbarkeit des Marketing-Gedankens auf den universitären Bereich, a.a.O., S. 247. 
dung bildet die Kompetenz. ${ }^{49}$ Identität und Kompetenz einer Hochschule legen damit die Basis zur Entstehung von Vertrauen bei den hochschulspezifischen Zielgruppen in bezug auf die Leistungsfähigkeit und Leistungsbereitschaft der Hochschulen. Eine Hochschul-Messebeteiligung kann in diesem Zusammenhang einerseits durch die Selbstdarstellung und die Präsentation von Exponaten die entsprechende Leistungskompetenz der Hochschule steigern. Andererseits wird der Erfolg einer Messebeteiligung durch eine bereits vorhandene Identität und Kompetenz der Hochschule wesentlich gefördert. Der integrierte Einsatz von Messebeteiligungen im Rahmen des Hochschulmarketing kann somit den Aufbau einer Hochschulidentität i.S.e. Marke forcieren und gleichzeitig von ihrer Existenz profitieren.

\section{Implikationen für Finanzmittelgeber von Hochschul-Messebeteili- gungen}

Vor dem Hintergrund der knapp bemessenen öffentlichen Finanzmittel für die einzelnen Hochschulen sind diese i.d.R. nicht in der Lage, Messebeteiligungen ohne zusätzliche finanzielle Unterstützungen durchzuführen. Neben eigenen Finanzmitteln aus Drittmittelprojekten sind Hochschulen im Rahmen der Durchführung von Messebeteiligungen vor allem auf die zusätzliche finanzielle Unterstützung sowohl öffentlicher als auch kommerzieller Institutionen angewiesen. Diese verfolgen i.d.R. mit derartigen Unterstützungen eigene Zielsetzungen, die vielfältig ausgeprägt sein können. Notwendige Voraussetzung zur Erreichung dieser Zielsetzungen ist eine erfolgreiche Messebeteiligung der Hochschul-Exponenten. Der Umfang der für Hochschul-Messebeteiligung eingesetzten Finanzmittel sowie deren Allokation sollten sich somit grundsätzlich sowohl im kommerziellen als auch im nicht-kommerziellen Bereich, der im folgenden im Vordergrund der Betrachtung stehen soll, am Erfolg von Hochschul-Messebeteiligungen orientieren.

Der Umfang finanzieller Unterstützungen von Hochschul-Messebeteiligungen aus dem öffentlichen Bereich wird i.d.R. über die Zuweisungen öffentlicher Finanzmittel determiniert. Derzeit werden auf Landesebene Systeme der Mittelzuwei- 
sung auf Hochschulen und innerhalb der Hochschulen auf Fachbereiche, Institute und Lehrstühle eingeführt, mit denen die Zuweisung von Finanzmitteln stärker nach Leistungskriterien erfolgen soll. ${ }^{50}$ Während in Nordrhein-Westfalen auf Landesebene bereits ein System der Mittelzuweisung auf die einzelnen Hochschulen eingeführt wurde, ${ }^{51}$ planen andere Länder wie Niedersachsen die Einführung derartiger Konzepte. ${ }^{52}$ Diese Systeme sehen vor, Finanzmittel proportional nach einem bestimmten Schlüssel auf die einzelnen Einheiten zu verteilen. Während der Schlüssel oftmals auf den gleichen Leistungskriterien basiert, wird deren Gewichtung in den einzelnen Konzepten sehr unterschiedlich vorgenommen. ${ }^{53}$

Diese Systeme führen in ihrer derzeitigen Ausgestaltung nicht zur intendierten Allokation der finanziellen Mittel, da mit den Verteilungsregeln oftmals nicht die höchstmögliche Effizienz erreicht wird. ${ }^{54}$ Eine implizite Kritik an diesen Ansätzen existiert insofern, als die Forderung besteht, eine Mittelverteilung nur auf denjenigen Budgetanteil zu beziehen, der nicht zur Absicherung der sogenannten Grundausstattung dient, weil sonst die Gefahr besteht, daß kleine und bisher ineffiziente Einheiten ihre Grundausstattung nicht mehr sichern können. Darüber hinaus wird kritisiert, daß eine zu Leistungskriterien proportional vorgenommene Verteilung möglicherweise existierende Verhältnisse festigen könnte, statt sie zu ändern. Die erfolgreichen Hochschuleinheiten erhalten hohe Mittelzuweisungen, wodurch sie ihr Niveau halten können. Würden jedoch die finanziellen Mittel den Hochschuleinheiten zugewiesen, die bisher noch nicht dokumentieren konnten, daß sie Finanzmittel insgesamt effizienter einsetzen können, so würde dies für das Gesamtsystem einen höheren Nutzen erzielen. ${ }^{55}$ Folglich sollten Landesregierungen ihre Finanzmittel den verschiedenen Hochschulen des Landes so zuweisen,

Vgl. Sönke, A., Optimale Allokation von Hochschul-Budgets, in: DBW, 59. Jg., Heft 5, 1999, S. 583.

51 Vgl. hierzu Fandel, G., Funktionalreform der Hochschulleitung, in: ZfB, 68. Jg., Heft 3, 1998, S. 241 ff.; Fandel, G., Gal, T., Umverteilung der Mittel für Lehre und Forschung unter den Universitäten, Diskussionsbeiträge des Fachbereichs Wirtschaftswissenschaft der FernUniversität Hagen, Nr. 248, Dezember 1997.

Vgl. hierzu Hilgenberg, F., Was kommt aus der Gieskanne? Ansätze finanzieller Anreizsysteme in den Ländern, in: Forschung \& Lehre, 1. Jg., Heft 7, 1994, S. $261 \mathrm{f}$.

Vgl. Sönke, A., Optimale Allokation von Hochschul-Budgets, a.a.O., S. 585.

Vgl. Behrens, Th., Globalisierung der Hochschulhaushalte - Grundlagen, Ziele, Erscheinungsformen und Rahmenbedingungen, Neuwied, Kriftel, Berlin 1996; Sönke, A., Optimale Allokation von Hochschul-Budgets, a.a.O., S. $583 \mathrm{ft}$.

Vgl. Sönke, A., Optimale Allokation von Hochschul-Budgets, a.a.O., S. 589. 
daß damit insgesamt ein maximaler Erfolg bzw. eine maximale Leistung bewirkt werden. Dies gilt ebenso für Hochschulen, die inre Mittel auf Fachbereiche, Institute und Lehrstühle verteilen, sowie auch für Fachbereiche, die ihre Mittel den Instituten und Lehrstühlen zuweisen. ${ }^{56}$

Übertragen auf die Allokation von Finanzmitteln auf Hochschul-Exponenten wird die einleitend angeführte Empfehlung, finanzielle Mittel insbesondere den Hochschul-Exponenten zuzuweisen, die die besten Voraussetzungen zur Durchführung einer erfolgreichen Messebeteiligung besitzen, nochmals bekräftigt. Der Erfolg einer Hochschul-Messebeteiligung - so zeigen die im Rahmen der vorliegenden Untersuchung gewonnenen Ergebnisse - wird von den jeweils angestrebten Zielsetzungen determiniert. Hochschul-Exponenten, die mit ihrer Messebeteiligung vor allem Projekt-, Beeinflussungs- sowie Informationsziele verfolgen, besitzen grundsätzlich die Voraussetzungen zur Durchführung einer erfolgreichen Messebeteiligung. Folglich sind Finanzmittel insbesondere den Exponenten zuzuweisen, die sich entweder als Projekt- und exponatorientierte Hochschul-Exponenten oder als Beeinflussungorientierte Hochschul-Exponenten charakterisieren lassen. Hingegen sind finanzielle Unterstützungen von Hochschul-Exponenten, die sich nicht aus eigener Initiative, sondern vielmehr aus der Pflicht ihres Status als öffentlichrechtliche, nicht-kommerzielle Institution heraus an Messen beteiligen, wenig sinnvoll, da sie das Potential von Messebeteiligungen i.S.d. Gesamterfolgs nicht ausreichend ausschöpfen. Finanzmittelgeber können einer Fehlallokation auf Hochschul-Exponenten, die sich als Pflichtrepräsentanten charakterisieren lassen, z.T. entgegenwirken, indem sie grundsätzlich keine Gesamt-, sondern nur Teilfinanzierungen von Hochschul-Messebeteiligungen vornehmen, da die Finanzierungsbereitschaft der Exponenten die Verfolgung eigener Messezielsetzungen signalisiert.

Der Erfolg von Hochschul-Messebeteiligungen hängt jedoch nicht nur von den verfolgten Messezielsetzungen ab, sondern wird ebenfalls von der auf diese Zielsetzungen abgestimmten strategischen und operativen Ausgestaltung des jeweiligen Messeengagements determiniert. Finanzmittelgeber von Hochschul-Messebeteiligungen sollten somit die von den Hochschul-Exponenten verfolgten Messezielsetzungen eruieren und die geplante Ausgestaltung des Messeengagements auf ihre Eignung zur Zielerreichung des Hochschul-Exponenten überprüfen. Wird 
die Zielerreichung durch die angestrebte Messebeteiligung forciert, ist eine finanzielle Unterstützung der Messebeteiligung sinnvoll. Steht hingegen das Messeengagement nicht i.S.d. verfolgten Messezielsetzungen, sollte eine finanzielle Unterstützung nur dann erfolgen, wenn die strategische sowie operative Ausgestaltung der Messebeteiligung von den Hochschul-Exponenten entsprechend an die Messezielsetzungen angepaßt wird.

Steht die finanzmittelgebende Institution vor der Entscheidung, entweder Messebeteiligungen oder andere Kommunikationsinstrumente von Hochschulen zu finanzieren, so ist die Entscheidung wiederum von den eigenen sowie von den verfolgten Zielsetzungen der Exponenten abhängig. Bei der Verfolgung von Projektzielen, deren Erreichung die persönliche Kontaktaufnahme mit Projektinteressenten voraussetzt, sind Messebeteiligungen aufgrund der sich auf Messen bietenden Kontaktmöglichkeiten anderen Kommunikationsinstrumenten überlegen. Folglich ist in diesem Fall die finanzielle Unterstützung von Messebeteiligungen sinnvoll. Wird hingegen bspw. eine Bekanntheitsgraderhöhung der Hochschule bei potentiellen Studierenden angestrebt, um diese Zielgruppe für die Aufnahme eines Studiums an der Hochschule zu gewinnen, so ist von der finanziellen Unterstützung von Messebeteiligungen abzuraten, da hier weder die anzusprechende Zielgruppe ausreichend vertreten, noch der Einsatz dieser Maßnahme nach Kosten-Nutzen-Relationen gerechtfertigt erscheint. Vielmehr sind zur Ansprache dieser Zielgruppe kostengünstigere Kommunikationsinstrumente zu wählen, deren Unterstützung durch die jeweiligen Finanzmittelgeber entsprechend möglich ist.

Vor dem aufgezeigten Hintergrund kommt dem Ausbau eines standardisierten hochschulübergreifenden Controllingsystems für Messebeteiligungen, das Vergleiche i.S.d. Benchmarking ermöglicht und den Erfahrungsaustausch i.S.d. Verbesserung des Wissensstandes fördert, eine zunehmende Bedeutung zu. 


\section{E. Zusammenfassung und Ausblick}

\section{Zusammenfassende Darstellung der Untersuchungsergebnisse}

Hochschulen sehen sich angesichts veränderter Rahmenbedingungen einer Verknappung öffentlicher Finanzmittel gegenübergestellt, die zu einer steigenden Relevanz der Drittmitteleinwerbung über eine Nachfragesteigerung im Bereich der Forschungsdienstleistungen führt. Zur Erreichung dieser Zielsetzung bedarf es des Austausches von Transferleistungen zwischen Hochschulen und an Forschungsleistungen interessierten Anspruchsgruppen. In diesem Zusammenhang gewinnen Messen als Ort des Zusammentreffens zwischen Ausstellern als Anbietern und Messebesuchern als Nachfragern von Forschungsergebnissen eine besondere Bedeutung. Vor diesem Hintergrund lag die grundsätzliche Aufgabe der vorliegenden Arbeit in der Analyse des Erfolgs von Hochschul-Messebeteiligungen, wobei die Untersuchung der hochschulspezifischen Messebeteiligungsziele und -zielgruppen als Grundlage der Erfolgskontrolle den Ausgangspunkt des Forschungsprojektes bildete.

Hochschulen sind in ein pluralistisches und komplexes Zielsystem eingebunden, wobei sie ihrerseits nicht über eine oberste Zielbildungsinstanz verfügen. Sowohl administrative und legislative Einflüsse führen zur Einengung der Autonomie bei der Festlegung hochschulspezifischer Messebeteiligungsziele der Exponenten. Bund, Länder und Kommunen können mit Hochschul-Messebeteiligungen Technologie-, Wirtschafts- und Standortförderungen anstreben. Diese Ziele, die teils neutral, teils konfliktär, oftmals jedoch komplementär zu den Zielsetzungen der Hochschulen stehen, werden i.d.R. implizit in den von Hochschul-Exponenten verfolgten beschaffungs- und leistungsmarktgerichteten Messebeteiligungszielen berücksichtigt. Die empirische Ermittlung der von Hochschul-Exponenten vorgenommenen Priorisierungen dieser Messezielsetzungen zeigte zunächst eine generell hohe Wichtigkeit aller hochschulspezifischen Messebeteiligungsziele aus Exponentensicht auf, wobei sich dennoch Prioritätsrangfolgen identifizieren ließen. So stuften Hochschul-Exponenten bspw. die Erhöhung des Bekanntheitsgrades des Exponates und des Instituts/Lehrstuhls/Fachbereichs, die Darstellung der Leistungskompetenz sowie den Erfahrungs- und Informationsaustausch mit Praxisvertretern als die wichtigsten Messebeteiligungsziele ein. 
Aufgrund der Vielfältigkeit und der hohen Anzahl hochschulspezifischer Messeziele wurde überprüft, inwieweit sich diese Zielsetzungen systematisieren und auf eine geringere Anzahl übergeordneter Zieldimensionen verdichten lassen. Die empirische Untersuchung zeigte, daß hinter den vielfältigen Einzelzielsetzungen einer Hochschul-Messebeteiligung eigenständige hochschulspezifische Messezieldimensionen stehen. Insgesamt konnten mit Informations- und Projektzielen sowie Exponatbezogenen, Instituts-/Lehrstuhl-/Fachbereichsbezogenen und Hochschulbezogenen Beeinflussungszielen fünf relevante Zieldimensionen für Hochschul-Messebeteiligungen identifiziert werden. Die Hochschul-Exponenten räumten dabei den einzelnen Zieldimensionen unterschiedliche Stellenwerte ein. So wurden den Exponatbezogenen sowie den Instituts-/Lehrstuhl-/Fachbereichsbezogenen Beeinflussungszielen die höchste Priorität beigemessen, während die anderen Zieldimensionen im Vergleich dazu eine geringere, aber dennoch nicht unwichtige Stellung im Zielsystem einnahmen.

Auf Basis der ermittelten Messezieldimensionen konnten drei hochschulspezifische Exponententypen empirisch abgeleitet werden, die sich in den Priorisierungen der hochschulspezifischen Messezieldimensionen signifikant voneinander unterschieden. Gemäß der von den jeweiligen Exponententypen primär verfolgten Zieldimensionen konnten als hochschulspezifische Exponententypen Projekt- und exponatorientierte Hochschul-Exponenten, Beeinflussungsorientierte HochschulExponenten und Pflichtrepräsentanten identifiziert werden. Bei Projekt- und exponatorientierten Hochschul-Exponenten stehen insbesondere Projektziele und Exponatbezogene Beeinflussungsziele im Vordergrund des Messeengagements, da sie durch eine Messebeteiligung vor allem die Akquisition von Drittmitteln anstreben. Beeinflussungsorientierte Hochschul-Exponenten verfolgen mit ihrem Messeengagement in erster Linie Exponatbezogene, Instituts-/Lehrstuhl-/Fachbereichsbezogene und Hochschulbezogene Beeinflussungsziele. Folglich ist die Messebeteiligung dieser Exponenten auf die Präsentation der eigenen Aktivitäten gerichtet, um dem im Hochschulrahmengesetz verankerten Gebot, die Hochschulleistungen gegenüber der die Hochschule alimentierenden Gesellschaft zu dokumentieren, gerecht zu werden. Die dritte Exponentengruppe im Rahmen der Messebeteiligung von Hochschulen bilden die Pflichtrepräsentanten, die im Vergleich zu den vorangegangenen hochschulspezifischen Exponententypen den Messezieldimensionen insgesamt eine geringere Bedeutung beimessen. Diese Hochschul-Exponenten beteiligen sich meist nicht aus eigener Initiative an Messen, sondern vielmehr aus der Pflicht ihres Status als öffentlich-rechtliche, nichtkommerzielle Institution heraus. 
Vor dem Hintergrund der aufgezeigten Ergebnisse wurde untersucht, inwieweit eine ausschließlich aus Exponentensicht vorgenommene und auf Grundlage exponententypspezifischer Messebeteiligungszielsetzungen abgeleitete Ausgestaltung von Hochschul-Messebeteiligungen ausreicht, um hochschulspezifische Messeziele zu erreichen. In diesem Zusammenhang deuteten die unterschiedlichen Priorisierungen der hochschulspezifischen Einzelzielsetzungen darauf hin, daß mit der Verfolgung bestimmter Zielsetzungen die Erreichung ausgewählter hochschulspezifischer Anspruchsgruppen angestrebt wird. Demzufolge wurde eine Überprüfung hochschulspezifischer Anspruchsgruppen als Zielgruppen von Hochschul-Messebeteiligungen erforderlich.

Die Diskussion verschiedener, in der Literatur aufgezeigter Ansätze zur Systematisierung von Zielgruppen im Hochschulbereich führte zur Ableitung von fünf hochschulspezifischen Messezielgruppen, die auf Grundlage von Wichtigkeiten vorhandener Austauschbeziehungen zwischen Anspruchsgruppen und Hochschulen gebildet wurden. Dabei konnten für die abgeleiteten Messezielgruppen Wissenschaft, Studierende, Wirtschaft, Öffentlichkeit sowie Staat aus Sicht der Hochschul-Exponenten klar abgestufte Priorisierungen ermittelt werden. Während Öffentlichkeit und Wirtschaft als primäre Messezielgruppen fungieren, stufen die Hochschul-Exponenten Zielgruppen wie Wissenschaft und Staat sowie auch Studierende insgesamt als weniger wichtig ein. Hinsichtlich exponententypspezifischer Zielgruppenpriorisierungen konnten nur leicht unterschiedliche exponententypspezifische Gewichtungen der einzelnen Zielgruppen festgestellt werden, was auf eine nicht konsistente, exponententypspezifische Planung von Hochschul-Messebeteiligungen schließen ließ.

Angesichts der sehr ähnlichen exponententypspezifischen Priorisierungen bestand eine Notwendigkeit darin zu überprüfen, ob eine auf Basis von Wichtigkeiten vorhandener Austauschbeziehungen vorgenommene Systematisierung von hochschulspezifischen Anspruchsgruppen zur Systematisierung von Messezielgruppen ausreichend ist. Angesichts der mit dieser Arbeit verfolgten Zielsetzung, auch Implikationen für die Messebeteiligung von Hochschulen abzuleiten, erschien eine zusätzliche Berücksichtigung verhaltensorientierter Kriterien unabdingbar, um aus Sicht der Hochschul-Exponenten verhaltenssteuernd agieren zu können. Zur Ableitung einer hochschulspezifischen Messeausgestaltung war es demzufolge erforderlich, das Verhalten von Messebesuchern zu analysieren und möglichst realitätsnah zu beschreiben. 
Das Verhalten der Hochschul-Messestand-Besucher wird von den Zielsetzungen determiniert, welche mit einem Besuch von Hochschul-Messeständen angestrebt werden. Vor diesem Hintergrund wurde eine Besuchertypenbildung auf Grundlage der theoretisch abgeleiteten hochschulspezifischen Besucherzielsetzungen Sammlung von Informationen, Beobachtung von Entwicklungen der Wissenschaft, Kontaktaufnahme zu Projektanbietern, Erfahrungs- und Informationsaustausch sowie Beziehungspflege oder -aufbau mit Wissenschaftsvertretern vorgenommen. Auf Basis dieser Besucherzielsetzungen konnten empirisch drei unterschiedliche Hochschul-Messestand-Besuchertypen gebildet werden. Gemäß ihrer primär mit dem Besuch eines Hochschul-Messestands verfolgten Zielsetzungen wurden die Bezeichnungen Intensive Hochschul-Messestand-Nutzer, Informationsorientierte Hochschul-Messestand-Nutzer und Hochschul-Messestand-Bummler gewählt. Während die Intensiven Hochschul-Messestand-Nutzer mit ihrem Hochschul-Messestand-Besuch sowohl Informations- als auch Kontaktziele verfolgen, stehen bei Informationsorientierten Hochschul-Messestand-Nutzern insbesondere Informationsziele im Vordergrund ihres Hochschul-Messestand-Besuchs. Dagegen messen Hochschul-Messestand-Bummler den einzelnen Zielsetzungen einen vergleichsweise geringen Stellenwert bei.

Im Hinblick auf die Zugehörigkeit der Hochschul-Messestand-Besuchertypen zu den einzelnen Zielgruppen von Hochschul-Messebeteiligungen konnten hinsichtlich der Zielgruppen Wirtschaft, Wissenschaft und Studierende signifikante, dagegen bzgl. der Zielgruppen Öffentlichkeit und Staat keine signifikanten Unterschiede konstatiert werden. Dabei zeigte sich, daß die von den Hochschul-Exponenten hoch priorisierten, auf die jeweiligen Zielgruppen fokussierten Messeeinzelzielsetzungen komplementär zum Verhalten der Hochschul-Messestand-Besuchertypen standen. Somit kann von einem „Fit" zwischen den Messeeinzelzielsetzungen von Hochschul-Exponenten und den Messebesucherzielsetzungen ausgegangen werden, wodurch grundsätzlich die Basis für eine erfolgreiche Hochschul-Messebeteiligung gelegt wird.

Zur Erfassung des hochschulspezifischen Messebeteiligungserfolgs wurden Ansätze zur Messeerfolgskontrolle von Hochschul-Messebeteiligungen analysiert. In der in diesem Rahmen angeführten Diskussion zur Unsicherheit über die Effizienz von Hochschul-Messebeteiligungen wurde herausgestellt, daß eine Hochschul-Messebeteiligung dann als effizient bezeichnet werden kann, wenn - nach der Ermittlung eines Messebeteiligungsnutzens (erste notwendige Bedingung) - der erzielte Nutzen die entstandenen Messebeteiligungskosten 
übersteigt (zweite notwendige Bedingung) und aufgrund einer alternativen Mittelverwendung kein höherer Nutzen hätte erzielt werden können (hinreichende Bedingung). Während erst nach Prüfung der hinreichenden Bedingung Erkenntnisse über die Effizienz von Hochschul-Messebeteiligungen vorliegen, können bereits nach Abschluß der Überprüfung der beiden notwendigen Bedingungen Aussagen über den Erfolg von Hochschul-Messebeteiligungen getroffen werden. Deshalb erschien die Durchführung einer zweistufigen Erfolgskontrolle sinnvoll. Dazu wurde auf eine in der Messeliteratur vorgenommene Unterteilung der Messeerfolgskontrolle in indirekte und direkte Erfolgskontrolle zurückgegriffen. Während die indirekte Erfolgskontrolle den Erfolg als Zielerreichungsgrad der verfolgten Messebeteiligungsziele auffaßt und damit ausschließlich eine Nutzenbetrachtung i.S.e. Effektivitätskontrolle vornimmt, wird im Rahmen der direkten Erfolgskontrolle, die den Erfolg i.S.v. Kosten-Nutzen-Relationen definiert, eine Effizienzkontrolle durchgeführt.

Im Rahmen der indirekten Erfolgskontrolle von Hochschul-Messebeteiligungen galt es, den Zielerreichungsgrad der von den Hochschul-Exponenten verfolgten hochschulspezifischen Messebeteiligungsziele zu ermitteln. Folglich wurde die Erfolgskontrolle auf Grundlage der empirisch analysierten Messezielsetzungen von Hochschul-Exponenten durchgeführt. Dabei wurden zunächst aus den hochschulspezifischen Messebeteiligungszielen Erfolgskriterien abgeleitet. Aufgrund der engen Verbundenheit der Erfolgskriterienauswahl mit der Wahl von Methoden zur Erfolgskontrolle wurden anschließend ausgewählte, für die indirekte Erfolgskontrolle von Hochschul-Messebeteiligungen grundsätzlich relevante Methoden analysiert. Dabei standen im Mittelpunkt der Analyse die Ziele und die wesentlichen Charakteristika der einzelnen Methoden. Darüber hinaus wurden Vor- und Nachteile der Methoden gekennzeichnet und deren Eignung zum Einsatz bei Hochschul-Messebeteiligungen überprüft. Dabei lag der Fokus - nicht zuletzt aufgrund des expandierenden Messeangebots und der damit einhergehenden Notwendigkeit einer verstärkten Messeselektion - insbesondere auch auf der messetypspezifischen Betrachtung (Hochschul-Messebeteiligungen auf Mehrbranchenmessen vs. Hochschul-Messebeteiligungen auf Fachmessen). Darüber hinaus wurde - soweit die einzelnen Methoden dazu geeignet waren - der indirekte Messeerfolg exponententypspezifisch analysiert. Das Phasenkonzept der Messebeteiligung bildete in diesem Zusammenhang den Ausgangspunkt der Analyse.

In der Vor-Messe-Phase können Hochschul-Exponenten die indirekte Erfolgskontrolle insbesondere zur Überprüfung ihres Messebeteiligungskonzepts (durch 
Einsatz des Messekonzepttests) sowie zur Erfassung der Erwartungen von Messezielgruppen (mittels der Durchführung von Vorbefragungen) nutzen. Aufgrund des mit dem Einsatz dieser Verfahren notwendigen Bedarfs an finanziellen und personellen Ressourcen wurden diese Methoden zur Erfolgskontrolle von Hochschul-Messebeteiligungen als eher weniger geeignet eingestuft.

Die indirekte Erfolgskontrolle in der Messe-Phase war auf die Erfassung des Geschehens auf dem Hochschul-Messestand gerichtet. Allgemein boten sich dazu sowohl Wegeverlaufsanalysen sowie Zählungen von ausgegebenen Informationsmaterialien, Hochschul-Messestand-Besuchern und persönlicher Kontakte als auch Erstellungen von Gesprächsprotokollen und Befragungen von Hochschul-Messestand-Besuchern an. Mit Ausnahme der Wegeverlaufsanalysen erwiesen sich diese Methoden zur Erfolgskontrolle von Hochschul-Messebeteiligungen als geeignet.

Die Zählungen ergaben in bezug auf die verfolgten Zielsetzungen eine eindeutige Überlegenheit von Hochschul-Messebeteiligungen auf Mehrbranchenmessen im Vergleich zu Fachmessebeschickungen. Die Ergebnisse der Gesprächsprotokolle sowie der Hochschul-Messestand-Besucherbefragungen zeigten hingegen keine eindeutige Überlegenheit eines Messetyps auf, so daß der Erfolg auf den einzelnen Messen stark von den jeweiligen Zielsetzungen der Hochschul-Exponenten abhängt. Die exponententypspezifischen Ergebnisse der eingesetzten Methoden zeigten, daß Projekt- und exponatorientierte Hochschul-Exponenten sowie Beeinflussungsorientierte Hochschul-Exponenten durch ihre Messebeteiligung die Erreichung primär verfolgter Zielsetzungen unterstützten. So konnten bspw. Projekt- und exponatorientierte Hochschul-Exponenten vergleichsweise viele projektbezogene Gespräche führen und damit die Erreichung ihrer hoch priorisierten Projektziele fördern. Beeinflussungsorientierte Hochschul-Exponenten konnten ihre primär verfolgten Beeinflussungsziele insbesondere über die insgesamt relativ hohe Anzahl an ausgegebenen Informationsmaterialien sowie die vergleichsweise hohe Anzahl an Kontakten zur Öffentlichkeit forcieren. Im Vergleich dazu wurden für Pflichtrepräsentanten relativ schlechte Ergebnisse ermittelt. Zwar konnte dieser Exponententyp vergleichsweise viele Gespräche mit Vertretern des Staates und damit seiner Pflicht als öffentlich rechtliche Institution zumindest ansatzweise nachkommen. Dennoch belegt die insgesamt geringe Anzahl an ausgegebenen Informationsmaterialien, realisierten Kontakten sowie geführten Gesprächen für Pflichtrepräsentanten einen im Vergleich zu den zwei anderen Exponententypen relativ geringen Messebeteiligungsnutzen. 
In der Nach-Messe-Phase wurde eine Gesamtbetrachtung der Erreichung verfolgter Messezielsetzungen vorgenommen. Während sich in diesem Zusammenhang die Anwendung von Einladungsanalysen, der Einsatz von VeranstalterBesucherstatistiken sowie die Nachmessebefragung von Hochschul-MessestandBesuchern für die Erfolgskontrolle von Hochschul-Messebeteiligungen als nicht geeignet herausstellten, wurde die Erfolgskontrolle auf Basis von FKM-Test-Auswertungen sowie Analysen des Messeprojektvolumens vorgenommen. Neben dieser eher objektiven Erfassung von indirekten Messeerfolgen wurde ebenfalls eine subjektive Erfassung des indirekten Messebeteiligungserfolgs auf Grundlage von Befragungen des wissenschaftlichen und nicht-wissenschaftlichen Standpersonals sowie auf Basis eines Messebeteiligungs-Nutzen-Index ermittelt. Insgesamt zeigten die Befragungen des Standpersonals, die Berechnung des Messebeteiligungs-Nutzen-Index sowie die Analyse des Messeprojektvolumens eine erfolgreichere Hochschul-Messebeteiligung an Fachmessen als an Mehrbranchenmessen. Die Auswertung des FKM-Tests hingegen deutete ähnlich erfolgreiche Hochschul-Messebeteiligungen für beide Messetypen an. Die exponententypspezifischen Ergebnisse der in dieser Phase eingesetzten Methoden belegen für Projekt- und exponatorientierte Hochschul-Exponenten sowie Beeinflussungsorientierte Hochschul-Exponenten eine erfolgreiche Messebeteiligung i.S. ihrer primär verfolgten Messezielsetzungen. So konnte für beide Exponententypen neben relativ guten Messebeteiligungs-Nutzen-Indizes eine hohe Messebeteiligungszufriedenheit ermittelt werden, wobei die höchsten Werte von den Projektund exponatorientierten Hochschul-Exponenten erreicht wurden. Zudem konnte ein relativ hoher Anteil dieses Exponententyps abgeschlossene bzw. zukünttig voraussichtlich abzuschließende Projekte vorweisen und damit die Erreichung von Projektzielen dokumentieren.

Grundlage der direkten Erfolgskontrolle von Hochschul-Messebeteiligungen, bei der Kosten-Nutzen-Relationen im Mittelpunkt der Betrachtung stehen, ist die Analyse der Messebeteiligungskosten. Die Kosten von Hochschul-Messebeteiligungen ließen sich differenzieren nach Standmiete und Nebenkosten des Hochschul-Messestands, Kosten für Bau, Ausstattung und Gestaltung des HochschulMessestands, Exponatkosten, Kosten für wissenschaftliches und nicht-wissenschaftliches Hochschul-Messestand-Personal sowie Kommunikationskosten. In Abhängigkeit von der gewählten Messebeteiligungsform hat der jeweilige Hochschul-Exponent unterschiedlich hohe Kosten zu tragen. Während bspw. bei Wirtschafts-, Regionen- oder Landes-Gemeinschaftsständen der jeweilige hochschulexterne Partner i.d.R. den größten Anteil der Messebeteiligungskosten trägt und 
damit die finanzielle Belastung für die Hochschul-Exponenten eher gering ausfält, haben Hochschul-Exponenten bei einer Präsentation auf einem Hochschul-EinzelMessestand meist alle anfallenden Kosten selbst zu tragen. Die empirische Analyse der Kostenstruktur der betrachteten Hochschul-Messebeteiligungen zeigte, daß sowohl die Standmiete und Nebenkosten sowie die Kosten für Bau, Ausstattung und Gestaltung des Hochschul-Messestandes als auch die Exponatkosten die größten Kostenanteile an den Gesamtkosten einer Hochschul-Messebeteiligung einnehmen, dagegen die messebezogenen Personal- und Kommunikationskosten vergleichsweise gering ausfallen. Insgesamt konnten für Mehrbranchenmessen höhere Messebeteiligungskosten als für Fachmessen festgestellt werden. Diese Kostendifferenz basiert vor allem auf messetypspezifischen Standmieten sowie Standnebenkosten. Deshalb wurde im Rahmen der direkten Erfolgskontrolle der Fokus insbesondere auf die messetypspezifische Analyse gelegt. Die in diesem Zusammenhang durchgeführten Kennziffernanalysen und Messekontaktwertrechnungen zeigten keine generelle Überlegenheit von Mehrbranchen- bzw. Fachmessen auf.

Im Anschluß an die Analyse des indirekten und direkten Erfolgs wurden intra- und inter-instrumentelle Vergleiche mit ausgewählten Vergleichsobjekten vorgenommen. Bei den intra-instrumentellen Vergleichen können grundsätzlich unterschiedliche Messebeteiligungen als Vergleichsobjekte herangezogen werden. Einerseits können hochschuleigene Messebeteiligungen, d.h. Messebeteiligungen an identischen Messen aus zeitlich vorgelagerten Perioden (Zeitvergleiche) oder Beteiligungen an verschiedenen Messeveranstaltungen bzw. -typen (Veranstaltungsvergleiche) als Vergleichsobjekte fungieren. Andererseits können Messebeteiligungen anderer Institutionen als Vergleichsmaßstab dienen, wobei sowohl Messebeteiligungen anderer Hochschulen (Hochschulvergleiche) als auch Messebeteiligungen von Unternehmen (Unternehmensvergleiche) als Vergleichsobjekte angeführt werden können. Insbesondere bei Hochschul- bzw. Unternehmensvergleichen erwiesen sich jedoch die oftmals unzureichende Datenbasis sowie die i.d.R. sehr unterschiedlichen Hochschul- bzw. Unternehmensstrukturen sowie Umweltsituationen für die Vergleichbarkeit als problematisch. Darüber hinaus wurden unterschiedliche Definitionen von potentiellen Vergleichskriterien als implizite Probleme des intra-instrumentellen Vergleichs identifiziert.

Im Rahmen von inter-instrumentellen Vergleichen wurden andere Kommunikationsmaßnahmen des Hochschulmarketing-Mix als Vergleichsobjekte herangezogen. Dabei konnte festgestellt werden, daß ein ausschließlich auf Basis von Kon- 
taktkosten vorgenommener Vergleich nicht ausreichend ist, sondern die Einbeziehung der jeweiligen Kontaktqualitäten eine unabdingbare Voraussetzung darstellt. Ein unter Berücksichtigung dieser Aspekte durchgeführter inter-instrumenteller Vergleich deutete auf eine Überlegenheit von Messebeteiligungen gegenüber alternativen Kommunikationsinstrumenten des Hochschulmarketing (z.B. Einsatz von klassischen Medien in Kombination mit Akquisitionsgesprächen, Beteiligungen an Symposien, Kongressen, Workshops, Seminaren und Diskussionsforen) hin, da durch den Einsatz derartiger Kommunikationsinstrumente die hochschulspezifischen Messezielsetzungen und -zielgruppen nicht in dem Grad erreicht werden können wie bei der Durchführung von Messebeteiligungen. Somit stellen derartige Kommunikationsinstrumente keine adäquaten Alternativen zum Messeengagement der Hochschulen dar.

Auf Basis der aufgezeigten Untersuchungsergebnisse konnte eine Reihe von Implikationen für Hochschul-Exponenten abgeleitet werden. Dabei wurden auf der Strategieebene die Auswahl des Messetyps und alternative Formen von Messebeteiligungen im Hochschulbereich diskutiert, während auf der operativen Ebene Messeaktivitäten in der Vor-Messe-, Messe- und Nach-Messe-Phase betrachtet wurden.

Auf der strategischen Entscheidungsebene konnten Empfehlungen zur Auswahl des Messetyps bei Hochschul-Messebeteiligungen sowohl hinsichtlich hochschulspezifischer Messeziele als auch hochschulspezifischer Messezielgruppen abgeleitet werden. Während für Projekt- und exponatorientierte HochschulExponenten die Beschickung von thematisch fokussierten Messen - wie Fachund Verbundmessen - zur Realisierung ihrer primär verfolgten Messebeteiligungsziele empfehlenswert erscheint, sollten Beeinflussungsorientierte HochschulExponenten und Pflichtrepräsentanten eher allgemeinere Messen - wie Universalund Branchen-/Mehrbranchenmessen - beschicken. Hinsichtlich der Erreichung von Messebesuchertypen konnte festgestellt werden, daß Intensive HochschulMessestand-Nutzer eher auf thematisch fokussierten und Informationsorientierte Hochschul-Messestand-Nutzer vorwiegend auf thematisch breiter angelegten Messen, Hochschul-Messestand-Bummler hingegen auf allen Messetypen ähnlich häufig vertreten sind. Die Präsenz hochschulspezifischer Messezielgruppen auf einzelnen Messen ist ebenfalls messetypabhängig. So ist davon auszugehen, daß auf hochschulspezifischen Messen - wie Wissenschafts- und Bildungsmessen Wirtschaftsvertreter unterproportional, dagegen Wissenschaftsvertreter und Studierende überproportional vertreten sind. Hingegen ist auf klassischen Messen - 
wie Fach-, Verbund-, Branchen- bzw. Mehrbranchen- und Universalmessen - oftmals eine genau entgegengesetzte Verteilung festzustellen.

Im Hinblick auf die Auswahl der Hochschul-Messebeteiligungsform konnten ebenfalls exponententypspezifische Empfehlungen abgeleitet werden. Von den sich insgesamt bietenden fünf Messebeteiligungsformen (Hochschul-Einzel-Messestand, Wissenschafts-, Wirtschafts-, Regionen- oder Landes-Gemeinschaftsstand) sind für Projekt- und exponatorientierte Hochschul-Exponenten grundsätzlich alle Messebeteiligungsformen zur Erreichung ihrer primär verfolgten Messebeteiligungsziele und damit zur Messebeschickung geeignet. Dabei bietet sich jedoch für diesen Exponententyp insbesondere die gemeinsame Präsentation mit Wirtschaftsvertretern an, da hierdurch das eigene Kooperationspotential dokumentiert und damit die Erreichung verfolgter Projektziele forciert werden kann. Dem Beeinflussungsorientierten Hochschul-Exponenten bieten sich mit dem Hochschul-Einzel-Messestand und dem Wissenschafts-Gemeinschaftsstand insgesamt zwei uneingeschränkt geeignete Messebeteiligungsformen. Demgegenüber erscheint für Pflichtrepräsentanten prinzipiell die Integrationsform des Landes-Gemeinschaftsstands zur Präsentation auf Messeveranstaltungen geeignet.

Auf der operativen Entscheidungsebene ließen sich zur Erreichung angestrebter Messebeteiligungsziele phasenspezifische Maßnahmen ableiten. In der VorMesse-Phase sind neben den vorbereitenden Arbeiten hinsichtlich der Auswahl des Exponates bzw. der auszustellenden Forschungsergebnisse, deren Präsentation sowie der Standgestaltung vor allem Maßnahmen zur Bekanntmachung der eigenen Messebeteiligung bei den potentiellen Zielgruppen sowie die Auswahl und Qualifizierung des Standpersonals von besonderer Relevanz. Während der Messe-Phase sollte insbesondere die Kontaktaufnahme zu den hoch priorisierten Messezielgruppen durch geeignete Maßnahmen forciert werden. So legt die Akquisition von Messebesuchern die Basis zur Intensivierung des Dialogs mit ausgewählten, hochschulspezifischen Zielgruppen, der wiederum die Voraussetzung zur Erreichung der mit der Messebeteiligung verfolgten hochschulspezifischen Zielsetzungen, wie Projektziele, darstellt. In der Nach-Messe-Phase sind vor allem Maßnahmen zur Aufbereitung des gesamten Messegeschehens erforderlich. In diesem Kontext nehmen für Hochschul-Exponenten Kommunikationsmaßnahmen einen besonderen Stellenwert ein, da durch diese die Erreichung von insgesamt hoch priorisierten Beeinflussungszielen besonders gefördert werden kann. Mit den jeweiligen, in den einzelnen Messe-Phasen eingesetzten Aktivitäten wird die Erreichung bestimmter hochschulspezifischer Messezieldimensionen 
unterstützt. Damit ergeben sich auch für hochschulspezifische Exponententypen entsprechende Implikationen bzgl. des verstärkten Einsatzes von Aktivitäten, die vor allem die Realisierung ihrer hoch priorisierten Messeziele beeinflussen können. Letztlich wird jedoch erst das Zusammenspiel der Maßnahmen innerhalb der einzelnen Phasen sowie die Integration aller Vor-Messe-, Messe- und NachMesse-Phase-Aktivitäten zu einer adäquaten Durchführung von Hochschul-Messebeteiligungen führen und damit den Erfolg einer jeweiligen Messebeteiligung bestimmen.

Neben dem gezielten und abgestimmten Einsatz von Messebeteiligungsmaßnahmen ist der Erfolg einer Messebeteiligung insbesondere auch vom integrierten Einsatz des Messeengagements im Rahmen des Hochschulmarketing abhängig. Der in diesem Zusammenhang auftretende Koordinationsbedarf bezieht sich sowohl auf die Koordination des Marketing mit den anderen hochschulspezifischen Funktionsbereichen als auch auf die Abstimmung aller Aktivitäten innerhalb des Hochschulmarketing i.S.d. Koordination der einzelnen Marketing-Mix-Instrumente. Messebeteiligungen sind dabei sowohl im Planungs- und Koordinationsprozeß als auch im Kontrollprozeß des Hochschulmarketing zu berücksichtigen und entsprechend zu integrieren.

Abschließend wurden Implikationen für die Finanzmittelgeber von HochschulMessebeteiligungen abgeleitet, die oftmals den Hochschul-Exponenten durch entsprechende finanzielle Unterstützungen Messebeschickungen erst ermöglichen. Die von den jeweiligen finanzmittelgebenden öffentlichen oder kommerziellen Institutionen zu unterstützenden Messebeteiligungen sind danach auszuwählen, inwieweit das von den Hochschul-Exponenten geplante Messeengagement zum Erfolg führt, nicht zuletzt auch deshalb, weil dieser letztlich den Erreichungsgrad der von den Finanzmittelgebern angestrebten Zielsetzungen determiniert. 


\section{Ansatzpunkte für weiterführende Forschungsarbeiten}

Angesichts der einleitend aufgezeigten Umweltveränderungen und der damit einhergehenden zunehmenden Bedeutung von Messebeteiligungen für Hochschulen wurde mit der vorliegenden Untersuchung angestrebt, den Erfolg von HochschulMessebeteiligungen zu analysieren, wobei die Betrachtung hochschulspezifischer Messeziele und -zielgruppen die Grundlage dieser Erfolgskontrolle bildete. Daran anknüpfend lassen sich verschiedene Ansatzpunkte für weiterführende Forschungsarbeiten identifizieren:

- Die durchgeführte Untersuchung zeichnet sich durch die Befragung von Hochschul-Exponenten und damit durch eine auf die Hochschulebene bzw. auf Ebenen innerhalb der Hochschulen fokussierte Betrachtungsperspektive aus. Dies erscheint vor dem Hintergrund der mit dieser Arbeit verfolgten Zielsetzungen gerechtfertigt. Darüber hinaus wäre es wünschenswert, die mit HochschulMessebeteiligungen verfolgten Zielsetzungen von Bund, Ländern und Kommunen den Messebeteiligungszielen von Hochschul-Exponenten in einer empirischen Untersuchung gegenüberzustellen.

- In der vorliegenden Arbeit wurde eine umfangreiche Erfolgskontrolle von Hochschul-Messebeteiligungen durchgeführt. Dazu wurden die hochschulspezifischen Messezielsetzungen als Grundlage der Erfolgskontrolle in vielen Facetten abgebildet. Für künftige Forschungsvorhaben wäre es interessant, die untersuchten Variablen auch zeitablaufbezogen zu erfassen. Derartige Längsschnittanalysen liefern wertvolle Informationen für die Ausgestaltung zukünftiger Hochschul-Messebeteiligungen.

- Auf Grundlage der gewonnenen Erkenntnisse wurden u.a. Implikationen für Hochschul-Exponenten abgeleitet, die sich sowohl auf strategische als auch operative Entscheidungstatbestände des Messeengagements beziehen. In diesem Zusammenhang wäre es wertvoll, die abgeleiteten Ausgestaltungsformen von Hochschul-Messebeteiligungen einer empirischen Überprüfung zu unterziehen.

- Die Ergebnisse dieser Arbeit sollen der Ausgestaltung von Hochschul-Messebeteiligungen allgemein sowie in bezug auf gemeinsame Messebeschickungen von Hochschul-Exponenten mit wissenschaftlichen, wirtschaftlichen oder öffentlichen Institutionen neue Impulse geben. Im Zusammenhang mit der Präsentation von Hochschul-Exponenten auf Gemeinschaftsständen ist es 
erstrebenswert, die komplexen Kooperationsbeziehungen zwischen Messestandausrichter und Hochschul-Exponenten zu analysieren sowie die sich im Rahmen derartiger Messebeteiligungen den Hochschul-Exponenten bietenden Ausgestaltungsmöglichkeiten zu diskutieren.

- Die durchgeführte Analyse weist einen bundeslandbezogenen Fokus der befragten Hochschul-Exponenten auf, welcher angesichts des verfolgten Untersuchungsziels gerechtfertigt erscheint. Dennoch ist es wünschenswert, die Ergebnisse der vorliegenden Arbeit durch ergänzende, bundesweit angelegte Untersuchungen zu überprüfen. Darüber hinaus sollte eine Betrachtung von Messebeteiligungen ausländischer Hochschulen vorgenommen werden, da diese neue Impulse für Messebeteiligungen deutscher Hochschul-Exponenten liefern kann.

Die Ausführungen zeigen den Bedarf an weiterführender Forschung zur Beantwortung vielfältiger Fragestellungen, die sowohl gegenwärtig als auch zukünftig für das Messeengagement von Hochschulen von besonderer Relevanz sind. Eine fundierte Beantwortung dieser Fragen erscheint um so bedeutender, als vor dem Hintergrund der aufgezeigten hochschulspezifischen Rahmenbedingungen ein steigender Stellenwert von Hochschul-Messebeteiligungen bei gleichzeitiger Betonung von Effizienzkriterien zu erwarten ist. Somit ist die Marketingwissenschaft im besonderen, aber auch die Betriebswirtschaftslehre im allgemeinen dazu aufgefordert, die Effektivität und Effizienz von Hochschul-Messebeteiligungen durch eine permanente Forschung kontinuierlich zu erhöhen. 


\section{Verzeichnis des Anhangs}

\section{Anhang I: Expertenworkshop zu Messebeteiligungen von}

Hochschulen

\section{Anhang II: Zusammensetzung der Hochschul-Exponenten- und} Hochschul-Messestand-Besucher-Stichproben.

Tab. A 1: Hochschul-Exponenten-Stichprobe differenziert nach Hochschulform

Tab. A 2: Hochschul-Exponenten-Stichprobe differenziert nach den acht am häufigsten vertretenen Fachbereichen

Tab. A 3: Hochschul-Exponenten-Stichprobe differenziert nach Beschäftigtenanzahl ihres Instituts/Lehrstuhls/Fachbereichs

Tab. A 4: Hochschul-Exponenten-Stichprobe differenziert nach Funktion des Befragten

Tab. A 5: Hochschul-Messestand-Besucher-Stichprobe differenziert nach hochschulspezifischen Messezielgruppen

Tab. A 6: Wissenschaftsvertreter und Studierende der Hochschul-Messestand-Besucher-Stichprobe differenziert nach Hochschulform

Tab. A 7: Wissenschaftsvertreter und Studierende der HochschulMessestand-Besucher-Stichprobe differenziert nach den Top 10 Fachbereichen

Tab. A 8: Wissenschaftsvertreter und Studierende der Hochschul-Messestand-Besucher-Stichprobe differenziert nach Funktionen

Tab. A 9: Wirtschaftsvertreter der Hochschul-Messestand-BesucherStichprobe differenziert nach Wirtschaftszweigen

Tab. A 10: Wirtschaftsvertreter der Hochschul-Messestand-BesucherStichprobe differenziert nach Anzahl der Beschäftigten im Unternehmen

Tab. A 11: Hochschul-Messestand-Besucher-Stichprobe des Inlands differenziert nach bundeslandbezogener Herkunft

Tab. A 12: Hochschul-Messestand-Besucher-Stichprobe differenziert nach Länderherkunft.

Tab. A 13: Hochschul-Messestand-Besucher-Stichprobe differenziert nach Alter und Geschlecht.... 
Anhang III: Fragebögen der empirischen Untersuchung.

Fragebogen 1: Teil 1 der Exponentenbefragung I in der Vor-Messe-Phase.

Fragebogen 2: Teil 2 der Exponentenbefragung I in der Vor-Messe-Phase.

Fragebogen 3: Teil 1 der Exponentenbefragung II in der Nach-Messe-Phase

Fragebogen 4: Teil 2 der Exponentenbefragung II in der Nach-Messe-Phase

Fragebogen 5: Befragung der Hochschul-Messestand-Besucher in der Messe-Phase. 


\section{Anhang I: Expertenworkshop zu Messebeteiligungen von Hochschulen}
Themen: - „Evaluierungen“ von Hochschul-Messebeteiligungen
- „Auslandsmessen“ und ihre Bedeutung für deutsche Hochschulen
- „Hochschulgemeinschaftsstände als Orte und Instrumente für Events"

Ausrichter: Unikontakt - Kontaktstelle Universität/Wirtschaft Ruhr-Universität Bochum

Zeit: $\quad$ 12./13. Juli 1999

Ort: Kiel

Teilnehmerliste:

Herr Baier

Herr Behnke

Herr Dr. Budach

Herr Ern

Frau Danne

Herr Gerster
Messebeirat der NRW-Hochschulen, Fachhochschule Dortmund

Dezernat Forschungstransfer und Haushalt, Bauhaus Universität, Weimar

Unikontakt,

Ruhr Universität Bochum

T.I.N.A. Brandenburg GmbH, Haus der Wirtschaft, Potsdam

Institut für Marketing,

Westfälische Wilhelms-Universität Münster

Hochschul-Gemeinschaft Messebeschickung RheinlandPfalz, IMG Innovations-Management GmbH, Mainz

Herr Dr. Hergenhahn

Abt. Technologietransfer - Messewesen, Johann-Gutenberg-Universität, Mainz

Herr Jacobs

Technologie-Transfer-Zentrale, Schleswig-Holstein

Herr Kauert O.-v.-Guericke-Universität, Magdeburg 
Herr Kern

Frau Löbkens

Herr Meier

Herr Milnikel

Herr Pambor

Frau Pohle

Herr Riebesell

Herr Dr. Schinke

Herr Dr. Schöller

Herr Dr. Steentoft

Frau Stephan

Herr Dr. Stracke

Herr Dr. Thelen

Herr Dr. Wejwoda
Hochschulreferat 4 - Messebeteiligungen, Technische Universität München

TuHH-Technologie GmbH, Techn. Universität Hamburg-Harburg

Wissenstransfer,

Technische Universität Berlin

Projektmanagement Bereich Halle 18, Deutsche Messe AG, Hannover

Wirtschaftsförderung Schleswig-Holstein $\mathrm{GmbH}$, Kiel

Büro für Forschungstransfer, Friedrich-Schiller-Universität Jena

Messe Bremen GmbH

Hochschulrektorenkonferenz, Bonn

Wissenschaftstransfer, Universität Frankfurt

Transferstelle, Christian-Albrecht-Universität, Kiel

Nieders. Ministerium für Wissenschaft und Kultur, Hannover

Abt. Forschungskontakte, Universität Tübingen

DAAD,

Bonn

TUD Transfer,

Technische Universität Dresden 


\section{Anhang II: Zusammensetzung der Hochschul-Exponenten- und Hochschul-Messestand-Besucher-Stichproben}

\begin{tabular}{|l|c|c|c|}
\hline Hochschulform & gesamt & $\begin{array}{c}\text { Mehrbranchen- } \\
\text { messe }\end{array}$ & Fachmesse \\
\hline Universität & $30 \%(13)$ & $23 \% \quad(7)$ & $43 \%(6)$ \\
\hline Fachhochschule & $46 \%(20)$ & $53 \%(16)$ & $29 \%(4)$ \\
\hline Gesamthochschule & $18 \%(8)$ & $13 \%(4)$ & $29 \%(4)$ \\
\hline Private Hochschule & $0 \%(0)$ & $0 \%(0)$ & $0 \%(0)$ \\
\hline Sonstige & $9 \%(3)$ & $10 \%(3)$ & $0 \%(0)$ \\
\hline
\end{tabular}

Tab. A 1: Hochschul-Exponenten-Stichprobe differenziert nach Hochschulform

\begin{tabular}{|l|rr|rr|rr|}
\hline Fachbereich & \multicolumn{2}{|c|}{ gesamt } & \multicolumn{2}{|c|}{$\begin{array}{c}\text { Mehrbrachen- } \\
\text { messe }\end{array}$} & \multicolumn{2}{|c|}{ Fachmesse } \\
\hline $\begin{array}{l}\text { Maschinenbau bzw. } \\
\text { Maschinenbauwesen }\end{array}$ & $18 \%$ & $(8)$ & $22 \%$ & $(7)$ & $7 \%$ & $(1)$ \\
\hline Chemie & $14 \%$ & $(6)$ & $10 \%$ & $(3)$ & $21 \%$ & $(3)$ \\
\hline Metallurgie/Werkstofftechnik & $14 \%$ & $(6)$ & $17 \%$ & $(5)$ & $7 \%$ & $(1)$ \\
\hline Elektrotechnik & $11 \%$ & $(5)$ & $13 \%$ & $(4)$ & $0 \%$ & $(0)$ \\
\hline Ingenieurwesen & $7 \%$ & $(3)$ & $10 \%$ & $(3)$ & $0 \%$ & $(0)$ \\
\hline Physik & $5 \%$ & $(2)$ & $7 \%$ & $(2)$ & $0 \%$ & $(0)$ \\
\hline Biologie & $5 \%$ & $(2)$ & $0 \%$ & $(0)$ & $14 \%$ & $(2)$ \\
\hline Geowissenschaften & $5 \%$ & $(2)$ & $0 \%$ & $(0)$ & $14 \%$ & $(2)$ \\
\hline
\end{tabular}

Tab. A 2: Hochschul-Exponenten-Stichprobe differenziert nach den acht am häufigsten vertretenen Fachbereichen 


\begin{tabular}{|c|c|c|c|}
\hline $\begin{array}{l}\text { Anzahl der } \\
\text { Beschäftigten }\end{array}$ & gesamt & $\begin{array}{l}\text { Mehrbrachen- } \\
\text { messe }\end{array}$ & Fachmesse \\
\hline 1-4 Beschäftigte & $30 \% \quad(13)$ & $30 \% \quad(9)$ & $29 \% \quad(4)$ \\
\hline 5-8 Beschäftigte & $7 \% \quad$ (3) & $10 \% \quad(3)$ & $0 \% \quad(0)$ \\
\hline 9-12 Beschäftigte & $9 \% \quad(4)$ & $3 \% \quad(1)$ & $21 \% \quad(3)$ \\
\hline 13-16 Beschäftigte & $11 \%$ & $10 \% \quad(3)$ & $14 \% \quad(2)$ \\
\hline über 17 Beschäftigte & $43 \% \quad(19)$ & $47 \%(14)$ & $36 \% \quad(5)$ \\
\hline
\end{tabular}

Tab. A 3: Hochschul-Exponenten-Stichprobe differenziert nach Beschäftigtenanzahl ihres Instituts/Lehrstuhls/Fachbereichs

\begin{tabular}{|c|c|c|c|}
\hline $\begin{array}{l}\text { Funktion der befragten } \\
\text { Hochschul-Exponenten }\end{array}$ & gesamt & $\begin{array}{l}\text { Mehrbrachen- } \\
\text { messe }\end{array}$ & Fachmesse \\
\hline $\begin{array}{l}\text { Professor/Direktor/ } \\
\text { Institutsleiter }\end{array}$ & $32 \% \quad(14)$ & $37 \% \quad(11)$ & $21 \% \quad(3)$ \\
\hline Akademischer Rat & $14 \% \quad(6)$ & $10 \% \quad(3)$ & $21 \% \quad(3)$ \\
\hline Wiss. Assistent & $1 \% \quad(1)$ & $3 \% \quad(1)$ & $0 \% \quad(0)$ \\
\hline Wiss. Mitarbeiter & $39 \% \quad(17)$ & $33 \% \quad(10)$ & $50 \% \quad(7)$ \\
\hline Wiss. Hilfskraft & $0 \% \quad(0)$ & $0 \% \quad(0)$ & $0 \% \quad(0)$ \\
\hline $\begin{array}{l}\text { Sonstige (v.a. Koordinator, } \\
\text { Arbeitsgruppenleiter) }\end{array}$ & $14 \% \quad(6)$ & $17 \%$ & $7 \% \quad(1)$ \\
\hline
\end{tabular}

Tab. A 4: Hochschul-Exponenten-Stichprobe differenziert nach Funktion des Befragten 


\begin{tabular}{|l|cc|cc|cc|}
\hline $\begin{array}{l}\text { Hochschulspezifische } \\
\text { Messezielgruppen }\end{array}$ & gesamt & $\begin{array}{l}\text { Mehrbrachen- } \\
\text { messe }\end{array}$ & \multicolumn{2}{|c|}{ Fachmesse } \\
\hline Wissenschaft & $12 \% \quad(142)$ & $12 \%$ & $(82)$ & $11 \%$ & $(60)$ \\
\hline Studierende & $20 \% \quad(243)$ & $25 \% \quad(172)$ & $13 \%$ & $(71)$ \\
\hline Wirtschaft & $62 \%$ & $(748)$ & $56 \%$ & $(381)$ & $70 \%$ & $(367)$ \\
\hline Öffentlichkeit & $5 \%$ & $(63)$ & $6 \%$ & $(41)$ & $4 \%$ & $(22)$ \\
\hline Staat & $1 \%$ & $(14)$ & $1 \%$ & $(6)$ & $2 \%$ & $(8)$ \\
\hline
\end{tabular}

Tab. A 5: Hochschul-Messestand-Besucher-Stichprobe differenziert nach hochschulspezifischen Messezielgruppen

\begin{tabular}{|c|c|c|c|}
\hline Hochschulform & gesamt & $\begin{array}{l}\text { Mehrbrachen- } \\
\text { messe }\end{array}$ & Fachmesse \\
\hline Universität & $43 \% \quad(175)$ & $47 \% \quad(125)$ & $36 \% \quad(50)$ \\
\hline Fachhochschule & $39 \% \quad(156)$ & $38 \% \quad(100)$ & $41 \% \quad(56)$ \\
\hline Gesamthochschule & $6 \% \quad(22)$ & $4 \% \quad(11)$ & $8 \% \quad(11)$ \\
\hline Private Hochschule & $1 \%$ & $1 \%$ & $0 \% \quad(0)$ \\
\hline Sonstige & $12 \% \quad(48)$ & $10 \% \quad(27)$ & $15 \% \quad(21)$ \\
\hline
\end{tabular}

Tab. A 6: Wissenschaftsvertreter und Studierende der Hochschul-MessestandBesucher-Stichprobe differenziert nach Hochschulform 


\begin{tabular}{|c|c|c|c|}
\hline Fachbereich & gesamt & $\begin{array}{c}\text { Mehrbranchen- } \\
\text { messe }\end{array}$ & Fachmesse \\
\hline Maschinenbau/-wesen & $27 \%(111)$ & $27 \% \quad(97)$ & $10 \% \quad(14)$ \\
\hline Elektrotechnik & $14 \% \quad(58)$ & $20 \% \quad(55)$ & $2 \% \quad(3)$ \\
\hline Ingenieurwesen & $11 \% \quad(44)$ & $9 \% \quad(24)$ & $15 \% \quad(20)$ \\
\hline Physik & $7 \% \quad(28)$ & $9 \% \quad(25)$ & $2 \% \quad(3)$ \\
\hline Chemie & $6 \% \quad(25)$ & $2 \% \quad(4)$ & $15 \% \quad(21)$ \\
\hline Ver-/Entsorgungstechnik & $5 \%(19)$ & $1 \% \quad(3)$ & $12 \%(16)$ \\
\hline Wirtschaftswissenschaften & $4 \% \quad(17)$ & $6 \% \quad(15)$ & $1 \% \quad(2)$ \\
\hline Umwelttechnik & $3 \% \quad(13)$ & $0 \% \quad(0)$ & $9 \% \quad(13)$ \\
\hline Geowissenschaften & $3 \% \quad(12)$ & $1 \% \quad(2)$ & $7 \% \quad(10)$ \\
\hline Physikalische Technik & $2 \% \quad(8)$ & $1 \% \quad(3)$ & $4 \% \quad(5)$ \\
\hline
\end{tabular}

Tab. A 7: Wissenschaftsvertreter und Studierende der Hochschul-MessestandBesucher-Stichprobe differenziert nach den Top 10 Fachbereichen

\begin{tabular}{|c|c|c|c|}
\hline Funktion & gesamt & $\begin{array}{l}\text { Mehrbranchen- } \\
\text { messe }\end{array}$ & Fachmesse \\
\hline $\begin{array}{l}\text { Professor/Direktor/ } \\
\text { Institutsleiter }\end{array}$ & $11 \% \quad(20)$ & $12 \% \quad(13)$ & $10 \%$ \\
\hline Akademischer Rat & $3 \% \quad(6)$ & $5 \% \quad(5)$ & $1 \% \quad(1)$ \\
\hline Wiss. Assistent & $9 \% \quad(17)$ & $10 \% \quad(11)$ & $8 \% \quad(6)$ \\
\hline Wiss. Mitarbeiter & $46 \% \quad(85)$ & $42 \% \quad(45)$ & $54 \% \quad(40)$ \\
\hline Wiss. Hilfskraft & $18 \% \quad(32)$ & $20 \% \quad(22)$ & $14 \% \quad(10)$ \\
\hline Sonstige Funktion & $13 \% \quad(23)$ & $12 \% \quad(13)$ & $14 \% \quad(10)$ \\
\hline
\end{tabular}

Tab. A 8: Wissenschaftsvertreter und Studierende der Hochschul-MessestandBesucher-Stichprobe differenziert nach Funktionen 


\begin{tabular}{|l|c|c|c|c|c|c|}
\hline \multirow{2}{*}{ Wirtschaftszweig } & $\begin{array}{c}\text { Hochschul- } \\
\text { Messestände } \\
\text { (gesamt) }\end{array}$ & $\begin{array}{c}\text { Mehrbranchenmesse } \\
\text { Messestände }\end{array}$ & $\begin{array}{c}|c| \\
\text { Fesamte } \\
\text { Messe }\end{array}$ & $\begin{array}{c}\text { Hochschul- } \\
\text { Messestände }\end{array}$ & $\begin{array}{c}\text { gesamte } \\
\text { Messe }\end{array}$ \\
\hline Energiewirtschaft & $11 \%(89)$ & $15 \%(57)$ & $11 \%$ & $8 \%(32)$ & $4 \%$ \\
\hline Entsorgungstechnik & $6 \%(48)$ & $1 \%(3)$ & k.A. & $12 \%(45)$ & $11 \%$ \\
\hline Maschinenbau & $16 \%(126)$ & $27 \%(106)$ & k.A. & $5 \%(20)$ & $8 \%$ \\
\hline Elektrotechnik & $3 \%(26)$ & $6 \%(23)$ & k.A. & $1 \%$ & $(3)$ & k.A. \\
\hline andere Industrie & $25 \%(196)$ & $28 \%(109)$ & $47 \%$ & $22 \%(87)$ & $18 \%$ \\
\hline Handwerk & $1 \%(9)$ & $2 \%(8)$ & k.A. & $0 \%$ & $(1)$ & $1 \%$ \\
\hline Handel & $2 \%(19)$ & $3 \%(12)$ & $9 \%$ & $2 \%$ & $(7)$ & $8 \%$ \\
\hline Dienstleistung & $14 \%(106)$ & $8 \%(30)$ & $13 \%$ & $20 \%(76)$ & $20 \%$ \\
\hline Andere & $21 \%(164)$ & $11 \%(45)$ & $20 \%$ & $32 \%(123)$ & $30 \%$ \\
\hline
\end{tabular}

Tab. A 9: Wirtschaftsvertreter der Hochschul-Messestand-Besucher-Stichprobe differenziert nach Wirtschaftszweigen

\begin{tabular}{|c|c|c|c|c|c|}
\hline \multirow{2}{*}{$\begin{array}{l}\text { Anzahl der Beschäftigten im } \\
\text { Unternehmen }\end{array}$} & \multirow{2}{*}{$\begin{array}{l}\text { Hochschul- } \\
\text { Messestände } \\
\text { (gesamt) }\end{array}$} & \multicolumn{2}{|c|}{ Mehrbranchenmesse } & \multicolumn{2}{|c|}{ Fachmesse } \\
\hline & & $\begin{array}{l}\text { Hochschul- } \\
\text { Messestände }\end{array}$ & $\begin{array}{l}\text { gesamte } \\
\text { Messe }\end{array}$ & $\begin{array}{l}\text { Hochschul- } \\
\text { Messestände }\end{array}$ & $\begin{array}{l}\text { gesamte } \\
\text { Messe }\end{array}$ \\
\hline weniger als 10 Beschäftigte & $18 \% \quad(143)$ & $16 \%(62)$ & $15 \%$ & $21 \%(81)$ & \multirow{2}{*}{$41 \%$} \\
\hline $10-49$ Beschäftigte & $18 \% \quad(144)$ & $19 \%(74)$ & $18 \%$ & $18 \%(70)$ & \\
\hline 50-99 Beschäftigte & $9 \% \quad(67)$ & $10 \%(41)$ & $10 \%$ & $7 \%(26)$ & $8 \%$ \\
\hline 100 - 199 Beschäftigte & $9 \% \quad(67)$ & $10 \%(38)$ & $9 \%$ & $8 \%(29)$ & \multirow{2}{*}{$20 \%$} \\
\hline 200 - 499 Beschäftigte & $12 \% \quad(94)$ & $10 \%(41)$ & $12 \%$ & $14 \%(53)$ & \\
\hline $500-999$ Beschäftigte & $9 \% \quad(73)$ & $9 \%(34)$ & $8 \%$ & $10 \%(39)$ & $7 \%$ \\
\hline $1.000-9.999$ Beschäftigte & $15 \% \quad(120)$ & $16 \% \quad(64)$ & $16 \%$ & $15 \% \quad(56)$ & \multirow{2}{*}{$24 \%$} \\
\hline 10.000 u. mehr Beschäftigte & $10 \% \quad(75)$ & $12 \% \quad(46)$ & $7 \%$ & $8 \% \quad(29)$ & \\
\hline
\end{tabular}

Tab. A 10: Wirtschaftsvertreter der Hochschul-Messestand-Besucher-Stichprobe differenziert nach Anzahl der Beschäftigten im Unternehmen 


\begin{tabular}{|c|c|c|c|c|c|}
\hline \multirow{2}{*}{$\begin{array}{l}\text { Regionale Herkunft } \\
\text { - Bundeslandbezogen - }\end{array}$} & \multirow{2}{*}{$\begin{array}{l}\text { Hochschul- } \\
\text { Messestände } \\
\text { (gesamt) }\end{array}$} & \multicolumn{2}{|c|}{ Mehrbranchenmesse } & \multicolumn{2}{|c|}{ Fachmesse } \\
\hline & & $\begin{array}{l}\text { Hochschul- } \\
\text { Messestände }\end{array}$ & $\begin{array}{l}\text { gesamte } \\
\text { Messe }\end{array}$ & $\begin{array}{l}\text { Hochschul- } \\
\text { Messestände }\end{array}$ & $\begin{array}{l}\text { gesamte } \\
\text { Messe }\end{array}$ \\
\hline Nordrhein-Westfalen & $56 \% \quad(626)$ & $43 \%(269)$ & $22 \%$ & $73 \%(357)$ & $54 \%$ \\
\hline Niedersachsen & $14 \% \quad(151)$ & $21 \%(133)$ & $29 \%$ & $4 \% \quad(18)$ & $9 \%$ \\
\hline Baden-Württemberg & $8 \% \quad(85)$ & $10 \% \quad(61)$ & $10 \%$ & $5 \% \quad(24)$ & $8 \%$ \\
\hline Bayern & $7 \% \quad(75)$ & $9 \% \quad(55)$ & $9 \%$ & $4 \% \quad(20)$ & $6 \%$ \\
\hline Hessen & $3 \% \quad(34)$ & $3 \% \quad(17)$ & $7 \%$ & $4 \% \quad(17)$ & $7 \%$ \\
\hline Rheinland-Pfalz & $3 \% \quad(30)$ & $3 \% \quad(20)$ & $3 \%$ & $2 \% \quad(10)$ & $5 \%$ \\
\hline Berlin & $3 \% \quad(29)$ & $3 \% \quad(18)$ & $3 \%$ & $2 \% \quad(11)$ & $2 \%$ \\
\hline Sonstige & $8 \% \quad(84)$ & $8 \% \quad(52)$ & $17 \%$ & $7 \% \quad(32)$ & $9 \%$ \\
\hline
\end{tabular}

Tab. A 11: Hochschul-Messestand-Besucher-Stichprobe des Inlands differenziert nach bundeslandbezogener Herkunft

\begin{tabular}{|c|c|c|c|c|c|}
\hline \multirow{2}{*}{ Länderherkunft } & \multirow{2}{*}{$\begin{array}{l}\text { Hochschul- } \\
\text { Messestände } \\
\text { (gesamt) }\end{array}$} & \multicolumn{2}{|c|}{ Mehrbranchenmesse } & \multicolumn{2}{|c|}{ Fachmesse } \\
\hline & & $\begin{array}{c}\text { Hochschul- } \\
\text { Messestände }\end{array}$ & $\begin{array}{c}\text { gesamte } \\
\text { Messe }\end{array}$ & $\begin{array}{l}\text { Hochschul- } \\
\text { Messestände }\end{array}$ & $\begin{array}{c}\text { gesamte } \\
\text { Messe }\end{array}$ \\
\hline EG & $58 \% \quad(55)$ & $57 \%(27)$ & $58 \%$ & $56 \%(23)$ & $58 \%$ \\
\hline Übriges Europa & $26 \% \quad(25)$ & $26 \%(12)$ & $19 \%$ & $27 \%(11)$ & $20 \%$ \\
\hline Afrika & $2 \% \quad(2)$ & $2 \% \quad(1)$ & $2 \%$ & $2 \% \quad(1)$ & k.A. \\
\hline Nordamerika & $4 \% \quad(4)$ & $6 \% \quad(3)$ & $4 \%$ & $2 \% \quad(1)$ & k.A. \\
\hline Süd-IMittelamerika & $4 \% \quad(4)$ & $4 \% \quad(2)$ & $4 \%$ & $5 \% \quad(2)$ & k.A. \\
\hline Asien & $4 \% \quad(4)$ & $2 \% \quad(1)$ & $11 \%$ & $2 \% \quad(1)$ & k.A. \\
\hline Australien & $1 \% \quad(1)$ & $2 \% \quad(1)$ & $2 \%$ & $0 \% \quad(0)$ & k.A. \\
\hline
\end{tabular}

Tab. A 12: Hochschul-Messestand-Besucher-Stichprobe differenziert nach Länderherkunft 


\begin{tabular}{|c|c|c|c|c|c|c|}
\hline \multirow{2}{*}{\multicolumn{2}{|c|}{$\begin{array}{l}\text { Alter und Geschlecht der } \\
\text { Hochschul-Messestand- } \\
\text { Besucher-Stichprobe }\end{array}$}} & \multirow{3}{*}{$\begin{array}{c}\begin{array}{c}\text { Hochschul- } \\
\text { Messestand } \\
\text { (gesamt) }\end{array} \\
16 \% \text { (191) }\end{array}$} & \multicolumn{2}{|c|}{ Mehrbranchenmesse } & \multicolumn{2}{|c|}{ Fachmesse } \\
\hline & & & \multirow{2}{*}{$\begin{array}{l}\begin{array}{l}\text { Hochschul- } \\
\text { Messestand }\end{array} \\
23 \%(154)\end{array}$} & \multirow{2}{*}{$\begin{array}{r}\text { gesamt } \\
9 \%\end{array}$} & $\begin{array}{l}\text { Hochschul- } \\
\text { Messestand }\end{array}$ & \multirow{2}{*}{$\frac{\text { gesamt }}{\text { k.A. }}$} \\
\hline \multirow{5}{*}{ Alter } & bis 25 Jahre & & & & $7 \% \quad(37)$ & \\
\hline & 26 bis 30 Jahre & $22 \% \quad(269)$ & $22 \%(152)$ & $18 \%$ & $22 \%(117)$ & k.A. \\
\hline & 31 bis 40 Jahre & $32 \% \quad(382)$ & $28 \%(189)$ & $34 \%$ & $36 \%(193)$ & k.A. \\
\hline & 41 bis 50 Jahre & $16 \% \quad(189)$ & $12 \% \quad(82)$ & $23 \%$ & $20 \%(107)$ & k.A. \\
\hline & über 50 Jahre & $15 \% \quad(179)$ & $15 \%(102)$ & $15 \%$ & $15 \% \quad(77)$ & k.A. \\
\hline \multirow{2}{*}{$\begin{array}{l}\text { Ge- } \\
\text { schlecht }\end{array}$} & männlich & $90 \%(1094)$ & $94 \%(637)$ & $94 \%$ & $86 \%(457)$ & k.A. \\
\hline & weiblich & $10 \%(116)$ & $6 \% \quad(43)$ & $7 \%$ & $14 \% \quad(73)$ & k.A. \\
\hline
\end{tabular}

Tab. A 13: Hochschul-Messestand-Besucher-Stichprobe differenziert nach Alter und Geschlecht 
Anhang III: Fragebögen der empirischen Untersuchung

Fragebogen 1: Teil 1 der Exponentenbefragung I in der Vor-Messe-Phase

\section{Institut für Marketing}

Prof. Dr. Dr. h.c. H. Meffert

\section{Hochschul- \\ Exponentenbefragung}

Fragebogen 1

Exponentenbefragung I

in der

Vor-Messe-Phase

- schriftliche Befragung - 
1. Sie werden sich mit einem Exponat auf einer Mehrbranchen-/Fachmesse beteiligen. Welches Exponat werden Sie auf der Messe ausstellen, und wie werden Sie dieses darstellen?

Exponatbeschreibung

Exponatpräsentation

$$
\begin{array}{ll}
\hline & \text { Product-Show (Produkt in Aktion) } \\
\square & \text { Dummy } \\
\square & \text { Computersimulation } \\
\square & \text { Videoaufzeichnung } \\
\square & \text { Sonstiges: }
\end{array}
$$

2. Wer wird auf der Mehrbranchen-/Fachmesse von Ihrem Institut/Lehrstuhl/ Fachbereich vertreten sein bzw. wer wird auf der Messe Ansprechpartner für das präsentierte Exponat sein?
Ansprechpartner auf der Messe
D Direktor / Professor
a Akademischer Rat
a Wissenschaftlicher Assistent
口 Wissenschaftlicher Mitarbeiter
a Wissenschaftliche Hilfskraft
口 Studentische Hilfskraft/Student
ㄱ Sonstige Funktion:

3. Seit wie vielen Jahren beteiligt sich Ihr Institut/Lehrstuhl/Fachbereich als Aussteller an Messen allgemein? Seit wie vielen Jahren ist Ihr Institut/Lehrstuhl/Fachbereich auf der Mehrbranchen-/Fachmesse vertreten?

Messebeteiligung des Instituts/Lehrstuhls/Fachbereichs

$\begin{array}{ll}\text { all- } & \begin{array}{l}\text { Mehrbran- } \\ \text { gemein } \\ \text { chen-/Fach- } \\ \text { messe }\end{array}\end{array}$

bisher noch nie seit 1 bis 3 Jahren seit 4 bis 7 Jahren seit 8 bis 10 Jahren seit über 10 Jahren 
4. An wie vielen Messen hat sich Ihr Institut/Lehrstuhl/Fachbereich als Ausstel-
ler im Durchschnitt der letzten Jahre beteiligt, und wie wird die Messebe-
teiligung in den kommenden drei Jahren sein? Wie viele von diesen Messe-
beteiligungen wurden unterstützt bzw. werden mit Unterstützungen geplant?

Messebeteiligungen im Jahresdurchschnitt

alle Messebeteiligungen

unterstützte Messebeteiligungen

\section{bis zukünftig}

heute (in den kommenden 3 Jahren)

5. Die Zielgruppen, die durch eine Messebeteiligung erreicht werden können, sind i.d.R. vielfältig. Bitte geben Sie für jede der folgenden Zielgruppen an, welche Bedeutung sie für Ihre Messebeteiligung auf der ausstellenden Mehrbranchen-/Fachmesse hat.

\begin{tabular}{|c|c|c|c|c|c|}
\hline & & & leut & & \\
\hline & $\begin{array}{l}\text { seh } \\
\text { geri }\end{array}$ & & & & $\begin{array}{l}\text { sehr } \\
\text { hoch }\end{array}$ \\
\hline & 1 & 2 & 3 & 4 & 5 \\
\hline Studenten & $\square$ & $\square$ & $\square$ & $\square$ & 口 \\
\hline Fachkollegen & $\square$ & 口 & $\square$ & $\square$ & $\square$ \\
\hline Praxisvertreter & $\square$ & 口 & $\square$ & 口 & $\square$ \\
\hline Forschungsförderungsinstitutionen & $\square$ & $\square$ & $\square$ & $\square$ & $\square$ \\
\hline Sonstige Zielgruppen: & & & & & \\
\hline 1. & 口 & $\square$ & 口 & $\square$ & $\square$ \\
\hline 2. & $\square$ & 口 & $\square$ & $\square$ & $\square$ \\
\hline
\end{tabular}

Welche Kontakte wurden in Anzahl der der Vergangenheit realisiert? Kontakte in der Messe-Phase
Anzahl der Kontakte, die in der Nach-Messe-Phase intensiviert wurden

- Studenten

口 Fachkollegen

口 Praxisvertreter

ㄱ Forschungsförderungsinst.

口 Sonstige: 
7. Hat Ihr Institut/Lehrstuhl/Fachbereich bereits Untersuchungen durchgeführt, um festzustellen, ob die angestrebten Ziele bzgl. der Messebeteiligung erreicht wurden?

口 Ja, immer.

口 Ja, aber nur bei ausgewählten Veranstaltungen.

- Nein, noch nie.

Wenn nein, bitte weiter mit Frage 9.

8. Wie werden der Erfolg bzw. die Wirkungen der Messebeteiligung erfaßt, und wie wurden diese Ergebnisse für die weitere Arbeit aufgegriffen? Bitte geben Sie an, wie zutreffend die folgenden Aussagen sind.

Die Aussage

Die Ergebnisse der Messebeteiligung

- werden dem Institutsdirektor/Lehrstuhlinhaber/Fachbereichsleiter präsentiert

- werden den betroffenen Mitarbeitern präsentiert

- sind schwer erfaßbar

- werden zur Beurteilung des weiteren

Engagements bzgl. der bewerteten

Messeveranstaltung herangezogen

- werden zur Überprüfung des gesamten Messe-Engagements des Instituts/Lehrstuhls/Fachbereichs genutzt

- werden zur Überarbeitung der Standkonzeption bzgl. weiterer Messeeinsätze genutzt

- werden selten als Basis zukünftiger Messe-Entscheidungen eingesetzt

- Sonstiges:

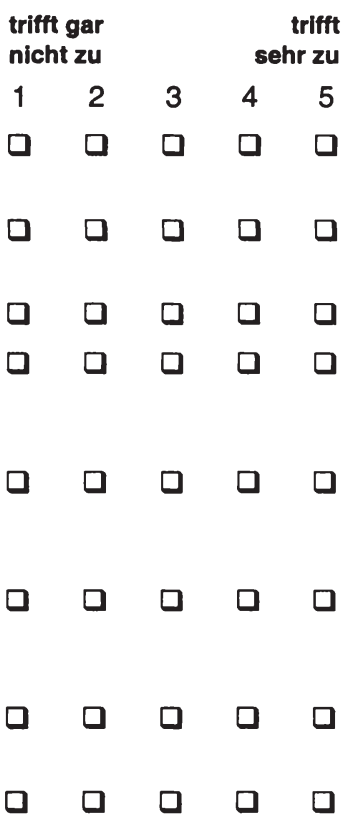


9. Im folgenden haben wir neben der Messebeteiligung weitere Instrumente zusammengestellt, die Sie möglicherweise zur Präsentation bzw. Bekanntmachung lhrer Exponate nutzen. Wie wichtig sind für Sie die einzelnen Instrumente?

\begin{tabular}{|c|c|c|c|c|c|}
\hline & \multicolumn{5}{|c|}{ Bedeutung } \\
\hline & \multicolumn{2}{|c|}{$\begin{array}{l}\text { sehr } \\
\text { gering }\end{array}$} & \multirow[b]{2}{*}{3} & \multicolumn{2}{|r|}{$\begin{array}{l}\text { sehr } \\
\text { hoch }\end{array}$} \\
\hline & 1 & 2 & & 4 & 5 \\
\hline Messen & 口 & 口 & 口 & 口 & $\square$ \\
\hline Symposien & 口 & $\square$ & 口 & 口 & $\square$ \\
\hline Kongresse & 口 & $\square$ & 口 & 口 & $\square$ \\
\hline Diskussionsforen & $\square$ & $\square$ & $\square$ & $\square$ & $\square$ \\
\hline Fachvorträge & $\square$ & $\square$ & $\square$ & 口 & $\square$ \\
\hline Öffentlichkeitsarbeit & 口 & $\square$ & 口 & 口 & $\square$ \\
\hline $\begin{array}{l}\text { Neuere Kommunikationsinstrumente } \\
\text { (z.B. Internet) }\end{array}$ & $\square$ & $\square$ & $\square$ & $\square$ & $\square$ \\
\hline \multirow[t]{2}{*}{ Sonstige: } & $\square$ & 口 & $\square$ & 口 & $\square$ \\
\hline & $\square$ & 口 & $\square$ & $\square$ & $\square$ \\
\hline \multicolumn{6}{|c|}{$\begin{array}{l}\text { 10. Neben der Möglichkeit, jedes Instrument einzeln und unabhängig von den } \\
\text { anderen Instrumenten zu planen und einzusetzen, kann der Instrumente- } \\
\text { einsatz auch i.S.e. integrierten Konzeptes aufeinander abgestimmt durch- } \\
\text { geführt werden. Wir möchten gerne wissen, in welcher Weise Sie Ihre Mes- } \\
\text { sebeteiligungen planen. }\end{array}$} \\
\hline
\end{tabular}

ja nein

Messebeteiligungen werden völlig unabhängig von den anderen Aktivitäten geplant und durchgeführt.

Es besteht ein übergeordnetes Konzept, in das auch die Messebeteiligungen integriert sind.

Messebeteiligungen werden insbesondere in ihrem zeitlichen Einsatz mit den anderen Instrumenten koordiniert.

Wir achten vor allem auf die formale Übereinstimmung von Messeauftritt und den anderen Aktivitäten.

Sonstiges: 
11. Abschließend wollen wir Ihnen noch einige Fragen stellen, die sich auf $\mathrm{Ihr}$ Institut/ Ihren Lehrstuhl/Fachbereich beziehen. Sie sind für die Auswertung des Fragebogens von wesentlicher Bedeutung.

a) Welcher Hochschulform gehört Ihr Institut/Lehrstuhl/Fachbereich an?

口 Universität

a Fachhochschule

․ Gesamthochschule

a Private Hochschule

口 Sonstige:

b) Welchem Fachbereich gehört Ihr Institut/Lehrstuhl/Fachbereich an?

口 Medizin

a Physik

a Philologie

a Chemie

․ Sportwissenschaften

a Biologie

口 Textil

口 Mathematik

․ Musik

․ Geowissenschaften

․ Theologie

a Wirtschaftswissenschaften

a Fotoingenieurwesen

a Rechtswissenschaften

ㅁ Elektrotechnik

a Sozialwissenschaften

ㅁ Metallurgie und Werkstofftechnik

a Geschichte

ㅁ Maschinenbau/Maschinenwesen

a Philosophie

- Physikalische Technik

ㅁ Erziehungswissenschaften

a Ver- und Entsorgungstechnik

․ Sonstige:

c) Wie hoch war 1997 die Anzahl der Beschäftigten an Ihrem Institut/Lehrstuhl/Fachbereich?

口 1- 4 Beschäftigte

口 5- 8 Beschäftigte

a 9-12 Beschäftigte

13-16 Beschäftigte

ㅁ über 17 Beschäftigte

d) Welche Funktion bekleiden Sie an Ihrem Institut/Lehrstuhl/Fachbereich?

口 Direktor

口 Akademischer Rat

口 Wissenschaftlicher Assistent

口. Wissenschaftlicher Mitarbeiter

a Wissenschaftliche Hilfskraft

口 Studentische Hilfskraft/Student

ㅁ Sonstige Funktion: 
Fragebogen 2: Teil 2 der Exponentenbefragung I in der Vor-Messe-Phase

\section{Westfälische Wilhelms-Universität \\ Institut für Marketing}

Prof. Dr. Dr. h.c. H. Meffert

\section{Hochschul- \\ Exponentenbefragung}

Fragebogen 2

Exponentenbefragung I

in der

Vor-Messe-Phase

- persönliches Interview - 
1. Für den Hochschul-Messestand wurde die Thematik "Energie und Umwelt“" gewählt. Wie beurteilen Sie dieses Thema, und welche Affinität besitzen Sie zu dieser Thematik?

Bitte geben Sie an, wie zutreffend die folgenden Aussagen sind.

Die Aussage

Das Thema "Energie und Umwelt" ist

- hoch aktuell

- zu eng eingegrenzt

- für die Praxis von besonderem Interesse

- für den Hochschulbereich insgesamt von besonderem Interesse

- für mein Institut/Lehrstuhl von besonderem Interesse

- für meinen Forschungsschwerpunkt mit hohen Affinitäten verbunden

- Sonstiges:

2. Wie beurteilen Sie das Umfeld der Messebeteiligten, in dem Sie Ihr Exponat präsentieren werden, zum einen in bezug auf die gesamte Mehrbranchen-/Fachmesse (a) und zum anderen bezogen auf den Hochschul-Messestand (b)? Bitte geben Sie an, wie zutreffend die folgenden Aussagen sind.

a) Mehrbrachen-1

Fachmesse

$\begin{array}{ccccr}\begin{array}{c}\text { trifft gar } \\ \text { nicht zu }\end{array} & \begin{array}{r}\text { trifft } \\ \text { sehr zu }\end{array} \\ 1 & 2 & 3 & 4 & 5\end{array}$

Das Umfeld paßt zur Thematik „Energie und Umwelt“.

Im Umfeld stellen zu wenig

Praxisvertreter aus.

Im Umfeld stellen zu wenig

Wissenschaftsvertreter aus.

Ich fühle mich in dem Umfeld wohl.

Keine Aussage möglich, da ich das Umfeld nicht kenne.

Sonstiges: trifft gar

nicht zu

$\begin{array}{lllll}1 & 2 & 3 & 4 & 5\end{array}$

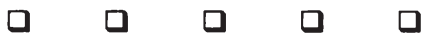

व 口

व $\square$

ㅁ

口

口

口

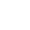

trifft

sehr zu

(2)

口

口

ㅁ

ㅁ

口

$\square$ 
3. Welche Bedeutung hat eine Unterstützung (finanziell, organisatorisch) für die Messebeteiligungen Ihres Instituts/Lehrstuhls/Fachbereichs heute?

\begin{tabular}{|c|c|c|c|c|c|}
\hline & \multicolumn{5}{|c|}{ Bedeutung } \\
\hline & \multicolumn{2}{|c|}{$\begin{array}{l}\text { sehr } \\
\text { gering }\end{array}$} & & \multicolumn{2}{|c|}{$\begin{array}{l}\text { sehr } \\
\text { hoch }\end{array}$} \\
\hline & 1 & 2 & 3 & 4 & 5 \\
\hline Kostenfreie Nutzung des Messestandes & 口 & 口 & 口 & 口 & 口 \\
\hline Aufbau des Messestandes & $\square$ & $\square$ & $\square$ & $\square$ & $\square$ \\
\hline Platzzuweisung auf dem Messestand & $\square$ & $\square$ & $\square$ & $\square$ & $\square$ \\
\hline Gestaltung des Standes & 口 & 口 & $\square$ & 口 & 口 \\
\hline \multicolumn{6}{|l|}{ Sonstiges: } \\
\hline & $\square$ & $\square$ & $\square$ & $\square$ & 口 \\
\hline & $\square$ & $\square$ & $\square$ & $\square$ & $\square$ \\
\hline
\end{tabular}

4. Welche der folgenden Leistungen sind nach Ihrer Meinung für die Unterstützung Ihres Messeauftritts zusätzlich wünschenswert?

zusätzlich finanzielle Unterstützung bzgl. Reisekosten

a umfangreicheres Catering auf dem Messestand

ㅁ größerer Freiraum bei der Gestaltung des Messestandes

ㅁ Zuweisung einer größeren Messestandfläche

a sonstiges:

5. Wie zufrieden sind Sie mit der Unterstützung Ihres Messeauftritts? Gibt es diesbezüglich bestimmte Gründe? Wenn ja, welche?

Ich bin/wir sind mit der

Unterstützung gar nicht

zufrieden sehr

zufrieden

zufrieden

Begründung: 
6. Wie wird sich die Höhe des Budgets für die Messebeteiligung für Ihr Institut/ Lehrstuhl/Fachbereich generell in Zukunft entwickeln? Wie hoch kalkulieren Sie Ihr Budget, welches Sie für die Beteiligung an der ausstellenden Mehrbranchen-/Fachmesse mindestens zur Verfügung haben müssen?

Das Messebudget wird zukünftig

\begin{abstract}
deutllch
geringer
\end{abstract}

unver-

ändert

$\square$

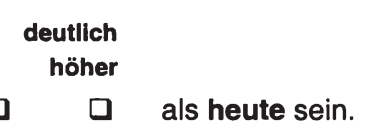

Das Mindestbudget für die Beteiligung an der ausstellenden Mehrbranchen-I Fachmesse beträgt.

$\begin{array}{ll}\square & \text { weniger als DM } 2.000,-- \\ \square & \text { DM } 2.000,-- \text { bis DM } 5.000,-- \\ \square & \text { DM } 5.000,-- \text { bis DM } 10.000,-- \\ \square & \text { DM } 10.000,-- \text { bis DM } 15.000,-- \\ \square & \text { über DM } 15.000,--\end{array}$

7. Die Ziele einer Messebeteiligung sind i.d.R. vielfältig und meist werden mehrere Ziele verfolgt. Bitte geben Sie für jedes der folgenden Messeziele an, welche Wichtigkeit diese für Ihre Messebeteiligung auf der Mehrbranchen-I Fachmesse hat.

Verbesserung des Hochschulimages

Verbesserung des Instituts-/Lehrstuhl-/Fachbereichsimages

Erhöhung des Bekanntheitsgrades des Inst./Lehrst./FB

Erhöhung des Bekanntheitsgrades des Exponates

Darstellung der Leistungskompetenz

Sammlung von Informationen

Beziehungspflege bzw. -aufbau mit Repräsentanten verschiedener Institutionen (z.B. Staat, Verbände)

Beobachtung von anderen Instituten/Lehrstühlen/Fachber.

Erfahrungs- u. Informationsaustausch mit Fachkollegen

Erfahrungs- $u$. Informationsaustausch mit Praxis-Vertretern Überprüfung d. „Marktreife“ eigener Produkte u. Leistungen Kontaktaufnahme zu Projekt-Interessenten

Einwerbung von Projektmitteln

Sonstige Ziele:

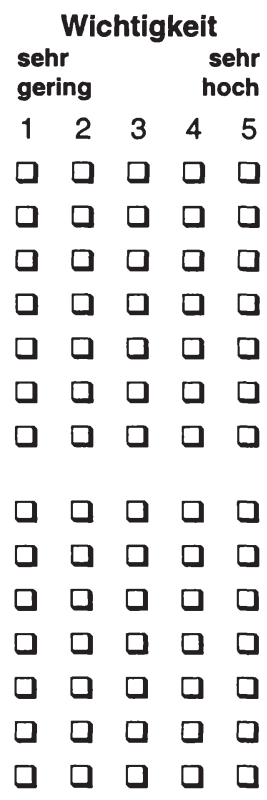


8. Die Erreichung der Messeziele ist im wesentlichen von den Aktivitäten des ausstellenden Instituts/Lehrstuhls/Fachbereichs abhängig. Dabei können die Aktivitäten der Vor-Messe-, der Messe- und der Nach-Messe-Phase zugeordnet werden. Welche der nachfolgenden phasenspezifischen Maßnahmen nutzen Sie für die Mehrbranchen-/Fachmesse, und wie wichtig sind Ihnen diese?

\section{Vor-Messe-Phase}

- Vor-Messe-Werbung durch Anzeigen in Fachzeitschriften

- Abfassung von Messevorberichten für die uni-interne und/oder regionale Presse

- Abfassung von Messevorberichten für die überregionale und/oder internationale Presse

- Verschickung pers. Einladungen (Direct Mail)

- Eintrag im Messekatalog

- Anzeige im Messekatalog

- Qualifizierung des Standpersonals

- Sonstiges:

\section{Messe-Phase}

- Werbeaktionen auf der Messe (z.B. Verteilung von Handzetteln, Luftballons)

- Außenwerbung auf dem Messegelände

- Nutzung des Messeinformationssystems für Besucher

- Präsenz des Institutsdirektors/ Lehrstuhlinhabers/Fachbereichsleiters auf der Messe

- Abhaltung von Pressekonferenzen

- Beteiligung bzw. Ausrichtung von Messebegleitveranstaltungen (Fachvorträge, Symposien, Podiumsdiskussionen)

- Sonstiges:

\section{Nach-Messe-Phase}

- Systematische Weiterverfolgung der Messekontakte

- Nach-Messe-Werbung mit den Messeergebnissen des Instituts/Lehrstuhls/Fachbereichs

- Abfassung von internen Messeberichten

- Abfassung von Messeberichten für die hochschul-interne und/oder regionale Presse

- Abfassung von Messeberichten für die überregionale und/oder internat. Presse

- Sonstiges:
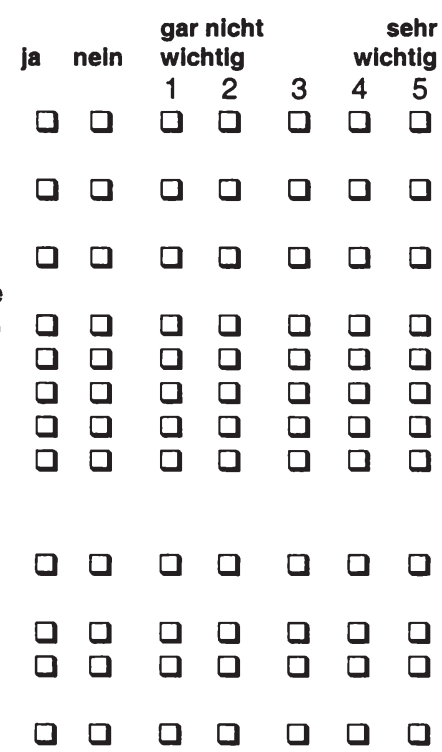

$\begin{array}{lllllll}\square & \square & \square & 0 & \square & 0 & 0\end{array}$
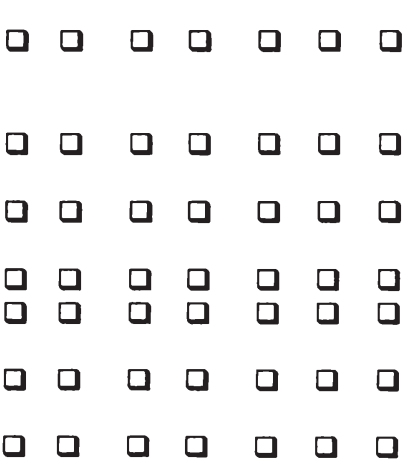
9. Welche und wie viele Kontakte planen Sie, auf der ausstellenden Mehrbranchen-/Fachmesse zu realisieren? Für den Fall, daß die angestrebte Kontaktzahl erreicht wird, wie viele dieser Kontakte werden Sie schätzungsweise in der Nach-Messe-Phase intensivieren können?

Welche und wie viele Kontakte planen Sie, auf der ausstellenden Mehrbranchen-/Fachmesse zu realisieren?

Anzahl der geplanten Kontakte in der MessePhase

a Studenten

口 Fachkollegen

a Praxisvertreter

- Forschungsförderungsinstitutionen

口 Sonstige:
Anzahl der Kontakte, die in der Nach-MessePhase vorauss. intensiviert werden können 
Fragebogen 3: Tell 1 der Exponentenbefragung II in der Nach-Messe-Phase

\section{Westfälische Wilhelms-Universität \\ Institut für Marketing}

Prof. Dr. Dr. h.c. H. Meffert

\section{Hochschul- \\ Exponentenbefragung}

Fragebogen 3

Exponentenbefragung II in der

Nach-Messe-Phase

- persönliches Interview - 
1. Zu welchen Zielgruppen hatten Sie in den letzten Messetagen wie viele Kontakte? Wie viele dieser Kontakte werden Sie schätzungsweise in der Nach-Messe-Phase intensivieren können?

Welche und wie viele Kontakte hatten Sie auf der Messe?

\begin{tabular}{ll}
\hline & Studenten \\
$\square$ & Fachkollegen \\
$\square$ & Praxisvertreter \\
$\square$ & Forschungsförde- \\
& rungsinstitutionen \\
$\square$ & Presse \\
$\square$ & Behörden \\
$\square$ & Privatpersonen \\
$\square$ & Sonstige: \\
\hline
\end{tabular}

Anzahl der Kontakte in der Messe-Phase

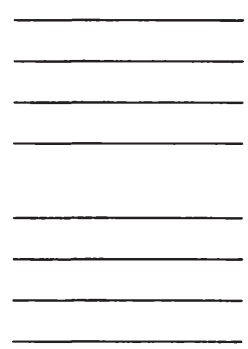

Anzahl der Kontakte, die in der Nach-Messe-Phase vorauss. intensiviert werden können

2. Welche und wie viele Gespräche haben Sie in den letzten sechs Messetagen mit den Messestandbesuchern geführt?

Ich habe Gespräche mit folgendem Charakter geführt:
Anzahl der geführten Gespräche mit Messestandbesuchern
Anzahl der Gesprächspartner, mit denen Sie vorauss. nach der Messe in Kontakt bleiben werden

口 Informationsgespräch(e) (z.B. Info.-/Erfahrungsaustausch)

Gespräch(e) der Beziehungspflege

口 Gespräch(e) über mögliche zukünftige Projektkooperationen

a konkrete Besprechung(en) zukünftiger Projekte

口 Sonstige:

3. Wie viele Personen wurden als Messestandpersonal in den letzten sechs Messetagen durchschnittlich eingesetzt?
口 1 Person
口 2 Personen
3 Personen
u über 3 Personen 
4. Welche und wie viele Informationsmaterialien haben Sie insgesamt in den Messetagen an die Messebesucher ausgegeben?

\section{Anzahl insgesamt}

口 Projekt-/Exponatbeschreibungen

․ Image-Broschüren des Instituts/Lehrstuhls/Fachbereichs

口 Image-Broschüre der Hochschule

口 Visitenkarten

口 Sonstige:

5. Wie viele Gesprächsprotokolle (Formulare) haben Sie in den Messetagen ausgefüllt?

Anzahl der ausgefüllten Protokolle:

6. Sind Sie mit der Messebeteiligung zufrieden? Bitte geben Sie kurz an, was Ihnen in bezug auf den Hochschul-Messestand besonders gut gefallen hat bzw. was Ihnen nicht so gut gefallen hat.

Ich bin/wir sind mit der

Messebeteiligung

Begründung:

\begin{tabular}{|l|l|}
\hline Was hat Ihnen bes. gut gefallen? & Was hat Ihnen nicht so gut gefallen? \\
\hline & \\
\hline & \\
\hline & \\
\hline & \\
\hline & \\
\hline & \\
\hline
\end{tabular}


Fragebogen 4: Teil 2 der Exponentenbefragung II in der Nach-Messe-Phase

\section{Westfälische Wilhelms-Universität \\ Institut für Marketing}

Prof. Dr. Dr. h.c. H. Meffert

\section{Hochschul- \\ Exponentenbefragung}

Fragebogen 4

Exponentenbefragung II in der

Nach-Messe-Phase

- telefonisches Interview - 
1. Mit welchen und mit wie vielen Messestand-Besuchern hatten Sie auch nach der Messe noch Kontakt?

Welche Art von Gesprächen wurden nach der Messe geführt?

\begin{tabular}{|c|c|c|c|c|c|c|c|}
\hline & \multirow[b]{2}{*}{\begin{tabular}{|l|} 
Anzahl \\
der \\
Kon- \\
takte
\end{tabular}} & \multicolumn{5}{|c|}{ Art der Kontakte/Gespräche } \\
\hline & & & \multicolumn{2}{|c|}{\begin{tabular}{|l|l|}
\multicolumn{2}{|c}{ Art der K } \\
Informa- & Gespr. \\
tionsge- & der \\
spräch(e) & Bezie- \\
(z.B. Info.- & hungs- \\
Erfahrungs- & pflege \\
\end{tabular}} & $\begin{array}{l}\text { Gespr. } \\
\text { über } \\
\text { mögliche } \\
\text { zukünf- } \\
\text { tige Pro- } \\
\text { jekte }\end{array}$ & $\begin{array}{l}\text { konkrete } \\
\text { Bespre- } \\
\text { chung } \\
\text { zu- } \\
\text { künftiger } \\
\text { Projekte } \\
\end{array}$ & $\begin{array}{l}\text { Son- } \\
\text { stige } \\
\text { (Wel- } \\
\text { che?) }\end{array}$ \\
\hline ㅁ & Studenten & & & & & & \\
\hline a & Fachkollegen & & & & & & \\
\hline 口 & Praxisvertreter & & & & & & \\
\hline$\square$ & $\begin{array}{l}\text { Forschungsför- } \\
\text { derungsinstitut. }\end{array}$ & & & & & & \\
\hline ㅁ & Presse & & & & & & \\
\hline 口 & Behörden & & & & & & \\
\hline 口 & Privatpersonen & & & & & & \\
\hline 口 & Sonstige: & & & & & & \\
\hline
\end{tabular}

2. Konnten Sie mit einigen Messestand-Besuchern bereits Projekte abschlieBen? (Wenn ja, wie viele und in welcher Höhe?)

Wenn nein, werden Sie voraussichtlich zukünftig Projekte abschließen können? (Wenn ja, wie viele und in welcher Höhe?)

\begin{tabular}{|c|l|l|l|}
\hline & \multicolumn{1}{|c|}{ Wie viele? } & Welche Höhe? \\
\hline$\square$ & $\begin{array}{l}\text { Ja, ich habe bereits Projekte } \\
\text { abgeschlossen. }\end{array}$ & \\
\hline$\square$ & $\begin{array}{l}\text { Nein, bisher noch nicht, aber ich werde } \\
\text { zukünftig voraus. Projekte } \\
\text { abschließen können. }\end{array}$ & \\
\hline$\square$ & $\begin{array}{l}\text { Nein, ich habe noch keine Projekte abgeschlossen und werde } \\
\text { voraussichtlich auch zukünftig keine Projekte abschließen können. }\end{array}$ \\
\hline
\end{tabular}


3. Inwieweit konnten Sie durch Ihre Messebeteiligung folgende Ziele realisieren?

\begin{tabular}{|c|c|c|c|c|}
\hline & \multicolumn{2}{|c|}{$\begin{array}{l}\text { reall- } \\
\text { siert }\end{array}$} & \multicolumn{2}{|c|}{$\begin{array}{r}\text { nicht } \\
\text { realisiert }\end{array}$} \\
\hline & 1 & 2 & 3 & 4 \\
\hline Verbesserung des Hochschulimages & a & 口 & 口 & $\square$ \\
\hline $\begin{array}{l}\text { Verbesserung des Instituts-/Lehrstuhl-/ } \\
\text { Fachbereichsimages }\end{array}$ & 口 & a & 口 & व \\
\hline $\begin{array}{l}\text { Erhöhung des Bekanntheitsgrades des } \\
\text { Instituts/Lehrstuhls/Fachbereichs }\end{array}$ & 口 & 口 & 口 & a \\
\hline Erhöhung des Bekanntheitsgrades des Exponates & ם & 口 & 口 & a \\
\hline Darstellung der Leistungskompetenz & 口 & 口 & 口 & 口 \\
\hline Sammlung von Informationen & 口 & 口 & 口 & $\square$ \\
\hline $\begin{array}{l}\text { Beziehungspflege bzw. -aufbau mit Repräsentanten ver- } \\
\text { schiedener Institutionen (z.B. Staat, Verbände) }\end{array}$ & 口 & 口 & 口 & $\square$ \\
\hline Beobachtung v. anderen Instituten/Lehrstühlen/Fachber. & a & 口 & 口 & 口 \\
\hline Erfahrungs- und Informationsaustausch mit Fachkollegen & $\square$ & $\square$ & $\square$ & 口 \\
\hline $\begin{array}{l}\text { Erfahrungs- und Informationsaustausch mit Praxis- } \\
\text { Vertretern }\end{array}$ & 口 & 口 & 口 & $\square$ \\
\hline $\begin{array}{l}\text { Überprüfung der "Marktreife" eigener Produkte und } \\
\text { Leistungen }\end{array}$ & 口 & a & 口 & $\square$ \\
\hline Anbahnung von Kooperationen mit der Wirtschaft & $\square$ & 口 & 口 & $\square$ \\
\hline Kontaktaufnahme zu Projekt-Interessenten & 口 & 口 & 口 & $\square$ \\
\hline Einwerbung von Projektmitteln & 口 & 口 & ם & $\square$ \\
\hline onstiges: & 口 & a & a & 口 \\
\hline
\end{tabular}

4. Welche der folgenden Maßnahmen haben Sie nach der Messe durchgeführt?

Systematische Weiterverfolgung der Messekontakte

ja nein

Nach-Messe-Werbung mit den Messeergebnissen

Abfassung von internen Messeberichten

Abfassung von Messeberichten für die hochschulint. u./od. regionale Presse

Abfassung von Messeberichten für die überregionale u./od. internat. Presse

Sonstiges: 
5. Wenn Sie noch einmal die Möglichkeit hätten, ein Exponat auf einer Messe auszustellen, würden Sie diese Möglichkeit wahrnehmen?

(Wenn ja, auf welchen Messen? Wenn nein, warum nicht?)

ㄱa, $\rightarrow$ Auf der bereits besuchten Messe?

․ Mehrbranchenmesse

a Fachmesse

Auch auf weiteren Messen?
口 Ja $\rightarrow$
a Allgemeinere Messen
Fachmessen
Nein $\rightarrow$ Warum nicht?

Nein $\rightarrow$ Warum nicht?

6. Sind Sie mit der Messebeteiligung insgesamt zufrieden? Bitte begründen Sie kurz Ihre Aussage.

Ich bin/wir sind mit der Messebeteiligung

$\begin{array}{lrrrr}\begin{array}{l}\text { sehr } \\ \text { zufrieden }\end{array} & \text { zufrieden } & & \begin{array}{r}\text { gar nicht } \\ \text { zufrieden }\end{array} \\ 1 & 2 & 3 & 4 & 5 \\ \square & \square & \square & \square & \square\end{array}$

Gründe für eher „NICHT-ZUFRIEDEN“ 
Fragebogen 5: Befragung der Hochschul-Messestand-Besucher in der MessePhase

\title{
Westfälische Wilhelms-Universität \\ Institut für Marketing
}

Prof. Dr. Dr. h.c. H. Meffert

\section{Hochschul-Messestand- Besucherbefragung}

\author{
Fragebogen \\ zur \\ Befragung der \\ Hochschul-Messestand-Besucher \\ in der \\ Messe-Phase
}

- persönliches Interview - 
1. Sie kommen gerade von einem Messestand. Was hat Ihnen auf diesem Messestand besonders gefallen?

2. Was bringen Sie mit dem gerade besuchten Messestand in Verbindung? Bitte wählen Sie im folgenden das aus, was Sie mit diesem Messestand verbinden, und geben Sie ggf. konkrete Beispiele an.

Mit dem gerade besuchten Messestand verbinde ich:

Konkrete Beispiele:

口 Institute/Lehrstühle/

Fachbereiche

- Hochschulen

Ministerien

口 Bundesländer

口 Städte/Gebiete

Exponate

口 Sonstiges:

3. Der von Ihnen gerade besuchte Hochschul-Messestand ist auf ausgewählten Messen vertreten. Wie oft haben Sie diesen Messestand bereits besucht?

Ich habe den Hochschul-Messestand bisher

noch nie besucht

ㅁ 1 bis 3 mal besucht

口 4 bis 6 mal besucht

口 über 6 mal besucht 
4. Wie finden Sie den Hochschul-Messestand insgesamt?

$\begin{array}{ccccccr}\text { sehr } & & & & \begin{array}{r}\text { gar nicht } \\ \text { gut }\end{array} \\ & 1 & 2 & 3 & 4 & 5\end{array}$

Den Hochschul-Messestand finde ich

Begründung:

5. Wie beurteilen Sie die Exponate, die auf dem Hochschul-Messestand ausgestellt werden? Bitte geben Sie an, wie zutreffend die folgenden Aussagen sind.

Die Aussage

Die Exponate auf dem HochschulMessestand sind:

- interessant

- innovativ

- wissenschaftlich anspruchsvoll

- für die Praxis von Bedeutung

- sonstiges:

․ Keine Aussage möglich

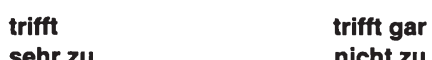

sehr zu nicht zu

6. Wie wichtig sind für Sie die folgenden Aspekte hinsichtlich des Besuches eines Hochschul-Messestandes?

Sammlung von Informationen

Beobachtung von Entwicklungen der

Wissenschaft

Erfahrungs- und Informationsaustausch mit Wissenschaftsvertretern

Beziehungspflege oder -aufbau mit Wissenschaftsvertretern

Kontaktaufnahme zu Projekt-Anbietern

Sonstige Ziele:

sehr

wichtig

1

1

ㅁ

$\square$

$\begin{array}{lllll}1 & 2 & 3 & 4 & 5\end{array}$

口 0 a

口 0

口

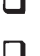

口 
7. Haben Sie auf dem Hochschul-Messestand Gespräche mit den Ausstellern geführt?

- Ja, ich habe Gespräche mit den Ausstellern geführt.

Die geführten Gespräche hatten folgenden Charakter:

Anzahl der
geführten
Gespräche auf
dem Hochschul-
Messestand

Anzahl der Aussteller, mit denen Sie vorauss. nach der Messe in Kontakt bleiben werden

口 Informationsgespräch(e) (z.B. Info.-/Erfahrungsaustausch) Gespräche Aussteller

口 Gespräch(e) der Beziehungspflege

口 Gespräch(e) über mögliche zukünftige Projektkooperationen

Gespräche
Gespräche
Gespräche
Gespräche
Gespräche

Aussteller
Aussteller
Aussteller
Aussteller
Aussteller

Nein, ich habe keine Gespräche mit den Ausstellern geführt.

8. Haben Sie neben diesem Hochschul-Messestand auch Hochschul-Messestände anderer Bundesländer besucht?

Ja, ich habe folgende Hochschul-Messestände besucht:

$\rightarrow 1$. Wie viele Gespräche mit Ausstellern haben Sie auf diesen Ständen geführt?

2. Was hat Ihnen an diesen Messeständen im Vergleich zum HochschulMessestand aus NRW besser oder schlechter gefallen?

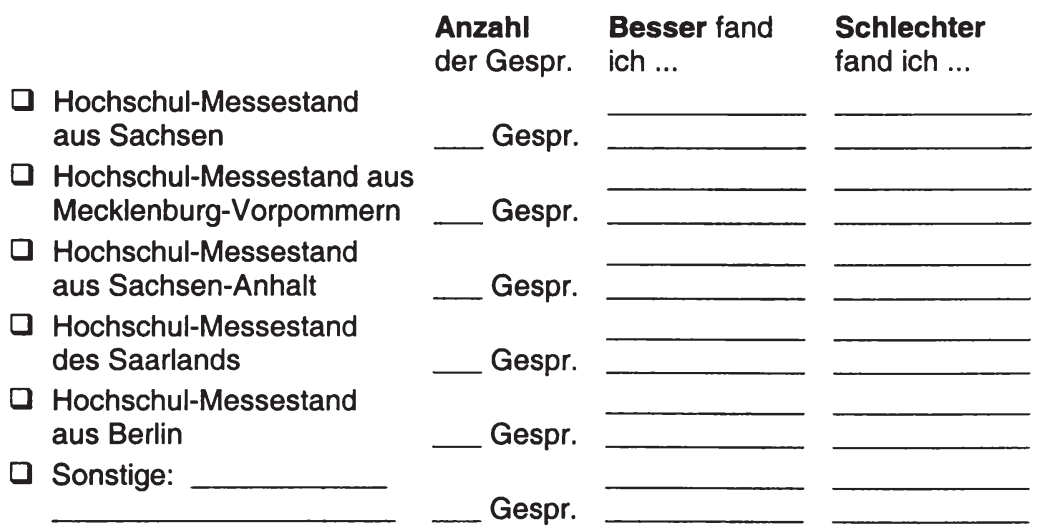

Nein, ich habe keine anderen Hochschul-Messestände besucht. 
9. Abschließend möchten wir Sie um einige statistische Angaben bitten. Sie sind für die Auswertung des Fragebogens von wesentlicher Bedeutung.

a) In welche der folgenden „Gruppen“ würden Sie sich einordnen?

- Student $\rightarrow$ weiter mit Frage b), c) u. i) $f$.

a Wissenschaftsvertreter $\rightarrow$ weiter mit Frage b), c), d) u. i) $f$.

a Praxisvertreter $\rightarrow$ weiter mit Frage e) ff.

a Forschungsförderungsinstitution $\rightarrow$ welche?

口 Sonstige:

b) Welcher Hochschulform gehören Sie an?

口 Universität

a Fachhochschule

a Gesamthochschule

口 Private Hochschule

口 Sonstige:

c) Welchem Fachbereich gehören Sie an?

$\begin{array}{llll}\square & \text { Medizin } & \square & \text { Wirtschaftswissenschaften } \\ \square & \text { Physik } & \square & \text { Rechtswissenschaften } \\ \square & \text { Ingenieurwesen } & \square & \text { Sozialwissenschaften } \\ \square & \text { Elektrotechnik } & \square & \text { Geschichte } \\ \square & \text { Maschinenbau/Maschinenwesen } & \square & \text { Philosophie } \\ \square & \text { Ver- und Entsorgungstechnik } & \square & \text { Erziehungswissenschaften } \\ \square & \text { Metallurgie und Werkstofftechnik } & \square & \text { Philologie } \\ \square & \text { Physikalische Technik } & \square & \text { Sportwissenschaften } \\ \square & \text { Chemie } & \square & \text { Textilwissenschaften } \\ \square & \text { Biologie } & \square & \text { Musikwissenschaften } \\ \square & \text { Mathematik } & \square & \text { Theologie } \\ \square & \text { Geowissenschaften } & \square & \text { Sonstige: }\end{array}$

d) Welche Funktion besitzen Sie an Ihrem Institut/Lehrstuhl/Fachbereich?

Direktor/Institutsleiter

ㅁ Akademischer Rat

․ Wissenschaftlicher Assistent

ㅁ Wissenschaftlicher Mitarbeiter

․ Wissenschaftliche Hilfskraft

a Sonstige Funktion: 
e) In welchem Wirtschaftszweig sind Sie tätig?

\begin{tabular}{llll}
$\square$ & Energiewirtschaft & $\square$ & Handwerk \\
$\square$ & Entsorgungstechnik & $\square$ & Handel \\
$\square$ & Maschinenbau & $\square$ & Dienstleistung \\
$\square$ & Bergbau & $\square$ & Behörde/öffentliche Einrichtung \\
$\square$ & Industrie & $\square$ & Andere \\
\hline
\end{tabular}

f) Wie würden Sie den Grad Ihrer Entscheidungskompetenz in Ihrem Unternehmen einschätzen?

a ausschlaggebende Entscheidungskompetenz

a mitentscheidende Entscheidungskompetenz

a beratende Entscheidungskompetenz

a keine Entscheidungskompetenz

g) Welche berufliche Position bekleiden Sie in Ihrem Unternehmen? selbständiger Unternehmer, Teilhaber, freier Beruf

- Geschäftsführer, Vorstandsmitglied, Behördenleiter o.ä.

口 Prokurist, Direktor, Abteilungsleiter, and. Angest. mit Leitungsfunktion o.ä.

․ Angestellter, Facharbeiter o.ä.

a Auszubildender, Praktikant o.ä.

a Sonstige:

h) Wie hoch ist die Anzahl der Beschäftigten in Ihrem Unternehmen?

$\begin{array}{llll}\square & \text { weniger als } 9 \text { Beschäftigte } & \square & 200-499 \text { Beschäftigte } \\ \square & 10-49 \text { Beschäftigte } & \square & 500-999 \text { Beschäftigte } \\ \square & 50-99 \text { Beschäftigte } & \square & 1.000-9.999 \text { Beschäftigte } \\ \square & 100-199 \text { Beschäftigte } & \square & 10.000 \text { und mehr Beschäftigte }\end{array}$

i) In welcher Region wohnen Sie?

\section{Inland}

․ Baden-Württemb.

a Bayern

a Berlin

․ Brandenburg

a Bremen

․ Hamburg

a Hessen

a Mecklenburg-Vorp.
․ Niedersachsen

口 Nordrhein-Westfal.

a Rheinland-Pfalz

- Saarland

a Sachsen

- Sachsen-Anhalt

口 Schleswig-Holstein

a Thüringen
Ausland

a EG

übriges Europa

a Afrika

- Nordamerika

口 Süd-/

Mittelamerika

a Asien

․ Australien

j) Dürtten wir Sie zum Schluß noch nach Ihrem Alter fragen?

Ich bin Jahre alt.

Geschlecht des Befragten:

u männlich

․ weiblich 


\section{Literaturverzeichnis}

Aaker, D. A., Building Strong Brands, New York u.a. 1996.

Achterholt, G., Corporate Identity: In zehn Arbeitsschritten die eigene Identität finden, Wiesbaden 1988.

Adam, D., Planung und Entscheidung: Modelle - Ziele - Methoden; mit Fallstudien und Lösungen, 4. Aufl., Wiesbaden 1996.

Alewell, K., Wissenschaftsmarketing, in: Tietz, B., Köhler, K., Zentes, J. (Hrsg.), Handwörterbuch des Marketing, Stuttgart 1995, Sp. 2776-2790.

Alewell, K., Marketing-Management für Universitäten: Umweltbezogene Führung von Universitäten, in: ZfO, 46. Jg., Heft 5, 1977, S. 263-274.

Amon, P., Messen + Ausstellungen: Messe-Ziele exakt formulieren und kontrollieren, in: Marketing Journal, 24. Jg., Heft 1, 1991, S. 56-60.

Amrhein, D., Die Universität als Dienstleistungsunternehmen: Innovative Organisationsstrukturen und Motivationskonzepte, Wiesbaden 1998.

Aries, L. A., Bereiten Sie Ihr Standpersonal intensiv auf das Messe-Verkaufsgespräch vor, in: Marketing Journal, 19. Jg., Heft 4, 1986, S. 365-368.

Ausstellungs- und Messe-Ausschuss der Deutschen Wirtschaft e.V. (AUMA) (Hrsg.), Messeplatz Deutschland 1999/2000 - Fachmessen, Termine, Fakten, Bergisch Gladbach 1998.

Ausstellungs- und Messe-Ausschuss der Deutschen Wirtschaft e.V. (AUMA) (Hrsg.), AUMA-Handbuch Messeplatz Deutschland 1999, Bergisch Gladbach 1998.

Ausstellungs- und Messe-Ausschuss der Deutschen Wirtschaft e.V. (AUMA) (Hrsg.), AUMA-Handbuch International 1999, Bergisch Gladbach 1998.

Ausstellungs- und Messe-Ausschuss der Deutschen Wirtschaft e.V. (AUMA) (Hrsg.), AUMA-Handbuch Regional 1999, Bergisch Gladbach 1998.

Ausstellungs- und Messe-Ausschuss der Deutschen Wirtschaft e.V. (AUMA) (Hrsg.), Die Messewirtschaft 1997/1998, Bergisch Gladbach 1998. 
Ausstellungs- und Messe-Ausschuss der Deutschen Wirtschaft e.V. (AUMA) (Hrsg.), AUMA-Leitsätze zur Typologie von Messen und Ausstellungen, Köln 1996.

Ausstellungs- und Messe-Ausschuss der Deutschen Wirtschaft e.V. (AUMA) (Hrsg.), Die Messekosten der deutschen Aussteller - Die Aufwendungen deutscher Unternehmen für Beteiligungen an Messen und Ausstellungen in Deutschland, Köln 1994.

Ausstellungs- und Messe-Ausschuss der Deutschen Wirtschaft e.V. (AUMA) (Hrsg.), Erfolgreiche Messebeteiligung - Made in Germany, Köln 1993.

Ausstellungs- und Messe-Ausschuss der Deutschen Wirtschaft e.V. (AUMA) (Hrsg.), Literatursammlung des AUMA, Köln o.J.

Axel Springer Verlag (Hrsg.), Verbraucher-Analyse 97/3, Hamburg 1997.

Bacher, J., Clusteranalyse - Anwendungsorientierte Einführung, München 1994.

Bachmeir, H. M., Marktforschung auf Messen, in: Meyer, P. W. (Hrsg.), Arbeitspapiere zur Schriftenreihe Schwerpunkt Marketing der Universität Augsburg, Bd. 23, Augsburg 1989.

Backes-Gellner, U., Zanders, E., Lehre und Forschung als Verbundproduktion, in: ZfB, 59. Jg., Heft 3, 1989, S. 271-290.

Backhaus, K., Messen als Institutionen der Informationspolitik, in: Strothmann, K.-H., Busche, M. (Hrsg.), Handbuch Messemarketing, Wiesbaden 1992, S. 83-97.

Backhaus, K., Erichson, B., Plinke, W., Weiber, R., Multivariate Analysemethoden: Eine anwendungsorientierte Einführung, 8. Aufl., Berlin u.a. 1996.

Bagozzi, R., Marketing as an Organized Behavioral System of Exchange, in: JoM, Vol. 38, No. 4, 1974, S. 77-81.

Bargehr, B., Marketing in der öffentlichen Verwaltung: Ansatzpunkte und Entwicklungsperspektiven, Stuttgart 1991.

Bauer, F., Datenanalyse mit SPSS, Berlin u.a. 1984. 
Becker, J., Marketing-Konzeption - Grundlagen des strategischen MarketingManagements, 6. Aufl., München 1998.

Behrens, K. C., Absatzwerbung, Wiesbaden 1963.

Behrens, T., Globalisierung der Hochschulhaushalte - Grundlagen, Ziele, Erscheinungsformen und Rahmenbedingungen, Neuwied, Kriftel, Berlin 1996.

Bender, I., Henning, W. (Hrsg.), Universitätsmanagement, Trier 1980.

Berekoven, L., Eckert, W., Ellenrieder, P., Marktforschung - Methodische Grundlagen und praktische Anwendung, 6. Aufl., Wiesbaden 1993.

Berghäuser, B., Messen + Ausstellungen: Entscheidungshilfen für die "richtige" Beteiligung, in: Marketing Journal, 16. Jg., Heft 2, 1983, S. 166-168.

Berghäuser, B., So lassen sich Messen messen, in: Marketing Journal, 10. Jg., Heft 9, 1976, S. 61-66.

Bergs, S., Optimalität bei Clusteranalysen, Experimente zur Bewertung numerischer Klassifikationsverfahren, Univ. Diss., Münster 1981.

Berndt, R., Hermanns, A. (Hrsg.), Handbuch Marketing-Kommunikation: Strategien - Instrumente - Perspektiven, Wiesbaden 1993.

Bertelsmann Stiftung (Hrsg.), Evolution im Hochschulbereich, Gütersloh 1990.

Birkigt, K., Stadler, M. M., Funck, H. J. (Hrsg.), Corporate Identity: Grundlagen, Funktionen, Fallbeispiele, 5. Aufl., Landsberg am Lech 1993.

Bleymüller, J., Multivariate Analysen für Wirtschaftswissenschaftler, Manuskript, Münster 1987.

Bleymüller, J., Gehlert, G., Gülicher, H., Statistik für Wirtschaftswissenschaftler, 10. Aufl., München 1996.

Block, H. J., Maßnahmen für eine Förderung des leistungssteigernden Wettbewerbs im deutschen Hochschulsystem, in: Helberger, C. (Hrsg.), Ökonomie der Hochschule I, Berlin 1989, S. 153-179.

Blohm, H., Messen und Ausstellungen als Mittel der Absatzförderung in der Schweizerischen Maschinenindustrie, Freiburg, Winterthur 1960. 
Böhler, H., Marktforschung, Stuttgart u.a. 1985.

Bolsenkötter, H., Ökonomie der Hochschule: Eine betriebswirtschaftliche Untersuchung (Bd. I), Baden-Baden 1976.

Bolsenkötter, H., Ökonomie der Hochschule: Eine betriebswirtschaftliche Untersuchung (Bd. II), Baden-Baden 1976.

Bolsenkötter, H., Die Hochschule als Betrieb, in: DUZ - Das Hochschulmagazin, 32. Jg., Heft 12, 1976, S. 650-653.

Bonoma, T. V., Get more out of your trade shows, in: HBR, Vol. 61, No. 1, 1983, S. $75-83$.

Brosius, G., SPSS/PC+ Advanced Statistics and Tables - Einführung und praktische Beispiele, Hamburg u.a. 1989.

Bruhn, M., Kommunikationspolitik, München 1997.

Budach, W., Einordnung der Messeteilnahme in das Hochschulmarketing, in: Budach, W. et al. (Hrsg.), Hochschulen und Messen: Praktische Hinweise für Messebeteiligungen durch Hochschulen, Bad Honnef 1992, S. 1-36.

Budach, W., Kayser, P., Krug, W., Meier, H.-G., Stracke, F. (Hrsg.), Hochschulen und Messen: Praktische Hinweise für Messebeteiligungen durch Hochschulen, Bad Honnef 1992.

Bundesministerium für Bildung und Forschung (Hrsg.), "Zahlenbarometer im Internet", in: Internet, http://192.76.176.139/deutsch/kap/s39.htm, Stand: 03.12.1999.

Bundesministerium für Bildung, Wissenschaft, Forschung und Technologie (Hrsg.), Grund- und Strukturdaten 1995/96, Referat Öffentlichkeitsarbeit, Bonn 1995.

Bundesministerium für Bildung und Wissenschaft (Hrsg.), Die Fachhochschulen in Deutschland, Bonn 1994.

Cavanaugh, S., Setting Objectives and Evaluating the Effectiveness of Trade Show Exhibits, in: JoM, Vol. 40, No. 4, 1976, S. 100-103. 
Chapman, E. A., Exhibit Marketing: A Survival Guide for Managers, New York u.a. 1987.

Christman, C., The Complete Handbook of Profitable Trade Show Exhibiting, Englewood Cliffs 1991.

Clausen, E., Mehr Erfolg auf Messen, Landsberg am Lech 1997.

Cunningham, M. T., White, J. G., The Role of Exhibitions in Industrial Marketing An Evaluation of the International Machine Tool Exhibition, in: IMM, Vol. 2, No. 3,1974, S. $238-251$.

Dawson, L. M., The human concept: New philosophy for business, in: BH, Vol. 12, No. 12,1969 , S. 29-38.

De Gijsel, P., Ziesemer, T., Hochschulreform in den Niederlanden in den achtziger und neunziger Jahren und ihre Implementierung, in: Grötzinger, G., Hödl, E. (Hrsg.), Hochschulen im Niedergang? Zur politischen Ökonomie von Lehre und Forschung, Marburg 1994, S. 99-125.

Deutsche Messe AG (Hrsg.), Das transparente Medium - Aussteller- und Besucher-Analysen Hannover Messe ‘94, Hannover 1994.

Deutsche Werbewissenschaftliche Gesellschaft e.V. (DWG) (Hrsg.), Messen als Marketinginstrument, Bonn 1983.

Diehl, J. M., Varianzanalyse, Frankfurt am Main 1977, S. 48 ff.

Dill, D., Sporn, B. (Ed.), Emerging Patterns of Social Demand and University Reform: Through a Glass Darkly, Oxford, New York, Tokyo 1995.

Dinkelbach, W., Ziele, Zielvariablen und Zielfunktionen, in: DBW, 38. Jg., Heft 1, 1978 , S. 51-58.

Dostal, G., Messen + Ausstellungen: So decken Sie Stärken und Schwächen Ihrer Messe-Beteiligung auf, in: Marketing Journal, 20. Jg., Heft 2, 1987, S. 140-144.

Doty, D. H., Glick, W., Typologies as a unique form of theory building: Towards improved understanding and modelling, in: AMR, No. 2, 1994, S. 230-251. 
Doyle, P., Gidengil, B. Z., A Review of In-Store Experiments, in: JoR, Vol. 53, No. 2, 1977, S. 47-62.

Doyle, P., Lynch, J. E., A Strategic Model for University Planning, in: Journal of the Operational Research Society, Vol. 30, No. 7, 1979, S. 603-609.

Doyle, P., Newbould, G. D., A Strategic Model for University Planning, in: Ryans, C., Shanklin, W. (Ed.), Strategic Planning, Marketing, Public Relations and Fund Raising in Higher Education, London 1986, S. 15-23.

Dudley, J. W., Successful Exhibiting, Holbrook 1991.

Dyllick, T., Management der Umweltbeziehungen - Öffentliche Auseinandersetzung als Herausforderung, Wiesbaden 1989.

Engelhardt, W. H., König, J., Nietiedt, T. (Hrsg.), Wissenschaftsmarketing, Hochschule und Region im Umbruch, Bochum 1993.

Erb, W. D., Differenzierung der wissenschaftlichen Hochschulen nach Größe, Alter und Angebotsstruktur, in: Giese, E. (Hrsg.), Aktuelle Beiträge zur Hochschulforschung, Giessener Geographische Schriften, Heft 62, Giessen 1987, S. 117-135.

Exner, W. F., Der Aussteller und die Ausstellungen - Erörterungen vor allem über die Messe von Wien 1873, Weimar 1873.

Fachverband Messe- und Ausstellungsbau e.V. (FAMAB) (Hrsg.), Jahresbericht `95.

Fahrmeir, L., Hamerle, A. (Hrsg.), Multivariate statistische Verfahren, Berlin, NY 1984.

Fahrmeir, L., Häußler, W., Tutz, G., Diskriminanzanalyse, in: Fahrmeir, L., Hamerle, A. (Hrsg.), Multivariate statistische Verfahren, Berlin, NY 1984, S. 301-327.

Fandel, G., Funktionalreform der Hochschulleitung, in: ZfB, 68. Jg., Heft 3, 1998, S. 241-257.

Fandel, G., Gal, T., Umverteilung der Mittel für Lehre und Forschung unter den Universitäten, Diskussionsbeiträge des Fachbereichs Wirtschaftswissenschaft der FernUniversität Hagen, Nr. 248, Dezember 1997. 
Fließ, S., Messeselektion - Entscheidungskriterien für Investitionsgüteranbieter, Wiesbaden 1994.

Földy, R., Die Messe als Markt - Beteiligungsstrategien für Aussteller, Wien 1990.

Forschungsland Nordrhein-Westfalen (Hrsg.), Messehandbuch, Aachen 1997.

Frackmann, E., Die Bedeutung von "Rankings" für den Wettbewerb im Hochschulbereich, in: Betriebswirtschaftliche Forschung und Praxis, 40. Jg., Heft 2, 1988, S. 139-162.

Frackmann, E., Informationsmanagement der Hochschule im Wettbewerb: Amerikanische Erfahrungen zu einer Betriebswirtschaftslehre der Hochschule, in: DBW, 47. Jg., Heft 6, 1987, S. 717-736.

Funke, K., Messeentscheidungen - Handlungsalternativen und Informationsbedarf, Frankfurt am Main u.a. 1987.

Funke, K., Ausstelleraktivitäten auf Messen: Eine kritische Analyse einschlägiger Darstellungen und Systematisierungsansätze in der Literatur, in: Arbeitsbericht Nr. 03/86 des Instituts für Wirtschaftswissenschaften der RWTH Aachen, Aachen 1986.

Gaugler, E., Hochschulen, in: Chmielewicz, K., Eichörn, P. (Hrsg.), Handwörterbuch der öffentlichen Betriebswirtschaft, Stuttgart 1989, Sp. 581-595.

Gelszus Messe-Marktforschung GmbH (Hrsg.), Mehr Erfolg auf Messen durch Messewirkungskontrolle, Dortmund 1994.

Gelszus Messe-Marktforschung GmbH (Hrsg.), Mehr Erfolg auf Messen - Wie Sie Ihren Erfolg messen und neue Potentiale entdecken, o.O., o.J.

Gelszus, R., Messen + Ausstellungen: Vergessen Sie die Messe-Wirkungs-Kontrolle nicht, in: Marketing Journal, 18. Jg., Heft 2, 1985, S. 172-177.

Gesellschaft zur freiwilligen Kontrolle von Messe- und Ausstellungszahlen (FKM) (Hrsg.), Bericht 1995, Köln 1996.

Gesellschaft zur freiwilligen Kontrolle von Messe- und Ausstellungszahlen (FKM) (Hrsg.), Satzung und Regeln, Köln 1994. 
Giese, E. (Hrsg.), Aktuelle Beiträge zur Hochschulforschung, Giessener Geographische Schriften, Heft 62, Giessen 1987.

Gluth, H., Keine schwarzen Messen - Erfolgskontrolle mit den Methoden der empirischen Sozialforschung, in: Beilage der FAZ, 23.01.1996, S. B7.

Goehrmann, K. E. (Hrsg.), Polit-Marketing auf Messen, Düsseldorf 1993.

Goehrmann, K. E., Natur- und ingenieurwissenschaftliche Hochschulinstitute sowie andere Forschungsstellen leisten wichtige Beiträge - Biotechnika: Horizonte rücken näher, in: HB, Nr. 182, 23.09.1987, S. 16.

Goehrmann, K. E., Die ungenutzten Instrumente der Wirtschafts-, Technologieund Strukturpolitik aktivieren - Messen können Technologie-Akzente gezielt setzen, indem sie Ergebnisse aus Forschungsprogrammen umsetzen helfen, in: HB, Nr. 249, 31.12.1985, S. 30.

Gopalakrishna, S., Lilien, G. L., Williams, J. D. et al., Do Trade Shows Pay off?, in JoM, Vol. 59, No. 7, 1995, S. 75-83.

Gopalakrishna S., Williams J., Identifying Important Factors in Lead Efficiency at Trade Shows, in: Trade Show Bureau (Ed.), Report No. SM 23, Denver 1993.

Goschmann, K., Die erfolgreiche Beteiligung an Messen und Ausstellungen von A-Z, Landsberg am Lech 1988.

Gräbener, W., Die Messepolitik als Marketinginstrument - dargestellt am Beispiel von Investitionsgüterproduzenten, Göttingen 1981.

Green, P. E., Krieger, A. M., Alternative Approaches to Cluster-Based Market Segmentation, in: JoMRS, Vol. 37, No. 3, 1995, S. 212-239.

Griffith, R. W., Management, 2. Aufl., Boston 1987.

Grob, H. L., Das Internet im Mittelpunkt einer computergestützten Hochschullehre (cHL), in: Hauff, M. (Hrsg.), media@uni-multi.media? - Entwicklung - Gestaltung - Evaluation neuer Medien, Münster u.a. 1998, S. 101-112.

Grob, H. L., Multimediale Lehre an der Universität, in: Forschungsjournal der Westfälischen Wilhelms-Universität Münster, 4. Jg., Heft 1, 1995, S. 38-42. 
Grob, H. L., CAL + CAT, Arbeitsbericht Nr. 2 der Reihe CAL + CAT des Lehrstuhls für Wirtschaftsinformatik und Controlling, Münster 1995.

Grob, H. L., Grießhaber, W., Computergestützte Lehre an der Universität, Arbeitsbericht Nr. 1 der Reihe CAL + CAT des Lehrstuhls für Wirtschaftsinformatik und Controlling, Münster 1995.

Groth, C., Das Leistungspotential von Fachmessen, in: Deutsche Werbewissenschaftliche Gesellschaft e.V. (DWG) (Hrsg.), Messen als Marketinginstrument, Bonn 1983, S. 57-71.

Groth, C., Die Wegeanalyse: Ein Instrument zur Verhaltensforschung bei Messeveranstaltungen und ähnlichen speziellen Märkten, in: GfK Mitteilungen zur Markt- und Absatzforschung, Heft 3, 1968, S. 87-90.

Groth, C., Lentz, I., Die Messe als Dreh- und Angelpunkt: Multifunktionales Instrument für erfolgreiches Marketing, Landsberg am Lech 1993.

Grötzinger, G., Hödl, E. (Hrsg.), Hochschulen im Niedergang? Zur politischen Ökonomie von Lehre und Forschung, Marburg 1994.

Gutenberg, E., Grundlagen der Betriebswirtschaftslehre, Bd. 1: Die Produktion, 24. Aufl., Berlin u.a. 1983.

Gutenberg, E., Die Unternehmung als Gegenstand betriebswirtschaftlicher Theoriebildung, Frankfurt am Main 1967.

Gutschke, J., Produktpräferenzanalyse: Ein modelltheoretisches und methodisches Konzept zur Marktsimulation mittels Präferenzerfassungsmodellen, Berlin 1995.

Gysin, R., Ansätze zur Universitätsplanung - dargestellt am Beispiel der Universität Basel, Basel 1973.

Haeberle, K. E., Erfolg auf Messen und Ausstellungen - Handbuch für Teilnahme, Organisation, Gestaltung, Technik, Stuttgart 1967.

Hagen, R., Erfolgskontrolle auf Verbrauchermessen - dargestellt am Beispiel der Analyse des Informationsstandes des Deutschen Bundestages, in: PuA, 10. Jg., Heft 7, 1983, S. 292-296.

Hammann, P., Erichson, B., Marktforschung, Stuttgart, New York 1978. 
Harpenau, J., Selbstdarstellung als Instrument im Wettbewerb zwischen Hochschulen: Ein Beitrag aus regionalwirtschaftlicher Sicht am Beispiel der Universität Hannover, Bern 1991.

Hartmann, H. (Hrsg.), Moderne Amerikanische Soziologie: Neuere Beiträge zur Soziologischen Theorie, 2. Aufl., Stuttgart 1973.

Head, J., Positioning, Objectives, and Selection Factors for Trade Shows, in: Trade Show Bureau (Ed.), Report No. MC24, Denver 1992.

Heinen, E., Einführung in die Betriebswirtschaftslehre, 9. Aufl., Wiesbaden 1985.

Heinen, E., Grundlagen betriebswirtschaftlicher Entscheidungen - Das Zielsystem der Unternehmung, 3. Aufl., Wiesbaden 1976.

Heinzel, M., Anforderungen deutscher Unternehmen an betriebswirtschaftliche Hochschulabsolventen - Zur Marktorientierung von Hochschulen, Wiesbaden 1997.

Helberger, C. (Hrsg.), Ökonomie der Hochschule I, Berlin 1989.

Hermeier, B., Konzept eines marketingorientierten Hochschulmanagement - theoretische Ansätze und empirische Befunde, Essen 1992.

Herrmann, A., Nachfrageorientierte Produktgestaltung: Ein Ansatz auf Basis der „means end"-Theorie, Wiesbaden 1996.

Herrmann, A., Wertorientierte Produkt- und Werbegestaltung, in: Marketing ZFP, 18. Jg., Heft 3, 1996, S. 153-163.

Hilgenberg, F., Was kommt aus der Gieskanne? Ansätze finanzieller Anreizsysteme in den Ländern, in: Forschung \& Lehre, 1. Jg., Heft 7, 1994, S. 261-262.

Hoche, K., Handbuch für Aussteller, 111 Messetips, München 1974.

Homans, G. C., Soziales Verhalten als Austausch, in: Hartmann, H. (Hrsg.), Moderne Amerikanische Soziologie: Neuere Beiträge zur Soziologischen Theorie, 2. Aufl., Stuttgart 1973, S. 245-263.

Hossinger, H.-P., Pretests in der Marktforschung - Die Validität von Pretestverfahren unter besonderer Berücksichtigung der Tachistoskopie, Würzburg, Wien 1982. 
Huckemann, M., ter Weiler, D. S., Messen Meßbar Machen - Die 5 trojanischen Pferde des Messe-Marketing, 2. Aufl., Neuwied u.a. 1998.

Janssen, J., Laatz, W., Statistische Datenanalyse mit SPSS für Windows, 3. Aufl., Berlin u.a. 1999.

Jaspersen, H., Messen beschicken oder Anzeigen schalten, in: Marketing Journal, 11. Jg., Heft 5, 1978, S. 456-462.

Jaspersen, H., Mehr Messe-Transparenz: Hüls, in: Marketing Journal, 20. Jg., Heft 2, 1977, S. 169-173.

Jaspert, F., Das synergetische Potential ist groß - die Messe als Teil der betrieblichen Absatzpolitik, in: Küffner, G., Mortsiefer, J. (Hrsg.), Messeplatz Europa: Messen als Bestandteil des betrieblichen Marketings, Frankfurt am Main 1990, S. 36-50.

Jaspert, F., Marketinginstrument Messe: Das synergetische Potential ist groß, in: BddW, Nr. 52, 1989, S. 7.

Josef, F., Effizienzsteigerung durch Messekontrolle: Wie man aus Fehlern lernen kann, in: MesseMarkt, o.Nr., Februar 1986, S. 56-57.

Kapferer, J.-N., Die Marke - Kapital der Unternehmung, Landsberg am Lech 1992.

Kayser, P., Typen von Messen und Formen der Messebeteiligung, in: Budach, W. et al. (Hrsg.), Hochschulen und Messen: Praktische Hinweise für Messebeteiligungen durch Hochschulen, Bad Honnef 1992, S. 49-65.

Kayser, P., Messebeschickung und Durchführung: Auswahl der Messe, in: Budach, W. et al. (Hrsg.), Hochschulen und Messen: Praktische Hinweise für Messebeteiligungen durch Hochschulen, Bad Honnef 1992, S. 67-75.

Keller, G., Academic Strategy: The Management Revolution of American Higher Education, Baltimore, London 1983.

Kinnebrock, W., Integriertes Event-Marketing: Vom Marketing-Erleben zum Erlebnis-Marketing, Wiesbaden 1993.

Kliche, M. (Hrsg.), Investitionsgütermarketing, Wiesbaden 1990. 
Kohle, G. U., Von der "Messe-Einladung“ zur Einladung mit Konzept, in: Marketing Journal, 24. Jg., Heft 5, 1991, S. 460-466.

Köhler, R. (Hrsg.), Empirische und handlungstheoretische Forschungskonzeptionen in der Betriebswirtschaftslehre, Stuttgart 1977.

Kook, W., Einstellungen zur Universität, Frankfurt, Berlin, Cirencester 1981.

Kortzfleisch, G. von (Hrsg.), Wissenschaftsprogramm und Ausbildungsziele der Betriebswirtschaftslehre, Berlin 1971.

Kosiol, E., Die Unternehmung als wirtschaftliches Aktionszentrum: Einführung in die Betriebswirtschaftslehre, Reinbek/Hamburg 1972.

Kotler, P., Andreasen, A. R., Marketing for Nonprofit Organisations, 5th Ed., New Jersey 1996.

Kotler, P., Fox, K. F. A., Strategic Marketing for Educational Institutions, 2nd Ed., New Jersey 1995.

Kotler, P., Levy, S. J., Broadening the concept of Marketing, in: Marketing Journal, Vol. 33, No. 1, 1969, S. 10-15.

Krebs, H., Messe als internationaler Marktplatz, in: Messen und Ausstellungen, Beilage der FAZ, Nr. 15, 19.01.1999, S. B3.

Kroeber-Riel, W., Konsumentenverhalten, 6. Aufl., München 1996.

Krueger, J., Messen und Ausstellungen: Die Kontakt-Kosten sichtbar machen, in: Marketing Journal, 17. Jg., Heft 5, 1984, S. 484-489.

Krug, W., Kosten - Finanzierung - Erfolg: Kosten, in: Budach, W. et al. (Hrsg.), Hochschulen und Messen: Praktische Hinweise für Messebeteiligungen durch Hochschulen, Bad Honnef 1992, S. 121-143.

Krug, W., Kosten - Finanzierung - Erfolg: Spannungsfeld Erwartungen - Realität, in: Budach, W. et al. (Hrsg.), Hochschulen und Messen: Praktische Hinweise für Messebeteiligungen durch Hochschulen, Bad Honnef 1992, S. 147-154.

Krumrey, H., Canibol, H. P., Studium für Maurer, in: Wirtschaftswoche, 46. Jg., Heft 18, 1992, S. 40-48. 
Küffner, G., Mortsiefer, J. (Hrsg.), Messeplatz Europa: Messen als Bestandteil des betrieblichen Marketings, Frankfurt am Main 1990.

Küng, P., Der Einsatz des Verkaufs an Messen, in: VM international, Heft 3, 1990, S. $45-49$.

Kustenaar, J., Messehandbuch - ein Leitfaden für Messebeteiligungen, Bern, Stuttgart 1983.

Lammert, N., Zwischen Lebenslügen und Überlebensstrategien - Vorschläge zur Reform des deutschen Hochschulwesens, in: Wirtschaftsdienst, 74. Jg., Heft 5, 1994, S. 223-226.

Leicher, R., Die Manöverkritik. Unerläßlicher Bestandteil der Messenacharbeit, in: Ausstellungs- und Messe-Ausschuss der Deutschen Wirtschaft e.V. (AUMA) (Hrsg.), Literatursammlung des AUMA, Köln o.J., S. 79.

Levy, S. J., Zaltman, G., Marketing, Society and Conflict, Englewood Cliffs 1975.

Liebl, F., Strategische Frühaufklärung, München 1996.

Lohmaier, P., Die Messe - Bedeutung und Problematik aus der Sicht der Aussteller, in: Arbeitspapier des Instituts für Absatzwirtschaft der Universität Augsburg Nr. 1/85, Augsburg 1985.

Meffert, H., Marketing - Grundlagen marktorientierter Unternehmensführung, 8. Aufl., Wiesbaden 1998.

Meffert, H., Herausforderungen an die Betriebswirtschaftslehre - Die Perspektive der Wissenschaft, in: DBW, 58. Jg., Heft 6, 1998, S. 709-730.

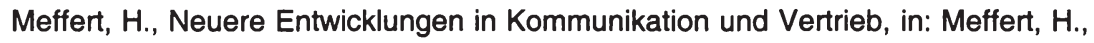
Necker, T., Sihler, H. (Hrsg.), Märkte im Dialog: Die Messen der dritten Generation, Leipzig 1997, S. 32-56.

Meffert, H., Einführung in die Themenstellung, in: Meffert, H., Wagner, H., Backhaus, K. (Hrsg.), Entwicklungsperspektiven der wirtschaftswissenschaftlichen Hochschulausbildung, Dokumentationspapier Nr. 102 der Wissenschaftlichen Gesellschaft für Marketing und Unternehmensführung e.V., Münster 1996, S. 1-5. 
Meffert, H., Messen und Ausstellungen als Marketinginstrument, in: Goehrmann, K. E. (Hrsg.), Polit-Marketing auf Messen, Düsseldorf 1993, S. 74-96.

Meffert, H., Marketingforschung und Käuferverhalten, 2. Aufl., Wiesbaden 1992.

Meffert, H., Einführung in die Problemstellung, in: Meffert, H., Wagner, H. (Hrsg.), Messemarketing - Bestandsaufnahme und Perspektiven, Dokumentationspapier Nr. 52 der Wissenschaftlichen Gesellschaft für Marketing und Unternehmensführung e.V., Münster 1989, S. 1-5.

Meffert, H., Messen und Ausstellungen als Marketinginstrument, in: Verlag Wirtschaft und Finanzen (Hrsg.), Messen als Marketinginstrument, Düsseldorf 1988, S. 7-30.

Meffert, H., Das System des Kommunikations-Mix, Münster 1979.

Meffert, H., Die Leistungsfähigkeit der entscheidungs- und systemorientierten Marketinglehre, in: Kortzfleisch, G. von (Hrsg.), Wissenschaftsprogramm und Ausbildungsziele der Betriebswirtschaftslehre, Berlin 1971, S. 167-187.

Meffert, H., Marketing, in: Management-Enzyklopädie, Bd. 4, München 1971, S. 383-413.

Meffert, H., Unternehmensziele, in: Schöttle, K. M. (Hrsg.), Jahrbuch des Marketing, Essen 1971, S. 22-34.

Meffert, H., Birkelbach, R., Frömbling, S., Die Westfälische Wilhelms-Universität Münster im Jahre 2010 - Bestandsaufnahme und Entwicklungsperspektiven, nicht veröffentlichtes Gutachten, Münster 1993.

Meffert, H., Bruhn, M., Dienstleistungsmarketing: Grundlagen - Konzepte - Methoden, 2. Aufl., Wiesbaden 1997.

Meffert, H., Burmann, C., Identitätsorientierte Markenführung - Grundlagen für das Management von Markenportfolios, in: Meffert, H., Wagner, H., Backhaus, K. (Hrsg.), Arbeitspapier Nr. 100 der Wissenschaftlichen Gesellschaft für Marketing und Unternehmensführung e.V., Münster 1996.

Meffert, H., Gass, C., Messen und Ausstellungen im System des Kommunikationsmix - ein entscheidungsorientierter Ansatz, Arbeitspapier Nr. 33 des Instituts für Marketing, Münster 1985. 
Meffert, H., Kirchgeorg, M., Marktorientiertes Umweltmanagement, 3. Aufl., Stuttgart 1998.

Meffert, H., Necker, T., Sihler, H. (Hrsg.), Märkte im Dialog: Die Messen der dritten Generation, Leipzig 1997.

Meffert, H., Robertz, G., Hauptstädte werden als Messeorte interessant, in: OWC, Heft 2, 1998, S. 22-26.

Meffert, H., Steffenhagen, H., Marketingprognosemodelle - Quantitative Modelle des Marketing, Stuttgart 1977.

Meffert, H., Ueding, R., Ziele und Nutzen von Messebeteiligungen, in: Ausstellungs- und Messeausschuss der Deutschen Wirtschaft e.V. (AUMA) (Hrsg.), AUMA Edition Nr. 4, Bergisch Gladbach 1996.

Meffert, H., Wagner, H. (Hrsg.), Messemarketing - Bestandsaufnahme und Perspektiven, Dokumentationspapier Nr. 52 der Wissenschaftlichen Gesellschaft für Marketing und Unternehmensführung e.V., Münster 1989.

Meffert, H., Wagner, H., Backhaus, K. (Hrsg.), Entwicklungsperspektiven der wirtschaftswissenschaftlichen Hochschulausbildung, Dokumentationspapier Nr. 102 der Wissenschaftlichen Gesellschaft für Marketing und Unternehmensführung e.V., Münster 1996.

Meier, H.-G., Technologietransfer via Messen und Ausstellungen am Beispiel des Forschungsmarktes Berlin, in: TU International, 28./29. Mai 1995, S. 4-5.

Meier, H.-G., Kosten - Finanzierung - Erfolg: Finanzierung, in: Budach, W. et al. (Hrsg.), Hochschulen und Messen: Praktische Hinweise für Messebeteiligungen durch Hochschulen, Bad Honnef 1992, S. 144-147.

Mellerowicz, K., Kontrolle in der Absatzwirtschaft, in: Tietz, B. (Hrsg.), HWAHandwörterbuch der Absatzwirtschaft, Stuttgart 1974, Sp. 1104-1116.

Merbold, C., Strukturerhebungen noch die Ausnahme - Transparenz läßt weiter sehr zu wünschen übrig, in: HB, Nr. 235, 07.12.1983, S. 17.

Möncke, A. F., Studenten im Wintersemester 1991/92, in: Wirtschaft und Statistik, 44. Jg., Heft 4, 1992, S. 245-251. 
Mortsiefer, J., Die Vorbereitung entscheidet über den Erfolg - Planung und Vorbereitung einer Messebeteiligung, in: Küffner, G., Mortsiefer J. (Hrsg.), Messeplatz Europa: Messen als Bestandteil des betrieblichen Marketings, Frankfurt am Main 1990, S. 51-61.

Mortsiefer, J., Informationen sammeln und auswerten - Die Abwicklung einer Messebeteiligung, in: Küffner, G., Mortsiefer, J. (Hrsg.), Messeplatz Europa: Messen als Bestandteil des betrieblichen Marketings, Frankfurt am Main 1990, S. $62-72$.

Mortsiefer, J., Messen und Ausstellungen als Mittel der Absatzpolitik mittelständischer Herstellerbetriebe, Göttingen 1986.

Mösch, B., Studierende im Wintersemester 1995/96, in: Wirtschaft und Statistik, 48. Jg., Heft 6, 1996, S. 375-382.

Müller, K., Die Messepolitik im Marketing der handwerklichen Zulieferer, Göttingen 1985.

Naumann, C., Erfolgreich auf der Messe: Handbuch für die Beteiligung an Messen und Ausstellungen, Heidelberg 1993.

Naumann, C., Die Arbeit nach der Arbeit - Messe-Nachbereitung und Erfolgskontrolle, in: Küffner, G., Mortsiefer, J. (Hrsg.), Messeplatz Europa: Messen als Bestandteil des betrieblichen Marketings, Frankfurt am Main 1990, S. 73-86.

Naumann, C., Ist es eine Messe wert? - Den Messeerfolg organisieren und kontrollieren, in: BddW, Nr. 170, 06.09.1982, S. 7.

Naumann, C., Was war die letzte Messe wert? - Kontaktwertmessung nach Punkten, in: m+a report, 55. Jg., Heft 1, 1974, S. 15-17.

Necker, T., Globale Herausforderung, Dienstleistungs- und Informationszeitalter, in: Meffert, H., Necker, T., Sihler, H. (Hrsg.), Märkte im Dialog: Die Messen der dritten Generation, Leipzig 1997, S. 18-31.

Nickel, H., Event-Marketing: Grundlagen und Erfolgsbeispiele, München 1998.

Nietiedt, T., Kommunikationspolitik für Hochschulen: Analyse und Instrumentarium, Frankfurt am Main 1996. 
Norius, M. J., SPSS für Windows: Anwenderhandbuch für das Base System 6.0, München 1994.

Norius, M. J., SPSS Inc., SPSS for Windows: Advanced Statistics, Release 5, Chicago, III. 1992.

Norius, M. J., SPSS Inc., SPSS for Windows: Professional Statistics, Release 5, Chicago, III. 1992.

Nowotny, H., Mass Higher Education and Social Mobility: A Tenuous Link, in: Dill, D., Sporn, B. (Ed.), Emerging Patterns of Social Demand and University Reform: Through a Glass Darkly, Oxford, New York, Tokyo 1995, S. 72-89.

o.V., Zwischen Erkenntnis und Vermarktung, in: Wirtschaftsspiegel, 53. Jg., Heft 8, 1998, S. 10-13.

o.V., Mitarbeiterschulung - Hymne in der Halle: Unternehmen bereiten sich mit ungewöhnlichen Methoden auf den Messeauftritt vor, in: Wirtschaftswoche, 49. Jg., Heft 1/2, 1995, S. 70-72.

o.V., Wirkungskontrollen für Messebeteiligungen, in: Fachverband Messe- und Ausstellungsbau e.V. (FAMAB) (Hrsg.), Jahresbericht`95, S. 21.

o.V., Hochschulfinanzen: Krisenmanagement, in: Informationsdienst der deutschen Wirtschaft, 20. Jg., Heft 18, 1994, S. 4-5.

o.V., Erfolgskontrolle - Umstrittene Methode, in: FAMA intern, Heft 2, 1985, S. 12.

Olson, J. C., Theoretical Foundations of Means-End Chains, Working Paper 174, Pennsylvania State University, Pittsburgh, PA 1988.

Olson, J. C., Reynolds, T. J., Understanding Consumer Cognitive Structures: Implications for Advertising Strategy, in: Percy, L., Woodside, A. (Ed.), Advertising and Consumer Psychology, Lexington, MA 1983, S. 77-90.

Peisert, H., Framhein, G., Das Hochschulsystem in Deutschland, hrsg. vom Bundesministerium für Bildung, Wissenschaft, Forschung und Technologie, 2. Aufl., Bonn 1997.

Percy, L., Woodside, A. (Ed.), Advertising and Consumer Psychology, Lexington, MA 1983. 
Perrey, J., Nutzenorientierte Marktsegmentierung: Ein integrativer Ansatz zum Zielgruppenmarketing im Verkehrsdienstleistungsbereich, Wiesbaden 1998.

Pidun, R., Messen und Ausstellungen: Messe-Promotion - einfach faszinierend und glaubwürdig, in: Marketing Journal, 23. Jg., Heft 1, 1990, S. 70-71.

Prüser, S., Messemarketing: Ein netzwerkorientierter Ansatz, Wiesbaden 1997.

Raffée, H., Integrierte Kommunikation, in: Werbeforschung und Praxis, 36. Jg., Heft 3, 1991, S. 87-90.

Raffée, H., Messen als Herausforderung für die Marketing-Theorie, in: Deutsche Werbewissenschaftliche Gesellschaft e.V. (DWG) (Hrsg.), Messen als Marketinginstrument, Bonn 1983, S. 73-95.

Raffée, H., Fritz, W., Wiedmann, K.-P., Marketing für öffentliche Betriebe, Stuttgart, Berlin, Köln 1994.

Rehorn, J., Markttests, Neuwied 1977.

Rehorn, J., Labortestmarkt und Minitestmarkt - Alternativen zum Prägen von Marktchancen, in: asw, 20. Jg., Heft 9, 1977, S. 51-60.

Reuther, K. H., Die betriebswirtschaftliche Bedeutung der Messen und Ausstellungen für die Konsumgüterindustrie, München 1956.

Rice, G., Using the Interaction Approach to Understand International Trade Shows, in: IMR, Vol. 9, No. 4, 1992, S. 32-45.

Robertz, G., Strategisches Messemanagement im Wettbewerb - ein Beitrag aus markt-, ressourcen- und koalitionsorientierter Sicht, Wiesbaden 1999.

Roloff, E., Messen und Medien: Ein sozialpsychologischer Ansatz der Öffentlichkeitsarbeit, Wiesbaden 1992.

Rosson, P., Seringhaus, F. H. R., Reaching Export Objectives through International Trade Fairs, in: Trade Show Bureau (Ed.), Report No. IN 6, Denver 1991, S. 2.

Rost, D., Messetransparenz - ein Imperativ, in: asw, 27. Jg., Heft 8, 1984, S. 30-32. 
Rost, D., Strothmann, K.-H. (Hrsg.), Handbuch Werbung für Investitionsgüter, Wiesbaden 1983.

Rost, D., Messen als Kommunikations-Problem, in: Deutsche Werbewissenschaftliche Gesellschaft e.V. (DWG) (Hrsg.), Messen als Marketinginstrument, Bonn 1983, S. 35-54.

Rost, D., Maßstäbe zur Beurteilung der Effizienz von Messen, in: VDI-Nachrichten, Nr. 115, 1973, S. 333-338.

Roth, G. D., Messen und Ausstellungen verkaufswirksam planen und durchführen, Landsberg am Lech 1981.

Ryans, C., Shanklin, W. (Ed.), Strategic Planning, Marketing, Public Relations and Fund Raising in Higher Education, London 1986.

Sashi, C. M., Perretty, J., Do Trade Shows Provide Value?, in: IMM, Vol. 21, No. 3, 1993, S. 249-255.

Scheitlin, V., Messebeteiligung erfolgreich gestalten, St. Gallen, Stuttgart, Steyr 1984.

Schmalen, H., Wagner, A., Empirische Untersuchung zur Messebeteiligung von Unternehmen, Arbeitspapier, o.Nr., Passau 1990.

Schmitz, U., Messepolitik eines Unternehmens der Investitionsgüterindustrie, in: Strothmann, K.-H., Busche, M. (Hrsg.), Handbuch Messemarketing, Wiesbaden 1992, S. 383-396.

Schober, K., Grundlagen des Fakultätsmarketing, Arbeitspapier Nr. 56 des Lehrstuhls für Marketing, WiSo-Fakultät Nürnberg, Erlangen-Nürnberg 1998.

Schober, P. M., Messen + Ausstellungen: Teilnehmen oder wegbleiben? - Eine kleine Entscheidungs-Hilfe, in: Marketing Journal, 21. Jg., Heft 4, 1988, S. $400-402$.

Schönemann, K., Messen und Kataloge: Die Sache mit den Katalogen, in: Der Fremdenverkehr, Heft 2, 1989, S. 16.

Schoop, K., Sandt, B., Die Messeerfolgskontrolle, in: Tietz, B., Die Werbung Handbuch der Kommunikations- und Werbewirtschaft, Bd. 2, Landsberg am Lech 1982, S. 1664-1679. 
Schöttle, K. M. (Hrsg.), Jahrbuch des Marketing, Essen 1971.

Schubö, W. et al., SPSS: Handbuch der Programmversionen 4.0 und SPSS-X 3.0, Stuttgart, New York 1991.

Schwenzner, J. M., Messen und Ausstellungen im Kreuzfeuer der Begriffe Reichweitenanalyse und Messeerfolgskontrolle, in: GfK-Mitteilungen zur Markt- und Absatzforschung, 18. Jg., Heft 1, 1972, S. 10-20.

Schwenzner, J. M., Andersen, E., Der Messe-Test, in: Wagner, K. (Hrsg.), Allgemeines Statistisches Archiv, Bd. 39, München 1955, S. 25-30.

Seitel, K., Erlebniswelt schaffen, in: Direkt Marketing, 32. Jg., Heft 5, 1996, S. 14-16.

Selinski, H., Begleitveranstaltungen von Messen, in: Strothmann, K.-H., Busche, M. (Hrsg.), Handbuch Messemarketing, Wiesbaden 1992, S. 485-499.

Selinski, H., Fair Play in Europa, in: Kliche, M. (Hrsg.), Investitionsgütermarketing, Wiesbaden 1990, S. 169-190.

Selinski, H., Messe- und Kongreßforschung (Teil II), in: PuA, 11. Jg., Heft 5, 1984, S. 212-215.

Selinski, H., Messe- und Kongreßmarketing, Berlin 1983.

Selinski, H., Sperling U. A., Marketinginstrument Messe: Arbeitsbuch für Studium und Praxis, Köln 1995.

Simon, H., Kucher, E., Akademischer Wettbewerb, in: asw, 31. Jg., Heft 31, 1988, S. 144-147.

Siskind, B., The Successful Exhibitor's Handbook: Trade Show Techniques for Beginners and Pros, 2. Ed., Vancouver, Bellingham 1994.

Sönke, A., Optimale Allokation von Hochschul-Budgets, in: DBW, 59. Jg., Heft 5, 1999, S. 583-598.

Spiegel-Verlag (Hrsg.), Messen und Messebesucher in Deutschland, Hamburg 1992. 
Spryß, W. M., Für Standmitarbeiter gibt es viele Stolpersteine, in: Beilage der FAZ, Nr. 20, 25.01.1994, S. 8.

Spryß, W. M., Messen + Ausstellungen: Mit dem "Messe-Diagramm" vorbereiten von den Zielen bis zum Controlling, in: Marketing Journal, 20. Jg., Heft 4, 1987, S. $368-370$.

Spryß, W. M., Nerb, G., Reuter, J., Messeziele - Messeeffizienz, Münster-Sarmsheim 1994.

Statistisches Bundesamt (Hrsg.), Hochschulen, in: Internet, http://www.statistikbund.de/basis/d/biwiku/hochtxt.htm, Stand: 03.12.1999.

Steger, U., Winter, M., Strategische Früherkennung zur Antizipation ökologisch motivierter Marktveränderungen, in: DBW, 56. Jg., Heft 5, 1996, S. 607-629.

Steinhausen, D., Langer, K., Clusteranalyse: Einführung in Methoden und Verfahren der automatischen Klassifikation, Berlin, New York 1977.

Steinmann, H., Schreyögg, G., Management: Grundlagen der Unternehmensführung - Konzepte, Funktionen, Praxisfälle, Wiesbaden 1990.

Stiege, J., Messebeteiligungen sollen gründlich bewertet werden, in: BddW, 24.04.1995, S. 1.

Stieger, H., Zur Ökonomie der Hochschule, Gießen 1980.

Stracke, F., Messebeschickung und Durchführung: Exponatauswahl, in: Budach, W. et al. (Hrsg.), Hochschulen und Messen: Praktische Hinweise für Messebeteiligungen durch Hochschulen, Bad Honnef 1992, S. 78-86.

Stracke, F., Messebeschickung und Durchführung: Präsentationsformen, in: Budach, W. et al. (Hrsg.), Hochschulen und Messen: Praktische Hinweise für Messebeteiligungen durch Hochschulen, Bad Honnef 1992, S. 87-92.

Stracke, F., Messebeschickung und Durchführung: Standbesatzung/Personelle Präsentation, in: Budach, W. et al. (Hrsg.), Hochschulen und Messen: Praktische Hinweise für Messebeteiligungen durch Hochschulen, Bad Honnef 1992, S. 94-97. 
Stracke, F., Messebeschickung und Durchführung: Begleitende Pressearbeit, in: Budach, W. et al. (Hrsg.), Hochschulen und Messen: Praktische Hinweise für Messebeteiligungen durch Hochschulen, Bad Honnef 1992, S. 98-103.

Stracke, F., Messebeschickung und Durchführung: Nachbereitung, in: Budach, W. et al. (Hrsg.), Hochschulen und Messen: Praktische Hinweise für Messebeteiligungen durch Hochschulen, Bad Honnef 1992, S. 104-106.

Stracke, F., Personelle Organisation der Messezuständigkeit, in: Budach, W. et al. (Hrsg.), Hochschulen und Messen: Praktische Hinweise für Messebeteiligungen durch Hochschulen, Bad Honnef 1992, S. 111-119.

Strothmann, K.-H., Im Verhalten unterscheiden sich Messe-Besucher beachtlich, in: Marketing Journal, 25. Jg., Heft 2, 1992, S. 164-169.

Strothmann, K.-H., Segmentorientierte Messepolitik, in: Strothmann, K.-H., Busche, M. (Hrsg.), Handbuch Messemarketing, Wiesbaden 1992, S. 99-114.

Strothmann, K.-H., Suchen Sie das Gespräch mit dem Standbesucher, in: Marketing Journal, 22. Jg., Heft 4, 1989, S. 388-389.

Strothmann, K.-H., Verbundveranstaltungen des Messe- und Kongresswesens im Investitionsgüter-Marketing, in: Rost, D., Strothmann, K.-H. (Hrsg.), Handbuch Werbung für Investitionsgüter, Wiesbaden 1983, S. 391-404.

Strothmann, K.-H., Messeforschung, in: Deutsche Werbewissenschaftliche Gesellschaft e.V. (DWG) (Hrsg.), Messen als Marketinginstrument, Bonn 1983, S. 97-107.

Strothmann, K.-H., Busche, M. (Hrsg.), Handbuch Messemarketing, Wiesbaden 1992.

Strothmann, K.-H., Roloff, E., Charakterisierung und Arten von Messen, in: Berndt, R., Hermanns, A. (Hrsg.), Handbuch Marketing-Kommunikation: Strategien - Instrumente - Perspektiven, Wiesbaden 1993, S. 707-723.

Taeger, M., Messemarketing - Marketingmix von Messegesellschaften unter Berücksichtigung wettbewerbspolitischer Rahmenbedingungen, Göttingen 1993. 
Täger, U. C., Ziegler, R., Die Bedeutung von Messen und Ausstellungen in der Bundesrepublik Deutschland für den Inlands- und Auslandsabsatz in ausgewählten Branchen, in: ifo-Institut für Wirtschaftsforschung e.V. (Hrsg.), ifoStudien zu Handels- und Dienstleistungsfragen, Nr. 25, München 1984.

Tanner, J. F., Chonko, L. B., Trade Show Objectives, Management, and Staffing Practices, in: IMM, Vol. 24, No. 4, 1995, S. 257-264.

Tauberer, A., Wartenberg, W., Serviceleistungen von Messegesellschaften, in: Strothmann, K.-H., Busche, M. (Hrsg.), Handbuch Messemarketing, Wiesbaden 1992, S. 235-247.

Tavenier, K., Marketing-Management in Universitäten, in: Engelhardt, W. H., König, J., Nietiedt, T. (Hrsg.), Wissenschaftsmarketing, Hochschule und Region im Umbruch, Bochum 1993, S. 111-124.

Tavenier, K., Strategisches Management in Universitäten, in: Bertelsmann Stiftung (Hrsg.), Evolution im Hochschulbereich, Gütersloh 1990, S. 55-71.

Tietz, B., Die Werbung - Handbuch der Kommunikations- und Werbewirtschaft, Bd. 2, Landsberg am Lech 1982.

Tietz, B. (Hrsg.), HWA-Handwörterbuch der Absatzwirtschaft, Stuttgart 1974.

Tietz, B., Bildung und Verwendung von Typen in der Betriebswirtschaftslehre, dargestellt am Beispiel der Typologie der Messen und Ausstellungen, Köln, Opladen 1960.

Tietz, B., Köhler, K., Zentes, J. (Hrsg.), Handwörterbuch des Marketing, Stuttgart 1995.

Tolman, E., Purpose Behaviour in Animals and Men, New York 1932.

Topf, C., Öffentlichkeitsarbeit im Rahmen des Hochschulmarketing - Interne und externe Informations- und Kommunikationsbeziehungen der Hochschulen, Frankfurt am Main 1986.

Trogele, U., Strategisches Marketing für deutsche Universitäten: Die Anwendung von Marketing-Konzepten amerikanischer Hochschulen in deutschen Universitäten, Frankfurt am Main u.a. 1995. 
Turner, G., Mangelnder Ansporn, in: Wirtschaftswoche, 46. Jg., Heft 42, 1992, S. 74.

Ueding, R., Management von Messebeteiligungen: Identifikation und Erklärung messespezifischer Grundhaltungen auf der Basis einer empirischen Untersuchung, Frankfurt am Main u.a. 1998.

Verlag Wirtschaft und Finanzen (Hrsg.), Messen als Marketinginstrument, Düsseldorf 1988.

Wagner, G., Innovatives Messe-Team-Training, in: Beilage der FAZ, Nr. 20, 25.01.1994, S. 5.

Wagner, K. (Hrsg.), Allgemeines Statistisches Archiv, Bd. 39, München 1955.

Wangen-Goss, M., Marketing für Universitäten: Möglichkeiten und Grenzen der Übertragbarkeit des Marketing-Gedankens auf den universitären Bereich, Spardorf 1983.

Weber, J., Hochschulcontrolling: Das Modell der WHU, Stuttgart 1996.

Wedel, P. Graf von, Messen - vom Markt zum Marketing, Frankfurt 1977.

Weintraub, D. K., Trade Show Exhibiting: The Insider's Guide for Entrepreneurs, o.O. 1991.

Weishäupl, G., Die Messe als Kommunikationsmedium - unter besonderer Berücksichtigung der Öffentlichkeitsarbeit und Werbung einer Messegesellschaft, München 1980.

Wenge, H. U., Müller, A., Das Management der Messe-Beteiligungen, in: Berndt, R., Hermanns, A. (Hrsg.), Handbuch Marketing-Kommunikation: Strategien Instrumente - Perspektiven, Wiesbaden 1993, S. 725-745.

Winnen, R., Beuster, A., Kontrolle des Messeerfolgs, in: Strothmann, K.-H., Busche, M. (Hrsg.), Handbuch Messemarketing, Wiesbaden 1992, S. 365-378.

Wissenschaftliche Gesellschaft für Marketing und Unternehmensführung e.V. (Hrsg.), Memorandum zur wirtschaftswissenschaftlichen Ausbildung in Deutschland, in: Meffert, H., Wagner, H., Backhaus, K. (Hrsg.), Entwicklungsperspektiven der wirtschaftswissenschaftlichen Hochschulausbildung, Doku- 
mentationspapier Nr. 102 der Wissenschaftlichen Gesellschaft für Marketing und Unternehmensführung e.V., Münster 1996, S. 57-58.

Wissenschaftsrat (Hrsg.), Empfehlungen zum Wettbewerb im deutschen Hochschulsystem, Köln 1985.

Wissenschaftsrat (Hrsg.), Empfehlungen zur Organisation, Planung und Förderung der Forschung, Bonn 1975.

Witt, F. H., Theorietradition der Betriebswirtschaftlichen Forschung, Wiesbaden 1995.

Wöllenstein, S., Betriebstypenprofilierung in vertikalen Vertriebssystemen: Eine Analyse von Einflußfaktoren und Erfolgswirkungen auf der Grundlage eines Vertragshändlersystems im Automobilhandel, Frankfurt am Main 1996.

Zahner, W., Directmail - Sein Einsatz im Marketing, Bern 1991.

Zerres, R., Ausstellen und Gewinnen: Der Kompass für die erfolgreiche Messepraxis, Woldert 1992.

Ziegele, F., Hochschule und Finanzautonomie: Grundlagen und Anwendung einer politisch-ökonomischen Theorie der Hochschule, 2. Aufl., Frankfurt am Main u.a. 1998.

Ziegler, R., Messen - ein makroökonomisches System, in: Strothmann, K.-H., Busche, M. (Hrsg.), Handbuch Messemarketing, Wiesbaden 1992, S. 115-126.

Zotter, R., Erfolgreiches Messemarketing: Leitfaden für Analyse, Planung, Durchführung, Nachbearbeitung, Erfolgskontrolle bei Messebeteiligungen im In- und Ausland, Zürich 1993.

Zurlino, F., Zukunftsorientierung von Industrieunternehmen durch strategische Früherkennung, München, Wien 1995. 


\section{SCHRIFTEN ZUM MARKETING}

Band 1 Friedrich Wehrle: Strategische Marketingplanung in Warenhäusern. Anwendung der Portfolio-Methode. 1981. 2. Auflage. 1984.

Band 2 Jürgen Althans: Die Übertragbarkeit von Werbekonzeptionen auf internationale Märkte. Analyse und Exploration auf der Grundlage einer Befragung bei europaweit tätigen Werbeagenturen. 1982 .

Band 3 Günter Kimmeskamp: Die Rollenbeurteilung von Handelsvertretungen. Eine empirische Untersuchung zur Einschätzung des Dienstleistungsangebotes durch Industrie und Handel. 1982.

Band 4 Manfred Bruhn: Konsumentenzufriedenheit und Beschwerden. Erklärungsansätze und Ergebnisse einer empirischen Untersuchung in ausgewählten Konsumbereichen. 1982.

Band 5 Heribert Meffert (Hrsg.): Kundendienst-Management. Entwicklungsstand und Entscheidungsprobleme der Kundendienstpolitik. 1982.

Band 6 Ralf Becker: Die Beurteilung von Handelsvertretern und Reisenden durch Hersteller und Kunden. Eine empirische Untersuchung zum Vergleich der Funktionen und Leistungen. 1982.

Band 7 Gerd Schnetkamp: Einstellungen und Involvement als Bestimmungsfaktoren des sozialen Verhaltens. Eine empirische Analyse am Beispiel der Organspendebereitschaft in der Bundesrepublik Deutschland. 1982.

Band 8 Stephan Bentz: Kennzahlensysteme zur Erfolgskontrolle des Verkaufs und der MarketingLogistik. Entwicklung und Anwendung in der Konsumgüterindustrie. 1983.

Band 9 Jan Honsel: Das Kaufverhalten im Antiquitätenmarkt. Eine empirische Analyse der Kaufmotive, ihrer Bestimmungsfaktoren und Verhaltenswirkungen. 1984.

\section{SCHRIFTEN ZU MARKETING UND MANAGEMENT}

Band 10 Matthias Krups: Marketing innovativer Dienstleistungen am Beispiel elektronischer Wirtschaftsinformationsdienste. 1985.

Band 11 Bernd Faehsler: Emotionale Grundhaltungen als Einflußfaktoren des Käuferverhaltens. Eine empirische Analyse der Beziehungen zwischen emotionalen Grundhaltungen und ausgewählten Konsumstrukturen. 1986.

Band 12 Ernst-Otto Thiesing: Strategische Marketingplanung in filialisierten Universalbanken. Integrierte Filial- und Kundengruppenstrategien auf der Grundlage erfolgsbeeinflussender Schlüsselfaktoren. 1986.

Band 13 Rainer Landwehr: Standardisierung der internationalen Werbeplanung. Eine Untersuchung der Prozeßstandardisierung am Beispiel der Werbebudgetierung im Automobilmarkt. 1988.

Band 14 Paul-Josef Patt: Strategische Erfolgsfaktoren im Einzelhandel. Eine empirische Analyse am Beispiel des Bekleidungsfachhandels. 1988. 2. Auflage. 1990.

Band 15 Elisabeth Tolle: Der Einfluß ablenkender Tätigkeiten auf die Werbewirkung. Bestimmungsfaktoren der Art und Höhe von Ablenkungseffekten bei Rundfunkspots. 1988.

Band 16 Hanns Ostmeier: Ökologieorientierte Produktinnovationen. Eine empirische Analyse unter besonderer Berücksichtigung ihrer Erfolgseinschätzung. 1990.

Band 17 Bernd Büker: Qualitätsbeurteilung investiver Dienstleistungen. Operationalisierungsansätze an einem empirischen Beispiel zentraler EDV-Dienste. 1991.

Band 18 Kerstin Ch. Monhemius: Umweltbewußtes Kaufverhalten von Konsumenten. Ein Beitrag zur Operationalisierung, Erklärung und Typologie des Verhaltens in der Kaufsituation. 1993. 
Band 19 Uwe Schürmann: Erfolgsfaktoren der Werbung im Produktlebenszyklus. Ein Beitrag zur Werbewirkungsforschung. 1993.

Band 20 Ralf Birkelbach: Qualitätsmanagement in Dienstleistungscentern. Konzeption und typenspezifische Ausgestaltung unter besonderer Berücksichtigung von Verkehrsflughäfen. 1993.

Band 21 Simone Frömbling. Zielgruppenmarketing im Fremdenverkehr von Regionen. Ein Beitrag zur Marktsegmentierung auf der Grundlage von Werten, Motiven und Einstellungen. 1993.

Band 22 Marcus Poggenpohl: Verbundanalyse im Einzelhandel auf der Grundlage von Kundenkarteninformationen. Eine empirische Untersuchung von Verbundbeziehungen zwischen Abteilungen. 1994.

Band 23 Kai Bauche: Segmentierung von Kundendienstleistungen auf investiven Märkten. Dargestellt am Beispiel von Personal Computern. 1994.

Band 24 Ewald Werthmöller: Räumliche Identität als Aufgabenfeld des Städte- und Regionenmarketing. Ein Beitrag zur Fundierung des Placemarketing. 1995.

Band 25 Nicolaus Müller: Marketingstrategien in High-Tech-Märkten. Typologisierung, Ausgestaltungsformen und Einflußfaktoren auf der Grundlage strategischer Gruppen. 1995.

Band 26 Nicolaus Henke: Wettbewerbsvorteile durch Integration von Geschäftsaktivitäten. Ein zeitablaufbezogener wettbewerbsstrategischer Analyseansatz unter besonderer Berücksichtigung des Einsatzes von Kommunikations- und Informationssystemen (KIS). 1995.

Band 27 Kai Laakmann: Value-Added Services als Profilierungsinstrument im Wettbewerb. Analyse, Generierung und Bewertung. 1995.

Band 28 Stephan Wöllenstein: Betriebstypenprofilierung in vertraglichen Vertriebssystemen. Eine Analyse von Einflußfaktoren und Erfolgswirkungen auf der Grundlage eines Vertragshändlersystems im Automobilhandel. 1996.

Band 29 Michael Szeliga: Push und Pull in der Markenpolitik. Ein Beitrag zur modellgestützten Marketingplanung am Beispiel des Reifenmarktes. 1996.

Band 30 Hans-Ulrich Schröder: Globales Produktmanagement. Eine empirische Analyse des Instrumenteeinsatzes in ausgewählten Branchen der Konsumgüterindustrie. 1996.

Band 31 Peter Lensker: Planung und Implementierung standardisierter vs. differenzierter Sortimentsstrategien in Filialbetrieben des Einzelhandels. 1996.

Band 32 Michael H. Ceyp: Ökologieorientierte Profilierung im vertikalen Marketing. Dargestellt am Beispiel der Elektrobranche. 1996.

Band 33 Mark Unger: Die Automobil-Kaufentscheidung. Ein theoretischer Erklärungsansatz und seine empirische Überprüfung. 1998.

Band 34 Ralf Ueding: Management von Messebeteiligungen. Identifikation und Erklärung messespezifischer Grundhaltungen auf der Basis einer empirischen Untersuchung. 1998.

Band 35 Andreas Siefke: Zufriedenheit mit Dienstleistungen. Ein phasenorientierter Ansatz zur Operationalisierung und Erklärung der Kundenzufriedenheit im Verkehrsbereich auf empirischer Basis. 1998.

Band 36 Irene Giesen-Netzer: Implementierung von Rücknahme- und Recyclingsystemen bei Gebrauchsgütern. 1998.

Band 37 Frithjof Netzer: Strategische Allianzen im Luftverkehr. Nachfragerorientierte Problemfelder ihrer Gestaltung. 1999.

Band 38 Silvia Danne: Messebeteiligungen von Hochschulen. Ziele und Erfolgskontrolle. 2000. 